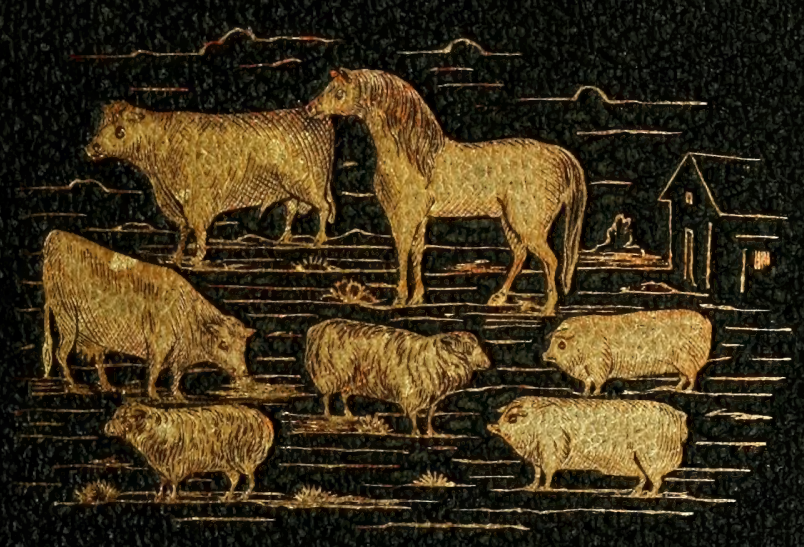




\section{LIBRARY OF CONGRESS.

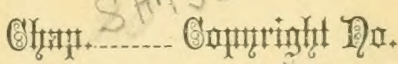 Shelf.L 28 \\ UNITED STATES OF AMERICA.}




. 


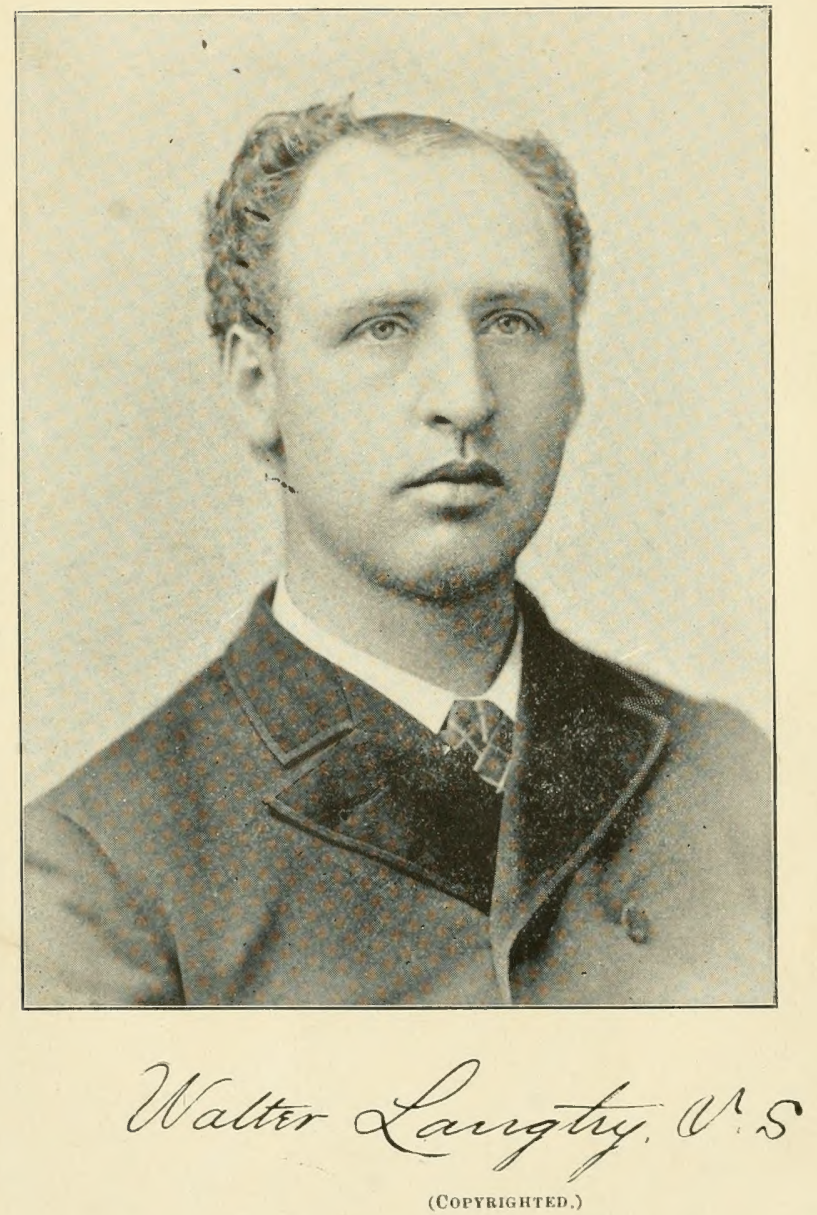




\title{
HOME TREATMENT
}

FOR

\author{
THE DISEASES
}

OF

\section{DOMESTICATED ANIMALS}

\section{A PRACTICAL WORK}

FOR

FARMERS, TEAUSTERS, MERCHANTS, MECHANICS, STUDENTS AND PRACTITIONERS.

A TREATISE ON THE DISEAJES OF HORSES, CATTLE, SHEEP, HOGS AND DOGS. THEIR CAUSES, SYMPTOMS, PREVENTION, AND CURE. ALSO THE LATEST AND MOST APPKOVED METHODS OF DELIVERING THEIR YOUNG.

FULLY ILLUSTRATED.
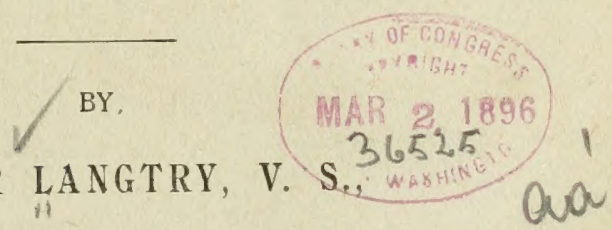

GRADUATE OF THE ONTARIO VETERINARY COLLEGE, TORONTO, CANADA. 
Copyrighted

by

WALTER LANGTRY, V. S.

FORT WAYNE, IND.

1895.

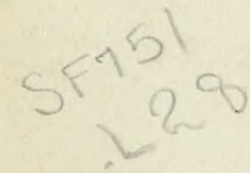




\section{NOTICE.}

For the diffusion of knowledge, all classes of literature should be placed at a very nominal price, so as to be within the reach of every one, and more especially should this be the case with medical works, so that persons may not only inform themselves, but render valuable assistance in all cases of emergency, as well as treat successfully those minor cases where medical skill would not be called upon. All such books should be written very plainly in the English language, carefully avoiding all technical terms, so that any person may read and understand what he is doing. This I have earnestly endeavored to do, and have placed the price at an extremely low figure, being satisfied with a small profit and depending upon a large sale, to recompense me for my time and trouble. The price being so low and the profit so small, book stores cannot afford to handle them. They will, therefore, be sold only through agents. Any person desiring this work after the agent has called, can procure a copy by writing to my address :

WALTER LANGTRY, V. S.,

Fort Wayne, INdiANa,

U. S. A. 


\section{PREFACE,}

In presenting this work to the general public, it is not to be presumed that many will take the time to read the preface, for only about one in a thousand receive close attention. But if the reader will take the time to give it a careful perusal, it will doubly repay him for his trouble. It is not my intention nor desire, to make every purchaser of this work a thorough veterinarian, but to supply the busy and industrious American, who is the owner or lover of domesticated animals, with a concise and comprehensive treatise of the diseases of live stock, according to the modern or advanced system of treatment, discarding the old, vulgar and incoupatible messes that have formerly been prescribed, and the poor, dumb brute has been compelled, through foree and ignorance, to endure that which it could not avoid. The advanced and successful theory and practice among the lower animals, so closely resembles that of the human family, that what is considered humane treatment for the one, also applies to the other, and I most respectfully invite you to ask your family physician to carefully peruse these pages, and obtain his opinion as to the correctness of treatment, and value to your library. It seems remarkably strange, from the number of books that are daily printed, that thr wants of the industrious 
farmer and owner of live stock, have been neglected in this line, and not supplied with a practical work, whereby he could not only avoid, but successfully and intelligently administer remedies and heal the wounds of his afflicted stock. The loss to the stock owner, amounts to an enormous sum annually, not only through diseases but during the period that they are producing their offspring, for a want of knowledge in assisting them at that critical time; and after an extensive practice in this line, for a period of eighteen years, and fully appreciating the desire of the stock-owner for a knowledge of this kind, I have endeavored to present it to them in this work as plainly as it is possible under the circumstances, for me to do in the English language, and if it will somewhat assist in mitigating some of the pain and misery, which the females are compelled to ondure, the author will greatly appreciate the fact and will consider himself amply repaid for his time and trouble. 


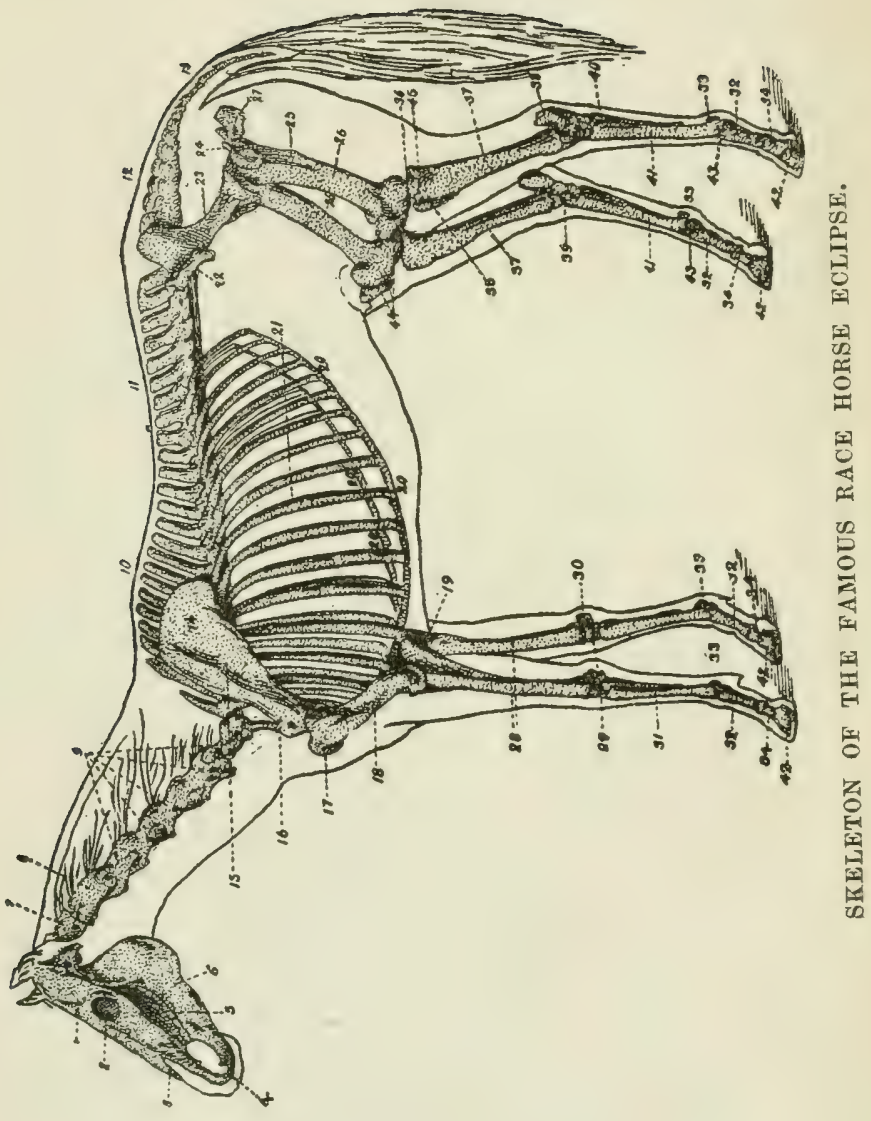




\section{EXPLANATION OF THE DIFFERENT PARTS OF THE SKELETON.}

1. Zygomatic arch.

2 Orbital cavity.

3. Face bone.

4. Incisor teeth.

5. Molar teeth.

6. Lower jaw.

7. Atlas, 1st vertebræ of neck.

8. Axis, 2d vertebrio of neck.

9. Cervical vertebræ.

10. Spinal process of back.

11. Dorsal and lumbar vertebre.

12. Sacrum.

13. Coccygeal or tail bones.

14. Scapula, or shoulder blade.

15. Acromion process.

16. Hollow of shoulder blade.

17. Superior tuberosity of the humerus.

18. Humerus, or arm bone.

19. Olecranon, or elbow bone.

20. Cartilages of the ribs.

21. Ribs.

22. Haunch, the external and anterior angle of the illium.

33. Os innominatum, or haunch bone.
24. Great trochanter.

25. Small trochanter.

26. Femur, or thigh bone.

27. Ischium, posterior angle of the 1llium.

28. Radius, or for $\theta$-arm bone.

29. Carpal, or knee bones.

30. Trapezium.

31. Metacarpal, or cannon bone.

32. Os suffraginis, or pastern bone.

33. Sesamoid bone.

34. Os coronæ, or small pastern bone.

35. Superior tuberosity of the tibia.

36. Stifle joint.

37. Tibia, or leg bone.

38. Os calcis, or point of hock.

39. Tarsus, or hock joint.

40. Head of small metatarsal bone.

41. Cannon, or metatarsal bone.

42. Hoof, or foot bone.

43. Fetlock joint.

44. Patella.

45. Fibula. 



\section{INTRODUCTORY,}

Since an ounce of prevention is worth a pound of cure, it will be well first to consider such rules of treatment and of diet, as will tend to maintain wood health in animals. The physical construction must be regarded, and the fact impressed upon the mind that they do not consist entirely of hair and hide and a few external organs, simply because such alone are visible; these but serve as an outer guard for a delicate, intricate and beautiful mechanism within. The wonderful work of digestion begins as soon as the food is taken into the mouth, for there it is ground by the teeth and mingled with the saliva; through the proper channels it reaches the stomach, and then enters the intestines. When dimested, the nutritive portions of the food are conveyed to the blood by means of absorbent ressels, whence occurs a process of purification, in which all waste particles are cast aside and the rich, life-giving fluid is propelled by the heart, through many hlood-vessels, to the various portions of the system, to supply worn-out tissues. The body is not interspersed with blood-channels alone, but there is also a tracery of nerves extending to every extremity, commonly called the nerrous system, and having two great centers; the spinal cord with which all lesser nerves connect, and the crowning splendor of animal existence, the brain, to which, in turn, the spinal cord is attached. There is also a structure of bones, termed the skeleton, to which cling in proper position, folds or layers of flesh, called muscles. The 
vast and marvelous mechanism of animal anatomy, is in itself a study, and since it can here be but briefly considered, it will be better to proceed at once to the consideration of the principles of hycriene and sanitation, and of proper nursing and medical treatment, than to dwell longer upon the art of dissection, We will therefore first regard such rules of diet and management, as will be conducive to the maintenance of health.

WATER.

Dysentery, diarrhera and many other diseases of stock, are very often occusioned by drinking water containing deconposing oranic matter, such as may sometimes be found in wells and ponds, situated near manure-heaps and cess-pools. It is important then, that water be free from impurities. When animals have. free access to water, they never drink to excess. Green food, owing to the juice it contains, lessens the quantity of water reyuired. When fed upon dry hay and grain, the thirst is necessarily greater.

Water from shallow wells, and river water, which is usually contaminated with sewage, is dangerous, while water from springs and deep wells, and upland surface water, is considered wholesome. Surface-water from land that has been cultivated, and stored rain-water, may be regarded with suspicion. Pure water can generally be determined by its bluish tint, its clearness, and lack of taste or smell, but the presence of certain poisons, bacteria, etc., can be detected only by microscopic and chemical examination conducted by an expert in this line. When at rest, the horse requires water at least three times a day; but when laboring, more frequently. The rule, under the latter circumstance, should be to give in small quantities and often. When a horse is warm, he may be allowed a few swal- 
lows of water and should then immediately be given a pound or two of hay. He should then rest about an hour before feeding. The danger in giving water to a warm or over-heated animal, is due to the excessive amount it will take while in this condition. Horses should not be allowed ice cold water, and while hot water need not necessarily be added, it should be seen to that it does not verge upon the freezing point. While it is not a good idea to give water thus cold, yet serious trouble is seldom produced, if the horse has not been deprived for too long a time. The same rule will apply to cattle, although they are not so susceptible to unpleasant effects from this source as the horse, having four stomachs while the horse has but one.

\section{FOODS AND FEEDING.}

Cleanliness should be considered both in preparing and administering food. The method of preparation, found by experience to be the best, should be adhered to and regular times of feeding should be observed. Oats do not pass from the stomach so quickly as hay and straw and should therefore be given after either of the latter, as they cannot then be forced by the hay or straw, which is more rapidly digested, into the intestines, before the stomach has performed its part in the work of digestion. When the horse is hungry or exceedingly tired, this manner of feeding is particularly commendable, since it requires more time to masticate hay, insuring a proper mixture with it of the saliva, and it cannot be bolted as are oats or other grains. - Water should not be given after feeding, as it washes the food from the stomach before it is prepared to enter digestion in the intestines. Before feeding then, is the proper time to give water. The selection of food should depend upon the nature of the labor required of the 
horse. Severe or hurried work cannot be expected of an animal with a full stomach. When labor of this nature is required, it is best that food be allowed in small quantity, about two hours before going to work. Even slow-working animals should not be gorged with bulky food lacking in nourishing properties, immediately before being put to labor. Grains should never be fed immediately after a hard day's work, as digestive disorders are apt to arise. Hay should first be given, with grains following. The same rule should be observed in feeding an animal that has been for any reason, deprived of food for sometime; also in this case, a less quantity should be given than would be consumed. If too much food is allowed, colic is apt to follow. For horses engaged mostly in slow work, chopped or cut hay, fed with crushed oats, ground corn, etc., is desirable. Diet should not be changed suddenly, as this is dangerous, but the desired change should be accomplished very gradually. Neither should there be an immediate increase in the quantity of food, if this is desired, but the amount required should be reached by dewrees. The quantity of food must be governed by the amount of labor to be performed. If the work is decreased or there is an entire cessation from toil for a few days, the food should be lessened. If the horse is to stand for sometime, the food should be of a laxative nature. Musty or moldy foods should not be fed, since from these frequently arise various diseases, such as bronchitis, lung trouble and heaves. Kidney disorders are apt to arise from the consuming of musty hay.

HAY.

Timothy, about a year old, fresh, clean, and of a greenish color, and possessing a pleasant odor, is the best hay for horses. If kept too long, however, it be- 
comes deficient in nourishment, hard, dry and more or less difficult of digestion. New hay also, is hard to digest, sometimes producing purging, and irritation of the skin. It should be mixed with old hay, if given at all. Second crop hay is not good for horses, but is considered by some persons, as good food for milch cows; it being claimed that it has a tendency to increase the flow of milk. Ten to twelve pounds of hay a day, is sufficient for the average lorse, when grain is also fed. Horses engaged in light work, should not be kept entirely upon hay, as they will fall off in flesh and become pot-bellied. This also applies to colts; unless the latter are allowed some grain in addition to their hay, they are apt to grow into lean, awkward creatures, never making as good horses, as others fed upon both kinds of food.

\section{STRAWS.}

When straws are used as an article of food, they should be chopped and mixed with crushed or ground graius, and hay. The straws mostly used, are those of rye, oats and wheat. Oats straw contains the greatest amount of nourishment and is the most easily digested. Bean and pea straws are sometimes fed to horses, and of these two, the pea straw appears to be preferable.

\section{CHAFF.}

When oat chaff is mixed with cut hay or corn fodder, it may be used as an article of food, but only in small quantities, as abundant feeding frequently produces a troublesome and sometimes fatal diarrhoea, in both horses and cattle.

Rye and wheat chaff should not be used. More or less trouble is occasioned by the beards becoming lodged in the mouth and throat, while in the bowels and stomach, they often form the basis of soft concretions. 
GRAINS.

Oats are the most easily digested, and contain the best proportion of nourishment. They are therefore preferable to all other grains. The best oats are a year old, clean, hard, short and plump. New oats are indigestible. Kiln-dried oats, though originally good, are not desirable, since this method of drying, injures them. Sprouted or fermented oats should never be fed, as they are injurious. For old horses and those having poor teeth, oats are best when crushed; in most instances, however, they may be fed whole. Crushed oats are also best for horses addicted to the habit of bolting their food, and in such cases, it is well to scatter the grain before them, or spread it in a thin layer. With the amount of hay already mentioned, the average horse will require about twelve quarts of oats daily. Moldy oats should not be fed, as they will give rise to very serious disturbances, often terminating fatally.

\section{WHEAT AND RYE.}

These grains should be given as food, in small quantities only, and should be crushed and mixed with hay or other grains. Digestive disorders, founder and similar troubles are produced in horses, if these grains are given alone, in any considerable quantities. Whon used as an article of diet, they should invariably be ground or crushed, and the amount given, should not be more than one-fourth of the quantity of grain allowed.

\section{BRAN.}

Wheat-bran is mostly used, when bran is desired. Various estimates are placed upon its value. It is best fed with other grain; it bestows freedom of action upon the bowels, and sour bran, therefore, should not be given, as it is productive of stomach and intestinal disorders. 


\section{MAIZE-CORN.}

This grain should not be used exclusively as food for young horses, as it is lacking in salts. It may be fed either whole or ground. For horses affected with lampas, it is generally given upon the cob, but if it is old corn and it is desired to give it in this manner, it should be soaked in clean water for some ten to twelve hours. Corn is better given in the ground form, from one to two quarts at a feeding, mixed with wheat-bran or crushed oats. A full feed of corn given to a horse unaccustomed to its use, is likely to give rise to acute indigestion. For this reason, small quantities should be given at first, and gradually increased until the lesired amount is reached.

\section{LINSEFD.}

When it is desired to keep the bowels open and improve the condition of the skin, ground linseed may occasionally be given with other articles of diet. When the digestive organs are inflamed or irritable, tea made of linseed is very beneficial.

\section{POTATOES-CARROTS-BEETS.}

Potatoes used as food for horses or cattle, are best steamed or boiled. If given in considerable quantities in the raw state, they are apt to produce indigestion. Like most other roots, they possess a small proportion of laxative properties. Beets are not used often as food for horses, and should not be used too liberally as food for cattle. Carrots, when fed in small quantities, are very beneficial and particularly so in sickness, as they improve the appetite, and to a slight extent, increase the action of the kidneys and bowels. They also contain alterative properties and when used as an article of diet the coat becomes glossy and smooth. 
GRASS.

If at all possible, every horse should receive a summer vacation, which he may spend in some good pasturage. This not only affords him his natural food, but gives him a brief season for rest and recuperation. Grass is indispensible to growing stock. It is not sufficient in itsef, as food for an animal engaged in labor, as purging results, and horses soon tire in travel or work. They also perspire easily. It should at first be given in small quantities to horses accustomed to grain and hay, but the best idea, is to let the animal crop it for itself. Chronic disorders sometimes disappear entirely, when the animal is upon grass; it also has a tendency to lessen fever, and wounds heal with greater rapidity than when upon grain. Last but not least, is the fact that an occasional change of diet, goes far toward maintaining a good condition in animals. It is not a good idea to continne the same routine of feeding, for this becomes tiresome, as it would with onrselves, were we subjected to a certain rule of diet. An occasional change is appreciated by animals, and consequently followed with good results.

\section{STABLING。}

Stalls should be large and roomy. Box stalls are best, for these allow such complete freedom from halter or strap, that the animal may choose any position desired, for comfort and rest. It is bad policy to economize in stable-room, by building narrow, uncomfortable stalls. There should at least be room for the animal to stretch out its limbs, so that it need not lie in a cramped position. A ground floor is the best, but upon floors of any kind, should be placed a good bedding. For this, clean straw is preferable. Ventilation should not be disregarded, but in order to secure this, it 
is not necessary that a flood of cold or damp, chilly air be allowed to rush upon the animal. In this, as in other matters, judgment must be used. Above all things, cleanliness must be observed, and there should be plenty of light. Food should be placed so that it can be reached without discomfort. Screen or netting properly arranged, aflords grood protection from flies. Floors of any kind should be level; if a board floor is used, there should be some means of drainage.

\section{HARNESS.}

This should be adjusted to the form of the horse, in as comfortable a manner as possible. Rubbing should be guarded against, particularly the friction of the collar upon the shoulders. The cruelty of tight checkreining cannot be too severely condemned. It not only cramps the horse into a painful position, but is senseless in the extreme, being a detriment to the natural beauty of the animal and a hindrance to him while at work.

\section{MUTILATIONS.}

The inhuman fashion of docking has happily almost entirely disappeared, as has also the slashing of ears. The mane, tail and fore-top, appear well if evenly trimmed, but there is no beauty in a stubby tail, a closely hacked mane and a raring fore-top; the latter should be either short enough to escape the eyes, or be carefully tucked away beneath some portion of the head gear. As to the recent style of clipping, it certainly does not seem reasonable to subject the horse to the chilling air of winter, deprived of his natural protection. The animal suffers as much as would his master, were he compelled to remain out any length of time, without a coat.

It may do well enough in mid-summer, or as soon as the weather becomes settled and warm. Nature, how- 
ever, has made ample provision, by granting a shedding or thinning of the hasr. at the proper time. But with all due care and consideration, animals will sicken sometimes from causes over which we have no control; it is the way of all tlesh, and when ailments occur, we must seek to administer in the best and most practical manner, the most efficient remedies.

\section{BATHS.}

For the cure of disease and likewise for the preservation of health, baths are important. When falling in temperature below $70^{\circ}$ Fahrenheit they may be termed cold baths: sucli, when used with discretion, ex ert tonic, stimulating or bracing effects. They contract the blood-vessels lying close to the skin and increase the excretion of waste particles. Cold baths should not bo too long continued, esperially when applied to young or weakly animals, as umlesirable and eren injurious results may ensue. A sea bath is better than fresh water at rest. By drying thoroughly, by hand-rubbing or blanketing, reaction is encouraged, and it may sometimes be well to give stimulants. If a lower temperature than $70^{\circ}$ Fahrenheit is required, the animal may be placed in a bath of that degree of temperature which may then be lowered to $60^{\circ}$ or lower; or may at once be placed in a bath, ranging from $60^{\circ}$ to $50^{\circ}$, or have cold water dashed orer it. Ten or fifteen minutes will suffice, in such cases, and the treatment may be given twice in a day. By this method, high fever may he reduced from $1^{\circ}$ to $4^{\circ}$.

TEPID BATHS.

From $65^{\circ}$ to $85^{\circ} \mathrm{Fahx}$, is the proper range for a tepid bath. They are henedual to heated and tired animals, producing a sense of comfort. Perspiration is encourawol, the skin is cleansel, and they also, 
more or less, allay thirst; likewise they are beneficial in removing stiffness, resulting sometimes after a hard day's labor.

WARM BATHS.

From $85^{\circ}$ to $97^{\circ}$ Fahr. is the proper range of temperature for baths of this kind. They are servicable in relieving chronic skin diseases and softening the skin. By their application, blood-vessels under-lying the skin are expanded and perspiration is promoted.

HOT BATHS.

The temperature in baths of this kind ranges upward from $97^{\circ}$ Fahr. As high as $110^{\circ}$ may be reached if increased by degrees. By their use, the temperature of the body is increased, the capillaries or blood-vessels of the skin are expanded, the perspiration and pulse are accelerated, and excretion of waste matter is hastenc d They are soothing to over-tixed muscles, useful in relieving colic and cramps, check such disorders as rheumatism, catarrh and weed attacks, and are of benefit in chronic affections of the skin.

\section{MEDICATION.}

Baths may be medicated. To stimulate the skin, salt or mustard may be added. Drugs of various kinds are used for the destruction of parasites of the skin and also for chronic skin diseases. In the latter instance, alkaline carbonates or potassium sulpheret; for the destruction of parasites, carbolic acid, tobacco juice, solution of arsenious acid may be used.

Not many stables, however, afford the convenient and elegant appliances facilitating the administering of baths. The next best method, and the one usually employed, is sponging with tepid, cold or hot water, as the case may require. Satisfactory results are thus obtained. In treatment of fever, the water should not at 
first, be of temperature lower than $85^{\circ}$ or $80^{\circ}$ Fahr. The sponging process should not last longer than three or four minutes; the animal should then be wiped dry and at once covered with a blanket. The sponging may be repeated within three or four hours, if necessary, especially should the temperature again have risen. After the first or second sponøing, the temperature of the water may be lowered to $65^{\circ}$ or $60^{\circ}$ Fahr. Sometimes a little acid added to tha bath is of benefit, and concurrent with this treatment, stimulants or antiseptic salines may be prescribed.

\section{FOMENTATIONS.}

In order to be of benefit, fomentations should be continued for several hours. Usually, water alone is used, but laudanum, vinegar, saline, and other remedies, suitable to the ailment, are sometimes added. Fomentations are usually hot, though cold applications are sometimes used. They are generally applied to bruises, inflammations, such as in kidney disease, or in diseases of the lungs as pneumonia and pleurisy; to sprains, wounds, or inflamed eyes. The temperature must of course be regulated to the nature of the disorder, and the portion of the body where it is situated. When applied to the eye, the degree of heat should not go beyoud $100^{\circ}$ Fahr. For bruises, external inflammations and disturbances of similar character, the water may be as hot as can be borne by the hand, and for pleurisy and other internal inflammatory affections, horse-cloths or pieces of flannel should be soaked with boiling water, partially dried, and spread over the surface around and above the affected parts and covered with a piece of rubber or any other material that will prevent evaporation. For the lesser ailments, a sponge or rag, a thannel rag is preferable, may be used, with 
some covering to retain the moisture. It is not advisable to use a sponge where there is any putrid or foul discharge, as it is apt to harbor disease germs, whereas, if a rag is used, it is usually burned, or destroyed in some other manner. Probably the most speedy and convenient method, is to wring a sheep-skin out of the water, and place the wooly side to the diseased part.

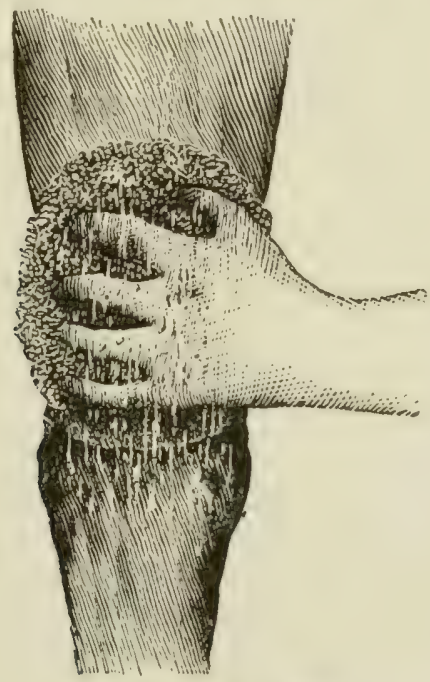

Fomenting a Bruise.

The temperature of the application should be kept as even as possible, and when the fomenting operation is completed, the affected portions should be nicely dried and wrapped, so as to prevent chilling. Mustard is sometimes applied to assist in sustaining the temperature, though this is apt to be somewhat painful to open, sensitive wounds. For this purpose, also alcohol or a mild stimulating liniment may be used. Guard against irritation, in the treatment of a raw sore or an open wound, as this will have a tendency to delay the healing process. 


\section{POULTICES.}

Boiled linseed-meal, oat meal, bran, bread, turnips, and dried hops where a light poultice is desired, are some of the substances used for this manner of moist application. Poultices may be either hot or cold; cold poultices are prepared in the same manner as are hot applications, and then allowed to cool. Hot poultices, however, are mostly used. They may be laid directly upon the wound, or affected part, or a very thin covering as a piece of old, thin muslin, may first be spread upon the part to be treated. This tends to prevent sticking, and for this purpose also, a light coating of wlycerine solution or vaseline, may be used. They should be remored every hour or two, and fresh ones supplied, and this changing should be accomplished with rapidity, so as to prevent chilling. Poultices encourage the excretion of pus and therefore should not be applied to fresh or to dry wounds where there is no suppuration. They should not be continued for toolong a time, as the result will be a ton great relaxing or softening of the affected parts. When the operation is 'ompleted, care should be taken to prevent chilling by "nveloping in flannel or some other suitable material. When heat is desired without moisture, hot salt or sand in bags, may be applied. Poultices are sometimes medicated. Opiates may be added to produce a soothing effect; a sprinkling of turpentine or mustard will encourage stimulation, and an antisentic tendency is produced by adding carbolic acid, yeast, charcoal or chlorinated soda.

\section{POWDERS.}

When not of too large a size powders may be dropped upon the tongue of the patient. The most satisfactory manner of administering, is to mix with some soft form of food, provided the flavor is not so disagreeable that the 
animal will refuse it. Most all medicines may be obtained in the form of powders, and while their action is not quite as quick as in liquid form, they are just as effective, and it kept in close stoppered bottles, will retain and impart their active principles for a great length of time.

\section{PLASTERS.}

In reterinary practice, plasters are not as a rule, a convenient method of treatment, owing to the fact that an animal usually shows a disposition to bite or rub them ofl, and thus there is danger of additional injury to the portion already affected. They are useful in affording protection and retarding eraporation, increasing artivity of the glands, and when applied in a thick form, tend to hinder motion of the diseased parts. When they are to remain for some length of time, the ingredients melted to softness, may be poured directly unon the skin, over-spread with ravelled lint or tow and wer the whole, a linen or leather bandige should be placed. Applications of this kind, are known as charees. For stimulation, mustard or cantharides may be added to the other ingredients, but if a soothing effect is desired, add opium or belladonna.

\section{IOTIONS.}

Fluid preparations intenclad for the hathing of external parts, are termed lotions. Various drugs are rised, with water or other fluids or mixtures of fiuids, as solvents. They are of benefit in swellings, sore joints and muscles, infiamed eyes, skin diseases and other external disorders. They may also be applied for stimulating effects upon internal organs. Besides pouring, or rubbing as in the application of liniments, lotions may 1.t applied by means of linen saturater? with the preparation and laid upon the part to be treated. 
OINTMENTS.

Lard, butter, or other fatty matters, form the basis of ointments. Taseline is very desirable since it does not become rancid and in itself possesses soothing and healing qualities. To the oily consistence employed, such drugs are to be added as are to be used in treating the ailment, and after being well mixed, the ointment is, as a general thing, ready for use. This manner of treatment is desirable for healin sores, skin disorders, and since the ingredients are capable of being absorbed by the skin, for introducing medicines, in certain instances, into the system. In order to facilitate absorption, lanolin or oleic acid is in the proper instances, used as a basis. When wax or resin is employed, it must be slowly melted and the other ingredients adder, and the mass should be stirred until well mixed and until it is of the proper consistence.

\section{VAPORS.}

For disinfecting the air-passages, destroying parasites therein, for soothing or stimulating, inhalations are sometimes used with good effect. This manner of treatment is beneficial in many ailments of the throat and breathing orans, as catarrh, sore throat, bronchial congestion. For these latter affections, vapor produced from pure water alone, or to which has been added a little chloroform, laudanum, belladonna, or whatever elie is designated for the ailment, often affords prompt relief. A hot bran-mash contained in a good sized nose bag, is a convenient method of producing steam, as also a bucket containing hot water, placed beneath the nostrils, the bucket and head of the animal being covered with sacking, or the covering may be omitted and a hot iron plunged into the water at suitahle intervals. For disinfection, and for irritability of the organs of breath- 
ing, fumes and gases from various drugs are sometimes employed, but these will be treated upon elsewhere. The practice of smoking with old shoes, rubber, wool, etc., is to be severely condemned as injurious, inhuman,

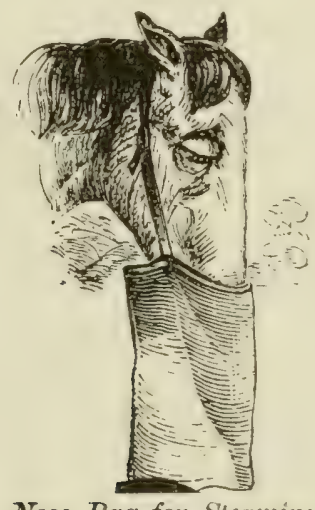

Nose Bag for Sleaminy.

and utterly senseless. To a human being suffering with throat or lung affections, this would be not only obnoxjous, but very prolific of injurious effects. It is none the less so to the fettered animal, unable to speak or offer any other sign of protest, and it must therefore stand patiently and endure, or if this becomes impossible, succumb to the torment.

INJECTIONS.

Liquid introduced into the rectum, whether pure water alone, or water with the addition of some medicinal element, or whether of some other fluid form, constitutes an injection. The object in administering, is to relax the bowels, for which it is safe and effectual, to maintain and hasten the action of purgatives, to cleanse of worms which may infest the posterior portion of the canal, and may be used with soothing as well as stimulating effect, influeneing both adjacent and remote 
organs. This is also a means of introducing medicines into the system, when there is difficulty in swallowing, as in sore throat, lock-jaw, or other affections producing a similar condition; they are not so rapidly absorbed by this method however, as when administered in the natural manner. This treatment is effective in constipation or in obstinate torpidity. In the horse, the hardened fecal matter may first be removed by the hand or spatula and the injection may then follow. From one and a half to five gallons of tepid water, may be injected into the rectum of a draught-horse, while a pint is sulicient for a dog weighing forty pounds. In this as in all else, judgment must be used, and the amount regulated to the nature of the disorder and species of animal. 'T'wo or three pints is a sufficiency for the ox or horse, when intended for retention and alsorption; three or four times this amomnt is required when evacuittion is desire?, and larser quantities in olstinate cases. By the addition to tepid water, of soap-castile is preferable, or raw linseed oil, the effect is increased. An injection composed of four ounces of turpentine and a quart of raw linseed oil, is effective in removing worms. The apparatus for giving injections are of various kinds, of which we will designate the barrel syringe amil the ball syinge as the most common. There is also Prof. Gamgee's funnel; a sort of rectangular arrangement, into the top or cup of which the liquid may be poured, and it will then follow the tube and enter the rectum. This method would no doubt be the most agreeable to the animal, as it does not necessitate the unpleasant pumping or forcing usually required to operate a syringe. The portion of the instrument introduced into the rectum, should be well greased with oil or lard and entered in a slow and careful manner, to prevent any unnecessary irritation to the parts. 


\section{SUPPOSITORIES.}

By combining with soap, cocoa-butter or lard, and wielding into a round form resembling a ball or cylinder, certain drugs may be introduced into the rectum, vagina and uterus. A suppository of soap placed in the anus, encourages acton of the bowels in young foals and calves.

\section{BOLUSES.}

Some medicines are conveniently arministered in the form of a bolus or pill. The remedy to be given, is mixed with a mass of sufficient consistency to allow molding into a round form: the cylindrical shape is better than the absolutely round ball, and it may measure

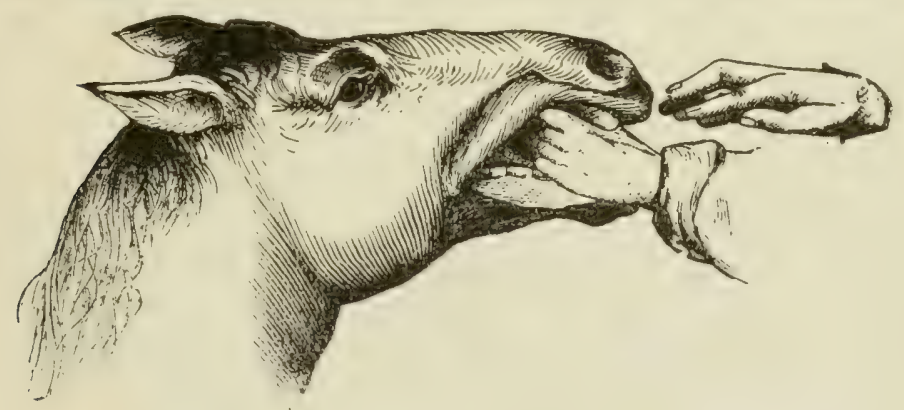

Giving " Pill

from a half to three-fourths of an inch in thickness, and two inches in length. There are a number of articles with which the medicine may be mixed and wielded into the proper form, but lard or linseed meal can probably be the most readily ohtained. In both mixing and administering, cleanliness should be observed and the balls must not be allowed to harden, as in this condition, they sometimes pass away from the animal without having been changed and absorbed. Wranping in oiled paper will preserve moisture, or fresh mes may be made as required. 
The bolus is usually given with the fingers, and the operation is simple. The ball is held by one end between the thumb (which supports it helow) and the fingers of the right hand, which is drawn together and rounded as much as possible. The tongue of the patient is gently lrawn out a little way by the left hand, and the ball passed rapidly along the roof of the mouth, and dropped on the back of the tongue. The hand must be quickly withdrawn and the hold upon the tongue at once released. Close the mouth, and slightly elevate the head which may be released as soon as the ball is swallowed. The operation should be performed in as rapid and careful a manner as possible and with a firm, steady hand, for a nervous doctor usually makes a nervous patient; neither should the animal be struck or abused. When, on account of viciousness, the ball cannot readily be administered by the hand, it is best to use a balling iron. This device is also conveniently used when the mouth is narrow, or when by reason of some affection, it cannot be sufficiently opened.

\section{DRENCHES.}

Fluid preparations given in a single good-sized dose, are termed drenches. When the flavor is not disagreeable, the animal may usually be induced to drink it mixed with its drinking water or with gruel. From two to six ounces, according to the size of the animal, is an average amount for dogs; one or two pints for horses, six to eight ounces for sheep, and for an ox one to two quarts. The mixture should be well shaken, so that the ingredients will be thoroughly mixed. In administering to a horse, the head should be raised to a moderate height, and the tongue secured so as to prevent the throwing out of the liquid, yet allowing sufficient action of jaws, lips, tongue and cheeks to permit swallowing. 
The tongue should not be drawn outward in a forcible manner, as in this position, it cannot assist in forcing lack the fluid, and furthermore encourages the danger of rhoking. To secure the head by means of ropes and pulleys fastened above, is an objectionable method; a letter way is to hold the head in position by means of a twitch, the noose of which is placed over the upper jaw within the incisor teeth, and an assistant, standing on the left side of the animal may raise and sustain the head by means of a stick attached to the noose, or the noose inay be slipped between the prongs of a stable fork. The hest method howerer, is to dispense with all fetters and entanglements, and use the hands alone, as first described. This requires lut one person, and is guickly and easily accomplished, at the same time allowing the most comfort and freedom to the animal. Drenching through the nostrils is not only unnatural, hut is attended with danger of death, as the fluid may enter either the channel to the lungs or the passage to the stomach, or both. The nostrils are to admit air, not food and drink or other matters foreign to them. The mouth has this office to perform. If, in administering a drench to an animal, it is seized with a fit of coughing, release it at once, as there is danger of choking. In administering to cattle, grasp firmly but not roughly by the nostrils, with the thumb and two fingers, in such a manner that there will be no interference with the breathing, and raise the head to a moderate height. The drench may then be given with the other hand. Aid is rendered in some instances, if an assistant grasps the horns and steadies the head. Large dogs may be backed into a corner, so that they cannot pull away, and the head held between the knees. Glass bottles are usually used to contain the fluid, but if a properly shaped tin vessel can be obtained, it will be all the bet- 
ter, as there is danger that the glass bottle may has broken, the contents spilled, and the mouth of the animal or the hands of the (iperator, cut by the fragment.. The drenching horn is also sometimes used. In all animals the nostrils should be left unobstructed to such an extent as to not interfere with breathing; choking should be guarded against, and the drench should be poured somewhat slowly.

DISINFECTION.

For cleansing a drain, pour into it a bucket of water in which has been dissolved a handful of copperas. When it is desired to eradicate disease germs, smoke the shed or stable with sulphur: if to this is added womil tar, the activity will be greatly increased. Place a heap of live coals in an iron kettle or other suitable vesses?, and lay the sulphur or tar and sulphur upon them. The gases will at once begin to generate. For thoronah disinfection, it is best to remove all living creatures, close the building tightly, arrange the vessel so that there is no danger of fire, and leave matters so, for an hour or two. The smoke will thus penetrate every crevice. A little of this gas inhaled by animals may be beneficial, but the suffocating power of sulphur fumes is well known, and it is therefore not to be trifled with. By all means, remove the animals if distress is indicated by coughing. Carbolic acid applied to the doorlintels and walls, forms a good disinfectant. Cloths wet with a weak solution of it and hung up, are an attraction and a destruction to disease germs floating about in the atmosphere. This acid is also disagreeable to flies.

\section{BLISTERING.}

Before applying a blister, shave the hair from the part, wash and dry well; then with smart friction continuel for a few minutes, apply the blistering prepara- 
tion. If the operation is properly accomplished, and the application is afterwards carefully watched, the blister should leave no permanent scar or blemish. If it proves to be too severe, remove it and wash the part gently and apply olive oil or lard freely. Tie the horse in such a manner that he cannot mnaw at the blister, and do not remove the loose skin raised by it; let this scale off naturally. This treatment is sometimes used to hasten the opening of an abscess, or to reduce an enlarged gland. When sufficient action has been aroused, further irritation may be discouraged by washing car:fully and applying olive oil or lard.

\section{FIRING.}

In many cases of chronic lameness and in obstinate bone diseases, firing is of great benefit. For this purpose, Williams' instrument for pyropuncture is to lis recommended, as it makes a deep impression and leaver scarcely any scar. The iron should he red hot, and applied with enough force to pieree the skin and enter the? discased part. Before the procedure begins, the hair should be shaved from the part to be treated, and the animal securely fastened. On the day following the operation, neats' foot or some other hland oil may be applied, to act upon the swelling, and this may hence forward be used daily, until the swelling is reduced. The animal should not be put to labor too soon, as the disease may return if this is done. A rest of several months is advisable.

\section{SLINGS.}

Unless suffering from diseased feet, it is hest to let the horse sustain himself, but when to all apyearances, exhaustion is so great that this is no longer possible, it is well, in some instances, to prepare a sling for support in maintaining an upright position. To accomplish 
this, a block and tackle may be securely fastened to a beam over-head and attached to the canvas or other stout material employed, and which has been wrapped two or three times at each end, around stout rollers, at least two inches thick, and securely nailed. If instead, a frame is to be used, set four posts, a little over three feet apart one way and five feet the other. On either. side and on a line with the middle of the body, place a strong horizontal bar. Lead the horse between the posts, place a bar in front at such a height that it will cross the lower part of the neck, and another between the hind posts, on a line with the stifle. Wind your material around one of the side bars, and nail it on the outside, then pass it beneath the body to the bar opposite, and secure in the same manner. Do not let the horse sink down into the sling until it is well secured. The cloth may be strengthened by small ropes, and it should be of such a width as to begin behind the forelegs, and clear the sheath in the male.

\section{BLEEDING.}

By diminishing the quantity of blood in circulation, bleeding lessens the action of the lungs and heart. If this manner of treatment is resorted to, it should be in the first stages of the disease, before there is much exhaustion. It is not a safe remedy and should be avoided if possible. In some instances, however, it is the only chance for life. In horses, the jugular vein is generally selected when blood-letting is desired. A fleam is preferable to a lancet, for this purpose, since with this instrument, there is certainty of tapping the vein to a proper extent. From a previously strong, healthy horse, one bleeding of from five to seren quarts, is sufficient. The quantity extracted, must le regulated to the size of the animal and the nature of the ailment. A rusty or unclean instrument should not be used, as it is likely to 
produce inflammation. The horse should be blindfolded; pressure should be brought upon the vein with the finger, until it grows tense, or to accomplish this, a stout string may be tied about the neck with pressure enough to bring the veins into prominence, but there should be nodragging upon the skin. The incision must be made above the point of pressure; the fleam should be laid lengthwise along the center of the vein, and should be given a quick, heavy blow, whereupon

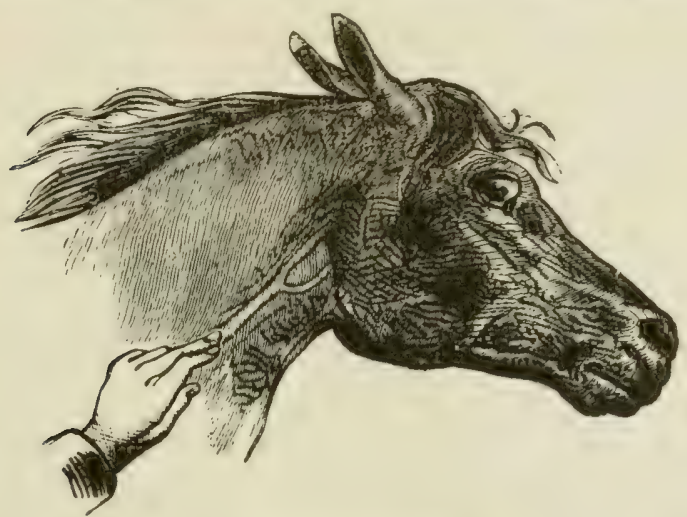

Raising the Vein.

the blood will spurt out. The pressure must be continued until a sufficient amount is drawn, when the string or finger, whichever has been employed, should be removed, and the flow will cease. During the operation and while the flow continues, the head should be elevated to a moderate height. When bleeding has ceased, a common pin is run through the margins of the wound and wrapped with thread crossed back and forth over the ends of the pin in the form of a figure eight. Care must be exercised in the pinning operation, so that there will be no dragging upon the skin, and no undue tightening of the thread ligature, and the vein must not 
be included. Usually in three days, the pin may be removed. It should first be twisted about to loosen adhesions, should there be any, and then withdrawn with one hand, while the other holds the skin in place below it, so that it will not be dragged back.

The ox and sheep are also generally bled from the

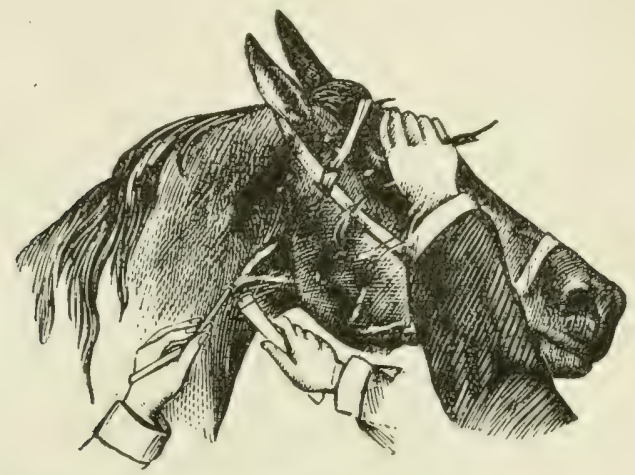

Blecding.

jugular, though sometimes in the sheep, the eye or cheek vein is tapped. In the pig, the ear nay be turned back and the veins pressed upon at the base, and broumit into prominence, when the incision can be made; or at cord may be tied around the foreleg above the knee, when the brachial vein, on the inside of the leg will ha seen, and can be lanced. The cut should be mads about an inch above the knee.

\section{SHOEING.}

While the art of shoeing belongs to another profession, which is closely allied to that of the veterinury surgeon, it may not be out of place here to make a few remarks on the various modes and manners in which the foot of the horse is treated by many blacksmiths, for want of a knowledge of one of the most intricate parts of the horse's anatomy. Far from it, is it $m y$ in- 
tention to a any injustice to the knights of the anvil, yet there are so many who undertake to practice this trade (1) profession, that have never given the parts sufficient study, or in whom there is not enough mechanical ingenuity, to properly place the shoe, and drive the inails in their proper position, after the foot has been lavelled and prepared for its reception. The somewhat weeping assertion has frequently been made, that the history of every horse, has been a record of human enleavor to destroy its usefulness, yet this assertion is not made without some foundation for its support, for where is the individual who questions the statement, "No font, no horse;" and yet there is no portion of this aniinal's anatomy, wherein he has been compellerl to sufler "' many ills, the cause of which, in the vast majority of instances, are traceable either directly or indirectly, to the manner in which the foot has been prepared and the ill-fitting shoe it has been comprlled to wear. Unfortunite indeed, has it been for that noble animal, that we have been obliged to invent some device for the artiticial protection of the foot, as the necessary work that is imposed upon it from day to day, causes a greater waste of horn than nature can replace during the intervals of rest it is allowed. The ordinary iron shoe, has so far, proven the best protection, and even this, when applied under the direction of, or hy a skillful mechanic, means a rertain amount of injury to the tissues of the foot. Every nail driven, means so much injury, and the larger the nail, the greater the injurious effects. There is however, one class of horses in this country, which, from the nature of the work they have to perform, and the condition of the ground they have to travel over, renders it unnecessary to furnish artificial protection for their feet, except under extraordinary circumstances. These are our agricultural horses; and when one of 
them, from the peculiar shape or condition of the feet, must receive attention, take it to a skillful mechanic, no matter how great the distance. It is strange, yet nevertheless true, that the average horse shoer has a mania for cutting, carving and rasping at the horse's foot, thereby taking upon himself the task of improving nature by means of art, which has always proven a dismal failure, for sooner or later, after being handled in this manner, you perceive a dried, contracted, pinched up or shriveled object instead of the broad, beautiful foot, with which nature in her ineffable wisdom, provided the animal. I will now endeavor to present to you, some of the pernicious and insane methods of preparing the foot for the shoe. Foremost among these, is the habit of trimming the frog. Now as the frog is nature's cushion and hoof expander, placed there to ward off concussion, through its elasticity, thereby furnishing the much needed protection to the other structures of the foot, which are possessed of a much smaller amount of expansion and contraction, it now being trimmed to a degree that deprives it of pressure, immediately commences to shrink, dry and harden, very soon presenting a pinched up appearance, when its usefulness to the foot is destroyed; then instead of being a protection to the foot, as in its natural state, discharging its allotted function, it becomes a menace rather than a benefit to this organ. Next among these, is the habit of paring out the sole of the foot, until it yields by the pressure of the operator's thumbs. As the sole grows very slowly, and rests upon a soft, velvety cushion, from which it is nourished and developed, consequently when it is pared down until it yields to light pressure, the part that still remains is only partially developed, and when exposed to dryness and heat, the delicate halfformed fibres contract to such a degree that their source 
of nourishment is greatly impaired, and the process of development is vastly retarded; then it is, that this mutilated and shrunken sole adds still more serious injury to the foot. Nor does the injury stop here. The operator proceeds to "open up the heels," as he calls it, by cutting out the bars and splitting down with the knife between the frog and the quarters. These bars are so arranged as to prevent the quarters from closing in on the frog, to prevent contraction at the heels. Thus, with the hars or lateral braces removed and a slice tinken out in the immediate vicinity of the heels, there is no other altemative, in a dry climate like ours in the summer, but to continuously contract, until the sensitive structures of the foot are naught but a constant source of irritation, pain and misery to the horse, the remander of its animal existence. Adding to this already mutilated foot, after the shoe has been placed upon it and the clinches marle, the rasp is brought into play, commencing up rery closely to the hair and rasping the whole external portion of the foot, thus robbing it of its natural layer of varnish, which was placed there by nature to prevent the evaporation of the moisture there secreted, for the nourishment and healthy maintenance of the parts. The condition of horses' feet depends greatly upon the care and management they receive from colthood up, and were they allowed the privilege of standing, while in confinement, upon some material containing a small amount of moisture instead of on a dry, wooden tloor, their feet would be foumd to be better shaped, tougher and less brittle, a conlition much better for the work to be exacted from them. Now in summarizing the conditions to which our horses' feet have been subjected, not only by the shoer, hut the owner, let us look together with more reason, and see if these evils cannot be abated in a great degree. As I 
have heretofore stated, the frow, sole and bars, were placed in position ly nature, to maintain the whole structure in a healthy condition, and as they hare been mercilessly mutilated with the drawing knife, that is the first instrument to be discarded from the shoer's outfit. The frog should never be touched with either knife or rasp, and as only the semi-detached portions of the sole should be removed, in order to level the foot for the proper adjustment of the shoe, the rasp is the only instrument that is necessary for the completion of that part of the work, for the shoe, when properly adjusted, has its bearing alike upon the horny wall, and the sole which is in immediate contact with it. It is a grave mistake to adjust a shoe and so concave the sole with the treacherous knife, that the only laring the fort has, rests upon the wall, and the wall only. After the bottom of the foot has been prepared for the shoe by levelling it off with the rasp, the next thing in order is to fit the shoe, using the best of judgment as to the proper weight of shoe the animal should wear. The only legitimate use of the shoe, is to prevent undue wear of the wall and sole, therefore the lighter the shoe, in proportion to the weiglit it has to sustain, the better. The plain shoe without calks, will allow the animal to rest in a natural position, consequently calks should be used only upon those horses where it is actually necessary, from the peculiar or difficult labor they have to perform. In such cases, a short calk answers the purjose much better than a long one, for the toe calks wear lown much more rapidly than the heels, resulting in a (1)pressed toe and an elevated heel. Also the relative positions of the bone structure of the foot are altered; the coffin hone beromes bruised and diseased, and Iossibly it may suffer during the balance of its existence. The weight of the shoe having been decided upon, it 
should now be so shaped as to conform to the size and shape of the foot, and not, as is too often the case, place on a shoe that is too small in circumference, then risp) down the foot to fit the shoe, in order to make it prewntable. The upper surface of the shoe, or that part that comes in contact with the hoof, should be perfectly Ierel; then with the two level surfaces coming in direct cpposition, it will require only a few nails, and these of a small size, to retain the shoe in position for a proper length of time, as the shoes should be changed erery four or five weeks. It is no uncommon sicht to see the shoer using heavy nails and a numerous lot of them driving them high up and clinching them firmly, and if remonstrated with or upbraided for doing such work, he will invariably tell you that if the shoes do not remain on for four or five months, the owner will go elsewhere to have his work done, thus losing his custom, which he cannot afford. Here it is clearly the owner's fault, either through short-sightedness or stinginess. $\mathrm{He}$ should be compelled to wear a pair of nicely fitting boots for a month, then supplied with a pair two sizes too small, and forced to wear them for the balance of the time the horse is compelled to wear his. I would venture the opinion that the horse would be taken to the shop immediately. A badly fitting shoe is as painful to the horse as the tight boot would be to the owner. After the shoes have been fitted and the nails driven and clinched, nothing remains to be done outside of rasping off the few slight projections that may extend over the shoe, but, just at this point, it is a common sight to see the rasp applied over the whole external part of the wall, robbing it of its layer of natural varnish, which was placed there to protect the foot, by liolding the moisture that is secreted within. When deprived of this covering, the moisture evaporates, the 
foot dries and hardens, and sooner or later contracts to a degree that is a source of irritation and pain. This habit, for such it is and nothing else, should be discarded, for nothing could be devised that is calculated to do a greater amount of injury, than this willful, wholesale and wholly uncalled for rasping. The various devices for shoeing to overcome deformities and habits, as well as for the production of speed, I will leave to the skillful mechanic, who understands that profession much better than I do, and who should receive the highest sense of praise, that can be bestowed upon those who follow this profession. 


\section{DISEASES OF THE HORSE}

THEIR

CAUSES, SYMPTOMS AND TREATMENT.

\section{DISTEMPER, OR STRANGLES.}

This is a disease peculiar to the horse, but may be communicated to man by inoculation. It seldom attacks a horse after maturity, but attacks colts from

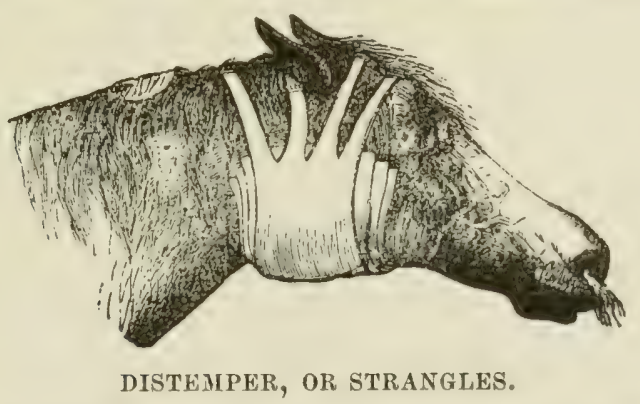

birth until they are five years old. The causes are many and varied. Among them are want of nutritious properties in the mother's milk, change from pasture to dry fced, irritation from teething, impure air, change from one climate to another, etc.

Symptoms:-The colt will appear dull and stupid; the eyes will begin to water and sometimes to matter; fever begins to rise; loss of appetite, coat begins to get rough; discharge from the nostrils; abscesses begin to 
form under the lower jaw. In irregular strangles abscesses may form anywhere on the body, or in the lungs or intestines, making the case very serious.

Treatment:-The treatment is very simple. In regular strangles place the animal in an airy, comfortable, loose box-stall and bed well. Blanket comfortably, jutect from exposure to cold to prevent severe bronAhitis or lung complications, and feed on soft food-such as bran-mashes, chopped feed, mashed turnips or car. rots. If the patient is constipated and the ferer high. give four ounces of glauber salts twice a day, as a laxative.

If there is a dry cough, steam the nostrils with scalded bran or oats, but do not smoke the colt. Poultice the throat with fried onions, boiled turnips or linseed meal, and when the abscesses soften, lance them to allow the matter to escape. If the abscesses remain hard, apply a good stimulating liniment, as,

Olive Oil............. four ounces.

Oil Turpentine..........two ounces.

Aqua Ammonia..........two ounces. Mix.

Apply morning and evening and gargle the throat with the following:

Chlorate of Potassium...... one ounce.

Nitrate of Potassium.......one ounce.

Hypo-Sulphate of Soda..... one ounce. Mix.

Make into twelve powders and give one powder every six hours.

When lung complications show themselves, apply mustard along the belly and sides. During convalescence, care must be taken not to expose the animal to cold, as a relapse might follow. Allow exercise but do not turn the exercise into work, until the animal has entirely recovered. 


\section{EPIZOOTIC INFLUENZA.}

This disease is rery feculiar and yet not uncommon in this country. In 1871 it traveled from east to west with such rapidity, that there is little reason to doubt that it is produced through atmospheric influences. It is much more dangerous in some localities than in others, according to the hycienic neasures employed. In localities that are low and malarious, with poor stabling, the disease is much more fatal than where the elevation is greater and the air dryer. But no location is exempt from the disease. In the same stable a num.

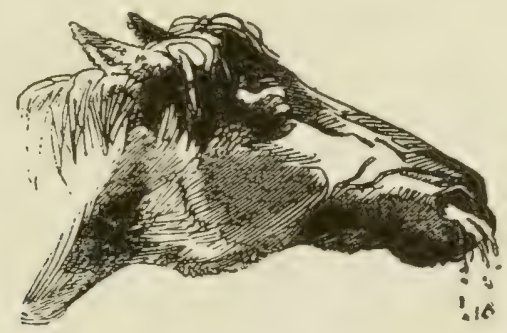

EPIZOOTIC INFLUENZA.

her of horses may be attacked, and very severely, while others remain perfectly healthy. Erery horse in a given stable may be affected, while their neighbors in a stable across the street escape entirely. It is my opinion that horses poorly cared for and ill-fed, are not only first attacked, but the first to succumb.

Symptoms:-The disease comes on very suddenly, with weakness and stupor, eyes swelled and watery. The membranes of the nostrils may be of a bright pink color, but are more frequently of a dull leaden hue. There is a dry cough, staring coat, ears and legs alternately hot and cold: patient is fererish: pulse accelerated-generally weak hut sometimes hard; sometimes 
a watery discharge from the nostrils, afterwards assuming a yellowish or greenish color; appetite lost; when made to move, will go with a swinging gait-frequently crackling of the joints will be heard, when the disease assumes a somewhat rheumatic nature, the legs swollen, hot and very painful when touched. In other cases the lungs or abdominal viscera may be seriously involved. If the horse is tucked up along the abdomen, with hard pellets of dung, covered with mucus, the bowels are attacked and your case is somewhat dangerous. If the

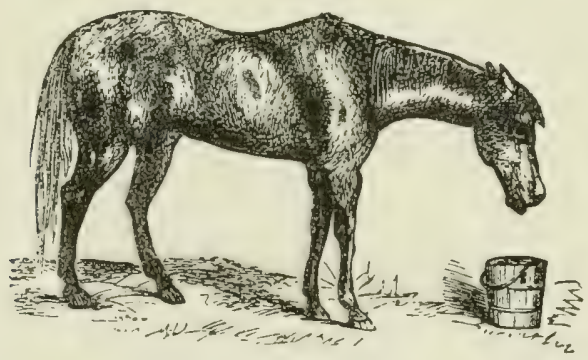

FIRST STAGE OF EPIZOOTIC INFLUENZA.

patient is tucked up in the flanks, with a ridge extending from the flanks to the breast bone, hurried breathing and short, painful cough, the disease has attacked some part of the thoracic cavity, - either the lungs or the pleura, probably both-and is extremely dangerous.

Treatment:- When first noticed, place in a comfortable, loose, box-stall, well aired but without draughts. Blanket well and bandage the legs. Relieve costiveness with a pint of linseed or castor oil and injections of warm water. If the fever is high give,

Quinine Sulph............ three drachms.

Nitrate Potass............... one ounce.

Bi-Carbonate Soda. one and one-half ounces. Mix. 
Make into six powders, of which give one every five or six hours. If the horse is very weak give,

Liq. Ammon. Acet...... eight ounces. Spts. Nit. Ath.......... four ounces.

Tinct. Nux Vomica.......two ounces. Mix.

Give two ounces every five or six hours in a little water as a drench. Bathe the throat with

Witch-hazel............... six ounces.

Tr. Arnica............... one ounce.

Aqua Ammonia........... one ounce. Mix.

Apply two or three times a day.

Feed on soft, nutritious diet and give plenty of pure, fresh water. If the joints swell, bathe with,

Alcohol..............eight ounces.

Witch-hazel........... four ounces.

Tr. Arnica............ four ounces. Mix.

Apply twice daily and bandage. If there are any lung complications, use mustard freely on the sides and read treatise on lung diseases. If the bowels are affected, give linseed jelly, slippery elm, etc.

\section{SPASMODIC COLIC, CRAMPS.}

Several direases of horses, - -such as affections of the kidneys, liver, spleen, etc., cause abdominal pain and are erroneously classed under the head of colic. I shall not treat of them here, but confine myself to spasmodic colic proper, which is a disease of the intestinal canal usually confined to the stomach and small intestines,an involuntary contraction of the muscular fibres, separate and distinct from inflammation, at the beginning causing intense pain in the abdominal region. There are many and various causes, - such as change of feed 
from oats to corn; too high feeding; sudden changes of temperature, from lot to cold; standing in the rain; drinking ice-cold water; innutritious food; in fact any-

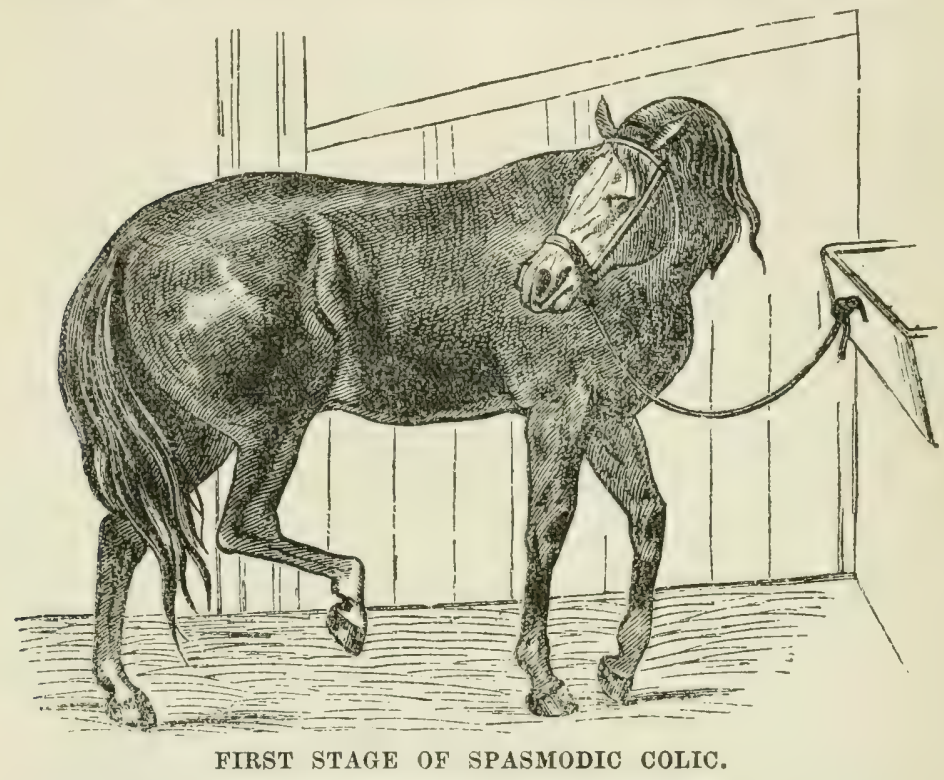

thing that will cause indigestion or irritation of the intestinal canal.

Symptoms:-The horse will generally appear uneasy, commence pawing and attempt to lie down, sometimes falling upon his knees, then raising himself up again. As the pain increases he will paw violently, suddenly drop down, roll around for a time, get up and stand apparently easy for a few minutes, possibly attempt to eat a few mouth-fulls. Then he is suddenly siezed again, paws violently, drops down again and rolls around-possibly rolls upon his breast and remains there for a time. Then commences tumbling and rolling again; gets up and shakes himself, and possibly tito 
cramps are over. If not the pain continues to increase in intensity. It will throw itself down, roll orer and over, jump up, whirl about, drop down again, paw or strike with the front feet, steam and sweat, and make

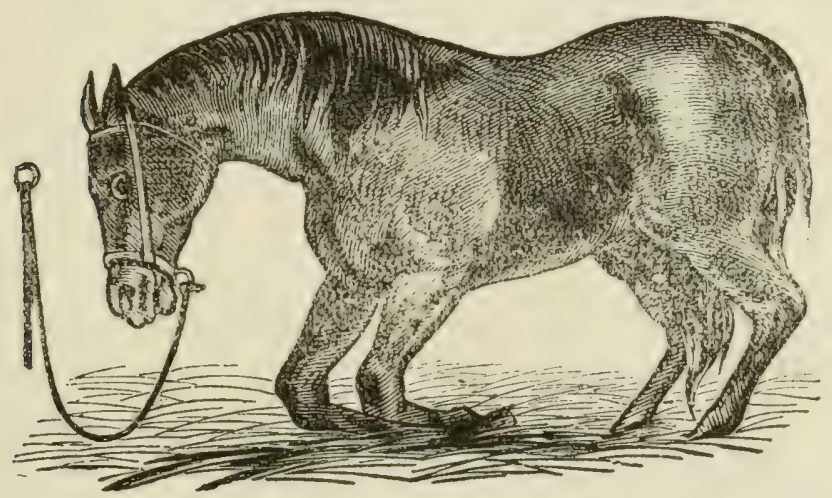

SECOND STAGE OF SPASMODIC COLIO.

frequent attemps to pass water. If a horse, frequently the penis will he partially erert; sometimes a small amount of water is passed, but more frequently none is passed until the spasm has passed off, when the urine will be voided freely, which has led many to suppose there was trouble with the kidneys and bladder when, in reality, it was only a symptom of colis. If the pain continues to increase in intensity, and the animal is not relieved, inflammation of the bowels (which is very dangerous) may result, or the horse may die from pain and exhaustion.

Treatment:-Place the animal in a dry, well-herded stall, where he is less liable to bruise himself. Rub the legs and abdomen and give,

Ginger............... half ounce.

Baking Soda.............half ounce.

Red Pepper............ half ounce: Mix. 
Make it into a tea and give at one dose. If this does not give relief in thirty minutes, repeat again, or give,

Barbadoes Aloes........... one ounce.

Chloral Hydrate........ one drachm.

Powdered Opium......... one drachm. Mix.

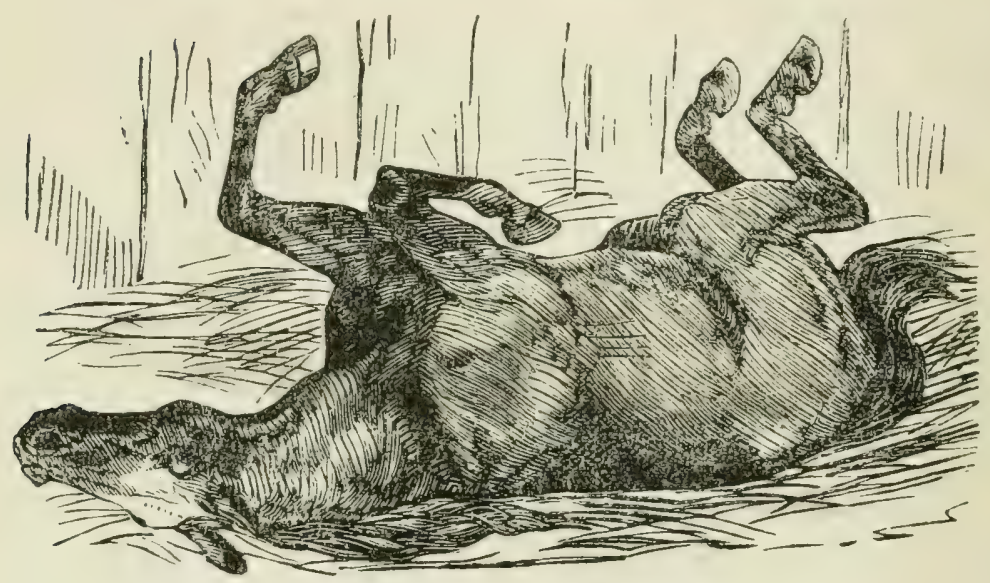

SPASMODIC COLIC-LAST STAGE.

If the pain increases, give,

Spts. Nitrous Ather, one and one-half ounces. Laudanum................ one ounce.

Oil of Peppermint.......... thirty drops. Spirits of Turpentine........ six drachms. Linseed Oil............. one-half pint. Mix.

Should the pain continue, repeat the latter prescription every forty minutes. If you have failed to give the aloes, after the pains have subsided it is good policy to give a good physic-either a pint of linseed oil or one ounce of barbadoes aloes and half ounce of ginger combined. 


\section{FLATULENT COLIC.}

Flatulent colic is a disease separate and distinct from spasmodic colic. In the former there is bloating, or distension with gas, of the bowels; in the latter there is no bloating whatever. Among the many canses are sudden changes of food, too long fastine, and food griven while exausted, new hay or grain, large quantities of green food, crib biting, sour, mouldy or other

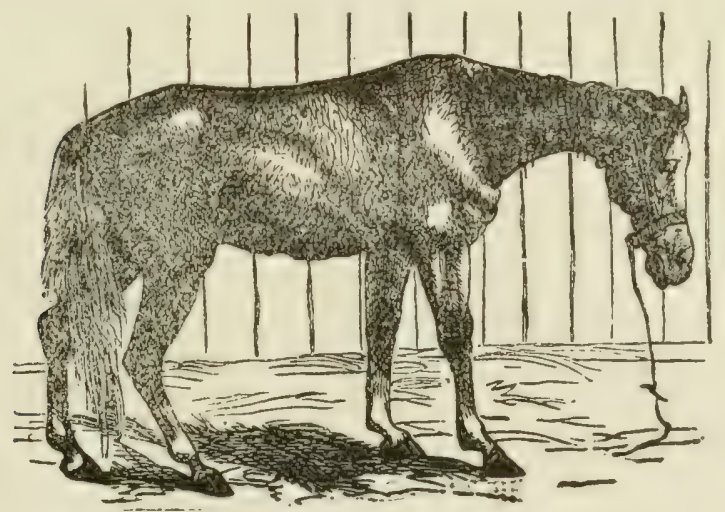

ADVANCED STAGE OF FLATULENT COLIC.

indigestible food setting up a ferment and creating a greater amount of gas than can be comfortably accommodated by the bowels, thus causing intense pain.

Symptoms:-The horse will hang his head and commence looking around at his flanks; soon he will begin pawing, then will lie down and roll, frequently looking at the flanks; the animal begins bloating and sometimes has belching of gas from the stomach; the bowels grow more distended, the pains are continuous and grow more severe, the breathing is shorter and the animal groans or grunts while lying down and often while standing; the legs and ears become cold, cold sweats break out over 
the body. The disease is usually of short duration and, if not speedily relieved, ends in death.

Treatment:-When first noticed, in the absence of other remedies, give two ounces of baking soda. If no relief is obtained in thirty minutes, give,

Chloral Hydrate........ two ounces.

Water...............eight ounces. Mix.

Give half in a pint of water and if necessary repeat in forty minutes, or give,

Linseed Oil............... one pint.

Spts. Turpentine......... two ounces. Mix.

If that fails to neutralize the gas, give

Liq. Ammonia Acet..... three ounces.

Chloral Hydrate....... four drachms.

Bi Carbonate Soda......two drachms.

Tinct. Aconite.........twenty drops.

Water.............. one-half pint.

Repeat every thirty minutes.

Give injections of hot water and castile soap every twenty minutes. If the bloating continues, tap with a trocar and canula, on the right side, between the hip and ribs, where it is most resonant on percussion. After recovery give a pint of linseed oil or castor oil.

\section{GLANDERS, OR FARCY.}

Glanders is a specific febrile disease peculiar to the horse, mule and ass; and, by inoculation, it can be communicated to man. Its causes are keeping horses in damp filthy stables, impure air, improper feeding, impaired nutrition, sequelx of "grease heels," sequelæ of epizootic influenza; also contagion, which is by far the most common. 
Symptoms:-Dullness, decreased appetite, eyes watering, fever rising from $102^{\circ}$ to $107^{\circ} \mathrm{F}$., coat staring, it. first watery discharge from the nostrils; discharge afterwards turns to a yellowish and later to a yellowish green, and is very tenacious; in the nostrils reddish ulcerations appear, later becoming purpleish; all over the body the lymphatic glands become enlarged, frequently breaking and discharging matter; the sub-maxillary glands enlarge and harden, later on becoming adherent to the jaw; breath foetid; by auscultation,

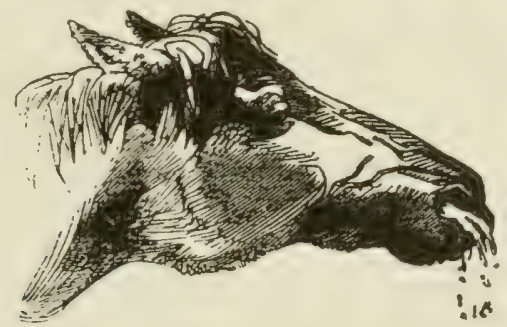

GLANDFRS.

crepitation of the lungs will be discovered; the discharge from the nostrils becomes very profuse and more greenish in color; fever now stands from $105^{\circ}$ to $107^{\circ} \mathrm{F}$; q quite frequently the limbs will swell,-more particularly about the joints.

Treatment:-The best treatment is the destruction of the animal as soon as thoroughly satisfied he has glanders. Separate all animals which have come in contact with him. If treatment be attempted, place the animal where he can have plenty of fresh air and try the sulphites - as sulphite of sodium, benzoate of sodium, arsenate of strychnia, carbolic acid; also vegetable and mineral tonics - as sulphate of iron, sulphate of copper, gentian, ginger, nux vomica, etc. 


\section{ANAEMIA, OR DROPSY.}

This is a term used to denote a deficiency of red corpuscles in the blood. The disease is caused by excessive bleeding, deteriorated food, keeping in illventilated, filthy stalles, without much light, effects of fevers, severe or protracted diarrhoea, leucorrhoea; it sometimes results from epizootic influenza.

Symptoms:-Pallor of the mucous membranes; is tucked up in the flanks, cardiac palpitation, disinclination to move, stagrering gait, paleness of eyes and eyelids, coat more or less rough; later on, if a horse, a swelling of the sheath-if a mare, swellings of a dropsical nature under the breast, slight at first, but gradually extending both backward and forward; in the horse usually extending from the sheath forward; the limbs begin swelling, sometimes reaching an enormous size; on scarifying the swelling, there will be an oozing out of serum, barely colored with blood, which may be continued for days, - you will also find the flesh of a bluish color; appetite sometimes fair, usually for hay but not for grain: bowels generally constipated, but sometimes a diarrhoa will set in which, if not checked, will commonly prove fatal; rumbling of the bowels is invariably a constant symptom.

Treatment:-If possible remove the cause as soon as can be give plenty of light and fresh air. If necessary, regulate the bowels with a pint of castor oil. Give strengthening and easily digested food-such as ground oats, beans, boiled flaxseed, etc. Administer such remedies as will tend to build up the system, as

Sulphate of Iron ......... two ounces.

Gentian .............. one ounce.

Nux Vomica............ one ounce.

Arsenic............thirty grains. Mix. 
Make fifteen powders; give one powder every five or six hours; or use any of the mineral or veretable tonics, as, cascarrilla, (quassia, gentian, sulphate of copper, etc. Regulate the kidneys with drachm doses of nitrate of potassium. Bed the animal well. When the sheath is much swollen, scarify lightly.

\section{EPISTAXIS, 0R BLEEDING FROM THE NOSTRILS.}

This disease seldom occurs. It is usually caused by hard ruming, sneezing, coughing, by external injuries, congestion of the lungs, bronchitis, or influenza.

Symptoms:-If the blood is merely dropping or running in a small stream from one nostril, some small blood ressel in the head is ruptured. If the blood flows in a stream and comes from both nostrils and is of a bright scarlet color, quite frothy and accompanied by a cough, the blood is coming from the lungs. If it comes away in dark colored clots, it is in all probability coming from the stomach.

Treatment:-If from some ruptured hlood ressel in the head, elerate the head and apply rold water orer it and down over the nostrils, or apply ice to the head. Inject weak solutions of alum water up the nostril, or a weak solution of sulphate of zinc; or plug the nostril with a sponge or cotton batting, but be sure and have a string tied to it to draw it out and only plug one nostril at a time. If the bleeding continues for some hours, give one drachm of sugar of lead in a pint of water, as a drench. If the bleeding is from the lungs and is accompanied by a cough, give,

Laudanum............ two ounces.

Raw Linseed Oil.......... half pint. Mix. 
Give at one dose. If the bleeding continues, take, Sugar of Lead........... one drachm.

Water....................... pint.

Give at one dose and keep the animal perfectly quiet.

\section{AZOTURIA, OR PARTIAL PARALYSIS.}

By some this disease has been described as a disease of the kidneys, by others as a disease of the spine and by others as a disease of the liver. But I have invariably found the whole system more or less aflected, from

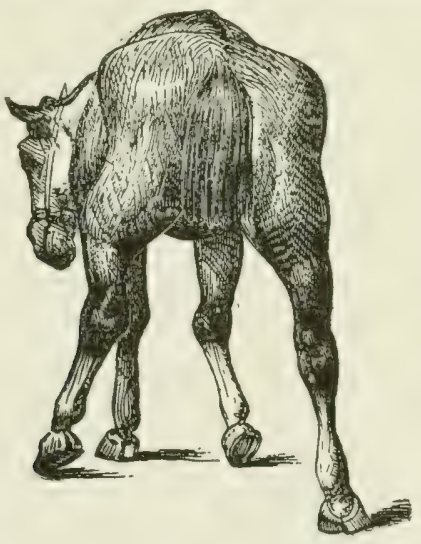

AZOTURIA.

an abnormal amount of nitrogen in the blood, which is caused by high feeding and want of exercise. As the animal is put to work, there is a greater amount of waste material cast upon the excretory orans than they can well dispose of; hence an excess of nitrogen is formed in the blood and is carried turough the system, invariably settling in the myolema or coverings of the deep muscles of the haunch, causing partial and sometimes complete paralysis of the hind extremities. 
Symptoms:- Invariably the horse leaves the stable in the best of health, and, as the driver says, "the horse was never feeling better." But it is probably not driven over half a mile, possibly five or six miles, when he is noticed to hang back, to be covered with profuse perspiration and to be stiff in his hind legs. Other symptoms, are looking back at the Hanks, anxious expression of countenance, breathing short, notrils distended and red, staring eyes, drooping of the hind legs and knuckling over at the fetlocks, as though there was no strength in them; after staggering around for awhile the animal falls and is unable to wet up,-although they sometimes keep upon their feet in a mild attack and, if so, are most likely to recover in a few days; if urine is passed it will be very dark, sometimes looks bloody, smells very strong and is very ropy.

Treatment:-If the horse remains very uneasy, grive four drachm doses of bromide of potassimn every hour until relieved; then remove to a comfortable place and bed well. Clean out the bowels well by giving,

Barbadoes Aloes..eight or ten drachms. Ginger..............two drachms. Mix.

Give in one pint of water as a drench. While the animal is perspiring, blanket well and keep sweating. Apply a fresh sheep-skin over the loins and cover well, or apply cloths wrung out of hot water over the loins, and be careful the animal does not take coll. If the kidneys do not act freely, give one ounce tinct. buchu compound, every six or seven hours in a little syrup as a drench. And in a few days give,

Tincture Gentian........ two ounces.

Tincture Nux Vomica......two ounces. Tincture Ginger.......... four ounces. Alcohol..............eight ounces. Mix. 
Give two ounces every four or five hours. Bathe the back and loins with a good, strong liniment once or twice a day, also rub from the stifle to the point of the hip. Turn the horse over from one side to the other every three or four hours, and occasionally sponge him off with dilute alcohol. If shod, remove the shoes and keep lying upon the breast as much as possible. I believe it bad policy in such cases to swing the horse, unless the slings can be mot under him before he goes down,-then it is advisable; but after he is down for a few hours, do not attempt to swing. If the patient lies upon his breast well and rests easy, you may look for recovery in a few days; if he lies straight out, the inevitable result is death in a few days. Should the animal not urinate properly, pass the catheter and draw off the urine. If in twenty-four hours the bowels do not respond to the aloes, wive a quart of raw linseed oil; also give injections of castile soap and hot water.

\section{NASAL CATARRH, OR COLD IN THE HEAD.}

This quite frequently arises from standing in a draught, from inhalation of irritating gases, or from decayed teeth.

Symptoms:-Discharge, watery at first, from the nostrils, also from the eyes; sneezing; later on a discharge of matter from the nostrils of a thick, mucilacinous state, of a yellowish white color, and it may be quite profuse. It is sometimes very foetid, especially when from a diseased tooth; sometimes ulcers will appear on the septum of the nose, when the discharce is of a muco-purulent character; often the appetite is lost and the animal becomes debilitated. 
Treatment:- Complete rest, with pure air and good food for a few dars should be granted. Steaming the nostrils with hot water or sealded bran, to which may be adred a little carbolic acid, four or five times a day and about fifteen minutes each time, will be of great benefit and cannot be over-estimated. Feed such food as the animal will partake, bran-mash, scalded oats or barley; green wrass if it can be procured, but if the patient prefers hay, oats, corn or other dry food, give it. If there is loss of appetite, gret liq. ammon. acet., sixteen ounces. Give two ounces with two drachms of chlorate of potash, in a pint of water, three times a day. After a few days give,

Powd. Sulph. Iron ....... two ounces.

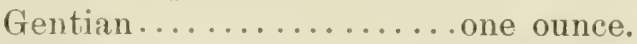

Quinine............ four drachms. Mix.

Make twelve powders. Give three powders each day. If the weather is cold, blanket and keep the animal warm. If from a diseaser tooth, the tooth should be extracted, and if there is matter in the frontal sinuses, they should be trephined and washed out with,

Listerine............ four ounces.

Sulphate of Zinc.... one-half drachm.

Water.............. four ounces. Mix.

Use morning and evening.

\section{CHRONIC ROARING.}

This is so called from the roaring, rasping, unnatural sound when the horse is put to work, and is caused by atrophy or wasting of the muscles on the left side of the larynx, by distemper, by epizootic influenza, by polypus in the nostrils; also by spasmodic constriction of the bronchial tubes from tonic contraction, mostly reflex of the involuntary muscular fibres; by fracture of the bones 
of the nose,-when the fracture is depressed; by an: extra amount of fat, by dropsical swellings, etc.

Treatment:-First, if possible, remove the cause. If that cannot be done, treatment is useless. If caused l:y atrophy of the muscles of the larynx, apply every fifth day, for three or four applications, a blister of

Vaseline..................... ounce.

Bin-Iodide Mercury....... one drachm.

Powdered Cantharides....one drachm. Mix.

If caused by polypus, have it removed. If from fat, reduce in flesh. In the majority of cases the roaring is. male during the inspiration. The horse seemingly inhales more air than the lungs can dispose of: consequently he roars and chokes down when put to work. By using a clasp over the nostrils, it holds them so he can only take in a certain amount of air at each inspiration; when put to work, the roaring will be stopped and the horse will do a reasonable amount of work. The clasp may be taken off after he has been working an hour, and, in the majority of cases, the animal will breathe freely. In order to keep horses from roaring badly, work them moderately every day. The operation of extirpating the collapsed cartilage and vocal cord, is the only way to give permanent relief, when the roaring is caused from wasting away of the muscles of the larynx, and as that requires a skillful practioner, it is quite unnecessary to describe it here.

\section{BRONCHITIS.}

This is an inflammation of the bronchial tubes. The mucous membrane alone may be affected or the whole tube may be involved. When the large tubes alone are affected, it is much less serious than when the smaller ones are involved. It is caused by standing in draughts 
when heated, inhaling irritating gases and smoke, and by drenching through the nostrils, whereby both thids and solids gain access to the parts. It occasionally follows influenza, distemper and other specific fevers, or even common cold or sore throat.

Symptoms :-Systemic depression, dullness; the appetite is partially or wholly lost, breathing quickened, redness and dryness of the mucous membrane of the nostrils, followed by fever and a hacking, barking coush, followed in a few days by a moist, rattling cough, with a light-colored discharge from the nostrils. Sometimes this discharge is tinged with blood. The pulse at first is hard and quick; later it becomes smaller and more frequent. By placing your ear to the side of the chest, you will hear a wheezing sound, if the small tubes are affected, and a rattling, suoring sound if the large tubes are affected. In a few days the cough becomes deeper and looser and the mucous which is secreted, is expectorated through the nostrils, although some of it may drop into the pharyx and be swallowed. The breathing is labored, short and quick but not necessarily painful. The bowels are generally constipated and the dung is covered with a slimy mucous.

Treatment:-Place the animal in a comfortable and airy stall and blanket well. Apply mustard to the sides and breast. Hand rub the legs until they are warm, then apply flannel bandages as high up as the knees and hocks. If the legs camnot be made warm by hand rubbing alone, apply whisky and red pepper mixed, and then bandage. Compel the patient to inhale steam from a bucket in which has been placed an ounce of turpentine, four or five times a day, and give

Bicarbonate of Potassium.two drachms.

Dover's Powders ....... one drachm.

Liq. Ammonia Acet...... three ounces. Mix. 
Give every four or five hours; and if the cough is very tight, give every three or four hours,

Syrup of Ipecac ....... four drachms.

Syrup of Squills.......... six drachms.

Tinct. Opii Camph........six drachms. Mix.

Continue until the cough softens.

If the bowels are costive, do not give physic, but give a few injections of warm water and castile soap. If the appetite remains passibly good, feed on soft diet, as bran mash, scalded oats, green grass, etc., and in cise the patient does not relish cooked food, feed anything that it will eat, and allow all the water the patient will drink. Give as it comes from the well. Do not think the disease can be cured in two or three days for it will likely last for two or three weeks. When the patient is making a gool recovery, and nothing remains except the coush and a slight discharge from the nostrils, disontinue all other remedies and grive the following:

Sulphate of Iron......... four ounces.

Gentian............ eight ounces.

Nux Vomica............two ounces. Mix.

Make twenty powders. Give one powder morning and evening in soft food or as a drench. Do not put the animal at work too soon, as a slight relapse may atevelop into a chronic case and terminate in an incurable case of thick wind.

\section{SORE THROAT, OR LARYNGO PHARYNGITIS.}

This disease affects the larynx and pharynx, situated at the upper part of the wind-pipe, and consists of an inflammation of the soft tissues of both.

Symptoms:-The disease is sometimes ushered in by a chill, immediately followed by fever; and if the attack 
is very severe, the nose will be elevated, to straighten the air passages. Swellings, sometimes very large externally, and most frequently noticed behind the jaws on the neck. Sometimes they are so extensive that the animal can scarcely breathe or can only do so with the rreatest difficulty; there will be a wheezing sound at each inspiration. During this period the heart becomes excited and its pulsations rapid. The mucous membranes in the nostrils will be of a bluish color, owing to the lack of sufficient fresh air in the lungs to oxidize

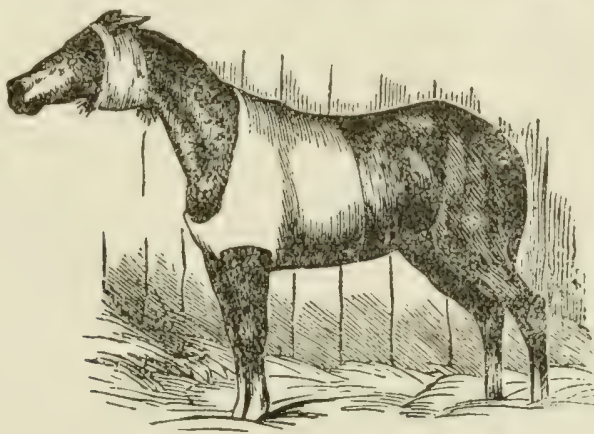

BANDAGE FOR LARYNGITIS AND INFLAMMATION OF THE LUNGS.

the blood. If the patient is in a box stall, it will wander around considerably and endeavor to keep its nose in the direction where there is most fresh air. If the animal attempts to eat, the food will be chewed and dropped out of the mouth; and, on attempting to drink, the water will be ejected through the nostrils. In some cases the cough is very loud, but in many,-especially when the internal swelling is very great, the cough will be very slight. Frequently there is a discharge from the nostrils and an abundant flow of ropy saliva from the mouth. 
Treatment:-Blanket well and place the patient in a romfortable and airy box-stall, devoid of draughts. Bandage the legs, and if possible give as a physic:

Aloes................seven drachms.

Ginger.............. one drachm. Mix.

Give at one dose. Then bathe the throat well with warm water and wrap it up in rugs or sheep-skins to keep it warm. Steam the nostrils with scalded bran or oats and give,

Solid Ext. Belladonna...... one ounce.

Chlorate of Potassium...... one ounce.

Pulv. Glycyrrhiza......... one ounce.

Gum Camphor....... one-half ounce.

Honey of Sim. Syrup.. one-half pound. Mix.

Give a table-spoonful three times a day. If the wather is in any way cold or disagreeable, be very cireful about bathing the throat with hot water, as the yitient may take cold. So, immediately after bathing, apply,

Olive Oil............ four ounces.

Turpentine............two ounces.

Aqua Ammonia........ two ounces. Mix.

Or,

Tincture Cantharides....three ounces.

Tincture Capsicum........ one ounce.

Rectified Spirits......... four ounces. Mix.

The danger from cold will be greatly lessened. It aiso acts as a good counter-irritant, reduces the swelling, removes the effusions by promoting absorption, and leaves the patient less liable to be "thick-winded," or "a roarer." The diet should be of soft food, as bran mash, or chopped feed mixed with a little linseed meal. Sometimes it is advisable to gargle the mouth with some cooling lotion; in such cases use, 
Powd. Chlor. of Potassium one ounce.

Powd. Nit of Potassium... one ounce.

Bi-Carbonate of Soda..... one ounce. Mix.

Make into twelve powders. Three times a day mix one powder with half a pint of water and wargle the mouth. If the swelling "points," open with a lancet and allow the pus to escape. Dress the cavity three times a day with,

Permanganate of Potassium, one drachm.

Water................ eight ounces. Mix.

\section{PNEUMONIA OR INFLAMMATION OF THE LUNGS.}

This consists of an inflammation of the lung substance caused by catching cold, inhaling irritating substances, injuries, drenching through the nostrils, badly ventilated stables, etc.

Symptoms:-The horse is generally taken with a chill : hangs his head; there is redness of the eyes and of the membranes of the nostrils, dryness of the mouth, a

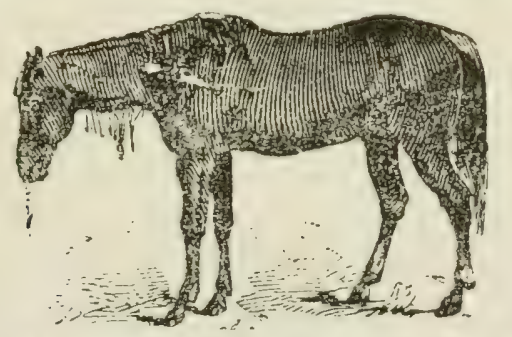

PNEUMONIA, OR INELAMMATION OF THE LUNGS.

cough, quite deep at first, which gradually shortens for a few days; pulse, at first generally full, gradually growing faster and weaker; rapid breathing; on the third day fever ranges from $103^{\circ}$ to $106^{\circ}$ and continued 
until about the sixth or seventh day, when it gradually declines; on the third or fourth it is likely there will be a discharge of mucous from the nostrils: about the seventh day when the fever subsides. the horse will

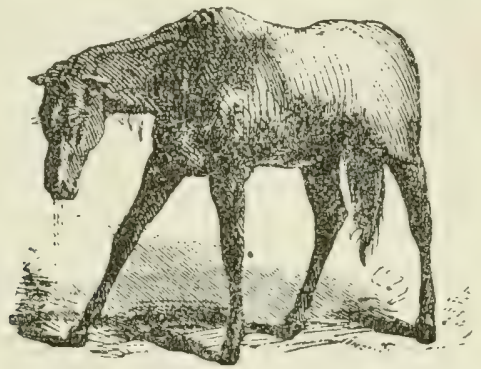

LAST STAGE OF INFLAMMATION OF THE LUNGS.

either get well or there will be great oppression in breathing, increased prostration, deeper cough, more copious expectoration, emitting a foetid, purulent odor; and death will result from the sixth to the twentieth day.

Treatment :-Place the animal in a comfortable, airy, loose box stall, devoid of draughts. Blanket the body to keep warm. Hand rub the legs and if warmth cannot be produced by hand rubbing alone, apply whisky and red pepper mixed; then bandage as high up as they are cold. Do not give a physic but open the bowels with soft foods and injections of warm water. Some practitioners will scoff at the idea of bleeding, nevertheless a plethoric, robust horse, in many instances, will be greatly benefited in the first three or four days by being bled, while an older and debilitated patient would succumb. Apply mustard to the sides and give the following:

Liq. Ammonia Acet...twelve ounces.

Spts. Aeth. Nit.......... four ounces.

Fluid Ext. Aconite...... one drachm. Mix. 
Give two ounces every three hours in a little water as a drench. Also give every four or five hours,

Quinine Sulph......... forty grains.

Nitrate of Potassium.... two drachms. Mix. Or,

Powd. Ext. Belladonna... one scruple.

Gum Camphor..........two scruples.

Nitrate of Potassium.... one drachm. Mix.

Give all the water the horse will drink,-just as it comes from the well, and feed on soft diet. If there is great prostration give tonic stimulants, as,

Alcohol ,.............e. eight ounces.

Tinct. Ginger .......... three ounces.

Tinct. Nux Vomica...... two ounces.

Tinct. Gentian.........three ounces. Mix.

Give two ounces as occasion demands, in half pint of water as a drench. During the period of convalesicence, good, nutritous food should be giren in limited quantities, and tonic medicines administered, as,

Sulphate of Iron......... four ounces.

Gentian.................six ounces.

Nux Vomica...........two ounces. Mix.

Make twenty powders. Give one powder morning and evening in soft food or as a drench.

\section{PLEURISY.}

This is an inflammation of the lining membrane of the thoracic cavity. (icnerally after a chill or cold stage, sharp pains hegin in the sides; the animal frequently will look at his sides and lie down very carefully, then rise again; the skin is hot, breathing short, also a short cough: no discharge from the nose; pulse hard and rapid; abdomen tucked up, and a ridge ex- 
tending from the hips to the breast-bone; on punching between the ribs, when the seat of inflammation is reached, the animal will evince pain by grunting; if the animal lies down it will invariably lie on the affected side, but they more frequently stand up. The second day, effusion of serum takes place; then the pulse will become slower and fuller, breathing will be easier and the animal seemingly is well again. And, if the eflusion is not very great, it will soon be absorbed and the animal is well. But, if the effusion is great, the

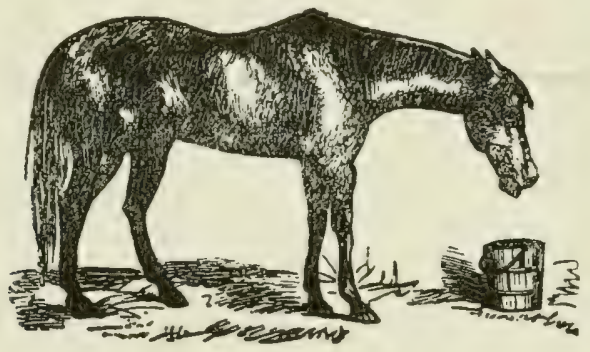

PLEURISY.

breathing becomes hurried and short; the pulse grows fast and weak; the ridge from the hip to the breast is more prominent; appetite almost completely gone, cold sweats break out and imminent danger is at hand.

Treatment:-Place the animal in a comfortable, airy, loose box-stall, free from draught. Blanket comfortably and warm the legs by hand rubbing, after which bandage with flannel to keep warm. Allow all the pure, cold water the animal will drink, and feed upon soft food, as bran-mash, boiled oats, etc. If it will not eat soft food, give it what it will eat. When the pain is severe, as is observed at the commencement of the attack, give the following drench :

Raw Linseed Oil.............. pine pint. Laudanum.............two ounces. Mix. 
If the pain continues, give ounce doses of laudinum every four hours. Then give, every six hours, the following:

Liq. Ammon Acet.....twelve ounces.

Spts. of Nitrous Ether.... four ounces. Mix.

Dose, four ounces in a pint of water as a drench. Also the following:

Powd. Potass. Nit........ two ounces.

Sodæ Bi-Carb........... two ounces. Mix.

Make into ten powders. Give one powder morning and evening in soft food, drinking water or as a drench. Hot flax-seed poultices applied to the sides are very beneficial, and in the absence of tlax-seed, good mustard plasters applied will assist in promoting absorption. If the patient becomes weak and debilitated, give stimu. lants, as,
Alcohol.............eight ounces.
Tr. Ginger............ three ounces.
Tr. Nux Vomica.........two ounces.
Tr. Gentian...........three ounces. Mix.

Give two ounces three times a day in half pint of water as a drench. Keep the bowels moving freely by frequent doses of raw linseed oil, which will assist in carrying off the effusion of serum that settles around the lungs.

But if the effusion of serum within the thoracic carity is very great and the breathing hurried, the chest should be tapped. Although the operation is simple and easily performed, it does not always result satisfactorily, probably because delayed too long. The proper place to operate is between the eighth and ninth ribs, as close as possible to the ninth rib. It should be done with trocar and canula, two-thirds the way down toward the bottom of the chest. After tapping give the following: 
Iodide of Potassium........ one ounce.

Whiskey................ pine pint. Mix.

Give a wine-glassfull every six hours; also give daily one ounce of tincture perchloride of iron. Apply a good mustard plaster to the sides or a good cantharides liniment. Or if the tapping is not resorted to, the latter prescription, as well as the perchloride of iron, may be given with excellent results.

\section{CHRONIC COUGH.}

A cough is not a disease but a symptom of a disease, usually of the throat, lungs, bronchial tubes or some portion of the air passages. A chronic cough is a

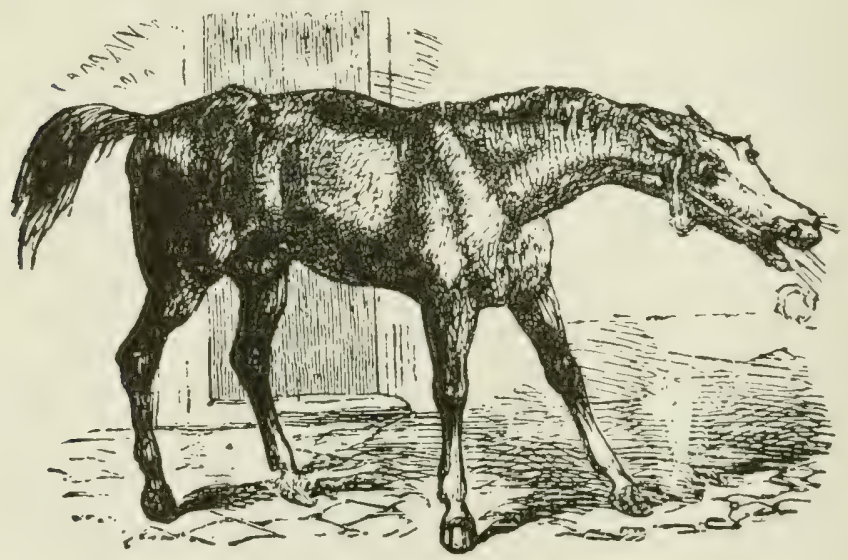

chroÑIC COUGH.

symptom of some chronic derangement of the air passages, the result of an incomplete recovery from some acute affection, as pneumonia, bronchitis, laryngitis, influenza, heaves, etc. It is also an accompaniment of heaves, and a symptom of diseases not classed 
with disorders of the respiratory organs, as diseases of the stomach, worms, etc. When inflammation of the membranes lining the throat and bronchial tubes becomes chronic, the parts are very irritalle and easily excited. Inhaling cold air suddenly, or dust, either from the hay or while on the road, or pinching the throat between the finger and thumb, will cause a heary fit of coughing that will last for a few minutes and then cease until irritated agaiu.

Symptoms:-The location of the diseased parts is determined by the mature am somal of the cough. The healthy cough is strong, full and often accompanied by a sneeze to clear the nostrils of whatever may be within them. The heave coush is at first syasmodic, becoming feeble, short and suppressed and accompanied by a grunt. The throat cough is light, short and hacking, and the chest cough is hollow deep and resonant, except during the acute, painful stages of inflammation, when it is short and almost noiseless.

Treatment:-Chronic cough in many instances is incurable, especially in cases of long standing; the more recent cases, however, often yield to treatment. When the irritation is in the upper part of the throat, great. benefit will be derived from the application of a good stimulating liniment, as,

Sweet Oil............ four ounces.

Aqua Ammonia......... two ounces.

Turpentine...........two ounces. Mix.

Apply thoroughly around the throat up to the ears once daily, and give internally,

Chlorate of Potassium.... two ounces.

Camphor Gum............ one ounce.

Solid Ext. Belladonna...... one ounce.

Molasses, or Honey..... eight ounces. Mix. 
Place a teaspoonful on the back part of the tongue three times a day, or give a teaspoonful of oil tar once daily, or,

Chlorate of Potassium .... two ounces.

Bicarbonate of Potassium.two ounces. Mix.

Make twelve powders. Give one powder morning and evening in half pint of water, or,

Gum Camphor............ one ounce.

Powd. Digitalis........... one ounce.

Calomel............... one ounce.

Linseed Meal............two ounces. Mix.

Make twelve pills. Give one pill every day for a week, then omit for a week, and repeat. Careful attention should be paid to the diet. Feed soft food; cut the hay and wet it, and mix chop feed with it. Allow an abundance of pure, fresh air. If grass is in season, turn out to pasture as much as possible, for the benefit resulting from this course, is sometimes wonderful, being often productive of more good than a course of medical treatment.

\section{CONGESTION OF THE LUNGS.}

Congestion is generally a fore-runner of inflammation of the lungs, although we frequently see cases where it never reaches the inflammatory stage; the patient either recovers or dies while the lungs are in the congested condition. Horses that are kept in the stable and fed and groomed well, without sufficient exercise, when taken out and put to severe exertion, are subjects for congestion of the lungs. In the same way when animals suffering from influenza, eatarrh or any other respiratory trouble, are put to severe work, congestion may be looked for. Or an animal unaccustomed to long 
drives, when driven a good distance on a hot day, may be stricken with congestion of the lungs; or it may result from badly ventilated stable, etc.

Symptoms:-When caused from over-exertion in an animal unaccustomed to severe exercise, the patient will stand with limbs out-stretched, neck extended. head depressed, anxious Jooking countenance, flapping of the nostrils, heaving in thank, more or less trembling over the whole body, with perspiration breaking out and

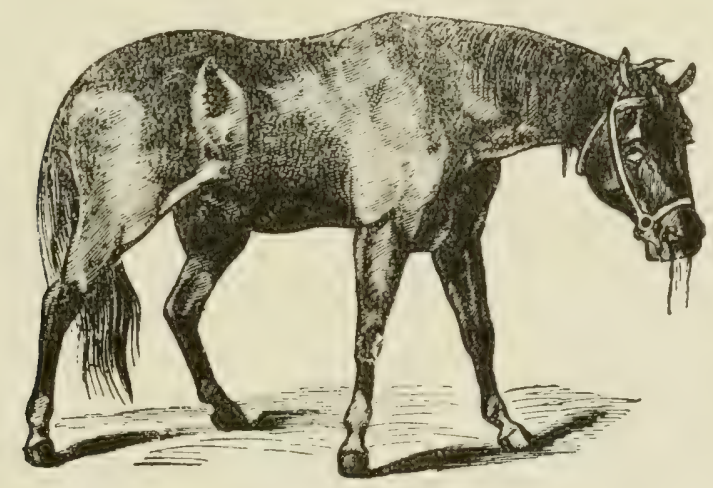

CONGESTION OF THE LUNGS.

rolling off in large drops. The limbs become cold, the eyes look blood-shot, the pulse is fast and feeble and the heart, as felt on the left side, is rapid and jerking. By placing the ear to the breast of the patient, you will hear minute crepitation or sharp, fine, crackling sounds. The nasal membrane will be of a deep red or blue wolor and the breathing will be quick, lahored and wheezy. If blood is extracted, it looks very dark.

Treatment:-As soon as congestion is observed and although the attack may be severe, by using very simple remedies, great relief may be obtained. If any wind is blowing, turn the animal's head toward it, then 
blanket well and rub the limbs thoroughly, or rub on some good, strong liniment and handage to keep warm. Bleeding at this time will be of great benefit in assisting to unload the over-charged lungs. Thorough rubbing all over the borly with cloths, hay or the hands, will tend to bring the hlood to the surface and assist in equalizing the circulation. Then give,

Whiskey............. four ounces.

Hot Water ............ . half pint. Mix.

Give every half hour until three or four doses are given. Or,

Liq. Ammonia Acetatis...t ten ounces.

Whiskey............... six ounces. Mix.

Give three ounces every hour in half pint of water. After the acute symptoms have sulsided, he very areful for a few days. Allow plenty of pure, fresh air and water, feed on soft food, to which may be added night and morning, one powder of

Nitrate of Potass.........two ounces.

Bi-Carbonate of Soda....... one ounce. Mix.

Make six powders. Blinket well to keep warm and allow only moderate exercise, for you should bear in mind, that for some time after an attack, another is liatble to occur from trifling causes and may terminate in a more serious complaint, inllammation of the lungs.

\section{ASTHMA.}

That a condition does exist, wherein a chronic spasm of the bronchial muscular tissues is seen as a distinct and separate affection from heaves, there is no longer doubt. From the strong similarity between the two diseases, and also from the fact that asthma is sometimes a precursor of heaves, the two ailments have become 
confounded and looked upon as synonymous. Asthma is hereditary in the majority of cases, and it may oceur at any age. It is also caused by fog, smoke, fumes from various things; from fatigue and overwork when the system is debilitated, irritation of the stomach from indigestion, etc.

Symptoms:-The difficult breathing, which is of a spasmodic charater, generally develops sudrenly and is fuite severe while it lasts, and may terminate rery speedily. Again it will last for months, unless relieved. There is also a wheezing sound accompanying inspiration; cough is short, quick and suppressed; great. anxiety of countenanee, which disappears to a sreat extent when expectoration commences. The chest and abdomen very much resemble those of heaves, in the lixed or set position of the ribs and the doulble lift of the flank during the respiratory act.

Treatment:-During the attack, the aim should be to give relief by relaxing the spasm, and what will accomplish this in one animal, will fail in another. You will therefore have to try several remedies, and when one is found that will answer that particular case, continue with it. Open the bowels with a quart of raw linseed oil, and give drachm doses of tincture of lobelia, every two hours, until five or six doses are given. If this does not give relief, try Hoffman's anodyne, two drachm doses every two hours; or two drachm doses of chloral hydrate, every hour, until five or six doses are given: dilute the chloral with a pint of water. Or give bro.. mide of potassium, two drachm doses every three or four hours, or half ounce doses of lauclanum every hour, until five or six doses have been given. Apply mustard plasters to the sides; feed on light, soft diet; leep the body warm and the stomach quite empty. After the 
most distressing symptoms have subsided and the trouble still lingers, feed the animal some cedar or hemlock boughs, as excellent results have been obtained from their use.

\section{QUINSY.}

This consists of an inflammation in the throat of a deep-seated character, tending to the formation of abscesses which are somewhat slow at development, but which subsequently break internally and are discharged through the nostrils.

Causes:- Some horses are seemingly predisposed to quinsy. Often it is the result of exposure to cold storms or standing in drafts when heated. It may also. be caused by neglected catarrh or anything causing an irritation and inflammation of the throat. Many rases of supposed influenza are really quinsy.

Symptoms:-Ferer is usually present from the outset. There is usually soreness of the throat in swallowing, with internal pain and swellings on one or both sides of the neck. Sometimes there is external swelling which can be plainly seen. Soreness of the throat increases day by day, until there is complete inability to swallow. The breathing is considerably affected; the symptoms become somewhat alarming, when suddenly the abscess bursts and matter gushes from the nostrils. The animal is immediately relieved and recovery soon follows :

Treatment:-Place the patient in a clean, comfortable stall devoid of drafts, and blanket well; then if possible, administer a pint and a-half of raw linseed oil, to be followed with, 
Nitrate of Potassium..... two ounces.

Sweet Spirits of Nitre.... two ounces. Tincture Aconite........ one drachm. Water............ to make one pint. Mix.

Give one ounce every two or three hours until the fever subsides, then give three or four times a day. Apply linseed meal poultices all around the throat, well up to the ears, changing them daily, and while changing apply quite freely to the throat,

Sweet Oil............ four ounces.

Turpentine ............ two ounces.

Aqua Ammonia........ two ounces. Mix.

Ferd on soft bran mashes or boiled food, and keep a hucket of cold water within reach of the animal, contimuonsly. If no abscesses form, the recovery will be rapid, or in case abscesses do form, as soon as they burst, recovery immediately follows. Two or three weeks frequently pass before the rupture takes place. Should the symptoms become rery alarming, the abscesses should be opened internally, and this requires the skill of a veterinary surgeon.

\section{SPASM OF THE DIAPHRAGM-THUMPS.}

The diaphragm is the principal muscle used in breathing, and is situated between the lungs and bowels, forming a sort of partition between them. Spasm of this muscle is caused by extreme and prolonged speeding upon the road or race track, also by hauling heavy loads a long distance on a hot day, or by doing any serere work on a hot summer day. The severe strain or orer taxation of this muscle causes an irritation of the nerves controlling it, and riolent and forcible contractions follow, which may be observed by 
a jerk or a thump in the region of the chest. It is sometimes mistaken for violent action of the heart, but by feeling the pulse and watching the thumping, it will be discovered that they do not act together.

Treatment:-If nothing better is convenient, give six ounces of whisky in a pint of hot water, repeating in one hour; or,

Chloroform............six drachms.

Whisky............. four ounces.

Water.................. pine pint. Mix.

Give at one dose and repeat in one hour if necessary, or,

Chloral Hydrate......... one ounce.

Sulphate of Morphia.... forty grains.

Water..................

Give half and repeat in one hour. After you have controlled the spasm, allow absolute rest for a week or ten days. If when put to severe exertion the thumps return, the animal should be worked either at very slow, light work, or turned to pasture for six months.

\section{HEAVES.}

This disease very closely resembles asthma in man, but is more continuous in its symptoms. One of the principal causes is over-feeding on clover hay ; but overfeeding with any bulky food, causing overloading and distention of the stomich, will produce heaves. Diseases of the lungs are also said to be a cause. I believe that large horses with small chests are more susceptible to this disease than those with large chests. Therefore, from mares that breal such colts with small chests, developing heaves at the period between six and ten years of age, the disease truly may be said to be hereditary. 
Symptoms:-No ferer, but short, hacking cough; sometimes discharge from the nose of a mattery substance; heavy, abdominal breathing, with a double lift of the flank at each inspiration; a wheezing sound is made when the animal is put to work; breathing very difficult on sultry, foggy days; the animal usually eats dirt and filth, and, invariably, when the animal coughs, wind will pass off from the bowels.

Treatment:-Keep the animal out of the stable both winter and summer and let it run on pasture as much as possible. When fed, dampen both hay and grain. If stabled, keep in a cool, well aired stahle. If the following recipe is followed daily, it will be of much benefit in checking the cough, which greatly benefits the animal :

Foenugreek...........two ounces.

Gentian ...............two ounces.

Ginger................two ounces.

Tartar Emetic..........two ounces.

Spanish Brown..........two ounces.

Sulphate of Morphia...... sixty grains.

Brown Sugar.......... four ounces. Mix.

Give a tablespoonful three times a day until the disease is checked; then give a spoonful once a day or every other day. Always gire the medicine in soft food; or give two ounce doses of raw linseed oil twice daily, to keep the bowels loose.

\section{LYMPHANGITIS, INFLAMMATION OF THE LYMPHATICS.}

This is a constitutional disease and most frequently occurs in fleshy, heavy-legged horses. It is caused by heavy feeding and want of proper exercise. Hence it is generally seen on Monday morning. The heavy truck horses are worked all week and highly fed, and 
on Sunday they receive their usual amount of food. As the system is not working enough to carry ofl the wasto material, the lymphatic glands become gorged and inflammation is the result.

Symptoms:-If seen at first, there will be noticed a (hill, with fever rising immediately afterward; pulse ranging from forty to seventy, hard and cord-like under the fingers; the horse is lame, either in the fore or hind leg, most freqently the latter; if the lameness is in the fore leg, press upon the inside of the fore leg, in the brachial region, close up to the breast, and the horse will evince great pain; if in the hind leg, press in the inguinal region, close up in the groin, and from there to near the stifle, and the horse will be so pained that he will raise his leg sideways, so high that he will almost fall over. The bowels become constipated and the urine scanty. The symptoms usually increase for twenty-four to thirty-six hours, then commence to abate. Occasionally, the lymphatic glands, high up on the inside of the leg, suppurate and pyemial supervenes and proves fatal. In severe cases, the skin remains hard, with a fibrous growth, which is known by the name of elephantiasis, or big leg.

Treatment:- If the case is a mild one, exercise moderately and apply hot fomentations to the parts affected. A recovery may be expected in two days. If the case is very severe, give immediately, or as soon as possible,

Barbadoes Aloes.......... one ounce.

Ginger...............two drachms. Mix.

Give in half a pint of water at one dose. Then apply fomentations of hot water and vinegar to the affected parts for twenty minutes, every two or three hours; after fomenting, wipe dry with a flannel cloth. 
As soon as the physic has operated, give two or three times a day, the following:

Powd. Potass. Nit.......... one ounce.

Bi-Carbonate Soda......... one ounce.

Resin ................ one ounce. Mix.

Make into twelve powders. As soon as the horse can walk with any comfort, take him out and exercise moderately. Should there be any swelling left in the groin or brachial region, apply tincture of iodine every day for a week; then cease for a few days and then repeat. Should there be a tendency to "big leg" (which frequently follows this disease), use the iodine freely and give the following:

Iodide of Potassium......two ounces.

Nux Vomica.............. one ounce.

Powd. Sulph. Iron ........ one ounce.

. ke into fifteen powders and give one powder at each meal. If that should fail, use a good blister along the lymphatic chain.

\section{STOMATITIS, OR INFLAMMATION OF THE MOUTH.}

This disease has various causes, such as stings of insects, snake-bites, injuries from the bit, injuries from pulling ropes through the mouth, giving medicines not thoroughly diluted, decayed or irregular teeth, etc.

Symptoms:-Swelling and redness of the mucous membrane lining the mouth; often small blisters will form in the mouth, allowing a discharge of serum, then look raw with ragged edges; sometimes several of these coalesce, forming a large sore; difficulty in swallowing; slavering; often a fœetid saliva dropping from the mouth. 
Treatment:-If the cause is apparent, remove it; if from any corrosive agent, wash the mouth with almond oil, sweet oil, or dilute glycerine. If the teeth are out of condition, attend to them at once. If the mouth is very sore, use a paste made of glycerine and prepared chalk and gum arabic; apply to the sores several times a day; or if ulcers are present, use a solution of chlorate of potash or alum, one ounce, to a pint of water. Wash the mouth three times a day with an ounce of the mixture. If the ulcers are very deep and not inclined to heal, mix thirty grains of nitrate of silver with an ounce of water and touch the sores with it every other day. Feed on soft food and give a saline cathartic, as,

Glauber Salts...........ten ounces.

Ginger............... Mene drachm. Mix.

Give at one dose in a quart of water. Also give drachm doses of nitrate of potassium, three times a day.

\section{TONGUE INJURIES.}

The tongue is a very important organ to the horse, for without its assistance, the act of eating and drinking is performed with great difficulty. The tongue is subjected to many injuries, as being pulled out forcibly to one side of the mouth by ignorant persons, while administering medicines or dressing the teeth; by being cut while rubbing against sharp edges of the back teeth, or against split teeth; by being torn with bit and halter chains. By falling and striking on the chin, the tongue may be badly bitten and it may also be injured by the breaking of a thin glass bottle in the mouth or by a bottle with a sharp or broken edge, used in drenching. 
Treatment:-Injuries to the tongue heal very quickly when the cause is removed. If the wound is caused by sharp edges of the teeth, dress them off with a file. If from other sources, and the injury is not sufficient to amputate the parts, dress the wound with the following:

Borax ............... half ounce.

Powd. Alum ............ half ounce.

Water................ Mix.

Apply to the sore three or four times daily. Before amputation is resorted to (which should be done by a veterinary suryeon) satisfy yourself that the parts will not heal or will cause great inconvenience, for it must. he remembered that the tongue may be almost cut or torn off, yet it will heal and be of great service. If eurly seen, a wound may be stitched together with gond results. Feed on soft, sloppy diet, and do not give hay or grain, unless it has been boiled sufliciently to soften it.

\section{LAMPAS, OR SWELLED GUMS.}

This is a red or swollen state of the upper gum and may he called moperly dentition fever. It usually is seen in colts from one to five years old, and is caused princiJally from teething; but may occur at any age from indigestion.

Treatment:-If in an old horse, give at one dose the following physic:

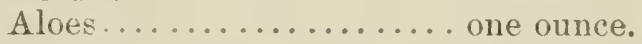

Ginger............. one drachm. Mix.

Afterward give a vegetable tonic to improve the direstion. If in a colt, scarify the gums,-but do not gu back of the second crease in the mouth. Feed corn on the ear and give, 
Nitrate of Potassium....... one ounce.

Chlorate of Potassium..... one ounce.

Bi-Carbonate of Soda.... two ounces. Mix.

Make into sixteen powders and give one powder at. each meal in the drinking water. If the bowels are costive, give a physic, -as one pint of castor or raw linseed oil.

\section{ENTERITIS, OR INFLAMMATION OF THE BOWELS.}

Causes:-Drinking ice-cold water, standing in drafts, giving too powerful cathartics; feeding new oats, new corn, or musty grain or hay; heavy fatiguing work, indirestion; spasmodic colic, when not properly attended to.

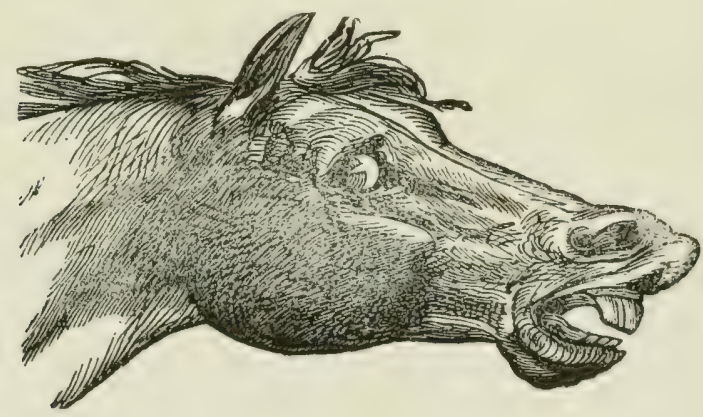

INFIAMMATION OF THE BOWEIS.

Symptoms:- The symptoms rery much resemble spasmodic colic; the animal will paw and lie down,very carefully at first; as the dise:tse advances, he will lie lown and get up frequently, roll upon his back, lie for a short time in that position, then hegin: tumbling around. The pains are very severe, and continuous; whereas, in spasmodic colic there are frequent intermissions. The membranes of the nose, mouth and eyes are 
congested and reddened, the month is hot and dry, respirations are increased, with a desire to drink small amounts of water. The legs and ears get cold. The pulse at first full and fast-ranging from sixty to seventy, very soon becomes smaller and weaker. Cold sweats break out on the body; an anxious expression of countenance appears; glassy, staring eyes; upon

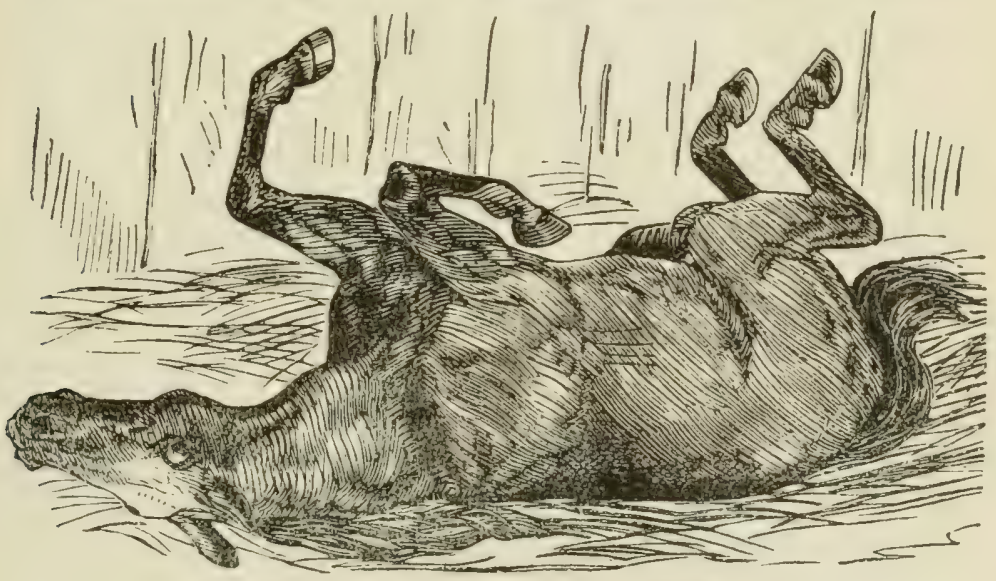

INFLAMAITION OF THE BOWHLS.

applying pressure to the abdomen the animal will evince pain. The bowels are generally costive but oceasionally jass small pellets of dunw senerally glazed with mucous () streaked with blood: but if occasioner by irritant food or medicine, purying and bloating may be present.

Treatment:- If the pationt is seen during the early stage, bleeding from the jumular rein, until an impression is made on the pulse, will often give relief. Give immediately-

Powd. Opium.......... one drachm.

Extract Belladonna...... one drachm. Mix.

Apply hot fomentations to the abdomen. Rub the legs with the following: 
Alcohol............... four ounces. Ext. Witch Haze!........ six ounces. Aqua Ammonia......... two ounces. Mix.

Apply bandages and if the pain continues, give drachm doses of powdered opium every thirty or forty minutes, until it ceases. Then apply the foregoing liniment to the abdomen and keep the patient well blanketed. Keep the bowels quiet, it makes no difference if they do not move for two or three days. Feed on light, soft food, as bran mash, linseed meal, etc. In the course of two or three days give sulphate of soda, eiglit ounces, and continue giving it morning and evening until the bowels move. Give walking exercise, and oceasionally warm injections to assist in moving the bowels.

\section{MUC0-ENTERITIS.}

This is inflammation of the lining membrane of the bowels. It is seen quite frequently in this country, though usually confined to localities where there is poor drainage and the hay and corn-fodder become musty; also where the stabling is poor and the horses are exposed to the inclement weather. Other causes are giving brisk eathartics, drinking impure or stagnant water, etc.

Symptoms:-The first symptom noticed, generally, is a staring coat; others are dullness, loss of appetite, and fever; sometimes pawing and lying down; tenderness of the aldominal region. The animal looks gaunt; abdomen is drawn up. Sometimes there is diarrhœa but more frequently constipation. Quite large balls of manure are passed, coated with mucous, sometimes very stringy like a large bundle of worms. 
Treatment:- When first noticed, give one quart of castor or linseed oil and change the diet immediately, give a gruel made from linseed meal or slippery elm tea. After the physic has operated, give the following:

Sulphate of soda........ one pound.

Quinine.............. four drachms.

Nux Vomica........... one ounce. Mix.

Make eight doses and give morning and evening. If diarrhoea sets in spontaneously, let it go twenty-four hours unchecked, as it is frequently beneficial. If the animal is suffering much pain, give ounce doses of laudanum until relieved; then immediately cease giving opiates and give more castor or linseed oil to move the bowels.

\section{SUPERPURGATION.}

This term is used to designate a diarrhøa or flux from the bowels, which is superinduced by and follows the administration of a physic. There is always considerable irritation of the bowels, and not infrequently inflammation supervenes, which latter symptom should always be looked upon with suspicion, as it is quite dangerous. The disorder is caused by giving too large doses of physic or by administering physic to horses suffering from debilitating diseases, as influenza, strangles, pneumonia, etc.; by driving horses some distance while purging, or by giving large quantities of cold water while a physic is operating. Great care and judgment should be exercised in giving physic to horses while suffering from diseases of the throat and chest. Before giving a physic, the animal should be prepared by giving two or three warm, soft bran mashes; immediately follow with the physic and after 
it hegins to operate, clothe the body and keep it warm, give small quantities of water slightly warmed and allow perfect rest until the purging ceases. After the horse has purged for twenty-four hours, the frequent evacuations may usually be stopped by feeding dry hay and oats, but should the purging still continue, give flour and water, or four ounces of brandy in a quart of milk to which add four eggs; repeat if necessary, five or six times a day and if this does not check it, use the following :

Tincture Catechu........ four ounces.

Laudanum ........... four ounces.

Tincture Ginger......... four ounces. Mix.

Give three ounces in half pint of water every three hours; restrict the drinking water and feed upon good hay and oats. If this manner of feeding constipates, give a pint of raw linseed oil, afterwards.

\section{CONSTIPATION.}

This is a condition of the bowels, where the fecal matter is abnormally retained, and when passed, is small in amount and hard in consistence. Constipation can hardly be considered a disease of itself but rather a symptom of a diseased condition, brought about by feeding upon bulky, innutritious, dry, fibrous food for a continuous length of time. The stomach and bowels become weakened, there is a dimunition in fluid material from defective secretion, and the dry ingesta contained within the intestines, causes a partially paralyzed condition of the muscular fibre of these.organs, with"loss of peristaltic action, which if not relieved, would bring on congestion or inflammation of the bowels. 
Symptoms:-Among the first symptoms noticed, is a gradual falling off in flesh, coat staring, distended abdomen or pot belly; swelling of the limbs, which disappears upon exercise; general lassitude and weakness, difficulty in passing manure, which is small, dry and hard and passed with much straining; appetite beromes imraired: pulse, small and weak, and a foul smell emanates from the mouth. In some instances there are colicky pains, but these are very rare.

Treatment:-The first step in the treatment of constipation, is to remove the cause. Place good, soft, nutritious food within reach and avoid giving drastic cathartics. If bran mash will be eaten, throw in a liandful of sulphate of soda or sulphate of magnesia, once or twice a day. Groom well and give moderate exercise. If the ailment does not yield to this treat ment in a few days, give a quart of raw linseed oil at one dose. After the bowels have responded to the above interference with medicines, they are liable to drop back into a torpid condition, and to relieve this, use the following:

Barbadoes Aloes......... two ounces.

Asafœetida...............two ounces.

Nux Vomica............. one ounce. Mix.

Make eight powders. Give one powder morning anul evening, for ten days, and allow all the water the animal will drink.

\section{INTESTINAL OBSTRUCTIONS.}

Accumulation of stercoraceous material in the intestinal tube, is sometimes noticed in horses. It consists of concretions of various kinds adhering to the walls of the intestines, sometimes assuming immense proportions before any symptoms of intestinal trouble are 
noticed. The position of these large concretions is in the large bowels, where they may remain for years, undisturbed, except perchance, the animal may have an attark of colic, and during the paroxysm of pain, while rolling and tumbling about, may displace the concre-

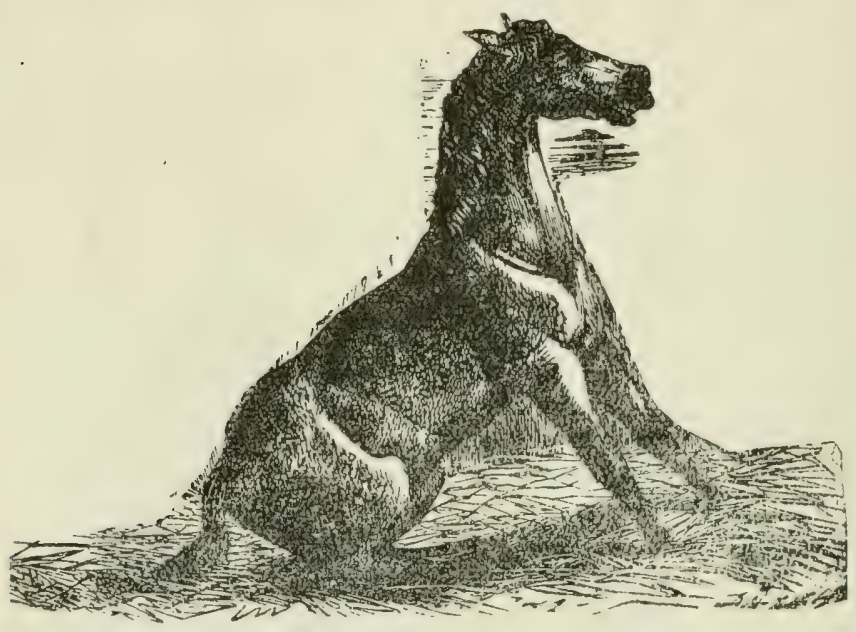

SYMPTOM OF INTESTINAL OBSTRUCTION.

tions and they may then become lodged in some other part, when grave symptoms are observed. Under this head, will also be included such troubles as invagination, where one bowel slips into another; strangulation of an intestine by another rolled round it, tumors and abscesses formed within the bowel, etc.

Symptoms:-When obstruction is complete, there will be severe colicky pains, without intervals, when free from pain, differing from inflammation of the bowels in that there is no rise of temperature at first. There may be slight diarrhœa, at first, which soon disappears, as only that manure will pass which is behind the obstruction. Frequently, the horse will sit upon its haunches, 
or if standing, press its haunches aninst the wall. Constipation or irregularities of the bow els, with occasional at tacks of colic, indicate intest inal concretions, and obstruction from accumulations of material, of whatever sort.

Treatment:- Whenever obstrution of the intestines is observed from the formoing symptoms, make an examination through the rectum, or last bowel; grease the hand and arm thoroughly, then pass it carefully, and if you can reach the obstruction, try to remove it. It is not advisable to give any physic in such cases. On the contrary, give medicines to relieve pain, and trust the rest to nature. If the animal will eat anything, give only soft, nutritious food, in limited quantities. To relieve the pain, use the following:

Chloral Hydrate......... two ounces.

Water.............. eight ounces. Mix.

Give two ounces every hour, in a pint of water until relief, or,

Laudanum ........... four ounces.

Tincture Belladonna......two ounces.

Sulphuric Ether........ two ounces. Mix.

Give one and one-half ounces every hour in a pint of water until reliof. If the pain is severe and bloating is obscrved, tapping the bowel with a trocar, and allowing the gas to escape, will give temporary relief. If invagination has taken place, the bowel may grow together and that position invaginated, slough off and pass away with the manure.

\section{DIARRHOEA.}

This disease is quite frequently met with among horses.

Causes:- living too heavy cathartics, drinking impure water, eating tender, green grass in the spring; 
indigestion, drinking ice-cold water, over-driving and watering while heated, exposure to cold rains; any irritant within the bowels; eating monldy or musty food, diseased condition of the teeth, pasturing on low marshy ground, exposure during cold nights, low damp stables, etc.

Symptoms:-There may be a soft discharge from the buwels, which may be completely overlooked for a considerable length of time because the animal retains its appetite; it may terminate in complete vecovery. But in the more severe cases, a watery discharge comes flooding from the bowels, with loss of appetite, staring coat, paleness of the mucous membranes, sunken eyes, unsteady or staggering gait, rapid breathing, small, weak and rapid pulse, rumbling of the bowels, - if the ear is applied to the side a rmmbling or churning sound is heard.

Treatment :- If the discharge is slight, give a pint of castor oil, which will generally effect a cure. But in the more aggratiated form, give a pint and a half of castor or linseed oil and one ounce of laudanum. Change the diet to good, nutritious food. If the purging continues, give wheat flour in water, starch water or white oak bark tea. If there is much bloating accompanying the diarrhoea, give a tablespoonful of baking soda three times a day, or give every four hours, two tablespoonfuls of the following:

Prepared Chalk......... four ounces.

Powdered Catechu......... one ounce.

Powdered Cinchona........ one ounce.

Powdered Gentian.......two ounces. Mix.

Allow a horse to stand for a few days before putting to work, as a second attack is worse than the first. 


\section{DYSENTERY.}

This is an agcravated form of diarrhea, tending to inflammation of the bowels. The "auses are like those of diarrhea,-such as feeding musty hay, drinking impure water, strong purgatives, exposure to cold rains; by being erowded in low, damp, dark stables; by grazing for a long time on low, wet, marshy pastures.

Symptoms:-Excessive watery discharce from the bowels, which has an offensive odor; the discharge is frequently streaked with hlood and passed with considerable straining; shreds of mucous membrane and blood are passed; the horse lies down a great deal; the appetite remains fair, and there is an unusual desire for drir' 'ng water; the ablomen is tucked-up and very tender; staring coat; sunken eyes; the animal soon becomes very weak; dejected appearance, generally.

Treatment:-First place the animal in a dry, warm, well-ventilated stall: brush and rub the skin thoroughly two or three times a day ; blanket well and bandage the legs: give good, pure water in small quantities and give at first,

Castor Oil.............ten ounces.

Laudanum..............two ounces. Mix.

Give at one dose; also,

Milk.................. que quart.

Whiskey ............ four ounces.

To which add four eggs.

Give three times a day. Give the patient soft and easily digested food, as linseed meal gruel, slippery elm gruel, etc. Administer the following:

Powd. Opium ........ one-half ounce.

Powd. Catechu............ one ounce.

Powd. Kino................ one ounce.

Prepared Chalk........ four ounces. Mix. 
Malie six doses and every four or five hours give one in : little sweet milk. Rub the abdomen with a cantharides liniment, or take mustard, mix with vinegar to form a paste, and apply to the abdomen. As the bowels begin to act better and are more regular and more nearly normal in their discharges, give,

Dover's Powder............ one ounce.

Quinine............ four drachms. Mix.

Make eight powders and give one powder at each meal. Bandage the legs and rub them with dilute alcohol; also keep the animal well blanketed and warm.

\section{GASTRITIS, OR INFLAMMATION OF THE STOMACH.}

Inflammation of the mucous membrane lining the stcmach, is rarely seen as an independent affection and is probably only seen as the result of irritation from impaction of the stumach, from over-feeding or overeating when fatigued, getting loose in the stable and having access to the grain hin, from foreign substances

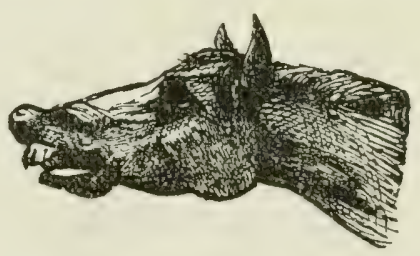

SYMPTOMS OF MNFLAIMATION OF THE STOMLCH.

given in the food, the administration of powerful and irritating drug without being sufficiently diluted with water or oil, by giving an over-dose of arsenic and by poisoning with lead. If horses are around newlypainted buildings or where paint kegs have been thrown away, they will sometimes lick and eat a sufficient quantity to produce gastritis, and not infrequently it 
results from drinking water which runs through new lead pipes.

Symptoms:- When occurring from inpaction and over-eating, colicky pains of a continuous character are usually observed. There is rapid breathing with a hard, wiry pulse, turning of the head around pointing to the stomach, and sometimes sweating along the neck, shoulders and sides. The desire for drinking water is somewhat intense and a turning up of the upper lip is sometimes observed. When arsenic is the cause of inflammation of the stomach, there will be loss of appetite with nausea and attempts to vomit, a desire for water and a frothy saliva in the mouth. Abdominal pain is present, a rumbling in the bowels is heard and sometimes diarrhoa supervenes with heary straining. The manure that is passed has a very offensive odor, sweat bedews the body, while the limbs are coll. The breathing is short and rapid, the pulse soon becomes very feeble and in a short time camnot be detected. Following the symptoms very closely, will be paralysis of the hind extremities and death. If the inflammation is caused by lead poison, colicky pains will be observed, with labored breathing, staggering gait, general paralysis, couvulsions and death. If there is chronic poisoning by lead, there will be an unthrifty appearance, diminution of appetite, a blue line extending around the margin of the gums, puffy swellings beneath the jaws, constipation, and a rough, staring condition of the hair. Abortion frequently occurs during chronic lead poisoning.

Treatment:-When we observe a case of inflammation of the stomach, arising from any cause, it is well to bear in mind that by reasons of the structures involved, its course is very rapid and also very fatal, although I believe there are more recoveries from this affection 
than from inflammation of the bowels. Soothing remedies should be given as quickly as possible and the stomach held in a state of repose. For this purpose give flaxseed tea, slippery elm tea, thoroughly boiled out meal gruel, etc., to which should be added as soon as can be obtained,

Powd. or Gum Opium...... one ounce.

Soft Water............ one quart. Mix.

Place over the fire and simmer down to one pint, then give two ounces every two or three hours. Hot applications along the abdomen just under the stomach, are attended with beneficial results. For this purpose blankets wrung out of hot water and held in that position for a few hours, is a good method, changing every few minutes to maintain the heat. After ceasing the Lot applications, a mustard draft should be applied around the region of the stomach. When the severe symptoms have subsided and the patient appears somewhat improved, administer a pint of raw linseed oil, which will assist in unloading the stomach of retained material and possibly the offending agent. When the whending agent is arsenic, give half dozen raw eggs well-beaten up with milk, raw linseed oil or linseed wruel every half hour for a few hours, or the administration of powdered charcoal, will be attended with beneficial results; or if obtainable, give two-ounce doses every ten minutes for an hour, of freshly prepared hydrated peroxide of iron. This is really the best antidote for arsenical poisoning. If the inflammation is caused from lead poison or any other of the irritant poisons, treat as above recommended, with milk, eggs, vil, linseed or slippery elm tea, etc., omitting the preparation of iron. But constantly bear in mind that the simach should receive soothing remedies, as the linseed sruel, etc., for a continued length of time. After 
recovery has taken place, feed only upon boiled fond and in limiter quantities, for a few months, or if in the proper season, turn out to grass.

\section{GORGED STOMACH-STOMACH STAGGERS-INDIGESTION.}

This derangement of the stomach is caused by teamsters feeding their horses a large feed after a heavy day"s work. The animal being very hungry, eats rapilly ; the food is not well masticated, and going into a strmach somewhat weakened by the day's work, is unable to digest it and indigestion is the result. It may

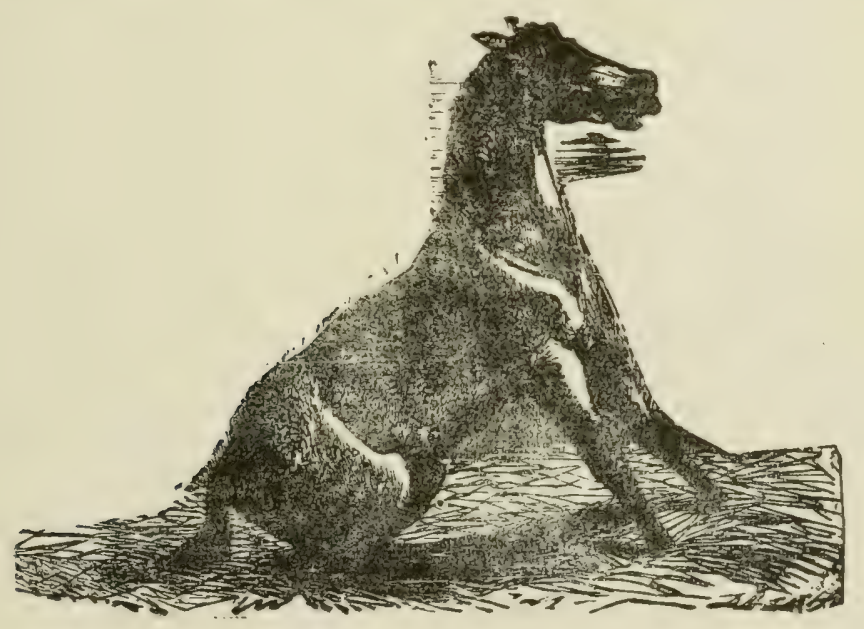

SIMPTOMS OF RUPTURE OF THE STOMACH.

also arise from eating damaged wheat, dried ripe vetches, and from eating rapidly of bulky food after fasting, etc. When we consider the small capacity of the stomach of a horse, holding only about, three crallons, we need not be surprised to see indigestion, for 
when the stomach beconies gorged, it has scarcely any other exit except to pass on, as it is very seldom that the horse vomits.

Symptoms:-These are generally sudden, but not always. The animal may step back from the manger and lie down, apparently resting. Suddenly, it will rise and stand for a while, without eating; then lie down again for a time. Soon it arises and becomes more restless by pawing with its fore-feet and lying down upon its breast, rolling over to its side, then upon its breast again and trying to vomit, at which it may make frequent attempts, and sometimes a sour smelling liquid discharge will come from the nostrils. There are frequent eructations, or belching up of gas from the stomach. The animal may tremble considerably, and cold sweats bedew the body. A thick slobber may be seen around the mouth. The bowels are usually costive, but sometimes a sliglst diarhoea is present. The pulse and respiration are both increased. If romiting does actually occur, it generally presages rupture of the stomach; at this stage the horse may sit upon its haunches, which is a langerous symptom, and an unfavorable termination may be anticipated.

Treatment:- When first noticed, give a heavy physic. As the stomach is distended with both food and girs, its action must be aroused to throw oft its contents. The following will suffice very well:

Powd. Barbadoes Aloes.....one ounce.

Calomel................ dre drachm.

Gentian.............three drachms. Mix.

Give at one dose. If the pain increases, give ounce doses of sulphuric ether in half pint of water every hour, until four doses are given. Or, aromatic spirits of ammonia, one ounce every two hours until three or four 
doses hare been given. Blankets wrung out of hot water and applied to the stomarlh and bowels, often give relief. If the former physic is not convenient, give a quart of raw linseed oil or a cuart of melted hogs' lard, as the stomach must be unloaded hefore permanent relief is to be obtained. Injections of castile soap and hot water are beneficial. A moderate amount of walking exercise, will also be of benefit. After relief has been obtained, feed on easily digested food, for a few days; also give the following, as much benefit will be derived therefrom:

Powd. Barbadoes Aloes.... one ounce.

Assafoetida..............ne ounce.

Gentian................ one ounce. Mix.

Make eight powders. Give one powder each day, until all are giren.

\section{HEMIPLEGIA-PARALYSIS.}

This form of paralysis is not frecuentl, seen among horses. It is usually the result of some brain trouble, as tumors in the lateral ventricles, pressure upon the brain from fracture of the skull, softening of the brain from poison in the blood, inflammation of the brain from emboli obstructing the blool-flow through the brain, etc.

Symptoms:-The attack may be sudden, with complete loss of motion of one side of the body, the animal falling down and being unable to rise. Again, the standing position is retained, but movements are made with great difficulty. The lips on one side will hang pendulous, the tongue protrude from one side of the mouth and there is difficulty in swallowing food or water. The urine may pass away involuntarily. 
Treatment:- The treatment of paralysis is very unsutisfactory and flattering results must not be anticipated. If due to an inflamed condition of the brain, bleeding will be proper. Carefully regulate the bowels by feeding soft food, in which may be given four to six ounce doses of sulphate of soda or epsom salts, until the bowels are regulated. Hand rubbing of the affected limbs and a strong liniment applied along the spine, may assist in restoration to health. The following will suffice :

Raw Linseed Oil........ six ounces.

Aqua Ammonia........ three ounces.

Oil of Turpentine....... three ounces. Mix.

Apply until blisters are raised.

\section{JAUNDICE, ICTERUS, 0R YELLOWS.}

Jaundice is caused by suppression or re-absorption of bile and is more of a symptom than a disease. Any caluse which tends to disturb the functions or tissue of the liver, as disturbed circulation from congestion or intimmation, deposits or growths, pressing upon the bile duct, catarrh of the bile duct, wall stones, or impurities in the blood from specific fever, etc.

Symptoms:-The first that is generally noticer, is the yellowness of the eyes and membranes of the nostrils and mouth, instead of the pale pink, as is observed in health. The tongue will be coated and an offensive smell emanates from the mouth. The bowels are somewhat constipated, the dung is dry, of a light color and has an offensive smell. When there is no fever present, the appetite will remain very good and the pulse will be but little affected. But when fever is present, the animal will appear drowsy, with loss of 
appetite, and seems greatly exhausted with moderate work, in fact the animal acts as if it wished to be left alone and does not care to move. Occasionally a dry itching, scurfy state of the skin is observed.

Treatment:-First discover the cause, if possible, then remove it. If from congestion, endeavor as quickly as possible to unload the liver, by giving such remedies as will have a direct action, as,

Barbadoes Aloes..........ne onnce.

Calomel.............. one drachm. Mix.

Give at one dose. The object is to rid the system of the excessive amount of bile and it is best accomplisher in this way. When fever is present, avoid giving such brisk physic and endeavor to move the bowels by giving,

Sulphate of Soda......... . s z ounces.

Water................. one quart.

Or linseed oil, one pint daily, until the bowels are loose. If there is an acid condition of the stomach, as evidenced by eating dirt or licking the walls, wive two to four ounces of lime water, twice a day. If the patient is weak and slow of recovery, give nitro-muriatic acid, one drachm, moning and evening, well diluted in trinkine water, and freed light, casily digestorl food, as bran mas', ground flaxseed, etc.

\section{CONGESTION OF THE LIVER.}

This disease is more prevalent during hot weather than at any other season, and is also seen in low, damp, malarial districts. It is caused from over-exertion, after eating a rich, hearty meal; sudden chills following specilic fevers; faulty dinestion, especially when rich food is given, without exercise; changes from a cold to a warm, damp region; chills in cold, damp nights after hot days, etc. 
Symptoms:-The animal will appear dull and disinclined to move. An muratural appetite is evidenced by the desire to eat dirt or lick at the walls of the stable. The appetite for food becomes impaired. The bow's are costive, and what does pass, is hard, scanty, of a light color and smells offensive. The mucous membranes of the nostrils and mouth assume a yellowish tint, as is also seen around the eyes. Frequently an offensive odor is observed around the mouth. The pulse is usually full and soft and the breathing but little affected. Pressure behind the right shoulder, frequently produces pain and sometimes there is lameness in the same shoulder.

Treatment:-Bleeding is frequently attended with good results, providing the pulse is full and distinct. The liver should be emptied as quickly as possible, and for that purpose, use the following:

Barbadoes Aloes.......seven drachms.

Calomel.............. one drachm. Mix.

Give at one dose, either in the form of a pill, or in a pint of water as a drench, to be followed up with

Epsom Salts............. one pound.

Bi-Carbonate of Soda.... four ounces. Mix.

Make twenty powders. Give one powder, morning and evening, in bran mash, chopped feed, or as a drench. The diet should be light, soft food of easy di_ gestion and given in limited quantities. Cloths wrung out of hot water and applied along the right side, behind the fore-leg, are very beneficial, or good mustard poultices on the right side, over the region of the liver, will assist greatly in affording relief. 


\section{HEPATITIS-INFLAMMATION OF THE LIVER.}

Although mankind is subject to many disorders of this gland, it may sound strange to say that diseases of the liver rarely attack the horse. Gall stones which accumulate in the gall sack in man, and cause so much trouble, are never seen in the horse for the very good reason that there is no gall-sack attached to the liver. They do, however, sometimes form in the liver itself. Inflammation of the liver may be either acute or chronic. The acute form is readily recognized, while the chronic form can seldom be detected during life by even a skilled veterinarian; it is mainly guess work, and for this reason, it will be unnecessary to treat of the chronic form in a work of this kind. In the acute form there will be great dullness with more or less pain, but not of a severe type. The animal hangs its head but generally remains in a standing posture. The bowels are constipated and the dung balls are small and of a clayish color, being sometimes covered with a slimy mucous. The urine is scanty and dark colored. Pressure applied to the right side over the liver, causes pain, consequently if the animal lies down it will lie on the left side. Sometimes there is lameness in the right shoulder and after a few lays' illness, the white of the eyes and tongue, turn a yellowish color.

Causes:-The prevailing cause is feeding too great an amount of rich, stimulating food in hot weather without sufficient exercise. Horses that are kept for family or pleasure driving, or the old family pet which has been pampered and highly fed in a warm stable, are the best subjects for these attacks, hence it is more often seen in the city than in the comntry. It is also caused by injuries, as blows or kicks on the right side; from foreign substances being swallowed, piercing the stomach 
and entering the liver, as nexiles, etc. It is also caused by the presence of worms in the liver; by gall-stones forming therein and passing through the ducts to the bowels, and by inflammation of surrounding structures, which extends to and envelopes the liver.

Treatment :-If detected in the earliest stage, alistract four or tive quarts of blood from the jugular vein, but if this is not done early, refrain from hlood-letting altogether. Then give a physic:

Powd. Aloes.............. one ounce.

Calomel.............. one drachm.

Ginger............. two drachms. Mix.

Give at one dose in a pint of water; or,

Epsom Salts.............................

Glauber Salts.......... one pound. Mix.

Dissolve in a gallon of water and give one quart every two hours. Apply hot cloths to the right side from the back of the girth to the last rib; or apply mustard freely over the same sp zce. Medicines that act upon the kidneys, should now be given. To reduce the fever, the following will answer admirably:

Saltpeter................ six ounces.

Powd. Nux Vomica........ one ounce. Mix.

Make twelve powders. Give three powders daily. Change the habits of the animal by griving a limited supply of soft food, as boiled oats, hran mash, etc., and as the patient improves, give exercise accordingly, and see that it does not get into such a condition acain through want of exercise and too high feeding.

\section{PARAPLEGIA-PARALYSIS OF THE HIND LIMBS.}

This form of paralysis affects both hind limbs and may result from tumors pressing upon the spinal cord, from parasites in the cord, from broken back or loins, 
from softening of the cord, inflammation of the cord, from bony growths, the result of sprains, pressing upon the cord; from over-feeding on certain herbs, seeds and grains, causing indigestion, etc. The treatment, for which see Hemiplegia, is very unsatisfactory.

\section{PROLAPSUS ANI, OR EVERSION OF THE RECTUM.}

Eversion of the last bowel, is generally seen as a sequel of some other disease; as occurring during the act of parturition or in parturient apoplexy, or following up certain cases of colic when they are somewhat protrated and accompanied with constipation, or in torpiclity of the howels, macrompanied with any other disease, we will often observe protrusion of the last bowel, or rectum. In the majority of cases, it is observed in horses that are in poor condition and fed on dry, innutritious food, or young colts that have been kept upon dry pastures or forl, after stabling upon dry food containing a super-abundance of woody fibre. When protrusion has taken place, the animal will not evince much pain, only when wanting to pass manure, which is generally very dry and hard; it will then evince pain and uneasiness, but after the freces have passed, the uneasiness disappears, and the animal moves on as though nothing had happened.

Treatment:- When the protruded bowel is not very extensive and has been observed before strangulation has taken place, it is quite easily reduced. Clean out the last bowel with the hand, which should first be well greased, then oil the protruded howel and press it back into place by gentle manipulation, after which apply the following: 
Powd. Opium .......... one drachm.

Tannic Acid............. drene drachm.

Vaseline................. Mixe ounce.

Insert enough in the rectum, morning and evening, to cover the protruded part. Place the animal on soft, nutritious diet and give mild laxatives to keep the bowels soft, as,

Sulphate of Soda........two ounces.

Epsom Salts............two ounces. Mix.

Give morning and evening into feed or as a drench. Should the protrusion become strangulated and swollen, scarify with the knife, grease or oil well, and return carefully as it is possible to do under the circumstances. Sometimes it is necessary to place a truss or bandage around the anus, to keep the bowel in place, but if watched carefully, and whenever it protrudes slightly, returned with the hand, continuing so to do until the bowels become regulated, favorable results may be looked for without any further treatment.

\section{SALIVATION OR SLOBBERS.}

This consists in an excessive flow of saliva, and is more frequently a symptom of some other trouble than a disease of itself. It is often seen in connection with irregular teeth, injuries to the tongue, from indiscriminate use of lobelia, mereury, colchicum, wild mustard, etc. Second crop of clover is one of the great causes of slobbering; foreign substances becoming lodged between the teeth, as parts of wood, nails, corncobs, etc.

Symptoms :-Fever and persistent discharge of saliva, or frothy masses hanging from the mouth: frequent 
attempts at swallowing, great desire for water, and disturbed digestion, causing colic.

Treatment:-First discorcr the cause, then remove it if possible. If from eating some obnoxious herbs or plants, or from eating second crop clover hay, remove from such pastures, or if in stable, change food; examine the mouth carefully, in corn districts, as sometimes corn cobs become fastened between the tecth, causing considerable trouble. If from irregular teeth, file off the sharp edges. If from injuries to the tongue or small fistules in the mouth, wash the mouth with,

Alum ................ one ounce.

Chlorate of Potash....... one ounce.

Water..................... pine pix.

Gargle the mouth two or three times a day; if somewhat persistent, paint the glands below the ears, once daily with tincture iodine and give one drachm of iodide of potash, morning and evening.

\section{GLOSSITIS, OR INFLAMMATION OF THE TONGUE.}

It is very seldom we encounter inflammation of the tongue, separate from injuries or administration of drugs not properly diluted, but when glossitis does exist as an independent affection, the tongue will become swollen and painful and look hard and black. Difficulty in swallowing is a prominent symptom, with a tenacious saliva adhering to the tongue, that has a disagreeable smell.

Treatment:-From the inability or difliculty in swallowing, it is out of the question to treat internally, which if we could, would be of much benefit, as with laxatives, fever powders, etc. But as we cannot do this, our attention must be directed locally. Scarify 
the tongue with the knife, and bathe with warm water to encourage bleeding; or steam the mouth with the ordinary nose-bag. Also wash the mouth with.

Borax.....................

Chlorate of Potassium....half ounce.

Water................ one pint. Mix.

Bathe the tongue three or four times a day. If the swelling does not extend backward and involve the textures of the throat, within two or three days, a speedy recovery may be looked for. If there is a desire to eat and the animal can swallow some, give fiaxseed gruel or soft bran mash.

\section{PURPURA HAEMORREAGIA.}

This aflliction usually occurs as a sequel of some lingering or debilitating disease, as distemper, influenza, epizuotic catarrh, lung troubles, etc., or it may originate spontaneously, not as the result of some prior disease, but from badly ventilated stables, where animals are compelled to breathe vitiated atmosphere arising from decomposed urine and manure; also in poorly fed horses, and such that are subjected to exhausting work, being placed in cold stables when perspiring freely, without blankets; exposure to cold rains and wind storms, when perspiring freely. It is more frequently seen in cold than in warm climates, and is very seldom seen when kept in well ventilated, warm, comfortable, clean stables, even as the result of a debilitating disease.

Symptoms :-Although it is a non-intlammatory disease, the first symptoms observed, are swellings appearing very suddenly on any part of the body, head or neck, only to disappear and re-ippear upon some other part. These swellings are hard, painful and pit slightly 
upon pressure. In some cases, these swellings app: al" around the head, eyes, nostrils, mouth and throat to an alaming extent, causing great difficulty in hreathing and eating. Sometimes one or more of the limbs will swell considerably, the swelling being very uniform and ending abruptly as though a cord had been tied aromed it. Small resicles appear, sometimes aromol the hocks and fetlock joints; these hurst and discharye an anber colored liquid, which scalds the hair ofl wherever it flows. Whenever the skin breaks over a swollen part, it leaves an ugly, unhealthy sore with a great tendency to sloughing. The pulse is weak and often double. The bowels are usually constipated hut easily excited by puratives, and blood streaks are often mixed with the manure. Small spots of a purple color are observed in the nostrils; these spots often slough and leave a raw, unhealthy looking sore, which discharges a dark colored liquid, frequently stained with blood. The urine is high colored and has a strong ammoniacal smell and quickly underwoes decompuition. Sometimes these swellings take place within the internal organs and to such an extent as to cause death from internal bleeding.

Treatment:-First and above all things, attempt to remove the exciting cause; if from badly-drained, illventilated stabling, remore to a well-rentilated, warm, comfortable place, as pure air, light and wamth are very necessary. If constipation is observed, the howels should be regulated but do not give a severe physic. The following will suffice:

Raw Linseed Oil.......... one pint.

Tinc. Terchloride of Iron, five drachms.

Spts. Turpentine..........ne ounce. Mix.

Give at one dose, to be followed up with, 
Chlorate of Potassium.... four ounces.

Nitrate of Potassium....... one ounce. Mix.

Make nine powders. Give three powders each day in soft food, drinking water or as a drench. Continue giving the above prescription for ten days to two weeks but diminish slightly in the dose. In the course of a few days, get the following:

Sulphate of Iron.......... one ounce.

Dilute Sulphuric Acid...... one ounce.

Water................. six ounces. Mix.

Give half ounce well diluted in water, twice daily, alternated with the potassium powders. If the swellings do not yield to this treatment in the course of a week, omit the last prescriptiou and give,

Raw Linseed Oil......twelve ounces.

Spts. Turpentine........ four ounces. Mix.

Give two ounces twice daily, alternated with the potassium powders. If there is much debility and loss of strength, give two to four ounces of whisky, two or three times a day or spirits nitrous ether one and one-half ounces, three times a day. For the treatment of the local swellings, do not scarify much, but if necessary to assist in breathing or eating, scarify around the head and throat. Theswellings around the head and throat may be bathed with hot water, to which may be added tincture muriate of iron or carbolic acid. Do not disturb the swellings on the other parts of the body or limbs, as the less they are interfered with, the better. Feed anything the patient will eat and give plenty of pure, fresh water.

\section{DYSPEPSIA.}

Dyspepsia is sometimes seen in horses that have been highly fed for a continued length of time, without 
proper exercise, and being stabled continuously and not allowed the privilege of a run at pasture.

Symptoms:-The animal will present an unthrifty appearance, the hair will look rough and the animal will loose flesh, growing very thin and hide-bound. The manure has a disagreeable smell, is quite hard and yellow in color. There is partial loss of appetite, although at times there is apparently great hunger, which is appeased by a few mouthfuls devoured in a greedy manner. The animal does not appear to relish its food and is generally spoken of as being "off its feed."

Treatment:-First clear the bowels of what material they contain, by giving a physic, as, raw linseed oil, one yuart. After a free evacuation of the bowels, give a good tonic, as,

Red Pepper.............two ounces. Gentian..............three ounces. Sulphate of Iron.........two ounces. Nux Vomica............two ounces. Saltpeter.............three ounces. Bi-Carbonate of Soda.... four ounces. Mix.

Give a teaspoonful three times a day in soft food; if the horse will not eat it, take a teaspoonful of the powder and put it into a pint of water, heat it up, making a regular tea of it, and administer three times a day. Give a complete change of food, as boiled wats or barley and bran mash, also give roots, as carrots, turnips, mangels, etc. If during the season of green rrass, turn out to pasture for a few months. Always examine the teeth thoroughly, and if any trouble exists, it should be attended to before any other treatment is given. 


\section{INTUSSUSCEPTION AND GUT-TIE.}

The slipping of one portion of a bowel into another like the turning of the finger of a glove partly wrong side out, sometimes occurs, and is most frequently seen in the small intestines, although it may also take place in the large guts. The part that slips in, may extend but a few inches and again a number of feet. This accident is most likely to happen in horses that are suffering from pains or spasms of the bowels, as spasmorlic or tlatulent colic, inflammation of the bowels, diarrheri, dysentery, or anything that will cause abdominal trouble or pain.

Symptoms :-There is no one special symptom wherely the trouble may be determined or located. Unless the parts become strangulated, there may be no symptoms displayed, except constipation and even then there may be a few scant passares of manure. Colicky pains may be present and severe straining may be observed. If strangulation of the bowel takes place, the animal will sufler intense pain unless relieved, and die within a few hours, and even when the pain is relieved, it may linger for a few days and then die. Cases have been reported where one portion of a bowel had slipped into another and become strangulated; the part of the bowel thus enveloped gradualy sloughed ofl, so that the ends of the bowel grew together, and the discarded portion passed away with the manul' ; a complete recovery resulted. Such cases are very rare; however, they have served as a warning and guide in our tratment of this affection. In such cases as terminate fatally, just prior to death the animal will sweat profusely; the legs and ears become cold and there is an anxious appearing conntenance and an occasional sigh; for a brief period of time there is cessation of acute pain, then the animal falls to rise no more. 
Traatment:-As soon as convinced that the bowels are invaginated, give such remedies as will relieve the pain and keep the patient quiet and comfortable, as,

Laudanum.......... eight ounces.

Sulphuric Ether....... eight ounces. Mix.

Give two ounces in a pint of water every hour until relieved, then wive at intervals, suficient to ke(ep) the patient quiet; or give one drachm of powdered or gum opium every hour until there is relief from pain, after which, just often enough to keep the patient quiet and let nature work its course. The giving of physic in this affection is liable to be productive of a crreat deal of harm and should, therefore, be avoided, but should the bowels slip back to their place and resume their normal functions, except being somewhat constipated, which they sometimes do when liept perfectly quiet, the administration of a pint of raw linsecd oil three times a day until the bowels become relaxed, will be of much benefit and should be given.

\section{PERITONITIS, OR INFLAMIMATION OF THE LINING MEMBRANE OF THE ABDOMINAL CAVITY.}

Causes:-Exposure to cold rains when heated; castration; any surgical operation in which the abdominal walls are piereed; kicks or blows; abscess of the liver or stomach; rupture of the womb while foaling: abscess of the womb.

Symptoms:-Pain in the abdomen, demonstrated by the patient's turning and pointing to the side; on pressing the abdomen at or near the aflected part, the animal will evince great pain; on being made to move, it seems stiff and in pain. If the horse lies down, it does so very carefully. As the pain is increased while down, 
it usually maintains a standing position. The pulse generally ranges from seventy to ninety beats per minute and is hard and wiry. The abdomen is tucked-up; the breathing accelerated and short, and quite frequently the animal will bloat; in three or four days dropsy of the abdomen appears, when the severer pains will cease and the patient will breathe more deeply and freely.

Treatment:-If acute peritonitis is seen at the outset, bleeding freely from the jugular vein will frequently stop the progress of dimase. Then give fifteen drop doses of fluid extract :a. . . te every hour until the disease is under control, or give,

Powd. Opium............ one ounce.

Calomel............ four drachms. Mix.

Make into eight powders and give one erery four hours. Apply hot cloths to the abdomen but be careful that the patient does not take cold. Mustard poultices applied to the abdomen will have the same effect. Physic must not be given during this disease. If the disease arises from abscesses of the stomach or liver, or from rupture of the womb, stimulating treatment should be tried, though the termination invariably is death. Apply diluted alcohol to the limbs and bandige thoroughy. Plare the patient where no draughts can strike it and blanket well. Feed on soft mashes or linseed gruel, boiled oats or barley, in restricted quantities.

\section{BOTS.}

These are the larve of the different species of gadfly, which pester horses in the summer. They fly about horses and deposit their egors upon their lews and sides: and by the horse biting or licking itself, they are 
taken into the stomach, when they fasten themselves to the mucous lining of the stomach or of the upper part of the small intestine (duodenum.) There they develop into the grul, or bot and subsist upon the gastric or intestinal juices, and when once they lose their hold they seldom regain it. If hut few in numbers, they never
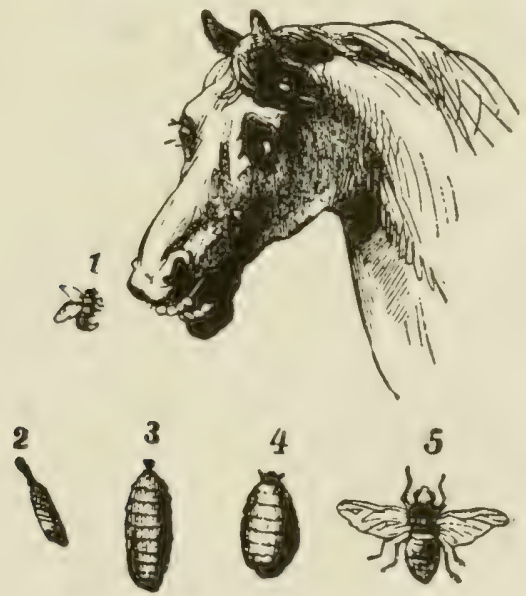

BoTs.

1.-Female Fly.

3. - The Bot.

2.-Egg.

4.-The Chrysalis.

5.-Male Fly.

do any harm. If existing in great numbers, they cause indigestion and colic; sometimes they almost stop up the duodenum. In such cases the horse will not thrive and will be troubled with indigestion; it will be weak and easily fatigued.

Treatment:-Feeding sliced potatoes is beneficial, or warm sage tea, two quarts. Follow it up with a brisk cathartic-

Aloes....................... ounce.

Ginger..............two drachms. Mix. 
This will sometimes carry ofl quite a number of the bots. But there is no remedy yet known which will destroy thr bot while in the stomach. In the early spring they pass away voluntarily and during the summer develop into the gad-fly.

\section{WORMS.}

A great many varicties of worms infest the intestines of horses. A thorough description of them is

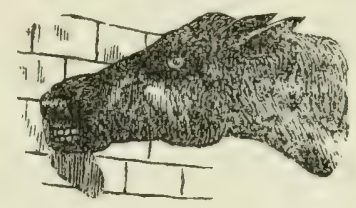

WORMS.

quite unnecessary. Suffice it to say they are of no bene. fit to the animal and the sooner they are expelled from the bowels the better.

Symptoms:-Rough, staring coat; ravenous appetite, rubbing the tail, loss of Hesh, big or pot-belly, licking

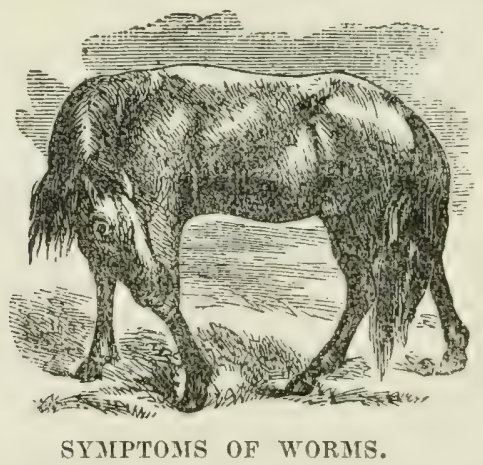

of dirt, pallor of the mucous membranes, sunken eyes, sometimes constipation, at others diarhora frequent 
switching of the tail; worms protruding or smished around the anus, leaving a white or mattery substance.

Treatment:-It is necessary to prepare the animal prior to niving any worm medicine. First, allow the animal to fast for twenty-four hours, then grive a soft bran mash, to be followed immediately with,

Barbadoes Aloes........... one ounce.

Santonine............. Mene drachm. Mix.

Give at one dose, either in pill or in half pint of water, as a drench. If this does not remove them all, repeat in five days, or you may use,

Raw Linseed Oil .... eighteen ounces.

Spirits Turpentine......... six ounces. Mix.

Give four ounces morning and evening. Immediately after the last dose is given, administer the following:

Barbadoes Aloes.......... one ounce.

Gentian............ two drachms. Mix.

Give at one dose in half pint of water as a drench. It is a well-known fact, that intestinal worms are mostly seen in horses that are in poor condition, and if allowed to remain in this condition, worms will rapidly ac'umulate again, even though they had all been expelled by the worm medicines. It is, therefore, necesiary to tone up the powers of digestion. This can be done with,

Ginger............... one ounce.

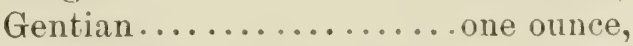

Nux Vomica........... one ounce.

Bi-Carbonate of Soda........ne ounce. Mix.

Give teaspoonful three times a lay in food or as a drench. Give good food and pure water and the animal will commence to thrive, then worms will cease accumuluting. A great many colts are lost every year from worms, when a few doses of prepared chalk wll remove 
them. Give a teaspoonful once a day for a week. If they become constipated, give four to six ounces of raw linseed oil. Prepared chalk given to aged horses, will assist in removing worms.

\section{DIABETES INSIPIDUS.}

Among the horses in this country, this disease quite frequently occurs and has various causes. Among them are feeding on musty hay or grain, exposure to wet and cold, blows on the top of the head, drinking ice-cold water while heated, eating too much malt from distilleries, the reckless administration of medicines that act upon the kidneys, new oats imperfectly cured, etc.

Symptoms:-An excessive discharge-amounting to several gallons within twenty-four hours, of almost colorless urine; excessive thirst; withholding water from the animal does not check the disease. Sometimes it comes on very slowly, at others it develops to an alarming extent within twenty-four hours; it varies in duration from a few days to months and even years. If it lasts for any considerable time, the animal will lose flesh rapidly. There will be pallor of the mucous memloranes, rough coat; skin drawn tight to the ribs. Death finally results from emaciation and prostration.

Treatment:-Change the diet to good, clean hay and grain, and give a liberal supply of boiled flaxseed in the drinking water. Also give,

Sulphate of Iron.........two ounces.

Gentian................. one ounce.

Nux Vomica............ one ounce.

Iodide of Potassium......two ounces. Mix.

Make into sixteen powders and give one powder at each meal. The drinking water should be restricted in quantity and of good quality. Give plenty of rest. 


\section{ALBUMENURIA-BRIGHT'S DISEASE.}

This disease consists of an inflammation of the kidneys either acute or chronic, with degeneration and shedding of casts of epithelium from the tubes of the kidneys. It occurs in horses of all ages.

Symptoms:-The horse walks with a straddling gait and passes a thick, ropy urine, which upon being boiled or tested with corrosive sublimate, acetate of lead, or nitric acid, coagulates in part in whitish thakes. The animal usually wastes in Ilesh; general health seems broken; dropsical swellings appear but vanish upon exercise.

Treatment:-Avoid any exposure to cold, wet or fatimue. Keep in a comfortable, warm box-stall and keep the body warm by blanketing. See that the bowels are kept loose by feeding on bran mashes or other soft food and if inclined to be costive, give a pint of raw linseed oil; also give half ounce doses of tincture chloride of iron three times a day, diluted in a pint of water. Use cloths wrung out of hot water, over the loins, frequently; if this does not assist in the flow of urine, give ounce doses of fluid extract buchu and juniper with acetate of potassium three times a day, but be persistent with the hot cloths, as this is better than giving medicines to act upon the kidneys.

\section{ACITES, ANASARCA, OR DROPSICAL SWELLING.}

Causes:-Obstruction to venous circulation, excess of water in the blood, disease of the heart, disease of the liver, disease of the kidneys, the action of cold and wet upon the skin, causing an arrest of execretion; but the principal cause, is neglected influenza; when it has con- 
tinued for some time, the system is debilitated and the blood is in a watery state.

Symptoms:-If following a prolonged attack of influenta or distemper, the blood is imporerished, paleness of the mucous membranes is observed around the mouth, nose and eyes; swellings appear, either at the breast and gradually spread backward under the belly, or commence between the hind legs or at the sheath, and gradually more foward. In the course of a few days, the whole of the lower part of the abdomen is swollen, so that it leaves a ridge on either side. The swelling pits, upon pressure, and if pricked with a knife, a yellowish fluid oozes out. The appetite is capricious, but hay will sometimes be fairly well consumed. The animal will lie down at first, but as the swelling increases, it will persist in standing, and if compelled to move, will walk quite stiff and sore. Pressure upon the swelling when it first appears, causes pain, but as the swelling increases, pain upon pressure diminishes.

Treatment:-First place the animal in a comfortable stall and bed well to entice it to lie down. Feed upon choice hay and grain. Give bran mashes to keep the bowels open. If the weather is cold, blanket well, and in the daytime turn out to exercise. Take a knife and make several small incisions into the swelling, to allow the serum to escape: or take a paddle and drive several small tacks through it, allowing them to protrude from one-fourth to three-eighths of an inch, then paddle the swelling with the sharp tacks; this will leave several small openings through which the serum will escape. Then give the following:

Sulphate of Iron.........two ounces.

Powd. Nux Vomica........ one ounce.

Gentian .............. one ounce.

Nitrate of Potass.........two ounces. Mix. 
Make sixteen powders. Give one powder morning, noon and night. If the urine does not pass freely, give the following:

Acetate of Potass....... four ounces.

Water.....................

Give four ounces every four or five hours. If the bowels are costive, physic witl,

Powd. Barbadoes Aloes.... one ounce.

Powd. Ginger.......... two drachms. Mix.

Give at one dose in half pint of water as a drench.

\section{HAEMATURIA, OR BLOODY URINE.}

Blood in the urine is not often observed in horses, but is frequently seen in both cattle and sheep. When seen in horses, it is generally caused by some violent strain or injury, as slipping while starting a heavy load or while rearing up and jumping, in this manner injuring or straining the psoas muscles, which are situated in the region of the kidneys below the back-bone. $\mathrm{O}_{\mathrm{r}}$ it may be caused by fracture of the back-bone over the kidneys, or from gravel in the kilneys or bladder, or in the channels leading from the kidneys and bladder, through which the urine passes. It may arise from (ongestion of the kidneys, or from ulcers and aloscesses: in the parts through which the urine passes, as well as in the kidneys, from the rupture of a small blood vessel in these organs; also from eating acrid plants which exert a powerful diuretic action. If the blood is coming from the kidneys, it will be uniformly mixed with the urine, but if from other parts. it may come away in clots or streaks.

Treatment:-Remove the causes if they can be ascertained and located. If from irritants in the food, 
change it immediately; if from gravel in the bladder, remove it; if from the rupture of a small blood vessel and it is within reach, tie it, and if from fracture or sprain, give the treatment required for these injuries. If the flow of blood is very great, apply cold water to the loins and keep the animal as quiet as possible. Give mucilaginous drinks frequently; as linseed and slippery elm tea; also give,

Tincture Chloride of Iron, eight ounces.

Water...............eight ounces. Mix.

Give two tablespoonfuls three times a day in half pint of water: or give a teaspoonful of sulphuric acid twice daily in drinking water, or ounce doses twice daily of spirits turpentine well diluted with raw linseed oil.

\section{PARALYSIS OF THE BLADDER.}

When the urine is withheld for a continued length of time, the bladder becomes over-distended and loses its contractile force, a condition which may last for a considerable period of time. It is generally brought on by some disease causing the animal to occupy a recumbent position, such as rheumatism, azoturia, etc. Many horses will fail or refuse to pass their urine while lying down, and all such cases should be relieved by having it drawn away twice daily with a catheter. When the bladder has lost its power of contraction, it is paralyzed, or partially so, and it will be observed that the urine dribbles away in small quantities, scalding all the parts with which it comes in contact. When causer by overdistension with urine, by keeping the bladler emptied, it will in the course of time, regain its contractile powors and normal condition. If it is caused by paralysis 
of the hind parts, while treating for this disease, do not allow the bladder to become distended but draw the urine away three times a day with a catheter.

\section{HYDROCELE-DROPSY OF THE SCROTUM.}

The scrotum is the sack or pouch which covers and contains the testicles. Dropsy, or a watery effusion within the scrotum, may arise from injuries to the parts, or disease of the testicles or lining membrane of the sack, and it may be the result of dropsy of the abdomen, as it has a direct connection with the abdominal cavity.

Symptoms:-There will be an enlarged condition of the scrotum, which organ will be soft and fluctuating under pressure of the fingers. The testicles will be felt floating in water. Sometimes the scrotum will retain the indentation of the finger after pressure. The liquid can be forced up into the abdomen by pressure; or by laying the horse upon its back, the fluid will gradually recede into the abdominal cavity.

Treatment:-If the efinsion of liquirl within the sack is very limited in amount, feed upon good, nutritious diet and give,

Jodide Potassium....... two ounces.

Water.................. one pint. Mix.

Give two tablespoonfuls morning and exening. But if there is a large amount of liquid within the sack, tap with a trocar and canula and allow the fluid to escape; immediately inject into the sack, one ounce of tincture jodine, and repeat this in a few days, if necessary. And in addition to the above preseription, administer a tonic, as, 
Sulphate of Iron........two ounces.

Nux Vomica............ one ounce.

Nitrate of Potassium......two ounces. Mix.

Make twelve powders. Give two powders each day. If the testicles are inflamed and enlurged, use locally,

Laudanum............. two ounces.

Goulard's Ext.......... two ounces.

Soft Water............ one quart. Mix.

Saturate a soft cloth and apply to the parts and keep. it wet with the above mixture. If it occurs in greldings as the result of injuries, dissect out the sacks and treat as an ordinary wound.

\section{LEUCORRHOEA, OR WHITES.}

Leucorrhœa consists of a discharge of mucous or natter from the mucous membranes of the vagina, from the neck of the womb, and not infrecuently from the womb itself.

Causes:-Generally irritation of the organ followed by relaxation, but relaxation alone will produce it. Want of nutrition attended with debilitating infiuences is a frequent caluse. Partial eversion of the womb or vagina, is also a cause.

Symptoms:-On opening the lips of the rulva, a sticky whitish or yellowish matter will be seen. Quite frequently it runs out of the ragina and accumulates on the tail and surrounding parts. As the disease progresses, the discharge emits an offensive odor, pulse grows feeble, mucous membrane pale, appetite partially suspended and a gradual wasting of the whole system. Mares affected with the whites are rery often secking a -male companion, but seldom ret with foal and when they do, they generally abort. Occasionally, to all 
appearances, an animal will be healthy and yet affected with the whites.

Treatment:-Feed on soft, nutritious diet, with tonies and stimulants, as,

Sulphate of Iron........ four urnces.

Gentian............ forir ounces.

Powd. Nux Vomica.......two ounces. Mix.

Make twenty powders. Gire one powder at each meal or as a drench. Use as an injection into the vagina or woml, as the case demands, twice daily, one of the following bowders dissolved in a quart of warm water:

Permanganate of Potassium, two ounces.

Sulphate of Zinc......... one ounce. Mix.

Make sixteen powders. Prerious to injecting, wash out the ragina and womb with warm water to remove all matter.

\section{STRICTURE OF THE URETHRA.}

This consists of a partial closure, at a certain place of the chammel through which the urine passes, and is caused by the healing of ulcers in gonorrhorat and whet, also ly using strong astringent injections in the treate ment of gonorhoa and gleet, or from irritating ingredients in the urine, as stone or crarel passing from the bladder.

Symptoms:-The trouble is demonstrated by the passage of urine in a fine stream. The act is accomplisher by hard straining; the animal will sometimes grom from pain; also painful erections of the penis are frequently observed. The passage of a small stream of urine, with straining and groaning" is sometimes noticed when the so-called bean is lodged in the end of the penis. 
Treatment:-Take a small sized catheter, oil it well and pass it beyond the stricture, using a catheter a little larger day by day, forcing it up past the stricture with rentle pressure, until the stricture has disappeared. Beyond the oiling of the catheter, there is no medicine necessary.

\section{NEPHRITIS, OR INFLAMMATION OF THE KIDNEYS.}

This disease is of infrequent occurrence but may be induced in various ways, - as by spraining the loins while pulling hard, carrying ton heary a weight, being

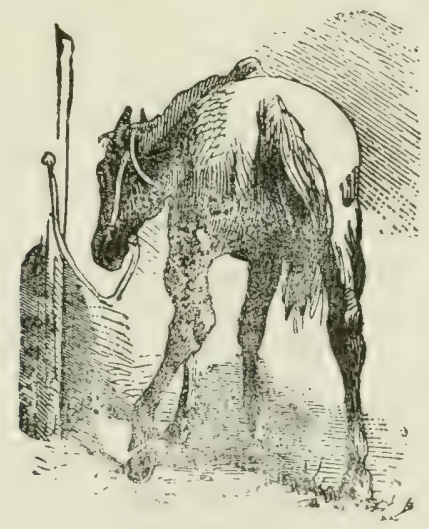

INFLAMMATION OF THE KIDNEYS.

heated and then exposed to the cold, absorption of cantharides when applied as a blister to large surfares, by the administration of severe diuretics, the presence of stone in the kidneys, etc.

Symptoms:-The animal walks with a straddling gait and with back "roached:" evinces pain similar to that of colic; if it lies down, will do so very carefully : small and frequent passiges of very highly colored urine; 
fever usually very hich, pulse cord-like to the fouch, and very fast; breathing very much accelerated: in the course of a few days, swelling of the limbs, which disappears upon exercising.

Treatment:- Put the animal in a good, comfortalile place, blanket warmly and persistently apply hot cloths across the loins. Give a good physic,

Sulphate of Soda.......... one pound.

Water............... two quarts. Mix.

()r give three pints of raw linseed oil, followed by drachm doses of Dover's powder, every three or four hours; or

Liq. Ammonia Acet....sixteen ounces.

Nitrous Ather..........two ounces.

Spts. Camphor..........two ounces. Mix.

Give four ounces every three hours to stimulate perspiration. Feed on soft diet and in a few days give fluid extract buchu juniper, with acetate potassium, half-ounce doses morning and evening. Keep the bowels loose all the time and give injections of warm water and laudanum every hour for a few hours.

\section{FOUL SHEATH.}

The sheaths of horses sometimes become very dirty, and should receive the owner's attention. The glands in the sheath secrete a fluid to lubricate the ports and when large quantities are secreted, it accumulates in the form of a black, gummy substance, filling up the sheath and preventing the animal from passing out the penis when urinating. Some horses seldom protrude the penis during the act of staling; when they do not, a sediment from the urine assists in creating a foul sheath. Sometimes when the sheath becomes foul, the 
p'nis becomes sore and scaly and the surrounding parts become swollen to a greater or lesser extent.

Treatment:-Take a sponge or soft cloth, some warm witer and castile soap and wash out the sheath thoroughly but carefully, and do not use too much force in drawing out the penis. It is well to cut off your finger nails to prevent scratching the parts. If the parts become injured while cleaning, oil with sweet oil, and should there be much swelling, bathe frequently with hot water and give the animal exercise. When washing out the sheath, always examine the end of the penis; here there is a small pouch just below the channel. through which the water passes; accumulations called beans, sometimes form and obstruct the flow of urine. These should always be removed.

\section{RETENTION OF URINE.}

This has various causes, - such as spasm of the neok of the bladder, calculus (or stone in the bladder) working its way into the urethra, paralysis of the neck of the bladder, over-distention of the bladder with urine. The more frequent cause is spasm. In spasmodic colic the neck of the bladder becomes constricted and is only relieved as the colic disappears.

Symptoms:-See treatise on colic for symptoms of spusm. From other causes the animal will walk with a straddling gait, frequently attempt to urinate, stand stretched out and groan when attempting to urinate; sometimes pain is so severe as to cause perspiration to break out all over the body; urine is passed in small quantities.

Treatment:- If from colic, treat for that. If from other causes, as calculus (stone in the bladder), pass 
the catheter and afterwarls remove the stone. If from spasm of the neck of the hlabler or from over-distension with urine, pass the hand per rectum and apply extract of belladonna, or give hot water injertions, or pass the catheter.

\section{CYSTITIS, OR INFLAMMATION OF THE BLADDER.}

Causes:-Retention and decomposition of urine, irritation by stone in the bladder; in the female, irritation and eversion caused by foaling; injudiraus use of diuretics; orasional heary and repeated blistering with cantharides; or application, by persons of little sense, of irritants to the neck of the bladder for the purpose of promoting a discharge of urine; irritation of a catheter or other foreign substance introduced from without, over-distension of the bladder with urine, sudden exposure while perspiring to cols and wet, or from disease extending from the vagina and urethra to the bladder.

Symptoms:-Frequent attempts to urinate, urine passed in small quantities, heavy straining; bearing. down pains, lying down and getiug up, quite frequently straining hard while lying; pulse not very much changed, fever ranging from $101^{\circ}$ to $104^{\circ} \mathrm{F}$.; if on pasture, the animal will wander around in a shiftless, uneasy manner. The penis hang from the sheath in the male and in the mare the vulva is frequently opened and closed, as after making water. Upon examination per rectum or vagina, there will be found a hard, round tumor-sometimes quite large, at others small-sensitive to the touch, and the neck of the bladder will be very much thickened.

Treatment:-Feed on soft, sloppy gruels or bran mashes. If there is intense pain, give drachm doses of 
powdered opium; also make a decoction of one drachm of powdered opium to one pint of hot water, and inject it into the bladder three or four times a day. Give internally, one quart of raw linseed oil; if that does not physic in twenty-four hours, repeat the dose. If the bladder is distended with urine, pass the catheter and draw the urine off. As the patient appears better and improving, give morning and evening half-ounce doses of thuid extract buchu juniper and acetate of potassium.

\section{GONORRHOEA-CLAP.}

In the male, this is inflammation of the urethra, and inflimmation of the vagina in the female. Its causes are unclean sexual intercourse, irritation by stone passing from bladder, injuries during copulation, or from passing a rough, unclean catheter.

Symptoms:-While passing urine the animal evinces pain; in the male, frequent erections of the penis; ocasional swelling of the testicles; aggravation by exercise; discharge of a yellowish white matter; occasional great swelling of the glans penis.

Treatment:-Frequently bathe the parts in hot water, give one quart of raw linseed oil, feed on soft mashes and good clean hay. Give drachm doses of balsam of copabia, morning and evening and use injections of hot water three times a day; or injections of-

Sulphate of Zinc.......twelve grains.

Water.............. eight ounces. Mix.

Or,

Sulphate of Copper..... eight grains.

Water............... eight ounces. Mix. Or,

Nitrate of Silver.........ten grains.

Water............ eight ounces. Mix. 
Use three times a day, any of these. If the testirles become inflamed, apply a poultice of tobacco.

If small ulcers make their appearance, fill them with calomel or cauterize with lunar caustic. Stallions afiected in this manner should be withheld, from service. Neither should mares be bred nor should they, if suffering from leucorrhoa or whites.

\section{EVERSION OF THE BLADDER.}

This occurs only in the female, and then only through excessive straining. Most frequently it hapjens when the patient is laboring rery hard to deliver herself of a colt, with a mal-presentation. If eversion tikes place, a red soft lictuating tumor will be seen, protruding between the lips of the vulva, varying in size from a goose egg to that of a sugar bowl.

Treatment:-If the eversion has just happened, take a smooth, round piece of hardwood about the size of your little finger, place it on the center of the tumor and press back through the channel through which it came; assist with your hand by pressing the tumor toward the center. If too large to return, wrap it tightly with a bandage to force the swelling down and when reduced enough, return as per direction given. Be very careful while returning, or you may rupture the bladder, when your patient is done for. After it is returned, bathe the parts with

Powd. or Gum Opium .... one drachm.

Warm Water............. Mix.

Give ounce doses of laudanum every two hours to prevent the straining. If the bowels become constipated, give one quart of raw linseed oil. Whenever you see the patient begin to strain, place your hand on 
the bladder and hold it firmly. Place the patient sc the hind parts will be a foot higher than the fore parts. A truss may be placed upon the patient, the same as for eversion of the womb.

\section{INFLAMMATION OF THE TESTICLES.}

This has various causes, among them blows, kicks from mares during copulation, excessive copulation, gonorrhea, catching cold while having distemper.

Symptoms:-Walks with a straddling gait; swelling of the testicles, accompanied by heat and tenderness upon pressure; loss of appetite; disinclination to lie down; sometimes the part is so painful that the animal breaks out in profuse perspiration; drawing up and letting down the testicle within the scrotum, etc.

Treatment:-Give the patient a good, comfortable, loose box-stall, well bedded, to entice him to lie down. Then give a good physic,-

Powd. Aloes...........nine drachms.

Powd. Ginger..........two drachms.

Water................ Mene-half pint. Mix.

Give at one dose. Then apply locally,

Goulard's Extract...... four drachms.

Laudanum........... four drachms.

Rain Water.............. one quart. Mix.

Keep the testicles wet continuously with the above mixture, by either putting it upon soft rags or cotton batting, and holding against the testicles by placing a suspensory bandage beneath them and tying it over the back. The bandage also acts as a support to the cord. Sometimes bathing the testicle with hot water and after each bathing applying solid extract belladonna and powdered opium, equal parts, or placing a boiled tobacco 
poultice in the bandage and keeping it on continuously, will give the desired result without further treatment. If pus or scrum can be felt fluctuating, make a free opening and allow it to escape. Then dress the wound with,

Carbolic Acid...........half ounce.

Water....................... pint. Mix.

Or,

Permanganate of Potash.two drachms.

Water................. one quart. Mix.

Apply two or three times a day. If gangrene takes place, castrate immediately, after which wash out the wound three times a day with the last prescription.

\section{CYSTIC CALCULUS, OR STONE IN THE BLADDER.}

Symptoms:-Frequent attempts to void the urine, oceasionally passing small quantities and suddenly stopping; blood may be passed in small quantities; on examination, per rectum, the stone, if of any size, will be found in the bladder; when the stones are small they sometimes will pass with the urine; occasionally hurd straining while passing.

Treatment:-This should never be attempted by an amateur, as it is not always successful in the hands of a professional. The operation is called lithotomy and is performed by cutting into the urethra in the perineal region, when the patient is a male. Previously pass the catheter and let it remain as a guide. Then pass a pair of spoon forceps, gently dilating the urethra, seize the stone and extract slowly. Afterwards close the wotsnd with sutures and treat as a flesh wound. ' In the female, cradually dilate the urethra, then pass the forceys, seize the stone and withdraw. In some cases the 
stone is very large: if so, introduce a pair of for and crush the stone, then remove the pieces. removing the stone, inject the bladder with warm w and a decoction of opium, three times a day for a days.

\section{INFLAMMATION OF THE MAMMARY GLANDS, OR UDDER.}

Causes:-Blows on the udder, lying on cold, ground, exposure to cold rains, neglect of milking, denly feeding rich food causing indigestion, etc.

Symptoms:-Loss of appetite, fever, hurried bre ing, constipation, shivering spells, sometimes the w bag is involved, but more frequently one-half quarter; the part of the bag affected, will present a appearance and be hard and painful to the touch; $t$ will be falling off of milk in that part and possibl the whole bag; frequently streaks of blood will from the teat while milking; patient walks in a sti straddling manner and, quite frequently, will go lia

Treatment :- If the case is very mild, bathe well camphorated soap liniment and a cure will be the re But in a more aggravated case give,

Epsom Salts..........twelve ounces.

Sulphate of Soda........eight ounces.

Ginger.............. one-half ounce. Mis.

Stir in a gallon of warm water and give at oned Bathe the bag freely with, camphorated soap lininf Or,

Witchhazel ......... fourteen ounces.

Laudanum.............two ounces. Mis

Use three times a day; or put one-half pound c phate of iron into one-half gallon of hot watet 
the the bag frequently; or poultice the bag with iseed meal and grease frequently with-

Lard, or Vaseline....... four ounces.

Solid Ext. Belladonna...... one ounce. Mix.

If matter forms, open and let it escape; inject the iscess with two drachms of permanganate of potas.m in sixteen ounces of water, twice a day. Somemes in the milder form, it is advisable to let a hungry If suck and burst it. If gangrene ensues, amputate lat portion of the bag.

\section{MPLE OPTHALMIA, OR INFLAMMATION OF THE EYES.}

Causes:-Feeding from a rack above the head, thus etting chaff, etc., into the eyes; blows from whips or icks, exposure to cold, obstruction of the lachrymal uct, irritating influence of a badly-ventilated stable.

Symptoms:-Whatever the cause, the eves will be ed, or blood-shot, and watery; swelling of the eye-lids; yes, hot and feverish; if not relieved, a white film preads over the eye; lids kept closed most of the time.

Treatment:-If a foreign body gets into the eye, move it, bathe the eye with hot water, and all will e well in a few days. A decoction of opium may be dded to the hot water. If from a blow or from xposure to cold, give a physic-

Powd. Alves............. one ounce.

Powd. Ginger........... one drachm.

Water............. one-half pint. Mix.

Bathe the eye with hot water and apply,

Zinc Sulphate......... four grains.

Atropia Sulphate........two grains.

Soft Water............two ounces. Mix.

Or, 
Sulphate of Copper...... four grains.

Water................ two ounces. Mix. Or,

Common Salt...........ten grains.

Brown Sugar........... ten grains.

Water............... two ounces. Mix.

Apply three times a day. Sometimes it is advisable to blister the temples with-

Cantharides............ one drachm.

Vaseline............. one ounce. Mix.

In removing a foreign body, apply to the eyes for a few minutes a solution of five grains of cocaine to onehalf ounce of water. Apply five drops and in two minutes repeat, when the object can be removed with ease.

\section{AMAUROSIS-PARALYSIS OF THE NERVE OF SIGHT.}

Amaurosis is a paralyzed condition of the nerve of sight and may be caused by pressure upon the nerve from tumors in the brain, injury or fracture of the skull causing pressure upon the brain or upon the nerve between the eye and brain, inflammation of the nerve of sight, excessive bleeding either internally or through accident, an over-loaded stomach, or excessive weight in the womb during pregnancy. It may be seen during a very high fever and is invariably observed during a severe attack of parturient apoplexy.

Symptoms:-Añ unnaturally wide dilatation of the pupil, which remains round and morionless. There is no contraction or dilation when brought to a bright light or placed in a darkened situation, as is observed in the healthy eye. The hest test is to bring the animal from a dark stall to a bright light; if the pupil does not contract at all but remains large and stationary, there 
is an attack of amaurosis, even though the eyes may look clear and bright. The animal is blind, and motions toward the eye as if the intention were to strike, will not cause any movement, such as winking or closing it, unless the hand causes a current of air. The ears arr on the alert and turn quickly if a noise is made, the animal steps hich with the front feet to avoid stumbling, the same as a blind horse.

Treatment:-All manner of treatment is useless, unless the disease is the result of some ailment or injury which is curable, as an over-loaled stomach, the abatement of high ferer, congestion of the hrain, excessive bleeding, etc., although there have been instances where the animal, fully reovering from excessive bleeding, failed to regain the eye-sight and remained blind through life. When the sight is not restored following the favorable termination of the disease, apply blisters behind the ears and around the temples, as,

Powd Cantharides.......one drachm.

Red Iodide of Mercury.... one drachm.

Vaseline... ......... one ounce. Mix.

Apply every five days until three applications have been made. Also give one drachm doses of powdered nux vomica, twice daily.

\section{OBSTRUCTION OF THE LACHRYMAL DUCT- WATERING EYE.}

Tears escaping from the eye and coursing down over the cheeks, are a symptom of inflammation of the eye, but if there is neither redness or swelling of the eye or eyelids, it is evident that there is some obstruction of the lachrymal duct. This duct is situated at the inner corner of the eye and extends downwards, opening into 
the nostril, and conveying the watery secretion of the eye, emptying it into the nostril. If this duct becomes obstructed in any manner, the tears will flow over the cheeks. It may be caused by a catarrhal affection; the duct may be closed by fracture of the bones of the face or from eversion of the eyelid, the duct failing to receive the tears, or it may be closed at the opening into the nostril, by dust and dry matter adhering to it.

Treatment:-If from the adherence of dust and dry matter, remove the obstacles with a wet sponge. If there are other obstructions, take a silver or an elastic probe about fifteen inches long and about the diameter of a knitting needle and insert it down through the duct once a day for a few days. If from inflammation or catarrhal affection, bathe the face frequently with hot water, for an hour at a time, also steam the nostrils. A good way to steam the nose, is to feed the animal hot hran and oats out of a nosebag. By examining the floor of the nostril about three or four inches up, a small opening will be observed, into which insert the end of a small syringe and inject warm water, which will come out at the inner corner of the eye; then inject the following :

Nitrate of Silver......twelve grains.

Water..............eight ounces. Mix.

Inject once daily for a few days. If fracture or disease of the teeth causes the obstruction, remove or treat them, to remove the pressure from the duct, and a cure will be perfected.

\section{TORN EYE-LIDS.}

This injury may arise from various sources; the most frequent are, from catching upon nails driven around 
the stable and from barbed-wire fences, or from the bites of other horses while playing, or in any other accidental way.

Treatment:-This consists in bringing the wound together with a few stitches, if possible, after which tio the patient so it cannot rub it and keep a linen cloth dampened with water over the eye; or if it in inflamed to any extent, take-

Chloride of Ammonium... two ounces.

Nitrate of Potassium......two ounces.

Water................... one pint. Mix.

Shake thoroughly until dissolved, then dip the cloths into the solution and apply to the inflamed part. If some part will not heal, take a pair of shears and eut it off, then apply the former solution.

\section{WARTS.}

Warts are an overgrowth of the papilla of the skin and are mostly seen on young horses, but horses of any ane are not exempt from them. They generally appear on the thin and delicate portion of the skin, as the sides

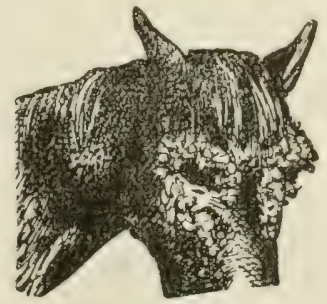

WARTS.

of the nose, the eye-lids, the inner parts of the hind legs, the sheath, abdomen and on the neck. They vary greatly in size from that of a grain of wheat to a large 
goblet. Some are flat and some pedunculated (that is fastened with a constricted neck). Sometimes they arequite tough and hard, and again some are soft and bleed easily. They always have a small beginning and graddally increase in size. Some warts, after attaining a certain growth, will remain the same size for years.

Treatment:-Warts should be removed while still small. If they have a constricted neck, twist or burn them off ; afterwards burn them with lunar caustic, blue vitriol or a red-hot iron. If they are flat, scrape them a little and dress with chloride of zinc or touch them with nitric acid once a day until they are destroyed, then grease until well. Sometimes warts are tied off. This is done by tying a strong string around them and in a few days another tied still tighter, and so continu. ing until the wart drops off. If these growths are on the lips or eye-lids, touch with nitric acid daily, but care must be exercised, so that the acid will not touch any part but the wart. Small warts that appear on young colts in the summer, usually disappear voluntarily in the winter.

\section{PERIODIC OPTHHALMIA, OR MOON BLINDNESS.}

Causes:-First and chief, want of judgment in breeding, as it is undoubtedly hereditary and horses or mares affected with it, should not be used for breeding purposes; living in low, marshy, ill-drained localities, irritation from teething; intestinal worms, etc.

Symptoms:-These are similar to those of simple ophthalmia, but are more marked. The first symptom usually noticed is swelling of the eyelids in the morn. ing, and, upon examination, the eye is found to be somewhat inflamed, with red streaks of an aggravated. 
appearance running across the eye-ball; at the lower part of the eye-ball will be seen a semi-circular whitish or yellow spot, possibly the size of a half nickel; the inner corners of the eye-brows are drawn down, forming almost a right angle; generally the eye is retracted within the orbit, and the haw, or membrana nictitans, pushed out over the eye-ball. These symptoms may pass off to reappear in the course of a few weeks or possibly months, and continue to reappear until the animal goes blind.

Treatment:-The treatment of periodic ophthalmia is simply palliative, unless the case can be removed to a dry, healthy climate. Give a physic,

Powd. Aloes..............ne ounce.

Powd. Ginger............ one drachm.

Water..................... Mix.

Bathe the eye with hot water and apply,

Atropia Sulph........... one grain.

Sulphate of Zinc.........two grains.

Water...................... Mix.

Put three drops into the eye three times a day. Good tonic treatment will invigorate the system and assist to ward off' another attack, as,

Sulphate of Iron......... four ounces.

Nux Vomica............two ounces.

Glauber Salts ...........two pounds. Mix.

Give tablespoonful morning and evening. But your best treatment is to dispose of your horse at your earliest convenience.

\section{EPILEPSY, OR "FALLING FITS."}

The exact cause of this disease is not known, but it undoubtedly arises from some trouble with the bowels or brain. For horses troubled with worms frequently 
have fits, and when once rid of the worms will cease having fits. Others, perfertly free from worms, have "falling fits" for years, owing to some structural change in the brain,-as tumors, abscesses, etc., pressing upon the nerve centers, causing a rush of blood to the brain. Fright will frequently cause "falling fits." A horse, subject to them, when frightened, will invariably fall over in a fit.

Symptoms:-Generally the first symptoms noticed will be movement of the ears backward and forward, loss of motion or staggering gait, head drawn backward, with twitching of the muscles of the neck. Then the animal will rear and fall over; when down, the head will be drawn backward and the muscles of the borly and neck will be rigid or trembling; sometimes the animal will kick and strike violently, remain for a minute or perhaps several minutes, then get up and move on; a wild or anxious expression of the countenance will always be noticed; the eves bulge out and are very red.

Treatment:-As soon as the fit is seen coming on, bleed from the nostrils and the animal will invariably rise and go on; or give by inhalation, chloroform or ether, which also relieves. Dashing cold water on the head during the fit is also beneficial. When aware that a horse has fits, attention should be directed to prevention as far as possible. If intestinal worms are suspected, clean the bowels out with a physic and get rid of the worms. In a plethoric horse, an occasional physic will benefit by lessening the quantity of blood and cooling him off. But if the patient is of weak constitution, give vegetable and mineral tonics, as,

Gentian.............. four ounces.

Powd. Nux Vomica.......two ounces.

Sulphate of Iron.........two ounces. Mix. 
Give teaspoonful morning and evening. In the summer, during hot weather, keep a wet sponge on top of the head. Feed on soft food. Above $u$ ll, do not whip or excite the animal. Drachm doses of bromide of potassium or sodium, given twice daily in drinking water, may be given with benefit.

\section{TETANUS, OR LOCK-JAW.}

The great majority of cases of lockjaw arise from puncturing the foot with a rusty nail, but it may be caused ly wounds of any description. Quite frequently it is caused by castration-no matter how neatly or

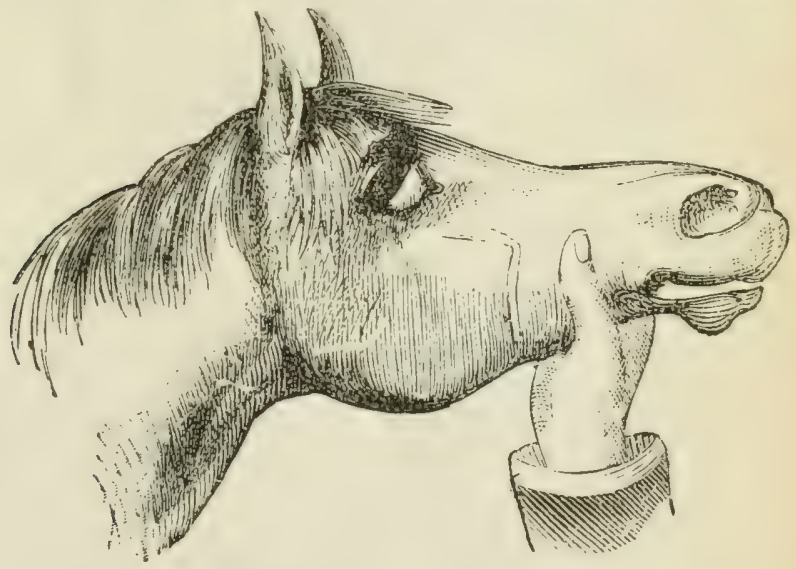

TETANUS, OR LOCK-JAW.

thoroughly performed. It may also arise from exposure to cold or wet, nicking or docking the tail, and from fractured bones. It will sometimes happen without any apparant cause, whatever, and sometimes from excessive frimht.

Symptoms:-The first observable sign is a great stillness; then the eyes are drawn back into the sockets 
and the haw, membrana nictitans, will almost cover the eyes; grinding of the teeth follows, with stiffness of the jaws and dribbling of saliva from the angles of the mouth; on approaching the animal, the eyes are withdrawn into their sockets, the haws protrude over the eyes, and the patient manifests great fear; the muscles along the neck are very rigid; the tail is slightly elevated; the animal will attempt to suck food or water. but the act of swallowing is extremely difficult and very little food or water reaches the stomach; breathing is usually accelerated, with the nostrils widely distended;

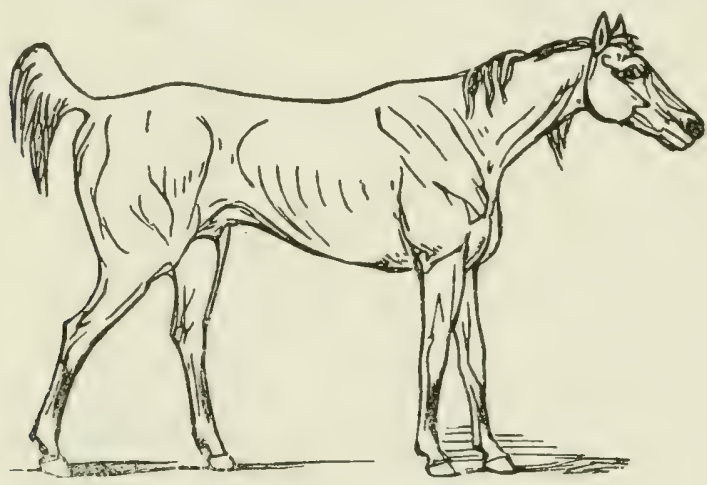

TETANUS, OR LOCK-JAW.

pulse, regular but hard and incompressible; fever, in some cases very slight, but in the more severe cases sometimes reaching $107^{\circ}$ to $108^{\circ} \mathrm{F}$. If compelled to move, the horse goes with a stiff, straddling gait; while standing, its legs are spread apart, acting merely as props for the body.

Treatment:-If possible, first ascertain the cause and remove it. If from a punctured foot, find the spot and pare it out to the bottom, and apply solid extract of belladonna to the wound. Put in a comfortable stall and, as soon as possible, place the slings under the 
animal to give it rest, but do so very quietly. Then, if you can, give a good physic-

Powd. Aloes...............................

Powd. Ginger........... one drachm. Mix.

Or, one pound of sulphate of soda. Give in drinking water or drench. Keep the bowels open hy giving oil or epsom salts, or sulphate of soda in drinking water. Keep the wound well greased with belladonna, every four to six hours. Give drachm doses of the solid extract of belladonna, every six hours, hy placing it between the back teeth, or on the hack part of the tongue. I have seen good results from giving two drachm doses of prussic acid every five hours, or the following:

Bromide of Potassium.... one drachm.

Chloral Hydrate....... one drachm. Mix.

Give with two ounces of liquid ammonia acetatis every four or five hours. All remedies of an irritating nature applied extermally, will do a great deal more harm than good. I have never seen any good results from their application, on the contrary, the patient invariably grew worse steadily. Good results are frequently obtained by covering the upper part of the head, neck and body with woolen blankets, kept saturated with very hot water. If the treatment is commenced, it must be kept up for six or eight hours every day, and until the acute spasms have subsided. Keep a pail full of pure, cool water in front of the animal, high enough so it can reach it without any special effort. It may not drink much but it will cool its mouth. If the disease is occasioned by a wound of any kind, poultice well with flaxseed and apply belladonna. (Ine thing I wish to impress upon the mind of the attendant: P'ace the patint in a dark stall, put the slings under it as soon as it is possible, and allow no one to go near it but your- 
self-no matter how anxious they may be to see it. While going around the patint, go as quietly as possible. Do your work as quietly as it can be done, as a little fright will sometimes place a convalescent in a worse condition than at first.

\section{PHRENITIS, OR INFLAMMATION OF THE BRAIN.}

Causes:-Blows, other injuries to the head, fracture of the cranial bones, exposure to excessive heat, clots of blood floating from some other part of the system and lodging in the brain; over-exertion, results of certain fevers, indigestion, tumors in the brain, etc.

Symptoms:-When the membranes covering the brain are atfected first, the animal will get very uneasy, in fact, uncontrollable; it will jump and plunge about, grind its teeth, climb up against the walls, strike with its fore feet-in fact, act as if it were crazy; the breathing will be accelerated, pulse, full and hard; occasionally sweat will break out over the body. If the brain substance alone is involved, the symptoms will vary considerably from the foregoing. The patient will act dumpish and sleepy; it will walk around the stall in a circle until it comes in contact with some object, then it will stand and press its head, possibly for hours, and may force it through ; pulse and respiration will be slow and full, eyes will have a dull or glassy appearance, appetite will be moderate-sometimes the patient will chew up a mouthful of hay and hold it in the mouth, possibly drop it out again ; it will drink a moderate amount of water; in a few days will look very gaunt. The skin of the head is invariably knocked off by their continual pushing and knocking of the head. 
Treatment:-Put the patient in a comfortable, airy, loose wox-stall. Bleed thoroughly from the jugular vein. Then give, to clean out the bowels, a brisk cathartic,

Powd. Aloes............. one ounce.

Powd. Ginger........... one drachm.

Water................ne-half pint. Mix.

Apply cold water or ice to the head and give,

Bromide of Potassium.....two ounces.

Nitrate of Potassium......two ouuces. Mix.

Make into twelve powders and give one every four hours. After the acute symptoms have subsided, mustard mixed with vinegar should be applied to the legs, and if the horse is not perspiring, apply heavy blanket. to promote perspiration. Give iodide of potassium, two drachm doses, twice daily; also one drachm of calomel, once a day.

If inclined to eat, give the patient soft food and all the drinking water it will take. As convalescence progresses, a few good blisters applied to the poll will often prove beneficial. If caused by a fractured skull, trephine and raise the bones to their normal position, thus relieving the congestion.

\section{SPINAL MENINGITIS, OR INFLAMMATION OF THE MEM- BRANES AND SPINAL CORD.}

The causes of this disease are similar to those of inflammation of the brain,-as external violence, disease of the bones of the spinal column, exposure to cold rains, spraining of the back by carrying a heavy person; also some specific virus in the blood; exposure to wet and cold when exhausted. 
Sympioms:-These are very different from inflanmation of the brain. The animal will have muscular spasms and partial paralysis of the parts behind the seat of the inflammation. In attempting to walk, the feet will be jerked up quickly and put down in a similar manner; patient moves with a staggering gait. Sometimes the pulse is rapid and the breathing greatly accelerated. Although its movements cause the animal great pain, it is unable to control them. In the course of a few hours, the patient will reel and fall over. Though conscious of all surroundings, it will evince great fear upon being approached, will be unable to regain a standing posture alone. Most commonly high fever is present, but frequently the animal will sweat profusely from intense pain.

Treatment:- When thoroughly convinced that the spinal cord is congested, put the patient in a good, comfortable place with plenty of bedding. Administer a physic,

Barbadoes Aloes.......... one ounce.

Gentian..............two drachms. Mix.

Give at one dose in a pint of water as a drench, to be followed with four ounce doses of sulphate of soda, morning and evening. Apply cloths wrung out of hot water, to the spine. In a plethoric animal, before paralysis exists, bleeding will be beneficial,-but not after the animal falls down from paralysis. Rub the back thoroughly with belladonna, or apply a bel lomna plaster. Give every two or three hours half drachm doses of tincture of ergot of rye. When certain that the congestion and inflammation have subsided, apply the actual cautery (red hot iron) from the withers to the rump, three lines on each side, an inch apart. Give grain doses of strychnia combined with two ounces of 
alcohol and four ounces of water, every four or five hours. Pay strict attention to the diet, giving soft, nutritious food. Keep the patient very quiet.

\section{CONGESTION OF THE SKIN.}

Causes:-Exposure to cold and rains, heing driven in the mud in the spring and fall, imperfectly fitting harness and collar, chafing during the summer months, etc.

Symptoms:-Redness of the skin, attended with itching: if between the thighs, the horse will straddle when standing or when first driven; if under the collar, tho horse will keep stretching and bobbing the head; if in other parts of the body, they will invariably rub themselves.

Treatment:-Bathe the parts well with hot water and castile soap, and apply three times a day-

Sugar of Lead........... one ounce.

Soft Water............. one pint. Mix.

Or, apply twice daily, this-

Tannic Acid............ one ounce.

Glycerine...........eight ounces. Mix.

Or, white oak bark tea, extract of witchhazel, salt and water, all of which will be found beneficial. Give two-ounce doses of glauber salts morning and evening.

\section{SUN-STROKE, OVER-HEATING.}

This disease attacks all kinds of horses during the summer heat, but is more prevalent in large cities than in small ones, or in the country. The principal causes are badly ventilated, filthy stables; poor food, causing derangement of the alimentary canal, and afterwards 
being driven in the hot sun; over-work and tight-fitting collars are also causes.

Symptoms :- Sometimes the horse will begin to hang back and seem stupid, hang his head and want to stop. If allowed to stop, he will prop himself with all four legs. The breathing is very rapid and heavy; skin, hot and dry; the head about the poll very hot, nostrils widely distended, pupils of the eyes contracted, eves bulged out and very red; if compelled to move, the animal staggers and sometimes falls; pulse very quick and weak. If the patient recovers, it is comparatirely useless in the summer, but will do all kinds of work in the winter.

Treatment:-As soon as noticed, take off the harness and collar and apply cold water to the head and along the spine. Sometimes it is advisable to apply cold water all over the body. If the patient is failing, give strong stimulants, as,

Carbonate of Ammonia..four drachms.

Whiskey............... one pint. Mix.

Give at one dose; or,

Aromatic Spts. Ammonia.. one ounce.

Water................ one pint. Mix.

Repeat every hour until the pulse grows slower and stronger.

Bathe and rub the limbs well with alcohol; or, better still, with mustard. Give injections of hot water containing a little ammonia. When the pupils begin to dilate and consciousness returns, give a quart of raw linseed oil.

After reaction has taken place and convalescence is established, the following tonic will be of great service.

Sulphate of Iron.........two ounces.

Gentian.............. two ounces.

Quinine.............. half ounce. Mix. 
Make sixteen powders. Give one powder morning and evening. It is good practice to wear large sponges wet with cold water on the top of the head of work horses during the heat of the summer, or bathe the head and nostrils with cold water frequently.

\section{IRRITATION OF THE SKIN-PRURITUS-PRURIGO.}

This affection is an inflamed condition of the skin, causing intense itching.

Causes:-It is caused by feeding to excess on hay and graiu, and not being worked regularly and kept in close

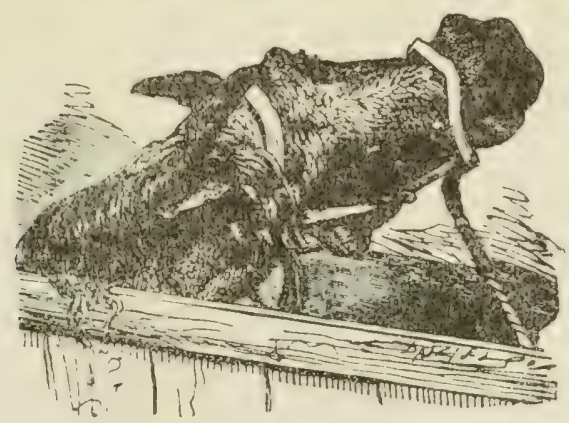

PRURITUS-PRURIGO.

stables. It is usually seen during the summer months but it is not uncommon to observe bad cases during the winter, where the stable is kept close and hot.

Symptoms:-Vesicles and patches of pimples will appear around the mane, neck, tail and rump and as the itching is quite severe, abrasions of the skin are sometimes observed, caused either by the biting, of the animal or by rubbing against some stationary object. Patches of the affected portions will sometimes dry up, and dry scabs or a surplus of scaly dandruff will peel 
off. Itching of the tail is very common among horses and seemingly becomes a habit with some. However, it is sometimes caused by worms, in the last bowel.

Treatment:-Feed more moderately, and give either steady work or turn out into the barn lot every day, for air and exercise. Give one pound glauber salts and wash the parts with,

Oil of Vitriol............. one ounce.

Carbolic Acid............. one ounce.

Soft Water............ two quarts. Mix.

Apply twice daily, or,

Salicylic Acid............. one ounce.

Borax................two ounces.

Alcohol.............. four ounces.

Water................... pine pint. Mix.

Apply twice daily, or

Oxide of Zinc.......... four ounces.

Vaseline............. four ounces. Mix.

Apply once or twice daily, or

Sulphur................. pone pound.

Oil Tar.................. one pint. Mix.

Apply once daily, washing it off occasionally with soft soap and warm water. If the case is very obstinate, give,

Sulphur............. eight ounces.

Powd. Nux Vomica........ one ounce. Mix.

Make sixteen powders. Give one powder morning and evening. If there are worms in the last bowel, inject,

Oil Turpentine........eight ounces.

Linseed Oil........... eight ounces. Mix.

()1 take three gallons of water, throw in half pound of quassia wood, let it steep thoroughly and inject occasionally. 


\section{SCRATCHES AND GREASE HEEL.}

Causes:-Continuous driving on muddy roads, too much! washing in cold water and not being rubbed dry ;

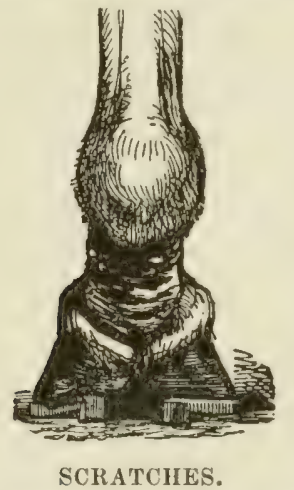

irritating fumes arising from the manure, running in dirty, wet barnyards, weak or deficient circulation, clipping the hair off from the heels, cold draughts, snow and freezing mud, working in deep, irritating mud, etc.

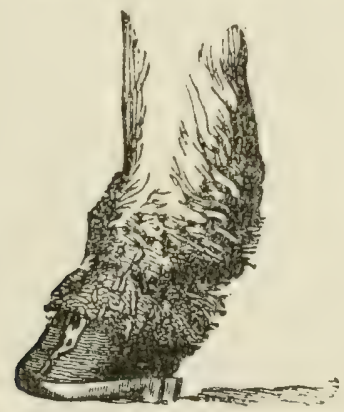

SCPATCHES.

Symptoms:-It is usually noticed in horses of lymphatic temperament, with thick, meaty legs, but may attack any horse. Cold, or any irritation of the lymphatic or of the subaceous glands will cause the heels to 
swell and crack open; small scabs will form and be very painful; when first started the horse will lift his heels very high, owing to the pain; there will be more or less fever, appetite usually good; after being driver. for awhile the horse gets over his lameness. If not atiended to, the case becomes more agcravated, the swelling increases; deep cracks will break across the

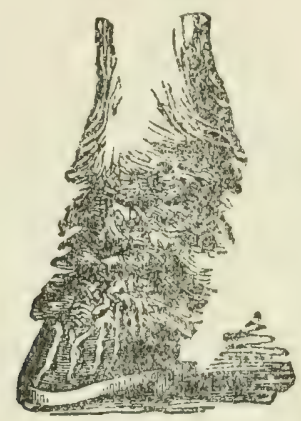

GREASE HEEI.

hoels, and a yellowish white, fotid discharge will come oozing out and run down the heels. Later on the cracks become larger and often coalesce, forming a very large sore which, when washed, looks red and aggravated. Then comes what is termed the "grapy" stage - small lumps of flesh will raise in bunches very much resembling a bunch of grapes. The discharge still contimues and is very foetid and offensive.

Treatment:-During the first stage, when the skin is swollen and tender, apply a hot bran poultice and regulate the diet. Feed on soft food and give at one dose,

Powd. Aloes............ one ounce.

Powd. Ginger.......... two drachms. Mix.

Quite frequently the disease will disappear. But as the disease becomes more advanced and small cracks 
appear with a discharge, continuous poulticing with hot bran will be very beneficial; also use a solution of,

Copperas.................ne ounce.

Soft Water...............ne quart. Mix. Or,

Sulphate of Zinc.......one-half ounce.

Sugar of Lead.............. one ounce.

Carbolic Acid......... four drachms.

Distilled Water............ one quart. Mix.

Apply three times a day. Also give the physic of aloes, and give three times a day, in soft food, a teaspoonful of the following:

Nitrate of Potassium....... one ounce.

Resin.................. oune ounce.

Bi-Carbonate of Soda ....t two ounces.

Sulphate of Iron.......... one ounce. Mix.

When the "grapy" stage appears, cut the knohs off with a hot iron or with a knife, and apply dilute muriatic acid, after which persist in the use of one of the former astringent lotions; or,

Vaseline.............. one ounce.

Oxide of Zinc......... two drachms.

Carbolic Acid...........thirty drops. Mix.

Apply three tines a day. A great many practitioners adrise cutting the hair short for the application of local remedies. I would not advise such a course, as, in my experience the short hair invariably proved a source of irritation.

\section{FACIAL PARALYSIS.}

This is caused by barly-fitting bridles, bruises, ete.

Symptoms:-Dropluing of the lip on one side, difficulty in eating and drinking, partial loss of motion and sensation on the affected side. 
Treatment:-Give drachm doses of nux romica three times a day. Apply to the side of the face the following blister, every four or five days, until it has been ai)plied four or five times:

Cantharides...........two drachms.

Red Iodide of Mercury....one drachm.

Vaseline.... one and one-half ounces. Mix.

\section{CHAFING BY THE HARNESS - SADDLE OR COLLAR GALLS. - SORE SHOULDERS.}

Chafing or abrasion of the shoulders is very commonly met with in the agricultural districts, and more especially so, in the spring of the year. Of late years, since the timber industry occupies so little of the attention of the farmer, his horses are either in the stable or barn lot, the most of the winter. Consequently, when they are put to work at plowing, in the spring, their shoulders are soft and tender, and easily chafed or galled. The majority of injuries of this kind, come from ill-fitting collars, being either too large or too. small, and not infrequently from having a rough, uneven surface. The harness sometimes becomes hardened if not frequently oiled, and when placed upon the animal, will not fit around the girth. The saddle pinching and pressing the skin, on either side of the back-bone, will produce sores.

Treatment:-First, ascertain the cause, if possible, then remove it. If the shoulders becone galled when first put to work, in the spring of the year, which is generally the case, look to the collars immediately and see that they fit perfectly; when the collars are fitting nicely and the shoulders are chafing, it is on account of the skin being tender; as the animal is generally soft 
at this season of the year, it sweats profusely; the sweat and dust accumulate on the collar, making it rough, and consequently, it irritates and chafes the skin. Every noon and evening the collar should be removed and cleaned, and the shoulders bathed with rold water. Continue in this manner until the shoulders bocome hardened to their work. If the harness pinches the back, place pads under it and oil it frequently to render it soft and pliable. The shoulders and back, when commencing to gall, should be washed every noon and evening with cold water, then wiped dry, and afterward bathed with tea made from white oak bark. If there is a raw sore, rest from work as much as possible, and while at work, pad the collar so it does not rub the sore. The tea of white oak bark will often heal the sore, or you may apply, three times a day, with a feather, compound tincture of benzoin, or,

Powd. Alum............. six ounces.

Iodoform.............. one ounce. Mix.

Or,

Sugar Lead ............. one ounce.

Sulphate of Zine.......... one ounce.

Soft Water............. one quart. Mix.

Shake well before using; or,

Carbolic Acid............. one ounce.

Raw Linseed Oil....... fifteen ounces. Mix.

Any of these preparations will quickly heal the sore, provided you keep pressure and friction from irritating it.

\section{MANGE.}

At the commencement this is purely a skin disease, and is caused by a small insect called acaria, which burrows into the skin and breeds. It forms small pus- 
tules which cause considerable itching. As the pustules break, a small scab is formed, and so intense is the itching that the horse will rub himself until the hair, and frequently the skin, are rubbed off. In severe cases

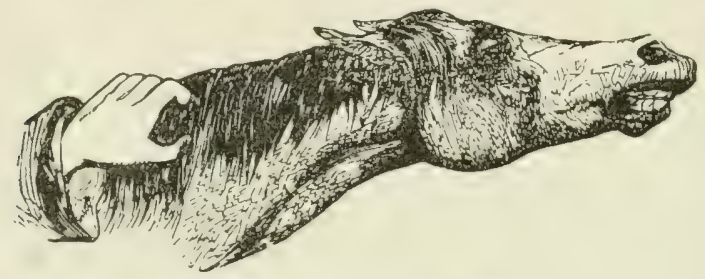

SYMPTONS OF MANGE.

the mane and tail will fall off, leaving the horse a pitiable object. Mange is very contagious-at least the acaria will crawl from one horse to another. It is even

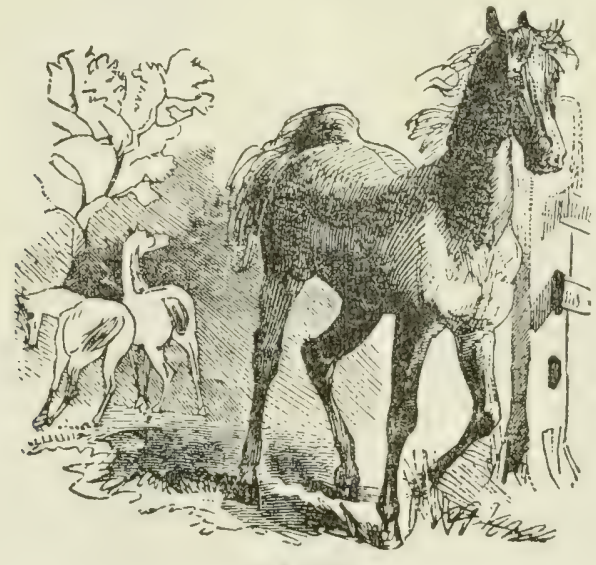

MANGE.

communicated to a healthy horse by his rubbing himself against a post where a mangy horse has been rubbing.

Treatment :-The disease is seldom noticed until scabs are formeil. They should he well niled with sweet oil, and, in twenty four hours, washed with warm water 
and "astile soap, until the scabs are removed. It' it is necessary, take a brush to accomplish this. 'Then apply tobacco liquor, prepared by boiling two unures of tobacen in a quart of soft water. The application

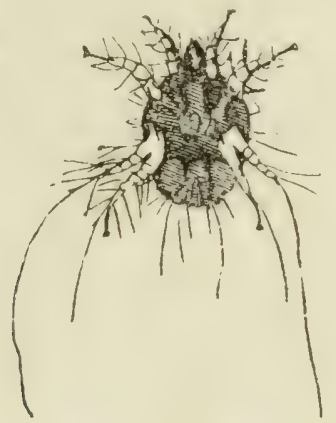

MANGE INSEOT.

should be used three times, with intervals of two weeks between each application; or the following liniment should be applied:

Linseed Oil...........eight ounces.

Sulphur..............two ounces.

Oil of Tar............. four ounces. Mix.

Apply with a brush and wash off in twenty-four hours. If thought necessary, apply a second or third time. Regulate the bowels and keep in as good spirits as possible. All harness, combs and brushes should be saturated with the tobacco liquor. The blankets should be boiled and the stable whitewashed, to which should be added half pound of chloride of lime to every gallon.

\section{LICE.}

These are small insects of a low grade, which seldom attack horses in good condition, but invariably appear on horses in poor condition. The animal will rub itself 
and the hair will look rough and staring; there will be pallor of the mucous membranes; sunken eyes; frequently an unsteady gait. An examination will reveal the cause. Quite frequently horses are kept close to a chicken house and become infested with chicken lice; these are very small and not easily detected but are very troublesome.

Treatment:-As lice seldom cover the whole body, examine and locate the place where they are. Apply diluted kerosene oil two or three times and they will generally disappear. Or, take one pound of stavesacre seeds and two gallons of soft water; simmer for two hours or till reduced to one gallon. Allow to stand twenty-four hours; then apply all over the animal. Two applications generally will suffice. Some prefer the use of tobacco in the same manner, but I am positive that the best results will be obtained from stavesacre seed.

\section{HIDE-BOUND.}

Hide-bound is not a disease but is a symptom of some disease, or of neglect. It may be caused by starvation, exposure, indigestion, irregular or diseased teeth, and by worms. Abuse is a frequent cause, also want of proper exercise, irregular feeding, and neglect of currying and rubbing, that the skin of the animal may be kept clean.

Symptoms:-The skin sticks as tightly to the body as though glued fast; the ribs show plainly and the skin can scarcely be moved from them. The hair looks rough and sands the wrong way; it is very dry, and the skin is dirty and full of dandruff. 
Treatment:-First remove the cause. If the teeth are sharp and irregular, take a rasp made for that purpose and level them down. If from exposmre, shelter in a warm, comfortable place, and if the want of goorl food is the cause, feed better and groom thoroughly, and the horse will soon begin to thrive and the skin will lonsen. In order to start a thriving condition in many horses, it is necessary to give them a physic, and for this purpose, a quart of raw linseed oil answers best. After the physic has operated, cut the hay for the animal and place chop-feed upon it, then seald it eithor with steam or hot water, and give with the ford a handful of ground flaxseed twice daily. If the manure smells badly, give a teaspoonful of baking soda in feed or water, once daily, and if the digestive powers are low, use the following:

Powd. Ginger.......... two ounces.

Powd. Sulphate of Iron... two ounces.

Gentian.............. four ounces.

Powd. Nux Vomica......two ounces.

Saltpeter............ four ounces.

Baking Soda........... four ounces. Mix.

Give a teaspoonful two or three times a day. Feed upon soft food-if scalded, so much the better. Also give regular exercise, either at light work or turn into a vacant lot or barn-yard. If it is auspected that worms are the cause of the ailment, see treatment for worms.

\section{SIT FAST.}

This condition is brought about by ill-fitting saddles, harness not properly adjusted, or badly-fitting collars, more especially on the top of the neck; or by any undue friction or pressure by anything the horse is made to 
wear. Being continuously kept under the saddle, and especially when the animal is mounted by a poor rider, the skin becomes bruised and calloused and becoms partly separated from the healthy skin; it then assumes

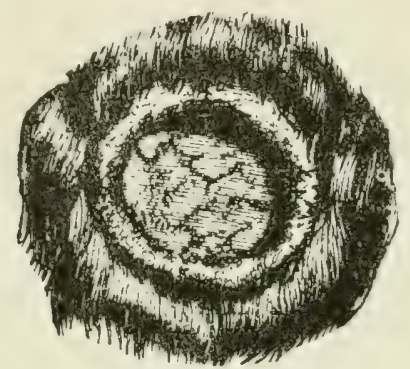

SIT FAST.

a tough, leathery, horny form, sinking deeply down into the soft tissue and is kept partly alive by the blood supply at the bottom.

Treatment:-The best mode of getting rid of this trouble, is to remove it with the knife. Cut it out clean, leaving nothing but healthy looking tissue. You may experience a little trouble in doing this, as they are very sensitive and the animal may strenuously resist the operation; but as there is no danger, be persistent in removing it, then use the following:

Sulphate of Iron....... four drachms.

Water............... eight ounces. Mix. Or.

Powd. Alum............two ounces.

Iodotivin........... four drachms. Mix.

Or,

Sulphate of Zinc...... four drachms.

Sugar of Lead........ four drachms.

Water............... eight ounces. Mix. Or, 
Carbolic Acid.........two drachms.

Listorine............... one ounce.

Water............... seven ounces. Mix.

Apply two or three times a day. Occasionally wash out the wound witl warm water and castile soap.

\section{ERYSIPELAS.}

Causes:-Anything which has a tendency to lower the vitality and ritiate the blood-such as diseases of the liver or kidneys, for they are great blood purifying organs, and any atrection of these organs tends to impair the blood by leaving within it the effete materials intended to be eliminated by them; feeding on musty or badly cured fodder, exposure to extreme licit, wounds, burns, scalds, bites of insects or reptiles.

Symptoms:-Isually eryipelas begins with ferer, quite frecuently preceded with a chill; the fever cradwally rising; loss of appetite; pulse ranging from fifty to eighty beats per minute. The skin and the connective tissues are the seat of the intlammation, which generally attacks the hind limbs, though the fore limbs, head and neck may be attacked. Owing to the attack, the swelling of the skin is sometimes very great. If it begins near the foot it runs very rapidly up the limb. The swelling is hard, but pits on pressure. In a white horse the redness is perceptible, but in horses of any other color the redness cannot be seen. Sometimes, though not always, the swelling is painful to the touch. Small vesicles appear and discharge a yellowish serum; they then form a small scab and in a few days peel off. If for the better, they leave a dry surface; if for the worse, a deep sore, and quite frequently deep cracks having a ragred, unhealthy appearance, and disinclined 
to heal. Sometimes pus will form under the skin, burrowing into the muscular tissues; sometimes it is very diffuse, and is re-absorbed by the system, when a fatal termination may be anticipated. When the swelling is great, the hair invariably drops off and leaves a shining surface.

Symptoms:-Open the bowels thoroughly withPowd. Aloes.............. one ounce.

Calomel.............. one drachm. Powd. Ginger........... one drachm. Mix.

Give at one dose; then follow up with tincture muriate of iron in half-ounce doses every two hours. If the swelling is odematous or pits on pressure, give--

Nitrate of Potassium....... one onnce.

Iodide of Potassium........ one ounce.

Powd. Nux Vomica....... one ounce. Mix.

Make into ten powders and give one three times a day. If there is much depression, wive alcoholic stimulants; but if they tend to irritate the skin, stop using them. As a local application, cover the swollen part with-

Sulphate of Iron......... one ounce.

Laudanum............ four ounces.

Soft Water.........twelve ounces. Mix.

Apply three or four times a day; or the parts may be painted twice daily with tincture muriate of iron, or tincture iodine, or the fluid extract of belladonna.

If pus has formed, make a free incision to allow it to escape, and dress the wound and abscess with one onnce of permanganate of potassium in one pint of soft witer, twice a day; also use the same solution on whealthy ragred sores; or,

Carbolic Acid............ one ounce.

Nitrate of Silver......twenty grains.

Glycerine.............ten ounces. Mix. 
Apply twice daily. When sores break out and become aggravated and when abscesses form, they should be washed twice a day with hot water and castile soap before the dressings are applied. The diet should be first-class in every particular but ground feed is preferable to solid grain. The animal should be kept in a clean, comfortable, airy place.

\section{BURNS AND SCALDS.}

A great deal depends upon the extent and severity of the injury. slight burns or scalds may be treated hy using a solution of one-half ounce of carbolic acid to one pint of water, applied frequently. But, if the burn is very deep, cover over with linseed oil or vaseline, to exclude the air. After the pain has ceased, open the blisters with a needle and allow the fluid to escape. Then press the skin down closely so that, if possible, it may adhere. If it does not adhere and an open sore results, dress it twice a day with carbolic acid five drachms in ten ounces of water; or sulphate of zine, one drachm, in one ounce of vaseline. Or,

Vaseline.............. one ounce.

Carbolic Acid........... one drachm. Mix.

Frequent washings with castile soap and hot water are also beneficial.

\section{INFLAMMATION OF THE FEET, LAMINITIS, FOUNDER.}

This disease has various causes, such as picking up a nail, having a nail driven too close while shoeing, driving on hard roads barefooted, drinking cold water when heated, eating tro much grain, feeding green corn, giv- 
ing a drastic cathartic, muco-enteritis, or from any affection of the alimentary canal or of the air passages.

Symptoms - When first noticed the patient generally will be standing with its feet well under the body, the fore feet both pointed forward, and so stiff that it is alnost impossible to move it, either forward or backward.

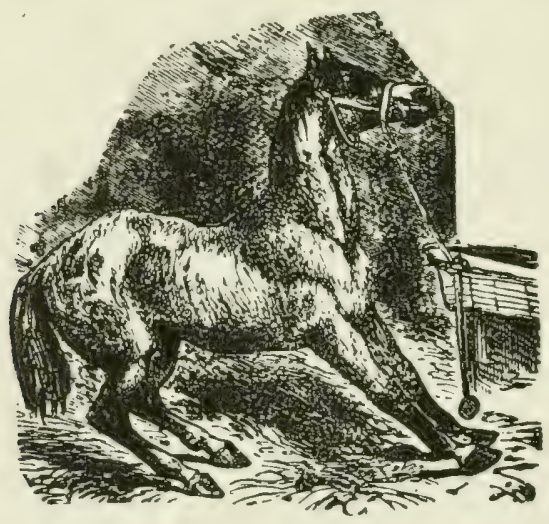

LAMINITIS, OR FOUNDER.

The fore feet almost invariably are the ones attacked; the hind feet are sometimes, but very seldom affected. The breathing is greatly accelerated; temperature varies from $103^{\circ}$ to $106^{\circ} \mathrm{F}$; ; attempts to move the animal cause so much pain that it will groan; if forced to move, it goes with a jumping, staggering gait, almost falling down, and rests very lightly on the heels of the front feet. The feet will be very hot and tender, and if they are struck with a hammer the horse will groan and almost fall down. Sometimes the breathing will be so accelerated that the nostrils are distended to their fullest capacity. Occasionally only one foot is attacked; in such cases the patient will keep the foot raised the most of the time. Sometimes in chronic cises, the feet 
contracted at the heels. Sometimes the wall and sole become greatly distorted; the healthy rings around the wall of the foot coalesce, the feet grow crooked and are become separated; an effusion takes place between the lamina and pedal bone, forcing it down and pressing the sole down along with it--sometimes penetrating through to the sround.

Treatment:-Some practitioners prefer hot applications to the feet, but my experience has forcerl me to conclude that cold applications are preferable to hot ones. Place the feet in a tub of cold water, putting straw in the bottom of the tub to ease the feet; in the heated months put ice into the water, and lieep the patient there for two hours at a time. Do this three or or four times a day. When out of a tub, or if a tub camnot be obtained, put Haxseed poultices on the feet and keep pouring in cold water occasionally. Change the poultice every twenty-four hours. Give a physic-

Powd. Aloes............. one ounce.

Powd. Ginger............ one drachm. Mix.

Give at one dose. Also prepare and give-

Saltpetre.............. sixteen ounces.

Glauber Salts.......... sixteen ounces. Mix.

Make into sixteen powders and give one three times a day. While the fever is high, rive tifteen drops of fluil extract aconite every hour or two until the fever subsides. After the patient recovers so it can walk armund, a little exercise on soft ground is beneficial; but too much exercise is harmful. Sometimes in the acnte stage, bleeding from the toe is very beneficial. But if this is done, let the blood run freely, though it is a practice to which I seldom resort. If properly attended to, a large inajority of cases will make complete recovery. After recovery is assured, shoe carefully and keep the feet soft and do not put the animal to work 
too soon. Sometimes it is good treatment to place slings under the patient, especially when it maintains a standing posture, to relicve the feet. If the patient chooses to lie down during the attack, bed well and allow it to remain in that position.

\section{CHOKING.}

Choking is most frequently found in horses that are very greedy eaters, and consists in the lodgment of dry food (most frequently corn or oats) within the throat.

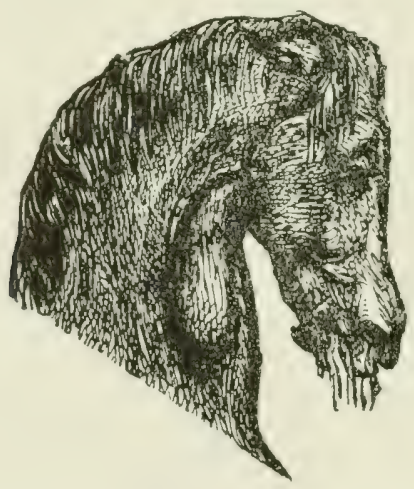

CHOKING.

Horses that are fed on ruots, such as carrots, mangels or turnips, quite frequently suffer from choking.

Symptoms.-In many cases the horse will be uneasy and the saliva will run from the mouth. If the obstruction be in or near the pharynx, the nose will be elevated, the patient will make frequent attempts at coughing and frequently if the obstruction be grain, portions of it will be ejected through the nostrils in a paroxysm of coughing. The breathing will be labored and difficult and accompanied by wheezing. If the obstruction be 
farther down the throat, the patient will breathe more easily and the frothy saliva will be less abundantsometimes entirely absent.

I have seen horses, where the obstruction was within the chest, that had not eaten for a day or more-as the owner said "were feeling a little off"," - that had eaten nore food and thus completely dilled the whole of the asophagus. During the whole time they seemed but little distressed other than being hungry and unable to eat. They would chew hay and drop it out of the mouth. Such cases usually terminate fatally.

Treatment:-If the obstruction be in the pharym, eirrate the nose. Put a month speculum in the mouth, incert the hand, and if possible, remore the object by fulling it up. If it be heyond reach and noticeable on the left side of the neck, an assistant will be of sreat strvice by pushing it up from the outside. If it cannot be gotten in this way, give the patient a little oil, as rali linseed, sweet oil, or fresh lard melted; then try and work it down. If the olsitruction be dry food, as oats, corn or hay, it probably will be softened and pass down. Sometimes while giving the oil, the horse will take a fit of coughing and eject both oil and grain by the nostrils. In such cases wait for a few minutes and repeat the dose; in that way the grain may be grotton rid of by coughing and the plug will pass on down. If no grain is coushed up or the obstruction is down in the chest, wive eight or ten ounces of melted lard or sweet oil and let it remain there for a few hours; the olstruction will sometimes pass away. Should it not move, place the speculum in the patient's mouth, elevate the nose and introduce a probang, which should be oiled wel] hefore using. Pass it down grently, and when you com to the obstacle, press quite hearily and as it starts 
send it clear into the stomach. Then give a little oil and for a few days feed on soft food.

If you find it impossible to remove the obstruction with the probang without seriously injuring the horse by rupturing the esophagus, and the obstruction can be felt on the left side of the neck, take a lancet and cut lengthwise of the neck, into the obstruction. Then carefully stitch up the œophagus (or gullet) with catgut, stitch up the integument (or skin) and feed on very soft or sloppy food for a week or two. Do not resort to the barbarous method of pushing a whip-stock, broom handle, or any other hard substance down the throat, as injuries to the throat are sure to occur, causing an immense amount of truble and sullering and in all probability the loss of the animal.

\section{CRIB-BITERS, WIND-SUCKERS OR STUMP-SUCKERS.}

This is a habit that is formed principally when the young animal is cutting teeth. It is caused by the pain

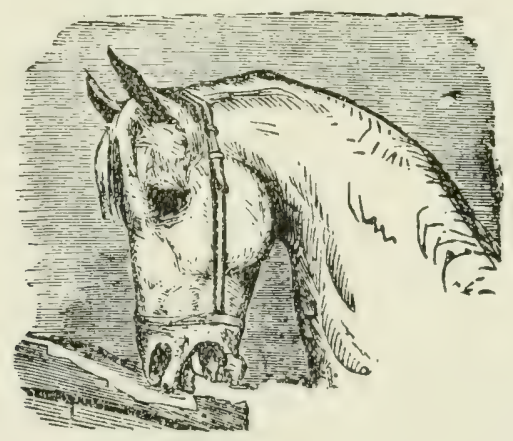

CRIB-BITER OR WIND-SUCKER.

of cutting and shedding teeth. To relieve itself of the irritation, the animal catches hold of the manger or any other object within reach. After a time, the habit ho- 
comes fixed, and when the animal catches hold of the object a culping sound is heard. It is then sucking air into stomach.

Any irregularity of the teeth, or decayed teeth, may cause a horse to form the same habit. When the habit is once formed, it is very hard to break it up. After the horse has been sucking wind for a considerable time, it causes indigestion and colic. They are unsafe horses to own for they may bloat up and die at any time.

Treatment:- Various devices have been used to break the habit, and sometimes with success. Atfend to the teeth and, after you get them in gom andition, mike a paste of red pepper and flour and daub it w the manerer; or fasten on the manger a sheep-skin with the woolly side out; or fasten a broad strap around the neck; or fasten some sharp object where the horse is in the habit of biting; or place small, revolving rollers just above the edge of the manger, so the teeth will slip off. Sometimes the habit may be broken by raising the tongue and cutting the membrane that fastens it to the lower jaw, about an inch, or by inserting a steel or silver ring throush the sume membrane and allowing it to stay there; the ring to be alout three-fourths of an inch in diameter. I have seen cases completely cured by this method.

\section{POLL EVIL.}

Poll evil consists of a swelling or an abscess just behind the ears upon the top and sides of the neck, and the irritation extends down to the first two or three bones of the cervical vertebrx. When the disease extends downward to the bones and affects the joints, the animal will carry the head and nock stiff. Among the 
many auses of poll evil, are badly fitting, heary halters and bridles, blows from the butt end of the whip, by the horse hitting its head against low beams or doors, from too high reining, etc.

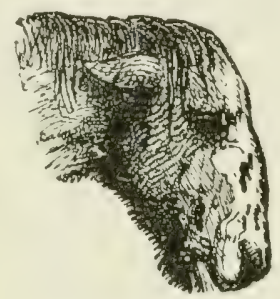

POLI EVIL.

Symptoms:-The first symptom observed is slight stiflness of the neck, with a swelling upon the poll, which is quite hard at first, later on becoming soft and fluctuating. The swelling may not increase much in size for some time, or it may increase quite rapidly. If there is no interference on the part of the attendant, it will eventually burst and discharge a whitish matter.

Treatment:-In the earliest stage, when soreness is present and the swelling has just begun so that it is, quite noticeable, the trouble may be aborted. First, give a pliysic, as,

Powd. Aloes............. one ounce.

Gentian.............two drachms. Mix.

Give at one dose in a pint of water, and give a small teaspoonful of saltpeter, three times a day, either in food, water or as a drench. Then cut off the hair and. apply the following as a blister:

Vaseline................... ounce.

Powdered Cantharides...two drachms. Mix.

Apply every fourth day for three times in succession. Then grease until well. By this treatment I have 
seen many cases aborted, and they required no other care except aroidance of the original canse. If this should fail to scatter the enlargenent, it wil! haten the formation of pus. The swelling should then be laid open with a knife. Make a bold incision and allow the matter to escape; then examine with a probe, and if no sinuses or pockets are found, you then have a simple, regular abscess to deal with. Dress the wound with,

Listerine............ four ounces.

Iodoform .............two drachins.

Glycerine............ four ounces.

Wash the sore with castile soap and hot water and apply the merlicine twice daily. If, however, while probing, you find pipes or sinuses, keep on exploring until you find them all; then take three or four grain quinine capsules, fill them full of corrosive sublinate and put one capsule into each pipe; its weight will wen. erally carry it down to the bottom. The capsule soon becomes dissolved and the medicine will cut out the pıpes. Grease the hair thoroughly over which the matter fiows, to keep the matter and medicine from excoriating the skin. After the pipes have come out, use the following:

Zinc. Sulph.......... four drachms.

Sugar of Lead ......... five drachms.

Water..................... Mine pint.

Or,

Carbolic Acid............half ounce.

Listerine............. four ounces.

Soft Water.........twelve ounces. Mix.

Apply once daily, after washina out thorouglily with castile soap and hot water. If, after it has healed up, another swelling appears, you may rest assured that you missed one or more of the pipes. You must then 
open again and go after what is left. Do not despair, for success will crown your efforts, and a complete cure will be effected.

\section{BRUISES.}

Bruises are caused by kicks, bites, falling upon hard, rough or uneven substances, etc, resulting in a crushed or pumiced condition of the flesh with the skin covering

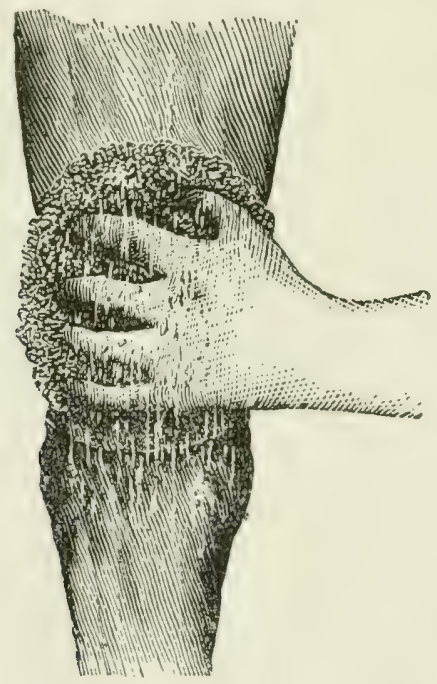

FOMLXIING A BPUISE.

it remaining unbroken. There is more or less subcutaneous bleeding, which may result in the formation of matter and a sloughing of the parts. If the bruise is not too severe, the formation of matter may be averted by fomentations of hot water, continued for a couple of hours, after which bathe freely with whiskey and camphor. If in a place where bandages can be used, a light bandage steeped in saltpeter water 'should be 
applied and the animal allowed rest for a few days, when the parts will resume their normal condition. If, however, the bruise is very severe, there is no other alternative save the formation of matter, and it should be hastened by the application of poultices of flaxseed meal, fried onions, bran, bread and milk, slippery elm or hops. While the sloughing of the parts is taking place, should there be a foul smell, powdered charcoal or jodoform, or a weak solution of carbolic acid, should be sprinkled over the poultice. After the parts have cloughed ofl, discontinue the poultices and use any of the following lotions:

Carbolic Acid............. one ounce.

Water or Linseed Oil........ one pint. Mix. Or,

Permanganate of Potash four drachms.

Water.................. Or,

Sulphate Zinc............half ounce.

Sugar Lead..............half ounce.

Water..................... Mine pint. Or,

Iodoform...............half ounce.

Powd. Alum............ four ounces. Mix.

Apply two or three times a day. If proud flesh makes its appearance, cut it out with a knife or apply burnt alum. Feed the patient on soft or green food and if symptoms of fever present themselves, give half ounce doses of saltpetre, three times a day. When the fever subsides and the discharge has been great, feed the best of nourishing food, with which may be nixed,

Sulphate of Iron......... two ounces.

Powd. Gentian.......... four ounces.

Powd. Nux Vomica........ one ounce. Mix.

Give a teaspoonful three times a day. 


\section{ABSCESSES.}

Abscesses consist of accumulations of matter within circumscribed walls and may be situated upon any part of the body or in the lungs, liver, kidneys, ovaries, $110 \mathrm{mb}$, bowels, etc. They are classed as acute, and cold or chronic abscesses.

Acute abscesses are most frequently met with in the muscular tissues or gland structures, following local inflammation; or they may be the results of bruises and injuries. The abscesses found most often in horses, are those affecting the salivary glands, the result of strangles or distemper. The glands behind and under the jaws are observed to gradually increase in size, anl become hard, hot, painful, and sensitive to the touch. As the abscess gradually increases in size, pus or matter begins to form and in a little while it becomes soft and fluctuating. This condition is discovered by pressing gently upon the center of the abscess with the fingers; it is then considered coming to a head. A small elevation is soon observed from which the hair slips off and is moistened with a yellowish fluid which oozes through the skin. In a short time the abscess breaks, its contents escape and the cavity gradually fills up by healthy granulations. When abseesses form in the muscular tissues, they are usually produced by bruises or some other form of injury. Sometimes, however, they are the result of irregular. strangles.

Whenever it is noticed that abscesses are forming, the aim should be to hasten development as rapidly ats possible, by hot fomentations ard poultices. When they are very slow in developing, a good stimulating liniment applied to the parts, is advisable, as,

Spts. Turpentine......... two ounces.

Aqua Ammonia......... two ounces.

Raw Linseed Oil........ four ounces. Mix. 
Apply twice daily. As soon as matter can be felt by pressure of the fingers, it will be well to open with a sharp knife, as low down as possible, to afford good drainage so that the matter may escape. But if not fully satisfed that the matter is near the surface, it will be better to encourage the ripening and allow it to open itself. An abscess located at a joint, should be allowed to open itself, or operated upon by a skilled veterinarian. It is unnecessary to squecze and pinch alsoesny when opened. Gentle pressure will remove all the old thickened matter, and if inclined to heal ton quickly, keep it open with a smooth piece of hickory or other hardwood, marle into a probe. Poultires and fomentattions are often heneficial after the alsocess has openerl, especially if there is much swelling. It is unnecessary to inject medicines into abscesses, unless a bad smell comes from the eavity, then it should be washed out with-

Carbolic Acid..............ne ounce.

Soft Water.............. one quart. Mix.

Use twice daily.

\section{COLD ABSCESS.}

Cold abscess is a term used to denote a large swellin $x$, the result of chronic inflammation, in the center of which there is usually a small accumulation of matter. These abscesses are generally situated on the neck in close proximity to the shoulder joint. When first observed, the swelling is quite large, and apparently increasing in size or growing (if the horse is working) until it assumes enormous proportions. It may be slightly warmer than the surrounding parts, but is not very painful when pressure is applied. The animal instear of exhibiting pain when moved about, acts as though the shoulders were stiff, or there is a stiffness in its 
movements. When relieved from work, this vast swelling gradually diminishes in size, until it leaves a large hardened mass, very much resembling a tumor, and remaining in that condition for a great length of time. There is now neither heat nor soreness and it neither grows nor diminishes, unless the animal is put to work, when the irritation from the collar will cause it to intlame, and increase in size again, but after a few days rest it will gradually recede to the same sized tumor as before commencing work.

Treatment:- The treatment as recommended for acute abscess, will fail to accomplish anything. We are therefore compelled to use more heroic measures. First, examine carefully to ascertain if matter is present, which is generally difficult to detect, for it is seldom that there is over two tablespoonfuls located in the center of the abscess. However, whether matter can be felt or not, make a deep incision into the center of the tumor, all the way through it if necessary, and allow the matter to escape. Then, whether matter is found or not, saturate a piece of sponge or cotton batting with turpentine and force it into the bottom of the cut; allow it to remain two or three days to excite the formation of matter; or pack the wound full of sulphate of zinc and plug it for twenty-four hours, then withdraw the plug and let it slough out; or take a red hot iron and fush it into the bottom of the cut; in fact anything to excite an inflammatory action and promote the formation of matter. If the opening is disposed to heal too quickly, open it up with some of the former preparations. After you have got it suppurating nicely, encourage it by applying poultices of flaxseed meal, bran, boiled turnips, bread and milk, slippery elm, ete. With this mode of treatment you will dispose of it and leave the surface smooth. 


\section{NASAL POLYPUS, OR NASAL TUMORS.}

'These pear-shaped tumors are frequently found in the nasal chamber and nay be attached anywhere along the nasal canal. They are, however, generally attached, high up in the nostril and hang by a slender neck.

Symptoms:- They sometimes grow down far enough to be olserved protruling from the nose. The breathing is olstructed more or less in that nostril. There is bulging of the nostril on the affected side if the tumor is large, according to its size. It prodnces a snuffling sound while breathing and sometimes a discharge from the nose.

Treatment:-Removal of the tumor can be accomplished by folding a copper wire through a gas pipe. Slip the wire over the tumor and pass it and the pipe up, until you reach the top of the tumor, then draw the wire up tightly against the pipe and twist the tumor off. Or if an ecrasure is obtainable, pass it up over the tumor and twist it off; or take a pair of forceps and pass them up to its attachment, then sieze it and twist it off. There will not be bleeding enough to do any harm. However, the blood may be stopped, by injecting up the nostril a solution of alum water or a solution of sulphate of iron, or the tincture of iron, or by forcing a plug of wool up the nostril, having a string attached to at by which to draw it out when the bleeding stops.

\section{TUMOR IN THE FALSE NOSTRIL.}

Small tumors are often observed in the false nostril (which is a small pouch just within the nostril and appears like a loop or fold of skin). These tumors are very slow of growth and contain a cheesy matter. They are usually about the size of a walnut and while they do 
not rause any pain, they are a blemish and sometimes Trome so large that they cause difficult breathing when the animal is put to severe exercise. They are observable on the outer side of the nostril, but by inserting

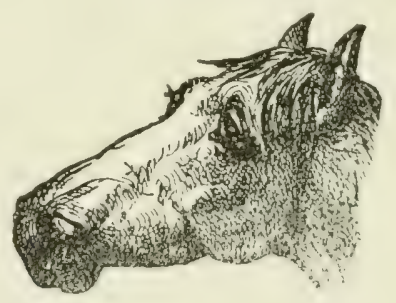

TUMOR IN THE NOSTRIL.

the finger up the nose, they will appear much larger on the inside. No danger need be apprehended in opening them and this can be done either from the outside or inside of the nostril, the latter being preferable. J'ass the knife up the inside of the nostril and when the tumor is reached, make a free incision; then squeeze the matter out and nature will generally perform the rest. Or you may syringe the cavity withSulphate of Zinc....... two drachms.

Water............. eight ounces. Mix.

Inject once daily for a few days. When the cut is made, blood sometimes flows freely for a short time, but this is of no consequence; it will soon cease.

\section{PHARYNGEAL POLYPUS, OR TUMOR IN THE THROAT.}

This is just the same kind of tumor as appears within the nostrils, only it is situated in the upper part of the throat. If it is located at the opening at the back part of the mouth, when the animal takes a drink, it is liabl" to drop into the larynx or upper part of the air channel, causing alarming symptoms by partially shutting off its breathing. 'Respiration is then accomplished with the 
greatest difficulty; efforts are made at coughing; the animal chokes until it falls upon its knees or falls down completely, when the tumor is generally dislodged from the larynx. The animal then breathes freely and appears as though there was nothing wrong. These frequent and sudden attacks, especially while drinking or eating, followed by almost instantaneous recoveries, should lead to an examination of the throat, which is done by holding the mouth open and passing the hand back into the throat, where the tumor will be found. If within reach, renove in the manner advised for removing nasal polypus. When the removal is accomplished, feed wholly upon soft food, for two weeks and after ear.h feed, give a few swallows of water, then pour some alum water into the mouth and allow it to run over the sore.

\section{WOUNDS ABOUT THE NOSTRILS.}

Wounds around the nostrils are quite a common orurence, and are generally caused by catching upon a nail or hook of some description, bites from other liorses, or by ruming against some object, etc. As quickly as possible after the accident, the wound should be stitched together. No loose parts should be cut ofl' and thrown away, but everything placed in its proper position. The skin around the mostrils is very thin and easily stitched, consequently any kind of a needle and any kind of white thread will answer. If not immediately given attention, the wound may swell considerably and difficulty may be experienced in getting the edges in direct opposition. If possible, place the stitches quite closely together. Occasionally a nostril is so badly lacerated, that it is with difficulty the parts are brousht together, and then a blemish may result. After the parts have been brought together, and secured 
with stitches, the animal should be tied so that it cannot rub the wound, for ten days or two weeks, and in the meantime keep the parts clean, and use,

Carbolic Acid......... four drachms.

Soft Water............... pone pint. Mix.

Apply twice daily, or dust it with iodoform once daily; or,

Sugar Lead.......... four drachms.

Sulphate Zinc.........two drachms.

Water................. one pint. Mix.

Apply morning and evening. After the parts adhere firmly, cut the stitches and carefully draw them out.

\section{NETTLE RASH OR SURFEIT.}

This is a frequent form of skin disease in the horse, and is caused from a sudden change of diet. It is fre-

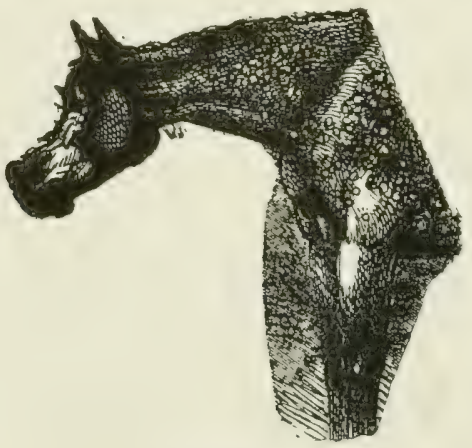

SURFEIT.

quently observed when horses are first fed upon grass. It may also be caused by sudden changes in the weather, taking a drink of cold water when heated, worms in the intestines, and is sometimes connected with the shedding of the hair. 
Symptoms:-The lumps rise quickly and appear on any part of the body, sometimes covering nearly the entire body, head and neck. The eyelids mat be closed and the lips greatly thickened. Sometimes the nostrils are swelled nearly shut. The swelling: are unequal in size, varying from a marble to a saucer and are flat upon their surface. It may be attended with diarrhea or slight colicky pains. The eruption breaks out very suddenly, the whole skin being covered in a short space of time, and it often disappears just as rapidly as it came, or it may linger for five or six days.

Treatment:-This is very simple and consists in cleaning out the bowels with,

Powd. Aloes............seven drachms.

Powd. Gentian.........two drachms. Mix.

Give at one dose in a pint of water. Also inive one ounce daily of glauber salts, for a week. Aply y to the skin twice daily,

Sugar Lead............two ounces.

Soft Water.............. one quart. Mis

\section{RINGWORM.}

Ringworm is most frequently seen in young horses that have been poorly kept through the winter and spring. It appears at the time of shedding and is a parasitic growth of a contagious nature, sometimes attacking several horses in the same herd. It may be rommunicated to other animals and also to attendants.

Symptoms:-It commences in a small pimple, at first, where the parasite has established itself. It then begins to form a circle which continues to spread daily. The hairs over the affected part stand erect, split up, break 
off and fall out, leaving a bare spot which is covered with a greyish scruff or dandrufl, very nuch resembling the coarse hulls of wheat bran. During its progress, the disease is not confined to one spot but may develop on

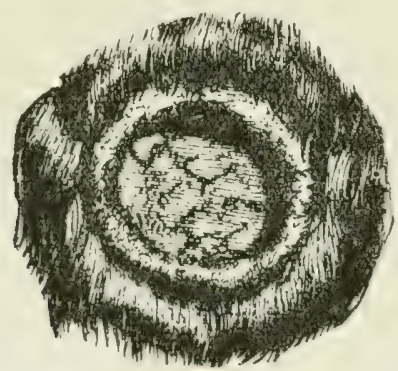

RINGWORM.

several parts of the body at the same time. If allowed to run undisturbed, it may prove very troublesome, $\mathrm{or}$ recovery may take place spontaneously within a couple of months.

Treatment:-Clip the hair closely over the affected parts, then wash with soft soap and warm water; liry the parts thoroughly and paint with acetic acid or tincture of iodine, twice daily until cured. Should these remedies fail after applying ten days, apply the following once daily:

Corosive Sublimate..... half drachm. Soft Water............. one pint. Mix.

\section{FISTULOUS WITHERS.}

This disease very much resembles poll evil, only it is located upon the shoulders, and is also very obstinate to treat. It usually comes from a bruise, from the 
saddle, from rolling, or striking the top of the shoulders against something; for instance, against the top of a low stable or shed.

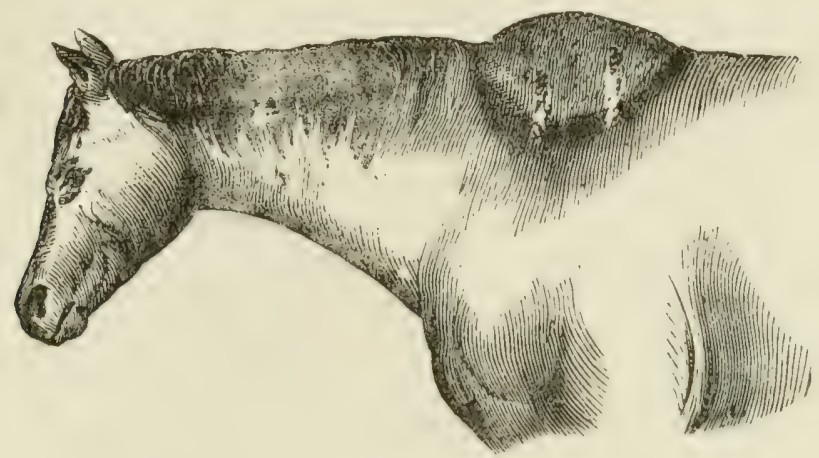

FISTULOUS WITHERS.

Treatment:-As soon as noticed, apply the same remedies as for poll eril, and make a dependent orifice to allow the pus to escape, treating the orifice as directed for the sinuses in poll evil.

\section{CASTRATION.}

There are various methods of performing this operation, but all tend to the same purpose, viz.: removing the testicles and preventing hemorrhage.

First cast the horse and confine him closely and tightly, drawing the hind feet well up along the sides. Have a good assistant to hold the head. Take hold of the testicle (always taling the smaller one first) and raise it well up. Hold it firmly and make a bold cut about three inches long, close to the dividing line of the testivles. . Then take hold of the testicle and cut off the white membrane at the back part of the testicle, but do not cut the spermatic cord or artery. Then if the ecraseurs are used, apply them and smash off the cord. If 
the clamp is used, fasten it on tighly and tie with a stout cord. The clamps should be grooved so they will have a firmer hold. Then cut off the cord and testicles, about a half inch from the clamp. Some prefer ligating, which is simply cutting out the testicle and tying the cord with a silk thread, to prevent hemorrhage.

If the testicles are not down in the scrotum, the horse is called a ridgeling.

If one testicle is down and the other is not, the one that is not down should be procured first. In order to get it, cast the horse and tie his hind feet close up alonn his sides. Then roll the patient upon his back and cut through the scrotum as if the testicle were there. ()il your hand and pass it up through the internal abdominal ring, where you will almost invariably find the testicle. Take hold of it firmly, draw it out and castrate by any of the usual methods.

If you do not find it between the internal and external abdominal rings, pass the hand on up through the external abdominal ring and you will fiml it, if he has one. Seize it, draw it out and take it ofl by any of the usual methods.

My preference is for the ecraseur, an instrument invented by $\mathbf{M}$. Chassaignac, of Paris.

Take the opposite testicle in the same manner and the operation is complete. Always cut well down toward the sheath, to allow the pus to escape freely to prevent any unnecessary swelling. If the clamps are userl, remove them in twenty-four hours and turn the horse out, or put him at moderate work, and he will do much better than if standing in the stable.

About the third day, run your finger up around the cord and break off any attachment, so that it will not adhere too low down, and thus prevent scirrhus cord from forming. 
Be careful before operating, to examine and see that the horse is not ruptured. If a rupture should exist, castrate by what is called the sowered method, which ronsist, in cutting through the skin only, being careful not to cut through the tunics or coverings of the testicle. Then castrate with the clamps. Place the clamps over the covering of the testicle, the testicle includerl, and clamp tightly. Then cut off the coverings and testicles together and allow the clamps to stay on until they come oft of their own accord. If the bowels should come out, return them and stitch up the internal alukminal ring with cat-gut. Then stitch up the scrotum, leaving a small opening for the escape of pus, and the operation will be complete.

\section{BLEEDING AFTER CASTRATION.}

Bleeding from the incisions in the scrotum, from the small blood vessels severed and especially from the small artery at the back part of the cord sometimes occurs in warm weather and may appear quite serious No fears need be entertained, as it seldom lasts only a few minutes and is easily stopped by splashing cold water into the wound.

Bleeding from the artery in the front part of the cord may he attended with danger, when the necessary precaution has not been taken to fasten it, to prevent bleeding. In such cases, the end of the cord should be found and the artery tied with a strong thread. If the end of the cord cannot be procured, pack the parts thoroughly with cotton batting or wool, which may he saturated with tincture of muriate of iron. Shove well ap into the wound; a blood clot may be formed in this 
manner and thus stop the bleeding. Allow the packing to remain two days, then withdraw it gently and but little danger need be apprehended.

\section{PHYMOSIS AND PARAPHYMOSIS.}

Phymosis consists in the glans penis becoming enveloped in the prepuce, thus heing hidden from view, and forming a soft, glazy swelling. It most frequently happens from an injury or as the result of castration.

Paraphymosis consists in the prepuce forming a constriction around the penis, just behind the glans. The glans penis will then be visible and frequently wreatly swollen. It also occurs from the results of castration, and from injuries of any description.

The treatment for phymosis, consists in applying hot fomentations and scarifying. When paraphymosis

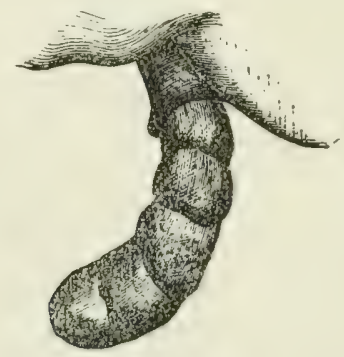

PARALTSIS OF 'THE PEYIS.

exists, apply hot fomentations and scarifying; also catch hold of the tumor and evert it by forcing it back through the constriction, then place it back into the sheath and place pledgets of cotton batting greased with vaseline, into the sheath, to prevent the penis from coming out. Usually within a few days the swelling 
disappears and the trouble is over. Sometime the swelling is so great that it cannot be everted immeriately: when such is the case, scarify and press the blood and serun out as much as possible, place a bandace around the body and suspend the penis to relieve the weight, occasionally pressing out the blood and sermm, and in a few days you will he able to evert it, when a speedy recovery will follow.

\section{AMPUTATION OF THE PENIS.}

It is rery seldom that we are called upon to porform this operation. But occasionally it has to be curne to save the patient and sometimes to be rid of the $u n-$ sightly appearance, which greatly depreciates the value of the horse.

Some of the causes justifying such an operation are paralysis of the penis, caused by blows from the whip or kick from a mare inring service; cancerous growth uron the penis; or anything which causes the penis to hang pendulous, with inability to retract it within the sheath.

The operation is performed by casting the animal and getting him under control. ('ommence to amputate by making a circular incision down to the urethra (or canal for the passage of urine.) Next cut the tissue off from the urethra for about an inch, which you must leave. Then cut off the urethra. If any small arteries present themselves, ligate them. sprinkle powdered per sulphate of iron upon the remaining part and allow the patient his freedom. Insert the catheter before operating and allow it to remain during the operation. Pass the catheter every day, until the wound is healed. 


\section{HERNIA, OR RUPTURE.}

Hernia is the protrusion, either of the bowels or of the mesentary through a natural or unnatural opening out of the abdominal cavity. There are several kinds of hernia, the most important of which I shall mention here.

Umbilical hernia occurs when the opening of the umbilical cord passes into the ablomen, which fails to

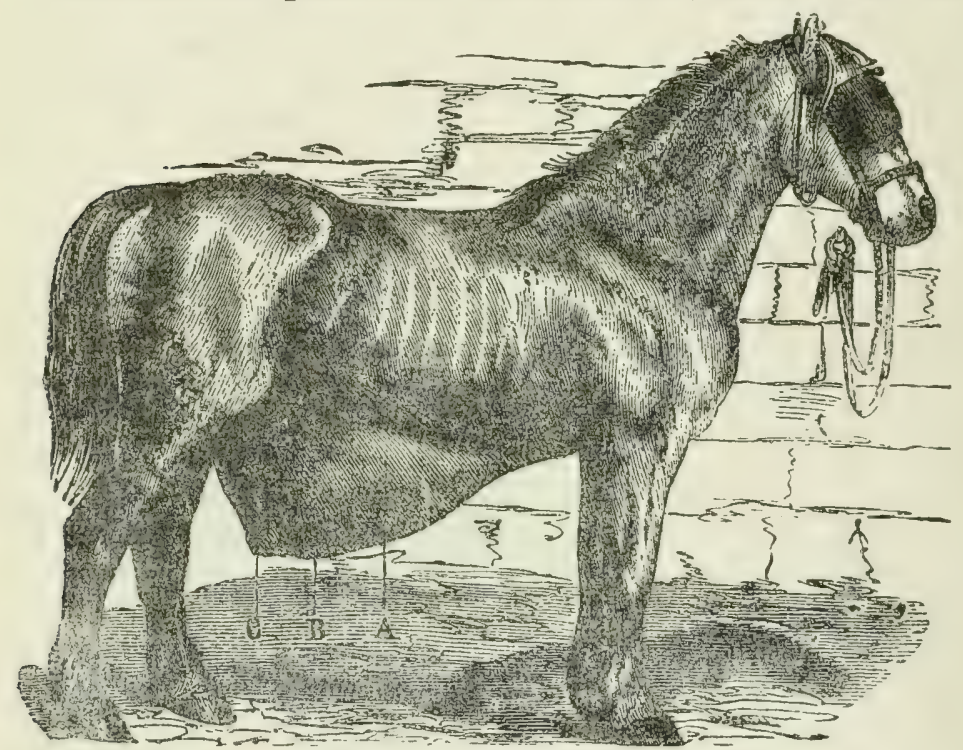

HERNIA OF THE UTERUS OR WOMB.

close up, and the aperture continues to increase in size. It is invariably seen in young colts, and, if it fails to close apply a bandage and keep it on for some time. A cure will generally be effected; if not, and the colt is a year old, fasten on a pair of clamps and allow them to slough off, or inject hypodermically, a solution of salt water in a few places around the base of the tumor; 
or another method and one that is very effectual. is to cut off the hair over the tumor and apply nitric acid enough to barely moisten the skin. Either of these set up a deep-seated adhesive inflammation and close ur, the opening.

Inguinal or scrotal hernia, consists in the passage of the bowels or mesentery down through the abdominal ring, into the scrotum. If in an entire horse, cut by the covered operation (see castration). If in a gelding, either open it up, stitch up the abdominal ring and then clamp, or, gather up as much of the scrotum as is possible and clamp, first returuing the bowels, and allow the clamp to remain until it sloughs off. A cure will most generally be effected.

Ventral hernia consists in an abrasion of the abdominal walls, allowing the bowels to escape through to the skin and forming tumors of rarious dimensions. It is readily recognized by its disappearance upon pressure, and almost immediate return on heing released from pressure; by its softness and the gurgling sound transmitted by the bowels. If the rupture is small, return the howels and fasten on a clamp, allowing it to slough off: or, bandage tightly when first noticed; or use some of the methods as given in umbilical hernia. Another method is to cast the animal, then return the bowels, cut through the skin and stitch up the abdominal wall: and apply a broad bandage.

Diaphragmatic hernia is when the bowels pas: through a rupture of the diaphragm, and press upon the Jungs. If any quantity of the bowels pass through, tho breathing will be difficult and a terrible gurolinw. wheezing commotion will be heard on applying the car to the chest. In such cases there is no remedy and the paitent will very soon die. If the rupture be small and a very small portion of the bowels protrude througl the 
diaphragm, the pain may be very intense but that terrible sound around the lungs will be wanting and, if kept quiet on opiates, the bowels may return and the patient do well.

In strangulated hernia, when the bowels beroms entangled in the mesentery, completely shutting ofl the passage, death is almost inevitable.

\section{ANEURISM.}

The term aneurism is used to denote the rupture or dilation of an artery, forming a fluctuating tumor, disappearing on pressure, but immediately returning. It may be caused by hard work or by a hard, straining full. By pressing the fingers upon the tumor, the pulsation may almost always be felt.

Treatment:-If the tumor is visible and can be operated upon, cut down and tie the artery at either side of the tumor, which should then be dissected out. Afterwards treat as an ordinary wound. But if the artery be very large, you had better let it alone.

\section{SEEDY TOE.}

This term is used to denote an unhealthy condition of the font, wherein the wall and sole of the foot partake of a mealy or dry, erumbling nature, thus separating them from the sensitive lamina of the os pedis, or bone of the foot. It is principally caused from laminitis but sometimes by a large clip being fastened to the front part of the shoe and being hammered down hard on the wall of the hoof. It may also be caused by an ill-fitting shoe being irregular upon its bearings upon the wall and sole at the toe. 
Trentment:- cut out the liseased parts and show wh a bar shoe: and pad it in the crevices to prevent dirt f:om getting in and accumulating.

\section{CORNS.}

At first these consist of a simple bruise of the sols of the foot and are usually found in the inner quarter of the fore foot, though they are sometimes found in the outer quarter. Some authors consider them to be horny tumors, but such I could never find. However, I an inclined to helirese that, from the constant irritation, they are the cause of infammation of the lateral cartilages and thus of the formation of side-bones. Soms-

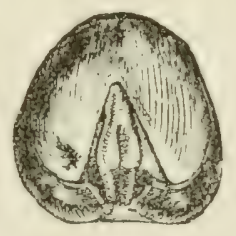

conss.

times the corns sulpumate and if an exit is not made for the pus to escape hy, it will ratuse a bulging of the coronet and will break and dischare there; or the pus will keep burrowing down towarl the toe and thus separate the wall from the sensitive sole. Barl shoeing, or allowing the shoes to remain on ton long, are the principal causes.

Symptoms:- Heat and lameness in the afferted font, and invariably pointing it forward; heat and tenderness aromu? the aflertud yaurter, and a sulky stmmbling gait when driven. If it is a suppurating corn, the horse will scarcely put the foot to the ground and, if it does, will merely rest it on the toe. Lpon tapping the quar- 
ter of the foot with a hammer, the animal generally evinces pain; also pressure from a pair of pincers will cause more or less flinching, and by taking off the shoe and paring down the quarters, blood streaks to a greater or less degree will be observed.

Treatment:-Take off the shoe and poultice the foot with ground flaxseed, or bran, for twenty-four hours; then pare down the corn until the sole between the bar and wall is compressible; then place a sponge saturated with crude oil or a seventy-five per cent. solution of carbolic acid over the corn and nail on the shoe, retaining the sponge in position and applying the crude oil or carbolic acid solution once or twice a week; or shoe with a bar shoe, using the sponge as directed. You may paint the wall and sole of the foot with crude oil once or twice a week, but avoid letting it get up into the hair around the coronet. If you suspect a suppurating corn, cut down and allow the matter to escape, then remove the dead horn as far around as the pus cavity extends. Afterwards apply compound tinct. benzoin twice a day, or tincture of iodine once daily. As soon as new horn "ommences to form, place a leather or felt pad over the bottom of the foot and shoe with a bar shoe.

\section{THRUSH.}

The principal causes of thrush are filth from standing in manure and urine in the stable, from poulticing with cow-dung, from wading around in a filthy barnyard, from grease-heel and scratches, and from hard work on rough, stony roads, causing bruises to the frog.

Symptoms:-There is a fortid discharge from the cleft of the frog, only in severe cases causing lameness and a feverish foot. 
Treatment:-First, remove the cause and keep in a clean, dry place; remove all ragged portions of horn and fill the crease in the frow with calomel a few times, and a cure is generally effected; or use powdered sul. phate of copper or burnt alum. If allowed to run undisturbed, it may develop into canker. This latter dis ease of the foot, is due to a regetable parasite which not only destroys the sole and frog, but invades the deeper structures and prevents the natural and healthy growth of the horn substance, which usually repairs an injury to these parts.

\section{CANKER.}

Causes:-As it is due to a parasite, certain conditions must present themselves before the parasite commences its work. As it is rarely ever seen in high, dry districts, it is quite evident that moisture paves the

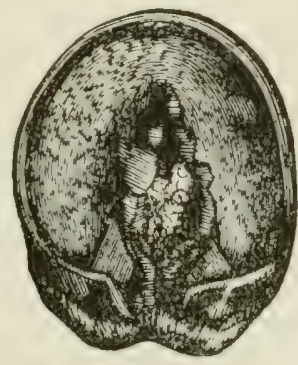

CANKER.

road for the germ to travel in. Among the many causes are filth from standing in manure and urine, when the frog or sole is injured; also from poulticing injuries, as pricks from nails, with cow-dung, or allowing them to run in filthy barnyards; also working on muddy roads and grazing on low, marshy pastures; from grease heel. 
Symptoms:-Canker is generally confined to one font, althugh all the feet may be attacked at the same time. When the disease follows an injury, which it usually does, and the soft tissues are involved, they show no inclination to heal; on the contrary, there is secreted from the inflamed parts, a foul smelling watery discharge, which gradually undermines the surrounding healthy horn and which, if not checked in its course, will completely destroy the whole frog and sole. Rapid growths spring out from the frog and sole, of a soft, spongy, unhealthy nature, and, if cut off, may grow from one to two inches in length in twenty-four hours. When cut "lose up to the foot, they bleed quite profusely. There is an offensive discharge and the disease stubhornly resists treatment. Lameness may at first be very slight, hut as the disease advances, it will be a prominent symptom.

Treatment:-First, remove to a clean, dry place, then cut out all of the disansed tissues or until it bleeds freely, pare down the sole quite thin next to the canker, removing all of the sole and frog that covers diseased parts. Next put on a shoe with quite a wide web. Then take some tow and saturate it with muriate of iron and cover the diseased part, and apply pressure by slipping sticks under the web of the shoe; it is very essential to apply heavy pressure upon the diseased parts. Medicines seemingly lose their force when applied for a few days in succession. It is therefore necessary to change from one remedy to another quite frequently. ('hoose any from the following list: Sulphate of copper, sulphate of zinc, sulphate of iron, chloride of zine, quicklime, chloride of antimony, creosote, calomel, carbolic acid, nitric acid, sulphuric acid, corrosive sublimate, nitrate of silver, or caustic soda. You may mix any one of these, equal parts, with animal chitroal, but 
very often you will be compelled to use the most severe remedies, undiluted. It will try your patience, but be persistent, and when you notice healthy horn commencing to form, cease using very severe remedies. From commencement to finish, the foot should be dressed once in twenty-four hours, bearing in mind that pressure should be applied continuously throughout the course of treatment.

\section{PRICKS FROM NAILS.}

This is of frequent occurrence from the carelessness of the horseshoer or from the mail splittmg while being driven; also from horses picking up nails while being driven, or while rumning at large-more frequently from the latter than from the former.

Symptoms:-Lameness, heat and tenderness in the foot aflecterl. If a nail splits when the smith is driving it, he will notice it at once from the horse flinching. If the nail be withdrawn and the owner not informed, the patient in a few days may be very lame and suppuration may take place hefore the real cause is found out; there will be heat and tenderness present. By tapping the foot with a hammer, you may find the exact place. Or, if a nail is driven close enough to press the sensitive laminx, the patient may not go lame for a few days, then it will he very lame, frequently holding the foot from the ground or morely resting it upon the toe. By tapping the foot with a hammer you will discover the spot, when you hit the nail that is pressing. Or, if the patient has picked up a nail and goes lame, by examining tho foot you will find either the nail penetrating the foot or the place whence it was withdrawn. 
Treatment :-If from a split nail or one driven too close, draw it out and poultice the foot for a few days with ground flaxseed; a cure will usually be effected. But if it suppurates, make an opening and allow the pus to escape, and treat as a suppurating corn (see corns). If the patient has picked up a rusty nail and you have withdrawn it, cut a hole into the bottom of the foot where the nail entered, about the size of a nickel and fill the opening 'with tincture of iodine, two or three times a day; but if the foot is very much inflamed, poultice for a day or two, also applying either tincture of iodine or Friar's balsam to the wound. If suppuration has taken place, remove all loose or dead tissue and wash out thoroughly with castile soap and hot water. Then dry it out and apply Friar's balsam or tincture of iodine, and protect the foot from dirt and filth. It is sometimes necessary to remove all of the frog and sometimes the sole. Be very careful if the horse has picked up a rusty nail, as tetanus, or lockjaw frequently ensues; also extensive inflammation and sloughing around the coronet and loss of the hoof, which in the majority of cases, renders the animal worthless.

\section{QUITTOR.}

The most frequent causes of quittor are horses calking themselves and catching cold, suppurating corns, pricks from nails, bruises, gravel working up through the feet, disease of the lateral cartilages, also quarter cracks, etc., etc.

Symptoms :-First a tender swelling around the coronet, bursting after a while and discharging a whitish fluid and showing no evidence of drying up or healing.

By proling, you will find fistulous openings running down into the foot in different directions. 
Treatment:-If much inflammation exists and a visible bulwing of the coronet, lance it and apply flaxseed poultices for a few days. Then into each sinus inject twice daily the following:

Zine Sulphate.......... one drachm.

Sulphate of Copper....... one drachm.

Water.............. four ounces. Mix.

After you have followed this up for about a week, and the foot is almost healed, stop the injections and apply Friar's balsam twice daily until a cure is effected. If the disease affects the lateral cartilages, or if sidebones are formed and they are affected, they may have to be removed.

Occasionally the sinuses burrow down deep into the soft tissues and a fistulous opening is established, which will not yield to the former treatment but will require more heroic measures. In such cases, fill the sinus full of corrosive sublimate, pounding it well in; allow it to remain for two days, then take it out and apply carbolic salve until well.

\section{GRAVEL IN FO0T.}

The feet of horses are often affecterl by a small gravel encased between the sensitive and insensitive parts. - It may become pressed in through an opening made in paring out corns, or into a large nail hole, or into the opening made from paring out the sole, from treading on a nail or being forced through a quartercrack; or by continuous pressure while traveling, a gravel may be forced through the parts where the sole and wall of the foot unite; or by being forced up between the frog and bars, etc. In whatever manner it gains admission to the sensitive parts, it causes inflammation and sloughing of the surrounding portions. 
Symptoms:-Lameness is a predominant symptom. If there be an opening, it will present a dirty appearance, look black and unhealthy and have a feetid smell. Upon tapping on the parts with a hammer, the animal will evince great pain. If much matter is formed, it is liable to work up and break around the top of the hoof close to the hair, when the lameness will partially or wholly subside.

Treatment:-First wash the parts thoroughly, then enlarge the opening by paring away the hoof, to make room to remove the gravel; examine very carefully to avoid pushing it in farther. When in contact with the gravel, it will be readily recognized by its sound and hardness to the touch. Work around it and dig it out as carefully as possible. Then wash the parts perfectly clean and inject into the wound twice daily-

Carbolic Acid.......... two drachms. Water................ half pint. Mix.

Or,

Sulphate of Zinc....... four drachms.

Water ..................... Mix.

Apply a hot linseed meal or bran poultice until the fever and lameness subside, after which, if the opening is protected to prevent dirt from getting into it, the animal may be put to work, still applying the aforesaid lotion if possible, until the parts are healed.

\section{PUMICED FO0T.}

This is a condition wherein the horny sole becomes separated from the sensitive sole or laminæ, by an effusion that accumulates as the result of inflammation of the parts. The bottom of the foot, especially toward the toe, crumbles away, exposing the sensitive tissues. 
It is generally caused by laminitis or founder when proper treatment has not been applied. The coffin-bone tips down at the toe causing pressure upon the sole, and prevents the formation of new horn. Other conditions may cause pumiced sole, but, as a rule, not to the extent as does founder. Punctured wounds of the feet and bruises of the sole occasionally cause an exudation, separating the sensitive from the insensitive parts, but the soft tissues are seldom exposed because new horn is developed, completely taking the place of that which has been separated.

Symptoms:-Exposure of the sensitive parts ain readily be recognizer. If it has not reached that stage, the foot will become convexed, or bulge down instead of being concaved or hollow. The sole of the fout, especially at the toe, readily crumbles away and there is lameness very similar to that of founder.

Treatment:-This disease is incurable. If the too pushes through, put the animal out of its misery. If the sole is convexed, by careful shoeing, keeping the pressure off the sole, and by keeping the feet cool, the animal may do light work on soft ground, but should never be driven on hard roads.

\section{BRUISES OF THE FROG.}

Causes:-Bruises of the frog usmally occur from horses stepping upon rough, hard, frozen gromd, or from travelling upon newly macadamized roads, thereby getting stones wedged in between the shoe and frog, which are frequently overlooked, and the frog becomes badily bruised. Flat feet, with low heels, are the best subjects for bruises. Cuts through the frog, from atepping upon glass or any other substance causing similar injury, are liable to cause suppuration beneath the frog. 
Symptoms :-Lameness, in proportion to the extent of the injury involving the sensitive parts. If the injury is quite severe, the animal walks wholly upon the toe or raises the foot completely and hobbles along on three legs. When the horse is standing quietly, the limb is set forward and flexed at the ankle, the toe resting upon the ground. Upon examining the foot, the injury may be detected, but if no opening exists, the matter will be observed working back toward the heels, seeking a soft place where it can break through and escape. The horn then becomes detached at its connection with the skin and looks somewhat white and bleached. As soon as the pus escapes, the lameness diminishes.

Treatment:- When lameness is first observed, examine the bottom of the foot carefully and if no puncture is discovered, bathe the foot in cold water to prevent the formation of matter; but if matter has already formed, pare the frog and sole quite thin to relieve pressure, make an opening to allow the pus to escape, then poultice for a few days with linseed meal, and pour tincture of iodine into the opening twice daily. If the pus has loosened much of the frog, remove the part that has become detached, with the knife. If the pus has worked its way up and has broken, and is discharging near the hair, a great portion of the frog has become separated and should be removed at once, after which dress three times daily with compound tincture of benzoin, tincture iodine or spirits turpentine, and if obstinate, with-

Chloride of Zinc......... one ounce.

Soft Water...........eight ounces. Mix.

Apply once or twice daily and pad with cotton batting. As soon as a thin layer of new horn has grown on, it may be padded with waste, a sponge or cotton 
batting, a piece of leather placed over the entire bottom of the font and a shoe nailed on, when the animal may be put at slow work.

\section{CALK WOUNDS.}

In all northern climates, when the roads become slippery, horses are necessarily shod with sharpened shoe calks. The heavier hreed of horses, used for teaming and hauling heavy loads, are compelled to

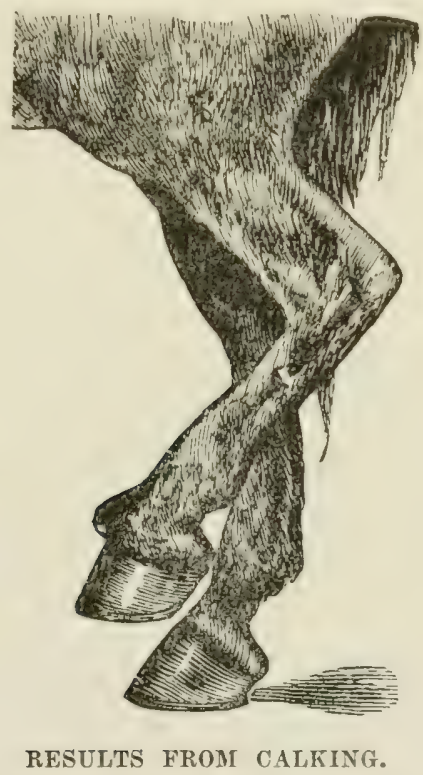

twist, turn and back up, when they frequently tread upon their mate, or upon their own feet, inflicting injuries of a somewhat severe character. In fact, any horse wearing sharp calks, is liable to tramp upon and injure either itself or another horse. Some horses while 
standing in the stable, rest with one hind foot upon and immediately in front of the other foot. These horses are liable to calk themselves at any time.

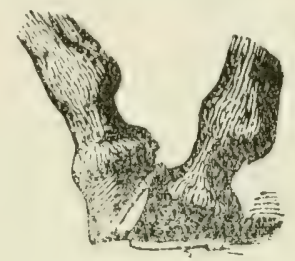

CALKING.

Treatment:- When horses rest one foot upon theother, a circular boot should be worn when in the stable, extending out four inches from the ankle, which will prevent calking. If the wound is not deep and there is little if any soreness, fill it with tar or vaseline. But where the wound is deep and there is great soreness, with considerable inflammation and suppuration, hot poultices should be applied for a few days. Beneficial results will be produced by sprinkling powdered saltpetre upon the poultice before applying it. Afterwards apply-

Sulphate of Iron......... half ounce.

Water..................... pint. Mix.

Use three times a day.

Or tincture benzoin compound may be applied twice. daily, with advantage. If the wound is deep between the horn and skin in front of the foot, cut away the detached horn and continue to apply either of the above. remedies.

\section{CONTRACTED HEELS.}

Contracted heels or hoof-bound, is a very common disease among horses in cities. They are generally kept upon hard, dry floors and the feet do not receive the 
necessary amount of moisture to keep the hoofs soft and pliable. When the hoof becomes dry and hard, it has a natural tendency from the conformation of the foot, to draw inward, and the continued inward pressure, causes a wasting away or shrinking of the soft tissues of the foot.

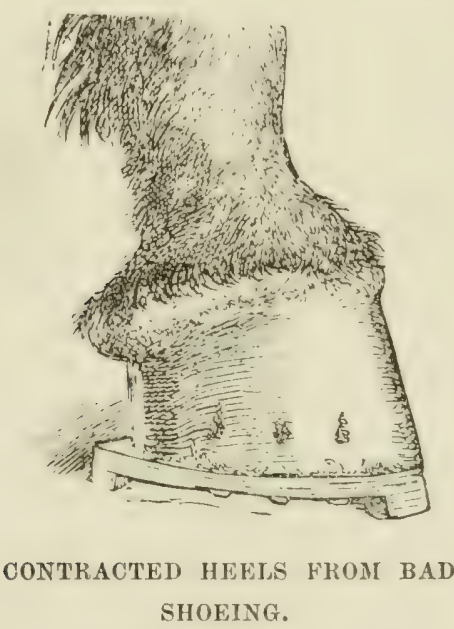

The fore feet are mostly liable to this affection, althouwh it may occur in a hind foot. ['sually, only one foot at a time is affected. When both feet are attacked, onc foot is worse than the other. Sometimes only one heel is contracted and when this is the case, it is generally the inner one.

Causes:-One of the common causes of contracted heels, is a want of knowledge of the structure of the foot, on the part of the horseshoers. They become entirely too free with the knife and rasp. The frog, heels and bars are cut away, the walls are rasped too much, high calks are put on and the nails driven too close to the heels. Another common cause is the removing of horses from pasture lands, where they 
receive moisture and daily exercise, and shipping them to cities, where they are apt to be placed on hard, dry floors. The feet become fevered on account of the change, the horn becomes dry and hard, the soft tissues gradually waste away and contraction is the result. Contraction is sometimes an accompaniment to other diseases, as ring bone, side bones, thrush, corns, navicular disease, sprains of the tendons, etc.

Symptoms:-The hoof becomes dry and hard, and loses its circular shape. The heels are higher and narrower, the sole is greatly concaved; the frog is greatly shrunken and cramped between the narrow heels. The bottom of the foot is smaller than at the hair, the heels are often ridged and scaly, with deep fissures running up between the quarters. In the first stages there is no lameness, but great uneasiness is observed, manifesting itself by the frequent shifting from one foot to the other. The animal will stumble considerably, especially when driven on hard, rough roads. When first brought out of the stable, there will be stiffness, and inclination to walk upon the toe, but after being driven a while, it will warm up and go with quite a free action. The toes of the shoes wear down very quickly and when the shoes are removed and the bottom of the foot pared, a dry mealy substance is observed where the sole and wall come together. When the disease is of long standing, lameness is present.

Treatment:-Preventive measures are much more effectual than curative ones. The feet should be kept moist, either by bathing or standing for a few hours laily upon some moist substance, as moist saw-dust, or a ground floor. Any of the parrafine ointments applied to both wall and sole two or three times a week, are very beneficial. One of the best remedies to keep the 
foot soft, is crude oil applied to both wall and sole twice a week but it should not be applied up against the hair. The rasp should not be used upon the wall to any extent and the knife should not be applied to the fror heels and bars. Level the heels down with the rasp, if necessary, and if there are loose pieces extending from the sole, remove them. Avoid putting calks on shoes except when absolutely necessary. Make the shoe the shape of the foot and not the foot the shape of the shoe. The shoes should be reset once a month and the feet will not become cramped nor grow too long. As to the cure of the disease, when it does exist, there is a great diversity of opinion. There are a great many shoes and other contrivances invented for the spreading of the heels, all of which may be of some ralue if properly and persistently used. About as good a course of treatment as can be employed, is to poultice the foot until coftened, then pare it down well and lower the heels; put a half shoe (commonly called a tip) on the toe of the foot; leave it the usual thickness in front but taper the ends of the shoe down to a thin edge, then take a rasp and commence just below the hair to file the wall very thin, gradually sloping down to the bottom of the foot, where it should be left its natural thickness. When this has been done, apply around the coronet,

Powd. Cantharides...... one drachm.

Vaseline.............. one ounce. Mix.

Apply and wash off the next day. Grease with lard for a couple of days and then turn ont to pasture. Leave in pasture for three or four months and about every two weeks apply the ointment. If the case is not of too long standing, a complete cure may be expected, but in those old chronic cases, where the frog is almost obliterated, the sole greatly concaved, with extreme 
wasting of the soft tissues of the foot, treatment is almost useless, and the animal had best be put out of its misery.

\section{KNEE SPRUNG.}

Knee sprung in reality is not a disease but is often the result of disease. In a perfectly formed limb, a line drawn from the elbow to the fetlock will touch the limb its entire length, but when the knees are sprung,

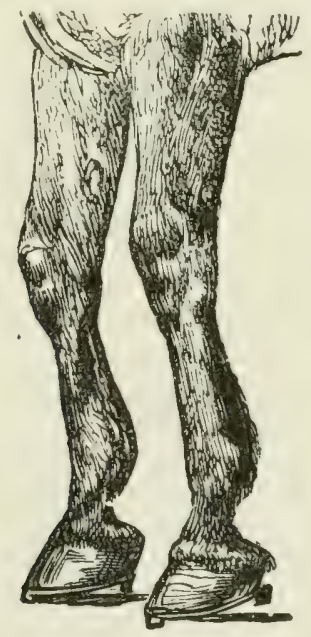

KNEE SPRUNG.

a line drawn from these points will pass at varying distances behind the knees, presenting a bowed appearance of the limb. Slight bending of the knee may be congenital; the colt being foaled with this deformity, may grow up and continue through life and yet not be considered a knee-sprung animal.

Causes:-One of the most frequent causes is too much driving before the animal has matured or received 
age sufficient to stand the work; or by hauling very heary loads with roung horses, but continuous hauling uf heavy loads will cause a weakness and sprung knees in horses of any age. Sprain and injury of the back, tendons or suspensory ligament, if not properly treated, is most certain to be followed by sprung knees. Sore feet, soreness in the knee joint, sore shins or any disease of the limb from the knee down, causing continued lameness whereby the animal rests the heel, will cause knes sprung, by the gradual contraction of the back tendons and ligaments.

Treatment:- When occuring in young horses from overwork or fast driving, if the case is not of too long standing, give absolute rest and use a stimulating liniment, as-

Tincture Cantharides.... four ounces.

Oil Turpentine......... two ounces.

Alcohol............. two ounces. Mix.

Apply twice daily with smart friction down the back of the limb, both above and below the knees, or,

Powd. Cantharides.... four drachms.

Vaseline............. two ounces. Mix.

Cut off the hair and apply along the back tendons, and in twenty-four hours wash off and grease every day with lard. While the medicine is on, tie the horse so he cannot bite it. Repeat every two weeks until three or four applications have been made. If there is thickening of the back tendons, use the latter prescription. The animal should rest for a few months and during that time it may be turned out to pasture. With this trouble, the danger of recurrence must he borne in mind for it is always liable to occur again. If it arises from other causes, see treatment for those ailments. In old, 
chronic cases, but little can be done. Sometimes the operation of tenotomy or cutting the tendons, may be performed, which see under head of "Sprain of the Flexor Tendons."

\section{SAND CRACKS.}

Sand cracks or quarter cracks most frequently comes from concussion, or from driving at a high rate of speed over rough roads, horses whose feet have weak walls, or whose feet have been weakened by rasping; also from ill-fitting shoes with uneven bearings; excessive dry-

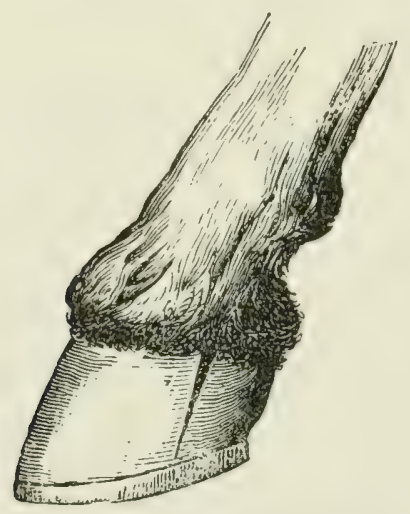

QUARTER OR SAND CRACK.

ness; also changing from damp to dry places, from taking horses from pasture and stabling them on hard, dry floors; from jumping, calk wounds, etc. The cracks invariably begin at the coronet and extend downward, sometimes into the quick, causing lameness and bleeding. Sometimes fungus growths will spring out of the crack near the coronet.

Treatment :-If sand crack appears in a strong footed horse, I would advise clinching with a nail or clasp, to 
hold the wall firmly together. But if in a weak footed horse, as it almost invariably is, commence at the coronet and cut the edges of the crack its full length, so they will not rub together. Then cut transversely (crosswise) at the coronet about three-quarters of an inch to each side of the crack to the quick, and apply a bar shoe, with even pressure all around. If the quarter is cracked all the way down to the shoe, you may take the bearing off from that quarter. Blisters applied around the coronet will now act beneficially. Use the following:

Olive Oil.............. four ounces.

Aqua Ammonia..........two ounces.

Tr. Cantharides..........two ounces. Mix.

Apply every three or four days. Also keep the entire hoof softened by painting it with crude oil two or three times a week. If a fungus growth springs out, cut it off and touch with nitrate of silver, or,

Chloride Zine......... four drachms.

soft Water.............two ounces. Mix.

Apply as often as you deem it necessary.

\section{CORONITIS, INFLAMMATION OF THE CORONET.}

Inflammation of the coronet, with suppuration, is not infrequently met with in this country, and in many instances is caused by frost bites. A great many horses are abused by being compelled to stand out-doors during the excessively cold weather which prevails during the winter season; and frequently we see cases of coronitis during that season. (oronitis may also be caused by standing in filthy wtables or by bruises. But from whatever cause, this disease is of a serious character. 
Symptoms:-There will he notieed a peenliar shuffling or sliding lameness; there will be bulging of the coronet, fever around the coronet and a tendency of the tissue to break and slough.

Treatment:- The treatment must be constitutional as well as local. Give the following physic,

P'owd. Aloes.............. one ounce.

Powd. Ginger........... one drachm. Mix.

Give at one dose. Also

Nitrate of Potassium .... three ounces.

Powd. Nux Vomica....... one ounce.

Bi-Carbonate of Soda.....two ounces. Mix.

Make fifteen powders and give two powders each day. The local treatment consists in washing the coronet thoroughly with castile soap and hot water, penciling the sores with nitrate of silver every third day and applying twice daily,

$V$ aseline..............two ounces.

Salycilic Acid.........two drachms.

Sulphate of Zinc......... one drachm. Mix. Or,

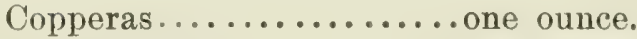

Carbolic Acid............six drachms.

Soft Water................ Mix.

Apply twice daily, or paint every day with muriate of iron or tincture iodine.

\section{NAVICULAR DISEASES, OR COFFIN JOINT LAMENESS.}

This disease consists in inflammation and alteration of the structures entering into the formation of the cofin joint. It is generally seen in horses that are used as roadsters, or on the track. It may be caused by hard driving, picking up nails, by nails being driven into the 
quick near the coffin joint, ly bill shoing, as uneven bearings, or contraction at the lieel; ly wearing the shoes too long and too much pressure upon the soles, by

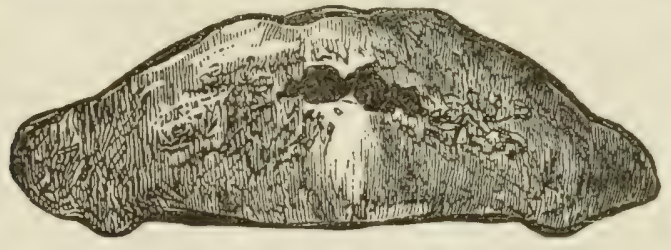

NAVICULAR BONE DISEASED.

gravel working into the foot, by strain of the flexor tendon where it passes orer the joint; also by concussions, bruises, or by standing too long in the stable.

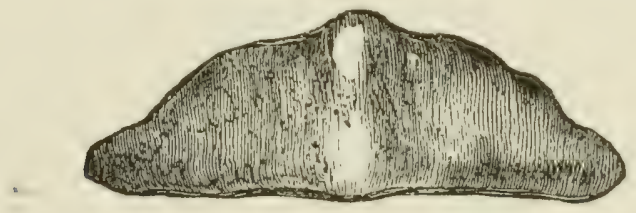

NAVICULAR BONE IILALTHY.

Symptoms:-Pointins of one or both fore feet; if one foot only be alfected it will be held in front of the other. and rest only upon the toe; if both are affected, the animal will change from one foot to the other and point alternately. There will be an inclination to stumble when first driven, but as the animal is warmed up, it will travel much better. The animal travels with a stumbling gait, the front part of the shoes being worn down when the heels of the shoes will he almost as good as new. This may go on for some time before the lameness becomes a prominent symptom. Afterwards will be fever in the heels, with more or less contraction of the hoofs at the heels, and a dryness throughout the foot, with lameness now as a prominent symptom. 
Treatment:- When first noticed, take off the shoes and turn the horse out to pasture on swampy ground, or fix up a stall with a clay floor, softened with water, and keep the patient standing there through the day. During the night, poultice the feet with flaxseed till you reduce the fever: then you may insert a frog seaton; or blister around the coronet with,

Cantharides.......... two drachms.

Vaseline ............... one ounce. Mix.

Apply every two weeks and keep the animal on damp pastures for three or four months, if possible.

Or you may use-

Tincture Cantharides..... two ounces.

Aqua Ammonia......... two ounces.

Sweet Oil............ four ounces. Mix.

Apply once daily for two or three weeks.

The treatment of navicular disease is generally nnsatisfactory; but, if the foregoing fails, neurotomy is the final resort. This may be performed either by the hich or the low operation. The high operation consists in cutting out a piece of the plantar nerve, just below the knee. The lower operation is cutting out a piece of the plantar nerve, at the pastern, between the fetlork and the foot. As the nervous communication is 'ut off, the lameness ceases, although the disease continues mubated. Nevertheless, to say the least, it is a humane operation.

\section{WOUNDS FROM BARBED WIRE.}

Wounds from barbed wire may be placed among the worst classes of injuries with which we have to deal. They are usually caused by horses running into a fence constructed of barbed wire. The skin is lacerated and torn and very frequently the muscles are diricied and 
torn in a frightfưl way, the injury depending greatly upsu the force and manner in which the animal comes in sontact with the wire. It is quite a common occur$\mathbf{r}$ me, to see the skin and lesh of a foreleg torn across at the forram to the bone, and the bone exposed from. there down to the knee, or to see the skin lacerated and

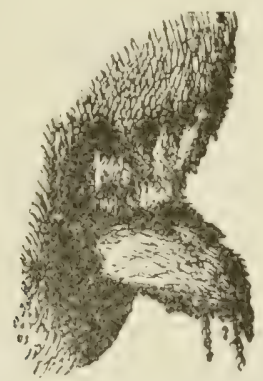

WOUNDS FROM BARBED WIRE.

torn around the hocks, and the tendons and ligaments exposed, also deep and serinus injuries around the fetlocks and feet. In wounds of this kind, the loss of blood, as a rule, is not very great, the hlood vessels beroming torn and twisted and thus preventing the flow of blood.

Treatment:-First examine the wound very carefully anl remove all dirt, splinters, or harbs from the wire, s? fomd flowing from any of the vessels, they should be pirked up and tied. If it is found dillicult to do this, take a needle-any ordinary sewing needle will answer -and pass it through the llesh close to the blood vessel on both sides of it, then tie it tightly around the vessel. The small amount of flesh thus tied with the bloon vessel, will do no harm. If the wound is not very large, it may be stitchul together, but rarely if ever, are stitches to be used in wounds of this kind. The 
surrounding tissues lecome so weakened and torn that the stitches will not hold, and only tend to irritate instead of assisting in holding the parts together. It is best to get the portions together as closely as possible and secure them in this position with bandages or sticking plaster. If the sticking plaster is used, shave the hair close to the skin so the plaster will hold, always leaving an opening for the exit of pus. When the skin is torn in shreds, the pieces may just as well be cut off, as they will not heal and will only slough off if left alone. If there is much swelling, frequent applications of hot water should be nsed until the swelling subsides; afterwards it will not be necessary to use hot water, except to wash off any matter that may acenmulate. The wounds may be dressed with any of the following:

Sugar Lead .......... four drachms.

Sulphate Zinc........ four drachms.

Water.............. one quart. Mix.

Apply twice daily, or,

Carbolic Acid........... half ounce.

Soft Water................ pine pint. Mix.

Or,

Carbolic Acid........... half ounce.

Raw Linseed Oil....... eight ounces. Mix.

Apply morning and evening. Or an excellent healing powder may be used if any unhealthy granulations called proud flesh, arise, as,

Powd. Alum.......... eight ounces.

Iodoform.............. one ounce. Mix.

Apply twice daily. Should any symptoms of fever arise, give half ounce of saltpetre twice daily, and feed upon soft diet. Keep the patient tied, so that it cannot bite the sore. If the wound is very bad, it is sometimes necessary to place the animal in slings. 


\section{SPLINT.}

Splint is a bony elargement seen most frequently on the inner side of the fore limbs, between the knee and lower third of the cannon bone. It is occasionally seeu on the outer side of the fore limbs, as well as upon the hind limbs between the hock and pasterns. It varies considerably in size, from that of a small buttermut

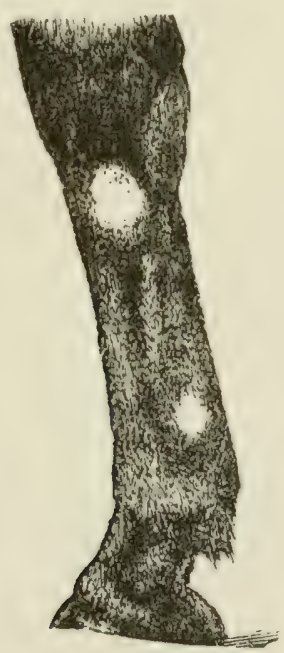

SPLINT AND SMALL WIND GALL.

down to a grain of corn, but the small nodule which is always found two-thirds the distance down the cannon bone from the knee, should not be mistaken for splint, as it is the natural development of the small cannon bone. There are three cannon bones in each leg, one large and two small ones, situated between the knee and fetlock and hock and fetlock. The splint occupies a position between the large and small cannon bones, sometimes extending entirely across the back part of the large bone, beneath the suspensory ligament." In 
this condition, permanent lameness may be looked for. $\mathrm{Th}_{1}$ regular splint frequently causes lameness while growing, but after becoming hard, rarely causes any annoyance, remaining only as an eyesore.

Causes:-The most common cause is too hard work, or too hard driving before the animal has fully matured. In the young animal, these bones articulate with a very limited movement and if strained or overtaxed, splint is formed. In older animals, these bones become united and it requires greater force to tear them apart so as to set up an inflammatory action resulting in splint. But there are other causes, as running: jumping, slipping, kicks, bruises to the bone, in fact anything that will sprain the ligamentous attachments of the large and small cannon bones. 'When splints are caused by bruises, they may appear anywhere on the bone.

Symptoms:-In the earlier stages, there will be lameness; as observed when the animal is trotting, the limb will be carried with an outward swing, accompanied by a very low nodding of the head, but while the animal is walking, the lameness will scarcely be perceptible, except when the splint is close up to the knee affecting the knee joint. Then lameness may be ohserved while the animal is walking. By benting the leg and pressing quite heavily along the bones, tenderness will be observel. Later on, by passing the hand down along the bone, an enlargement will be felt. When the enlargement is an inch or more below the knee, it seldom amounts to much, but if close to the knee so as to affect the joint, it frequently causes permanent lameness.

Treatment:-In many instances it is adrisable to let it aloue, but if they are quite tender and feverish, use sone mild lotion, as, 
Witchhazel.............. six ounces.

Tincture Arnica............ne ounce.

Laudanum.............. one ounce. Mix.

Apply three times a day, after bathing with cold water. After the fever and soreness have been reduced and the animal still remains lame, use a blister, as,

Red Iodide Mercury...... one drachm.

Cantharides............ one drachm.

Vaseline.................... Mixe ounce.

Make two or three applications, allowing a week to intervene between each application. Then allow two or three weeks rest, if possible. If lameness and wroness upon pressure is great, the trouble lies moler the arfrring of the bone; a small opening made with a small blade of a knife, cutting through the correme immotiately over the lump, will relieve the tension. Treat as above specified.

\section{SORE SHINS.}

This is an affection of the cannon bones in the fore legs of young horses, and is mostly seren in race horses and trotting horses under four years old. It is caused by the amount of hard work they are forced to perform when their bones are as yet unable to stand it. The bones do not become fully developed until the age of five or six years, and if subjected to hard training while in the state of devclopment, they are unable to stand the shocks and concussions caused by severe and continued speeding. Sore shins may inclucle the entire bone but generally aflect only the anterior part of the lower third thereof. It is also caused by kicks either from the attendant or from other horses; or by grooms becoming vexed while curying over the shins and hit- 
ting them three with the edge of the curry comb. because the animal is restless from pain.

Symptoms :-If in the thoroughbred, lameness will be observed after a gallop; if in one les, that foot will be placed in front of the other, or pointing. If occurring in both lews, the animal will be restless, shifting its weight from one leg to the other. An animal affected in this mimner, acts sore and moves with a short, stiff, stilty gait. The parts are very sensitive when pressed. upon. There is more or less swelling along the bone, which at first is soft and doughy upon pressure; after a time it becomes hard, like bone. The soreness may extend the entire length of the bone, but if caused by an injury, it may be confined to the injured portion alone, and also as a usual thing, soreness arising from overwork, settles in the lower third of the bone, just above the fetlock joint.

Treatment:-When the lamenesss is first noticed, bathe the parts with cold water for half an hour to an hour at a time, three times a day. Immediately after apply,

Spts. Chloroform........two ounces.

Laudanum.............. two ounces.

Tr. Arnica.............two ounces.

Witchhazel............ ten ounces. Mix.

Apply a loose bandage and moisten it occasionally with this liniment. Continue in this manner until the soreness disappears completely, then, if necessary, apply a few blisters, allowing ten days to two weeks to intervene between each application. The following will suffice :

Red Iodide of Mercury....one drachm.

Cantharides...........two drachms.

Vaseline or Lard..........two ounces. Mix. 
Apply with smart friction. In twenty-four hours wash off, and grease daily with lard, until another application of the blister is made, after which grease as before. Absolute rest is imperative and a complete restoration of the parts may be effected in a fow weeks, without the use of blisters. Bad cases, however, will need the blisters and continued rest for sereral months. The constitutional treatment consists of a physic, given at the outset, of,

Barbadoes Aloes.......... one ounce.

Gentian...............two drachms. Mix.

Give at one dose, in half pint of water. Feed on soft food and give a teaspoonful of powdered saltpetre twice daily.

\section{WIND GALLS-WIND PUFFS.}

This disease consists of dilated burser situated at the back part of the limbs, at the fetlock joint, or immedi-

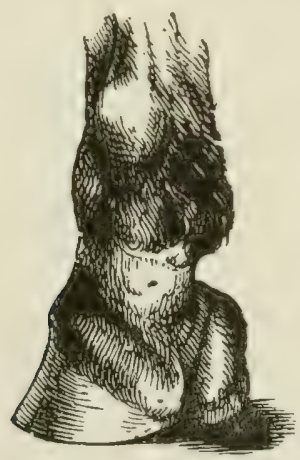

WIND GALL.

ately above the joint. They are more often seen on the hind than on the fore limbs and are usually caused from over exertion and sprains. They appear as soft tumors of various sizes, being somewhat tense, when the animal 
stands firmly upon the afflicted limb. They are usually painless and seldom caluse lameness, but if an inflammatory action takes place, they become painful and lameness results. They may swell so large as to interfere with the action of the tendon, thus causing lameness, or sometimes they change their condition and become calcified or bonelike, then lameness will follow, although this condition is seldom seen.

Treatment.- When they first appear, bathing with cold water and bandaging, will keep them under control, followed with a few applications daily of tincture of iodine. If they do not yield to this treatment, use the following blister:

Red Iodide of Mercury.... cne drachm.

Powd. Cantharides...... one drachm.

Vaseline............two ounces. Mix.

Cut off the hair and apply with smart friction. Allow it to remain on for twenty-four hours, then wash off and grease every day for five days, when apply again. Use in this manner until four applications have been made, or,

Cottonseed Oil.......... five ounces.

Croton Oil........... ten drachms.

Oil Camphor......... three drachms.

Oil Turpentine........ tive drachms.

Oil Origanum..one and a-half drachms.

Coal Oil.............ten drachms.

Sulphuric Acid........ half drachm. Mix.

Apply once a day with smart friction, until thoroughly blistered; apply a little hogs' lard for a few days, then repeat the liniment. The treatment of wind cralls is sometimes a little unsatisfactory, as they will often reappear. 


\section{SWELLED LEGS-STOCKING-ODEMA.}

This is more of a symptom than a disease and is frequently the result of defective kidneys, liver or heart, weak circulation, or lymphatic irritation from standingupon a hard floor, without exercise, etc.

Symptoms:-When the animal is at work or turned out to pasture, there will be little or no swelling: whon standing in the stable, the lower part of the limbs will

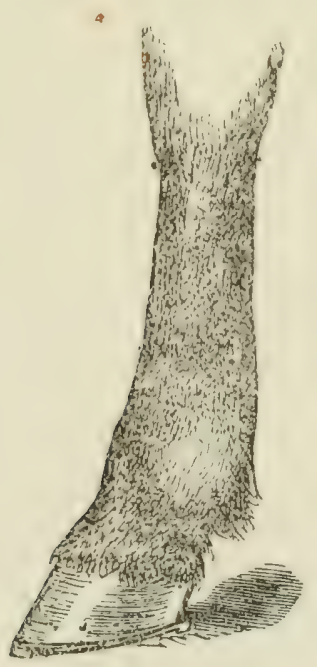

SWTLIEI HEGS.

become swollen, but the swelling will disappear upon exercise. The animal will apparently be enjoying excellent health, pulse normal and appetite good, yet swelling of the limbs every morning.

Treatment:-If from standing upon the hard floor, change to a dirt floor, and if from want of exercise and you have no work for the animal, turn into a vacant lot, for a few hours each day. Give some tonic to tone (ip) 
the system as well as medicines to operate upon the kidneys, as,

Sulphate of Iron........two ounces.

Quinine Sulphate..... four drachms.

Gentian............... Mixe ounce.

Mako sixteen powders. Give two powders each day, or,

Nitrate of Potassium.....two ounces.

Powd. Nux Vomica......... one ounce.

Bi-Carbonate of Soda...... one ounce. Mix.

Make twelve powders. Give two powders each day. Frequent hand rubbings of the swollen parts, with a few applications of witchhazel, will be found to be very beneficial.

\section{COCKED ANKLES, OR KNUCKLING.}

Causes:-All kinds of heavy work and hard pulling, especially on hilly roads, or not being properly shod anil

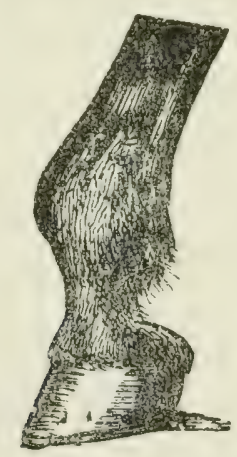

KNUCKLING OVER, OR COCKED ANKLES.

pulling and slipping on smooth pavements; also fast driving upon hard roads or race tracks. It is most frequontly seen in horses with-very straight pasterns, and especially in the hind pasterns of horses somewhat ad- 
Tanced in years. It is also produced by disease or sprain of the suspensory ligament, and by shortening of the tendons at the back of the leg, as well as diseases of the fetlock joint. Many young foals knuckle over so harly, that they walk upon their pasterns for a short time, but as the muscles, ligaments and tendons gain a little strength, they adapt themselves to their functions and force the joints to their place.

Treatment:-In the case of young foals, it is seldom necessary to interfere, as in a few days or weeks they will straighten themselves. But if they walk over on the pastern joint and do not straighten themselves in a few days, take some rubber hose or belting padded with cotton batting or wool, and make a brace to extend from the foot to the knee, fasten it on, with the leg perfectly straight but do not tie too tightly; allow it to remain for a week or ten days, examining frequently to see that it does not chafe the leg and as soon as the colt will stand straight, remore it. When knuckling occurs in young horses, from fast driving upon hard roads, remove the shoes, then apply a few blisters from below the knees behind the legs, down around the fetlock, and turn out to pasture. You may use either a liquid blister or a salve; either of the following will suffice:

Spts. Turpentine.........two ounces.

Aqua Ammonia.......... two ounces.

Olive Oil............. four ounces. Mix. Or,

Oil Cedar..............half ounce.

Oil Tansy............... half ounce.

Oil Hemlock........... half ounce.

Oil Wormwood............half ounce.

Alcohol................ten ounces. Mix.

Apply morning and evening for a week or ten dạ̦s. Or, 
Powdered Cantharides.. four drachms. Vaseline .............two ounces. Mix.

Apply every four days with smart friction, until three or four applications have been made; then grease every day with hog's lard until healed, or use the firing iron which should only be attempted by a qualified surgreon. When knuckling has become chronic, relieve the tendous by proper shoeing, which is best done by raising the heels and lowering the toe. For the hind feet, a long heel shoe with high calks answers best.

\section{SIDE-BONES.}

This consists in solidification or ossification of the lateral cartilages, situated on either side of the foot just above the heel. It may be caused by shoeing with too high calks, by pricks of nails, by corns, by uneven shoeing, by cutting away the bars of the foot, by bruises to the heels or soles of the feet, causing a deep irritation or inflammation.

Symptoms:-Lameness, with short, stilty gait and a want of elasticity in movement. On pressing upon the cartilage on either side of the heel, you will find it hard and unyielding, instead of soft and pliable; and the pressure will cause pain. Sidebones are almost always found in the heavy breed of horses. Whether in heary or light horses, it is considered an unsoundness.

Treatment:-The treatment consists in absolute rest, and shoeing with a bar shoe. If lameness continues, blister with-

Cantharides........... two drachms.

Red Iodide of Mercury .... one drachm.

Vaseline.... one and one-half ounces. Mix. 
Apply every five or six days until four applications have been made. If that should fail, use the firing iron and blister immediately. If this fail, perform neu. rotomy by cutting out the plantar nerve.

\section{CURB.}

This disease is always seen at the posterior aspect if the hock and consists of a sprain of the calcaneo cuboi! ligament, or sprain of the tendon which passes down the back part of the hock, or one of its sheaths or cov-

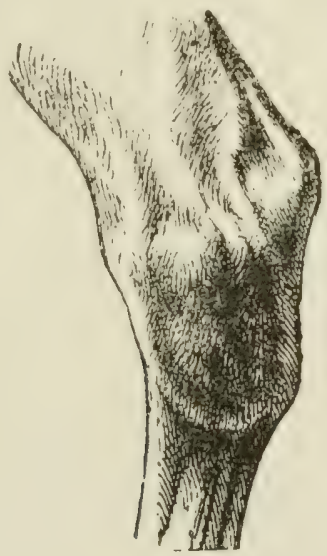

CURB.

erings. It is generally caused by horses jumping and rearing, by backing up violently, or by slipping. It sometimes appears as a small very hard nodule; or it may be very large and soft, with heat and tenderness, and more or less lameness. The lameness does not depend upon the size of the curb. Sometimes the curb is very large with no lameness; at other times it is very small with considerable lameness present. Sometimes it is congenital, and in such cases it is not an unsound- 
ness. It is best observed by standing over it and lookinz itraight down the hind leg, from the point of the

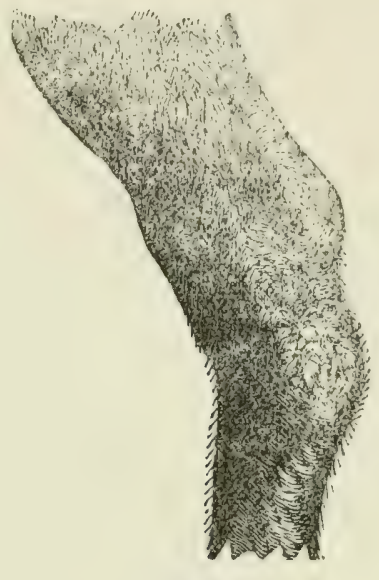

CURB.

hock to the fetlock, which should present a straight line, but when a curb is present you will observe the enlargement about three or four inches down from the point of the hock.

Treatment:-First and foremost-rest. Apply highheeled shoes, hot fomentations and a liniment composed of-

Alcohol.............. four ounces.

Tinct. Iodine.......... two ounces.

Witchhazel.............two ounces. Mix.

Apply after bathing. If this fail to remove, apply-

Tinc. Myrrh et Capsicum ..eight ounces.

Red Iodide Mercury.... two drachms.

Iodide Potassium.......two drachms. Mix.

Apply after bathing with hot water, or,

Powd. Cantharides...... one drachm.

Red Iodide of Mercury... one drachm.

Vaseline.............. one ounce. Mix. 
Apply once a week until four or five applications have been marle. Sometimes it is necessary to use the firing iron; when such is the case, use the sharp pointed or needle iron and fire quite deep, after which apply the above blister. Sometimes a curb can be removed by friction. Take a bone or smooth piece of hard wood and rub the curb, thorounh once or twice a day, and in the course of time, a small curb will disappear.

\section{CAPPED HOCK.}

This consists of a serious abscess, situated on the point of the hock, between the skin and tendons in areolar tissue. It is wencrally alused by the horse kicking the point of the hock against some hard substance, or by

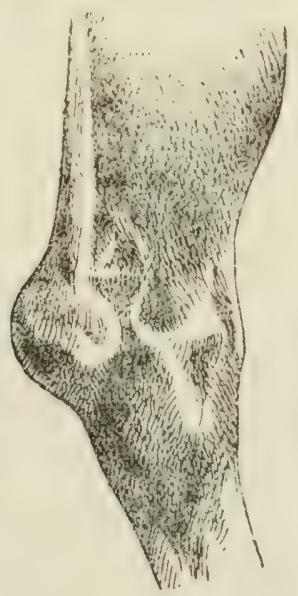

CAPPED HOCK.

bruises on the point of the hock. The size varies from that of a walnut to that of a good, large bowl. The abscess is soft and fluctuating. It seldom causes any lameness, but in rare instances it does. 
Treatment:-First of all prevent the cause; if from kicking in the stable, which it generally is, change the patient to a stall where it cannot injure itself. Then apply high heeled shoes, and foment with hot water until the inflammation subsides. Then open with a lancet from below and allow the serum to escape. Afterwards inject tincture of iodine into the sack and apply a cold bandage; if thought necessary, repeat in two or three days. If it heals up and there is no serum accumulating, but just a slight thickening of the parts, apply,

Rectified Spirits........ four ounces.

Tincture Iodine......... two ounces.

Witchhazel.............two ounces. Mix.

Foment with hot water and apply the liniment twice daily ; or,

Tr. Iodine............ two ounces.

Oil Hemlock............half ounce.

Oil Wormwood...........half ounce.

Oil Origanum............. one ounce.

Alcohol.............. four ounces. Mix.

Foment with hot water and apply twice daily. Have plenty of patience and perseverance as the complete removal is sometimes very obstinate.

\section{THOROUGH PIN.}

This is a disease of the sheath of the flexor perforans tendons, and is usually caused by a sprain. It sometimes causes considerable lameness; at other times the lameness will be very slight, and, possibly, none at all. This tendon plays down the back part of the hock to the inside of the os calsis or point of the hock, and any sprain or irritation may cause an extra secretion within the sheath of the tendon and a bulging out on each side of the back part of the hock, in the hollow or gambrel 
space. It is usually a soft, fluctuating tumor and if pressed on one side, will cause a greater enlargement on the other side.

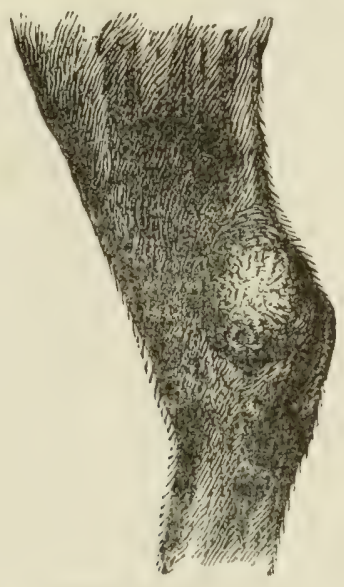

THOROUGH PIN.

Treatment:-If there is lameness, give absolute rest and apply hot fomentations for a few days. Then blister with,

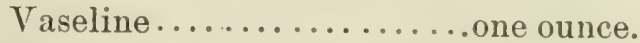

Cantharides............ one drachm.

Red Iodide Mercury . one-half drachm. Mix.

Or apply,

Tincture Myrrh........ four ounces.

Capsicum............ four ounces.

Iodide of Potassium...... one drachm. Mix. Or,

Cotton Seed Oil... . . four ounces.

Croton Oil.............. one ounce.

Oil Camphor..........two drachms.

Oil Turpentine......... four drachms.

Oil Origanum............ one ounce.

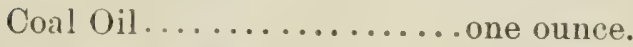

Sulphuric Acid......... forty drops. Mix. 
Apply once daily with smart friction. If this should fail to remove it and the lameness has subsided, leave it alone unless it be a valuable horse. Then, if the swelling is great, open it and allow the fluid to escape, afterwards injecting tincture of iodine into the sack, until you are satisfied the sack is destroyed. Then keep cold water running over the hock, or apply a sponge on oither side of the hock and keep it wet with cold water to keep control of the swelling: and keep this up until the swelling subsides. Immediately after opening give physic,

Powd. Aloes.............. one ounce.

Powd. Ginger........... one drachm. Mix.

Give at one dose. Feed on soft feed and give,

Saltpetre............. four ounces.

Bi-Carbonate of Soda......two ounces.

Glauber Salts............. one pound. Mix.

Give two tablespoonfuls two or three times a day. It will take about three months to efiect a cure. If the limb swells very much and the patient does not lie down, place slings under it to give rest and relieve the other leg.

\section{TUMORS.}

Tumors belong to a class of diseases known as hypertrophies or enlargements, and should not be confounded with inflammatory exudations. Tumors constantly grow or increase in size independent of the surrounding tissues, with the exception that they derive their nourishment from the blood, whilst an inflammatory exudation depends on a morbid state of the parts, and increases as long as the morbid state continues, then decreases to a greater or less extent. Tumors are di- 
vided into several kinds or classes. But to speak of them separately is quite unnecessary in a work of this kind. We will treat of them in a general sense or take up those which are most frequently met with in veterinary practice. These are fibroid, fatty, cystic and "ancerous tumors. Any of these tumors may be seen on almost any part of the animal, and for the removal of them, such remedies as iodine, mercury and stimulating embrocations, are used with more or less success. The best mode of disposing of them, is to dissect them out and afterwards treat them as an ordinary wound. (See wounds.) Should a fibrous tumor make its appearance in the nostrils in the form of a nasal polypus, it may be removed by an ecraseur, an instrument of French invention for removing excresences; or you may take a small iron with an eye in the end of it, fasten a heary corl through it, and slip it up over the tumor until you have reached the top of it, then commence twisting and continue until you have twisted it off; pull the tumor out and you have completed your work.

\section{INCISED AND CONTUSED WOUNDS.}

An incised wound is the result of a cut from any sharp instrument. If an artery or vein cut, it should be taken up and tied. Then wash out any dirt that may have accumulated and bring the edges of the wound together, and fasten with stiches. This may be done by using a curved needle and white linen or silk thread, or catgut sutures. The best mode is by the interrupted suture, viz. : by taking stitches one-half to three-fourths of an inch apart, tying a knot at each stitch and cuttiug the ends about one-half inch long. Afterwards apply Friar's balsam, or- 
Vaseline.............. one ounce.

Carbolic Acid...... one-half drachm. Mix. $\mathrm{Or}$,

Carbolic Acid........ one-half ounce.

Raw Linseed Oil....... eight ounces. Mix.

Apply once or twice daily. Should the stitch break out, the wound will heal by granulation. When this occurs bathe with tepid water and rastile soap occasionally, and apply twice daily the following:

Powd. Alum........... four ounces.

Iodoform.............. one ounce. Mix.

Use sparingly.

Contused wounds are those where the tissues are bruised or torn, and always require a careful and thorough examination, for fear some foreign body may be lodged deeply within them. This, if not removed, will be a constant source of irritation and thus prevent the wound from healing. After all foreign bodies are removed, wash out thoroughly and stitch the edges of the wound together as best you can, then if possible, bandage below the wound to assist in bringing and holding it together. As these wounds always heal by granulation, begin at once to use compound tincture of henzoin, or,

Sulphate of Zinc....... four drachms.

Sugar of Lead.......... five drachms.

Soft Water............ one pint. Mix. Or,

Powd. Alum.......... four ounces.

Iodoform.............. Mne ounce. Mix.

Apply morning and erening. If it suppurates much, wash with hot water and castile soap. Occasionally an enlargement or inflammatory exudation is thrown out, sometimes called "proud flesh." When this happens, 
touch every third day with nitrate of silver, or burnt alum, until it disappears. If any portion of the skin lecomes detacheu or fails to heal up, cut it off with i knife.

\section{IRREGULARITIES OF THE TEETH.}

Sometimes from various causes, the teeth of a horse become very irregular. I have seen the molar teeth of a horse serrated like the teeth of a saw, so that they fitfed nicely together. Such animals could not wrind their hay, and had to be fed altogether on soft food. The upper jaw is always a little wider than the other, just enown to fetch the molars nicely together. But. sometimes the upper jaw is so wide that the teeth do not come together, consequently there is an irregular growth. At other times the surface of one tooth is larder than that of its fellow on the opposite jaw; the result is that one tooth grows long and its opyosite is worn away. In such cases the projecting tooth should be cut off. I remember seeing a horse six years old, belonging to a Mr. Butler, near Spencerville, Indiana, almost dead from starvation, though it had plenty of hay and grain in the manger. The cause was rregular teeth. The horse was so poor it could not stand, and so weak that it could not raise its head, and was in fact, dying when I put it out of its misery. Had I secn the horse before it became so weak as to be unalile to stand an operation on its teeth or to eat afterwards, it might. have been saved.

When the teeth become irregular so tha sharp corners lacerate the tongue or cheeks, they should be rasped off with a guarded rasp. Any person can do this with a little care. The surface of the teeth should never be rasped smooth; as the horse has to grind his food, the teeth should be rough. 


\section{TOOTHACHE-DECAY, OR CARIES OF THE TEETH.}

Decay of the teeth is generally caused by blows, by biting hard substances, such as nails; or from fool becoming lodged between the teeth, or between the cheeks and teeth.

Symptoms:-An excessive flow from the mouth of a saliva, very ropy in consistency; intense pain, exhibited when the tooth is struck by an instrument, by redness and swelling of the gums: by dropping of half-chewed food from the mouth, by the presence of whole grain in the manure, by suddenly stopping while chewing food, throwing the head to one side and slightly opening the mouth; also by suddenly stopping and throwing the head to one side when drinking cold water. Other symptoms, are unthrifty appearance of the animal; rough staring coat, weakness and emaciation, with profuse sweating on very little exercise, swelling of the jaw. If in the fang or root of the upper molar, there will frequently be a futid discharge from the nostril on the affected side. If the root of the lower molar, there may be, in connection with the swelling, a fistula of the lower jaw.

Treatment:-First make an examination of the teeth by looking into the mouth, or explore with the hand. If food or any other substance is lodged around the teeth, remove it, and if you cannot decide which tooth is aflected from looking into the mouth, press upon the teeth separately through the cheeks and usually when the aflected tooth is pressed upon, the animal will evince pain. If the tooth is badly affected, it should be removed. This may be acomplished by taking a pair of forceps and fastening onto the tooth, then twisting and working the tooth until it appears loose. Then place a small piece of wood beneath the handles of the 
forceps close up to the tooth: pry down on the handle and the tooth will come out, unless it should he so hadly decayed that it lraks off. When this happens the best mode of treatment is to trephine the jaw, just above the fangs of the tooth, and punch the latter out. The punch should not be less than a quarter of an inch in diameter. Place the punch on the fangs of the tooth anil hit it a few taps with a hammer, when the tooth will drop out. The after treatment (onsists in feeding solt food for a few days and washing out the cavity with a ten per cent. solution of carbolic acid twice a day.

\section{PARROT MOUTH.}

This is a peculiar combition of the mouth, where the upper jaw is much longer than the lower. (innseguently

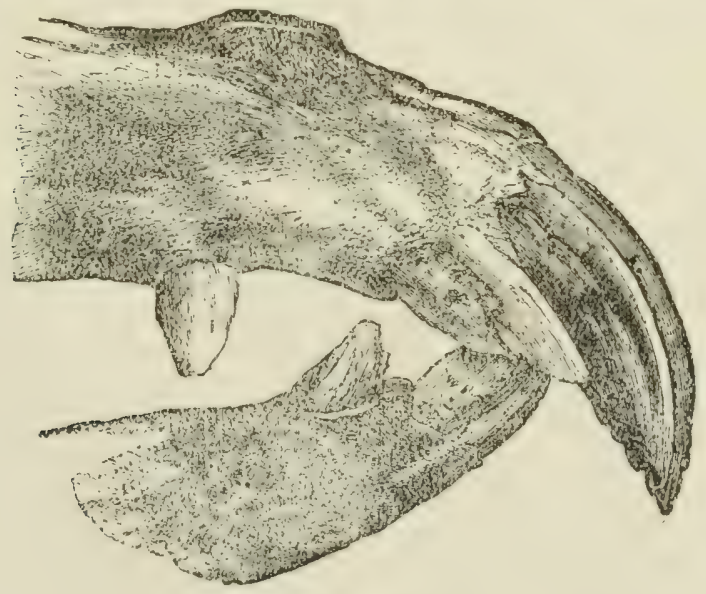

PARTOT MOUTH.

the upper and lower incisors fail to meet, and the upjer incisors grow down to an enormous length. The incisors of the jaw also grow to such a length that they press 
upon the bars of the roof of the mouth, sometimes to such an extent as to injure the horse. In such eases the teeth must be filed or rasped down. Parrot mouth is a great inconvenience, as the animal cannot graze; it will, however, do well when fed in the stable.

\section{WOLF, OR SUPERNUMERARY TEETH.}

Supernumerary teeth, more frequently called "wolf teeth," are frequently seen just in front of the molars in either the upper or lower jaw. They are small, conical shaped teeth and of little or no importance whatever, except where they grow to a sufficient length to disturb the horse while eating. Many persons labor under the mistaken idea that "wolf teeth" cause blindness in horses. This can hardly be the case. But horses having hereditary opthalmia, or which are otherwise prone to weakness of the eyes, may be greatly disturbed during dentition fever; and after such fever is abated, all symptoms of ophthalmia disappear; and the credit is erroneously given to the extraction of the "wolf teeth." But when called upon to extract them, (lo so for the price and for the owner's consolation-but not for any great benefit to the animal.

\section{APOPLEXY.}

Apoplexy is a rupture of a blood-vessel in the brain. The vessel being in a weakened or diseased condition, breaks or ruptures and allows the blood to escape into the brain structure, where it forms a clot.

Symptoms:-A stroke of apoplexy comes on very suddenly. The animal will fall, if the extravasation of blood is great. There is a sudden loss of both feeling and motion; difficult breathing is observed and there is 
inability to arouse the patient from its stupor. The action of the heart seems regrular at first, but gradually grows quicker and weaker, and if the shock is very heary, the organ soon ceases to beat. If the ruptured vessel is small and the escape of blood very limited, unconsciousness may be complete for a short time, possibly a few minutes, or an hour or more. After this, a general or local paralysis is observed. During the period of insensibility, the breathing is labored, in fact stentorian. The pulse is slow and full and the head hot. The location of the paralysis, which is sure to follow apoplexy, depends upon the portion of the brain pressed upon by the extravasated blood.

Treatment:-I'lace the patient in a cool, quiet, comfortable place and kecp the head elevated, and cool with wet cloths or a sponge until the temperature becomes natural. Rub the limbs thoroughly and bandage, and if necessary to warm them, apply mustard. After the animal regains consciousness so that it will swallow, give the following:

Powd. Aloes............. one ounce.

Calomel............... one drachm.

Powd. Gentian.........two drachms. Mix.

Give at one dose in a pint of water as a drench. Also give,

Iodide of Potassium.......two ounces.

Bromide of Potassium....two onnces. Mix.

Make sixteen powders. Give three powders each day for a couple of weeks.

\section{PARALYSIS OF THE LIPS.}

Paralysis of the lips is seen in all classes of horses, and is the result of an irritation and inflammation of the serentl pair of nerves, called the portio dura. It is 
very seldom, indeed, that the nerves on both sides of the ficcere aflected. The disease is generally confined to ons silk. During the time the inflammatory action(neuritis) is in progress, the nerve loses its functional ability and the exudation from the inflammatory action, pressing upon the nerve, prevents it from regaining that ability and thus the paralysis continues. Examination along the course of the nerve will frequently reveal a small, hard nodule, or the inflammatory exudation which is the seat of the trouble. The causes of this disease may be found in badly fitting or heavy halters and bridles, blows, kicks, etc. The lip will hang pendulous and flabby, with inability to raise it to its proper place. The animal will drop food from the mouth and have great difficulty in getting enough to eat, as it has to gather it. with its teeth instead of with its lips. When drinking, it pushes its head deep into the bucket to drink.

Treatment:-The treatment consists of removing from the nerves, all pressure in the form of halters and bridles, and place the horse in a loose box stall. Feed on soft food and apply hot fomentations to the side of the cheek. Immediately afterward apply,

Tincture Cantharides.....t two ounces.

Tincture Iodine.........two ounces.

Tincture Capsicum........ one ounce.

Alcohol..............three ounces. Mix.

Apply morning and evening along the side of the face for about two weeks. Then, if no progress is noticeable, apply,

Vaseline.................. one ounce.

Cantharides.........one-half drachm.

Red Iodide of Mercury.... one drachm. Mix.

Use thoroughly every fourth day, for three applications. Afterwards grease with hogs' lard every day until well. Should this fail, apply the actual cautery, or insert seatons; or you may try acupuncturation. 


\section{GOITRE-BRONCHOCELE.}

This is an enlargement of the thyond whond, which is situated on the under side of the neck just hehind the angles of the lower jaw, at what is sometimes called the throat-lateh. These glands when enlared assume varying proportions, from those of a hen's egg to the size of a sugar bowl. When wreatly enlarwed, they sometims press heavily upon the winlpipe and cause difficult. breathing. It is most frequently seen where animals

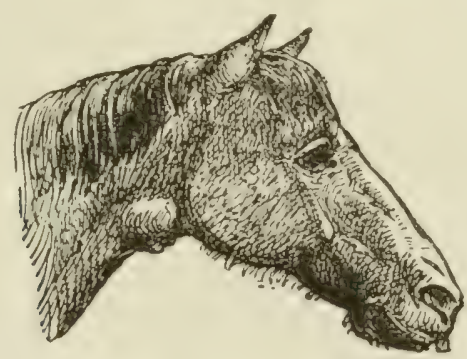

BRONCHOCELL.

graze and drink water off of magnesian linestone formations,although low situations and damp, high localities are producing causes. The disease appears as a hard lump or tumor on the side of the neck. The enlargement is movable, rrows very slowly and is not rery sensitive.

Treatment:-Remove the "ause, if it is ascertainable, feed on good food, and wive drachm doses of iodide of potash once daily; also cut ofl the hair and paint the lump with tincture of iodine once daily for a continued length of time. If this does not check the growth and reduce it, and it should be of such a size as to interfere with the breathing, take a hypodermic syringe and inject half an ounce of iodine into the center of the tumor, once a week for a few weeks; or if need be, it ('in be dissected out, but this operation requires the skill of a veterinary surgeon. 


\section{PILES-HAEMORRHOIDS.}

This disease though somewhat rare in horses, is deserving of mention, as it is a source of considerable ammorance when it does exist. It is due principally to constipation, but may be observed to follow the intense straining of dysentery, or the severe and prolonged labor pains attending difficult parturition, or foaling. Injuries might also be considered a cause. Whenever bright red irregular tumors are observed, varying in size from a small marble to that of a hen's egre, protruding after the passage of manure or when the animal is lying down, you have a case of piles on hand. Often when piles are present, the animal will rub the tail and rump against a post or other stationary object until it bleeds or scabs are formed on the parts, and not infrequently blood streaks are observed on the manure.

Treatment:-First direct your attention to the condition of the bowels, which should be kept soft by feeding on mashes and chop feed with a liberal supply of salt, but physic should be avoided. When the tumors protrude beyond the anus, wash them off thoroughly with warm water then barely cut into them with a knife, squeeze them out nicely, and deep into the wound put a few drops of,

Sulphate of Zinc....... four drachms.

Water................ two ounces. Mix.

Afterwards grease the tumor with vaseline and return it within the anus. One tumor each week is enough to operate upon. If considered preferable, they may be tied off by using fine white silk or hemp thread. Place the thread around the tumor close up to the bowel and tie it as tightly as it can be comfortably drawn and allow the tumor to slough off. If the tumors do not protrude, oil the hand and pass it into the 
bowel, then sieze the tumor and by rentle traction it may be brought to the surface for the operation of removal. If the tie method is chosen, only two at a time should be operated upon and these allowed to come away before another operation is undertaken.

\section{RABIES-MADNESS-HYDROPIOBIA.}

Madness is credited with arising spontaneously in the dogn, fox, wolf and cat but does not attack the lareer animals, save only as the result of inoculation. In regard to spontaneous origin in any animal, I entertain serious doubts. That a poison or virus may be intro-

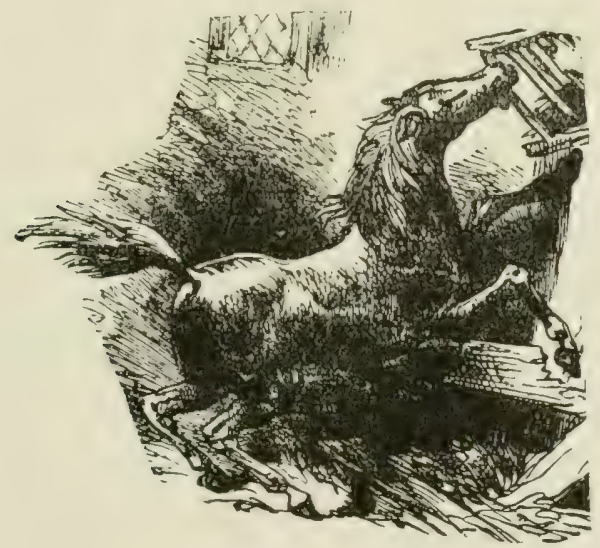

RABIES-MADNESS.

duced into the system which will act upon the nerve centers, causing severe symptoms with destructive tendencies, there is no doubt, but it must be done through inoculation. "One dog may bite another and the one. bitten may become affected with rabies, while the one inflicting the wound, shows no symptoms of the disease 
whatever, but at the time the bite was given, the saliva was unhealthy and contained a virus which became aborbed into the system, affecting the nerve centers and producing the disease. It is communicable to all the lower animals, but all animals bitten by a mad dog do not become affected with rabies. While statistics show that one in eleven of the human family, having been bitten by a rabid animal, was stricken with rabies I helieve that even then, it was caused by fear and continued worry over the possible results of the injury, rather than from inoculation from the bite itself.

Symptoms:-The period of incubation extends from a few days to a few months. It is usually observed in the horse in from fifteen to ninety days, twenty to thirty days in cattle, twenty to seventy days in sheep, twenty to fifty days in swine, and in man its development is questionable unless he brings it upon himself by continued anxiety and worry about the bite and the disease itself. The first thing observed, is irritation of the original wound; although the injury may have healed completely, the animal commences to rub and bite at it and soon a new sore is formed. If a horse, it now becomes irritable, frantic and vicious. The penis will protrude with frequent crections. A mare will act as though desiring the stallion. The patient will neigh, paw, strike, kick, bite the manger and surrounding objects, while the slightest noise made by an attendant seems to be exaggerated by the afflicted animal; it may then try to destroy everything around it. There will be periods of calm and rest, when the attacks of rage and wild, vicious manoeuvering will again return. In attempting to eat or drink, the act of swallowing is prevented by spasms of the throat. If the disease is allowed to run its course, paralysis of the limbs will follow, and death. 
Treatment:- When first bitten, burn the wound deeply with a red-hot iron, or nitrate of silver (lunar ('austic), caustic potash or carbolic acid, or cut out a piece of the flesh around the wound and then burn it. When the disease has once developed, it is too dangerous a risk to attempt to do anything, except to destroy the animal, thus putting it out of its misery as well as preventing a loss of the property it would destroy while in a frenzied condition.

\section{HYSTERIA.}

This disease is seldom seen in the domesticated animals and when appearing, occurs only in the female. It consists of an excitable condition of the whole nervous system, occurring about the time the mare is in soason, which wives rise to the theory that it is caused by the ovaries and womb. The nervousness is exhibited by trembling of the muscles, wrinding the teeth, champing the jaws and sometimes fomming at the mouth. The patient acts as though delirious. It may paw, kick or strike, or it may fall or lie down and refuse to arise if forced, but may get up voluntarily if left alone. There is rolling of the eyes, and when in a recumbent prition, the animall will sometimes be perfectly still, and again will paw or strike; in fact, the actions are at times so peculiar, that it is almost impossible to describe them.

Treatment:-Give a quart of raw linseed oil and leave the patient alone in a comfortable place, where it cunnot injure itself. Also sive two drachm doses of bromide of potassium, every two or three hours until the physic operates, when, as a rule, the symptoms sv'sile. 


\section{STINGS OF BEES, WASPS AND HORNETS.}

Horses when in a sweating condition, should nercr be brought close to bees, especially when the bees are swarming. There is something in either the smell of the horse at that time or the salts of the sweat, that attracts their attention. A whole swarm of bees has been known to attack horses at such a time, with fatal re: ults. The sting of bees is very irritating to horses, on ircount of the barbed stinger being left in the skin and the poisonous material given off from it.

Treatment:-If the imberded stinger can be found, it should be immediately extracted, either with the finger nails, a pair of forceps or a knife point, then immediately covered with an application of wet clay, or bathed with ammonia or baking soda and water, or,

Sugar of Lead............ one ounce.

Water.................. Mix.

\section{HIP LAMENESS.}

Hip lameness occurs in a manner similar to that of the shoulder. The causes leing about the same, it will not be necessary to recupitulate them. The symptoms vary from those of shoulder lameness, materially. When the hip joint is affected, there is a hop and a catch in the lame leg and the movement of the aflected quarter will be greatly lessened. While at rest, the foot of the lame leg in bad cases, will be held clear from the ground. Heat may be felt near the joint and pressure upon the parts will cause pain. The muscles over the hip soon become atrophied or wasted away, causing what is termed swinney. 
Treatment:-The treatment is just the same as recommender for shoulder lameness, including absolute rest for sometime, and with the exception of placine a high-heeled shoe on the aflected liml, to enable the parts to be maintained in a state of rest.

\section{RUPTURE OF MUSCLES.}

That muscles do become ruptured or torn is perfectly true, nevertheless these cases are seldom seen, except it may be in the muscle called flexor metatarsi which is situated above the hock on the inner side of the limb and whose action is to flex the hock. Muscles are ruptured during a severe sprain or over-exertion, as in starting a heavy load and slipping, while the muscles are at full tersion.

Symptoms:-Severe lameness is observed if the animal is forced to move and while the leg is raised, the hock will present the appearance of a dislodged hamstring. Some soreness, heat and swelling will be detected at the seat of the rupture. If the foot is placed squarely under the animal, it will stand upon it in a natural attitude, but there is an unwillingness to move either forward or backward. If forced to move foreward, the foot will he jerked upward and backward so high that a crease will be left in the muscles above the hock, presenting the appearance of a broken limb.

Treatment:-The care and attention necessary for a ruptured muscle should be about the same as for a fractured bone. Place the animal in closely fitting slings, boxing it in so as not to armit of any movement, either sideways, backwards or forwards, and keep in that position for about two months. In that time, the ends of the muscles will have grown together, when 
exercise should be given, or the animal may be put to moderate work so that the muscles will again develop. If there is much soreness, heat and swelling, ocurring shortly after the rupture has taken place, it is best combatted with frequent fomentations of hot water, and between each bathing, use-

Laudanum............. one ounce.

Tinct. Arnica..........two ounces. .

Witchhazel.........thirteen ounces. Mix.

Apply until all the inflammation has subsided, then cease using anything and allow nature to complete the cure while the animal is resting in the slings.

\section{CHRONIC CATARRH-NASAL GLEET, OR OZOENA.}

Nasal gleet consists of a suppurative inflammation of the lining membrane (Schneiderian membrane) of the nostrils and of the sinuses in the bones of the front part of the face. It is sometimes confined to the nostrils alone, but more frequently the sinuses are also affected. The principal cause, is a catarrhal inflammation of the membrane, but it may also arise from ulcerated teeth. It may also be caused by injury to the face, enlargement of the fangs of the teeth, abscesses forming in the sinuses ; disease of the facial bones, or the lodging of foreign bodies in the nostrils.

Symptoms:-A constant discharge from the nostrils or more frequently from one nostril, as one side of the head alone is more frequently affected. This discharge is of a bluish white color and tenacious, very much resembling the discharge of glanders. But the glanderous ulcerations are absent, although abrasions or excoriations of the membrane are present, owing to irritating influence of the discharge. The sub-maxillary glands 
may he enlarged and if adherent to the jaw, should be looked upon with suspicion. Heat over the sinuses will generally be observed, and sometimes a bulging out in their locality. Percussion on the side affected, will froduce a dull sound, compared to that given hy its fellow on the opposite side. This discharge is sometimes very offensive and when that is the case, the teeth should be examined thoroughly, as it is indicative of disease of the fangs.

Treatment:- When the lining membrane of the nostrils alone is affected, throw a spray up the nostrils of a five per cent. solution of listerine, and administer internally-

Sulphate of Copper........ one ounce.

Nux Vomica............. one ounce.

Iodide of Potassium........ one ounce. Mix.

Make into twelve powders and give one morning and evening. This will often effect a cure. But if the smuses are affected and pus is contained therein, trephine the sinuses and evacuate all the pus. Then wash out thoroughly with a five per cent. solution of listerinc. twice daily. Also throw up the nostrils a spray of the sime solution. If there is any diseased bone, or if there. are any foreign bodies or a diseased tooth, a remoral must be affected. This is best accomplished by trefhining, as it opens the cavity so that treatment can be directly applied. The following will suffice for a wash:

Carbolic Acid............ one ounce.

Soft Water.............. Mene quart. Mix.

Or,

Sulphate of Zinc.........six drachms.

Soft Water.............. one pint. Mix.

Syringe through the opening once or twice daily. The system should be kept up on tonies, as 
Sulphate of Iron......... four ounces.

Powd. Nux Vomica......two ounces.

Gentian...............six ounces.

Saltpetre.............three ounces. Mix.

Give a teaspoonful morning and evening. Give the most nutritive food obtainable and a moderate amount of exercise.

\section{BONE SPAVIN.}

Bone spavin consists in an irritation, sufficient to cause inflammation of the bones of the hock joint. When the inflimmation exists between the bones, iroducing lameness, but without any osseous deposit

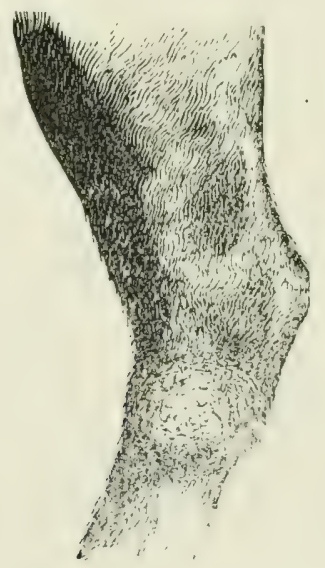

SPAVIN.

thrown out to form an enlarement, it is called occult spavin. But when there is an exudation of a bony deposit thrown out, causing an enlargement on either the inner or outer aspect of the hock and inducing lameness, it is called a bone sparin. This may occur from various causes, but in the majority of cases it is the 
result of a wrench or sprain. Still I am inclined to believe in the hereditary tendency to sparin. Whether it he in the peculiar conformation of the hock which is marked and transmitted by certain sires, or if it be an ossific diathesis in the blood, I am umable to tell. But I have seen a colt not to exceed three months old, with well-defined ringbones and spavins causing lameness, without any apparent reason therefor. And I could not

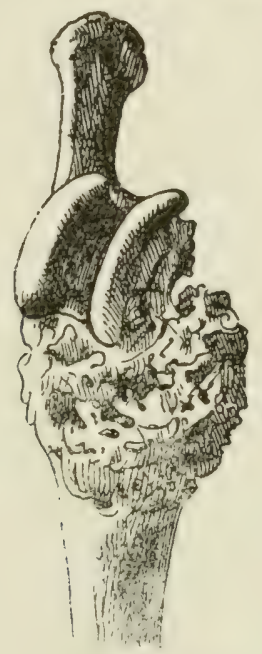

BONE SPAVIN.

attribute it to overgrowth, with the consequent extra amount of weight to carry, for, in the majority of rases coming under my observation, it happened in small breeds of horses and the colts were only in moderately good keeping condition. Sometimes a small, bony enlargement is situated at the internal aspect of the hock. It is called jack spavin and seldom does very much harm, merely serving as an eye-sore. A horse with jack spavin should not be considered unsound, as lameness very seldom results therefrom. The symptoms 
of spavin are lameness of a peculiar character. The animal, when lirst drisen after rest, will walk on the toe of the affected limb, with the fetlock bent forward and with a hopping movement of the hind parts. After driving a short distance, it improves very much in its gait; the lameness diminishes and sometimes after the animal is driven some distance, the lameness disappears altogether. In other cases, after driving, the lameness.

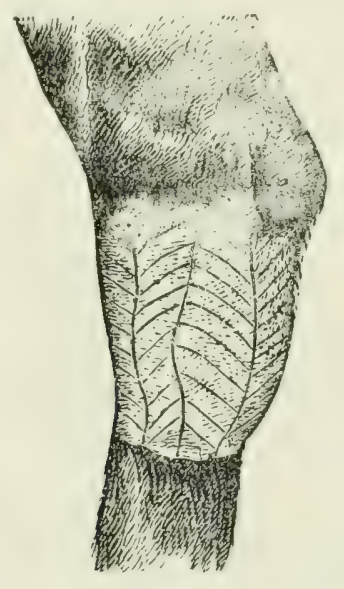

SPAVIN BONE FIRED.

will diminish but still be quite perceptible. If in the stable, the animal is made to turn around in the stall, it does so wlth a stumbling, hopping movement. On very forcibly flexing the leg at the hock, then dropping the foot and starting the horse on a trot, it will often go off on three legs. By sitting in front of the horse and looking toward the hock, you will perceive the enlargement, if there be any. By holding the hand over the anterointernal part of the hock, you will invariably detect fever.

Treatment :- Various methods of treatment are used for spavin, but one rery essential thing, and one upon 
which all must agree, is that the animal must have absolute quiet and rest. If the spavin be just started, mild counter irritation will often eflect a cure; in this case, apply-

Tinct. Cantharides.......two ounces.

Spts. Turpentine.........two ounces.

Sweet Oil........... four ounces. Mix. Or,

Cottonseed Oil ........ four ounces.

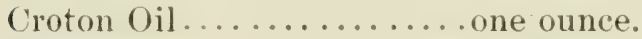

Oil Camphor..........two drachms.

Oil Turpentine....... four drachms.

Oil Origanum.......... one drachm.

Coal Oil.................. ounee.

Sulphuric Acid......... forty drops. Mix.

Apply once daily for six or eight weeks. If the lamenese does not cease in that time, apply

Vaseline.............. one ounce.

Red Iodide of Mercury... one drachm.

Powd. Cantharides ...... one drachm. Mix.

Apply thoroughly; wash off in twenty-four hours and grease with fresh lard or oil, every day for fire days. Then apply the foregoing prescription again, wash off as before and continue this treatment for three applications. Give the horse complete rest and tie it while the medicine is on, so it cannot bite or rul, the place. In the course of five weeks, if this fails, apply the actual cautery (firing irons) and immediately afterward apply the before mentioned salve, as directed. If it be an occult spavin or if the true hock joint, that is the large cuneiform and astragalus bones are affected, a failure to cure is almost certain and the animal remains lame as long as it lives. The sole object in the treatment of bone spavin, is to prevent the horse from going 
lame and this is accomplished only when the inflammation exists between the cuneiform and metatarsal bones, and you establish complete union between those bones thus preventing irritation from their gliding motion.

\section{BOG SPAVIN.}

This sometimes consists of an inflammation of the hock joint, but not always. I have seen bog spavin gradually developing upon horses, without the existence of any perceptible inflammation. This kind is generally

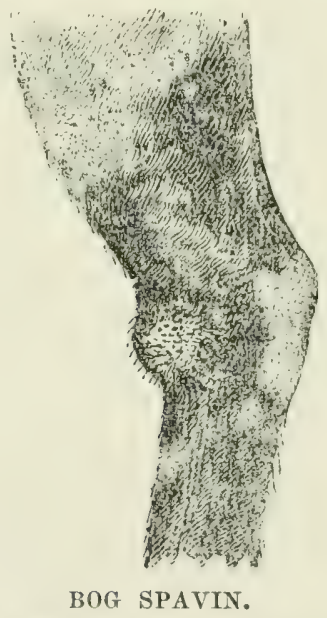

found in horses which have weak or very upright hocks. It consists of a fluctuating swelling, situated at the antero-internal and upper part of the hock, and composed of synovia or joint oil. It is nature's remedy to prevent disease by having an abundance of oil to lubricate the joint during work or violent exercise. This slould be considered merely a blemish and not a disease. But when it is caused by overwork, sprains or 
wounds it is considered to be more serious. It is then accompanied by heat and lameness, varying in their intensity. When how spavin occurs from these caluse, or from rheumatic trouble, absolute rest should be given, and the part fomented with hot water three times a day for a few days. Then apply

Tinct. Cantharides.......two ounces.

Tinct. Iodine........... one ounce.

Tinct. Capsicum........two ounces. Alcohol..............three ounces. Mix.

Or the following blister:

Vaseline.............. one ounce.

Red Iodide of Mercury.... one drachm.

Powd. Cantharides ..... one drachm. Mix.

After the lamenes ceases, stop treatment and allow nature to take its course. Sometimes bog spavin canses thoroughpin and when this is the case, treat as for bug spavin alone.

\section{BLOOD SPAVIN.}

This is a distension of the large vein that passes down over the anterior and internal part of the hock, and is caused from pressure upon the vein, arresting or freventing a free circulation of blood, thus damming it up to a certain extent, and in this manner causing a distension of the coats of the rein to a greater or lesser extent, leaving an enlargement that is soft to the touch and can usually be rubbed away. They seldom produce lameness and cause no harm. When examined with the hand, a blood spavin is found to be soft and pliable and by rubbing it for a time, it will disappear. No treatment will give better results than frequent rubbing. 


\section{OCCULT SPAVIN.}

When inflammation exists between the bones of the aock, producing lameness without any enlargenent to sight or touch, it is called an occult spavin, or hidden spavin. A deposit of bone material may form a partial or complete union of the small bones of the hock, causing intense lameness, and yet the hock to all appearances, will be perfect and smooth.

Causes:-It is caused in the same manner as bone spavin; by concussion to the ends of the bones, slipping while at play, hauling or starting a heavy load. In the majority of cases, it is the result of a wrench or sprain sufficient to cause a laceration of ligamentous attachments in the internal pait of the hock, and in many other ways by which the hock receives injury. Yet spavin may occur without any injury. My own experience has convinced me, of the hereditary disposition to spavin in certain families of horses, it being transmitted from one generation to another and sometimes occuring at a very early age.

Symptoms:- The same symptoms are observable in o'vult as in bone spavin. When first driven, after rest, will walk on the toe of the affected limb with the fetlork bent forward and with a hopping movement of the hind parts. While at rest, the animal frequently stands upon its toe with the heel raised and the hock flexed. If made to stand over in the stall, it stands on the toe of the affected limb and hops over. After hilving been driven and allowed to cool off, take hold of the toe of the affected limb and raise it up high, forcibly flexing the leg at the hock, holding it in that position for a minute, then dropping the foot and starting the animal on a trot, it will often go on three legs. This is one of the best tests for occult spavin. In the majority 
of cases of spavin, after driving a short distance there is much improvement in gait; the lameness diminishes and sometimes, after driving some distance, the lameness disappears altogether. In other cases the lameness is continuous. The treatment for occult spavin is just the same as for bone spavin, but more persistene may be necessary, as it usually takes a greater leneth of time to effect a cure, and not infrequently the lameness continues through life.

\section{STIFLE-JOINT LAMENESS AND DISLOCATION OF THE PATELLA.}

Stifle-joint lameness is usually detected by the manner in which the horse brings the foot forward, which is

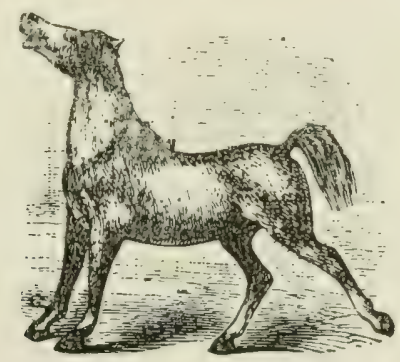

STIFLE-JOINT LAMENESA.

done with difficulty, the leg swinging outward with a circular motion and dragering the toe (although they do not always drag the toe). The foot is sometimes raised clear of the ground, but with the circular motion, and placed flat on the ground. Whilst the animal is at rest the leg is usually in a semi-flexed position. Heat around the stific joint is quite a prominent symptom, but is sometimes wanting. Sometimes quite a prominent symptom is a fluctuating swelling or dropsy of the "oint. 
If you hare dislocation of the patella, or what is more commonly called "stitled joint," you will observe that while the horse is standing in the stall, it may stand squarely upon both feet; but, if you force it to walk the stifled leg will fly backward and the animal is powerless to bring it forward. If you examine the joint you will find the patella on the outer sule of the legand; the groove in the femur may be felt quite plainly.

Treatment:-When lameness is present in the stithejoint, without dislocation, wive absolute rest and aply ly hot fomentations of water three or four times a day, to allay the inflammation. Then apply

Aqua Ammonia..........two ounces.

Spts. Turpentine........two ounces.

Olice Oil............. four ounces. Mix.

Apply three or four times daily, until you blister thoro:aghly. Then allow to rest for three or four days and repeat several times in this mamer. If the patella is dislocated, fasten a rope or strap around the fetlock, then pass it through the collar and draw the leg forward: no matter if the patient struggles hard, force it forward. Then place your hand behind the bone, which will be felt at the outside of the leg, and force it forward, when you will feel it drop into place and the patient will have full control of its leg. Apply

Tincture Cantharides.....two ounces.

spirits Turpentine.......two ounces.

Aqua Ammonia........... one ounce.

Sweet Oil............ four ounces. Mix.

Ur, use the former prescription, three.or four times a day for two or three days; allow the patient to rest for a week and all will be well. If the patient kicks it out or it drops out of its own accord immediately a ter you have placed it in position, you must apply one of the former preseriptions until it blisters thoroughly, 
and the swelling will force it into position and strengthen the ligaments that have been relaxed. Then allow a longer rest than would ordinarily be required, or apply the following:

Vaseline............... one ounce.

Powdered Cantharides...two drachms. Mix.

Wasl! off in twenty-four hours and grease every day for threx days; then apply again and grease every day until well.

\section{RINGBONE.}

Ringlone rery much resembles sparin, except in location. Ringlone consists of an osseous deposit upun the upper and hower pastern-bones. When the deposit is

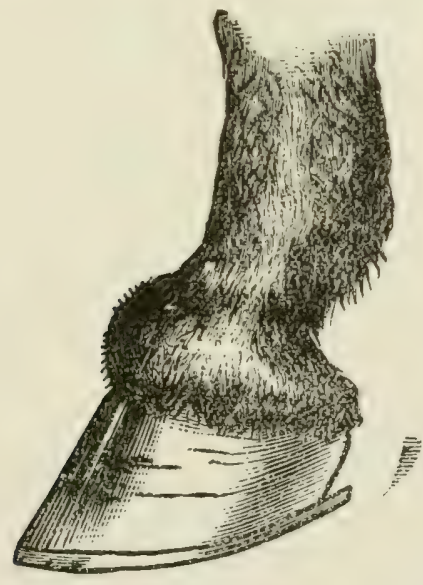

RINGBONE.

around the middle of the large pastern and not involring the joint, it is called an osselet or false ring-bone, and is seldom the cause of lameness; it should not be looked upon as an unsoundness, as it malls for notreatment what- 
ever. True ringbone acts very dillerently from the false, and oreasions more or less lameness. It may affect two very important articulations, viz, the articulation between the large and small pastern, or between the small pastern and the coftin bone. The former articulation is the one most frequently affected, and where complete unim is most easily obtained; here less stifness will be the result if a cure results, than where the lower joint is

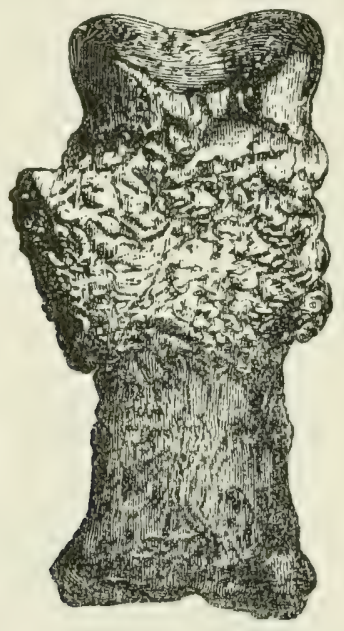

RINGBONE.

diseased. But in either location, the disease is difficult to treat with any satisfaction and it is sometimes incurable, the lameness continuing through life. "Sometimes the deposit completely encircles the joint; at others is on one side, and directly in front of the joint. The degree of lameness does not depenl upon the size of the enlargement but more upon its location. If it is situated immediately in front of the pastern, the lameness_, will be greater than when situated on either side. The lameness is greatest, when the deposit is between the lower pastern and the coffin bone. When between 
these two bones, it frequently causes a bulging of the foot and not seldom causes the foot to turn inward, forming what is known as reel-foot. The lameness of ringbone alway precedes the deposit and is caused by inflammation existing in the bone or in the synovial membrane surrounding it. Ringbone is occasioned by sprain; by fractured bone; and by the hereditary tendency, referred to in the treatise on spavin.

Treatment:- When first noticed there will be no enlargement but considerable lameness and fever. Then apply hot water freely to allay the inflammation and after bathing, apply the following:

Spirits Chloroform.......two ounces.

Laudanum.............two ounces.

Witchhazel........... four ounces.

Tincture Aconite...... one-half ounce. Mix.

If the lameness continues after ten days' treatment apply-

Vaseline ..............two ounces.

Red Iodide of Mercury...two drachms.

Cantharides.........three drachms. Mix.

Make three applications, with intermissions of four or five days between each two applications. Give absolute rest and if the patient is inclined to walk upon the toe, use high calks upon the heal of the shoe. But if inclined to walk upon its heel, use a thin-heeled bar shoe. If limeness continues, use the firing irons. Fire it deeply as illustrated in spavin and in a few days apply-

Vaseline.............. one ounce.

Powd. Cantharides.....two drachms. Mix.

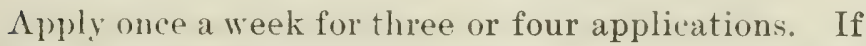
all these fail, the cutting out of the plantar nerves may be performel with varying success. 


\section{SPRAIN OF THE SUSPENSORY LIGAMENT.}

This is a very large ligament composed of white fibrous tissue, and extends from the knee in the foreleg and from the hock in the hind leg, down the back part of the cannon bones. Just above the fetlock it divides and becomes attached to the sesamoid bones, helow which it reunites and joins the flexors of the foot. Sprains of this ligament happen only through severe

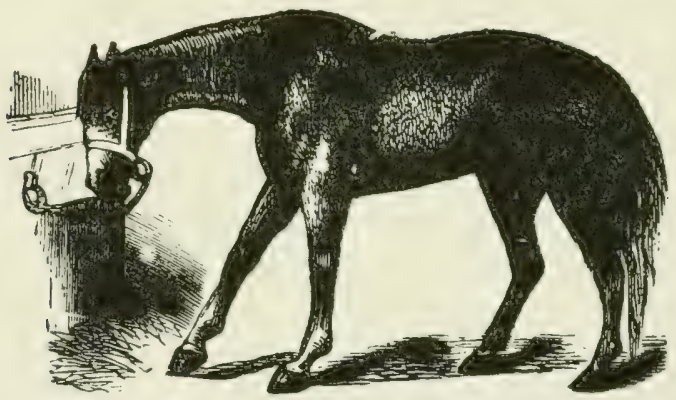

POSITION OF THE FOOT IN LAMENESS.

exertions, and sometimes the strain is sufficient to cause rupture of the ligament. This generally happens near the dividing point, just above the fetlock. The most prominent symptoms are lameness, heat, and swelling beneath the flexor tendons and down behind the cannon bones, with swelling of the leg at the fetlock joint. The animal stands or walks upon the toe, the fetlock joint is flexed to relieve the ligament of tension. If the ligament is ruptured, the fetlock will spring down close to the ground and the toe will be turned upward. The rupture may occur higher up, or at the attachment of the ligament to the sesamoid bones, or even below the fetlock; the result is the same. This is what is meant by the term, "broken down." 
Treatment:-Absolute rest in a comfortable box stall, well badied, to entice the patient to lie down. Bathe with cold water, after which apply bandages and keep wet with cold water. After the fever has subsided apply to remove the swelling,

Tincture Iodine.........two ounces.

Red Iodide of Mercury...two drachms.

Tincture of Myrrh and

Capsicum............. six ounces. Mix.

Apply three times a day, or

Powdered Cantharides .. two drachms.

Vaseline.............. one ounce. Mix.

Apply every two weeks until three applications have been made. If the ligament is ruptured, bandage the ley in a set of splints, so as to hold it in a proper position. Heavy belting makes a good support for the limb, by cutting it to tit, and packing the space between the fetlock joint and the heel to form a prop. This will hold the limb in proper shape. Or put on a bar shoe and fasten an irom brace to it, bending it to fit the fetlock and extending it up to the knee. I'ad it well with cotton batting and bandage. After complete union is secured, remove the support and hlistrer a few times to remove the swellixg. Horses that have once "broken down," or ruptured thr suspensory liwament, shoulil not. be put on the track nor at heary pulling; but they may be used to grood advantage for ordinary work, after a rest of six or eight months.

\section{SPRAIN OF THE FETLOCK JOINT AND SESAMOIDITES.}

It is possible to have sprain of the fetlock joint or of its lateral ligments, without injury to the sesamoid bursa; but such cases, I think are very rare. Owing to the particular manner in which the sesamoid bones are 
placed behind the joint, over which the flexor perforans tendons play, I am constrained to believe that any exertion sufficient to cause sprain of the fetlock joint, will, at the same time produce inflammation of the sesamoid bursa.

Symptoms:-If the sprain is very slight, lameness will scarcely be perceptible, appearing only while traveling upon uneven ground and the joint is twisted a little; but if the sprain is severe, the lameness will be so intense that the animal will go on its toe or hobble on three legs. The joint swells and is quite hot, and when the animal is resting, the leg is flexed at the joint, the toe resting upon the ground.

Treatment:-Apply cold water frequently and bandage until the fever subsides, then use

Tinct. Iodine .......... two ounces.

Witchhazel.............two ounces.

Alcohol.............. four ounces. Mix.

Apply morning and evening and bandage, or

Vaseline.............. one ounce.

Powdered Cantharides.. two drachms. Mix.

Apply every ten days until three applications have been made. Complete rest should be given for a month. If you consider the ligaments ruptured, a plaster of paris bandage should be applied, the same as for fracture, and allowed to remain on for a month. If any swelling remains, a few applications of the above blister will greatly assist in removing it.

\section{SPRAIN OF THE FLEXOR TENDONS.}

Sprain of the flexor teudons, like the sprain of the suspensory ligament, is caused by heary fulling and fast running or trotting; also by wearing shoes with 
high toe callis. The symptoms observed will be lanseness. heat and swelling at the back part of the leg, and the peculiar way in which the horse moves the limb, which is with a stiff. stubby movement; the toe touches the ground first and the horse bears most of its weight

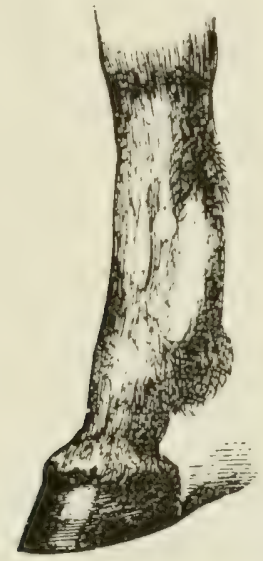

BACK TENDONS TUICKENED FROM INJURY OR SPRAIN.

upon it, seldom bringing the heel to the ground during the first stages. After it beemes chronic, or the mores severe symptoms have subsided, the heels will be brought to the sround. there will be a shortening of the tendons and a knuckling over of the fetlock joint. The swelling, which is a prominent symptom, will diverge from the seat of the sprain and extend up and down the tendon, involving the thecæ, or sheaths of the tendons to such a degree, that the tendons with difficulty pass through them.

Treatment:- Shoe the horse with a bar shoe without calks, to wive the shoe an even pressure when standing. This will prevent shortening of the tendons, which is likely to occur with high heel calks, which only afford 
temporary relief. Apply cold bandages, occasionally pouring on cold water until the fever subsides. Then apply one of the following mixtures, three times a day:

Tincture Iodine.......... one ounce.

Red Iodide of Mercury.............

. one and one-half drachms.

Tincture Myrrh and Capsi-

cum............... Meven ounces. Mix.

Or,

Tincture Cantharides...... two ounces.

Tincture Iodine.......... one ounce.

Tincture Capsicum.......two ounces.

Alcohol..............three ounces. Mix.

Or,

Oil Cedar............ four drachms.

Oil Hemlock......... four drachms.

Oil of Tansy .......... four drachms.

Oil of Wormwood...... four drachms.

Alcohol............... eight ounces. Mix.

If shortening of the tendons should take place, tenotomy may be performed, if the patient is a young horse. But if an old horse, the operation had better be let alone. The operation is performed by casting the horse and securing it firmly. Then loosen the leg to he operated upon ; fasten it as straight out as possible by a rope or strap tied to the fetlock. Make a small incision midway between the knee and the fetlock, the smaller the incision the better. Pass the knife tlat-wise beneath the tendons, taking care to keep outside the blood vessels, until you feel the knife azainst the skin on the opposite side. Then turn the erlge of the knife towards the tendous and cut outward very slowly until you have severed the tendons. Then withdraw the knife. Be very careful to muly cut the skin in one place and that a very small opening, where the knife enters. Place 
your knee against the leg and forcibly straighten it, to break up any adhesions which may have formed. After the operation, keep the leg straight by putting on a shoe with a high toe-calk; or fasten a piece of iron to the shoe and let it extend six inches in front of the foot. If the toe should turn up a little, have no fear, for when the tendons grow together it may shorten a little too much. After union has taken place, remove the thickening with a few applications of the last prescription.

\section{ELBOW LAMENESS.}

Disease of the elbow is not an infrequent occurrence.

Symptoms:- While standing, the horse holds its leg in a peculiar manner-carried well back, the lew llexed and resting upon the toe. In walking, the limb is brought forward with difficulty and when putting it to the ground to make the step, the patient drops the head and shoulder as if about to fall. There may not be very much heat or swelling. If there is fracture of the point of the elbow, you will see the excessive dropping of the head and shouller; the leg will be flexed or seemingly in a pendulous condition, with heat and swelling and pain upon pressure. Sometimes "capped elhuw" causes lameness, hut not always; when it does, you do not have such excessive dropping of the head.

Treatment:-If there is lameness, without swelling or heat, apply three times a day of-

Oil of Cedar......... four drachms.

Oil of Wormwood...... four drachms.

Oil of Tansy.......... four drachms.

Oil of Hemlock........ four drachms.

Alcohol.............eight ounces. Mix. 
Allow sufficient rest and a cure will result. If there is fracture of the elbow, swing the patient up immediately and feed on soft food. Apply splints, and bandage from the knee up above the elbow to hold it in position and keep the patient swung up until complete union has. taken place, which will be in from six to ten weeks. Broad rubber belting, makes a good splint and bandage combined. If the lameness comes from capped elbow (usually caused by the horse lying with its elbows resting. upon the heels of its shoes), remove the shoes and place a roll of sheepskin, or other soft material, around the back part of the fetlock, to prevent the feet from irritating the bruise. Then apply hot water to the elbow until the inflammation subsides, when the lameness will usually disappear. But, to remove the enlargement, make a free incision low down to allow the fluid to escape: then apply some irritating substance to cause it to slough out. After it heals and there is a small nodule left, dissect it out and treat as an ordinary wound. Widen the stall and allow plenty of room for the animal to lie down.

\section{CARPITIS, OR INFLAMMATION OF THE KNEES.}

Inflammation of the knees may arise from different causes, such as concussion, rheumatism, sprains, etc. The animal is observed to hold the leg stiff and to exhibit intense pain upon either flexion or extension of the knee. If the animal walks, it will carry the leg stiffly. Sometimes there is a swelling of the knee; at others, no swelling whatever is perceptible. If the disease exists between the bones, there will be no swelling until an exudation is thrown out, and when this happens, a stiff knee is almost rertain to be the result. 
Treatment:-Give complete rest and, if thought necessary, place the patient in slings. Use applications of cold water until the fever subsides; then apply a strong blister, as-

Vaseline................. one ounce.

Red Iodide of Mercury.... one drachm.

Cantharides........... one drachm. Mix.

Use repeatedly. If there is a fluctuating swelling in front of the knee, puncture it and allow the fluid to escape, after which apply a wet bandage and keep it wet. Should it fail to disappear, apply the foregoing blister.

\section{BROKEN OR BRUISED KNEES.}

Whenever you see a bruise or a scar upon the knees of a horse, the animal, as regards value, should he looked upon with suspicion, unless the horse has crout, free action and the bruise is known to be purely acci-

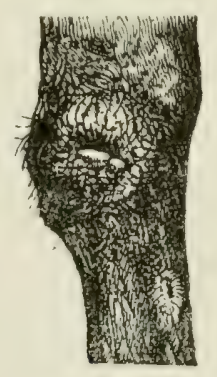

BROKEN KNEE.

dental. For a majority of cases arise from faulty action of the fore legs, vertigo or fits, "speed cut" or interfering, diseased feet or navicular disease; over-rear hing: or from bad shoeing, and possibly from the driver"s awkwardness. The disease is more frequently noticed in the form of bruises than as broken bones. 
Treatment:- When bruises exist, without any abrasion of the skin, apply cold water frequently, with dressings of vaseline. Give rest, with grood, deep bedding, and probably no other treatment will be necessitry. But when the skin is lacerated, if none of the leeper tissues are involved, wash the wound well and bring its edges together with heavy court-plaster, leaving an opening below for the escape of pus; give rest and tie the horse up so it cannot lie down, to prevent it from breaking any of the alhesions while Hexing the leg. Never use any stitches, as any movement of the knee will tend to tear them out. Where, besides the laceration of the skin, deeper tissues are involved, so that the tendons and synovial membrane are torn, and "open joint" is the result, you have a more serious case in hand. The wound may set up an extensive inflammation, the surrounding parts become greatly swollen, followed by so extensive a sloughing of the parts as to expose the bone. There will then be consillerable constitutional disturbance, high fever, loss of appetite, howels constipated, diflicult voiding of the urine, and a fortid discharge from the wound. It is then as serious as a broken bone and the animal should be destroyed, unless valuable for breeding purposes. If the animal is to he preserved, force the leg out straight and fasten it with splints to maintain it in that position, leaving the knee exposed for treatment. Wash it out three times a day with tepid water and castile soap aud apply one of the following three times a day:

Zine Sulphate.........two drachms. Acetate of lead........ four drachms. Carbolic Acid.........two drachms. Soft Water.............. one pint. Mix. Or, 
Permanganate of Potassium. . ..................... drachm.

Tannin................ drachm.

Distilled Water........... pone pint. Mix. Or,

Listorine..............two ounces.

Carbolic Acid........... one drachm.

Distilled Water............ six ounces. Mix.

\section{SHOULDER SLIP OR SWINNEY.}

This disease generally termed "swinney," consists in atrophy, or wasting away, of the antea and postea spinatus muscles. A brief description of the parts

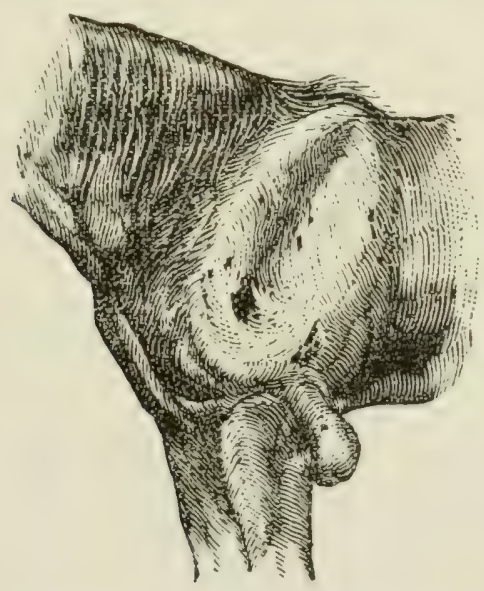

SWINNEY AND CAPPED ELBOW.

forming the shoulder joint, will facilitate an unclerstanding of the matter. The glenoid carity, or hollow in the lower end of the shoulder bone, is very small compared with the head of the humerus, which plays within it, thus giving it great freedom of motion, espe- 
cially as the joint is not bound down with inelastic ligaments, but by muscles and tendons only. The antea and postea spinatus muscles occupy the outer surface of the shoulder blade and extend down over the external and lateral surface of the joint. The sub-scapularis muscle occupies the inner surface of the shoulder blarle and extends down over the inner and lateral surface of the joint. The coraco humeralis nuscle extends dow: over the front of the joint. So it will be seen that, when the external muscles are wasted away (atrophied) there is nothing left strong enough to hold the joint to its proper position, and it has an outward movement or slipping-but not a dislocation. It may be caused by it bruise, heavy pulling, or walking on uneven ground, as the furrow horse is compelled to do while plowing. It is more frequently seen in young horses, used for plowing, especially in the spring when the muscles are soft and flaceid.

Symptoms:-There are generally swelling, heat and tenderness, all along the external surface of the shoulder blade; but in the majority of cases, the patient show: no lameness until the swelling and heat have subsirled and atrophy, or wasting of the muscles, has begun. This generally takes place in a week or two, from the time of the injury. The hollow space is usually the full length of the shoulder blade, and is sometimes very deep, so that the spine of the bone is prominent.

Treatment:-During the inflammatory stage, while there is heat and swelling, foment well with hot water and after the swelling has disappeared, apply a mild stimulating liniment, as

Ammonia............. one ounce.

Turpentine ............ two ounces.

Swect Oil............ five ounces. Mix. Or, 
Oil of Cedar........ one-half ounce.

Oil of Tansy ......... one-half ounce.

Oil of Hemlock....... one-half ounce.

Oil of Wormwood..... one-half ounce.

Alcohol...............ten ounces. Mix.

Use three times a day, rubbing in thoroughly. Do not be afraid of rubbing, as it stimulates the muscles to development. Use at light work on level ground, or turn out to pasture, for the patient needs exercise to develop the muscles. Do not be discouraged, but he persistent in your treatment, as it frequently takes six months, and sometimes a year, before the hollow is filled up and the muscles fully developerl. If it seems very slow, apply at intervals the following:

Vaseline............. one ounce.

Red Iodide of Mercury... one drachm.

Cantharides.. one and a-half drachms. Mix.

If you are persistent, success will crown your etlort and reward your trouble.

\section{ATROPHY OF THE MUSCLES-SWINNEY.}

This consists in a shrinking or wasting away of the muscles and is most frequently seen at the shoulders and hips. It may, however, manifest itself upon any part of the limbs or body. It is a aused by sprains, bruises, severe and continued pressure, paralysis, rhematism, continued lameness of the foot or limb or anything in fact, whereby a certain set of muscles do not receive sufficient exercise.

Symptoms:-A thattencd or hollow space will be observed instead of the full, round, plump muscles. When occurring at a certain point, compare it with the 
same point on the opposite side, and a flattening or wasting away will be observed.

Treatment:-Atrophy of the muscles and swinney mean one and the same thing; therefore, the same treatment may be used as outlined under the head of "Swinney." Any manner of treatment, however, will in some instances, fail to redevelop the muscles but it is well to be persistent for a continued length of time.

\section{SHOULDER LAMENESS.}

Shoulder lameness may arise from different causes, but the most frequent are kicks, bruises, sprains and rheumatism. It is characterized by the peculiar manner in which the patient holds the leg, which is flexed

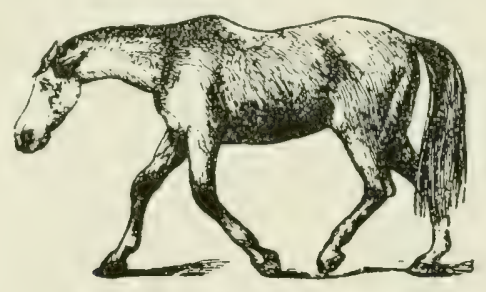

SHOULDEIR LAMENESS.

and resting upon the toe; also the great difficulty with which the leg is brought forward in walking, with at the same time, a dragging of the toe. If from kicks or bruises, the lameness will be observed at the injured place; but if from sprain of the coraco humeralis muscle, which plays on the front of the joint in the form of a tendon, there will be observed flexing of the limb and great difficulty in bringing it forward, with swelling heat and tenderness upon pressure at the point of the shoulder. As inflammation of a tendon frequently results in formation of bone material, you will readily ${ }_{g}$ ee the need of prompt and careful treatment. 
Treatment:-Put on a very high heeled shoe and aII'!y frequent fomentations of hot water; or place a rug on the point of the shoulder and keep applying hot water until the inflammation has subsided. Then remove the shoes and keep quiet on a level floor, and apply the following three times a day:

Tincture Iodine.......... two ounces.

Witchhazel............two ounces.

Alcohol............. four ounces Mix.

Or,

Oil of Cedar........ one-half ounce.

Oil of Hemlock...... one-half ounce.

Oil of Wormwood.... one-lialf ounce.

Tincture of Iodine........ one ounce.

Alcohol.............eight ounces. Mix.

Allow the patient to rest for sometime, and use only at light work for at least four months.

\section{RHEUMATISM.}

This is a peculiar disease, and none too thoroughly understood. It attacks the fibrous structure, such as muscles, tendons, etc. It is apt to move suddenly from one joint to another, from one set of muscles to another, or to some internal organ, as the heart. It is seldom that acute rheumatism terminates in chronic rheumatism, or that the reverse occurs. Chronic rheumatism is met with most frequently among the domesticated animals.

Symptoms:-The patient will appear dull and stupid with an inclination to be let alone and not to be moved about. If the affection be in the limbs or joints and the animal be forced to move, it either goes very lame or is very stiff. The parts affected may be swollen and hard, 
or, if in the joint, soft and fluctuating; or there may not be any swelling, but a crackling noise at the joints when moved. There is generally fever with a full, hard pulse, increased temperature, hurried breathing, costiveness and scanty, high-colored urine. If acute rheumatism, the disease will generally terminate in about six or eight weeks; but, if chronic, it may last until warm weather comes.

Treatment:-Give as a physic-

Powdered Aloes.......... one ounce.

Powdered Ginger........ one drachm. Mix.

Administer at one dose; and, if the pain be excessive, give ounce doses of laudanum, to be followed by-

Salicylic Acid...........two ounces.

Bi-Carbonate of Soda......two ounces. Mix.

Make sixteen powders. Give one powder three times a day, to be followed by-

Acetate of Potassium....... one ounce.

Bi-Carbonate of Soda....... one ounce.

Nitrate of Potassium.............

........ one and one-half ounces. Mix.

Make twelve powders and give one powder three times a day. Apply hot cloths, hot bran, hot corn, hot salt, or make the following liniment:

Spirits of Chloroform.....two ounces.

Laudanum.............. two ounces.

Tincture of Arnica....... two ounces.

Witchhazel............two ounces. Mix.

Apply two or three times a day.

Or,

Aqua Ammonia.........two ounces.

Spirits of Turpentine..... two ounces.

Sweet Oil............ four ounces. Mix. 
Apply two or three times a day. Lou may also give, with benefit, liquid ammonia acetate, two ounces three times a day; but the treatment is seldom very satisfactory.

\section{SPRAINED BACK.}

Sprained back is not of frequent occurrence, but it may happen from the hind feet s'ipping, while the animal is pulling hard to start a heavy loal, or from its struggling when cast in the stall. The psoas muscles,

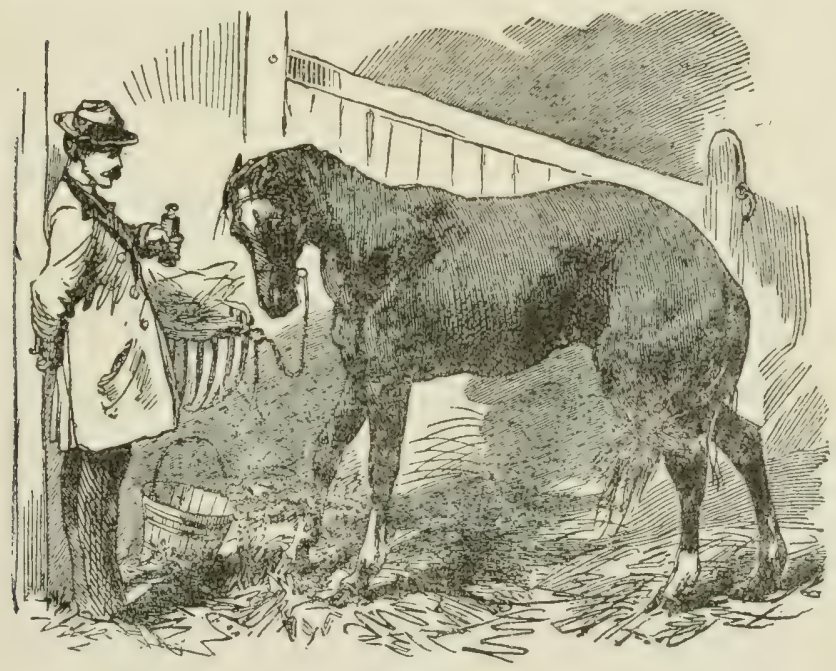

SPRAINED BACK.

which are the seat of the sprain, are situated underneath the lumber vertebre, and are attached anteriorly to the heads of the last ribs and dorsal vertebræe and posteriorly to the brim of the pelvis and internal trochanter of the femur; their action is to bend the haunch upon the pelvis, and when the horse is pulling, 
to produce the appearance of a "roached back." When sprained, these muscles lose their tone and power, and instead of a "roached back" there will be the reverse, except there will be swelling over the loins, which is frequently the case. If both the psoas muscles are strained, the animal will be unable to rise from a recumbent position, though when placed on its feet, it may stand; it will walk with a drunken, staggering gait and a knuckling over of the fetlocks. It is easily distinguished from broken back by the power to move its limbs, and the sense of feeling in the hind extremity. Examination per rectum, reveals heat and swellinir, with pain upon pressure along the spine. After these symptoms have passed away and the animal is restored to usefulness, there will be a weakness of the back for some months, or until the muscles are again fully developed and regain their natural power and tone.

Treatment:-If the patient is able to stand, place the slings under it, but if unable to stand, you had better let the slings alone. Apply frequently, hot fomentations across the loins and afterwards use-

Tincture of Arnica....... four ounces.

Chloroform............. one ounce.

Laudanum............. two onnces.

Witchhasel............three ounces, Mix. Or,

Aqua Ammonia..................

......... one and one-half ounces.

Turpentine...one and one-half ounces.

Olive oil.............. five ounces. Mix.

Or,

Tincture of Iodine.......two ounces.

Oil of Hemlock........ one-half ounce.

Oil of Wormwood..... one-half ounce.

Oil of Origanum.......... one ounce.

Alcohol.............. four ounces. Mix. 
Apply three times a day. If the pattient is dragerng along and not improving very rapidly, in the course of a month apply a good blister across the loins and it will help materially to ievelop the muscles, and to regain their natural tone and power. A blister may be applied every two or three weeks; the following will answer nicely:

Vaseline.............two ounces.

Red Iodide of Mercury....

......... one and one-half drachms.

Powdered Cantharides...two drachms. Mix.

\section{WOUNDS AT THE JONTS-INFLAMMATION OF THE JOINTS, OR OPEN JOINT.}

An open joint may be caused by a puncture from a knife, from splinters of wood, or by the prongs of a fork; or by bruises, by kicks, or by lacerations from falling on a hard or rough surface. It is always attended with more or less danger.

Symptoms :-If the opening is small with but a tritling leakage from the joints, the patient will not at first exhibit any distressing symptoms; but in the course of a $f \circ w$ days it will manifest pain by going very lame and possibly, not resting upon the liml) at all but holding it up and keeping it in continuous motion, on account of severe pain. The leakage will then be more profuse and accumulate on the outer edge of the wound. The patient will now lose its appetite, and have very high feror with constipation. The pulse will be quick, hard and-wiry; and, from extreme pain, the patient will break out in a sweat. If at this stage the patient does not receive prompt attention, the disease will continue in force, and from between the opening and the accumulation on the outer edge of the wound, a thin watery 
discharge, accompanied by streaks of blood and small scales of cartilage and bone, will issue. The ends of the bone entering into the formation of the joint are now undergoing a destructive change, and a cure can only be effected by a consolidation of the joint. A stiff joint is inevitable; and if it be a joint requiring great motion the patient had better be destroyed. During the whole time, the tissues surrounding the joint will be enlarged and inflamed, sometimes very hard and at others soft and doughy.

Treatment :-Two things are very essential to a favorable termination. The first is endeavoring to heal the wound by the adhesive process (or first intention) and thus prevent suppuration. The second, is preventin the air from penetrating the joint, thus avoiding extensive inflammation. Never probe around in the wound to find if there be any dirt, gravel or any other foreign substances lodged there, but wash it out carefully with a sponge, and syringe with tepid water; if any foreign substance be found, remove it carefully. If the tissues are lacerated, cut off the small particles that will not adhere, but do not cut the skin, and bring the edges of the wound together with stitches of catgut; then place half a teaspoonful of iodoform upon the wound and bandage. Immediately after, place the animal in slings, as it not only affords the patient complete rest but prevents the stitches from being torn out by the flexing of the limb in lying down. If complete union is effected and there is enlargement of the joint with some infiammation apply a good blister. If there is not complete union of the parts and they burst out, and the synovia, or "joint oil," comes oozing out, accompanied by pus, you then can only expect to heal by granulation. The best method to secure this is to apply a good blister, as it not only sets up a healthy 
inflammation but frequently produces a swelling sufficient to bring the edges of the wound together, thus excluding the air and, before the swelling subsides, forming granulations enough to close the wound, and so effecting a cure. The following is a good blister :

Vaseline............. two ounces.

Powdered Cantharides..four drachms. Mix.

Apply every third day for four applications. If much swelling remains after the wound is healed, apply

Tincture Iodine.........two ounces.

Oil Hemlock.............half ounce.

Oil Wormwood...........half ounce.

Oil Origanum............ one ounce.

Alcohol.............. four ounces. Mix.

Apply once or twice daily with smart friction.

If small abscesses form around the joint and cause much pain, open them; if they do not cause much pain, allow them to burst. The constitutional treatment should be to open the bowels with a physic-

Aloes.................. one ounce.

Ginger.............. one drachm. Mix.

Give at one dose. Then give three times a day one powder of the following:

Quinine Sulphate...... four drachms.

Nitrate of Potassium......two ounces.

Bi-Carbonate of Soda...... one ounce. Mix.

Make into twelve powders. For the first few days feed on a soft diet, as bran mash, chopped feed, etc. Afterwards feed on the most nutritious diet in limited quantities, to build up the strength.

\section{STRINGHALT.}

This disease consist: of an involuntary spasmodio contraction of the muscles of the hind leg. The horse raises and lowers the foot in a peculiar manner, with a 
quick, jerky motion, which it cannot control. Tho causes are not thoroughly understood. It is by some attributed to injuries; by others to inflammation of the nerves, and others still attribute it to tumors variously situated-as in the brain, in the spinal cord, in the muscles of the legs, etc.; and some attribute it to a peculiar condition of the blood. These theories are all

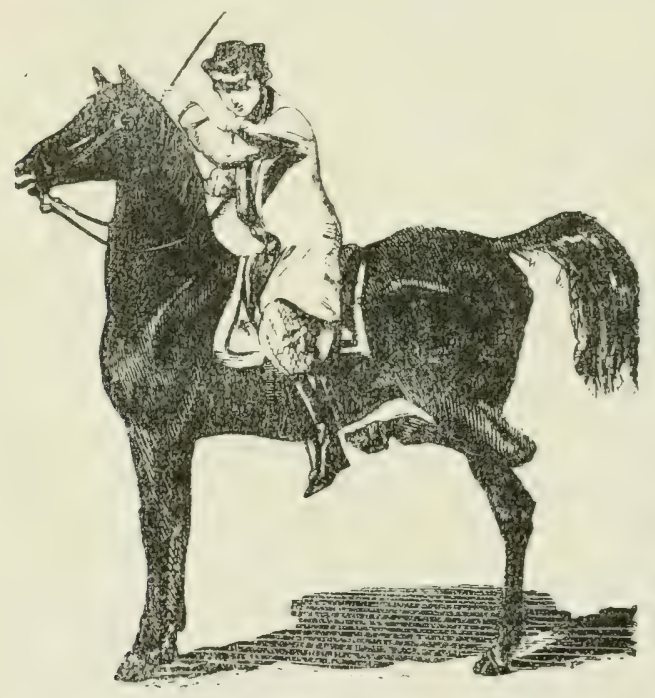

STRTNGHALT.

guppositions; possibly all are, to a certain extent, true. The disease generally comes on very slowly but may dovelop very rapidly, even in so short a time as twenty-four hours. At first the foot may be slightly elevated, with a quick, jerking motion, and bronght to the ground in the same manner. This may be noticed in the winter season, and during the heated months the animal may travel all right. When this is the case, it may be regarded as a rheumatic form of stringhalt. If it does not improve during the summer months, as age advances the distase 
gradually continues to grow worse, until the horse strikes the belly with the fetlock. The disease is purely nervous in nature, as, for instance, should the animal pick up a nail with the foot, the stringhalt is $m$ ifested in a severe form, and extreme pain is manifested. Except for the rheumatic form, there is no treatment that will benefit the patient. In that case, treat for rheumatism; or when first noticed, give strychnia in full doses for a few months.

\section{ARTHRITIS, SYNOVITIS, OR INFLAMMATION OF THE JOINTS.}

This may be due to sprains of the ligaments extendince into the joint, and adjoining the synovial mem. brane; to concussion of the bones extenting to the joint, to wounds admitting air into the joint; to rheunuatism, to scrofula, to calcareous deposits in the joints, elc. From whatever cause, the whole joint becomes involved and the symptoms have such a close similarity that they may be considered alike.

Symptoms:-The joint will be swollen and of a doughy consistency. There will he lameness with more or less fever, according to the severity of the attack. If the joint is movel, the patient will exhibit exeruciating pain. Sometimes the animal will hold the foot clear of the ground, in a fixed position. Often from pain the patient will break out in a sweat, the fever running high and the pulse being quick and wiry. The tissues surrounding the joint become greatly swollen, with increased heat and tenderness of the parts.

Treatment:-Place the patient in slings and give a physic to open up the bowels. Then apply hot fomentations to the joint frequently, with thanel bandages, and the following every three or four hours: 
Sulphuric Ether........ two ounces.

Laudanum............ two ounces.

Chloroform............. one ounce.

Spirits Turpentine..... three ounces. Mix.

If this does not quiet the pain and the swelling is obstinate, apply a blister-such as for open joints, and administer three times a day half-ounce doses of fluid extract buchu and juniper, with acetate of potassium.

\section{RICKETS.}

This is not an inflammatory disease, but consists of a softening of the bony structures. It is most frequently seen in young animals, those from a few days to a few months old. The real trouble, is a lack in the bones of such inorganic material as the phosphate and carbonate of calcium. Whenever the bones become deficient in these materials, they are cartilaginous in texture and easily bent; in this way occurs the deformity of rickets. The causes are mal-nutrition, or a deficiency of the elements necessary to build up the already deficient bony structure. Although hereditary in its character, it may be developed by not allowing the colt to suck the mare at will only permitting it, perhaps, to suck three times a day when the mare, hot and feverish, returns from work. Such treatment will cause irregularities of the howels, the milk passing through them in a half-digested curd-like mass, white in color and very sour to the smell. This will cause great depletion, and rickets is the result.

Treatment:-Feed regularly on good, nutritious diet, change the pasture, bandage the legs to keep them straight, give small doses of phosphate of lime; keep in a dry, sunshiny, airy place; give cod-liver oil and, to check the scouring, four to six ounces of castor oil. 


\section{TEETH.}

HOW TO TELL THE AGE OF HORSES BY THEIR TEETH.

Persons should familiarize themselves as much as possible, with the various shapes and changes which the teeth of horses undergo, from colthood to extreme age, so that they may not be deceived when purchasing a horse, which is quite frequently the case with those persons who have never given the teeth any attention. I have known cases where a fifteen and a sixteen year old horse had been sold for a seven and an eight year old, and the deception or falsehood was accepted by the purchaser, as he was himself unable to tell anything about their ages. I shall here endervor to explain as nearly as possible, the various changes that take place, from foaling time to twenty years of age, so that the purchaser of a horse can determine very closely, the age of the animal he desires to buy, and not be too badly

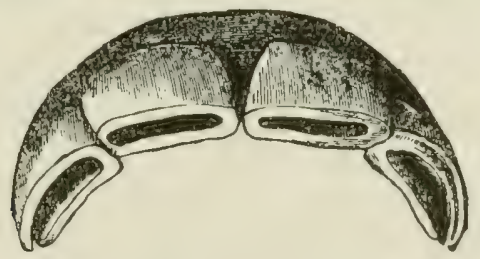

TEETH OF A FOAL.

deceived by the seller. When the colt is foaled, it frequently has four front teeth or nippers, two above and two below. If these are not present at foaling time, they make their appearance within ten days afterwards. In the course of five or six weeks, four more incisor front teeth, make their appearance alongside of the first four; these are called the middle teeth. Then a lapse 
of six or seven months takes place before the last four incisors make their appearance, and they are designated as corner teeth.

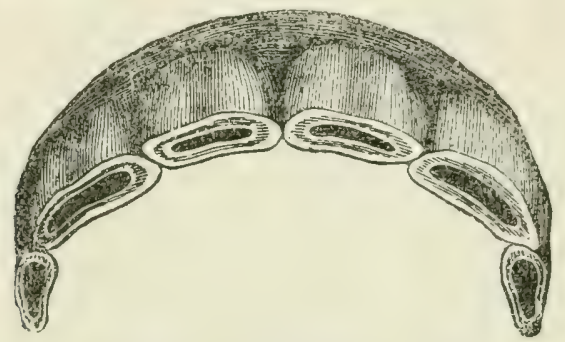

TEETH AT ONE YEAR OLD.

It is then possessed with twelve incisors, six above and six below, which is considered a full set of front teeth. After this,

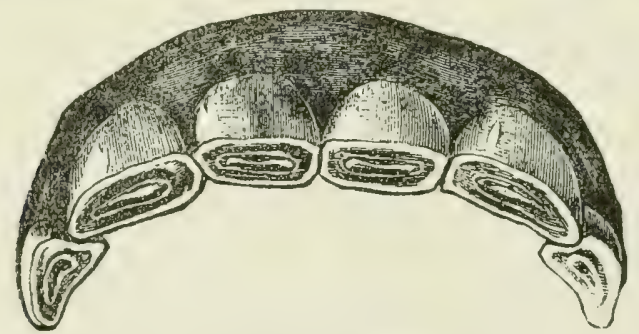

TEETH AT TWO YEARS OLD.

the only distinguishing mark to be observed, is that the wearing surfaces become somewhat smoother every few months, until

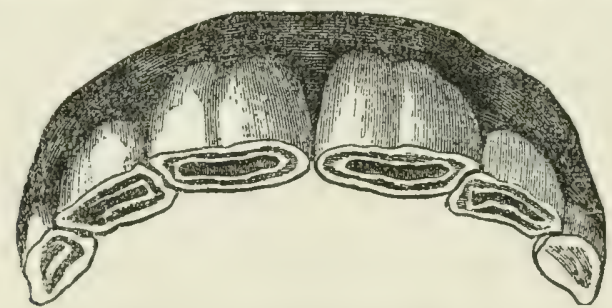

TEETH AT THREE YEARS OLD.

the colt isitwo and one-half to three years old; at this age the four central incisors or colt teeth are shed and four permanent 
incisors are or horse teeth, are coming through the gums, to occupy their place, and in the course of a few weeks, they will have attained sufficient growth, to be of service while eating. At from three and a half to four years, four more colt incisors

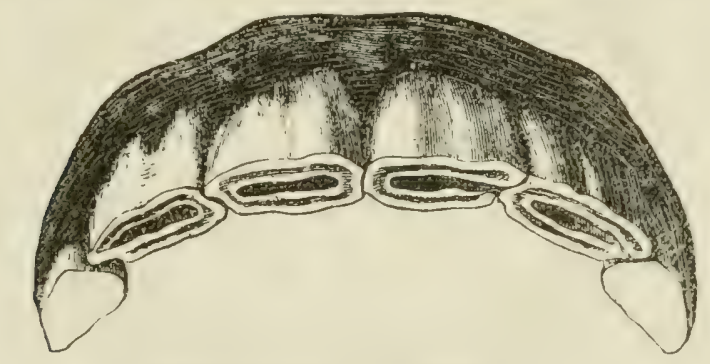

TEETH AT FOUR YRARS OLD.

give way, and their place is taken by the same number of permanent incisors, or horse teeth. The front or nipper teeth, now commence to show wear on the outer edge. At from four and a half to five years, the colt teeth have all gone and four more

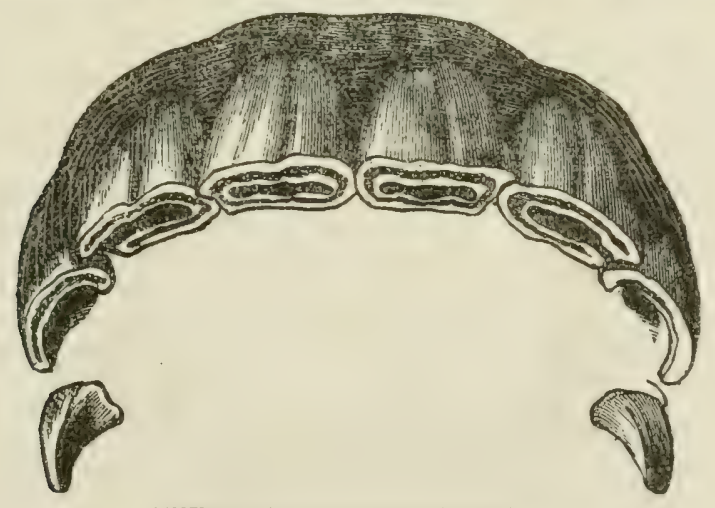

TEETH AT FIVE YEARS OLD.

permanent or horse teeth, have taken their places. The nippers now show wear on both outer and inner $\epsilon$ dges, and the middle teeth show wear on the outer edges. The horse is now said to have a full mouth or a complete set of permanent teeth. I have 
not said anything about the back or molar teeth, for the simple reason that they are seldom examined with reference to variation, in order to ascertain the age of the animal. The frout or

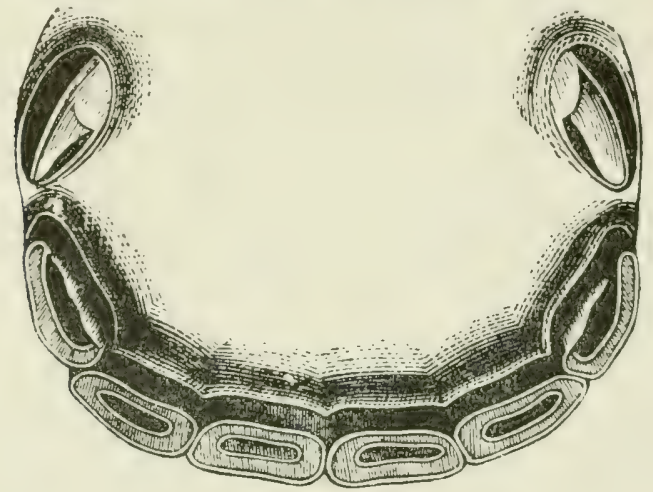

LOWER JAW A'T SIX YEARS.

incisor teeth, are considered the indicators, and to them I will confine myself. At six years of age, the nipper teeth in the

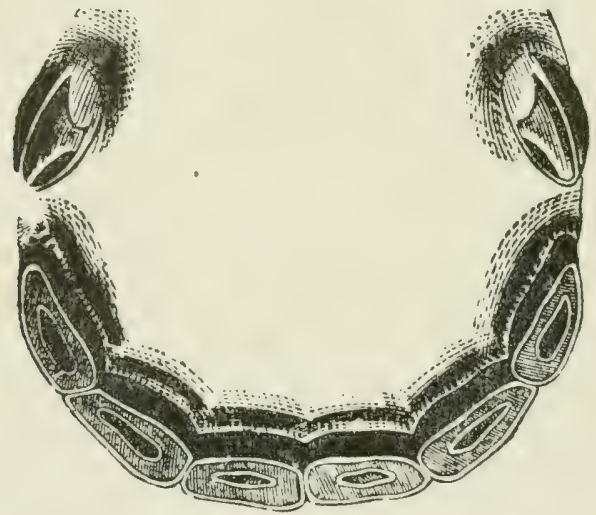

LOWER JAW AT SEVEN YEARS.

lower jaw have worn down, until the large cavity or mark in the center of the teeth, is nearly gone, and the inner edge of the corner teeth, is in full wearing, unless it should be what is 
called a shell tooth, then it is not considered to have an inner edge. At seven years, the nippers and middle teeth in the lower jaw, are worn quite smooth; the cavity or mark in the

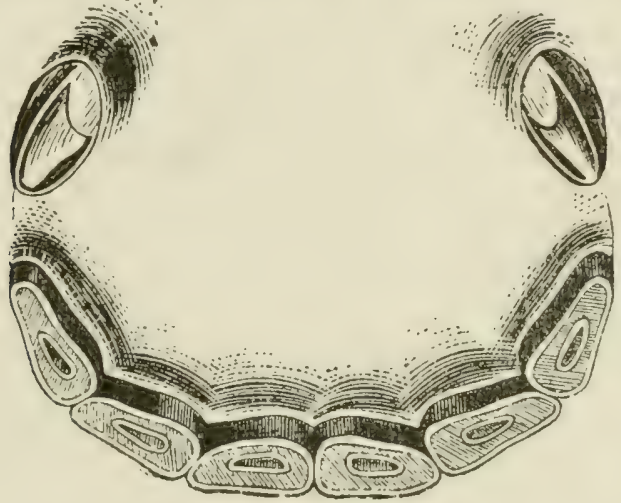

LOWER JAW AT EIGHT YEARS.

center of these teeth has disappeared, except in certain cases, when a slight trace of it is left. The corner teeth in the lower

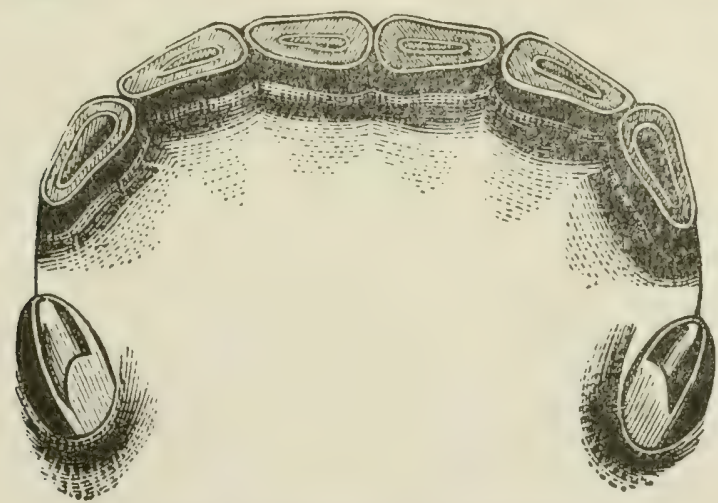

UPPER TEETH AT NINE YEARS.

jaw, at eight years of age, lose the cavity in the center, through wear, although a slight trace of it may yet be seen. All the incisor teeth in the lower jaw, now present a well worn, or 
smooth appearance. At nine years, the mark or cavity in the nippers of the upper jaw disappears, and the wearing surface of these two teeth, becomes quite smonth, but the marks in the

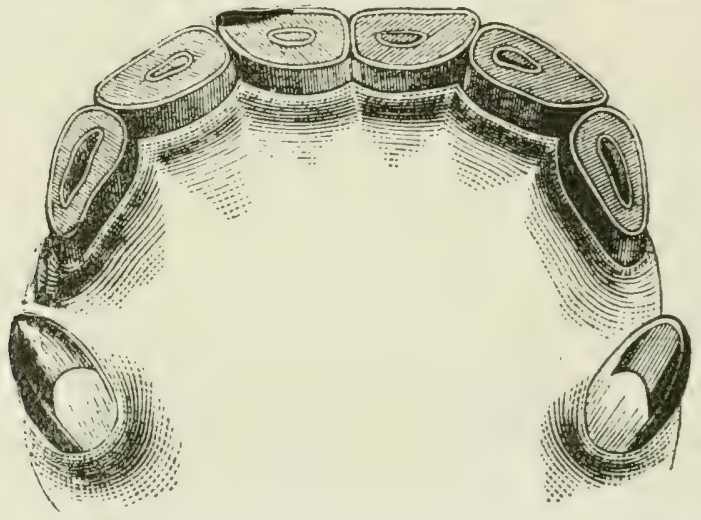

UPPER JAW AT TEN YEARS.

balance of the upper teeth, are plainly visible. At ten years of age, the middle teeth of the mpper jaw, lose their mark, and

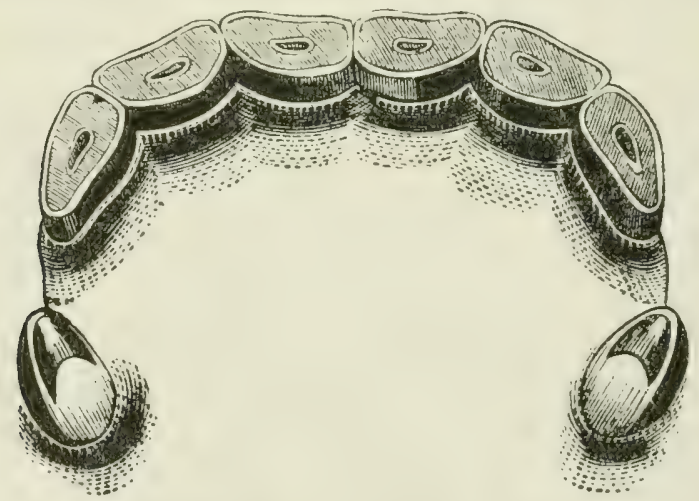

UPPER JAW AT ELEVEN YEARS.

they become smooth through wear. At eleven years of age, the corner teeth in the upper jaw, lose their mark, and the wearing surface of all of the upperincisor teeth becomes smoth, 
and the cavity or marks obliterated - the teeth commencing to assume a little difference in shape. The gums are commencing to recede from the teeth, which gives them the appearance of being much longer. They are also a little deeper from their

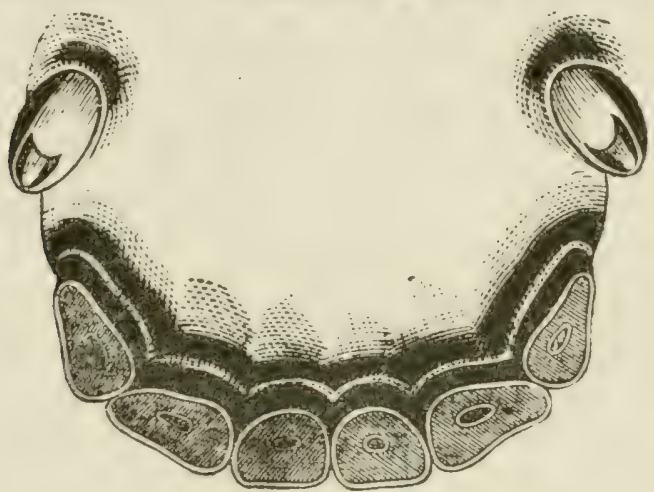

LOWER JAW AT TWELVE YEARS.

outer to their inner edge, in proportion to their breadth. At twelve years of age, the nippers of the lower jaw are almost round. They are about two-thirds the depth from the outer to

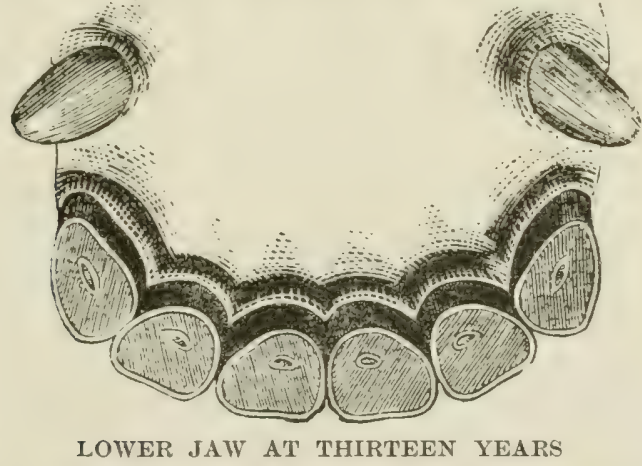

the inner edge, that they are in breadth, and the middle and corner teeth, are assuming the same shape. Thirteen years of age, shows the middle as well as the nippers of the lower jaw, to be almost perfectly round; the distance from the outer to the 
inner edge, measuring about the same as the breadtb. Fourteen years, presents all of the incisors of the lower jaw, as being nearly round, measuring about the same in depth that they do in breadth. When fifteen years old, the nippers of the upper

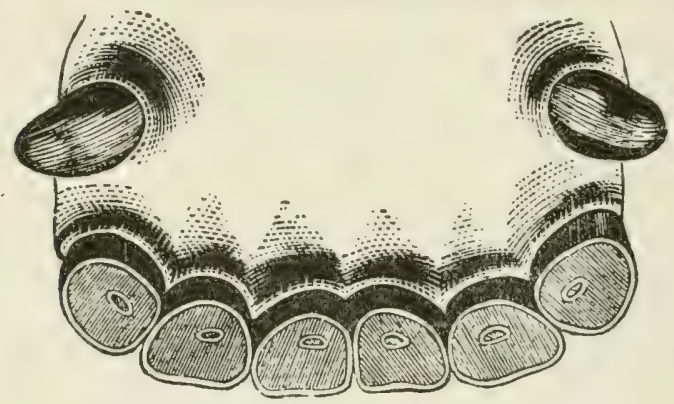

LOWER JAW AT FOURTEEN YEARS.

jaw are nearly round, measuring about the same in depth that they do in the breadth. At sixteen years, the middle teeth of the upper jaw have become nearly round, the nippers measuring

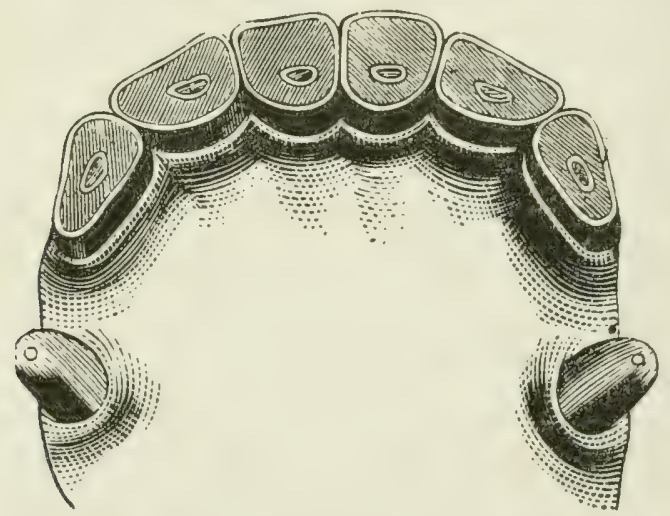

UPPER JAW AT FIFTEEN YEARS.

a little more in depth than they do in breadth. Seventeen years of age, presents all of the incisors of the upper jaw, as being nearly round, the nippers and middle teeth being a trifle greater in depth than they are in breadth. At eighteen yeears, 
the nippers in the lower jaw have become triangular in shape,

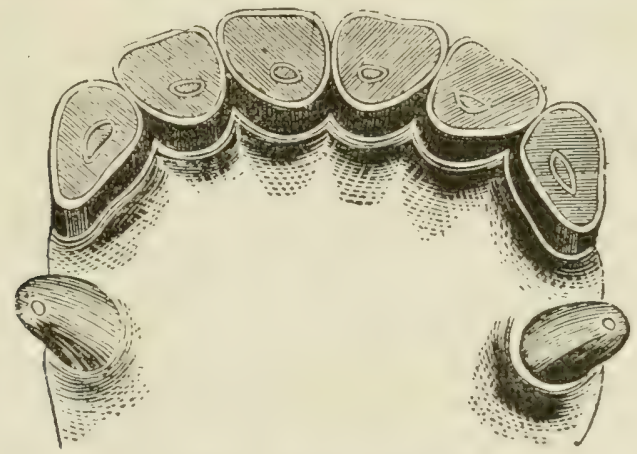

UPPER JAW AT SIXTEEN.

being about one fifth greater in depth than they are in breadth.

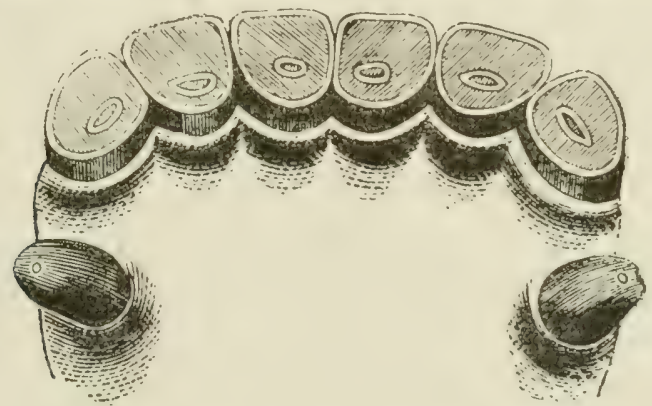

UPPER JAW AT SEVENTEEN YEARS.

Nineteen years, presents the middle teeth of the lower jaw as

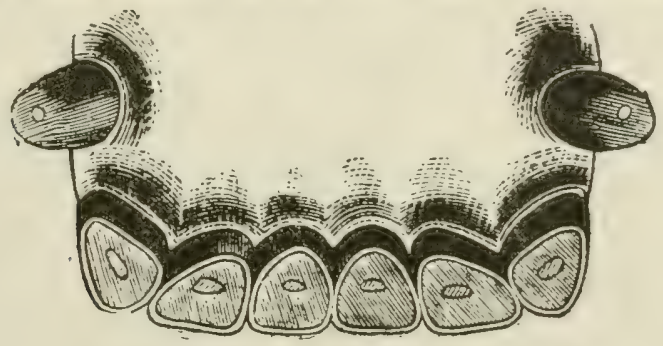

LOWER JAW AT EIGHTEEN YEARS.

being triangular in shape, increasing in depth and diminishing 
in breadth. At twenty, all of the incisors in the lower jaw have become triangular in shape, measuring about one third more in

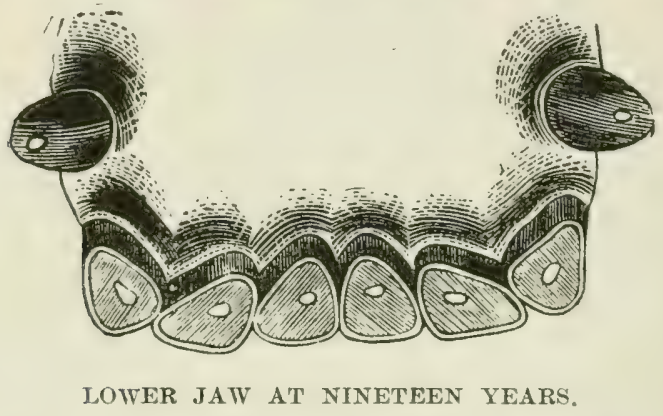

depth than they do in breadth, and as the animal grows older,

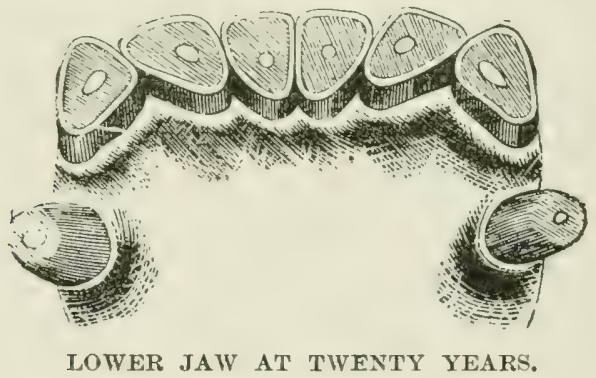

they increase in depth and diminish in breadth, or become very irregular. 
CATTLE DEPARTMENT, 


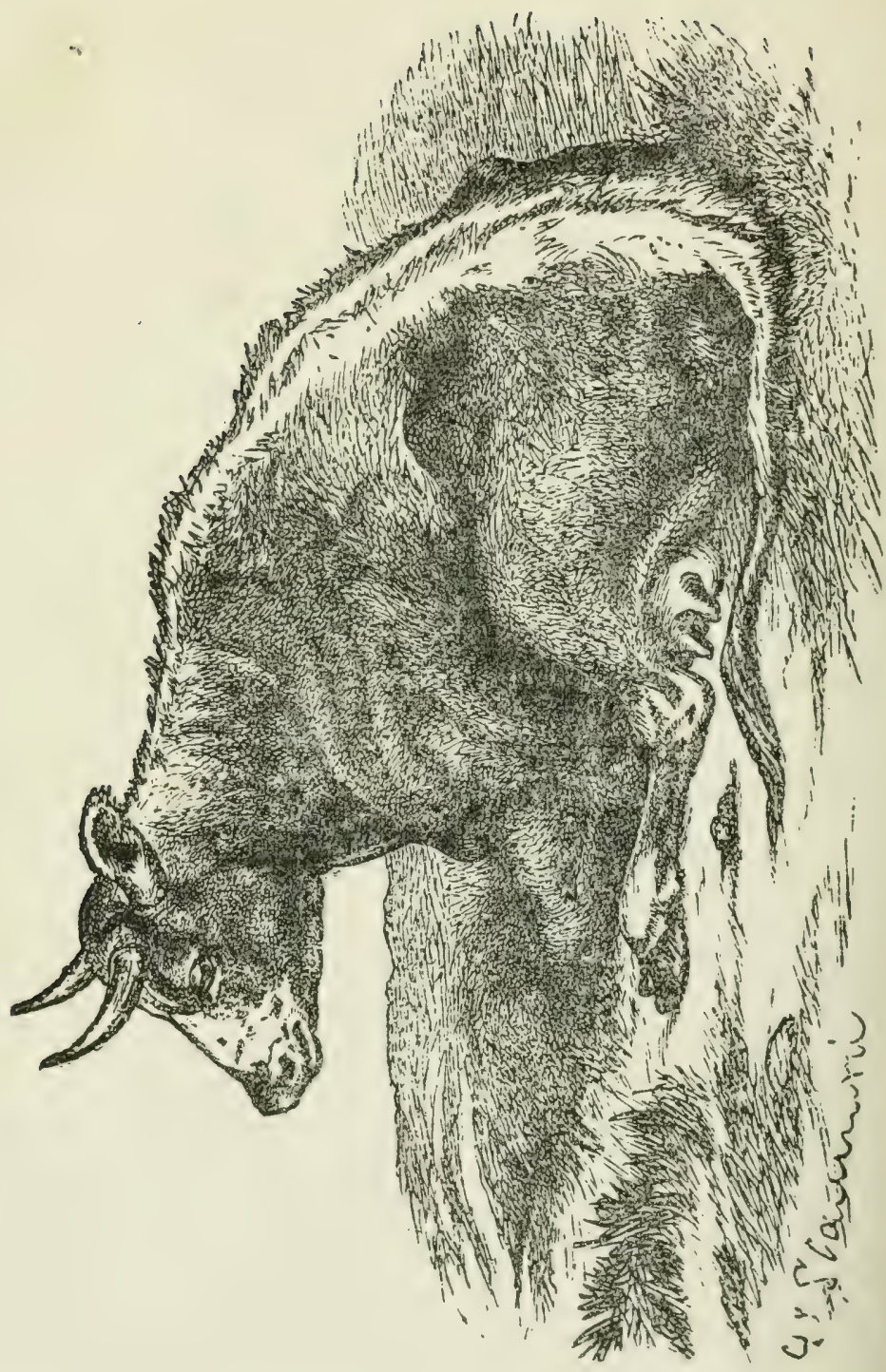




\section{DISEASES OF CATTLE,}

THEIR

CAUSES, SYMPTOMS AND TREATMENT.

\section{SORE MOUTH, OR APTHA.}

Aptha consists of small vesicles or sores in the mouth, around the lips and cheeks and sometimes upon the tongue. They become ulcerated and are quite troublesome.

Symptoms:-Sometimes there is considerable fever, but not always. There is usually dropping or quidding of the food. The mouth will feel hot with saliva dropping from the lips, and upon examination, the vesicles and ulcers will be revealed.

Treatment:-Give a mild physic, say a quart of raw linseed oil and apply one of the following applications:

Chloride Zinc......... two drachms.

Water.................... Mix. Or,

Powd. Alum........... two ounces.

Water................... pine pint.

Or rub powdered alum into the sores, which will often work the best.

\section{GLOSSITIS, OR INFLAMMATION OF THE TONGUE.}

This discase is quite often met with among cattle, and is either supeficial or deep-seated in character. When of a superficial nature, caused by the administra(295) 
tion of medicines not thoroughly diluted or by drinking lye or mineral acids, as cattle will do sometimes when thirsty, the inflammatory action may follow to the stomach and produce death, but if confined to the

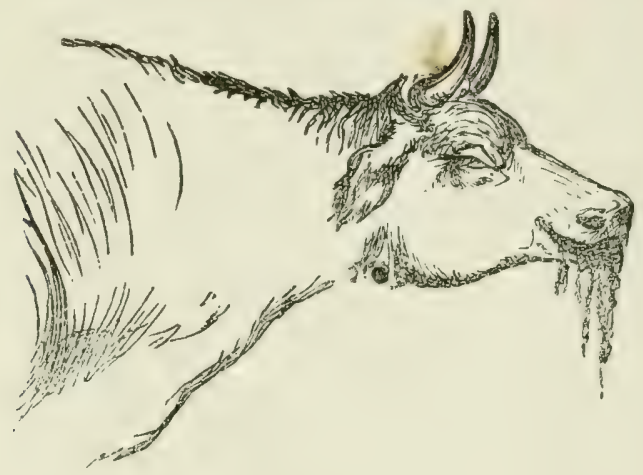

GLOSSITIS, OR INFLAMMATION OF THE TONGUE.

tongue, no unfavorable results need be looked for; but when of a deep-seated character, it is very obstinate and with difficulty attended. It usually comes from direct injuries and deep-seated ulcers, and sometimes abscesses form spontaneously.

Symptoms:-The tongue will be more or less swollen and of a deep red color. Sometimes swollen to such an extent that it will protrude from the mouth. There is great difficulty in swallowing, laborious breathing, saliva driveling from the mouth, tongue hot and the pulse quickened. Sometimes a foetid smell will be noticed in the mouth.

Treatment :-If of a superficial nature, mild solutions, such as,

Powd. Alum............. one ounce.

Water................... pne pint. Mix.

Wash the mouth three times a day; or, 
Vinegar.................six ounces.

Honey ............... four ounces.

Chlorate of Potassium...... one ounce. Mix.

Apply three times a day. Feed soft food as, bran mash, etc. If deep-seated in character, the treatment will be more severe. Give physic,

Epsom Salts.......... sixteen ounces.

Powd. Jamaica Ginger...two drachms. Mix.

Give at one dose in half gallon of water. Keep chlorate of potassium in drinking water continuously. Feed soft food, allowing plenty of water. If any foreign substance is lodged in the tongue, remove it, or if an abscess forms, open it and allow the matter to escape. If from deep-seated ulcers, take,

Chlorate of Zine........two drachms.

Water................ four ounces. Mix.

Apply to the ulcers with a feather or small brush, every three or four days until the ulcer looks healthy, or pencil it well with lunar caustic. Sommes starifying the tongue with the knife, will be attended with great benefit. Also give as a tonic,

Sulphate of Iron......... two ounces.

Powdered Gentian........two ounces,

Powdered Nux Vomica..... one ounce.

Nitrate of I'otassium............

$$
\text { ......... one and one-half ounces. Mix. }
$$

Make twelve powders. Give one powder morning and evening in a pint of water or beer.

\section{ULCERATED TONGUE.}

Ulcerations of the tongue, fauces and cheeks occur from inflammation excited by injuries, diseased teeth, lodged food, corn cobs or sticks between the teeth in the 
mouth; by trying, whilst the animal is choking, to force apples, potatoes, or other substances down the throat with a stick.

Symptoms:-Constitutional disturbances will be noticed from the very first, as the animal experiences great difficulty in masticating and swallowing food. Emaciation will follow, attended by a rough, staring roat, sunken eyes and a dejected appearance generally. On examining the mouth, the cause will appear in the form of one or more ulcers on the back part of the tongue or fauces, having a ragged appearance and emitting a sour, disagreeable smell.

Treatment:-Feed on soft, nutritious diet, as bran mash, chop feed, corn meal, green grass, etc. With 2 sponge or cloth fastened to a stick, apply to the ulcers the following, once a day:

Sulphate of Zinc....... one drachm.

Butter of Antimony...... one drachm.

Alcohol............. eight ounces. Mix.

Or,

Nitrate of Silver..... one-half drachm.

Water.............. eight ouncés. Mix.

Or,

Chloride of Zine........ one drachm.

Water................ten ounces. Mix.

Keep in a cool place and allow plenty of cool drinking water. A cure will generally result.

\section{DIARRHOEA.}

Diarrhoea is observed in cattle as well as in all other animals, and its causes are many and various.' 'Among them are eating irritating substances; eating too large quantities of food; eating to excess of sloppy bran or linseed meal; changing from dry to green food; the inju- 
dicious or continued use of purgative medicines; indigestion or disordered stomach. Diarrhoea in calves may be caused by giving very rich milk in large quantities, or by feeding sour milk; the latter curdles in the stomach, and passing on into the bowels in a halfdigested state, acts as an irritant, producing what is known as the "white scours." Whilst diarrhea is a disease, it is sometimes a boon to the animal, being nature's effort to cast off some deleterous matter that has impregnated the system.

Symptoms:-The most prominent symptoms are a copious discharge, of a liquid consistency, from the bowels, accompanied by flatulence and continued straining; sometimes a good appetite, lut more frequently it is diminished; cramping pains, staring coat; and the animal will stand all drawn up; if the patient be a calf whose diarrhoea is caused by sour milk the freces will emit a sour odor; the animal will lose flesh rapilly and have a dejected appearance generally.

Treatment :-Too much cannot be said against the use of astringent remedies at the commencement of diarrhœa unless the cause be known to be the use of purgatives, bran or linseed meal. The reason for this is that there is generally within the bowels some irritant which nature is trying to expel. Instead of giving remedies to prevent it, give half a pint or a pint of linseed or castor oil to aid in removing the offending object; it will usually effect a cure. If not, you may then resort to astringents and give one of the following every four or five hours until a cure is effected:

Tincture Catechu ....... two ounces.

Tincture Kino.......... two ounces.

Tincture Opium......... two ounces.

Tincture Ginger......... two ounces. Mix. 
Give two ounces at a dose in a little water as a drench ; or,

Powdered Chalk.......... one ounce.

Powdered Catechu.....three drachms.

Powdered Kino......... one drachm.

Powdered Opium.... one-half drachm. Mix.

Give at one dose; or,

Prepared Chalk..........six dracnms.

Powdered Opium....... one drachm.

Tannic Acid............ one scruple. Mix.

Give at one dose. If, after giving these remedies, the bowels become too much constipated, give a pint of raw linseed oil, or castor oil. Feed on dry food, give no grain and limit the amount of drinking water to half gallon at each drink. For calves give one-fourth the amount prescribed above.

\section{ACUTE DYSENTERY.}

This disease consists in an inflammation of the mucous membrane of the bowels, with ulceration and hemorrhage, and is sometimes called bloody flux. It is very often the sequel to diarrhea and has similar causes. The symptoms, too, are about the same, only of a more aggravated form. There is great constitutional disturbance with a liquid or watery discharge, intermingled with blood, from the bowels." The animal appears very dull, with clammy mouth, flurred tongue, sunken eyes, discharging a thin, watery or mattery discharge; rapid, small and wiry pulse, dry, harsh, staring coat, great emaciation, with a hide-bound appearance. The animal grunts, grinds its teeth and stands with its back arched. Pain in the bowels is manifested by uneasiness and frequent straining: the straining is some- 
times so hard, that it forces the bowel out, when it presents a red and inflamed appearance. Sometimes, but not always, there is considerable bloating on the left

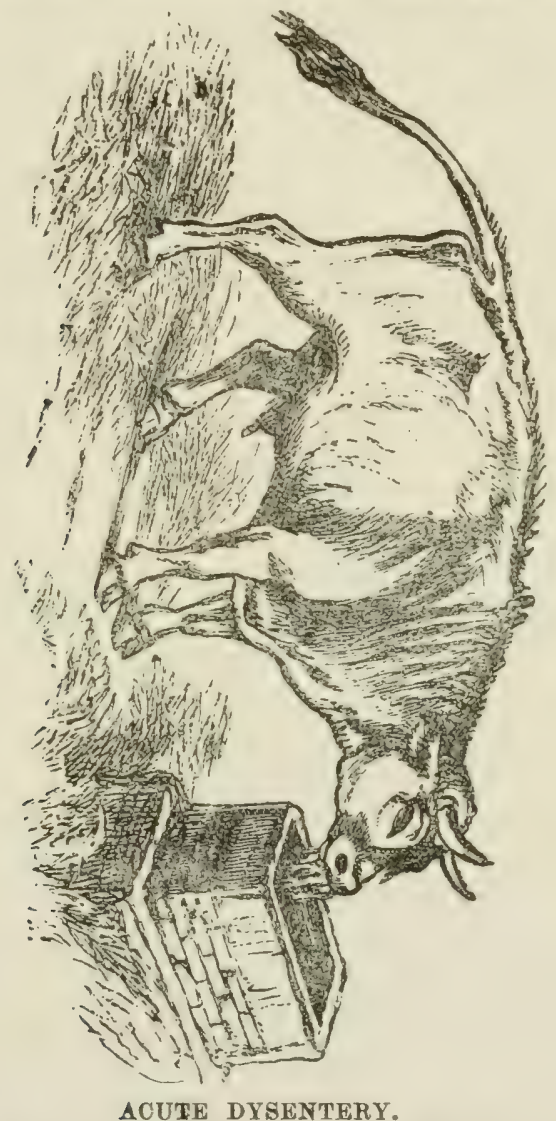

side. The horns, limbs and ears are cold. If a cow she loses her milk. Loss of appetite is noticed, with suspended rumination and increased desire for water.

Treatment:- Place the animal in a good, comfortal,le piace, well bedded, and blanket well. Feed choice, 
clean, nutritious food and allow drinking water sparingly. Give the following:

Castor or Linseed Oil....... one quart.

Powdered Opium........ one drachm.

Powdered Catechu....... one drachn. Mix.

Give at one dose, and afterwards give every four or five hours till the bowels stop running off and the patient stops straining, of the following :

Calomel........... one-half drachm.

Powdered Opium.......two scruples.

Powdered Kino......... one drachm. Mix.

Give in a pint of linseed tea; or,

Powdered Opium........two scruples.

Prepared Ohalk ....... five drachms.

Powdered Catechu...... four drachms.

Ginger.............. two drachms. Mix.

Give in a quart of linseed gruel; or,

Muriate of Morphia.......ten grains.

Powdered Camphor...... one drachm.

Prepared Chalk..........six drachms.

Whites of three hard-boiled eggs .... Mix.

Give in a pint of linseed or oatmeal gruel; or,

Tincture Opium........... one ounce.

Tincture Catechu...... one-half ounce.

Tincture Kino........ one-half ounce.

Pure Carbolic Acid.......thirty drops.

Sweet Spirits of Nitre............

.......... Mne and one-half ounces. Mix.

Give in a pint of linseed gruel.

Sometimes acute dysentery subsides and takes a chronic form, when the length of time for recovery will be prolonged. Select one or two of the foregoing prescriptions containing chalk, and continue its use twice daily; the results will be very satisfactory. But, if the 
chronic form is the result of old age and general debility, the bowels become so weakened that any treatment is of little avail and death closes the scene.

\section{WHITE SCOURS IN CALVES.}

This disease is caused by an acid condition of the stomach. Calves that are not allowed to nurse their mothers and especially the first milk after calving (called colostrum) which acts as a mild physic and prepares the stomach and bowels for the reception and proper digestion of food, are susceptible to white scours. When calves are not allowed the first milk of the mother, they become constipated, which condition is followed by an acid secretion from the lining membranes of the stomach and bowels, and when milk is swallowed it soon curdles; the cheesy material acts as an irritant and the whey passes off' in the form of white, half-fluid maunre, which is properly termed white scours.

Treatment:-The first object is to counteract the acid secretion and restore the bowels to a natural condition. This is best accomplished by giving a mild physic to remove offending agents, as,

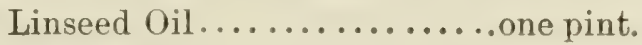

Laudanum............... one ounce.

Brandy................two ounces. Mix.

Give two ounces three times a day until the bowels act in a somewhat natural manner. The food should recei immediate attention. Give milk and linseed gruel, half and half, until the bowels are in a condition to receive milk alone. Or, you can constantly diminish the gruel and add more milk daily, until the desired results are accomplished. Sometimes white scours as- 
sumes a chronic form, when more active astringents are called for, and when such is the case, use the following;

Prepared Chalk..........two ounces.

Powdered Catechu......... one ounce.

Powdered Ginger...... four drachms.

Powdered Opium........ one drachm.

Peppermint Water......... one pint. Mix.

Give one ounce three times a day.

\section{TYMPANITIS, HOVEN OR BLOATING.}

This is a common disease, or rather accident, among cattle and has been pretty generally observed by almost every person who is in the habit of keeping or handling them. The complaint is usually observed in cattle which are greedy eaters and have just been turned out

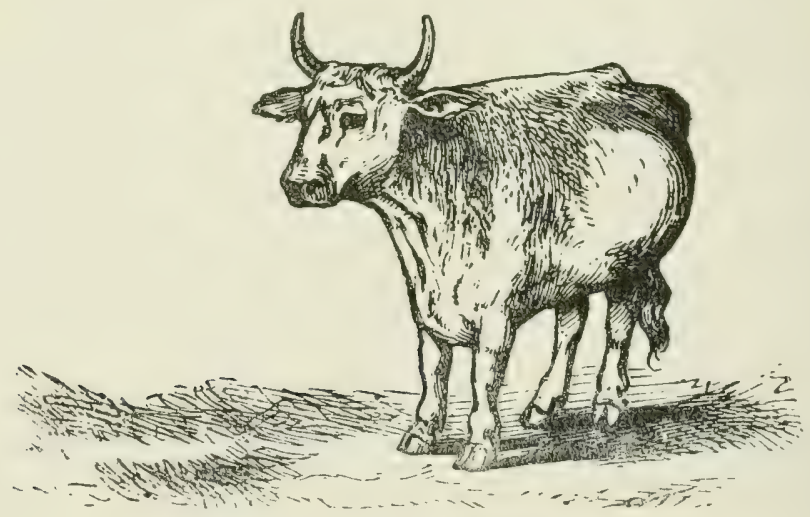

ACUTE TYMPANITIS, OR BLOATLY.

to feed on red clover pasture of rank growth and covered with moisture or dew. The greedy animal eats more than the stomach can handle and force up for mastication.' In the rumen, or first stomach, which is merely a receptacle for food, a fermentation takes 
place. wases are formed and the paunch is distended to an enormous size and is notired on the left side. If relief is not speedily obtained, rupture of the stomarh or bowels takes plare and death ensues. Cattle that have been fed on dry food, and are turned upon green pasture that is wet from rain or dew, are most liable to hecome alfected. Great care should therefore be exercised, in not allowing the cattle to eat too much such grass, liefore being removerl to a place of confinement, where they may ruminate or "chew the end." Cattle fed on frozen turnips, carrots or other roots, are subject to tympanitis. So, also, cattle which are choking, or have any foreign substance within the asophlatsus, are liable to bloat: but on removal of the olusmetion, the bloating disappears. I have seen a case where the cow had attempted premature birth. Thr culf was dead and partially decayed within the won!h; rumination was suspended, appetite lost, rough coat, dejected appearance generally. Bloating was a prominent symptom. An examination by the vagina revealed the cause.

Sympioms:-The symptoms are developed very rapidly. After being turned on clover or wet grass, a swelling appears on the left side and gradually increases in severity. The bloating becomes greater, breathing is most difficult and the patient moans and grunts; the eyes become blood-shot, saliva drivels from the mouth, and the back is arched. The animal seems riveted to the spot on which it stands, or staggers around and falls to rise no more.

Treatment:- If the case is not too far advanced, sive the following at one dose:

Aqua Ammonia.......... one ounce.

Tincture of Ginger.......two ounces.

Tincture of Anise......... one ounce.

Water................... quart. Mix. 
Or give the ammonia and water together, omitting the other drugs. Afterwards place a large gag in the mouth to keep it wide open and fasten it there by ropes tied around the horns or neck; this will permit the free outlet of eructations of gas from the stomach. If the symptoms increase in severity, it is best to tap the stomach with a trocar and canula, which is done as follows: Fasten the animal so that it eannot gret away, then shove the beast against the stall or fence. Then, standing on the left, somewhat in front to prevent being kicked, measure about half way hetween the last rib and hip bone, and about six inches from the lumbar vertebre. Now plunge the instrument inward and downward and withdraw the trocar leaving the canula, through which the gas will come rushing out. The canula may be fastened there, and, if necessary, kept in position for twenty-four hours. The trocar and canula should be eight or nine inches in length and threeeightlis of an inch in diameter. If you have no instrument, use an ordinary jack-knife, as there is very little danger from such an operation. After discharging the gas, to empty the stomach, give the following,

Epsom Salts..........twelve ounces.

Glauber Salts.......... four ounces.

Powdered Ginger...... four drachms.

Water............... one gallon. Mix.

Give immediately. Sometimes the hollow probang is introduced into the stomach, to allow the gas to escape; but this is not very practical, as you cannot allow it to remain in very long and the opening is liable to berome plugged with food. After giving the physur you may give some brandy, whiskcy or ale, to tone up the stomach. 


\section{PLEURO-PNEUMONIA CONTAGIOSA.}

For forty years, or so, this disease has been noticed in different parts of the eastern states, but through precautionary measures, it has not become at all widely spread in the rest of the country-for which stock owners may be deeply thankful. The means now employed to prevent a spread of the disease by quarantining imported cattle for a period of three months will, no doubt, lessen, if not completely check, the ravages of this malady. Floating rumors sometimes derlare the existence of contagious pleuro-pneumonia in some of the western states; but upon the best authority I am informed upon thorough examination, both ante and post mortem, by qualified veterinary surgeons, such rumors fail to establish themselves. At the same time considerable alarm may exist. P'leuro-pneumonia epizootica is as contagious among cattle as smallpox is among men. Bedding, food, blankets, manure--in fact, anything used around an animal affected with pleurojoeumonia-brought in contact with a healthy animal, is liable to communicate the disease. The period of incubation varies with different animals. The disease may develop in a week or ten days and may not appear under twelve or fifteen weeks-though during this time there will appear some symptoms. There may be a slimht, husky cough, pulse somewhat accelerated, with a raise in temperature to possibly $103^{\circ} \mathrm{F}$. Yet the animal may thrive and lay on flesh and, if a milch cow, the flow of milk may not be diminished. After the disease becomes further advanced, respiration is quickened, the temperature reaches $108^{\circ} \mathrm{F}$., appetite ceases, rumination is suspended, the mouth is hot, there are chills with more or less shivering, milk diminishes, bowels are coustipated, urine is high-colored, breath somewhat offensive, pulse accelerated to possibly one 
hundred or more per minute, coat staring, with yellow skin. The cough becomes more frequent and troublesome, the nostrils are dilated, with a slight, offensively smelling discharge, there is great emaciation; on applyinc the ear to the windpipe a rushing sound of air is heard; at the base of the lungs a loud sound may be distinguished but still further back, on acrount of the infiltration or consolidation of the lung tissue, no sound at all or only a wheezing sound can be heard. If the disease be confined to one side or to one lung only, on the opposite side the respiratory murmur is much louder, on acrount of the double work on that side. No treatment should be attempted, but the infected animals slould be destroyed and their carcasses burned, as som as the identity of the disease is established beyond doubt, in order to prevent the spread of the contagion.

\section{ALBUMINURIA, INFLAMMATION OF THE KIDNEYS, OR BRIGHT'S DISEASE.}

This disease is not very frequent, except as the result of injury, when it sometimes occurs in a violent form. Still it may arise from bad feeding, from feeding poorly matured or marsh hay, which causes derangement of the digestive organs and irritation of the kidneys through reflex action of the brain. This form is seldom observed among the lower animals. But when an injury is received upon the brain or spinal cord, albuminuria is frequently the result.

Symptoms:-Generally the first symptoms observed are the animal's standing with its legs gathered under it, and the back arched; or the legs will be somewhat stretched out, the hind legs wide apart, and the back sumken or straight. The animal is loath to move, appears stifl, and if compelled to move, does so with a 
painful, straddling motion. The bowels are usually constipated and the breathing accelerated. The urine is thick and dark colored, and sometimes passed with difticulty ; if tested for albumen, it will be found in varying amounts. The attack is usually very sudden, and, if not speedily relieved, the patient usually dies from blood poisoning.

Treatment:-If there is inability to void urine, it should be drawn away. In the cow this is easily done with a straight catheter; but in the ox, owing to the curvature in the penis, it can only be done by making. an incision into the urethra below the anus, through which pass the female catheter. Then immediately give a strong physic to unload the bowels and keep their contents in a liquid state to relieve the kidneys of a share of their work. Give the following,

Epsom Salts........... sixteen ounces.

Sulphate of Soda......twelve ounces.

Ginger.................. oune ounce.

Warm Water one and one-half gillons. Mix.

Keep hot cloths across the loins, and mustard embrocations may be applied arross the loins to advantage. Keep in a comfortable place, and feed on soft, nutritions diet, as, bran mash, scalded chop feed, linseed gruel, etc. After the bowels have been relieved, give the following:

Powdered Opium........... one ounce.

Calomel............ four drachms. Mix.

Make eight powders. Give one powder morning and evening, in thick linseed gruel.

\section{EPIZOOTIC APHTHA, OR FOOT AND MOUTH DISEASE.}

It is proper to state here, that a simple form of aphtha appears in young animals during dentition, when small vesicles or blisters break out around the 
mouth, on the tongue, and sometimes around the feet. But this is in no way contagious and calls for little or no treatment except the application of some healing lotion. But epizootic aphtha is a different disease and highly contagious, often fatal; and like smallpox and measles in man, is seldom seen twice in the same individual. It is purely an eruptive fever and destined to run a certain course, until a favorable termination may be reached, despite all treatment.

Symptoms:-The symptoms vary in intensity according to the condition, temperament and habits of the animal. It develops rapidly from the commencement and is generally ushered in by a chill which is succeeded by a staring coat, dry cough, quick, hard pulse, and a temperature ranging from $103^{\circ} \mathrm{F}$. to $106^{\circ} \mathrm{F}$, the appetite is poor and sometimes entirely suspended. Thick, ropy saliva drivels from the mouth, and, as the vesicles rupture, it becomes mixed with hlood. Epon examining the mouth, vesicles varying in size will be seen on the tongue, lips and cheeks. The feet are not always attacked, but generally are. If so, there will be swelling around the coronets covered with small blisters, also small blisters between the coronets. The swelling presses the toes apart and, on standing or walking, causes great pain. The blisters or vesicles soon burst, discharge their contents and, in the majority of cases, scab over. Sometimes the teats and udders of cows become affected, break out, swell, and prove a great source of irritation.

Treatment:-Give a mild laxative, as, Sulphate of Soda.........ten ounces. Ginger............. one-half ounce.

Foenugreek......... one-half ounce. Mix.

Give in a quart of warm beer. The mouth should be washed twice or thrice daily with the following: 
Chlorate of Potassium...... one ounce.

Powdered Alum.......... one ounce.

Water.................. Mue quart. Mix.

If matter forms around the fect, apply the following twice daily :

Iodoform..............

Vaseline............ two ounces. Mix.

Grease the parts; or use,

Salicylic Acid........ one-half ounce.

Iodoform.............. one ounce.

Taseline ............ two ounces. Mix.

Grease the parts thrice daily. If the udder is swelled and painful grease with,

Gum Camphor............. one ounce.

Lxtract of Belladonna...... one ounce.

Vaseline............. five ounces. Mix.

[se twice daily. If the appetite fails or the animal cinnot eat, dreucls with gruel tio or three times a day, into which may be mixed a quirt of beer.

\section{CANCEROUS ULCERS.}

When the veterinary sureon's attention is called to these cases, he can do lith mure than to advise concern-

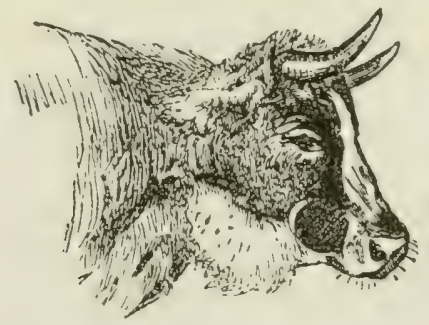

LUMP JAW.

ing the best mode of destroying the animal. These ulcers generally appear as small, hard nodules, situated upon oither the upper or lower jaw, principally the lat 
ter. Usually they are at first movable, the akin playing over them with perfect ease. As they gradually develop in size, they become adherent to the bone and may continue to grow for months before breaking. When one breaks, it discharges either a thin liquid, or

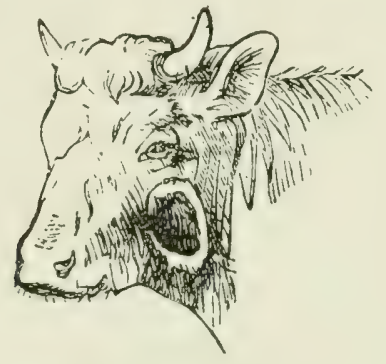

TUMOR REMOTED.

pus, and presents an ugly, ragged appearance, of a fibrous nature and sometimes bleeds profusely. It may partially heal up, then it will swell up and discharge, and lumps of tissue will slough away. Gradually becoming worse, it eventually affects the teeth, causing

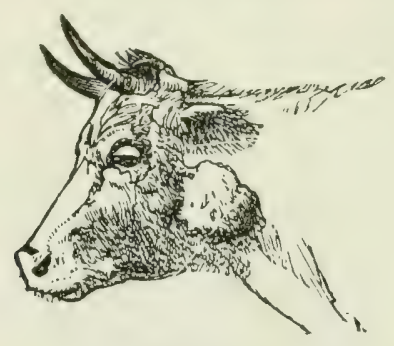

LU:IP IAW.

them to fall ont and establishing an opening into the mouth, throunh which the ford will drop out. There is no special treitment save to fatten the animal for the butrher-that is when the swelling is first noticed. 
After it has broken out and discharged for some time, the flesh ought not to be used. Still, destroying the animal is a humane act.

\section{HAEMATUREA, OR BLOODY URINE.}

This disease is seen amongst all breeds of cattle and 1s caused by injuries over the back and loins: by cattle jumping upon each other, by eating herhs which cause

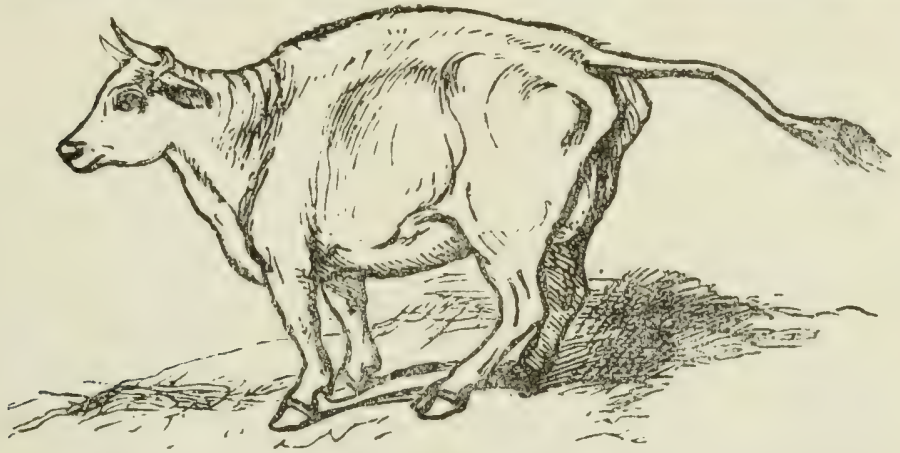

TRAUMATIC HEMATUREA.

Irritation of the kidneys, by the admmistration of painful diureties; from stones in the bladiler, rupture of small blood vessel's in the kidneys, or vagina.

Symptoms:-The symptoms develop very rapidly. The animal appears weak, scarcely able to rise, and when standing the back is arched; there is straining, the animal voids small quantities of urine mixed with blood globules and albumen; pulse quick, snall and wiry; appetite and rumination partly suspended, leos and horns are alternately lot and cold, mouth hot, howels constipated, tlow of milk decreased; on applying pressure along the back, the animal evinces pain; if complled to walk, it moves with a straddling gait. 
Treatment:-Apply hot fomentations across the loinc and give the following:

Epsom Salts.......... sixteen ounces.

Sulphate of Soda........eight ounces.

Ground Ginger........ one-half ounce.

Gentian............. Mne-half ounce. Mix.

Give in a gallon of water at one dose; to be followed with tonics, as,

Tincture Per-Chloride of Iron.......

...................... ounce.

Tincture Cinchona....... four ounces.

Tincture Nux Vomica.....two ounces.

Tincture Opium......... four ounces.

Infusion of Quassia ....... five ounces. Mix.

Give two ounces, three times a day. Feed on a soft nutritious dict and the drinking water should be rendered mucilaginous by linseed.

\section{PHRENITIS, INFLAMMATION OF THE BRAIN.}

Inflammation of the brain usually results from injuries to the head, or as the sequel of fevers caused by eating buckeyes; also from indigestion, from exposure to: the hot sun, from extensive inflammation of the ear, etr.

Symptoms:- sometimes an animal sullering from inflammation of the brain will appear stupid, pressint its head for a considerable time against a wall, fence or some stationary object, and occasionally running about wildly as if it were both drunk and blind. Others appear as though seized with a fit of madness and act fiercely; the eyes are inflamed and staring-constantly: watching. The animal in rushing about, will fall dowis. in almost any position. The breathing will be slow. and the pulse, strong and full at first, will gradually de... 
cline with throbbing of the temporal arteries. The dis ease generally occurs during the hot months.

Treatment :-Give a brisk physic:

Epsom Salts...... twenty-four ounces.

Ginger................ six drachms. Mix.

Put in a gallon of warm water and give at one dose. Bleed from the jugular rein, while the pulse is full, from one to two gallons. Apply ice-cold water to the head constantly. If the limbs are cold, apply friction or some stimulating liniment and bandage, and give,

Bromide of Potassium......six ounces.

Sulphate of Soda......twelve ounces. Mix.

Make twelve powders. Give one powder every two or three hours in a pint of water, and as convalescence is taking place, apply a grood blister on the head, back of the horns.

\section{GONORRHOEA, OR CLAP.}

This disease is not very frequently met with in reterinary practice, but sometimes occurs and is a source of consilerable annoyance to both practitioner and patient. Bulls and cows suffer considerably in passing water. The animal strains frequently and only a small quantity of urine at a time is passed; the animal occasionally stamps its feet and lashes its tail from side to side; the bowels are constipated; if a bull, a swelling appears around the sheath; a discharge of matter, at first light in color and then assuming a thick white or yellowish white color, is seen dropping away; sometimes the penis swells up and becomes fiery red in color.

Treatment:-As soon as noticed give a brisk physic: Epsom Salts.......... sixteen ounces. Sulphate of soda..........six ounces. Ginger.............. seven drachms. Mix. 
Give in a gallon of warm water. Bathe the parts well with hot water and into the affected portions inject hot water frequently, or inject the following:

Iodoform............. two drachms.

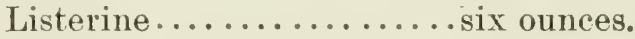

Water................ten ounces. Mix.

Use three times a day; or,

Sulphate of Copper......... one ounce.

Water................ Mene quart. Mix. Or,

Calomel.............two drachms.

Lime Water...............ne pint. Mix.

Inject twice daily and give the following:

Balsam Copaiba.........two ounces.

Tincture Cubebs..........two ounces.

Spirits, of Camphor.......two ounces.

Tincture Kino.......... two ounces.

Morphia............ forty grains. Mix.

Give tablespoonful three times a day. If chancres appear, they should be touched with lunar caustic and afterward covered with iodoform or calomel twice daily. Cattle thus affected should not be bred until a complete cure is effected, as it is communicable to other animals. Great care should be used in thoroughly cleansing the hands, as the discharge is very poisonous.

\section{CATARRH, OR COLD.}

This is the term used to denote an inflamed condition of the lining membrane of the nostrils, sinuses of the head and upper air passages; it may extend to the eyes and throat. The causes are change of climate, sudden changes of weather, bad ventilation, deficient 
drainage, being turned from a warm stable out into a cold storm, etc.

Symptoms:-The lining nembrane of the nostrils is at tirst reddened and dry, soon becoming moist with a thin, watery discharge. The eye-lids become swollen and red and discharce a watery secretion, soon assuming a mattery appearance. The forehead and butts of the

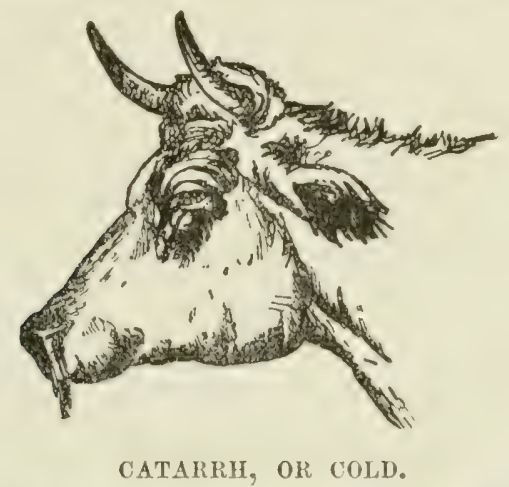

horms are very hot. The animal snuffles and sneezes and sometimes coughs. The breathing is somewhat difficult. The animal occasionally shivers. Appetite and rumination are partly suspended. The urine is high-colored. Coat is rough and staring. Sometimes a light diarrhora is present and later on a thick, viscid discharge from the nostrils.

Treatment:-Give as a mild laxative-

Epsom Salts ......... twelve ounces.

Ginger ............ one-half ounce.

Gentian.......... one-half ounce. Mix.

Give in a half gallon of warm water. Keep the patient in a comfortable place, blanket well and feed on hot mashes. Give one of the following powders three times a day : 
Chlorate of Potassium.... one ounce.

Nitrate of Potassium....... one ounce.

Bicarbonate of Soda........ne ounce. Mix.

Make eight powders. If the discharge becomes stopped before a favorable termination is reached, steam the nostrils with scalded bran or oats and add a little vinegar to the hot water.

\section{CHOKING.}

This is a common accident among cattle that are fed on such roots as turnips, carrots, potatoes, beets, etc., et $\because$. In greedy eaters it is also caused whilst eating grain or chaff; in the hurry to eat, the food is not thoroughly mixed with saliva, and heing swallowed about

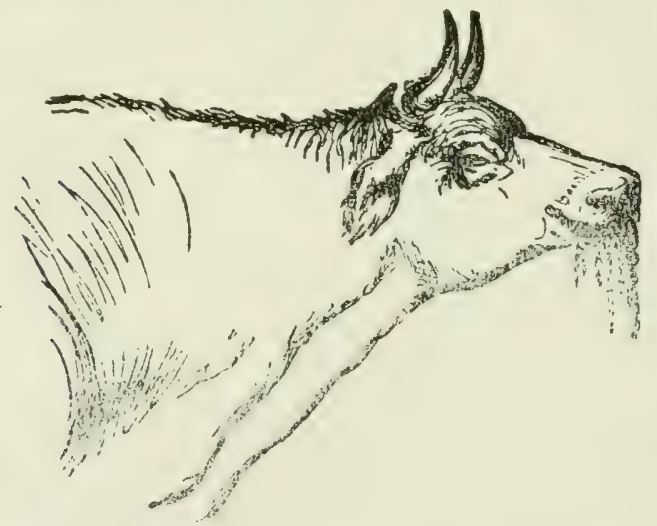

INJURIES TO THE GULLET FROM USING BROOM STICKS AND WHIP STALKS WHILE CHOKING.

half dry, it frequently becomes lodged in the osophagus. Choking also occurs from leather, clothing, etc., of which cattle frequently attempt to eat.

Symptoms:- If the obstruction is high up in the throat or in the pharynx, the animal will slaver consid- 
orably-the saliva running profusely from the mouth. There will be difficult breathing and inuch uneasiness.

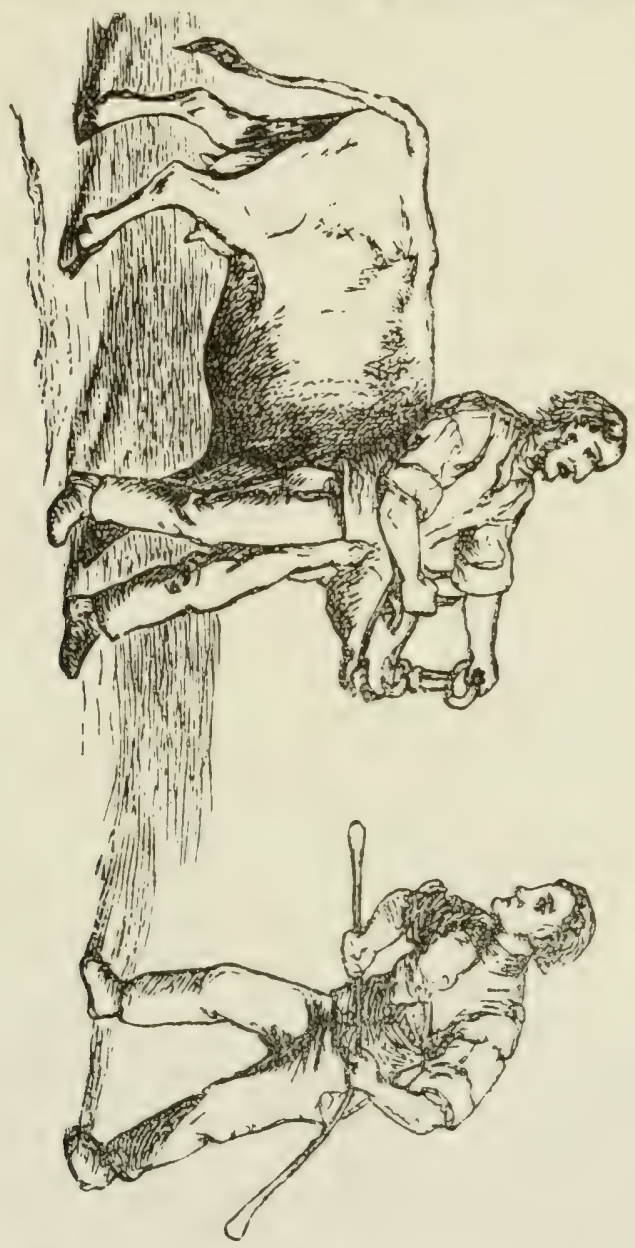

THE MANNER OF USINE THE PRORANA IN CHOKINY.

The head will be held low down with the nose straight out; sometimes attempts at conghing are made. The 
eyes are staring and blood slot. If the obstruction is lower down the neck or within the chest, the symptoms are not so severe. The breathing may be but little altered and the slavering much less and probably absent. If the animal attempts to drink and the obstruction is large, the water will be ejected through the mouth and nostrils; but if the obstruction is small, it may pass by it and go to the stomach. Bloating is a prominent symptom-the left side will sometimes be greatly distended.

Treatment:- If the obstruction is within the pharynx place a gag in the mouth and remove it with the hand or with a pair of forceps. If you fail to withdraw it, give a half pint of sweet oil or melted lard; administer carefully, and the obstruction may pass downward or be expelled in an attempt to cough. Should this fail or should the animal be in great distress, pass the probang immediately. This should be done as follows: Place gag in the mouth and have an assistant hold it; then grease the probang and pass it slowly down the throat. When you come to the obstruction, press heavily but slowly, and when it starts force it on into the stomach. If the obstruction is low down or within the chest, give six or eight ounces of sweet oil or melted lard, also a quart or two of water, and it will frequently pass on. If it does not, pass the probang, but do not attempt to force it down with sticks or whip-stalks as you are liable to produce great injury, by rupturing the resophagus. When this occurs and inflammation sets in, apply hot water to the side of the neck, and give internally-

Powdered Alum...... one-half ounce. Powdered Opium......three drachms. Warm Water............ one pint. Mix. 
Give wineglassful three times a day. Sometimes it is advisable to apply a blister along the side of the neck. The following will answer very well:

Aqua Ammonia........ two ounces.

Spirits Turpentine.......two ounces.

Linseed Oil........... four ounces. Mix.

Apply two or three times a day, rubbing it thoroughly. Feed on soft food, allow drinking water at pleasure and fatten for the butcher, as there will always be more or less trouble.

\section{CHRONIC INDIGESTION.}

This is a disease of the fourth stomach and is caused by irregular feeding, bad ventilation, feeding innutritious and badly-coured fodler, exposure to cold and rain, feeding on marsh grasses, etc.

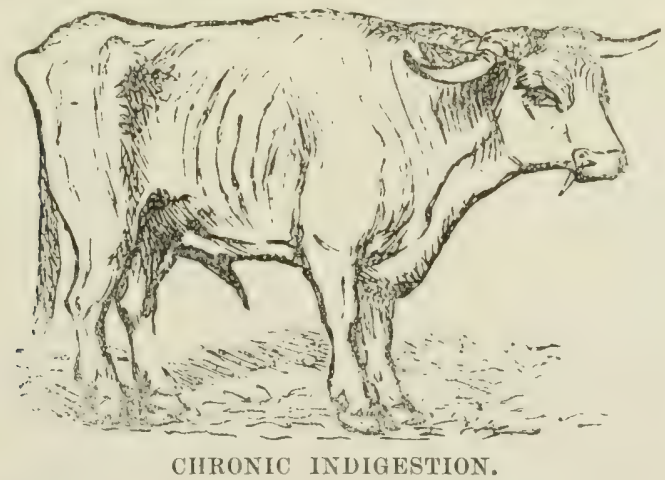

Symptoms:-At first the animal will have a whimsical appetite and eat irregularly; later on, they will sometimes be discovered eating dirt, filth, straw, manure, etc. - The skin becomes harsh and dry, with a. staring coat. The dung will be passed in small lumps 
which will he hard and dry and rovered with mucous. The loss of flesh will be rapid and the creature at the flanks will look as thin as a sunfish. The blood becomes depleted, with pallor of the mucous membranes; and if

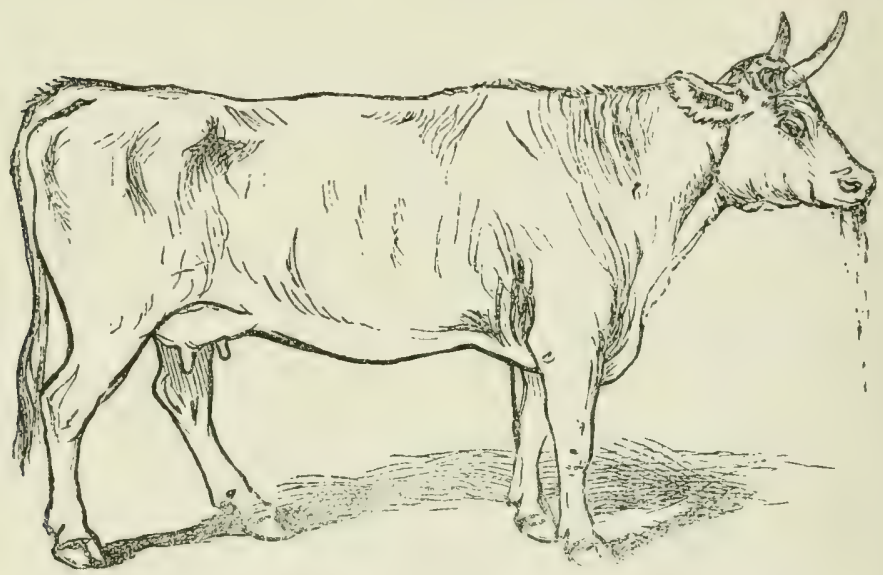

DROPPING OF THE CUD, OR INDIGESTION.

the disease is allowed to progress, diarrhøa will supervene and death will result. But, if attended to in time, a favorable termination may be looked for.

Treatment:-Put the animal in a comfortable place and give a mild laxative.

Epsom Salts......... twelve ounces.

Powdered Ginger...... one-half ounce.

Powdered Gentian.... one-halfounce.

Aromatic Spirits of Ammonia.....

................. two ounces.

Water.................... quart. Mix.

Give at one dose, and afterwards give-

Powdered Nux Vomica....two ounces.

Powdered Jamaica Ginger. two ounces.

Powdered Gentian...... four ounces.

Powdered Fonumreek.... two ounces. Mix. 
Give desertsponful two or three times a day in soft ford. Feerl on soft, nutritions food in small quantities, often repeated; or feed ronts, as turnips, carrots, beets, etc., with a little linseed meal occasionally. Give plenty of fresh drinking water, with a teaspoonful of soda, three times a day.

\section{COLIC.}

This disease is seldom seen in cattle and when it occurs, the animal is not very violently attaclied. ('ompared with the same disease in the horse, the symptoms are very milıl. The animal will appear a little uneasy, stamp with its hind feet, twist its tail around from side to side, and possibly lie down and remain quiet; sometimes it will turn its head around to its side. The breathing and pulse remain about normal.

Treatment :-Administer a physic; the following will answer very well:

Epsom Salts........... sixteen ounces.

Powdered Ginger.......... one ounce.

Powdered Gentian.... one-half ounce.

Warm Beer............. one quart.

Warm Water........... one quart. Mix.

Give at one dose. Bathe the lower part of the abdomen and allow the animal to rest. If it still appears uneasy give the following;

Tincture Opium........ two ounces.

Tincture Ginger......... one ounce.

Tincture Capsicum.... one-half ounce.

Whisky........... one-half pint. Mix.

Give at one dose, and, if the pains still continue, repeat in two hours. 


\section{ENTERITIS, OR INFLAMMATION OF THE BOWELS.}

Among cattle this disease is not very frequent, but when it does occur it is usually very fatal. The causes are obstruction of the bowels, feeding on musty hay, drinking ice-cold water, administration of powerful, irritating medicines, change of food, etc.

Symptoms:-The animal will be seized with colicky pains; will strike at the ahdomen with the hind feet; there is suspension of appetite and rumination; pulse, at first full and hard, gradually growing quick, small

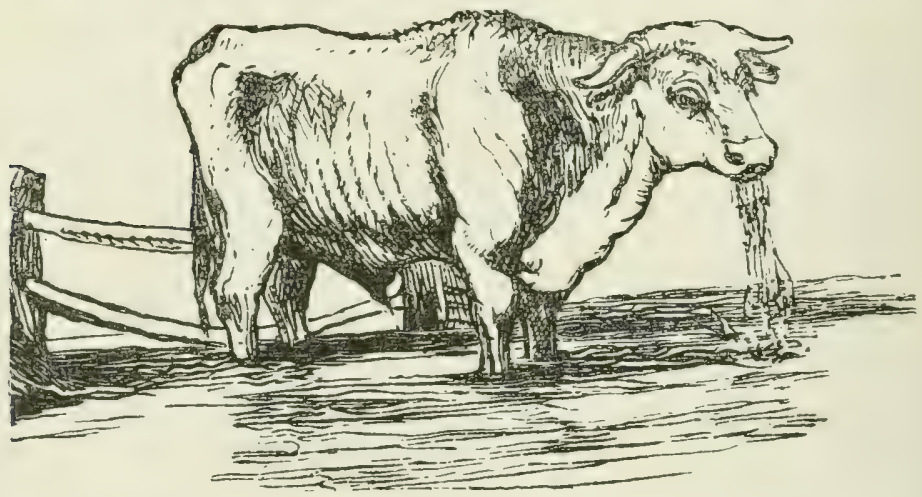

ENTEITTIS, OR INELAMMATION OF THE BOWELS.

and wiry : the animal lies down and rises up frequently; pain is constant: there is moaning; nose hot and dry; mucous membrane very red; anxious expression of countenance.

Treatment:-Place the animal in a comfortable stall, well bedded. If the disease is caused by obstruction of the bowels give a mild laxative-as a pint and a half of sweet oil, or a pound of sulphate of soda in half a gallon of water. Follow up with drachm doses of powdered opium, to relieve the pain, or twenty-drop doses of fluid extract aconite, every thirty minutes until 
relief is obtained. Apply to the abdomen blankets wrung out of hot water and rub mustard on the abdomen and legs. Blanket well and feed on soft bran mashes for a few days, to relax the bowels. Avoid giving brisk purgatives as evil results are liable to follow their use.

\section{AFFECTIONS OF THE RETICULUM, OR SECOND STOMACH.}

The reticulum, or second stomach, of cattle is sometimes the seat of trouble. It is this stomach which presents a honeycombed appearance. The usual trouble here is the accumulation of foreign bodies-such as pins, nails, shoe soles, pieces of tin, wire, etc.-which cows are liable to pick up. Many of these articles find a resting place in the rumen, or first stomach, but some pass on into the second stomach. Needles or nails sometimes pierce the stomach and pass out to the ribs or on through the flesh and form an abscess, finally sloughing their way out of the animal's body. ()thers may pass forward through the diaphrim and pierce the heart, causing instant death. Others may pieree the walls of the stomach and excite an inflammation there; and they may be incased in tumors which have formed around them, there to remain for months, or even years; or abscesses may form and break, assisting to destroy the life of the animal. It may not show any signs of illness and yet have within the stomach quite an accumulation of bits of iron, etc. But, if tumors and alscesses form, the animal will begin to lose flesh; an intermittent diarrhoea will be noticed, with rough, staring coat and yellow skin, full of dandrull, impailed appetite, sunken eyes, and aversion to movement.' The treatment is unsatisfactory, as the offending object is 
difficult to locate. All that can be done is to feed on good, soft nutritious diet, and, if able to locate the irritating substance, operate and remove it.

\section{CONJUNCTIVITIS, OR INFLAMMATION OF THE EYES.}

Inflammation of the eyes arises from rarious causes, such as blows from sticks or stones, being struck with a whip, dirt or chaff getting into the eye, foul stables, exposure to cold, etc.

Symptoms:-Swelling of the eyelids, with water dropping from the inner corners; eyelids closed most of the time, intolerance of light, impaired appetite, feverishness, conjunctiva swollen and red, with a whitish film covering the anterior portion of the eyeball; if from a bruise, red streaks of blood will be seen radiating from the seat of injury.

Treatment:- If a foreign body is in the eye, remove it. This is much easier said than done, but the following solution will greatly assist in the operation:

Cocaine .............. ten grains.

Soft Water.............. one ounce. Mix.

Place a few drops into the eye every thirty seconds, for twa or three minutes; the eye becomes insensible and a thorough examination may be made. After the removal of any foreign substance, bathe the eye a few times with hot water, and the trouble is usually at an end.

If from other causes, give a physic,

Epsom Salts.......... sixteen ounces.

Ginger ............. one-half ounce.

Foenugreek............ one-half ounce. Mix.

Give at one dose. Then bathe the eyes three or four times a day with hot water; feed on soft food and apply twice daily, one of the following lotions: 
INFLAMMATION OF THE LITER, OR IIEPATITIS.

Sulphate of Zinc........ four grains.

Sulphate of Atropia ........ one grain.

Soft Water............two ounces. Mix.

Or,

Powdered Opium........ one drachm.

Hot soft Water...........six ounces. Mix.

Or,

Sulphate of Zinc........ four wrains

Water.................two ounces. Mix.

Or,

Atropine.............. one grain.

Sulphate of Copper....... four grains.

Water...............two ounces. Mix. Or,

Sulphate Morphia.......... one grain.

Cocaine.............. live grains.

Water................ one ounce. Mix.

Keep the patient in a clean, clark, airy stable; if at pasture, and only one eye is affected, bandage the affected eye.

\section{INFLAMMATION OF THE LIVER, OR HEPATITIS.}

Inflammation of the liver is most frequently the result of high feeding in warm stahlws, without sufficient exercise to keep the circulation of the blood active. The bowels herome constipated and the liver suffers from an extra amount of blood sent to it, causing congestion and resulting in inflammation.

Symptoms:- When the liver is attacked with congestion, the animal exhibits no distressing symptoms. But when inflammation sets in, the pulse is sow and intermittent; respiration slow, the mucous ne ibranes are pale or tinged with yellow; bowels constipated; the 
dung hard and black; urine high colored; coat staring, dry and hard to the touch; horns, ears and legs cold. On applying pressure to the right side, behind the last rib, the animal will evince pain and try to get away

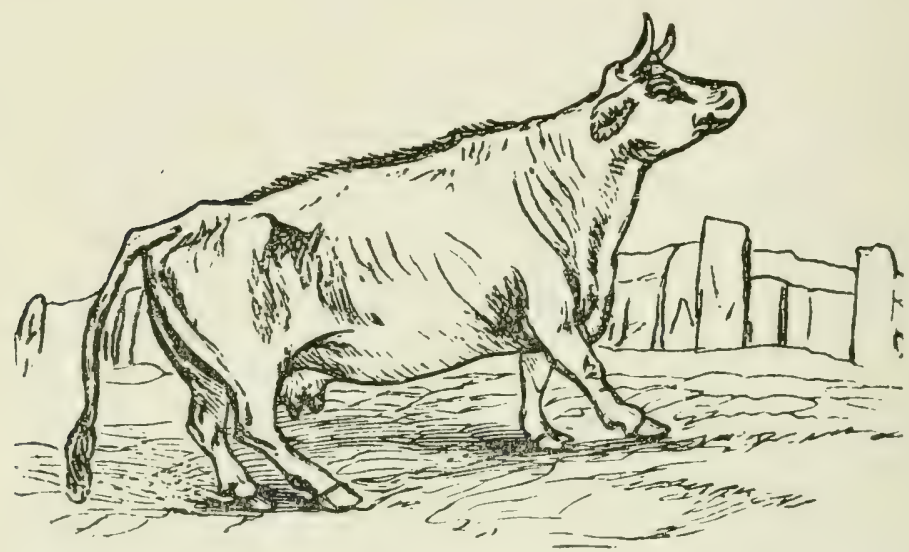

FAINTING FIT FROM INFIAMMATION OF THE LIVER.

from you, and sometimes moan; you will also feel a hard tumor-like substance.

Treatment:-At the outset give a brisk catharticEpsom Salts.........twenty ounces.

Aloes................... one ounce.

Calomel ..............two drachms.

Ginger.............. Mne ounce. Mix.

Give in a gallon of warm water at one dose. After the physic hasoperated, give morning and evening, for a week or ten days, in a pint of gruel, the following:

Iodide of Potassium...... one drachm.

Calomel.............twenty grains.

Extract of Taraxacum...two drachms.

Nitrate of Potassium....two drachms. Mix.

If the bowels become costive, give a physic. When convalescence is established, great care in feeding 
ghould be observed. Give highly nutritious food, in limited amounts, and such as is easily digested, as, scalded bran mash, linseed meal, boiled barley, turnips and choice, clean hay. sometimes much benefit is derived from blisters applied on the right side.

\section{DISLOCATION OF THE PATELLA, OR STIFLE JOINT.}

Among cattle this is quile a common affection, asually resulting from slipping on the ice, or in stables where the drop or receptarle for manure is very deep and the floors not well cleaned.

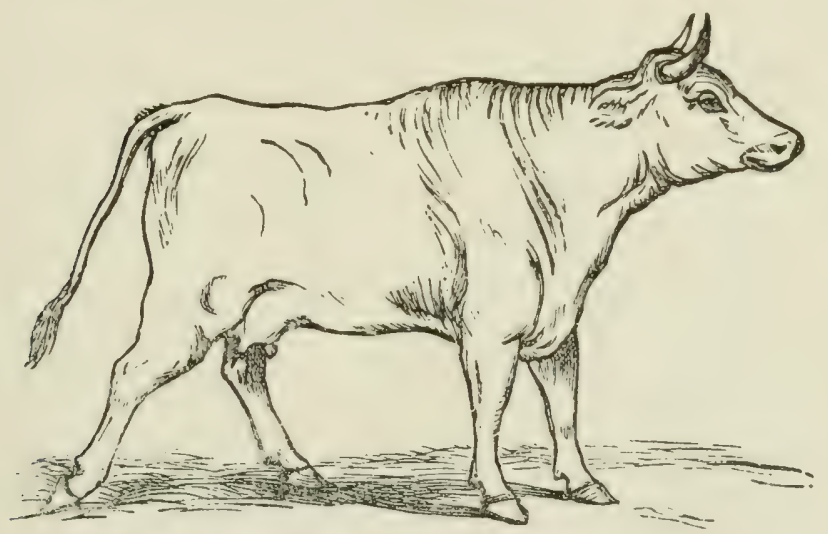

DISLOCATION OF THE PATELLA, OR STIFLE.

Symptoms:- When once seen it will be readily recognized again. The animal can walk only on three legs: the stitled one will be dragged along behind and will be still and rigid. Where the patella should be, when in position, a hollow spot will be observed. There will be inability to flex the affected limb with more or less swelling around the joint. 
Treatment:-Fasten a strap around the fetlock of the disabled leg, pass it forward, hetween the front legs, then forcibly draw it forward, when the patella will often drop into place; if not, press forward on the bone (which is always on the outside) and it will readily drop into place. Then apply a blister, as,

Aqua Ammonia.........two ounces.

Spirits Turpentine.......two ounces.

Linseed or Olive Oil..... four ounces. Mix.

Apply around the stifle morning and evening until the ligaments are strengthened.

\section{JAUNDICE.}

This is quite a common disease among cattle, and is caused by a re-absorption of bile into the system. The bile is secreted in the liver and emptied into the gall sack, and from there it is emptied into the duodenum or first bowel, to increase the peristaltic action of the bowels and complete digestion. There are many diseases of the liver that will produce jaundice. Gall stones passing through the duct and becoming lodged, will prevent the bile from passing into the bowels; it then becomes re-absorbed and diffused through the whole system.

Symptoms:-In ordinary or acute cases of jaundice, either suddenly or after a few days, you will notice a yellowness of the eyes and the lining membrane of the mouth and nose, and a general listlessness is apparent. The bowels will be constipated and what does pass, will be dry, hard, dark-colored and covered with mucous. The urine is high-colored and stains linen yellow. linmination is generally suspended and the tongue heavily coated. Sometimes bloating will be present, but not always. Acute jaundice is not very often fatal. 
Treatment:-When supposed to be functional disturbance of the liver, the object is to restore that gland to a normal condition, or if gall stones be the cause, they may be removed by the same course of treatment, viz:

Epsom Salts.........twelve ounces.

Powdered Barbadoes Aloes..one ounce.

Podophyllin Resin..... trio drachms.

Ginger............. two drachms. Mix.

Give at one dose in two quarts of water. A fter the physic has operated, give the following:

Ohlorate of Potassium...two drachms.

Podophyllin Resin....... one drachm.

Extract of Taraxacum .... one drachm.

Ginger............. one drachm. Mix.

Give in a quart of water or gruel, night and mornIng, for four or five days. When convalesence takes place, feed carefully on easily digested food, such as bran mash, clover hay, malt, barley, etc.

\section{FITS FROM EATING BUCKEYES.}

Buckeyes are nuts which grow on trees, in a great. many parts of this country. In the autumn, when they fall to the ground, they are a source of great annoyance to the farmers who use forest pasture lands. Some cattle are particularly fond of these nuts and eat them readily, seldom, however, eating them a second time if they eat many the first. When once observed, the symptoms will never be forgotten. For a while the animal will appear dull and stupid; respirations natural; pulse, at first full and quick, gradually growing small, weak and rapid. When the animal attempts to walk,-it staggers around like a drunken man, striking all around with its forelegs and falling in almost any position, as if in a tit 
After lying a short time, it gets up very awkwardly and may stand perfectly quiet; but, if forced to walk, it will stagger around until it either regains its balance or falls to the ground. The animal may lie perfectly quiet, stretched out on the ground for two or three days, and if relief is not obtained, death ends the scene. By pressure on the stomach, you will generally feel the

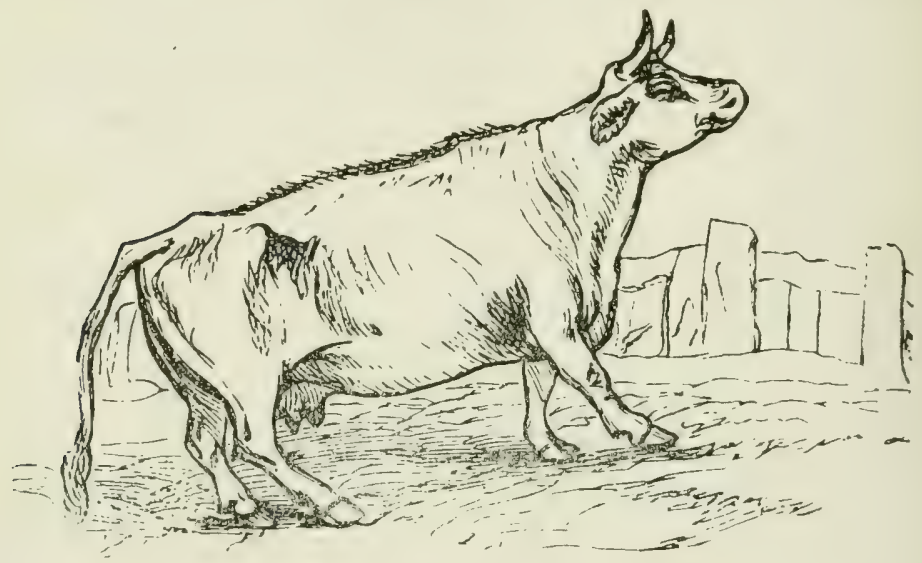

FITS FRON EATINA BUCKEYES.

oflending agents. There will be involuntary twitching of the muscles and grinding of the teeth.

Treatment:- When first noticed, give a brisk physic to expel the buckeyes-

Epsom Salts..........twenty ounces.

Powdered Aloes............ne ounce.

Ginger.............. one ounce. Mix.

Put in a gallon of water and give at one dose. Give per rectum, frequent injections of warm water. If within twenty-four hours the animal is not convalescent, rumenotomy should be performed (see Inpaction of the liumen:) after which give one of the following powders three times a day: 
Bromide of Potassium.... two ounces.

Nitrate of Potassium....... one ounce.

Ginger................ one ounce.

Gentian..............two ounces.

Bi Carbonate of Soda.....two ounces. Mix.

Make twelve powders. Feed on soft food, as, bran mash or linseed gruel. The balance of the herd should be mored to other pasture, or the nuts should be gathered and destroyed.

\section{IMPACTION OF THE OMASUM, OR FARDEL-BOUND.}

Impaction of the manifolds, or third stomach of the ox, is sometimes met with; more especially when the cattle are poorly kept during the winter season and are fed on innutritions food, containing a great amount of

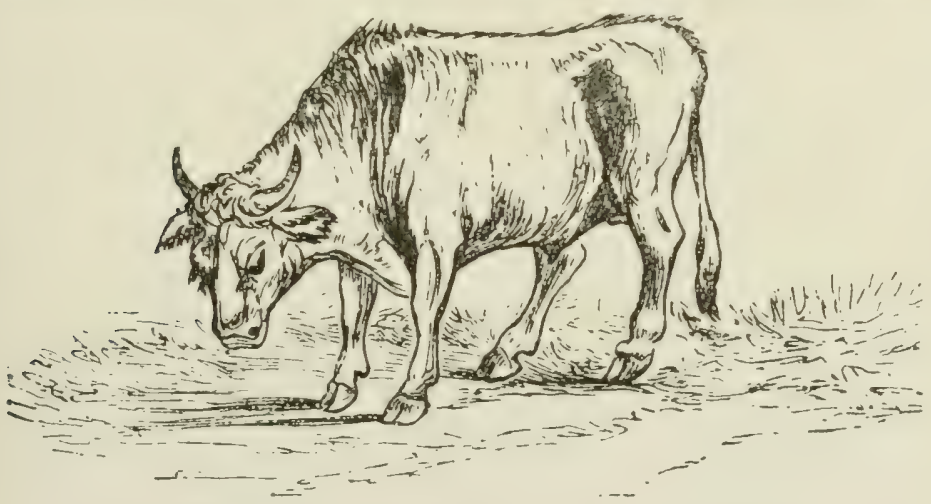

MMPACTION OF THE OMASTM, OL THIRD STOMACH.

woody fiber. Even soft, nutritious food, when thoroughly masticated, appears very dry when passing through the manifolds of the third stomach. "Consequently, when the food is innutritious, dry, possessing a vast amount of woody fiber, it is liable to become impacted while passing through this stomach. 
Symptoms:-At first the animal may be uneasy, but this is only of short duration, as it soon grows dull and stupid, resting its head on the manger or on other objects. The head is carried low down; appetite and rumination are suspended; if in a cow, the flow of milk decreases; the tongue protrudes from the mouth, eyes are staring and blood-shot; colicky pains arise; the tail is switched from side to side. The first stomach sometimes fills up with gas; then the breathing is accelerated. The eyes are affected and will bear the touch of the finger without closing the lid. Sometimes the brain is affected, when the animal will rush around as thounh it were mad, fall down and struggle as if in a fit, when death puts an end to its misery. Sometimes the dung passed while the animal is straining will be thin, and frequently blood and mucous are passed. The urine is high colored; pulse at first full and natural but gradually growing weaker. The disease may last but a day, or it may continue for a week.

Treatment:-Give a good, brisk cathartic, as the following:

Epsom Salts...........two pounds.

Calomel.............. two drachms.

Gentian................ Mene ounce. Mix.

Give in half a gallon of warm beer. Follow this up with hourly injections of warm water and castile soap, as long as deemed advisable. Allow all the water the animal will drink, but allow no food until the bowels are moved by the physic. If the first stomach bloats up, draw off the gas with a probang or trocar and canula. Rub the legs with some stimulant as mustard and alcohol. Blanket well to keep the animal warm and give the following stimulant every four hours, in a quart of warm beer: 
Aromatic Spirits of Ammonia....

two ounces.

Tincture- Nux Vomica...two drachms.

Tincture Gentian......... one ounce. Mix.

Never bleed in this disease as it is dangerous, even while the animal has a full pulse. Be careful after giving the first dose not to give any more physic for forty-eight hours. Then, if the bowels do not move, give half of the first dose.

\section{HIDE-BOUND.}

A great many diseases of the alimentary canal in cattle, cause great depletion in flesh, whereby the skin assumes an unhealthy appearance; the cellular tissue loses its elasticity, and to all appearance, the skin is fastened to the bones. Then the animal is said to be hide-bound, when, in reality, the discase exists in the alimentary canal, and the tightened appearance of the skin is only a symptom of some other affection, such as indigestion, diarrhœa, disease of the liver, or failure of the secretory glands in the intestines to perform their work. Some animals present a pincherl-up, poor, unthrifty appearance, without any teudency to take on flesh. Such animals may have some derangement of the system over which medicines would have no control, and may be classed as worthless, as you cannot make beef out of them. And they are useless in the dairy. The treatment should be to find out the real rause of the trouble, which is generally in the alimentary canal, and if this is properly treated, the skin will soon assume a healthy appearance. But I know of no disease among the lower animals which could properly be called hidebound. 
Treatment:- When it appears without any assignable ('aluse, disease of the digestive organs may be suspected and give as a mild physic-

Sulphate of soda........ one pound.

Powdered Gentian ....... one ounce. Mix.

Give at one dose in a gallon of water, to be followed up with-

Powdered Nux Vomica.... one ounce.

Powdered Gentian...... four ounces.

Powdered Foenugreck... four ounces.

Bi-Carbonate of Soda.... four ounces. Mix.

Give a dessertspoonful three times a day, in soft food.

\section{IMPACTION OF THE RUMEN, OR FIRST STOMACH.}

This affection is quite common among cattle. It is caused by cattle eating too large quantities of food which they may particularly like and of which pre-

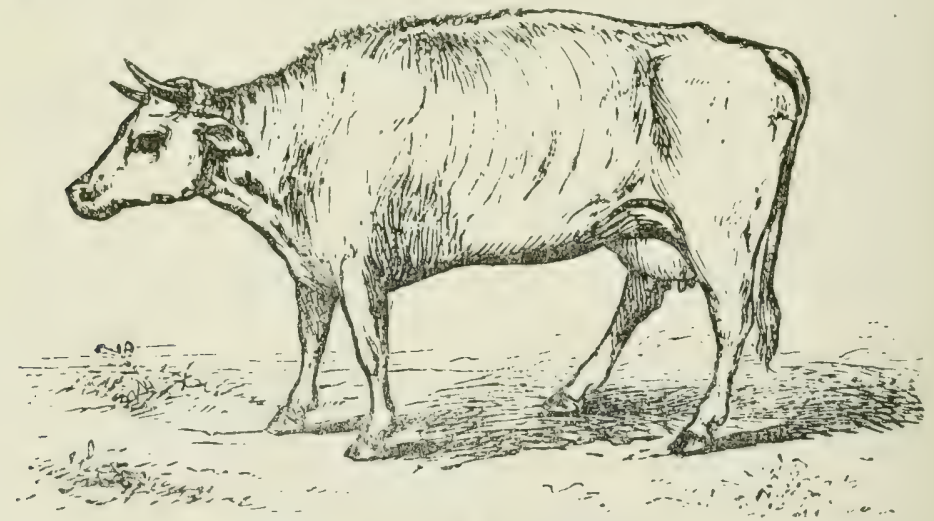

IMPACTION OF THE RUMEN.

viously they may have been deprived. Cattle are particularly fond of "lover, and if deprived of it for some time, and then obtaining free access to it, they are lia- 
ble to eat so much that the stomach becomes so gorged that the museular coats become partially laralyzed. Or cattle that have gained access to a large quantity of grain, will devour a large amount of it, werloading the stomach and causing impaction, with more or less tympanitis.

It is also caused when cattle are fed ujom innutritious food, or food containing a vast amount of woody fibre.

Symptoms:-Usually, at first, the symptoms are not severe. The animal will appear dull, and possibly, somewhat distressed; bowels constipated, appetite and rumination suspended, nose dry and mouth hot; pulse full at first but growing gradually faster and weaker; horns, ears and lecessow cold: labored respiration; sometimes the animal will groan or grunt, and the breath be fortid or sour; paleness of the risible mucous membranes; if lying down, the animal usually lies on the right side. By pressing the finmers on the left side the contents of the stomach will be felt like a hard mass within it and will pit upon pressure, leaving the indentions of the fincrers there for some time. ()in percussion the resonant sound is alsent, mless tympanitis supervenes; lut if no gas is present, the doughy feel of the stomach remains.

Treatment:-If there is tympanitis, relieve by giving the following:

Liquor Ammonia....... two drachms.

Tincture Ginger....... one-half ounce.

Water............... one quart. Mix.

Or, pass the hollow probang, or tap with the trocar and canula; after which give at one dose in a gallon of warm water the following:

Epsom Salts..... twenty-four ounces.

Ginger............... one ounce. Mix. 
Give repeated injections of castile soap and warm water, and every four hours give the following as a drench :

Aromatic Spirits of Ammonia ......

two ounces.

Tincture Gentian........ one ounce.

Warm Beer.............. one quart. Mix.

If the bowels fail to respond in from twenty-four to thirty-six hours, rumenotomy should be performed, as later the animal may become exhausted and unable to stand the operation. The operation should be performed as follows: First prepare yourself with the necessary instrumenti, which consist of a scalpel or an ordinary sharp jack-knife, two pairs of hooks, needles, white silk thread of the heariest kind, some clean, white linen cloths, a sponge and a pail full of clean, warm water. Next fasten the animal by ropes with the right side against the stall or fence; be sure to fasten it securely, so it cannot get away; have three or four assistants to hold it up and steady it. Commence the operation by making an incision through the skin midway between the last rib and the point of the hip, and about five inches from the hones which protrude from the hack (vertehra). The incision should he downward and about eight inches in length. Then divide the muscles successively and cut through the peritoneum. Lastly the walls of the stomach should be dirided about the center: first make a small opening into the stomach; then secure the edges of the stomach with hooks and gradually pull it outward, while the incision is being lengthened both upward and downward, but not to the extent of the external opening." The edges of the wound in the stomach should be drawn outward to cover the external wound so as not to allow any of the food to fall into the abdominal cavity, as 
PNEUMONIA, OR INFLAMMATION OF THE LUNGS. . 33 ?

that would surely cause the death of the patient. The hand is then introduced and about three-fourths of the contents of the stomach remored, leaving about onefourth hehind, be careful not to leave any hard, dry lumps. Iou may then pour into the stomach through the opening the following:

Epsom Salts............ten ounces.

Warm Beer............. one quart.

Warm Water............ one quart. Mix.

Next wash off the wound and sew up the stomach with the uninterrupted suture, bringing the edges neatly together. Then stitch up the muscles in layers, taking deep stitches with the uninterrupted sutures. Lastly sew up the skin in the same manner. Immediately after, apply the following ointment:

Vaseline............ four ounces.

Iodoform............ six drachms. Mix.

Continue its use until the wound is healed, or use some other healing lotion. The food should be light and soft, of the most nutritious kind, and given in small quantities. If the wound heals except a small opening, scarify its edges and stitch them together again.

\section{PNEUMONIA, OR INFLAMMATION OF THE LUNGS.}

Inflammation of the lungs may have various causes, such as exposure to cold and damp, being kept in filthy, ill ventilated stables, a morbid condition of the bloodlungs, in their endeavor to throw off its impurities, the becoming altered in tissue, with inflammation as the result; injuries etc.

Symptoms :-The disease is generally ushered in with a shivering chill, which is not always noticed, after which the animals appear dull and stiff. The skin is dry and harsh, coat staring, nose dry, mouth hot; pulse, 
at first full and strong-from fifty to sixty beats per minute-later becoming small, weak and rapid; mucous membranes are red; slight cough ; anxious countenance; breathing short, quick and chiefly abdominal, the chest and ribs being held, as much as possible, in a fixed position; bowels constipated; urine scant and high colored; patient lies down most of the time; dullness on percussion; on applying the ear to the sides of the chest no

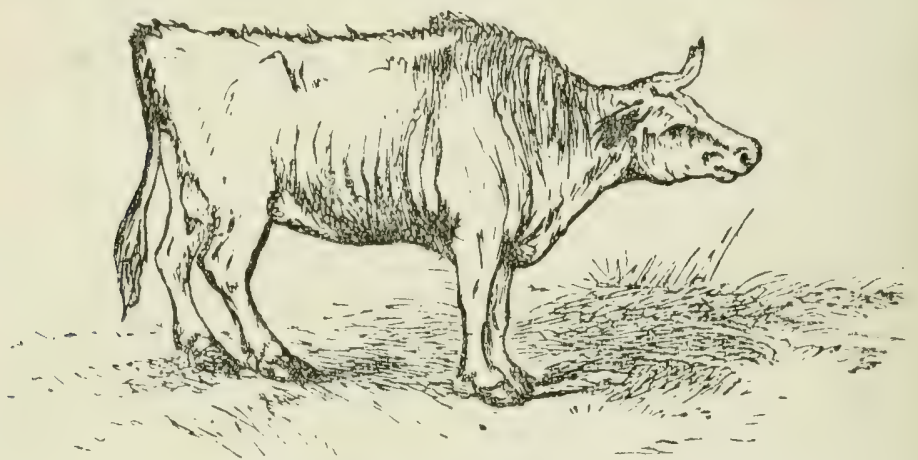

INFLAMMATION OF THE LUNGS.

respiratory murnur will be heard over the affected part, but the unaffected portion will be heard with increased force; as the disease advances the legs and ears hecome cold, the breath becomes foetid and shorter, and an unfavorable termination seems imminent.

Treatment:-When first seen while yet the pulse is full, bleed to the amount of two quarts; but, should the pulse be weak, do not hleed, and give every three or four hours, two ounces of the following, in a little water as a drench:

Liq. Ammonia Acetate.. twelve ounces.

Sweet Spirits of Niter..... four ounces.

Fluid Extract Aconite.... one drachm. Mix.

Also give the following powders every four or five hours: 
Quinine Sulphate......three drachms.

Dover's Powder........... one ounce. Mix.

Make into twelve powders. If the bowels are constipated give-

Epsom Salts..........twelve ounces.

Ginger..............ne-half ounce. Mix.

Give in two quarts of warm water. Apply mustard freely to the sides of the rhent; feed on soft food; blanket well: allow the patient to breathe cold, fresh air and give plenty of fresh, cold water; if the limbs become cold rub them thoroughly with dilute alcohol and bandage.

\section{CYSTITIS, OR INFLAMMATION OF THE BLADDER.}

Inflammation of the bladder is a very rare disease and is occasioned sometimes through diflicult parturition, or injuries from various sumrces. The mine of

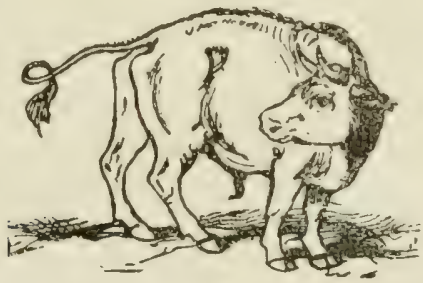

INFLAMMATION OF THE BLADDER.

cattle is alkaline, but from disturbed digestion and the injudicious use of drugs of various kinds, it sometimes becomes acid, thereby causing inflammation of the bladder.

Symptoms:-The animal exhibits great uneasiness. Colicky pains are apparent with efforts to void urine, and sometimes, to vomit. The head will frequently be turned toward the flanks and severe constitutional dis- 
turbance will be manifest. Occasionally, the urine will be voided frequently.

Treatment:-In this disease, it is always policy to examine the bladder and urethra carefully, to ascertain whether there be a calculus or stone causing these symp. toms, which may be dislodged or removed. If the bladder is distended with urine, pass the catheter and draw it away, after which give the following:

Raw Linseed Oil.........two ounces.

Or,

Epsom Salts.........twenty ounces.

Powdered Gentian.....three drachms. Mix.

Give at one dose in a gallon of water. Mustard plasters placed over the loins are beneficial, as are also injections thrown into or around the bladder, of the following:

Tincture of Opium....... four ounces.

Fluid Extract Belladonna. four ounces. Mix.

Put one ounce into a quart of warm water and inject every three or four hours. Flaxseed tea may be given freely. If pain continues give-

Liquid Ammonia Acetate.... one pint.

Fluid Extract Aconite...two drachms. Mix.

Give two ounces every three or four hours.

\section{EVERSION OF THE BLADDER.}

Among the cattle this is not a very common aftliction, except during difficult labor. However it may occur from other causes, as administration of powerful diuretics; use of too much cantharides, either internally or externally; any irritation of the bladder causing continuous straining. By violent attemps to pass water, followed by continuous straining, the vagina is ruptured 
and the bladder is everted, when it appears as a red, fluctuating tumor gradually filling with water, as it is unible to discharge its contents owing to the twisted condition of the neck of the bladder.

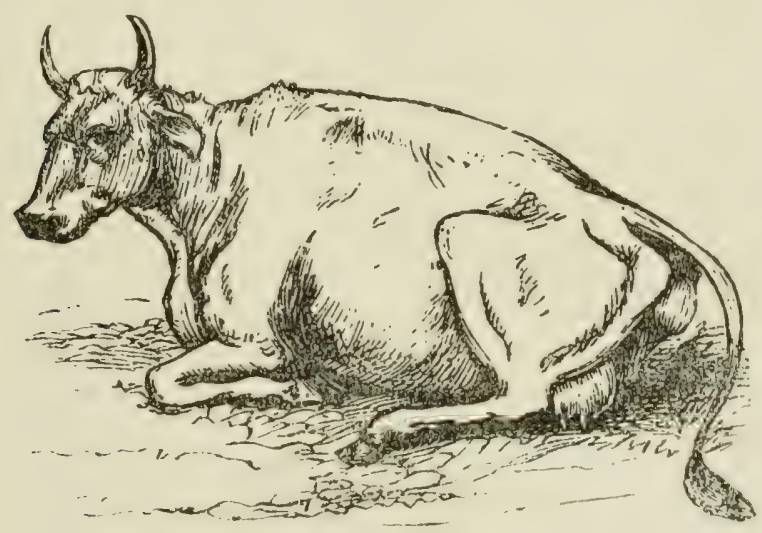

EVERSION OF 'THE BLADUER.

Treatment:-Oil the hands with sweet oil and carefully replace the bladder; then draw off the urine and, while the catheter remains in position, inject a decoction of opium and warm water. Then, if possible, stitch up the rupture in the vagina and allay the irritation and overcome the straining by giving drachn doses of powdered opium every two hours until relieved; or you may give twelve-grain doses of sulphate of morphia until relieved; afterwards give a physic:

Epsom Salts.........twelve ounces. Sulphate of Soda........ four ounces.

Ginger................ six drachms. Mix.

Give at one dose in a gallon of water. To assist in preventing the straining tie a rope or strap tightly around the body immediately over the loins. 


\section{EVERSION OF THE VAGINA.}

With cows this is quite a common occurrence and is the result of diflicult parturition, the animal receiving some injury therein; also is caused by tumors in the vagina or womb, struining after calving, retention of the placenta or afterbirth; swinging animals so the whole weight is upon the abdomen, ete.

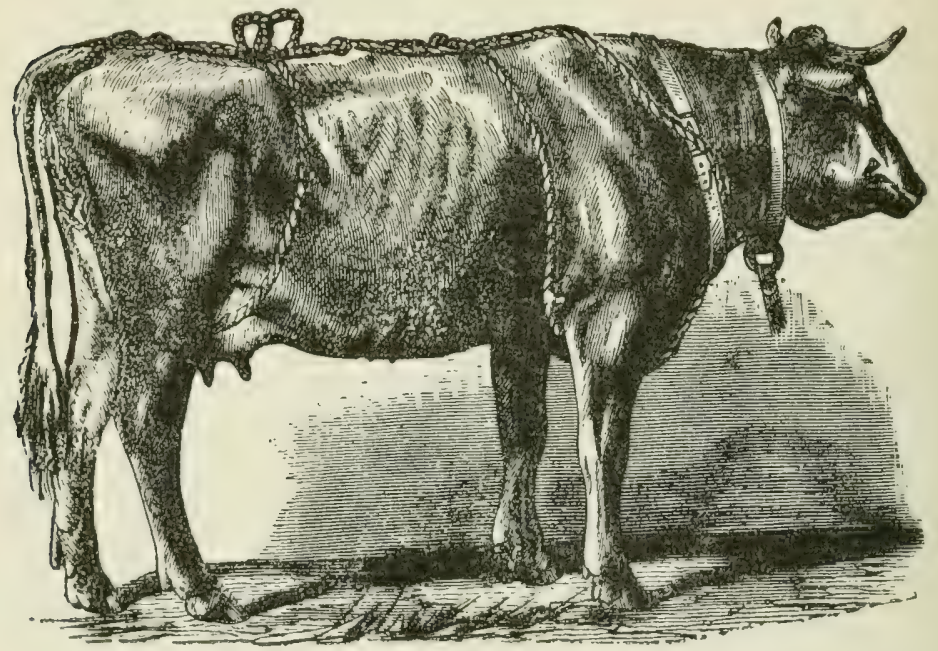

TRUSS TO PREVENT EVERSION OF TIIE WOAB.

Treatment:-First ascertain the cause and, if possible, remove it; then oil the hands with sweet oil and carefully press the vagina into position; then wash it thoroughly with--

Gum, or Powdered Opium.........

.................. drachm.

Hot Water............... one pint. Mix.

Place the animal in a stall, with the hind parts elevated fifteen or twenty inches above the front: Apply a truss made of rope, fasten it around the neck and 
bring the ropes straight down along the back; pass one down on each side of the tail to the udder, and on each side between the udder and hind leg; fetch it up past the flank and tie it just over the loins to the rope coming down the back. Then make a few circles around the body and tie the ends of the rope securely. Thus you have the animal harnessed safely so that it cannot strain any, as the back will be kept in a straight line. Inject the above preparation of opium into the vagina every three or four hours and give drachm doses of powdered opium every hour unt il the straining ceases. If the bowels become constipated, give a physic as-

Epsom Salts............. one pound.

Gentian................half ounce. Mix.

Give at one dose in a gallon of water, and feed on soft, nutritious food.

\section{DROPSY OF THE UTERUS, OR WOMB.}

Occasionally vast accumulations of serum or fluids, are found within the womb. This, at the present time is none too thoroughly understood, although various theories have been presented.

Symptoms :-The symptoms very much resemble those of pregnancy. The abdomen will begin to enlarge, and the owner usually considers her with calf, from the fact that the cow appears in a healthy condition, but as the period of gestation goes by without any signs of calving, the conclusion is soon reached that something is wrong. Upon examination the womb is found distended to a considerable extent and by pressure and manipulation, it will be found to contain a watery substance.

Treatment:-Open up the neck of the womb with the finger, if possible, and allow the fluid to escape. If that 
cannot be done, puncture with a trocar and canula; it will then escape. Allow the canula to remain until you have injected one of the following:

Tincture of Iodine........ one ounce.

Water................. Mix. Or,

Permanganate of Potassium.......

..................two drachms.

Water................two quarts. Mix.

Inject through the neck of the womb, if it can be opened. The injection may be used every other day, until it has been used five or six times, then if the fluids continue to form, the operation of allowing it to escape will have to be repeated, as often as occasion demands.

\section{LEUCORRHOEA, OR WHITES.}

Leucorrhoea consists of a chronic discharge of mucopurulent matter from the mucous membranes of the vagina, and, not infrequently, from those of the womb. Generally irritation of the organ followed by relaxation is the cause; but relaxation alone will produce it. Want of nutrition attended with debilitating influences is a frequent cause. Sometimes, to all appearance, an animal will be healthy and yet affected with leucorrhoea. On opening the lips of the vulva a sticky, whitish or yellowish matter will be seen; quite frequently it runs out of the vagina and accumulates on the tail and surrounding parts. As the disease progresses the discharge emits an offensive odor; pulse grows feeble; mucous membranes pale; appetite and rumination suspended; mouth clammy; gradual wasting of the whole system. Cattle affected with leucorrhoea are quite frequently seeking a male companion, but seldom get with calf, and if they do, generally abort. 
Treatment:-Feed on soft, nutritious diet with tonics and stimulants. Give twice daily two ounces of the following, diluted in water:

Aromatic Spirits of Ammonia ...... ................. four ounces.

Sweet Spirits of Niter..... five ounces.

Tincture Ginger......... five ounces.

Tincture Muriate of Iron.. two ounces.

Strychnia ............ two grains. Mix.

Use as an injertion per vaginmm twice daily one of the following powders, dissolved in a quart of warm water :

Permanganate of Potassium........

.................. one ounce.

Sulphate of Zine...... four drachms. Mix.

Make into eight powders; previously wash out the vagina and womb to remove all matter.

\section{PLEURISY.}

The term pleurisy is used to lenote an inflammation of the membrane covering the heart and lungs, which is also the lining membrane of the chest. Sometimes pleurisy occurs in pnemmonia or inflammation of the lungs, on account of the proximity of this membrane to the lung tissue. Sometimes it occurs as a separate and independent disease. The causes are exposure to wet and cold, filthy stables, sudden changes by being turned from warm stables into the cold, injuries to the chest, etc.

Symptoms :-The animal appears dull and stiff, without desire to walk around. Fever is present from the beginning of the attack. The breathing is chiefly abdominal, the chest being held, as far as possible, in a 
fixed position. The patient seldom coughs, as the act is very painful. The pulse is wiry, ranging about sixty beats per minute. The head is carried low with drooping ears. Appetite and rumination are suspended; nose dry; eyes about half closed. On applying the ear to

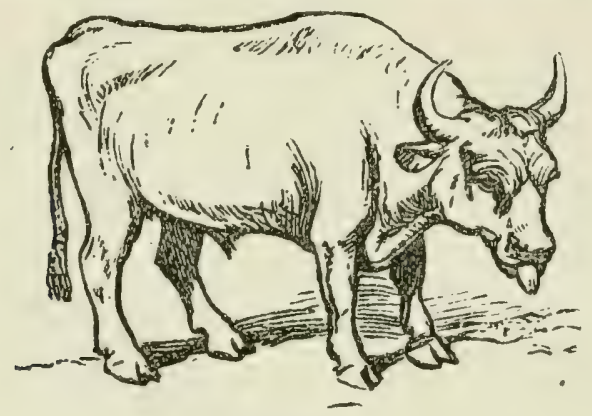

PLEURISY.

the part affected, a grating sound is heard, although the lungs give forth a healthy respiratory murmur; pressure between the ribs causes great pain, sometimes causing the animal to groan or grunt. While breathing, inspiration is short, becomes painful, while the expiration is more easily performed and therefore lengthened.

Treatment:-Put the animal in a good, comfortable place and blanket well. Apply poultices of linseed meal to the chest; in its absence, poultice freely with mustard. Give every two hours, two ounces of the following mixture :

Liquid Ammonia Acetate......... ................twelve ounces.

Sweet Spirits of Niter.... four ounces.

Fluid Extract Aconite...two drachms. Mix.

Regulate the bowels by giving a pint and a half of raw linseed oil, and injections per rectum, of warm water, every two hours. Also give one of the following powders every six hours : 
Nitrate of Potassium.....two ounces.

Chlorate of Potassium..... one ounce.

Iodide of Potassium....... one ounce. Mix.

Make into twelve powders. If it terminates in resolution the disease will last but a few days; but if it terminates in hydrothorax, or water in the chest, it may last some considerable time and require a different treatment. If there is much effusion into the chest, it should be drawn off with a trocar and canula. The place to pass the trocar and canula is between the eighth and ninth ribs; after they are inserted withdraw the trocar and allow the canula to remain until all the fluid runs out. Sometimes bands of lymph plug up the canula; these should be removed with a long needle. After the fluid has all drained out, remove the canula. Then administer tonics and stimulants to support the system. Give every six hours one of the following powders:

Powdered Digitalis..... six drachms.

Saltpeter... one and one-half ounces.

Sulphate of Iron.........two ounces.

Powdered Nux Vomica..... one ounce. Mix.

Make eight powders. Give in a pint of warm beer. Also give twice a day, drachm doses of iodide of potassium, with four ounces of brandy and a little water. Feed on soft, nutritious diet.

\section{SORE TEATS.}

Around the teats some cows are more tender than others. The skin is tender and easily cracked, forminc open and indolent sores which are hard to heal. These sores may occur at any season of the year and are most commonly seen on cows which have just calved. 
(nw-pox is liahle to break out on the teats at any time, and to cause considerable annoyance. It first forms a round blister which bursts and discharges a bluish liquid. It then scabs over with a thick crust which cracks open during milking, or comes loose' around the edges; or comes ofl altorether, when it leaves a small

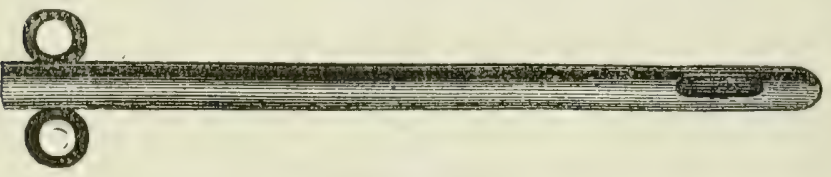

TEAT SIPHON.

depression in the structure of the teat. It gires consid erable annoyance to the milkman, as the sores frequently bleed and his hands become covered with matter, and blood frequently gets into the milk. When the teats are in this condition calves should not be allowed to suck, as it aggravates the sores and tends to make the cows professional kickers. The following ointment will be found to be very beneficial, especially during the season of flies:

Vaseline ............ two ounces.

Carbolic Acid........... one drachm.

Iodoform............ two drachms. Mix.

Apply twice daily; or,

Vaseline............. two ounces.

Salicylic Acid........... one drachm.

Iodoform ............. one drachm. Mix.

Apply twice daily. Great care should be exercised in milking such animals, and if badly affected the teat siphon should be used.

\section{INJURIES AND OBSTRUCTION IN THE TEATS.}

There are many ways in which the teats become injured, causing an obstruction to the flow of milk or a 
complete stoppage, giving rise to inflammation of the udder. The ends of the teats frequently become injured from barbed wire, or from warts forming there, and often from the annoyance of warts in milking, the milkman seizes them and pulls them off, thus causing an abrasion of the skin, at or in the channel through which the milk flows, and as the healing process takes place, the channel grows together, shutting off the flow of milk from that quarter of the bag. When this condition is noticed, the teat siphon should be forced through the channel and kept in position, if possible, until the wound is healed. If the siphon cannot be kept in this position, it should be forced through three or four times a day, until the wound is healed and the opening remains.

Another and more frequent cause of ohstruction is ohserved in the small tumors that sometimes form in the teats, varying in size from a pea to a good sized bean. They are generally morable. Various methods have been employed for their removal; sometimes they can be hroken up with a knitting needle, or pass a shielded bistoury up the teat and cut them out, but neither of these methods have proved satisfactory to me. The most successful way to manage them, is to fasten the cow and cut down directly onto the tumor, then dissect it out, bring the wound together with a few stitches, and use the following:

Taseline ................................

Iodoform............ four drachms. Mix.

Apply twice daily, then cover with cotton batting and bandage lightly and loosely. Draw away the milk with a teat siphon for a few days, and all will be well. Sometimes strictures exists in the passage of the teat and greatly diminish the flow of milk. When such a condition is observed, pass a smooth blunt instrument, 
as a knitting needle or a teat siphon, up the teat, two or three times a day, gradually increasing the size, until the stricture disappears. Occasionally we find in fresh milch cows, the passage up close to the udder, cemented over, so as to allow no milk whatever, to enter the teat. In such cases, pass the teat siphon up until you come to the obstruction, then force it through and rotate it, to break the obstruction away, and there will afterwards be but little trouble.

\section{COW-POX.}

Every case of eruption or sores upon the udder and teats of cows, should not lead the casual observer to conclude that it is a case of genuine cow-pox. Indeed, cow-pox seems to be a disease originating spontaneously with, and confined to milking cows, as bulls are not subsubject to it and neither are heifers, until they have undergone the change in life and become mothers. Cow-pox is infectious, and it may be communicated to man through inoculation, after which, if he be susceptible to small-pox, it will only be in the mildest form.

Symptoms :-At first there may be some fever, which, as a rule, will scarcely be noticed, but which will be followed in a few days by an eruption upon the udder and teats consisting of small, red spots, which are painful and hot. The spots at first are small, but within a few days, contain a bluish liquid that soon becomes converted into a yellowish matter. By the process of milking, these spots, which have formed pus tubes, become broken and discharge their contents, and if not broken that way, will burst spontaneously, after which a scab forms and in the course of ten or twelve days drops off, leaving the teat somewhat tender for a short space of time. During the eruptive and scabby stage, 
the milk should be drawn with the teat siphon, to save yain and trouble, and the attendant should not milk other cows.

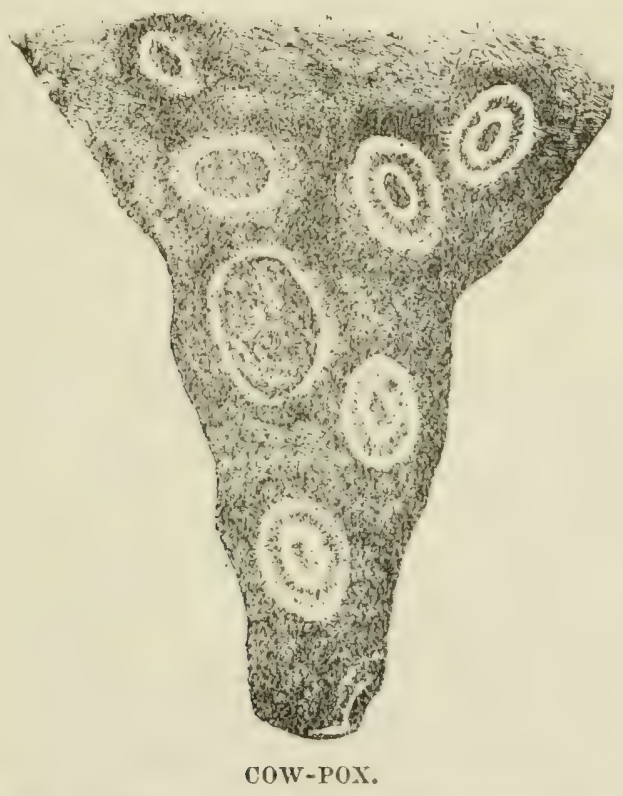

Treatment:-The treatment is very simple. If in the winter, feed on soft food and give plenty of fresh, cold water; should the udder become swollen, painful and hard, foment well with hot water and poultice thoroughly with flaxseed or bran. It is rery seldom that any treatment is needed.

\section{MAMMITIS, OR INFLAMMATION OF THE UDDER.}

Infiammation of the udder arises from many causes, such as bruises, wounds, bites of insects and reptiles: allowing the animal to go umilied for a day or so: 
taking eold; rhemmatism and other fevers; being overheated: allowing a superabundance of milk to remain in the udder previous to calving. The last named is the most frequent cause among cattle of all ages, but especially with young cows which are having their first calf.

Symptoms :- Inflammation of the udder may exist in a mild form for a few days and then subside, without any alarining symptoms beyond a slight increase in heat around the affected parts. But with the severe form, there will be considerable fever and dulness. The animal will have a capricious appetite and rumination he partially suspended; bowels slightly constipated; the udder becomes very hot, is swollen and tender; milk will be decreased and sometimes totally suspended; skin covering the udder is very red and slistening; if only one or two quarters of the bag are affected, they will appear and feel like hard tumors and may continue swollen for some time. The operation of milking is performed with difficulty on account of the painful condition of the udder. The milk from the affected quarters will be thick and curd-like and frequently streaked with blood. If not relieved suppuration takes place within the udder, the hardened parts becoming soft and discharging matter during the operation of milking; or the affected quarter may point and break externally, in which case its usefulness is generally gone forever.

Treatment:-If properly attended to when first noticed, mammitis will gonerally result satisfactorily. But if left unattended for some time the opposite state of affairs is certain. Put the animal in a good, dry, comfortable place, free from drafts, and administer in a gallon of warm water, the following:

Epsom Salts.............. one pound. Ginger................ one ounce. Nitrate of Potassium.... two drachms. Mix. 
Afterwards apply to the udder, for two hours at a time, fomentations of hot water; to each gallon of water used, add four ounces of powdered sulphate of iron. Immediately after bathing apply a hop poultice as hot as can be borne; or a poultice of fried onions will do as

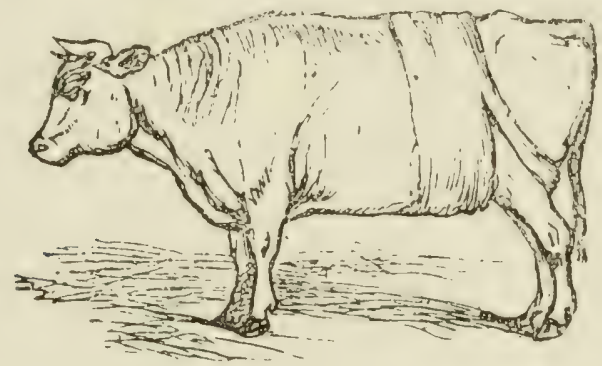

BANDAGE FOR THF SUPPORT OF THE UDDER.

well. Use a broad bandage and tie it up around the back to support the bag. If the animal shows signs of high fever, administer the following draught, every four or five hours :

Liquid Ammonia Acetate. four ounces.

Fluid Extract Aconite... fifteen drops.

Sweet Spirits Niter....... one ounce.

Water.................. one quart. Mix.

Milk the patient frequently, but if it causes much pain, insert the teat siphon. Occasionally rubbing the lang with fluid extract belladonna is frequently benefirial. If the udder still remains hard, the following lotion will be found of benefit:

Rectified Spirits...........six ounces.

Witchhazel.......... eight ounces.

Spirits of Camphor.......two ounces. Mix.

Apply three times a day. This can be used from the commencement of the attack until the end. If mortification sets in, cut out that quarter of the bag and dress with the following: 
Vaseline.............. four ounces.

Iodoform............ five drachms. Mix.

Apply thrice daily; or,

Chloride of Zine........ two drachms.

Water................. one quart. Mix.

Apply thrice daily; or,

Listerine.............. six ounces.

Carbolic Acid ........ two drachms.

Water................. one pint. Mix.

Apply frequentiy. Feed on soft, nutritious diet and keep as clean as possible.

\section{SPLENIC APOPLEXY.}

This disease seldom occurs, except among cattle which are fed for beef and fattened rapidly. It consists: of a poisonous ferment created within the blood itself, during an unnaturally plethoric condition of the system and it is demonstrated by the extravasation of blood throughout the intestinal canal, brain, serous membrane, pericardium, ete., as found in post morteli examination. That it is poisonous is shown by the fact that dogs and pigs have died within a few hours after eating the flesh, and that men have died from cutting themselves while skimning animals dead from this disease.

Symptoms :- Great excitement, eyes staring, mucous membraner red, mouth hot and dry, urine and manure mixed with blood, colicky pains, breathing accelerated and short: pulse, from full and hard becomes quick and feeble and almost imperceptible. These symptoms ar" not always observed, as the disease is of such short duration; the animal may be left to all appearance perfectly well in the evening and found dead in the morning. 
Treatment:- When seen in the first stitge. wive immerdiately the following:

Epsom Salts........... sixteen ounces.

Glauber Salts........twelve ounces.

Calomel............. two drachms.

Ginger............... one ounce.

Warm Beer, two quarts; or Warm

Water............... one gallon. Mix.

Constantly apply cold water or ice to the head and give every two hours the following:

Carbonate of Ammonia..two drachms.

Gingrer.............. one drachm.

Beer............... one quart. Mix.

Bleeding is sometimes beneficial.

\section{STRANGULATION OF THE INTESTINES, OR “GUT-TIE."}

This disease is seen only in steers and work oxen. When the animal is castrated, the spermatic cord recedes and becomes adherent to the abdominal ring. The cord is united to the pelvic bones by a fold of peritoneum, which by pressure is sometimes ruptured and allows the gut to pass in and out and thus hecome strangulated.

Symptoms:-The animal appears dull; colicky pains are present; appetite and rumination suspented; frequent straining with great effort, but nothing passed save a little mucous and possibly blood; pains become more violent and the animal kicks at its belly with the hind feet, lies down and quickly rises again; pulse small and quick; the animal joints its nose to the affected side; moaning and crinding of the teeth frecuently occur; pressure on the right side causes great pain; the 
back is curved downward: administration of purgatives produces alarming symptoms.

Treatment:-The only sure treatment is by operating. Place the animal with its left side against a stall and fasten there securely. Make an incision in the right side, large enough to adnit the hand. Pass the hand down to examine the abdominal rings and trace up the cord. When you come to the strangulation, sever the adhesions of the cord and the intestine is then liberated. Draw out the cord and cut off two or three inches, then return it, and treat as an ordinary wound.

\section{WARBLES, OR GRUBS UNDER THE SKIN.}

This is really no discase but simply the manner in which an insect, called the ostrus bovis, or gad fly,

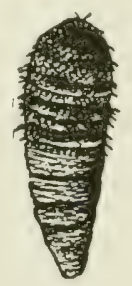

GRUB FROM COW'S BACK.

develops itself. During the warm months of summer, this insect flies ahout and attacks cattle, hy piercing the skin and depositing an egg in the orifice. In the course of time a swelling is observed, caused by the deposition of lymph and the development of the larva from the egg, which is in time discharged by the process of suppuration. It may fall upon the ground or in the stable. If the temperature is sufficiently warm to develop it, it puts forth wings and soon bewins to deposit eggs for the continuance of its race in the manner 
already described. Sometimes there are a number of these tumors that acquire the size of a butternut, and suppurate and discharce to such a derree that they call

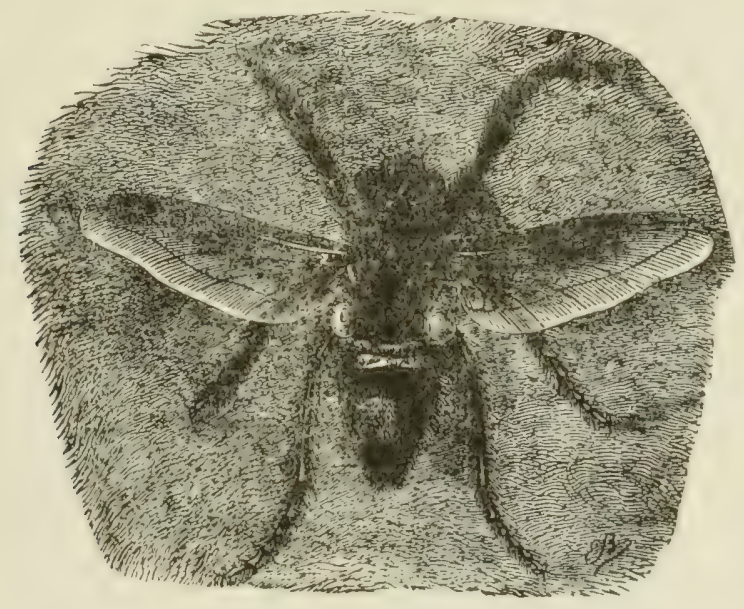

CRTRUS BOVIS, OR GAD FIY.

for treatment. This consists of pressing them out and using some mild astringent lotion: the following will suffice :

Zine Sulphate.........two drachms.

Acetate of Lead........two drachms.

Water...............ten ounces. Mix. Apply twice daily.

\section{LICE.}

Cattle are affected by several kinds of lice, but thuse chiefly mot with are the small, red lice and the large gray ones which have black spots on their backs and sides. They are most frequently seen on poor, debilitated cattle that are poorly fed and cared for. Their 
propagation is very rapid; when an animal hecomes infested, they are soon numbered by thousanris and the whole herd is speedily possessed of them. They may be speedily exterminated by the use of some of the following remedies: Take one pound of stavesacre seeds, soft water two gallons; place over the fire and simmer down to one gallon. Sponge the animal all over and repeat every third day until three applications have been made. Or, take leaf tobacco one pound, water two gallons; boil thoroughly and apply every other day, for four or five times. Or, take blue ointment and rub it back of the horns and down the back every thind day, for three applications; this is the most dangerous and the animal must be kept tied so it cannot lick itself; also protect it from storms. If the stavesacre :an be obtained, it will be foum to be the most effectivo.

\section{RINGWORM.}

Ringworm in cattle, is an aflection that seldom calls sorth much attention. It is uswally seen in poorly fed

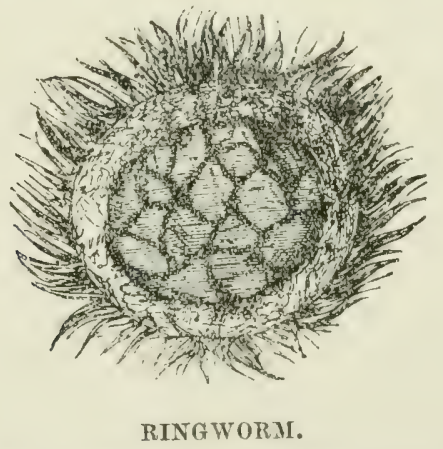

rattle, although it sometimes makes its appearance on cattle in good condition. It is caused by a small parasite and is somewhat contagious. 
Symptoms:-The first symptoms observed, are rouml patches about the size of a nickle, which sometimes coalesce, but the well-defined ring will be observed. They generally appcar around the head, neck, eyes and nose and sometimes on the shoulders, loins, hips an? udder.

Treatment :-If the animal is in poor condition, feel on good, nutritious food and remove from other cattle. As cleanliness is very necessary, bathe with soft soap and warn water, after which use one of the following prescriptions to kill the parasite:

Nitrate of Silver........ drene drachm.

Water.............. four ounces. Mix.

Apply every day for three or four days. $O \mathbf{y}$,

Chloride of Zinc....... four drachms.

Soft Water........... four ounces. Mix.

Apply once daily for three or four days. Or, paint the parts thoroughly with tincture of iodine, several times; or, apply accotic acid morning and erening for a few days. If the ringworm is close to the eyes be rery careful not to let any of the merlicine get into them.

\section{WARTS.}

Every person that is familiar with the care and handling of stock, has noticed these excrescences which make their appearance on any part of the body, but most frequently about the eyes, mouth, udder and belly. Warts are a skin disease in every sense of the word and do. not affect the blood in any way, hence constitutional treatment is useless. They vary greatly in size, from a small pea to a large goblet, and are best removed with the knife. Cut them oft beneath the skin and apply 
either chloride of zinc or corrosive sublimate in powder, to the raw surface, and they are not liable to grow again.

\section{WORMS.}

Cattle suffer more or less from worms, which accumulate in the intestines. Those most commonly seen are the large found worms, called lumbricoides, although tape-worm is occasionally discovered in cattle.

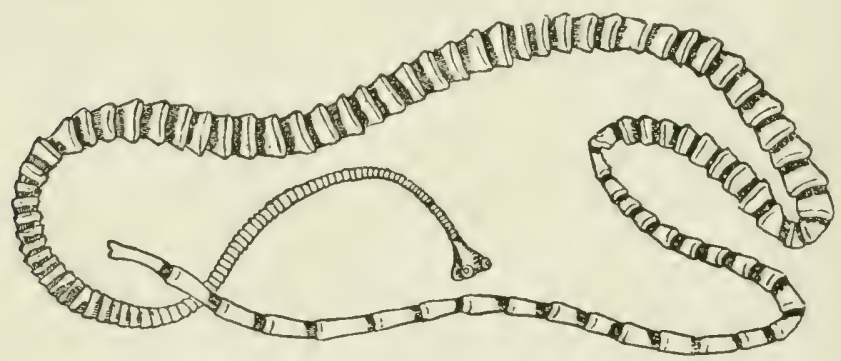

TAPEWORM.

Symptoms :-One of the most prominent symptoins, is the finding of worms or links, if tape-worm is suspected, in the manure, also an irregular or ravenous appetite. There is a falling off in condition, general weakness, with a hide-lound appearance, and sometimes diarrhœea with colicky pains, is observed.

Treatment:-If tape-worm is present, keep both food and water away from the patient for twenty-four hours, then give two quarts of pumpkin seed tea, and within two hours after, give the following:

Epsom Salts............. one pound.

Gentian............ four drachms. Mix.

Give at one dose in a gallon of warm water. If aflicted with other kinds of worms, feed on soft food, 
as bran mash, etc., for a few days, then give the following before you feed in the morning:

Linseed Oil.............. two pints.

Oil of Turpentine........two ounces.

Oil of Male Shield Fern....half ounce. Mix.

Give at one dose. If one dose iails to remove them, repeat in five or six days.

\section{TETANUS, OR LOCKJAW.}

This disease is not so common among cattle as it is among horses, but is to be worse dreaded as it is more often fatal. It is one of those nervous disorders that. are none too thoroughly understood. There are two kinds of tetanus, trammatic and idiopathic. The former

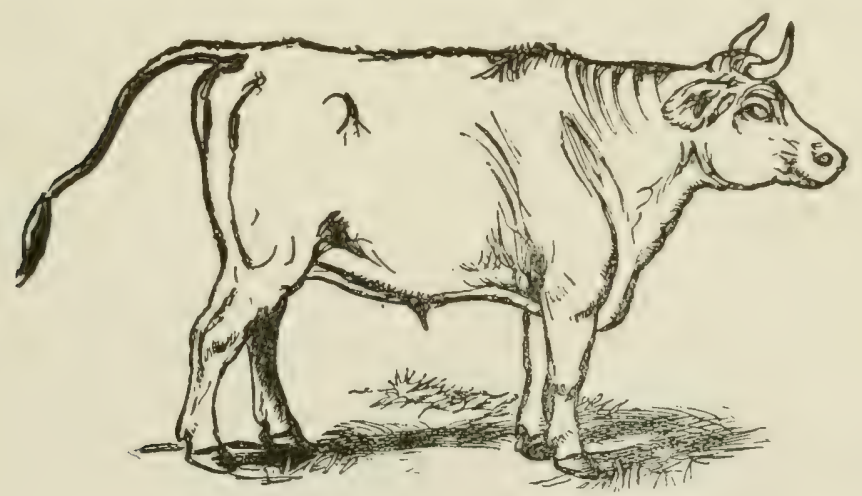

IOCKJAIT.

wiginates from wounds, pricks of nails, castration, or a skin abrasion from surgical operations, etc. The latter is of obscure origin and may he produced from exposure to cold, irritation of the bowels from drastic cathartics, from fright, etc. But from whatever source, the symptoms are about the same. 
Symptoms:-At first the casual observer might think there was nothing specially wrong, except that the animal was lacking in appetits: but the symptoms gradually increase in force and intensity and it will then be noticed that the animal becomess somewhat stifl, with the nose protruding. The musles along the neck and back become rigid and stifl, the tail is slightly elevated, the limbs rrow stiff and act like props for the body; the mouth is closed, but there may be slight motion of the jaws. There is partial, and sometimes complete inability to swallow and saliva will drivel from the mouth. There will be grinding of the teeth, and if the head is elevated, the haw or membrana nictitans will shoot out over the eyes. Sometimes the patient will appear hungry and make attempts to eat but will only suck the food after it has gotten it within the mouth. At all times, the animal is very easily frightened. There is usually more or less fever, sometimes reaching $107^{\circ}$ or $108^{\circ}$ Fahrenheit. If compelled to move, it will do so with a straggling, staggering gait.

Treatment:-First ascertain the cause, if possible, and if from the prick of a nail, pare the wound to the bottom and fill it with solid extract belladonns. Or if from a wound of any other kind, apply the belladonna frequently. Put the animal in a comfortable place and hed well, as cattle frequently lie down during this disease. Give a brisk physic, if the animal can swallow, as-

Sulphate of Soda......twelve ounces..

Epsom Salts.......... eight ounces. Mix.

Give at one dose in two quarts of water, or give-

Epsom Salts........... sixteen ounces.

Barbadoes Aloes......... one ounce. Mix.

Give in two quarts of water, after which give- 
Bromide of Potassium..three drachms.

Chloral Hydrate........two drachms.

Water................. one pint. Mix.

Give three times a day or place drachm doses of solid extract belladonna back between the teeth or on the tongue, three times a day. It will gradually become dissolved and taken into the system. Apply no irritating medicines whatever, such as blisters, etc., as they do more harm than good. Allow them to suck at water and gruel as much as possible, to maintain their strength, and one thing above all others is to be impressed upon the mind of the attendant, that is, to leep the animal isolated and in a dark stall, no yerson to go near it but the attendant himself, and then only to give medicine and food. Approach very carefully, as fright will cause a relapse. Some cases, when isolated in a dark stall and left alone, free from noise and fright, will recover without the use of any medicine whatever.

\section{BLACK LEG, OR QUARTER-ILL.}

This disease is rarely seen in the eastern, middle, or southern states but is frequently seen in the west. It is seldom ever seen in animals over three years of age, but is observed occasionally, in animals beyond this age. It is purely a blood disease and is caused from too high feeding of animals, taken from a half-starved or poor condition. The reason it is most frequently seen in the western states, is because of the immense herds in that section receiving a scanty amount of provision, during some of the severe winters. They are-consequently very thin in the spring, and when turned upon the rich, nutritious buflalo and bunch grasses, they thrive so rapidly that a sudden change in the quality of the blood 
takes place, having a great tendency to decomposition. Although called black leg, it must not be taken for granted that the limbs are the only parts that become aflected, for the seat of the disease, while purely a blood affection, will he noticed at the point of the shoulders, the neck, back, loins, tongue and thighs.

Symptoms:-The symptoms in the first stages are seldom noticed, but when observed, the eyes will look bloodshot, the urine will be highly colored, costiveness, with blood streaks in the manure; a full, quick pulse and hurried breathing; then if in the quarter, the animal will appear lame, the quarter will commence to swell, and by presing the hand down over the swelling, it will emit a crackling sound as though there was air beneath the skin. If located in some other part of the body, there may not be any lameness, lut the crackling sound beneath the skin, when pressed upon, will be apparent. This disease runs its course very rapidly, seldom lasting twenty-four hours and frequently terminating fatally within twelve hours.

Treatment:-If seen in the early stages, abstract two gallons of blood and give the following physic:

Epsom Salts.......... sixteen ounces.

Sulphate of Soda....... eight ounces.

Powdered Ginger........ two drachms. Mix.

To be given at one dose in two gallons of water. Plunge a knife deeply into the swellings, in several places, and pour turpentine into the wounds. Give the following stimulant:

Sweet Spirits Niter........ six ounces. Aromatic Spirits of Ammonia ...... one ounce.

Tincture Ginger.......... one ounce. Mix. 
Give two ounces every two hours in a pint of water or beer. Should a recovery take place, and the ulcerations prove troublesome, apply

Chloride of Vine....... two drachms.

Sulphate of (Copper..... four drachms.

Water................ one pint. Mix.

Apply twire daily. Attention should be directed toward the prevention of further attacks among the herd. As it is not contagious, no fears need be entertained from that source. Insert a seaton into the brisket below the neck and just in front of the forelegs. Keep the cattle in yards at niwht where no food can be had, and allow only moderate feeding in the daytime, until they become habituated to the rich, succulent food or pasture.

\section{RHEUMATISM.}

Rhematic lameness in rattle is quite frequently observed, and is one of those diseases that is none too thoroughly understood. It attacks the muscles, tendons and fibrous structures around the joints, as also the heart, bowels, bones, lungs, or any other part of the animal. There is a specific condition of the blood, rausing constitutional disturhance. It is undoubtedly hereditary with some animals, as is observed by followinc down through certain families. It also arises from other sources, such as being poorly kept, and exposed to cold and storms in winter; from exposure to the cold rains in the spring of the year, by housing in damp, wet, filthy stables, etc.

Symptoms:-In the acute form, the first signs are general uneasiness and stiflness, staring coat, appetite diminished, rumination partially or completely suspended, the bowels constipated and the urine high col- 
ored. Suddenly one of the joints will become swollen, hot and tender and probably the following day that joint will apparently be in healthy condition and another joint affected, and so continue to clange about from joint to joint, or to the heart, lungs, bowels, bones, muscles, ete. When rheumatism assumes a chronic form which we most often see in cattle, it remains stationary in whatever part affected, as in a joint or whole limb. When in a joint, as in the hock, it becomes swollen, hot, .hard, very sensitive to the touch and causes intense pain. As time passes by, bone material is formed and the joint becomes solid. In other cases, the tissues above and helow the joint, become swollen, hot and painful. Abscesses form and discharge matter. There is continued fever and loss of flesh. The animal will lie down a good portion of the time.

Treatment:-Move the bowels with the following laxative :

Epsom Salts............. one pound.

Ginger............. four drachms. Mix.

Give in a gallon of water. Next attend to the joints. Cold applications are not desirable in swellings of this nature, but warm applications are always soothing and prove beneficial. Cloths wrung out of hot water and applied to the swollen joints, give relief. Heated oats or salt sacks applied, are also beneficial, or use the following liniment:

Olive Oil............ four ounces.

Spirits of Turpentine......two ounces.

Aqua Ammonia.........two ounces. Mix.

Apply two or three times a day.

Or,

Soap Liniment......... four ounces.

Oil of Turpentine....... four ounces.

Laudanum............ two ounces. Mix. 
Apply two or three times a day. If the pain is intense, give ounce doses of laudanum until rest is obtained, to be followed with-

Quinine..............six drachms.

Salicylate of Soda.......... one ounce. Mix.

Make nine powders. Give three powders each day. Or,

Sulphate of Potash........ two ounces.

Sulphuric Acid.......... one drachm.

Water.................. one pint. Mix.

Give as one dose, morning and evening. Bed well and hlanket the animal, and arrange to keep cold draughts from striking it. Feed anything it will eat, but soft food is preferable, as hran mashes, hoiled oats or corn, clover hay, green grass, etc. While the foregoing are worthy of trial, all may prove a failure; if a favorable termination lias resulted, immediately fatten for the butcher, as a recurrence may be expected.

\section{DE-HORNING.}

As horned cattle frequently do considerable harm by goring other animals, it is just and proper to deprive them of these weapons of defense. It also makes them more docile while feeding. The operation is very simple; first confine the animal securely, then take a fine, sharp saw and saw off the horns close up to the head, taking off a small portion of the skin where the horn and skin come together; then put on some pine tar and it will quickly grow over. Have no fears about the bleeding, as it will do no harm. When raising calves, the horns can be prevented from growing by cutting off the hair over where the horns start to grow; moisten the parts a little and pencil with caustic potash. Do this when the calf is two or three weeks old. Two applications will suffice. 


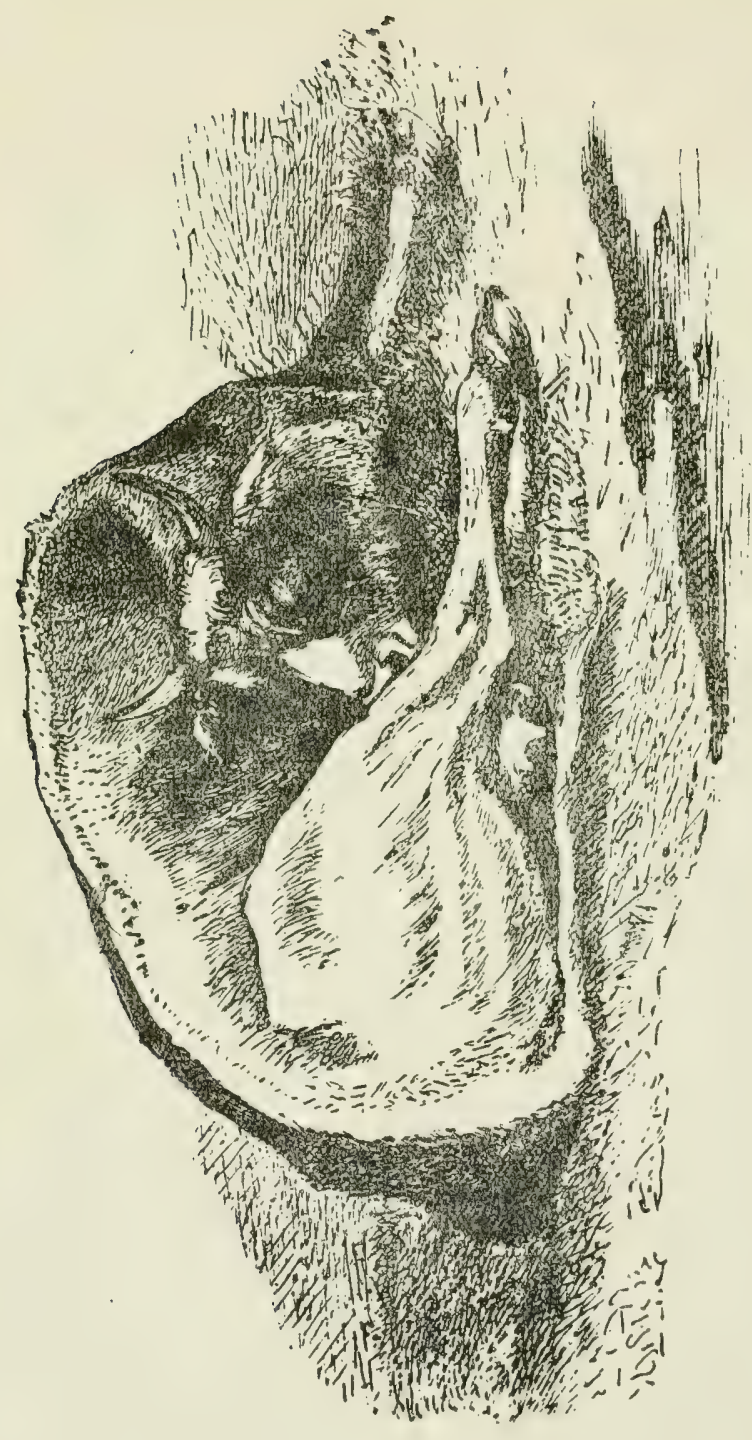




\section{OBSTETRICS}

WND

\section{DISEASES OF THE GENERATIVE ORGANS IN THE MARE AND COW,}

CARE OF FOALS AND CALVES.

\section{STERILITY-BARREN HORSES AND CATTLE.}

The loss to farmers, as well as to owners of stallions and bulls, on account of sterility, is sometimes enormous. Sterility exists both in males and females. If a number of females are hred to a certain male animal and fail to conceive, there is something wrong with the male and he is considered sterile. Again, certain females may be bred to a number of male animals, without conceiving, then the fault is with the female and she is considered barren. In the male, sterility may be due to many causes, as hardening of the testicles, the result of inflammation; softening of the testicles from being pampered, and liberally fed upon starchy food without sufficient exercise; imperfect dovelopment of the testicles, as in the case of ridglings. Change of climate has sometimes a marked influence on the generative ormans, at times only for a season and again it renders them wholly sterile. Self-abuse, continued for a length of time, also renders animals incapable of impregnating females. There are also several diseases of the generative organs, which tend to pro- 
duce sterility, as inflammation of the membrane covering the penis, ulcerations on the penis, warts on the end of the penis, paralysis of the penis, tumors affecting either the testicles or penis, tumors affecting the excretory ducts of the testicles, enlarged prostrate glands, injuries or disense of the back and loins rendering the animal unable to mount; serving too many females within a few days, will sometimes render the male sterile for the balance of the season. Sterility in the female, has also a variety of causes. It results from imperfect development of the ovaries; changes from one climate to another, will sometimes render females barren; closure of the neck of the womb by inflammatory adhesions; by the male during the act of copulation, exciting and irritating the womb, causing straining and ejection of the semen; catarh of the womb followed by a discharge of mucous; leucomhor or whites, a fatty condition of the ovaries in females that have been pampered, highly fed and are very fat and not receiving enough exercise; tumors in the ovaries or fallopian tubes; low condition, with imperfect nutrition of the ovum; poor feeding and chronic weakening diseases; from mares being advanced in years when bred for the first time. All these conditions should be met and attended to, if the owner wishes to breed and increase the quantity of his live stock. If from imperfect development of the testicles, as in a ridgling, castrate at once and convert into a good work horse. When there is softening of the testicles. from being too fat and fed upon starchy food, give plenty of exercise or work, and avoid feeding corn, wheat, or other starchy food, and feed oats, bran mash, cottonseed cake, etc. When there is hardening of the testicles, grease them with a weak iodine ointment, to which should be added some camphor and extract of bella- 
donna. Where self-abuse has been continued for a considerable length of time, castration is the most profitable method to pursue. Ulcerations of the penis, are best combatted by filling the ulcers with calomel, a few times. Warts should be extirpated with the knife and afterwards touched with lunar caustic or acetic acid. Too much stress cannot be placed upon the evil of serving too many females in a short space of time. One service a day, is all that should be allowed during the season. Two services may be permitted in one day, by allowing five or six hours to elapse between each service, but this should not be continued daily. The indiscriminate and injudicious methods practiced by some owners of sires by allowing four, five and sometimes as high as eight services in a single day, is very liable to cause at weakness in the testicles, so that the fluid serreted thereafter for a considerable length of time, will be deficient in impregnating properties, and although the animal may seem vigorous and continue in service, the female will fail to conceive, through no fatult of her own. Closure of the neck of the womb, if by spasm, is best treated by greasing the arm with water and castile soap, vaseline, or hogs' lard. Reach into the female, and as soon as you come to the neck of the womb, insert one finger first, then two fingers, and continue in this manner until the hand can be passed through. Ordinarily, a very small opening will suffice. Females will become impregnated when the opening is no larger than will admit a lead pencil. If the clusure is due to inflammatory adhesions, greater force will have to be used. If you cannot force a finger through, take a small-bladed penknife and make a few incisions into the neck of the womb, then force an opening either with the finger or some round-pointed instrument, like a scratch-awl, and immediately serve with the male. If 
the neck of the womb is four or five inches in length, which is sometimes observed, a portion of it should be excised, after which place a small sponge into the neck of the womb until it heals, to prevent its growing together. When the womb is excitable and irritative, causing straining and ejection of the seminal fluids, it is best to give the female a good run or a hard day's work-something to compel the animal to become fatigued, then breed immediately and she will likely conceive. If from a fatty condition of the ovaries, where the animals are very fat and pampered up, place them to work and work them hard for a month or six weeks, feeding on a limited quantity of oats and hay, but if from too low a condition, and over-work, reverse the conditions. If from catarrh of the womb, leucorrhøa or whites, see treatment for these ailments. If from diseased ovaries, the animal should be spayed. If only one ovary becomes affected, that ovary should be taken away, when the animal will possibly breed all right. When mares become greatly advanced in years, say seventeen to twenty, without ever raising a colt, they should not be bred, as the pelvic bones becone solidified and diflicult delivery is the result, probably ending in the loss of both foal and mare.

\section{ABORTION IN CATTLE.}

Abortion, slinking or slipping of calves, to a breeder of fine stock is a great misfortune. It not only deprives him of the profit on the increase of his herd, but the animal fails to give her full quota of milk, which she would otherwise do, if permitted to run the full period of gestation, the system thus undergoing the necessary change. 
Causes:-Abortion is due to many causes. It is sometimes caused mechanically, such as being injured by unruly and vicious animals, horning or bunting them; also by cattle that are continuously; bulling, junping upon them; from certain herbs obtained in the pastures

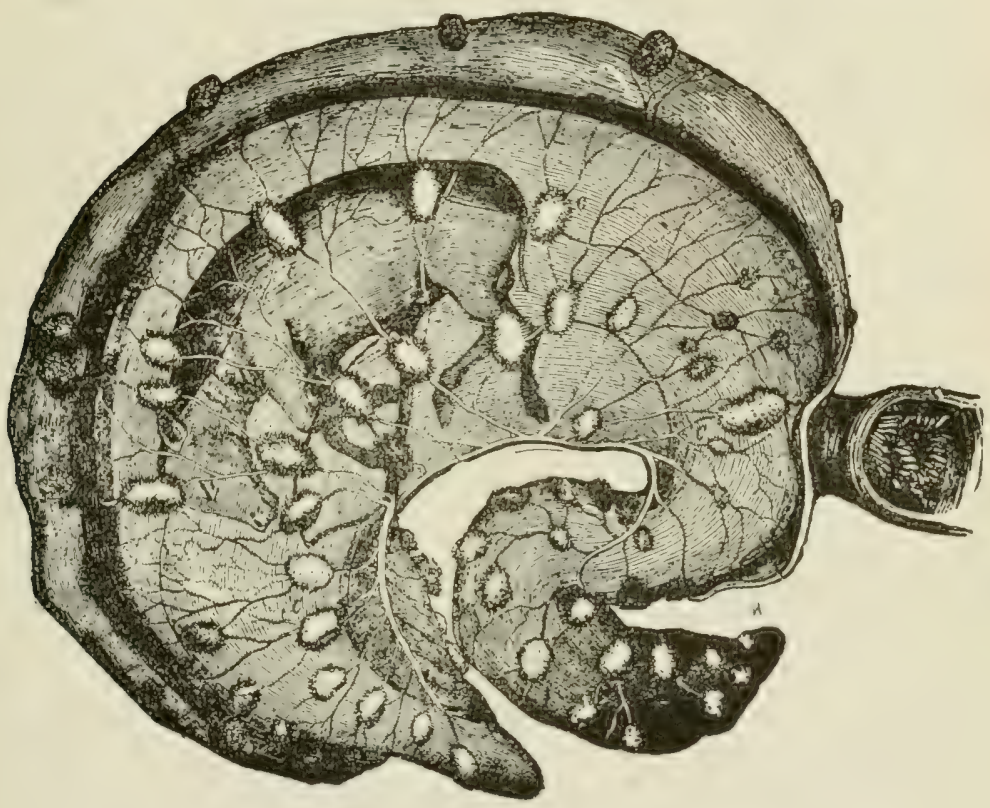

CALF IN WOMB AT FIVE MONTHS.

or in the hay; from too close in and in breeding; from eating frozen roots, drinking ice water, slipping into too deep a trench constructed as a receptacle for the manure; also from smelling other cows that had aborted or had premature births, and from the last cause it seems almost like an epidemic in some herds. It is generally considered an abortion, when the cow loses her calf within the first six months of gestation, and during the last three months, a premature birth. 
Symptoms:-It is very seldom that any symptoms precede abortion. Usually the animal will abort the calf and not miss a meal, but with premature birth, we will quite frequently notice a loss of appetite, suspended rumination, dullness in appearance and a disinclination to be with the balance of the herd. At first there is a yellow, glairy discharge from the vagina, sometimes red and streaked with blood; if the calf is not soon expelled, decomposition soon takes place and an offensive smell emanates from the vagina, with a whitish colored matter and small portions of the placental membranes. Colicky pains and great uneasiness are sometimes noticed.

Treatment:-First isolate the animal from the balance of the herd to a comfortable place, then make an examination, by greasing the hand and arm thoroughly with hogs' lard and passing it into the womb. Remove the dead calf and all of the membranes, after which wash out the womb with carbolized water, say half an ounce of the acid to two quarts of warm, soft water, or permanganate of potassium, one drachm to two quarts of warm water, once a day for three or four days. Give the following physic:

Epsom Salts..twelve to sixteen ounces.

Powdered Fœnugreek, or Ginger....

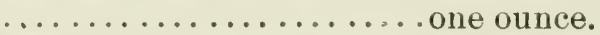

Water................. one gallon.

Give at one dose, to be followed with-

Aromatic Spirits of Ammonia..... ..................ten ounces.

Tineture of Cardamon.... four ounces. Tincture of Jamaica Ginger.two ounces. Mix.

Give two ounces every three or four hours in a pint of water, or you may give four quarts of beer each day, 
until the appetite returns. At all times, give warm bran mashes and entice the animal to eat anything she will, and give all the water she will drink.

\section{NECESSARY AID IN NORMAL PARTURITION.}

When animals are about to deliver their young, they should have suitable places provided for them-more especially large animals, as the mare and cow. As the act of delivery is performed with a certain amount of pain and uneasiness, they should be left untied, and provided with a large, comfortable hox stall, eontaining a sufflcient amount of bedding to prevent slipping and injuries; or if in proper season, they will do better in a pasture field or meadow. When delivery commences, it is seldom that the aid of man is necessary, yet at the same time, he should make an examination to see that the little animal is started right-that is with the two front feet tirst, the head resting upon and between the legs, the upper part of the head and backbone resting against the mother's backbone; or if the hind feet start first, it will be delivered in that position; but see that its backbone rests against the mother's backbone. If it lies in either position, do not meddle too much, but allow some time to elayse he fore aid is rendered, except it be a mare, then do not delay too long, for if the colt is not delivered voluntarily within an hour from the time of commencement, aid should be rendered immediately or the little animal may he lost. As it receives its nourishment and supply of air through the coverings or afterbirth, thence through the umbilical or navel cord, should these become detached from the mother's womb, the colt quickly dies from inanition or want of air, if not brought into the external world. Sometimes: females will act very sick, and the straining or labor 
pains will be exceedingly hard or heavy. Usually in such cases, the mouth of the womb has not dilated sufficiently to allow it to start, or the other surrounding tissues have not become sufficiently relaxed. In such cases, you may give ounce doses of laudanum to assist in quieting the pains; or half ounce doses of chloroform or chloral hydrate, as well as applying hot cloths to the belly; or give a liberal amount of walking exercise, in fact anything to attract or divert her attention for the time being, to allow the parts to become relaxed. Placing a drachm of belladonna on the mouth of the womb, will often cause it to open rapidly. Use quiet and soothing measures, and in the course of from six to twenty-four hours, the delivery will be comparatively easy. However, when parturition or delivery is retarded, it is sometimes a question whether the young animal is living or dead; this is frequently diflicult to determine, and as my experience has been I presume, almost equal to that of any person of my years, having attended over twelve hundred cases in eighteen years of practice, I have resolved that there is but one absolute or positive symptom of the death of the young animal, prior to birth; that is, when upon examination, you discover bloating or puffiness of the skin, and the hair slips off readily, decomposition has set in and there can no longer be any doubt but that the young animal is dead. 'Many and varied are the symptoms spoken of and cailed infallible signs of the youngster's death, but all have proven failures in my own experience, with the exception of the one referred to above. It is perfectly true that in fully sixty per cent. of the cases which I attended, the foals were dead, and about two per cent. of the mares succumbed, but many of these cases were confined in labor for from twenty-four to forty-eight hours before I reached them. The foal seldom lives 
over four hours within the mare, after the first expulsive eflorts or foaling have commenced, while a calf may live for two or three days. The placental membranes or afterbirth, do not become detached so readily in the cow as in the mare, hence the longer lease of life in the calf, after the labor pains have started. If, after the water bag is ruptured and part of the fluid has escaped, the natural expulsion of the young animal should be waited for. I will here remark that it is never necessary to rupture the water bag in the cow artificially, as it is rery thin and ruptures easily itself. But with the mare it is quite diflerent. The membranes are quite thick and firm and not infrequently the foal is dropped enveloped in the sack without it being ruptured, when the young animal would quickly perish. Therefore, when the water bag presents beyond the vulva or opening as a large sack, it may be torn with the fingers or cut with a linife or scissors, after which some assistance may be needed. If the forefeet and head are presented, take hold of the feet, and at each labor pain of the mother, draw steadily and firmly upon the feet in the direction of the mother's hocks, so as to follow the natural curve of the channel through which it has to pass; occasioually moving it from right to left, will assist greatly in working the shoulders and hips through the passage. When the young animal comes with the hind feet first, it will be delivered in this way easily, and will be recognized by the pasterns bending upward instead of downward, as in the front feet also by the feel of the hocks, which is vastly different from the knee. If assistance is rendered draw in the direction of the mother's hocks, the same as in the front presentation. 'But when the hind feet are delivered first, immediately after delivery, seize it by the hind legs and raise it clear from the ground with the 
head hanging downward, so that any fiuids remaining in the nostrils and wind pipe, will escape. Many foals and calves are lost annually, through neglect to empty the fluids from the wind pipe; the lungs are too weak to force air in through these fluids. When colts and calves are born head first, these fluids escape, as the head hangs downward during delivery, but when the heald comes last, these fluids are liable to remain in the wind pipe, and death quickly ensues. Sometimes after birth, life is apparently suspended in the young animal. When this is observed, clean out the mouth and nostrils and dash some cold water upon its head, or take wet, cold cloths and beat them against the body and chest. Also take hold of both forelegs, draw them well forward to elevate the ribs, then gradually bring them back again, continuing to repeat this operation to invite artificial breathing; also starting a little blood from the navel, may assist in restoration to life. When the roungster is lively, but unable to stand and nurse the mother, you may draw some milk in a clean bottle and administer it. Always give the first milk of the mother as it acts as a mild physic, and if this has escaped, wive a teaspoonful of castor oil. In assisting the youngster to nurse, do not place the hand under the breast bone; as it is quite soft and yielding, you may do considerable harm, even causing a quite lively colt to become sick and die. Many are lost in this way, the owner considering that he was doing an act of kindness. When it is necessary to hold them up to nurse, eatch one arm around the forelegs and breast, and the other arm around its haunches, and you will not injure your colt in the least. After the colt is a few days old, it is necessary for it to receive some exercise; therefore, allow it to run in the barn lot, or if in season, turn the mare and colt to pasture. Calves should be treated in 
a like manner. The mother should receive some attention after delivering her young, as she is generally irritated and somewhat fatigued. She should be kept comfortable, in an airy place but free from draughts. If she has been sweating, she should be rubbed dry-in fact a good rubloing is very beneficial at this time as it equalizes the circulation, allays restlessness, and hastens the contraction of the womb. Sometimes it is necessary to blanket the animal, as they are cery susceptible to cold at this period. Give the mother a gallon of warm gruel, and hed liberally. Allow her to remain alone for an hour or so, when you may gire her some more soft, easily-digested food, then if necessary, assist the colt to nurse. Feed upon soft, easily digested food for ten or fifteen days and allow her perfect rest during this time, in order to permit a gond flow of milk to be fully established and the complete recorery of the animal. If parturition has been prolonged and the animal has suffered considerably, and the womb and other organs have become bruised and inflamed, she should rest for a greater length of time, and care should be taken to prerent inflammation of the womb). A few injections into the vagina will now be beneficial. Take

Permanganate of Potassium....... half ounce.

Water.............. one gallon. Mix.

Inject once daily, using a quart each time. “Apply warm cloths over the loins, feed on soft food, and gire four ounces of glauber salts daily, to keep the bowels soft. Also keep in a clean stable, where the atmosphere is pure. When animals are either old, or weak and debilitated, and the labor has heen prolonged with some bleeding following, stimulants should be given, as whisky, brandy, wine, etc. Rul) the body and limbs thoroughly and supply nlenty of bedding; and as the 
mothers contract colds quite readily at this time, shelter Ehould be prorided when turned to pasture, to protect against cold storms, for it should be borne in mind that cold and dampness are dangerous to the health of the animal, for some time following parturition.

\section{PARTURITION-ITS SYMPTOMS, ETC.}

During parturition, from the earliest symptoms developed by the attempted expulsion of the fœtus until the fœtus and membranes are delirered, is called labor. Symptoms of approaching labor and the termination of the period of gestation, are sometimes observed for sev-

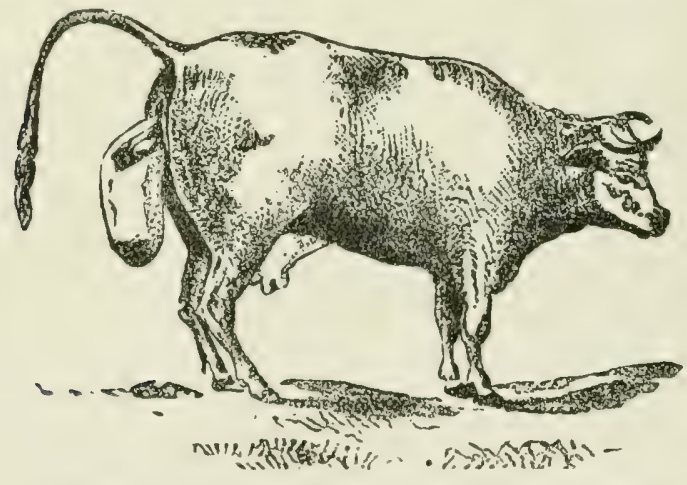

NORMAL PARTURITION, OR NATURAL LABOR.

eral days before the event transpires. One of the most important symptoms is enlargement of the mammary glands-sometimes to such an extent that swellings, from the size of a saucer to that of a large plate, appear in front of the bag, also behind the bag up between the hind legs to the lower part of the vulva. " Later on, if pressure is applied to the teat, it yields a watery secretion which sometimes escapes unaided and accumulates around the lower part of the teat, forming a gum-like 
substance or wax. The vulva increases in size and its lining membrane l, rmes rerldened; sometimes a whitish, glairy secretion is formed and discharger as a long, stringy, sticky substance hanging from the vulva and sticking to the tail and legs. The croup, on either side of the tail, looks hollow and feels soft and pliable from relaxation of the broad ligaments. The hack is somewhat sprung downward, the abdomen distended and hanging low down. The animal walks about carefully and is somewhat inclined to leave the balance of

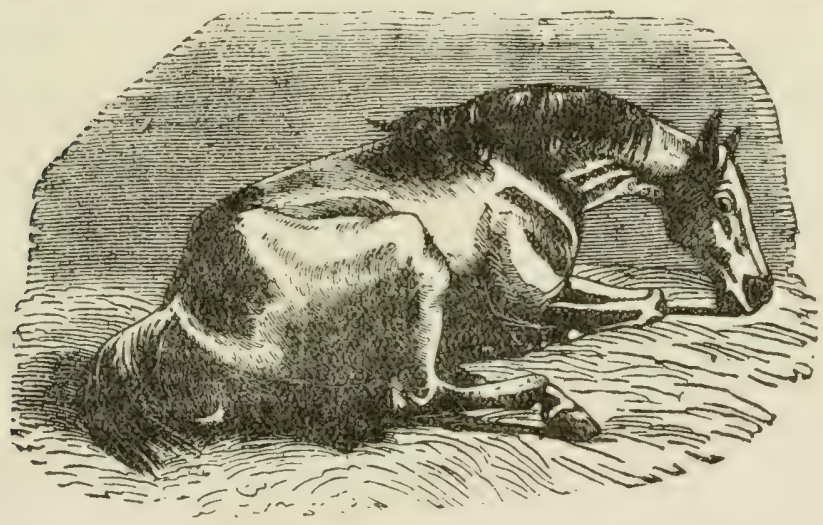

NORMAL PARTURITION, OR NATURAL PRESENTATION.

the herd. In mares, swelling of the hind legs is frequently seen. As the time for the expulsion of the fotus draws nearer, the animal has pains of a colicky nature; gets up and lies down frequently, and, if permitted, usually will seek a place of seclusion. As the uneasiness and pain increases, the os uteri, or mouth of the womb, continues to dilate until the womb and vagina form one large continuous passage. The membranes are now observed externally in the form of a water bag, which on pressure, is usually ruptured. The pains become more severe and frequent. The animal 
takes a full breath (as if preparatory to making a deep sigh), filling the lungs, and, holding it there for a time, brings the diaphragm and abdominal muscles into action to assist the uterus in the expulsion of the fœtus. If the animal is standing, it brings its four feet close together, arches its back, elevates the tail, and takes a deep inspiration, the same as if lying. These labor pains are continued for a time, when the forefeet and head are observed to be passing. The spine of the fotus should be resting against the spine of the mother, and the breast and abdomen against the lower part of the vulva. In the mare, a few more labor pains and all is over; but in the cow, labor is more prolonged and an extra effort is made when the chest is forced through the vagina to the outer world. As soon as the young animal is born, the umbilical cord is torn apart, and, if bleeding much, should be tied; but this is seldom.necessary. The remaining fluid now escapes from the womb and is sometimes tinged with blood, from the sudden separation of the membranes. Sometimes the fretus is delivered encased in the membranes, the uterine passage being sufficiently large to admit it, and the membranes being very thick and strong. When such is the case, the fortus sometimes ruptures them when struggling, or the mother tears them apart with her teeth; if not speedily ruptured, the young creature soon expires for want of air. After the young animal has been delivered, the next act is to get rid of the fortal membranes -" the after-birth." In the mare, the placenta will have such delicate attachment that slight contractions of the womb, together with the weight of the protruded part cause speedy expulsion. But in the cow, the placenta is not readily expelled; its attachment is by cotylredons instead of villi, and the contractions of the womb do not so readily detach them. There may be 
from forty to a hundred attachments, and from four hours to three or four days are required before the membrane is expelled, and then it may have to be remored artificially. If the mother is not seen during the act of parturition, or for some time after, the afterbirth may never be seen, as cows, and sometimes matres, devour it as soon as expelled.

\section{CARE AND FEEDING OF PREGNANT ANIMALS.}

The measures for preservation of lealth in animals during pregnancy, are those which should be observed generally with animals which are not pregnant, except a few extra precautions which should be taken, in order that the mother may pass the periods of gestation and delivery in safety. When an animal has been served by the male and has concoived, she should not be allowed to ascociate with the mate (ither in the stable or pasture, as attempts on the part of the male at coition and casual excitement on the part of the female, may do considerable harm, and even produce abortion. With such animals as are kept at work-as the mare-it is bad policy to work them hard, especially after pregnancy is well advanced. Great care should be taken not to put them in a position where their sides are apt to receive blows, such as from heary wagon tongues, etc. Neither should they be pampered and nursed in a stall, without exercise. The work should be slow, and if at all slippery, the mare should be well shod. They should not be used for saddle purposes, but if used, spurs should not be worn. Galloping, jumping, or in fact, anything producing excitement should be avoided. Should the patient be a mare used exclusively for breeding purposes, she should have a box stall and a yard in which to exercise. It is not at all necessary to cease working a mare for the two 
or three months previous to foaling; better to give slow, light work, so that they will have exercise up to the day of foaling. The food is a most important factor of success. Milch cows or working animals should be well fed, as not only their appetite but their physical rondition demands it. Some animals are prone to lay on flesh when pregnant; with such you should be very careful about feeding, as it may prove troublesome, especially if allowed to proceed to the extreme. It may retard the growth of the foetus, produce abortion, cause difficult parturtion, or give rise to some other serious trouble, which will be more likely to take place during the last few months of pregnancy than at first. The food should be clean, of wood quality and easily digested. Damp, bulky, mouldy or fermentable food should be discarded, as evil results are nearly certain. Grazing is heneficial if the pasture is nutritions, if not, supply the deliciency. The animals should be protected from foggr, dimp or stormy weather. They should have all the clean, pure water they desire, for when at liberty with free access to water, they seldom drink much at a time. The water should not be too cold and ice water is to be strictly avoided. When animals are housed, strict cleanliness must be olscrved, and when near foaling time, they should be placed in roomy box stalls. If one animal aborts and there are others pregnant on the same farm, the one aborting should be removed, as the excitement amongst the others is liable to produce similar results. The stall in which abortion took place, should be white-washed and strewn with slacked lime, to destroy the smell. Harsh or cruel treatment on the part of the attendant or groom should be suppressed. loung animals, about to bring forth their first offspring, should be handled very gently. Their udders and teats should be handled frequently, thus preventing 
aversion to suckling their young. As much as possible, surgical operations and the administration of medicines should be avoided. Above all things, avoid giving drastic purgatives, as the irritation they produce is apt to cause contractions of the womb, resulting in abortion, and endangering the life of the mother. The stables in which pregnant animals are kept, should have floors as nearly level as possible, for if the incline is at all great, the gravid uterus pressing backward, may cause eversion of the vagina and abortion. The doors should be wide to prevent the animal striking or crowding together its sides on entering or going out.

Cows that are rich, heavy milkers, and more especially about the time they produce their third or fourth calf and from that period on, are very susceptible to parturient apoplexy or milk fever, which being very fatal, should receive special attention. When we know that the subjects of it are hearty feeders, fleshy, and good milkers, we have every reason to caution the owner to take preventative steps to combat or ward off the malady, by a strict attention to feeding-more especially during the last months of pregnancy and immediately after delivering their calves. For two months before calving, the food should be soft, to prevent constipation; and when the animal is quite fleshy, it should be given in limited quantities, in order to reduce the condition. The cow should be allowed to take daily exercise, as it greatly assists in preventing an attack of this disease. Extracting the milk by hand for a few days before calving, if the bag is full and large, should not be neglected. After calving, allow drinking water in a limited amount, and within four hours or as quickly after as possible, give a physic, asEpsom Salts............. one pound. Powdered Ginger...... four drachms. Mix. 
Dissolve in one gallon of warm water and give at one dose. If these directions are carefully followed and more especially the physic given immediately after calving, parturient apoplexy, or milk fever, will very seldom be seen. I will here add that it is more frequently seen in the rich milking, delicate Jersey, than any other breed; but no class of cattle are exempt from this terribly fatal complaint.

\section{PARTURIENT APOPLEXY-MILK FEVER.}

This disease is seen in all breeds of cattle and generally attacks cows which give large quantities of rich milk. It is seldom seen during the first, second or third calving period, but usually from that time on, and almost always in cows that are highly fed and in good flesh. It usually occurs within three days after calving, and is very fatal-fully one-half of those attacked die. If recovery does result great care should he taken at the next time of calving, as a recurrence of the attack at that time is frequent and generally proves fatal.

Symptoms:-Usually between the first and third day after calving, the cow will stand around dull and stupid; appetite, rumination and milk will be suspended-or only a slight flow of milk present; the cow walks as if weak across the back, staggers around shaking hor head; as the disease advances she falls to the cround unable to rise again. She may rest in a recumbent position with her head carried around to her side, or she may lie stretched out upon her side with her head lying upon the ground. Sometimes the head is shalien viciously from side to side, endangering those alout her and being liable to break ofl her horns. Ocoasionally, but not often, a few kicks with the feet are given. 


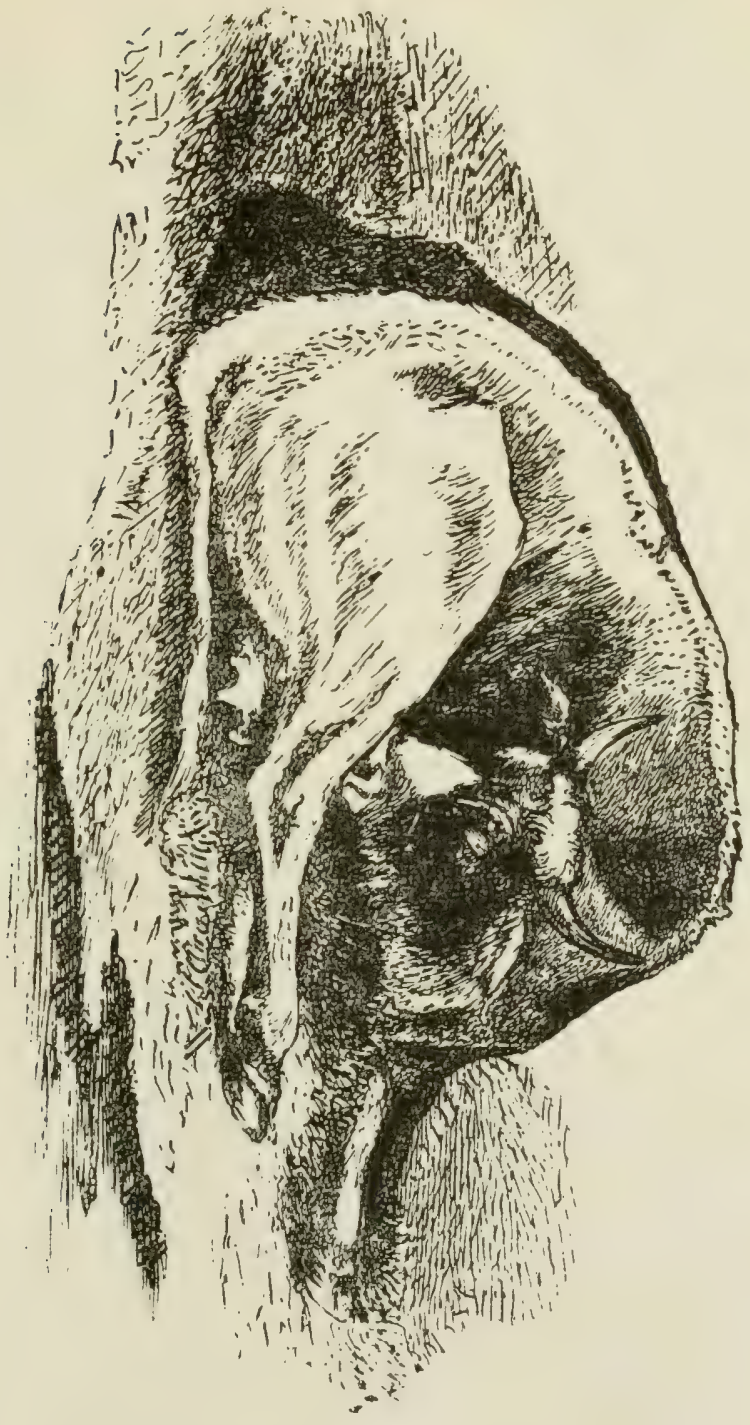

PARTURIENT APOPLEXY. 
The eyes become glassy and staring, soon growing insensible to the touch, with total blindness. Sometimes the eye-lids will quiver and jerk for some time. The pulse, at first full and soft, later on becomes slower and feebler, gradually becoming almost imperceptible. The breathing, at first almost normal, becomes generally slower, prolonged and difficult; and frequently a rattling sound is heard within the wind-pipe. At the commencement of the attack, the temperature will ranse from $103^{\circ}$ to $104^{\circ} \mathrm{F}$., and as the disease proyresses will gradually $\operatorname{sink}$ to $3^{\circ}$ or $4^{\circ}$ below normal. At first the lowels may appear lose, but soon become constipated, and the urine is usually retained within the bladder.

Treatment:-I wish here to impress upon the reader's mind that to be successful in the treatment of parturient apoplexy demands persistent attendance until death has actually occurred. I have seen cases, where the owner considered treatment useless and momentirily looked for death, make complete recovery. If the patient is seen when the pulse is still full and strong, hleed to the amount of six quarts; but if the pulse is weak and the animal down, with the eyes insensible to light, do not bleed. As soon as possible give the following physic :

Epsom salts.........twenty ounces.

Calomel............. one drachm.

Croton Oil........... twenty drops.

Ginger............... one ounce.

Warm Beer.......... one-half gallon. Mix.

If the animal has become unconscious, give very slowly as it is liable to run down the wind pipe. Then draw away the urine by means of a catheter, or by slipping the finger well oiled into the neck of the bladder, the urine will frequently be passed. (xire injections of warm water every hour. Constantly apply 
rugs wrung out of hot watel orer the loins and apply ice-cold water or ice hags to the head, as the brain is badly congested. Frequently draw away the milk and keep the body and limbs warm, by friction and blankets. Keep the animal in a position as nearly erect as possible, and give the following every two hours until recovery begins, when the time should be lengthened:

Aromatic Spirits of Ammonia ..... ................... eight ounces. Spirits Nitrous Ether .... four ounces. Tincture Nux Vomica.... two ounces. Tincture Gentian..........six ounces. Mix.

Give four ounces in a quart of warm beer or water, or a half pint of hot whisky every two hours. This may seem like heavy dosing with stimulants, but it is the proper thing to do. Should the physic operate and the animal begin to appear conscious of her surroundings, feed un soft and sloppy diet, until convalescence has been established. Prevention is the best and safest treatment for parturient apoplexy. When an animal is known to be a heavy milker and is fleshy or plethoric in condition, she should be fed previous to calving, on a laxative diet and but sparingly. The milk should be drawn away for ten days previous to calving; immeciately after calving give the following drench:

Epsom Salts..........twelve ounces.

Glauber Salts............ six ounces.

Ginger............. one-half ounce.

Fonugreek.......... one-half onnce.

Water........... one-half gallon. Mix.

If these directions are followed you will not be? troubled with parturient apoplexy. 


\section{OEDEMA, OR SWELLING IN PREGNANCY.}

In some of the coarser breeds of animals and, occasionally in some of the finer, during pregnancy swellings appear around the udder, extending forward as far as the breast and backward and upward as high as the vulva. The hind legs will begin swelling just above the hoof, and the swelling will extend upward to the hocks or even to the groin. These swellings are serous or watery in character, diminish rapidly during exercise and increase upon rest. The disease is not of much conseriuence, being caused by deficient circulation occasioned by the pressure of the fotus upon the crural vessels. It is never seen in the cow, as in her the circulation through the mammary veins is more complete than in the mare. In mares it sometimes appears three or four months previous to foaling; but when at pasture where they can get regular exercise, it rarely occurs. It depends very much upon the conformation and temperament of the individual animal, as well as on season and hygienic management. No fear need be entertained, as it disappears in a few days after foaling. Should it occasion any inconvenience, give exercise. Or, bandaging, the application of a mild stimulating liniment, or scarification may be resorted to. Howerer, this is very seldom necessary.

\section{EVERSION OF THE WOMB.}

Eversion of the womb can only happen when the os uteri, (or mouth of the womb) is dilated. Consequently it happens only shortly after parturition or during the act of parturition. It is liable to occur amongst all breeds and all classes of animals. Mares seldon suffer from it, though it is quite common among cattle. I 
cannot say whether or not it be from peruliar conformation, but some cows are more subject to this aflection than others. The uterine ligaments of the cow are so extensive and elastic, that a few extra eflorts or labor pains after the frotus is expelled, send the womb along

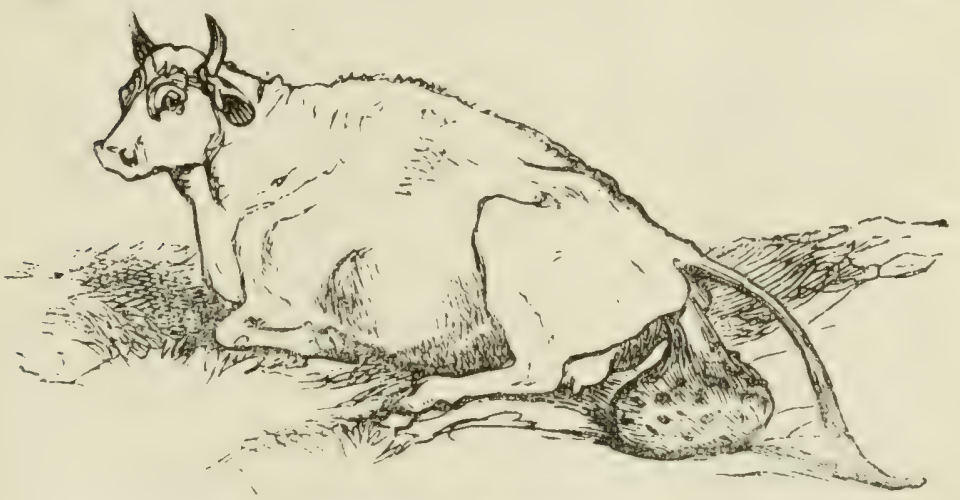

EVERSION OF THE WOMB.

with it, or shortly after. If eversion of the womb is thought probable, the animal should be watehed and before the womb can he everted, a pessary should bo introlured and the womb maintained in its position. A truss should be wasl to prevent straining and the following drench should be given at one dose:

Sulphuric Ether........... one ounce.

Tincture of Opium.......two ounces.

Brandy ..............two ounces.

Water.................. Mix.

Previous to inserting the pessary, remove all of the after-birth.

should the womb be inverted and lying on the ground, or langing from the animal as it stands, gather It on a clean linen sheet, remove all the membranes and wash it thoroughly with warm water, to which may be 
gided a mild solution of listerine. Then commence to replace it. Oil your hand thoroughly, then close your fist and place it in the hollow spot or center of the womb. Press inward slowly and carefully. If the animal strains be very careful, for fear of rupturing the womb. Have your assistants press on the sides of the

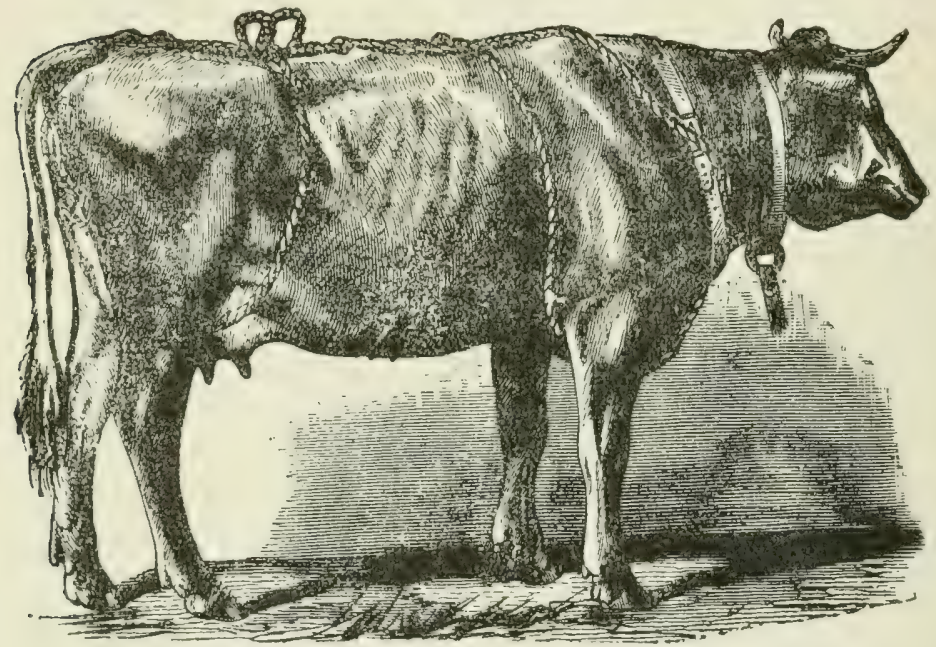

TIUSS TO PREVENT EVEREION OF THE WOMZ.

womb towards your arm. In this way the womb an he? returned. This should be done as quickly as possible after eversion has taken place. As soon as the womb is replaced, insert a pessary and keep it there from four to ten days. The pessary should be made of light word about twenty-two inches long and two inches thick, with the end inserted covered with a smooth, oiled cloth and the outer end having a hole bored through it, in which to pass a rope to hold the pessary in position. Fasten a truss made from rope around the patient, (as per illustration) but do not fasten it too tightly. Leave 
loose enough to allow the patient to get up and down. Feed on soft food to prevent constipation, and give

Powdered Ginger.......... one ounce.

Baking Soda........... one ounce.

Saltpeter..............two ounces. Mix.

Make six powders and give one powder every six hours, made into a tea as a drench.

It is a rather difficult task to return the womb and maintain it in position. But be persistent in your work and your efforts will be crowned with success, at least if the patient is a cow. In mares, fully threefourths of the cases prove fatal.

\section{POST-LABOR PAINS-AFTER PAINS.}

The painful sensations of the womb, after the expulsion of the firtus and secumblines (which are frepuently olserved are sometimes termed after pains. They are due to contractions of the womb which continue for some time, in order to reduce its volume and cast off the membranes. They are seldom seen after an easy delivery and, when they are seen, last but a short time. But when there is difficult or protracted labor, the after pains may last twenty-four or forty-eight hours; or, if some of the placental membranes are attached, the straining is liable to be prolonged. The animal will arch its back, contract the abdoninal muscles and strain violently, as though trying to deliver another fortus. If retention of the membranes is the exciting cause, tiney should be removed at once, as otherwise the after-pains will be continued and result in eversion of the womb. After an exploration to discover the cause of the afterpains, and removal of the membranes, should you find any bruises or lesions of the vagina or womb, wash 


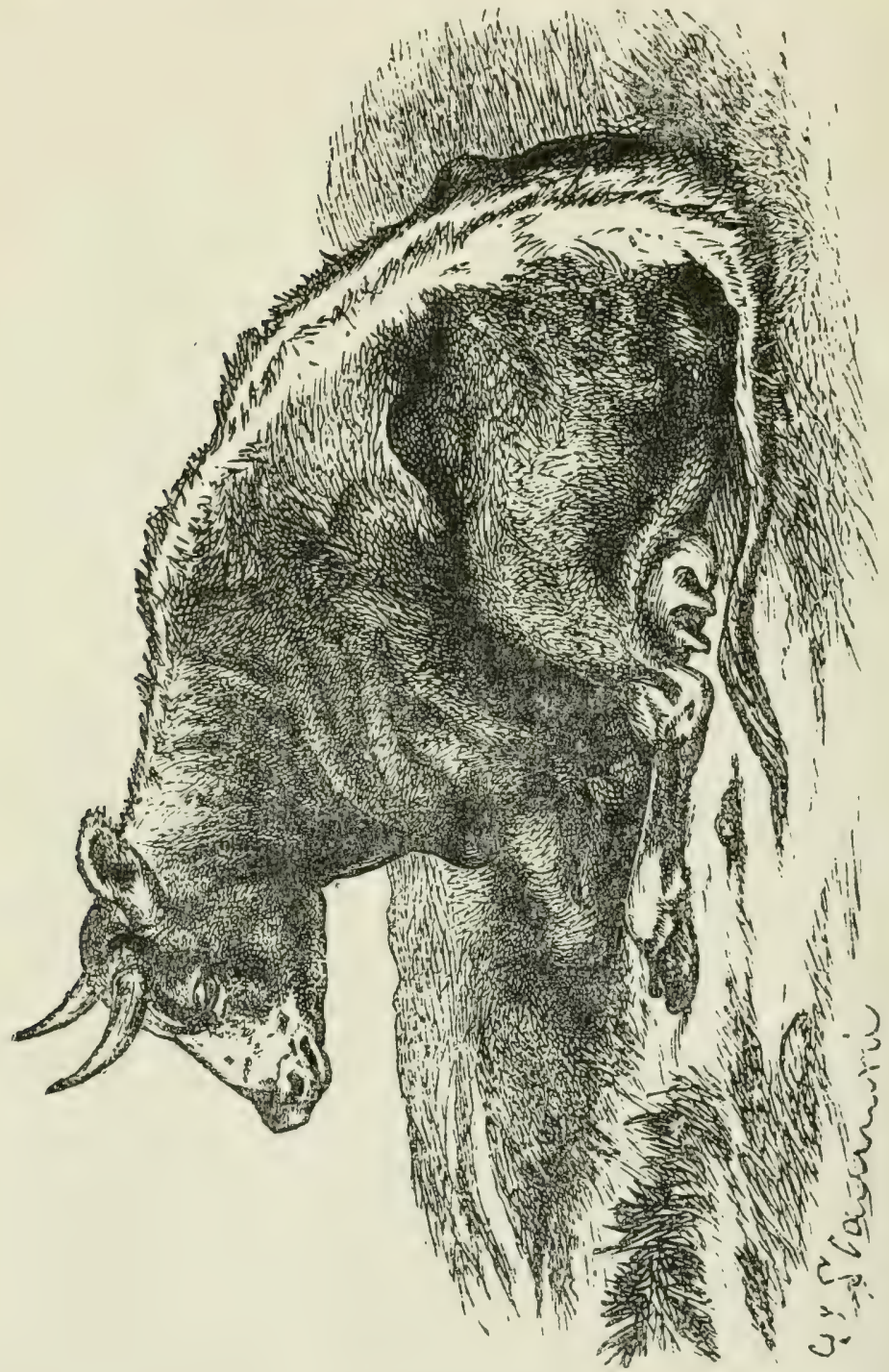

AFTER PAINS. 
out thoroughly with warm water, and afterwards with a tifty per cent. solution of permanganate of potassium. Also give at one dose, the following mild physic:

Sulphate of Soda......twelve ounces.

Ginger............. one-half ounce.

Warm Beer............. one pint. Mix.

Then apply a truss, such as is used for eversion of the womb.

\section{PREMATURE LABOR PAINS.}

Premature labor pains frequently oceur in the lower animals and are sometimes attended with considerathe danger. Various things are supposed to cause them, some of which are exposure to cold rains; injuries: administration of certain drugs; feeding upon rye grasses, etc. I am inclined to helieve that the quality and quantity of the food, exerts upon the uterus a greater influence in producing premature labor pains. than any thing else. Musty, mouldy, indigestible or fermentable food, will cause derangement of the bowels and, through sympathy, involuntary contractions of the womb. Frozen food or ice-cold water acts similarly. The symptoms are similar to natural labor except that in premature labor, the animal almost always strains while standing, while in normal labor the position may be either lying or standing, and frequently both positions are taken alternately, while straining. During natural labor, the mouth of the womb is open, while, during premature pains, it is closed for a considerable length of time. The straining is intermittent and sometimes very forcible and, if not overcome, results iu abortion.

Treatment:-Give drachm roses of powdered opiun! 
every hour, until the straining stops; then give at one dose, the following mild physic:

Magnesia Sulphate .... twelve ounces.

Ginger............. one-half once.

Water................ two quarts. Mix.

If this fails to act in thirty-six hours, repeat the dose.

\section{POST-PARTUM HEMORRHAGE, OR FLOODING.}

Bleeding from the womb, or "flooding," after abortion or after delivery at full term, occasionally occurs among domesticated animals and is very fatal.

Causes:-Injuries to the womb during expulsion of the fotus; premature removal of the placenta; partial

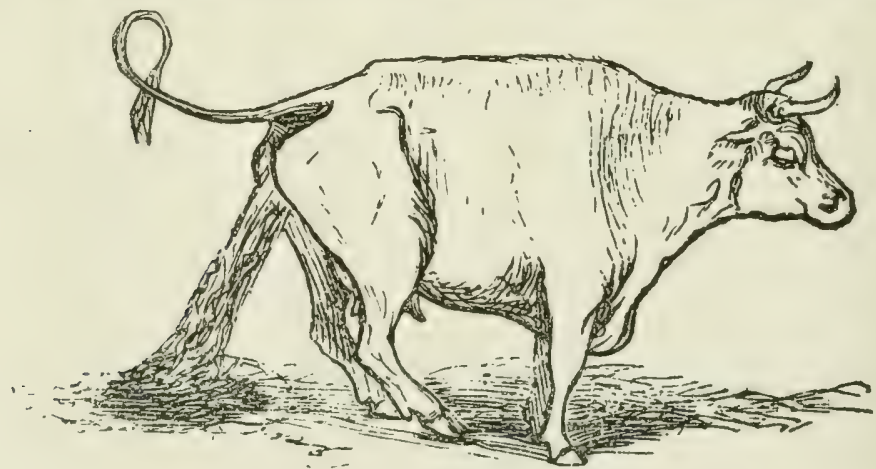

POST-PARTUM HEMORRHAGE, OR FLOODING.

adhesion of the placental membranes; feeble development of the uterine walls and insufficient contraction of the womb. If a slight dribbling stream is noticed, an examination of the vagina will probably reveal slight lesions there, which seldom do any harm. But if the blood issues forth in torrents, and there is a quick weak pulse, pale mucous membranes, increasing prostrution, 
staggering gait, chilliness of the surface, coldness of the limbs and breaking out of a cold elammy sweat, the bleedinw is great and, if unrelieved, will terminate shortly in death. The treatment must be very prompt and eflective or death will speedily follow. First, examine to ascertain the cause. If part of the membranes still arlhere, at once remove them; but do so carefully or you will increase the hemorrhage. Remove all clots of blood. Then apply cold water over the loins. Also dip your arm into cold water, insert it into the womb and move it aromd, which sometimes will induce contraction of the womb and thus stop the bleeding. If not, take a soft cloth or a sponge, dip into cold water, and incort in the womb; or inject cold water into the womb; (1) nse injections of cold water and per-chloride of iron. If there is much prostration, the following will prove rely bencficial :

Brandy................. one pint.

Tincture of Per-Chloride of Iron.... ................ four ounces. Mix.

Give three ounces every two hours, in a half pint of hot water as a drench, after which place the latient in a cood comfortable stall, well bedded, and feed on soft nutritious food.

\section{RETENTION OF THE PLACENTA-AFTER-BIRTH.}

Beyond a certain period after the expulsion of the foetus, the retention of the placenta or "after-birth" whould be considered unnatural and to require some attention. With mares this seldom occurs, but in cows, owing to the peculiar attachment of the membranes, it is quite frequent. In the mare the placenta is aftached ly fine villi, which are easily detached and the placenta is only retained by abnormal adhesions. - In the cow 
the placenta is attached by cotyledons, varying from forty to one hundred in number, which are so constructed that entanglement of the membranes is liable to occur at any time after calving. From this peculiarity of construction, abnormal adhesions are more frequent than in the mare. The natural mode of expelling the placenta, is by the contraction of the womb, although I have seen cases where the womb, had contracted and the os uteri, or mouth of the womb, completely closed, and the foetal envelopes, completely encased within the womb, and could only be removed by forcible dilation of the mouth of the womb. But these are exceptional cases. The general rule, where there is retention of the membranes, is that the womb will contract, but its mouth will remain open with part of the membranes protruling through it. More frequently the membrames are visible, hanging down from the vulva. In the mare, the placenta, if not expelled by the efforts of nature, should not be allowed to remain undisturbed, but should be removed within twelve hours after foaling. With the cow, danger is less imminent, and unless straining or hemorrhage are prominent symptoms, the placenta shomld not be disturbed for at least from one to three dars, during which time-if not detached naturallydecomposition is liable to occur. If this be the case, it should be immediately removed or chronic inflammation of the womb and vagina may follow and leucorrhoen (or whites) be the result. When the placenta is not expelled shortly after calving, the following may assist its removal:

Gentian ........... one-half ounce. Ginger............... one ounce. Foenugreek............. one ounce. Anise................ one ounce. Bi-Carbonate of sola..... one ounce. Mix. 
Make two powders and give morning and erening. After a lapse of three days, it is advisable to remove the placenta by a manual operation. First see that there are no abrasions of the slin on your hand or arm, through which absorption of putrid matter can take place and so lead to blood poisoning. Then grease your hand and arm and pass between the placenta and the walls of the womb. As you meet the cotyledons, unfasten the membranes in the same manner in which you unbutton your coat; proceed to the next, and so on until you lave unfastened them all. I'se gentle traction with the other hand all the time, as some of them will be detached by traction alone. When you have detached the placenta from all the cotyledons, it will drop out easily. After the placenta is detarhed, when there is a very foetid odor, wash out the womb thoroughly with a soft cloth and warm water, to which may be added a drachm of permanganate of potassium. Then feed on a nutritious diet, to which add a dessert spoonful of glauber salts morning and erening. In the mare, take the same precautions before remoring the placenta. Grease the arm thoroughly, then pass the hand gently between the placenta and womb, separating the attachments slowly and carefully, using traction with the other hand. If the attachments are very close or abnormally adhered, break them down, when the plarenta is easily removed. Keep her quiet for a time; feed soft food to which add twice daily a teaspoonful of-

Glauber Salts.........eight ounces.

Saltpetre........... four ounces. Mix.

\section{HERNIA OF THE UTERUS, OR WOMB.}

This accident is not freguent, though it sometimes occurs. During the eighteen years of my practice I 
have worked extensively in obstetrical cases, and have seen only five mares with hernia of the womb and not one cow. Hernia of the womb consists of a breaking down or separation of the abdominal muscles through which the womb and contents pass until they come in contact with the skin, which from its elastic nature, is carried down'sometimes within fifteen to

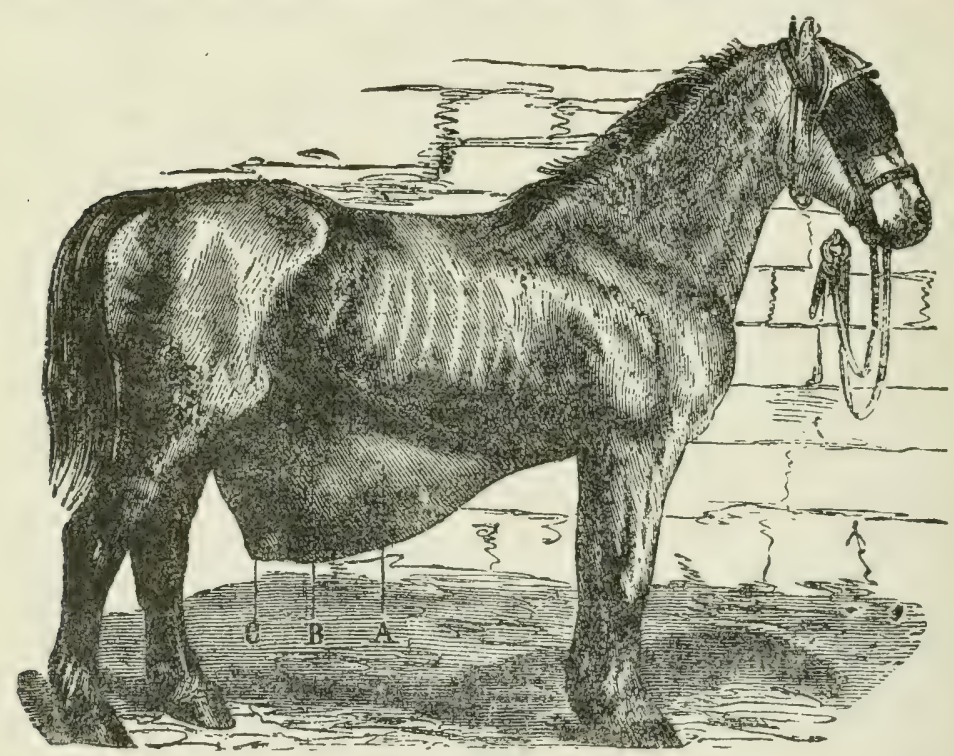

HERNIA OF THE UTERUS (OR WOMB).

oighteen inches from the ground. The bag and teats are generally carried down with the skin and frequently form the lowest point of the tumor. The causes of uterine hornia are none too thorougly understood, but that which seems to me the most reasonable supposition, is that the uterine muscles become ruptured or relaxed, thus allowing the gravid uterus to rest entirely upon the abdominal mus.les. These become weakened from the abdominal strain, after which the energetic movements 
of the fœtus, materially assist in forming the rent in the abdominal muscles. In the first case of this descripti in that I ever saw, I was somewhat puzzled, as the owner did not know whether the mare was in foal or not; and I was unable to ascertain any signs of life, though the mare presented the appearance of being with foal. I then determined upon exploring the tumor by inserting a small trocar and canula. In a moment the amniotic fluid began to escape and soon the foetus began to struggle. I immediately withdrew the canula and applied bandages. In about a month after, I delivered her of a dead colt fully developed. The operation was rather difficult and the mare died in a few hours. A post mortem examination revealed a complete rupture or rather lacerations of the oblique muscles as well as of the rectus muscles and linea alba. Had the mare lived she would have been useless either for work or for breeding purposes.

This accident seldom takes place until within the last two months of pregnancy. When it befalls a cow, I would recommend that she be fattened and sold to the butcher. But mares, which are not used for food and can be utilized in no way other than breeding or working, may be bred again. I have known one mare, which I was called to deliver on account of a wrone presentation, and which had a comparatively bad uterine hernia, to bring forth two living foals, entirely unassisted, during the next two years after my attending her. After this she was sold, left the neighborhood, and I lost trace of her. When females are found to be in this condition previous to time of delivery, they should be supported with broad bandages. At the time of delivery, trouble should be anticipated and they should receive prompt attention. If unable to deliver spontaneously, have a number of assistants to place a broad blanket 
under the abdomen and raise it up as high as possible. Then should the presentation be the buttock, the operator should push it a little toward the side opposite to the legs and seize the hind legs, one at a time, and bring them into the natural passage. Fasten a quarterinch rope, well greased, around the pasterns, separately. Then pull with considerable force, the assistants still holding up the abdomen, and you may expect a safe delivery, providing there are no malformations. If it be a head presentation, fasten a rope around the neck and lower jaw; then push the head in and to one side. Next endeavor to bring the forefeet up into the natural passage, fasten a rope around the pasterns, then draw the head back betwen the front legs and pull. Use plenty of fresh hogs' lard if the membranes are in the least dry, also have all ropes or straps well greased while using them, to assist delivery. If you are unable to deliver in this position, make a deep, soft hed of straw and, if the animal will lie down, all the better. If she will not lie down herself, lay her down as easily as possible. Then secure her limbs to prevent her doing injury and roll her on her back; have plenty of assistants to hold her there, with her hind quarters raised higher than the fore. Have some handy assistant to try to reduce the tumor while the operator is trying to seize the parts which are most convenient. If there be a head or a breast presentation secure it in the manner prescribed before and, when everything is straight, apply traction and complete the delivery. But, in case you cannot deliver by the natural passage, and the colt is living and would prove more raluable than the mother, you can secure the animal and perform the Crsarean section, which is to open the abdomen sufficiently to deliver the foetus in that way, after which I believe it profitalle to destroy the mare and 
raise the colt on cow' milk. But in the case of the row, you may stitch up the wound after removing the afterbirth; then stitch up the integument (skin) and try to save her. If she lives, she should be fattened for slaughter.

\section{RUPTURE OF THE PERINEUM.}

Generally, this accident is the result of difficult parturition, though sometimes it is caused by unruly stallions. Is a result of parturition, rupture of the vagina is a frequent occurrence, and sometimes we meet with rupture of the perineum and rectum combined. In other cases the perineum may be ruptured and the sphincter muscles undisturbed; while in some cases the rupture will extend clear out, severing the sphincter muscles. If the fotus be not in a natural position, the uterine contractions driving it backward may force the limbs or the head through the perineum and present them at the anal opening. If assistance is not at liand and the parts presenting are not returned and placed in the proper chammel, the expulsive efforts of the mother will cause them to tear the perineum and sphincter muscles asunder and thus deliver the fotussometimes dead and sometime living-but leaving an unsightly appearance, as the vagina and rectum are onc continuous slit. The results vary, owing considerahly to the extent of the injury. If the laceration is not great, no serious results need be anticipated. But if the laceration be great and the sphincter be torn through, a suppurative inflammation may occur with a continuous discharge of pus, smearing the tail and legs and causing the animal to present an unsightly appearance. Others seem to experience no inconvenience, a partial recovery takes place, the animal appears per- 
fectly healthy, may breed again and bring forth its progeny without any evil results.

Treatment:-Trim off evenly the lacerated margins, bringing them together with the cat-gut suture, using the interrupted stitch. Then introduce a tampon or plug into the vagina to support the septum during defecation. Apply cold water frequently to keep down the inflammation, and twice daily use the following dressing all along the laceration:

Vaseline............ four ounces.

Carbolic Acid..........two drachms. Mix.

Should the stitches tear out, you may replace them a second or third time. After that it will be useless. While the stitches are intact, place the animal in a narrow stall to prevent her lying down. If there is much bleeding when the laceration occurs, arrest it with cold water; if that fails, apply tincture of persulphide of iron. Feed on soft, sloppy food, and wive frequent injections of pure water, to soften the dung and thus relieve the strain on the perineum.

\section{METR0-PERITONITIS, OR INFLAMIMATION OF THE WOMB AND PERITONEUM.}

This disease affects animals of all ages, and results from difficult parturition, retention of the fortal membranes, inversion of the womb, exposure to wet and cold, drinking ice-cold water, etc.

Symptoms :-The disease may occur from within a few hours after parturition up to the fifth or sixth day' At first the animal will appear dull with loss of appetite; visible mucous membranes injected red; secretion of milk dimished: pulse quick, full and hard: bowels constipated; urine diminished in quantity; colicky pains 
are present, manifested by frequently lying down and gretting up until lying down becomes too painful, from the pressure of the abdominal muscles on the womb; striking at the belly with the hind feet; pointing with the nose at the flanks; respiration accelerated; abdomen tucked up. As the disease advances, the pulse srows small, feeble and wiry; the animal becomes insensible of objects around her and plunges about with great violence; death shortly follows.

Treatment:-First examine the womb. If any of the membranes adhere, they should he removed at once. Wash the womb out thoroughly with warm water, to which add a little carbolic acid-one part of acid to forty of water. This should be repeated twice daily. If the bowels are constipated and the patient is a mare, give the following physic at one dose in half a pint of water, as a drench:

Alnes................. one ounce.

Calomel............... one drachm.

Ginger............. one-half ounce. Mix.

For the same condition in a cow, give at one dose in a gallon of water, the following:

Magnesia Sulph.......twenty ounces.

Calomel ..............two drachms.

Ginger................ one ounce. Mix.

After which give every four or five hours one of the following powders:

Quinine Sulph........ four drachms.

Salicylic Acid......... four drachms. Mix.

Make into six powders. Or the following, griven every four hours, will be found beneficial:

Liquid Ammonia Acetate......... three ounces.

Fluid Extract Aconite...twenty drops. Mix. 
If the animal's temperature remains high, place a sheet, wet with cold water, around the body and keep wetting it with a sprinkling can for an hour, after which rub the animal dry and place warm, dry blankets over it. When convalescence has occurred good food and tonics should be administered. Give one of the following powders three times a day:

Powdered Sulphate of Iron........ .......... one and one-half ounces.

Nux Vomica...........two ounces.

Ginger................. one ounce.

Potassium of Nitrate............

......... one and one-half ounces. Mix.

Divide into sixteen powders. While the pain is very severe, the appliration of mustard to the abdomen is very beneficial. After the disease has abated, if there is a chronic dischare from the womb, the treatment given for leucorrhma will suffice. The person examining the womb should be very careful, and sec that there are no fresh sores on his hands or arms and wrease them thoroughly to prevent inoculation and blood poisoning.

\section{UMBILICAL HEMORRHAGE, OR BLEEDING FROM THE NAVEL.}

Bleeding from the umbilical cord, of an alarming nature, is not a very common accident, nevertheless it sometimes occurs and occasionally so profusely as to cause death. It generally happens immediately after birth or within a short period afterwards and may continue for some time in small quantities, but sufficient to produce great debility. The bleeding is generally ralised by the cord's being cut or broken ofl too close to 
the abdomen; it may be due to a raricose condition of the cord. sometimes, after the cord is tied, when sutliciently long enough to admit of it, the mother will nip it off closer and so induce bleeding.

Treatment:-When the bleeding is slight and not likely to be continued, little or no treatment is requiresl. If possible, seize the cord and tie; if not apply tannic acid, alum, or per-chloride of iron and bandage, after placing some cotton batting saturated with Monsell's solution of iron upon the cord. Or you may take a needle and thread and pass it through the skin on both sides of the cord, then tie it tightly and you will completely close the opening, hut in doing this be careful not to penetrate or include the intestines.

\section{PERSISTENCE OF THE URACHUS, OR URINE PASSING FROM THE NAVEL.}

What is understood by the urachus, is the canal formed by the middle portion of the allantois, which passes through the umbilical ring and communicates with the bladder through which the urine is conveyed to the allantoid sack, during foetal life. After birth the urachus should become obliterated and the bladder should be retracted and further developed within the pelvic carity, the urine then passing through the natural channels. It sometimes happens the urachus does not close up and disappear, and the urine is passerl through the navel. It more frequently occurs in male; than in females. In the latter it generally comes by drops and quite frequently ceases spontaneously, whils in the male it usually rums in a stream and is more diatgerous. 
Treatment:-In a great many cases no treatment is required, the escape of urine ceasing in a few days after birth; but, where treatment is demanded, a cure can usually be effected. If the urachus is sufficiently long, seize it and tie it. If this cannot be done, grasp the cord between the thumb and fingers and raise it up; then pass a stitch in a circle clear around the cord and tie it tight. The urachus is then closed. But be careful not to gather any more skin than is actually necessary into the stitch. If the urethra, or natural channel for the urine to pass by, is not opened, get the longest human catheter obtainable and pass it to open the channel.

\section{UMBILICAL HERNIA, OR RUPTURE OF THE NAVEL.}

Rupture of the navel is most frequently congenital, although it sometimes ocurs two or three months after birth, when it would be called accidental hernia. A small, round tumor, situated at the navel opening and varying in size from that of a bird's egg to that of a goose egg or even larger, is observed. It is usually soft and fluctuating and easily reduced within the abdominal cavity by manipulation with the fingers, just as readily returning when the hand is removed. Sometimes it presents a doughy or hard appearance, when probably the intestines within the sack are filled with alimentary matter. When the intestines are returned, the opening will present a circular or elliptical shape, and around the edges it will be tense and hard to the touch.

Treatment:-In a great majority of cases when the tumor is not very large, if allowed to remain undisturbed nature will bring about a complete cure varying in time from three months to three years of age. I have 
applied a broad bandage with very gratifying results and would recommend it strongly. Constriction of the tumor is an excellent method of effecting a cure. First cast the animal and secure it firmly, place it on its bark and maintain it in that position. Then manipulate the tumor until the intestines are returned to the abdominal cavity. Next grasp the skin over the tumor and raise it up as far as you can stretch it, and fasten a pair of clamps as close to the abdomen as possible. Fasten them tightly. Then put a couple of wooden pegs through the skin, protruding beyond the clamp, to keep it from slipuing off when the animal is standing. In this way you set up an adhesive inflammation, forming a plum filling up the opening by the time the clamp sloughs ofl. Some practitioners recommend the application of nitric acid to the tumor and report good results; but I have never tried it. But to inject into the sub-cutaneous connective tissue a few drops of salt water, around the rupture, will, in a great many cascs, through irritation set up a healthy inflammation, throwing out a plastic sulsstance, filling the cavity, thus foreing the intestines back within the abdominal carity and completely jolusgring up the opening. This operation can be done with the ordinary hypodermic syringe, care being taken not to insert it too deep.

\section{RETENTION OF THE MECONIUM, OR CONSTIPATION SHORTLY AFTER BIRTH.}

The contents of the intestines previous to birth is ealled meconium. This is generally expelled immerliately after birth; but when it is retained for any length of time, serious results follow if left unreliered.' This occurs, perhaps, more frequently with the colt than with any other animal, but occasionally happens with 
calves. It generally oceurs in animals that are weakly and not thoroughly developed. It is grenerally caused by the mothers being fed on dry fodder for a length of time, or by her milk heing thin and not rich in those purgative elements so necessary for the newly loru animal. Hence, it is generally seen in those colts and calves that come in the winter and early spring, previous to the mother being turned out to pasture.

Symptoms:-iWithin a few days after birth, the little animal appears uneasy and refuses to suck; shows symptoms of colic, lies down and rolls around, oceasionally looking toward the abdomen; when standing, the back is arched and attemps to pass manure and water are frequent; pulse and respiration are frequent and sometimes the respiration is labored; the eyes are injected red; grinding of the teeth is a frequent symptom.

Treatment:-The proper treatment is to attend to the food and condition of the mother, previous to parturition. The diet should be soft and nutritious. Immediately after birth, the young animal should be fed the first milk the mother gives, as it contains purgative elements so necessary for the movement of the bowels. If the bowels do not move, remove all the fuecal matter you can with the oiled finger, after which give frequent injections of warm water and castile soap, or injections of oil. Feed the mother on a soft, sloppy diet, to which may be added a mild physic. Should the bowels firil to respond, give two ounces of castor oil, to which you may add a dessertspoonful of brandy with a little sweetened water; or you may give every two or three hours, two ounces of cod-liver oil with a little brandy and a little sweetened water. Keep the little creature warm with blankets, rub the limbs with dilute alcohol and bandigi.. 


\section{DIARRHOEA IN COLTS.}

This disease consists of a watery discharge from tho: bowels, differing somewhat both in color and ingredients from the regular natural discharge. (2uite often it will be strongly acid, as is demonstrated by its scalding the hair off from the surrounding parts and emitting an offensive odor.

Causes:-It is produced from many causes; such as an altered condition of the mother's milk through some unknown or unforseen agency; or by the mother eating too rich and highly nutritious food, or just the reverse -by being furnished with a scanty amount of poor, innutritious food, or bad, deficient, improper food: also from an excessive amount of lime salts in the food; milk that is too rich in fatty constituents; permanent stabling; defective stabling; chills or stable miasma. It is most frequently seen where the young creature is permanently housed, and seldom observed when out to pasture, thus indicating very strongly that the oflending agent is in or about the stable, and is properly called a stable miasma. Another circumstance must be borne in mind, that is, when one young animal becomes badly affected, other colts or calves that are around the same stable, soon berome affected, thus giving rise to the theory of contagrion, or infectious nature of the complaint.

Symptoms:-This disease generally makes its appearance quite suddenly and without any direct or assignable cause, as the food and water as consumed by the nother, may not have been changed or altered in the least. : But within eight or ten rlays after birth, the diarrhœa manifests itself in a riolent form. - The passaces are very watery in character, and have a sour, offensive smell. They are eitheryellow-tinted, whitish, or a grayish green color, but more often whitish. The 
bowels move quite frequently and with some force, as the discharge will squirt some distance. Sometimes the animal will cease sucking, while others again, will nurse as though there was nothing the matter, except that they will switch and rub the tail, from the irritation produced where the hair and sometimes the skin has been scalded off from the effects of the discharge from the bowels. In those cases where they cease to suck, they fail in strength very rapidly and are nearly always lying down. The eyes are sunk quite deeply within their orbits and appear indifferent to surrounding objects; the hair looks dry and staring. There is neither bloating nor rumbling of the bowels, and the belly does not seem sore nor painful when pressed upon. There may be some straining, and as the end approaches, the little creature remains immovable, and dies without even a struggle.

Treatment-As is the case with all other diseases, preventative measures are always better than curative treatment. Attention should be given to cleanliness and diet at all times, but more especially is this the case, when this disease has appeared in the stable before, or is now present; and all pregnant stock should be removed from the stable, when this is the case, a month prior to producing their off-spring, in order to render them secure. The stable or shed in which this disease has occurred, should be thoroughly washed and disinfected with carbolic acid, chloride of lime or sulphuric acid, paying particular attention to the floor, where the infectious material may remain for months.

It is good policy not to use the stable after disinfecting it, for the balance of the summer. The curative measures employed, are many and varied." Such as have proven beneficial, I will here insert. First, change the mother's diet. If fed too highly, reduce a little; or if 
scantily fed, increase her rations, and if in season, do not fail to turn out to pasture. If the colt or calf is strong and lively, give an ounce of castor oil to clean the bowels of any irritant that may be within them, to be followed with a couple of raw eggs, every four hours until six or eight eggs are given, and if this fails to check it, a few doses of white oak hark tea may be given. Or give,

Prepared Chalk.......... one ounce.

Powdered Catechu..... four drachms.

Powdered Opium........ one drachm. Mix.

Make fifteen powders. Give one powder every three or four hours, in a little milk. Or better still, give-

Powdered Opium.........ten grains.

Tincture of Cardamon...ten drachms.

Sulphuric Ether........two ounces. Mix.

Give dessertspoonful every four to six hours, in four to six ounces of linseed tea or gruel.

$\mathrm{Or}$,

Laudanum.............. one ounce.

Tincture of Catechu....... one ounce.

Sweet Spirits of Niter...... one ounce.

Paregoric.............. one ounce. Mix.

Give dessertspoonful ever hour or two until relief.

Burnt flour sometimes aids materially. Keep the patient warm and comfortable and if pains in the bowels are exhibited, apply cloths wrung out of hot water to which may be added some camomile tea or flowers, to the belly, which will greatly assist in easing the pains. 


\section{PRESENTATIONS AND POSITION OF THE FOETUS.}

We have before spoken of normal parturition, where the foetus was presented in a natural position and the mother had power to expel it. We will now consider the various presentations and positions of the footus during parturition. They should be well studied, as the different modes of delivery are of much importance practically, to the obstetrician. When we remember that during gestation the fotus in the womb assumes so many different positions, it is not surprising that in delivery so many different presentations occur. Notwithstanding, there is supposed to be some agency or influence, so induce the foetus to assume a natural position and presentation at delivery, this is not always the case; and, instead of the fotus being in a favorable position, it frequently requires assistance to change the false position assumed by the young animal at the termination of gestation.

What is understood by presentation, is that part of the fœetus which presents itself first at the pelvic inlet, or that part of the foetus which the hand of the operator touches when making an examination as to positionwhich may be the head, forefeet, hocks, breast, abdomen, sides, etc. Presentation may vary exceedingly, as any part of the foetus may occupy the space at the mouth of the womb. I will condense and simplify as far as possible, the description of these presentations and positions, in order not to confuse the mind of the reader.

Presentations may be grouped in four classes, viz.: Anterior Presentation, when the head, neck, chest, forefeet or forelimbs are presented; Shoulder and Loin Presentation, when any portion of the body is presented, as the shoulder, withers, back, loins or hauuches; 
Breast and Ahdominal Presentation, in which the limbs are most frequently felt first, possibly all four feet, or only three of them, and sometimes only one; Posterior Presentation, in which the breech or croup is facing the inlet and the presence of the limbs there only constitutes a different position.

The different positions in which a fotus may be found with any of these presentations, are numerous. For instance, with anterior presentation, the withers of the foetus may rest against the spine of the mother, and the breast against the lower part of the pelvis; or it may be just the reverse; or the fotus may be lying on its right or left side, when its ribs would rest against the mother's spine and against the lower part of her pelvis, the breast facing either the right or left ileum, and the withers the opposite; the head may deviate to the right or left; one limb may be retained, or crossed over the neck; or both limbs may be retained, etc.

\section{ANTERIOR PRESENTATION.}

This is the only natural presentation of the fotus, and in this presentation, the different positions of the young creature frequently require the aid of art to complete the delivery. But when the presentation is natural and nature has brought about the necessary changes in the generative organs of the mother, delivery is comparatively easy and successful. In this position the fotus at the inlet should present the forefeet first, with the head lying upon or between them, the withers toward the spine of the mother, and the breast resting upon the pubes. When the fotus is found in this position, it should be left undisturbed for a time, as the contractions of the womb gradually increase in force. 
The abdominal muscles are now brought into play, the animal makes a few extra efforts, and, generally, the foetus is expelled. But, if the mother is lahoring hard and the progress is very slow, man's intervention is

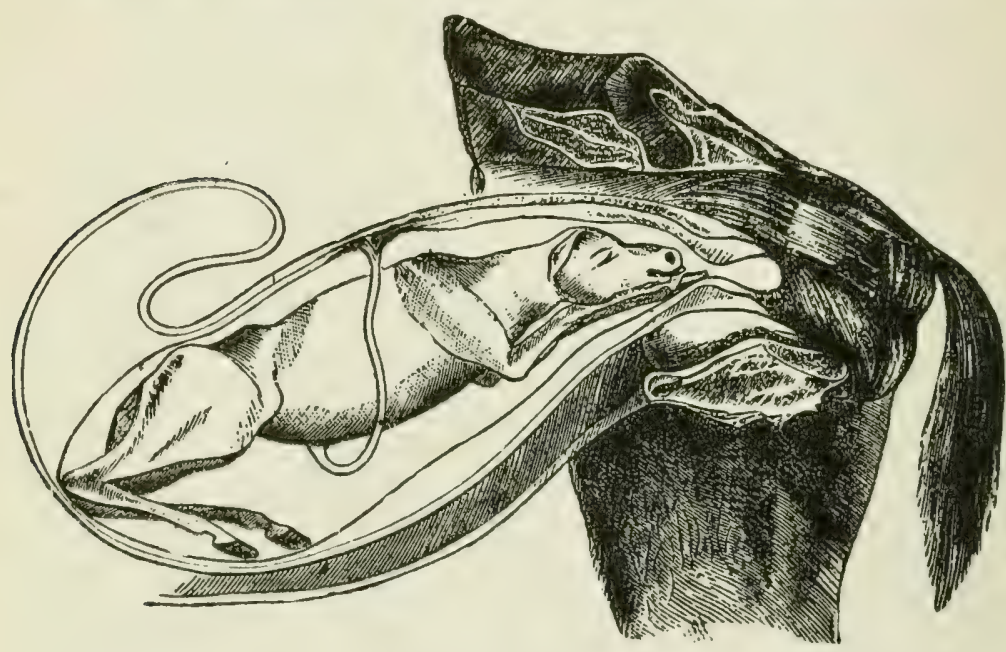

ANTERIOR PRESENTATION.

necessary. You should then seize the young animal by the forelegs, and, at each succeeding labor pain, use traction until the little creature is born. In using traction, always pull outward and downward towards the mother's heels. Immediately after the birth, tie up the navel about three inches from the abdomen. Then allow the mother to have care of her young.

\section{FORE LIMB CROSSED OVER THE NECK.}

It is not at all uncommon to find colts and calves in this position, and delivery very much retarded or incomplete without assistance. Thouch I have no doubt that delivery has been completed in this position 
without assistance, the atses where the mother has not. been more or less lacerated, are exceptional. In this position, instearl of the shoulders being lodger in the hollow spare along the side of the nerk, they are held back along the side of the chest, increasing the transverse diameter als it enters the pelvic inlet, instead

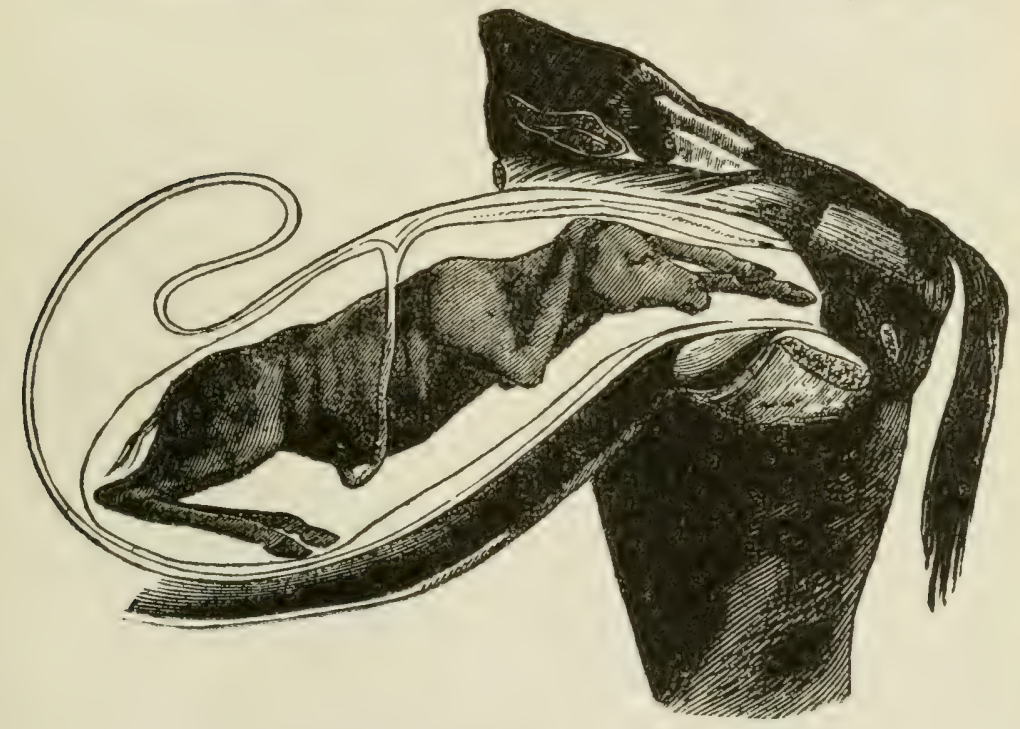

FORE LIMB CROSSED OVER THF NECK.

of diminishing it, thus retarding the delivery. But if the limbs are crossed over the neck up at the poll, it is still more dangerous. In all complications tending to retard delivery, the mother becomes more excited and the labor pains more violent and irregular. Then with the limbs crossed at the poll, the feet are pushed against the roof of the ragina, causing laceration of that part. Sometimes they are pushed through the roof of the ragina into the rectum, producing a rupture of the perineum. In the more farorable cases, the limbs are 
crossed far cnough over the neck to allow the knee to become flexed, turning the feet downward. There is then lout little danger of rupture of the perineum, but the labor is protracted and severe and certain to result in contusions of the vagina, to a greater or less extent. This position is frequently recognized hefore an examination is made. If the forefeet are seen pressing against the roof of the vagina, at the outlet, the probabilities are that both legs are crossed over the neck. If only one foot is sen pressing the roof of the vagina, or if both feet are olsserved on one side of the head, the one leg shorter than the other, only one leg is crossed. If only one leg is crossed, it may be placed in position without much difficulty and without the trouble of pressing the fœetus hack into the uterine carity. Seize the leg above the fetlock, raise it up and draw it to its proper side; then place one hand on the head of the foetus and hold it firm, with the of her hand extend the leg forcibly, when it will readily straighten out into position. Delivery may then take place without help, or a little help with the head and limbs may be beneficial. When both fore limbs are crossed over the head, the case is more difficult to handle. If the feet are visible, fasten a half inch rope to each separately, then force the fnetus back within the womb by pressing upon the head. Then the operator should place the feet to their proper sides, if possible. If he cannot do this, bring the ropes to their proper sides, and, while the operator holds the head back firmly, let an assistant pull the rope on one side until that limb is brought into position. "Then take hold of the other rope and fetch that into position. Then place the head straight in a line above and between the limbs, and delivery will be eflected with but little help. 


\section{FORELIMBS FLEXED AT THE KNEES.}

In this position, the difliculty, as a rule, is easily overcome. When the lator pains first commence, if the fect are not lifted high enomgh to enter the pelvic cavity, they become lodged aminst the lower border of the pubes; and, as labor-progresses, the head is forced onward, the feet being retained, the fore legs become

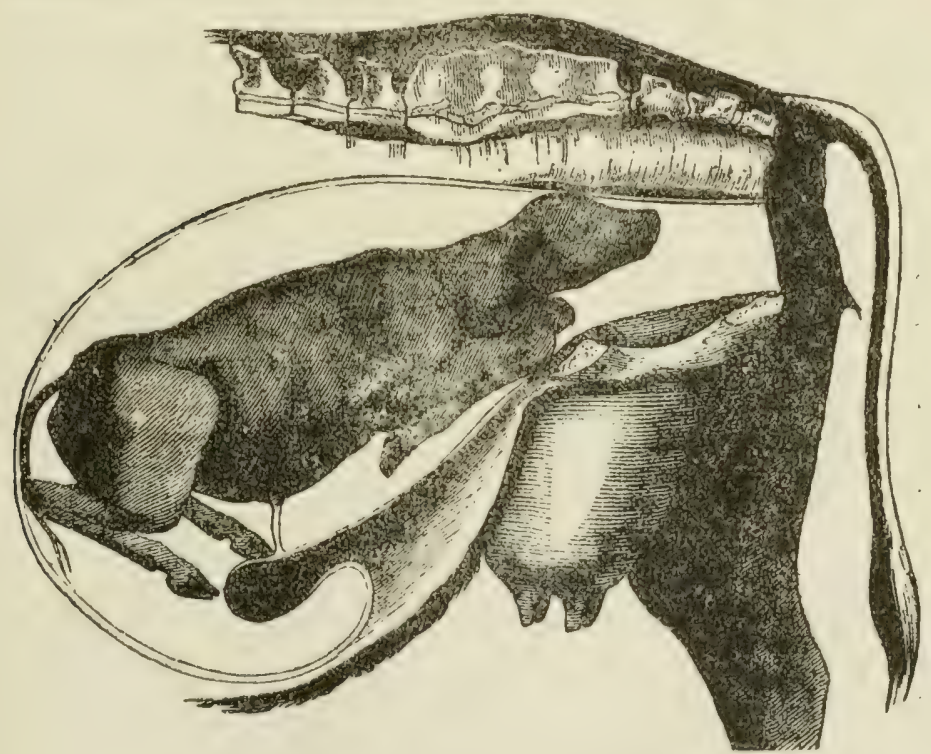

FORELIMBS FLEXED AT THE KNEES.

flexed at the knees and there they remain with the knees pressing up against the lower part of the neck. If an examination is made before labor has progressed any length of time, the nose and knees will be felt at the same time. But if labor has progressed any length of time, the head will be forced farthrr out. Then the operator, on exploring, will first come in contact with the head, for sometimes it is advanced in the passage 
even as far as the outlet. The object now to be obtained is to straighten the forelegs, in order to complete the delivery, as the vertical diameter of the pelvis will not admit of delivery with the fore limbs beneath the breast. The operator will have but little difficulty, if called early enough, or before the head has passed any great distance into the vagina. If the head has made but little progress, the operator, upon examination. will feel the krrees as readily as the head. While ascertaining the position of the legs, you will almost always find that one has greater liberty than the other, and, if selzed by the fetlock, can be thrown easily into its natural position. Then seize the other leg just below the knee, raise it up with the lower part of the hand, while pressing backward with the upper part of the hand, and that leg can be brought easily into position. Use the right hand to handle the left foreleg, and the left hand for the right foreleg. If the head has been forced well into the passage or presented beyond the outlet, the case will have to be handled differently. First fasten a small, well greased rope around the neck, then grasp the head, and, during the intervals between the labor pains, force it back into the womb. Then grasp the legs separately below the knees and raise them up, pressing backward at the knee and forward further down the leg, and in this way bring them into position. If they cannot be brought up in this manner, fasten a small rope around the fetlock of each leg, then bring them into position separately. The operator must take hold of the knee and force it back into the womb, while an assistant draws on the rope. In this manner, you will force the leg upward and outward, into the proper position. Then fetch the other limb up in the same manner. If the head is presented beyond the outlet and the little creature is dead, the only thing to be 
looked after is the preservation of the mother. First eut off the head close to the skull, then cut a hole through the skin and flesh about six inches back on the top of the neck, and guide the knife so as to pierce between the first two bones of the neck. Then pass a small rope through the first bone of the neeclis and

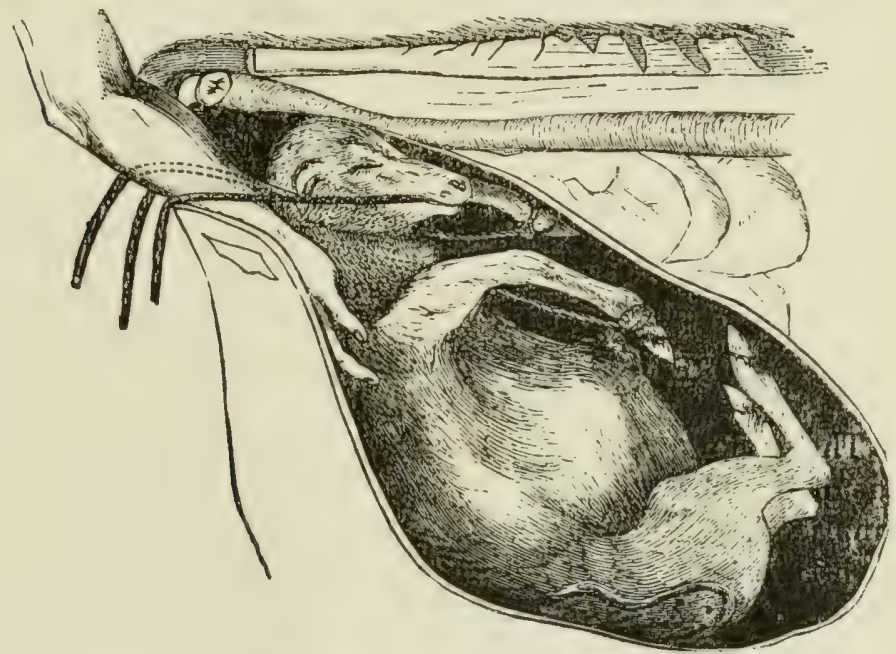

FORELIMBS COMPLETELY RETAINED-REVERSEU POSITION.

through the opening, and tie it. You then have a hold that will stand a pull. You must then force the foetus back into the womb, raise the forefeet as described, fetch the neck into position and deliver.

If the colt or calf is lying upon its back, with the legs turned upward within the womb, it will be impossible to extract it in that position. It must be turned, which will prove a difficult task. In this position a great deal depends upon the judgment of the operator. If the mother is lying down, the hind parts should bo raised higher than the fore quarters. Seize the futus and fasten a small rope around each fore fetlock. and 
another around the jaws. Then, if the fotus is slightly turned to the left, let the operator pass his right hand under the left shoulder. Have the rope attached to the right leg, pass out on the left side of the head. Then, at each labor pain, draw tightly upon the rope, and with the hand under the shoulder, raise up with a rotary movement. In the course of time you will completely turn the little creature around to a proper position. Proceed in a manner precisely the reverse, if the fœetus is turned to the right. When the proper position is gained, delivery will be easy. If the mother can be kept in a standing position, the fœetus can be much more easily handled.

\section{FORELEGS COMPLETELY RETAINED.}

The complete retention of one or both of the fore. legs of the fotus within the womb, with head presentation, is frequently met with in veterinary practice. With the mare the case is somewhat serious, the colt usually dying before delivery is completed. But with the cow the danger is not so great; the calf, in all probability will be delivered alive. The position is, no doubt, assumed during birth in the same manner as knee flexion. It is possible for the fœetus to be delivered in this position, but it would have to be very small, and the vagina of the mother very large and roomy. This position is more frequently seen in the colt than in the calf, a fact probably due to its having much longer legs. In making an exploring examination, to determine the true position, the hand will touch first the head, sometimes at the inlet, at others further outward within the raginal passage. If both limbs are completely retained, the head may be forced well into the vaginal canal or 
even to the outlet, at each labor pain, but retracting immediately after each pain ceases. Sometimes it is with the greatest difficulty that the forelegs are reached, owing to the rast dimensions of the womb, although the arm may be introduced without trouble. When the

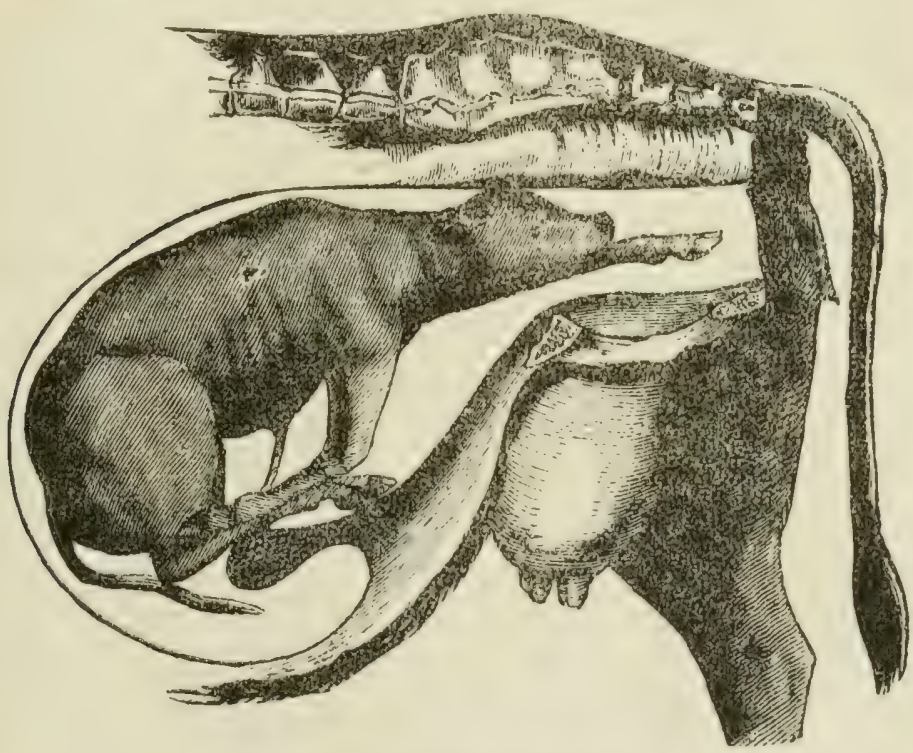

ONE FORELIMB RETAINED.

legs can be felt, they are usually lying immediately under the abdomen or beside the chest and flanks. The main object now, is to reach the legs with the hand and bring them forward to the knee position first. In order to do this, it may be necessary to force the head back within the womb.' If so, first place a rope around the neck and lower jaw, then foree it back within the womb during the intervals between the labor pains. Then pass a rope around the formarm of the colt or calf, pushing it well down toward the knee, and use gentle traction until you bring the knee 
up to the passage. You may then fasten the rope to the fetlock, and have an assistant to use traction upward and outward, while you grasp the knee and force it upward and backward within the womb. Next bring the other leg into position, in the same way. In this manner the forelegs are lrought into proper position. Then you should find the head and place the hand

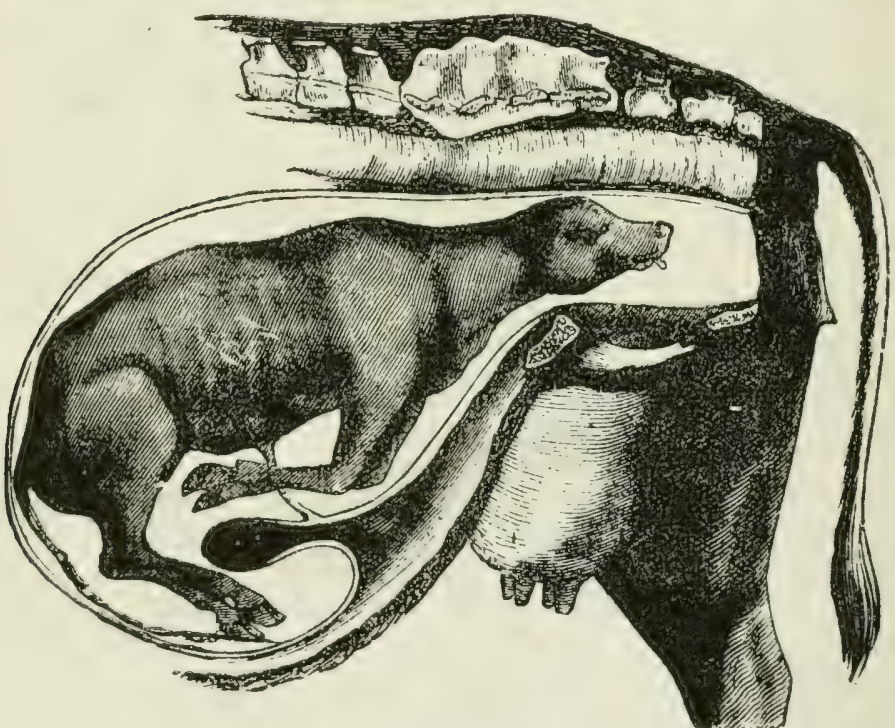

FORELIMBS COMPLETELY RETAINED.

under the nose and lower jaw, and have an assistant pull upon the rope which was fixed to the jaw, until the head is brought into position, but little force may be required after this to complete the delivery. If the head presents beyond the outlet and the foetus is dead, you should remove the head before forcing it back within the womb, (this operation is described under the head of "Forelegs Flexed at the Knee.") Then proceed as before described. Indeed it is sometimes nec- 
essary to decapitate a living foetus, in order to save the mother. To place a rope around the neck in this position and apply force without placing the fuetus in position, is brutal, and the law for the prevention of cruelty to animals, should be rigidly enforced in such cases.

\section{DOWNWARD DEVIATION OF THE HEAD.}

Downward deviation of the head, may exist from mere tlexion of the head on the first hone of the neck,

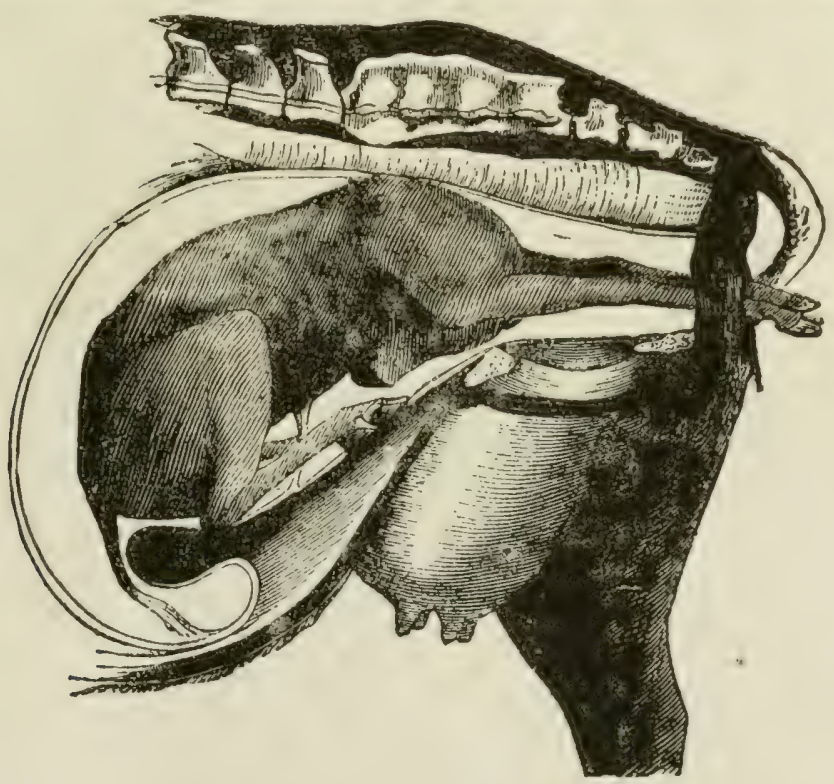

DOWNWARD DEVIATION OF THS HASW.

the nose being caught on the lower bone of the pelvic inlet. But when the downward deviation is great, the upper part of the head comes in contact with the lower border of the inlet and is forced down 
beneath the chest and abdomen. The cause usually, is premature rupture of the futal membranes, and the nose or head catching on the pelvic border as the fuetus is entering the inlet. If the feet are in a proper position and the nose catches on the lower border of the inlet, the contractions of the womb will force the upper part of the head and neck into the genital passage, along with the feet. Then the operator, on passing the hand to explore matter's, will come in contact first with the feet and next with the top of the head. During the intervals of labor, he should press backward and upward against the top of the head; then pass the hand down under the nose, seize the lower jaw and raise upward and outward. The head will be easily thrown into the natural position, when delivery may be completed in the usual way. But, if the pains have forced the head and neck outward, so that they are firmly wedged in the passage, take a crotch and place it against the shoulder, and force the foetus back within the womb a distance sufficient to aldnit of raising the nose and placing the head in a proper position, to allow a natural birth. This may take some time, but can be accomplished.

When the top of the head rests against the lower border of the inlet the case is a little more diflicult, but can be brought into position hy passing the hand down to the nose and fastening a rope to the upper jaw. Then let the operator press backward on the side of the neck and an assistant draw upward and outward on the rope, and most frequently the lead will be brought into position. But, if labor has been progressing some time and some wise fool has been pulling at the legs, the head will be forced down underneath the chest and, possibly as far back as the abdomen. You then have one of the most critical cases to deal with. Incleed, so critical is 
the case, you may consider yourself extremely fortunate should you complete a delivery and save the life of the mother. You may take a couple of blunt crotches, and place them against the shoulders of the fortus and push it back within the womb. If you succeed in this, you may reach the hear, which you may fasten with cords, as before described, and raise it up. If you fail in this, gou may return the forelimbs and try to turn the fnetus around and deliver the hind feet first. Failing in this, you may cut off the head, when you feel the neck, and then delicer: or, amputate the legs at the shoulder and then deliver. L'se plenty of fresh lard and handle the knife carefully.

\section{LATERAL DEVIATION OF THE HEAD TO THE RIGHT OR LEFT.}

In this position, the head is carried either to the right or left side of the fœetus, and the forelegs pre. Eented either in the genital passage or at the outlet. It

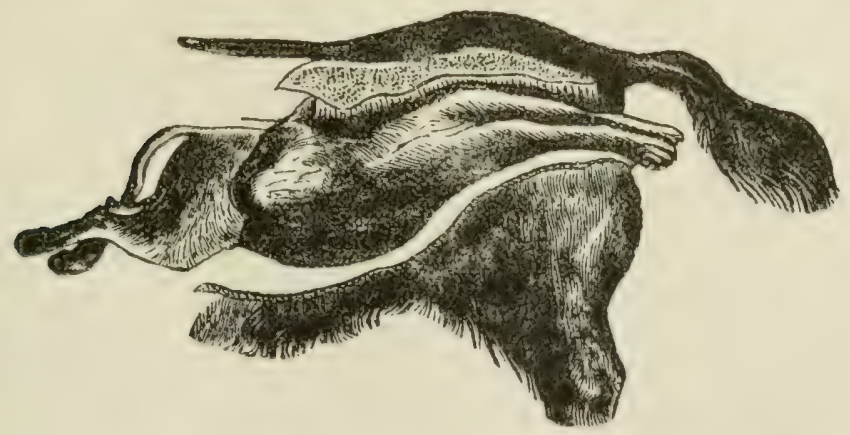

LATERAL DEVIATION OF THE HEAD TO THE PIGHT OR LEFT IN AN UPWARD POSITION.

is a very difficult case to handle. It may be caused by uterine contraction taking place too strongly, before the 
mouth of the womb is thoroughly dilated. If the nnse is not in a straight line with the opening, when partially dilated, the forefeet may pass through and as the uterine contractions increase in force, the head is forced off to one side. "Sometimes it rests back on the shoulders, at others down along the back or sides, and

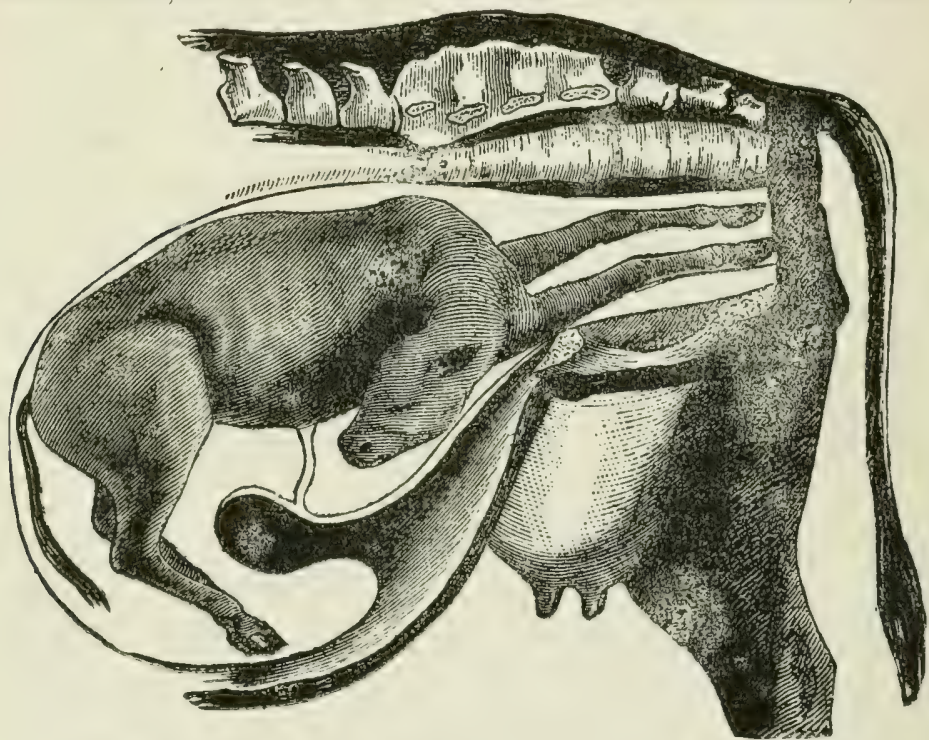

LATERAI DEVIATION OF THE HEAD TO RIGHT OR LEFT-

DOWNWARD POSITION.

it may be down by the flanks of the mother. I have delivered two cases when the deviation was to the right side and the colts were wry-necked and could not be straightened after delivery. One was born dead and the other, to my knowledge, lived for two years with a crooked neck. It was then sold and taken out of the neighborhood. As a rule, it is not hard to distinguish this position of a colt or calf. The forelegs will be presented in the genital canal, but birth is retarded and on 
the side to which the head is turned, the leg will appear shorter than the other. On being passed beyond these, the hand comes in contact with a bulky mass occupying the whole space of the inlet, rendering access to the uterine cavity difficult. Upon examination this will be found to be the neck; and, by tracing it up, the ears,

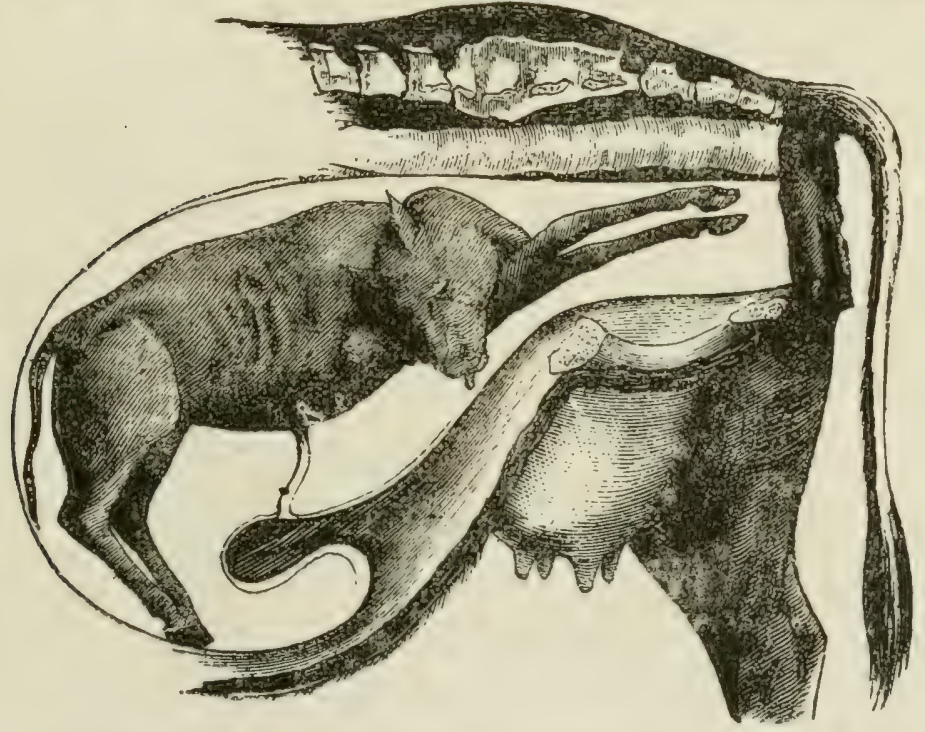

LATERAL DEVIATION OF THE HEAD TO THE RIGHT OR LEFT.

eyes and frequently the nose can be felt, -if a calf. But, if a colt, it is seldom that the nose can be felt if the uterine contractions have continued for any length of time. The colt's head will be pushed hack toward the chest, abdomen or flanks, when it scarcely can be reached. This position is not only frequent but serious, as it is impossible for birth to occur without assistance, and this requires strength, perseverance and ingenuity. The object now is to get hold of the head and hring it into a favorable position, and then complete the 
delivery. But this eannot be done very easily, especi ally when the head is lying along the abdomen or down in the flank and beyond reach of the operator. If the head can be felt hy the hand, you should catch hold of the cheeks, by putting your hand into its mouth, and raise it into position. Failing in this, fasten a rope around the lower jaw, then secure the legs with ropes and push the foetus back into the womb. Then have an assistant pull on the rope attached to the jaw, while the operator pushes inward on the neck just at the bend. The head will readily be brought into the vaginal passage. Then draw up the feet and complete delivery. In case the feet present and the head cannot be reached by the operator, first fasten small ropes to the fore fetlocks. Then return the legs to the womb, pressing them to the opposite side from that to which the head is bent. Then if the head is turned to the right, press against the left shoulder, and vice rersa if it is turned to the left. With the hand or a crotch, you can fasten a rope around the neck. Pass the rope along the neck as near the head as possible, then twist the rope until it presses deeply into the flesh of the neck, care heing taken not. to entangle the membranes in the rope. Then press inward on the shoulder and draw outwards and sideways on the neck and you will draw the head backward toward the natural position. Continue in this until you reach the head, when you should fasten the rope to the head or lower jaw, after which you can easily bring it into position. Then draw up the feet and complete the delivery. 'sometimes an advantage is sained by securing the legs with cords, then placing a crotch against the neck or upper part of the chest, and forcing the foetus back into the womb, while the operator fastens a blunt hook into the eye or mouth of the fœetus and uses outward traction on the head. But if the 
mouth can be reached, I would prefer catching the hold there. If the fortus is dead and the membranes are dry, use copious injertions of warm water. l'erseverance and good judgment will nearly always eflect a delivery in this position. Let the knife for dissecting purposes, be the very last resort.

\section{DEVIATION OF THE HEAD UPWARD AND BACKWARD.}

In this position, the head will he found more or lese oxtended along the bark, or slightly deviated to one

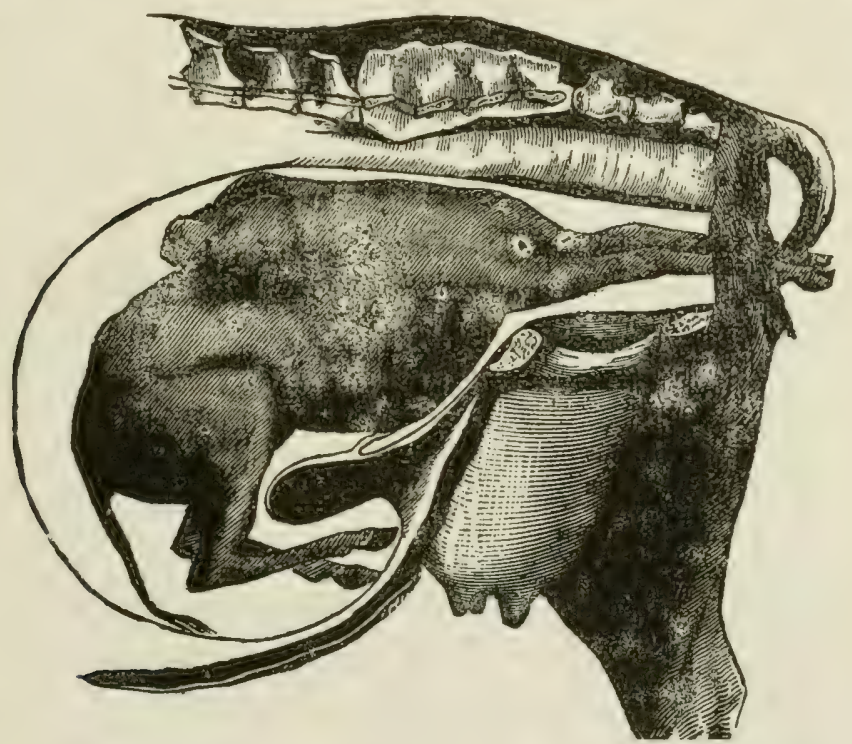

DEVIATION OF TIIE IIEAD UPWARD AND BACKWARD.

side, with the inferior border of the lower jaw resting against the mother's back. "In this position the head may be forced through the womb into the rectum, causing rupture of the periueum, and the foetus may be 
delivered in that way. (On examination, the forefeet will first be felt, possibly well advanced into the vagina, and beyond them at the inlet, the hand meets the chest and, above, the lower part of the neck which, if trared backward, will lead to the head more or less back on the withers and loins or very slightly inclined to one side, the lower jaw facing the back of the mother. In order to place the head in a natural position, it is necessary to force the fotus back within the womb by pressing the chest downward. Should the hear mot irop down into the natural position, introduce the hand and seize the head by the mouth or lower jaw, and pull it downward, slightly sideward and outward, into the natural passage. If this cannot be done with the hand, place a small rope around the lower jaw and have an assistant pull, while the operator guides the head with his hand until it is brought into the natural passage, when delivery will be completed easily. This is not a difficult position and can be rectified with ease and safety.

\section{HIND LEG DEVIATION-ANTERIOR PRESENTATION.}

In this position, the operator may consider the case a natural presentation, which it is, as far as the head and forefeet are concerned. Birth may be proceeding favorahly, the forelegs, head and body being normal and in a natural position, protruding well beyond the outlet; when, suddenly, the foetus becomes locked and wedged within the passage, notwithstanding thei mother increases her efforts at straining; and powerful traction upon the foetus, fails to bring it beyond a certain point.

The operator should pass his hand underneath the abdomen of the fortus, along the genital passage, until he reaches the inlet, where he will find the obstruction. 
Here he may find the thighs of the foetus turned w ward from its flanks, forming too broad a surface in pass thromgh the genital canal. Sometimes he will find tl: hind legs flexed forward, underneatin the belly of thr fortus, and the fect or fetlocks caught under the brim of the pelvis at the inlet, so that to cleliver hy force, would cause the hind legs of the fuetus to force their way throngh the tissues into the ablominal cavity of the mother, before delivery could be rompleted: and this might cause the reath of the mother and ofl-spring.

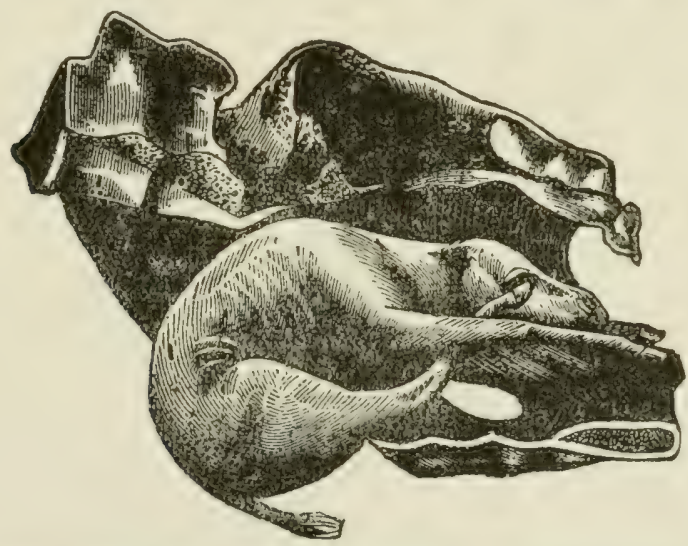

HIND LIMB DEVIATION-ANTERIOR PRESENTATION.

A safe delivery can be eflected only by the proper adjustment of the hind legs. This is all the more difficult, when the foetus is well advanced in the passage and much traction has been employed. If, upon examination, the thighs are extended outward from the flanks of the fuetus, it should be forced back within the womb and the limbs drawn as closely together as possible, with the hand. If this cannot be done, you may take a half-inch rope, well greased, and fasten it around the body of the foetus with running noose. Then the operator should work the 
rope just beyond the rump of the fotus, or midwily between the rump and the hocks. He must hold it there, while an assistant draws tightly on the rope and forces the thighs together. The delivery can then be completed. If, however, the foetus cannot be forced back into the womb to adjust the thighs, cut it off close up to the mother: then force the remainder back within the womb and deliver the hind feet first. But, if upon examination, the hind feet or fetlocks are found to be caught below the brim of the pelvis at the inlet, you should pass your hand between the belly of the fortus and the genital canal, until you come in contact with the feet or fetlocks. Then pass the hand with the palmer surface against the feet or fetlocks, and extend it backward and inward as far as the arm will reach, when traction may be applied to the foetus and delivery completed.

\section{DEVIATION OF THE HIND LEGS INTO THE GENITAL CANAL - THE ANTERIOR PART OF THE BODY PRESENTING.}

In this position we have a serious case to deal with. Generally, the principal object is to save the life of the mother. The foetus generally is dead or in a dying ("ondition, before help is thought necessary to complete the delivery. Outward appearances may reveal nothing but a natural presentation,- - the forefeet and head being presented and sometimes so well advanced, that part of the chest will be presented to view beyond the outlet, when progress ceases, notwithstanding the - riolent efforts of the mother are increased and forcible traction has been used. The operator now has wreat difficulty to ascertain the cause of the delay, the dilliculty depending upon the size of the foetus and the amount of space in 
the genital canal occupied by it. The hand should be introduced to ascertain the cause of delay, which may be very difficult. When the feet are found, they should be examined to make certain they are the hind ones. Sometimes the hind legs are well advanced in the passage, even as far as the hocks; but

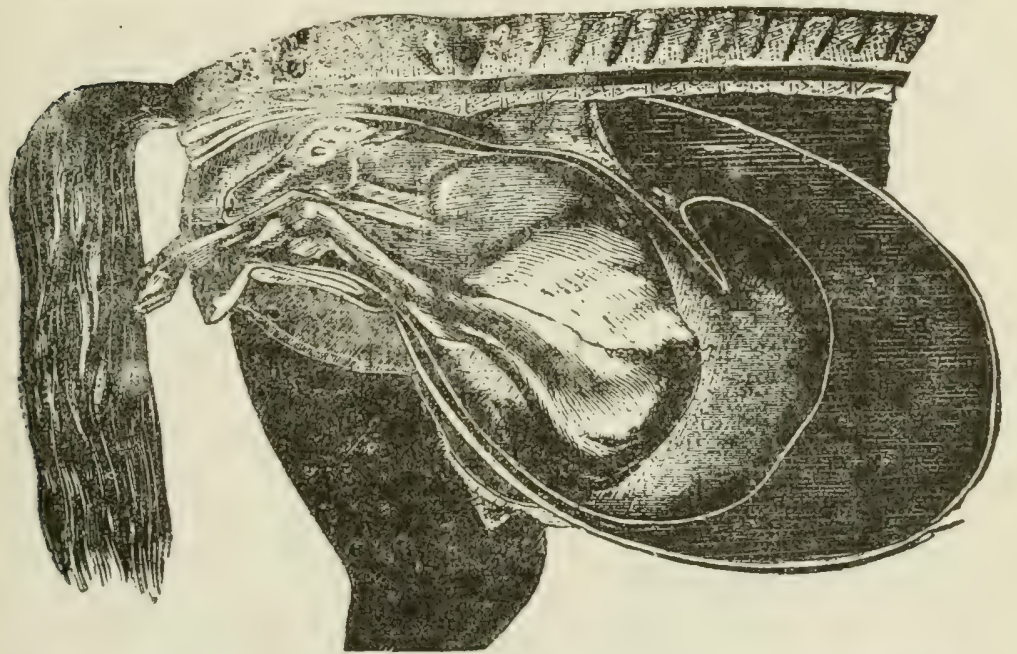

SLL OF THE LIJBS ENTERIXi( TIIS PASSAGE, THE ANTERIOR PART OF THE BODY PIESENTING.

this depends greatly upon the size of the foetus and the capacity of the mother. It is impossible to extract the fotus until this position is rectified, no matter what amount of force may be used; and violent force is certain to injure the mother. It is, therefore, necessary to ascertain the exact state of affairs before using any force. As to saving the life of the fietus, should it be living, your decision generally must be unfavorable. In a great majority of cases the young animal, if not already dead, must be salcriticed to save the life of tho mother. 
If attention is given to the mother before the foetus has adranced far into the passage, and the hind feet are found mingled with the forefeet, the operator will have little difficulty in pushing them back to the lower border of the pelvis. Then push them downward and inward and immediately after grasp the forefeet and heid and hasten the delivery, which can be done successfully. Or the operator may hold the hind feet back, while an assistant extracts the foetus. But if the foetus is well adranced, the forelegs, hear and chest being beyond the outlet, you may try and force it back within the womb until you can reach the hind feet. Then, if possible, push them back within the womb, either with the hand or with a crotch. Then, the head and forelegs being in good position, delivery can be effected easily. When the foctus has advanced so far and is very large, it is very seldom possible to force it back into the womb or even to introduce the hand beside it. The only recourse left then, is to cut the fortus off close up to the mother; then force the remainder back within the womb, turn it around and deliver the hind feet first.

\section{POSTERIOR PRESENTATION.}

In posterior presentation, as in anterior presentation, the foetus may assume several positions, in only one of which, a spontaneous and natural delivery can result. This is when the back of the fnetus is next to the back of the mother, the abdomen next to the lower part of the pelvis and the thighs occupying the horizontal diameter of the genital canal, the hind legs being fully extended outward and entering the inlet, first gradually dilating the mouth of the womb. Birth in this position without assistance, is more frequent with cows than 
with mares, and the calves are generally alive. With the mare, parturition is more prolonged and laborious, and the foal quickly dies after rupture of the memhranes, or is suffocated immediately after birth for want of attention. Colts that are foaled in this position, should be raised by the hind legs until the heal is clear (yll from the wround, to allow the escape of any lluid that may be in the wind-pipe. I have seen as much as

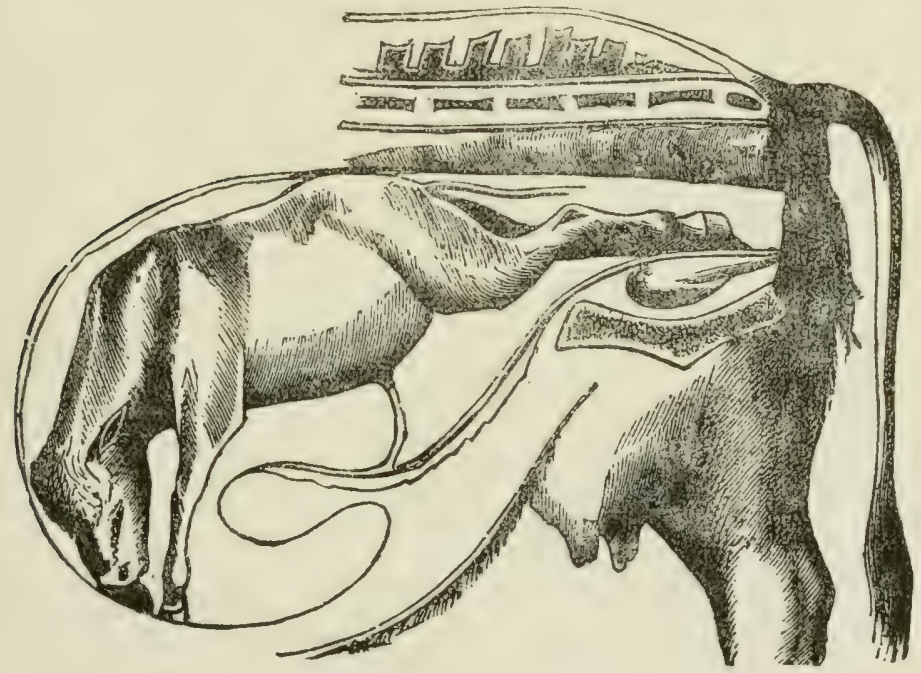

POSTERIOR PRESENTATION, OH HIND FEET PRESENTATION.

a pint escape, and the little animal begin to breathe and do well. When colts are foaled with an anterior Iresentation, the head and neck hang down so that if any fluids have accumulated in the wind-pipe, they escape spontaneously. With the posterior position, it is just the reverse, hence the necessity of raising the lind quarters and allowing the fluid to escape. The foal sometimes perishes quickly after rupture of the membranes. It is therefore necessary, in a posterion: 
presentation, when everything is favorable, to hasten the delivery to save the life of the colt.

In the posterior reversed position, when the hind feet are presented with the foetus lying on its bark within the mother, its belly against the back of the mother, and its back upon the lower bone of the pelvis, there is danger ahead and trouble to be anticipated. As a consequence of this reversed position, the hind legs (which enter the passage first) are directed upward and

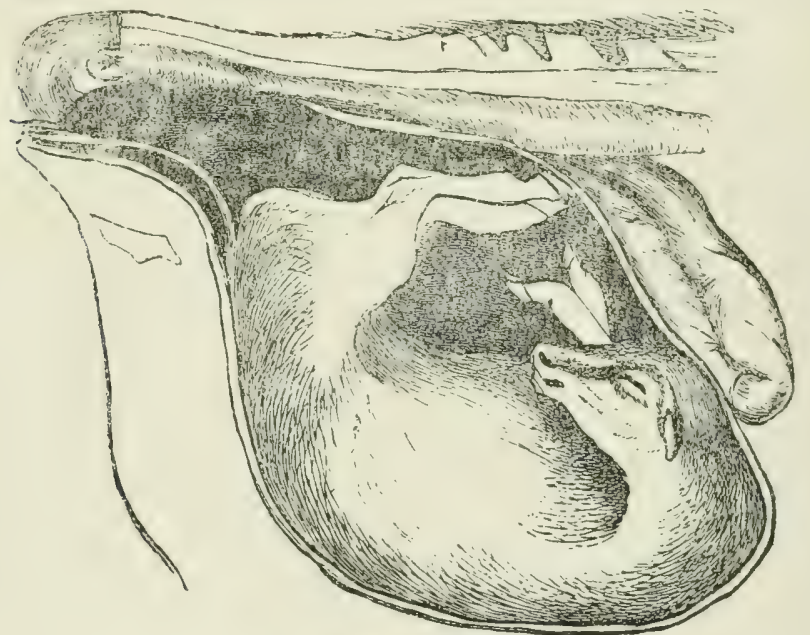

HOCK PRESENTATION-REVERSED POSITION.

outward, and are the parts first met by the hand in making an examination. The operator can make no mistake, if he notices whether the back or front of the hind leg is uppermost. This can be told easily from the shape of the feet or the position of the hocks, the print of the latter being downward, and the bend upward in the same direction as the wall of the hoof. Whereas, the bend of the knee is in the same direction as the sole of the foot. The obstacle to birth, lies in the buttocks 
resting against the lower border of the pelvis, whilst the legs are extended upward towards the mother's back and, to a certain extent held rigid. There is danger of lacerating the perineum; or the feet may be pushed through into the rectum, causing rupture of the perineum. Besides this, the body of the foetus itself forms a curve exactly opposite to that of the genital canal, so that it cannot easily accommorlate itself to the bony canal through which it must pass. From these circumstances, the position not only is unfavorable to birth, but must be remerlied, or serious accidents may follow.

When the foctus is found in this position, the operator must take great care in conducting the feet through the genital canal, to prevent lacerations of the perineum. Sometimes, in order to bring the feet beyond the outlet, it is necessary to pass the hand and raise the horks above the brim of the pelvis. As soon as the feet alpear heyond the outlet, they should be secured with ropes, and, while an assistant seizes the legs and attempts to turn the foetus on its belly, the operator should pass his hand under its rump and assist in turning it over so that its back rests against the mother's bark. When this position is gained, extract the foetus, -if necessary, by forcible traction. If the fotus is small, or if the rump has passed into the cenital passage it should be drawn far enough into the passage to have the rump beyond the outlet; then twist it half way round, or until its back is uppermost, and complete the delivery.- If the fœtus be a colt, delivery must be completed within a half hour; otherwise it is likely to die before entirely born. If it be a calf, it may live for several hours and be born alive. 


\section{HOCK PRESENTATION.}

This presentation is iiable to happen when there is a posterior presentation, and the womb contractions force the fortus toward the inlet, before the hind legs are completely extended. Consequently, the back part of the legs below the hocks, comes in contact with the brim of the pelvis. The croup, heing above, naturally forces the legs into a cramped position. The labor"

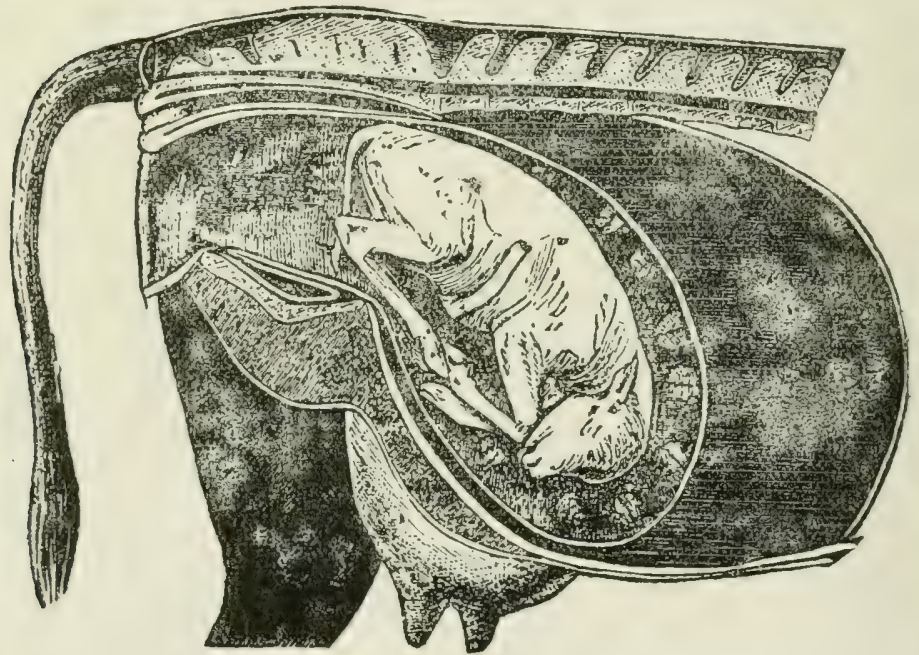

HOCK PRESEN'TA'TION.

pains continuing, the croup has a tendency to descend and, with the horks, to enter the pelvic inlet. The legs now heing thoroughly thexed, the croup pressing upon them they become jammed tightly in the inlet, forming too large a mass to advance further. Therefore labor is suspended and the animal becomes exhausted by continuous straining.

On making an examination in hock presentation, the point of the hork is alway's the tirst part encoun- 
tered: but it may be found at different points. Sometimes it is well advanced in the inlet, the croup and body of the foctus being still within the womb. In other cases, the body and croup are in the inlet and thoroughly wedged in the passagre. As a rule, if the mother has run the full period of gestation, birth cannot take place until the deriation has been rectified; and until

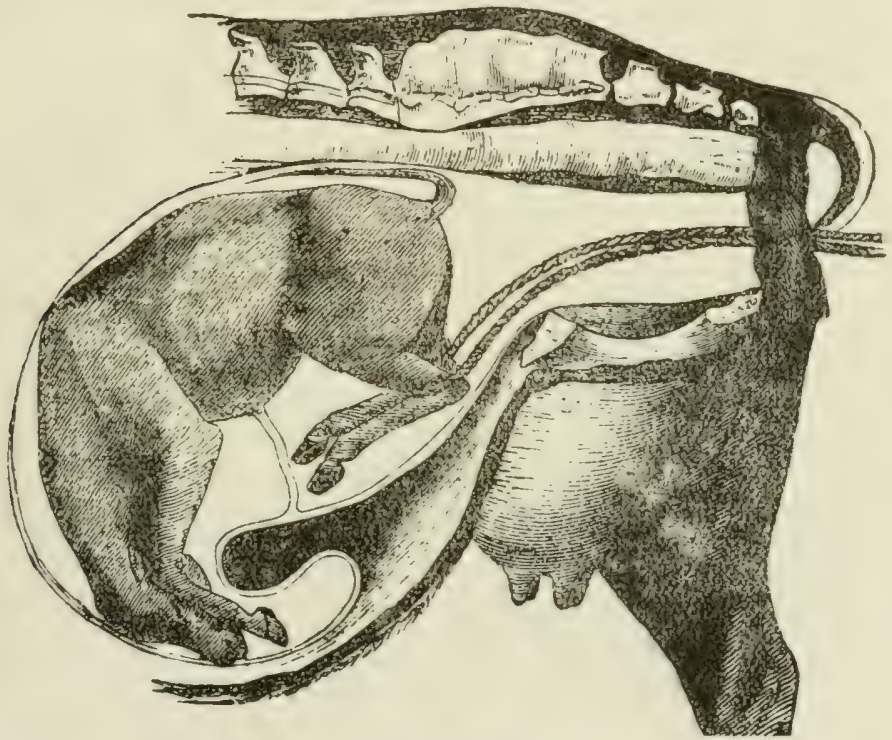

HOCK PRESENTATION-HOCKS CORDED.

this has been done, a forcible attempt at delivery is likely to take the life of the mother. Therefore it is necessary to place the hind legs in a favorable position, by extending them in the genital passage. This is more easily done with the calf than with the colt, owing to the shortuess of the legs of the former. If the offspring be very small and the mother large and roomy in the genital canal, the fortus ma be delivered in this posi- 
tion, by extending its legs forward underneath its abdomen. But this would be a very exceptional case.

The best mode of completing delivery, is to so adjust the foetus that the hind legs are completely extended outward. This is quite easily accomplished, if an examination be made before the foetus has entered the pelvic inlet, or, at the most, the points of the hocks, and raising them upward, at the same time pressing inward, the operator can draw the feet into the pelvic inlet. Or, if the operator has secured the feet by ropes previous to handling the hocks, as he raises and forces them inward an assistant can pull on the ropes and bring the feet outward into the genital canal, and can then complete the birth with ease. But at a later period, when labor is more advanced and the croup is well advanced or wedged in the inlet along with the hocks, the case is more troublesome. First, fasten a rope around each hock separately; then apply pressure to the croup, forcing it inward and upward until you force it within the womb, or far enough inward to secure the feet with ropes. Then take hold of the hocks and press upward and inward, when an assistant may draw upward and outward on the ropes and bring the feet into position.

If there is much trouble in forcing the foetus back and it is known to be dead, or if it must be sacrificed to save the mother, ropes may be fastened to the hind legs and the hocks drawn as near the outlet as possible. Then amputate the legs at the hocks, remove the letached parts, and the remainder is easily brought into position for delivery. If the mother persists in lying, you may elevate her hind quarters by packing straw under her. If thought necessary, place the hobbles on the hind feet, then place a rope over a high beam and draw up the hind parts. This sometimes, will ureatly lesson the danger to the operator and assist him in extracting the foetus. 


\section{THIGH AND CROUP PRESENTATIONS.}

The cause of this mal-position, is the same as that operating in the hock presentation. It occurs frequently with hoth mares and cows. When the thigh and croup are presented and the legs are flexed slightly forward, the mass is too large to enter the pelvic inlet. But, as labor progresses, the croup is forced into the pelvic

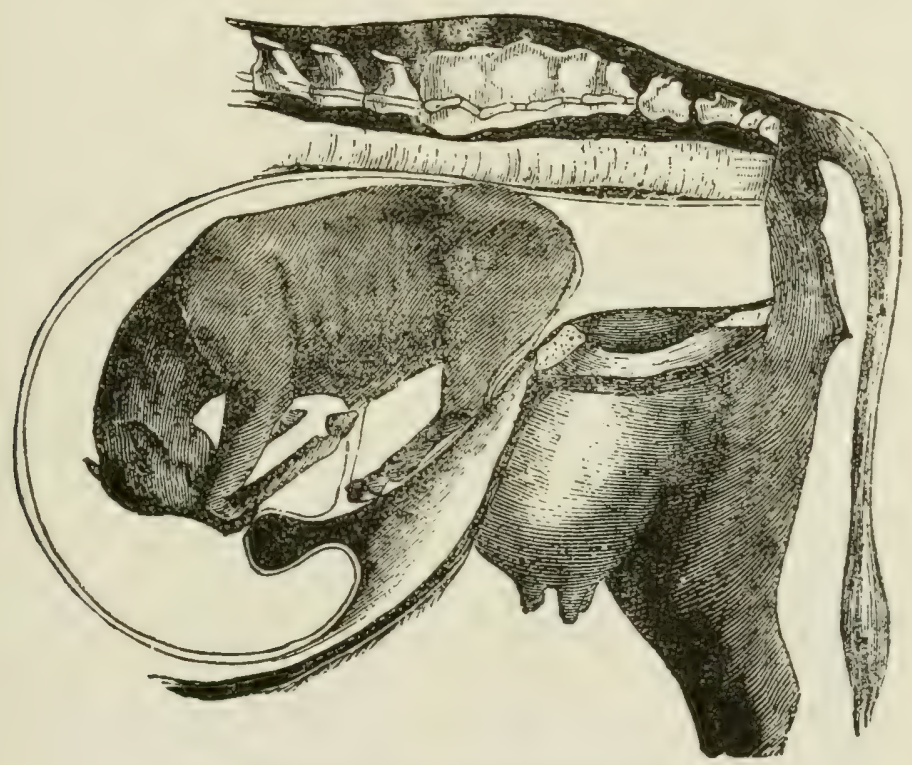

THIGI AND CROUP PHESENTATION.

inlet, while the thighs are forced downward and the legs forward until the latter are pressed up against the abdomen. In this position birth is possible; but the foetus must be very small and the pelris of the mother large and well-formed. In this position, upon examination, the hand first encounters the tail and buttockis of the foetus, then the thighs, and possibly the hocks. But if labor is somewhat adranced, the legs will be 
pressed up along the abdomen, when the hocks will be leyond the reach. The croup and haunch may have bissed some distance into the pelvic inlet, yet it is very unusual for any part of the foetus, except the tail, to be discernible externally, no matter how long labor may hatve been progressing. This mal-position is the most dilicult encountered in veterinary obstetrics. Sometimes the mother perishes without being delivered. Though a living calf sometimes is delivered in this posi-

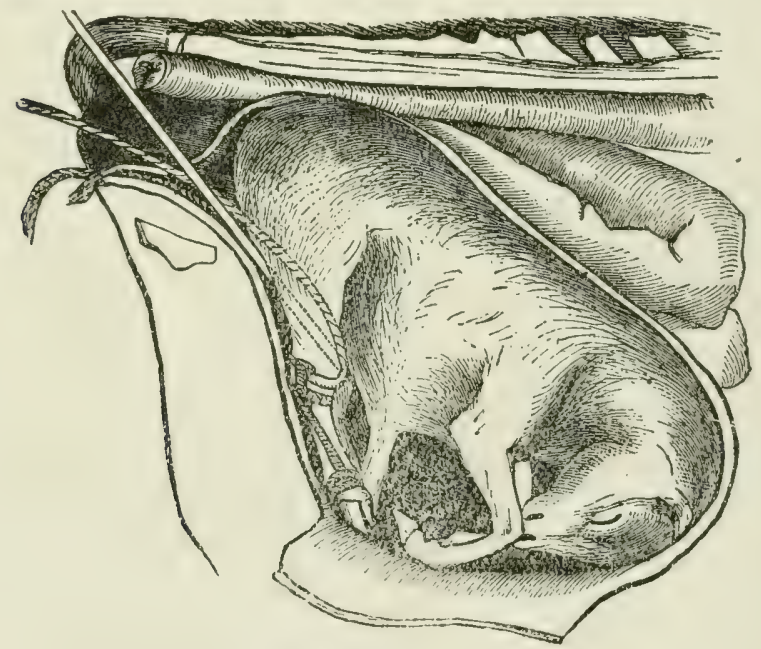

THIGH AND CROUP PRESENTATION-THE HOCK COIDED.

tion, a living foal is very rarely obtained; and only too often, the mare dies after the foal has been taken away. The chance of saving either mother or off'spring, depends greatly upon the length of time the mother has been in labor, and the amount of injury or irritation sustained by the genital organs through improper handling. The chances are lessened if it be the first foal or calf, or if the fotus be unusually large. To complete birth, it is necessary to extend the 
limbs of the fortus backward, as in ordinary breech Iresentation, the hind feet first so that the mother's efforts with slight assistance from the operator, will clfect a delivery. This will prove a difficult task. Though in some instances, where labor has not advanced very far and the foetus is still in the uterine cavity and can be moved around so that the feet can be reached and brought into the genital passage, it is not very difficult. When labor has progressed far enough to force the croup into the pelvic inlet, delivery will be completed only by hard work and perseverance. "If possible, place a small rope around rach

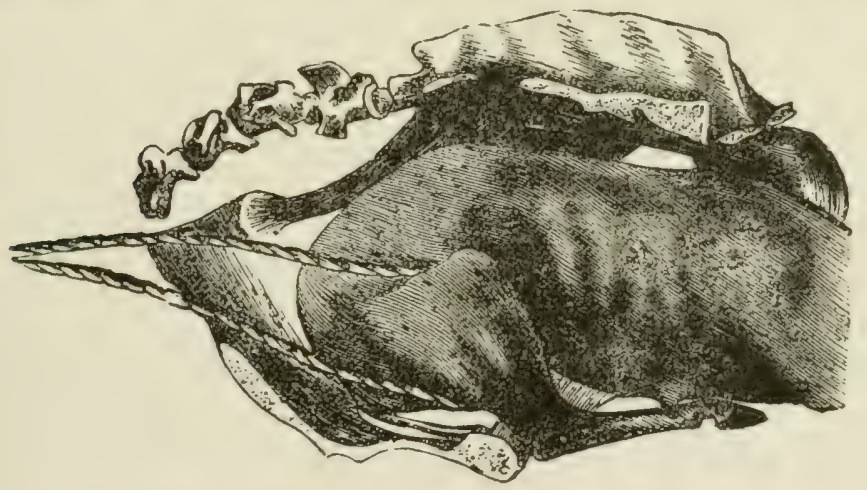

THIGH AND GROUP PRESENTATION-THE THIGHS CORDED.

leg separately, as low down as the hock. Then take a repeller, or use the hand, placing it against the croup, and force it inward, the assistant drawing upward and outward upon the hock. Continue in this way until you have a hock presentation. Then fasten ropes to the fetlocks, and while the operator seizes the hock and presses upward and inward, the assistant may draw upward and outward on the fetlocks, until they are brought into the passage, when the delivery may be completed. But if the croup and thighs are completely 
wedged in the genital passage, and can not be moved, either inward or outward, which sometimes is the case, no recourse but embryotomy is left. This is a very tedious manner of completing delivery. Take a guarded knife and cut through the skin and muscles until you come to the hip joint. Then detach that limb and remove the other in the same manner, after which you can insert hooks in the cavity of the hip-joint and extract. Or, place a rope completely around the body of the foetus and extract. Or, sever the muscles from the bones of the haunch as much as possible, then fasten a hook into the bones and extract them. After you have severed the haunch bones, remove the bowels. Then fasten a rope to the remaining skin and complete delivery.

\section{TRANSVERSE PRESENTATION.}

The fotus is in a transverse position when, upon examination, the hand of the operator first encounters the shoulders, withers, sides, flanks, haunches, loins, back, breast, belly or all of the limbs collected together. The possibility of the fuetus assuming a transverse position within the womb, is by some strongly disputed, but entirely without reason, because the womb is a soft, yielding membraneous sack, capable of assuming almost any position; so it readily appears that its transverse diameter may be increased at the expense of its length. 'Though transverse presentation sometimes is observed, it is not nearly so common as an anterior or posterior presentation. Still it is quite as necessary that the operator should understand something of this presentation, as with it, hirth is impossible when unasristed. No matter whether the foetus presents in the transverse vertical or transverse horizontal position, 
version must be accomplished in order to change it to either an anterior or posterior presentation. For in one of these ways only, can the foetus be brought into the pelvic inlet and delivery be fliected. This presentation

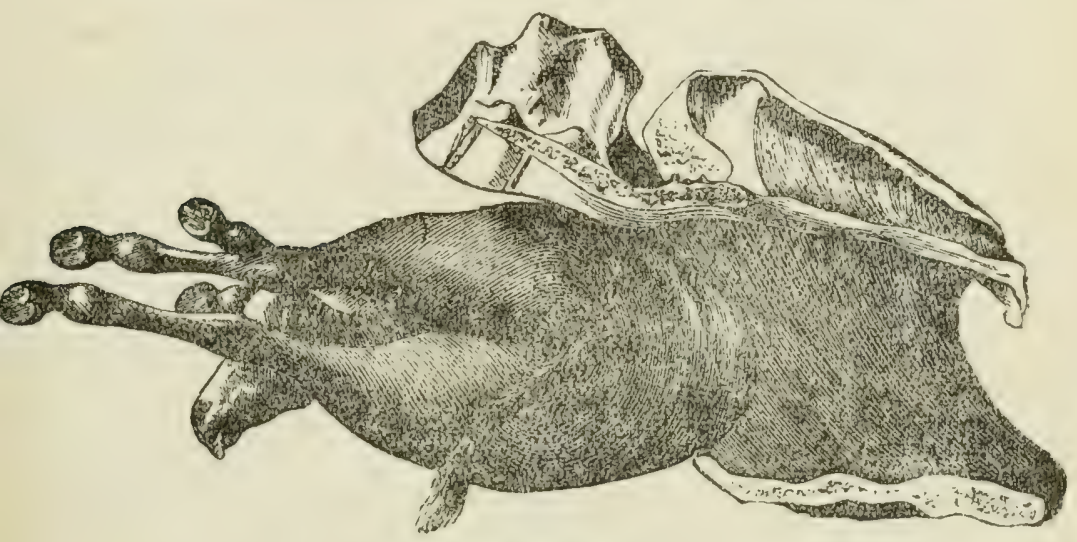

'TKANSTEKSE IPESEXTATION.

occurs more frequently in the mare than in the cow, perhaps, owing to the stronger contractions of the womb of the former not allowing proper adjustment of the fortus, should it not be in a position exactly favorable at the commencement of labor.

\section{SHOULDER AND LOIN PRESENTATION.}

When labor has continued for some time with little or no progress, and upon examination, the hand of the operator comes in contact with the shoulder and loins, he knows that he has to deal with a transverse presentation. If labor has lastcil some time, the straining has been energetic and the waters have escaped, it sometimes is a difficult matter to decide what part of the foetus the hand encounters first. There is first felt a more or less rounded surface, that has no special char- 
acteristic by which it may be recognized. The hand should then be introduced farther into the womb and search should be made for some part of the foetus that can be recognized. Then study the position of the young creature in the womb. The presentation having been recognized, the position must be determined by ascertaining the direction in which the head lies. With the foal, this is accomplished by feeline for the mane and tracing it as far as the arm can reach. It may lie in

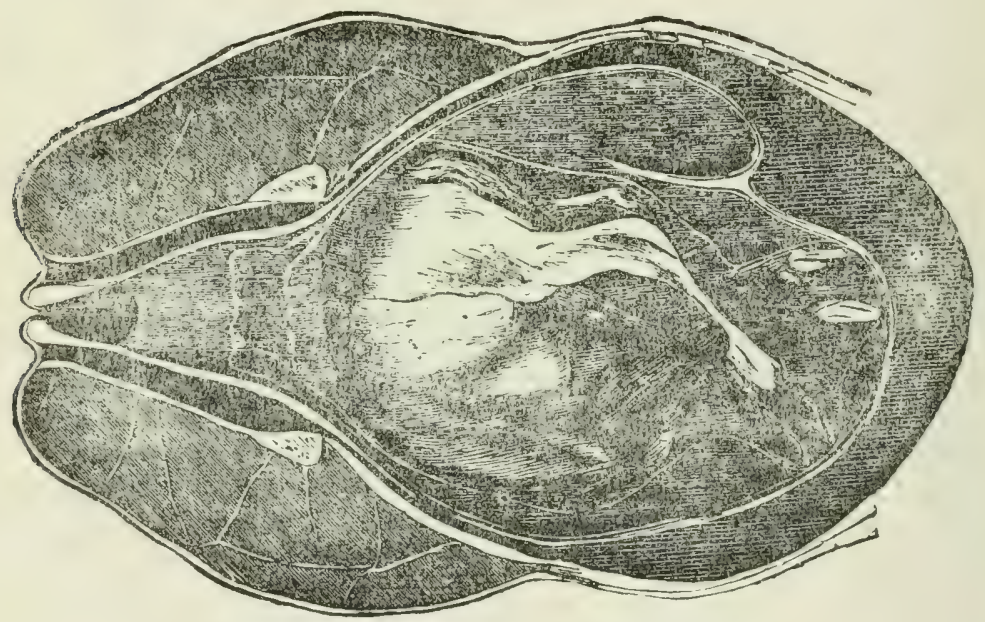

SHOULDER AND LOIN PIESENTATION.

the right or left flank of the mother, or up along her back, or down toward the floor of her belly, the legs assuming different positions in a direction opposite to the head; although sometimes one leg is found crossed over the neck. To learn the exact position, is absolutely necessary, before venturing upon any attempt at extraction, as in nearly every case, the presentation is a ditlicult one and requires all the skill anid judgment of the operator to rectify it. But if labor has not continued any length of time before the operator discovers 
the presentation, and if the membranes are soft and yielding from the natural lluids, the position, generally, is easily converted into an anterior or posterior presentation, and a living fotus is lelivered. If labor has continued for some time and the patient is a mare, you may depend upon it, the foal will be dead. But with the cow, a living alf may be delivered-the tenacity of lite and the puwer of endurance heing wonderful. The

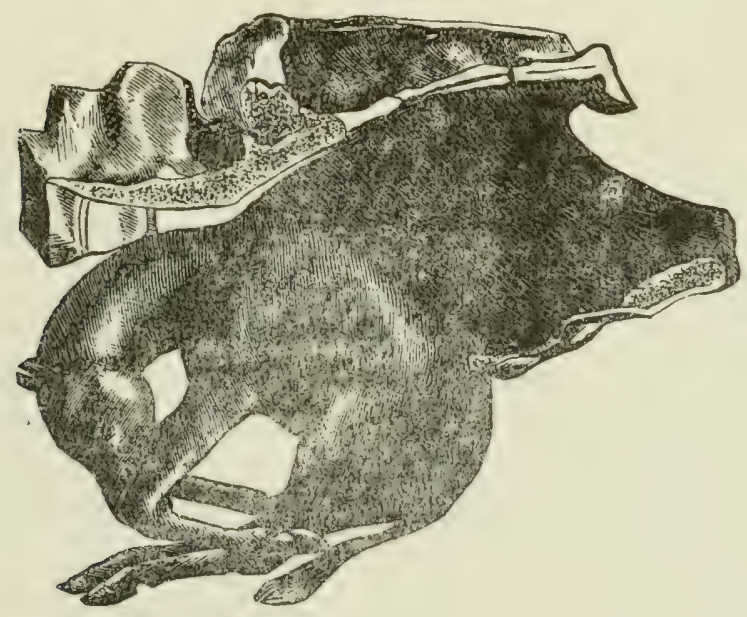

SHOULDER ANI IOIX PLESENTATION.

principal object is to convert the transverse presentation into either an anterior or a posterior presentation, for only in this way, can delivery be eflected. If the foetus lies or can be moved so that the head and foreless are most convenient and can with the least difficulty be brought into the pelvic inlet, the position should be converted into an anterior presentation. If the hind leys are most accessible and can be brought into the inlet. with less difliculty, convert the position into a posterior presentation. But to do either, is generally a diflicult task, as the presentation offers nothing to take hold of 
ardvantageously, or to which ropes could be attached. If labor has progresser for some time and the memTranes are dry, before attempting to turn the fortus, it is a good treatment to inject warm water into the womb, to moisten the membranes, which will greatly aid the operator in his efforts at version. The fœetus must not be pushed directly forward into the womb, but rather obliquely and in a direction opposite to that of the parts we wish to bring into the inlet. If the mother be lying down, the operator will find it greatly to his adrantage to raise her hind quarters very hich. Ile should be persistent in his work, use frequent in ioctions of war'm water, and, in the great majority of cise's, the foetus will glide around the inner surface of the womb until a convenient part presents itself. Then the forelegs and head, if they are the parts sought, or the hind legs, are brought into the genital canal by the hand or by ropes, when slight traction will complete the delivery. When all these maneuvers have failed, embryotomy should be performed in such a mamer as the operator deems most adrantagens. At the best it will be a difficult, tedious and laborious task, seldom attended with satisfactory results. The mother wererally perishes, either during the operation or shortly afterward.

\section{BREAST AND ABDOMINAL PRESENTATION.}

This presentation is quite common in both mares and cows, but more frequently occurs in the former. Generally the foetus is lying on its side with its legs flexed, or cxtended, or presented at the inlet, or engaged in the passage. There is no difficulty in diccovering and reconnizing this position. As the op rator passes the hand to explore the parts, it first en... un' ers the feet-usually 
all four of them. They may be within the womb, or one or more of them engaged in the passage. In the majority of cases, either the hind parts or fore quarters are near the passage, a condition recognized by the legs of that part being further advanced than those of the other. The position must now be ascertained, which can be done readily if the head can be felt. But this, generally, is beyond reach and the position can be

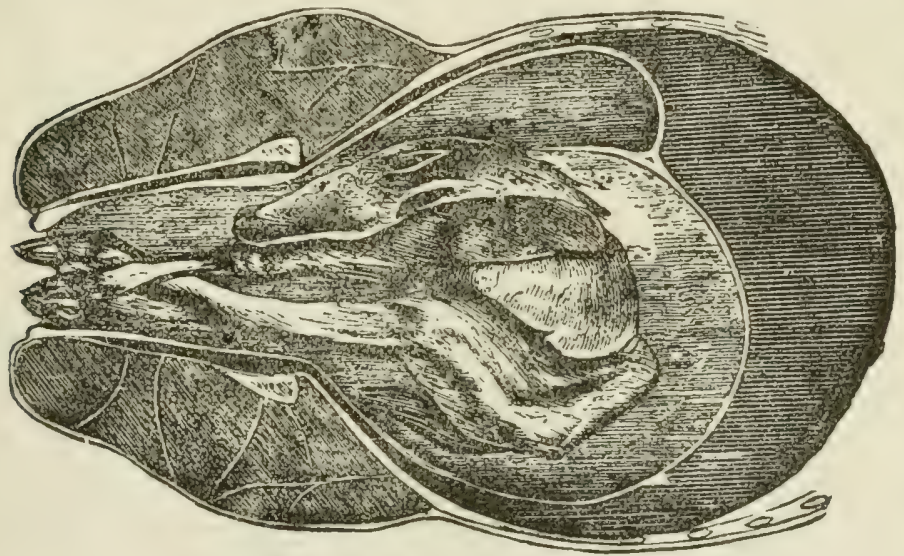

BREAST AND ABIOMINAT, PRESENTATION.

determinesl only by a "allerial cxamination of the limbs. In this we must distinguish hetween the hind legs and foreleas, as these alone can show the direction of the head. To do this, the operator should remember that the hind leg flexes in the direction of the wall of the foot, whereas the knee flexes in the direction of the sole of the foot. The hock can also be recognized by its flatness. It nust be remembered that, in this presentation, the foreleys and hind legs are across each other, when engaced in the genital passage. The hind legs, crossing the forelegs, are pointing towards the anterior part of the body of the 
foetus; while the forelegs, crossing the hind legs, point toward the posterior part of the foetus. This is not a very serious presentation, as a rule. The gravity of the case depends somewhat upon the length of time labor has continued. As it is impossible for birth to occur spontaneously, while the foetus is in this position, if the mother has not received the necessary aid for some

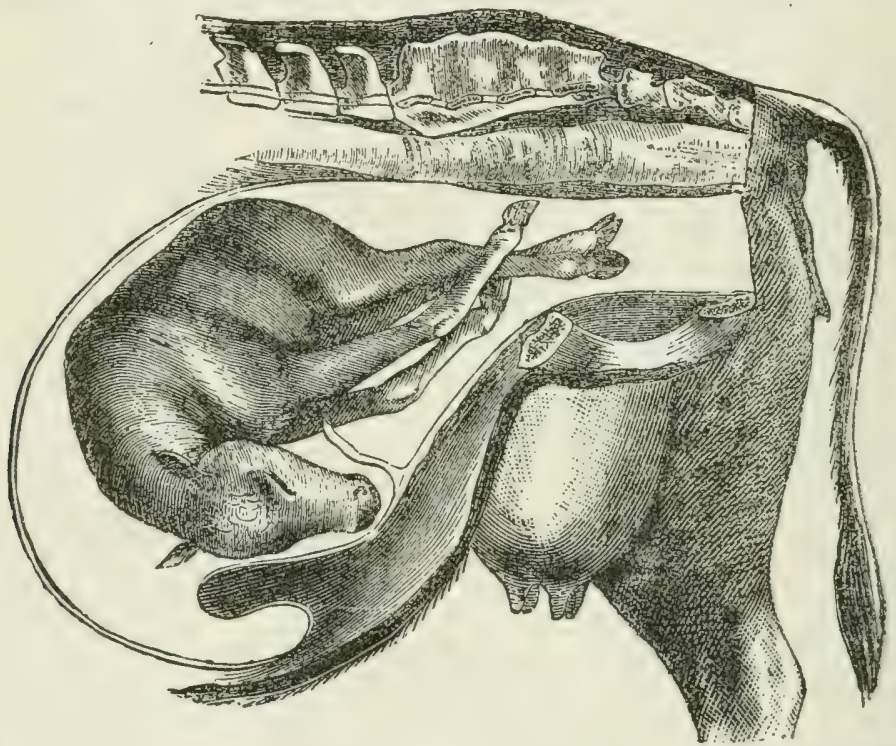

BREAST ANU ABDONINAL PRESENTATION.

time after the commencement of labor, her continued straining may not only cause the death of the colt, but the mother's death, from prostration. When the legs have been distinguished, fasten ropes around the ones you wish to extract first. Generally the operator will find it most advantageous to convert the position into a posterior presentation and deliver the hind feet first. If only one hind foot and one forefoot are encaged in the passage, fasten a rope around the one hind foot and 
search for the other one; secure it and bring it into the passage. Then return the foreleg to the womb, as far in as possible, apply traction and deliver. But if all four legs are chlaged in the passage, fiaten ropes to the hind feet; then return the forefeet as far as possible within the womb. Sometimes this is more easily and more advantagenty ancomplished by thexing the forelegs at the knee, until the foot rests against the elbow; then force it within the womb in a downward direction, while the assistant draws upon the hind feet. The foetus will straighten out generally and birth will be completed. Even if the head be engaged along with all four legs in the passage, I would recommenu returning it to the womb in a downward direction along with the forelegs, and that delivery be made with the hind feet first. When the foetus is partially delivered, say the hind legs, croup and hips are without or beyond the outlet, the operator should pass his hand between the belly of the foetus and the floor of the genital canal, to ascertain whether the foreleng have become cramper or fastened within the inlet too soon. If so, straighten them by pusing them inward, for if not strainhtener. serious rewults might follow. In delivering the fortus with a posterior presentation-that is the hind feet first-the back of the fortus should be against the bark of the mother.

But in a breast and abdominal presentation, if it is fouml impossible to force the foetus back into the womb and the foetus is dead, there should be no hesitation about resorting to embryotomy. The best way to commence, is to cut off the forelegs at the knces or elhows and remove them; the lower part of the legs being removed, the remander of the fuetus can be readily pushed back into the womb, and by applying traction to the hind legs, delivery can be effected. 


\section{TWIN FOETUS-IN DIFFERENT PRESENTATIONS.}

With mares and cows it is not an uncommon occurrence for them to bring forth twins, though it is rarely, indeed, that assistance is needed to complete delivery. Usually, when the mother is carrying twins, either they are expelled before the full period of erstation is completed, or each is much sma!ler than if she were carrying only one. Indeed, birth usually is much easier with

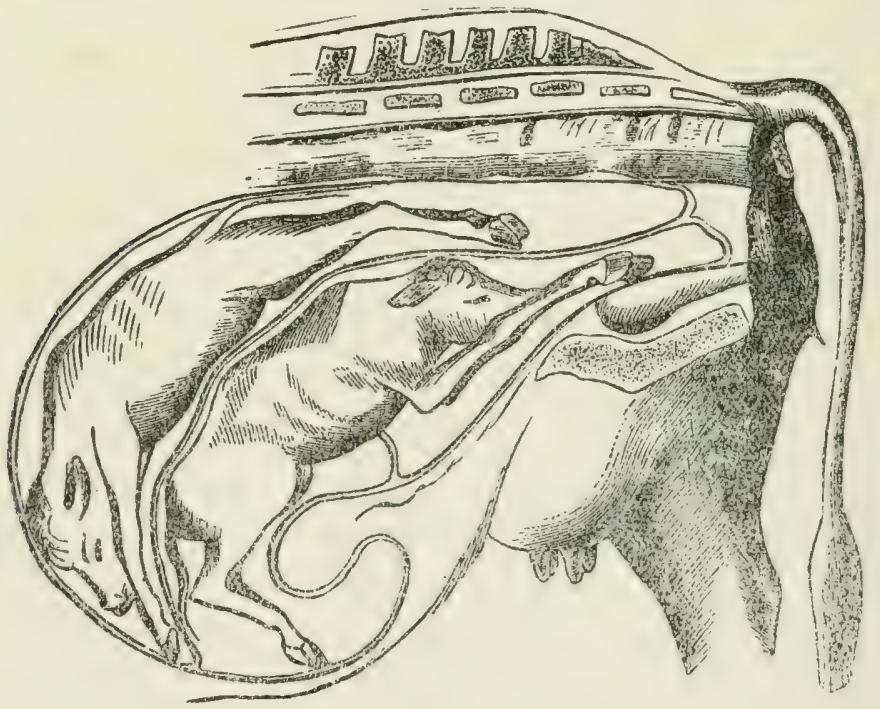

TWINS IN DIFFWRET POSITION.

a twin pregnancy than with only one. When the most advanced foetus is expelled, a variable period elapses before the second hirth. During the interval the mother generally is uneasy and pays little or no attention to the one already born, but manifests a peculiar anxirty, which is a good indication that delivery is not complete. But when the second fortus is born, the mother generally turns her attention to her ollspring. This is the general 
srder of 1 win births. But it is not always so fortunate. In some cases, after the first one is horn, a long interva! occurs before the birth of the second one, which is usually fatal to its existence. This suspension of laho: in twin birth is generally due to a mal-position of the fetus. Difficult paturition from this cause also happens when the twins are alont equally distant fiom the mouth of the womb, and are presented at the inlet together. Of course, they cannot pass through the canal together, hence the difliculty. Lum aximination, the operator will experience great difficulty in determining the positions of the two foetuses. This can be done only by careful examination, and then the operator will find himself somewhat embarrassed. For example, the forefeet of one foctus may be presented with the head of the other; or the head and one forefoot may present with one forefoot of the other; or one hind foot of each fotus may present together; or a forefoot of one with the hind foot of the other, ete. In some instances the legs of the twins are so interlaced, that they are separated only with the weratest diffindty. ()ne thing is certain, only one can be delivered at a time. Thereiore, they must be separatod and one pushed back into the womb, while the other is betus deivered. When the one is born, the other, if in a wrong position, must he straightened and remored. This is quite easily written but not so easily performed. If assistance is not aflorded before both foetuses become werlued into the pelvicinlet, the operator will experience great trouble in adjusting them. But if distinguished hefore entering the inlet, lut little trouble need be anticipated. Select the one most favorably situaterl, fasten ropes to the head and forelegs or to the hind lews, as the rase may be, and arrange them in a direction proper for relivery. Then let an assistant draw the fixtus toward the outlet while 
the operator pushes the other back within the womb. After delivering the first one, search for the other, which probably will be in a mal-position, requiring adjustment before it can be born.

\section{DOUBLE-HEADED MONSTROSITIES.}

Very seldom, indeed, do we witness double-headed monstrosities with mares or cows. In the former I have neither seen nor read of any; but in the cow I have seen

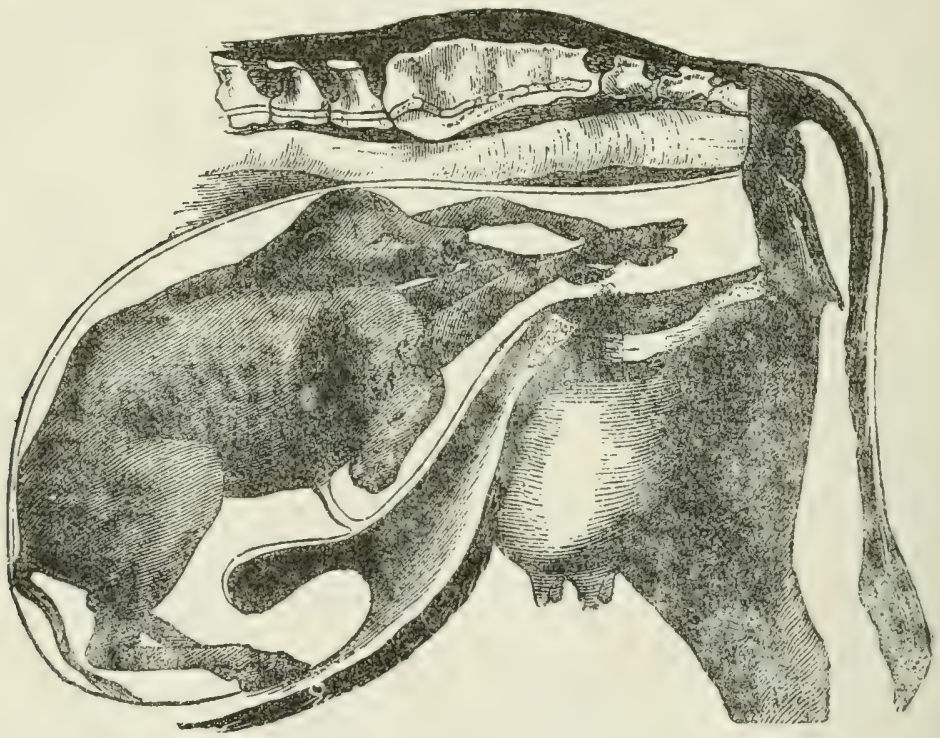

DOCRTE-IIEAHED MONSTROSITY.

one and read of others. But they are very rare, indeed. Sometimes these monstrosities are born alive and live a considerable time. The one that I saw, was living at three years of age, to all appearance healthy and a good feeder. It was placed on exhibition. She only used one mouth while feeding. The other mouth was well formed and had a full set of teeth, but she had little 
control of the lower jaw. Though, if the mouth was opened with a little assistince, it would close spontaneously. Both heals were well-formed, uniting at the neck, both mouths emptying into the one cesophagus.

The existence of this mal-formation renders birth more or less diffirult and sometimes impossible, according to the size of the heads. But sometimes birth oceurs without assistance and with little difficulty. An examination will reveal the condition, when the operator will adopt the measure necessary to complete the delivery. If the mother is large and the genital canal well formed, forcible traction may complete the delivery. But if, in the judgment of the operator, this will not do, embryotomy must be resorted to. Cutting off one or both of the heads may be necessary before delivery can be completed. If the heads are united closely, split them with a chisel and then remove them; or, in such cases, bone forceps may be wsed. If it be a double head and neck, cut ofi the neck, as low down as possible, and remove it first; then remove the balance. There are several other forms of monstrosities but, as they so seldom occur, a description here is quite unnecessary. Suffice it to say, that if it be impossible to deliver them by moderate traction, amputate such parts as cause the obstruction and deliver.

\section{IIYDROCEPHALUS, OR WATER ON THE BRAIN.}

This anomaly is found in colts and calves, both. The enomous size of the head, is the obstacle to a natural idivery. If with an anterior presentation, birth will be delayed, and, on making an examination, the operator will soon discover the deformity. But, if with posterior presentation the operator discovers this deformity, he should endeavor to change the position to in anterior presentation, and, after l, ringing the nose into the inlet, 
he should plunge a knife through the head, making a free incision to allow the water to escape. After which, on applying traction, the tissues gradually tlatten down and birth is completed. - But if it be nesenterl hind feet first and birth is complete with the

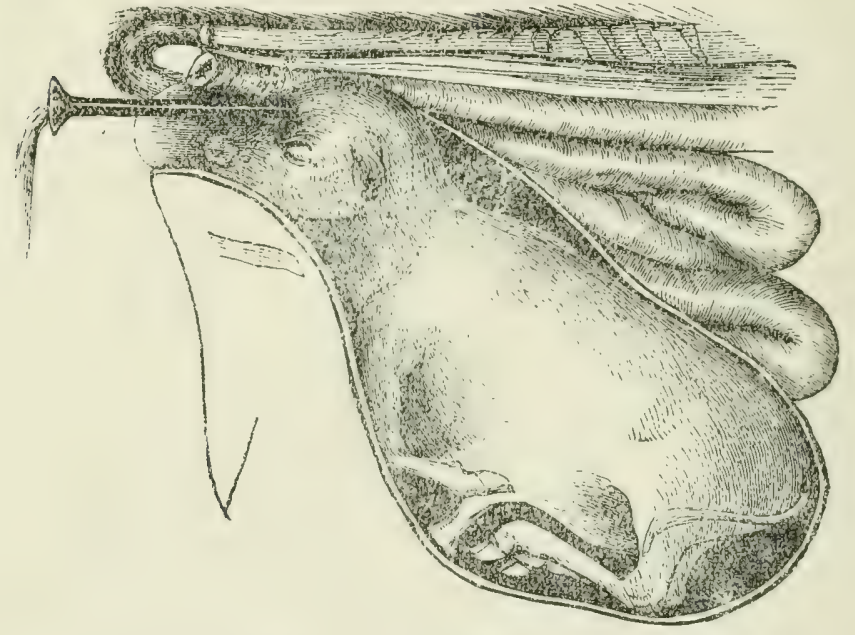

HYDROCEPIIALUS, OR WATER ON TIE BRAIN.

exception of the head, which is too large to pass through the genital canal and prevents delivery, the operator should pass the hand along the neck and examine the head. If hydrocephalus is recognized he should open the head with a knife or trocar and canula, and allow the fluid to escape, after which the tissues will llatten down and traction will complete the delivery.

\section{ASCITES, OR ABDOMINAL DROPSY.}

With this disease, the ablomen of the foetus will be swollen to such an extent that it cannot enter the genital passage, though the head and forefeet may be pre- 


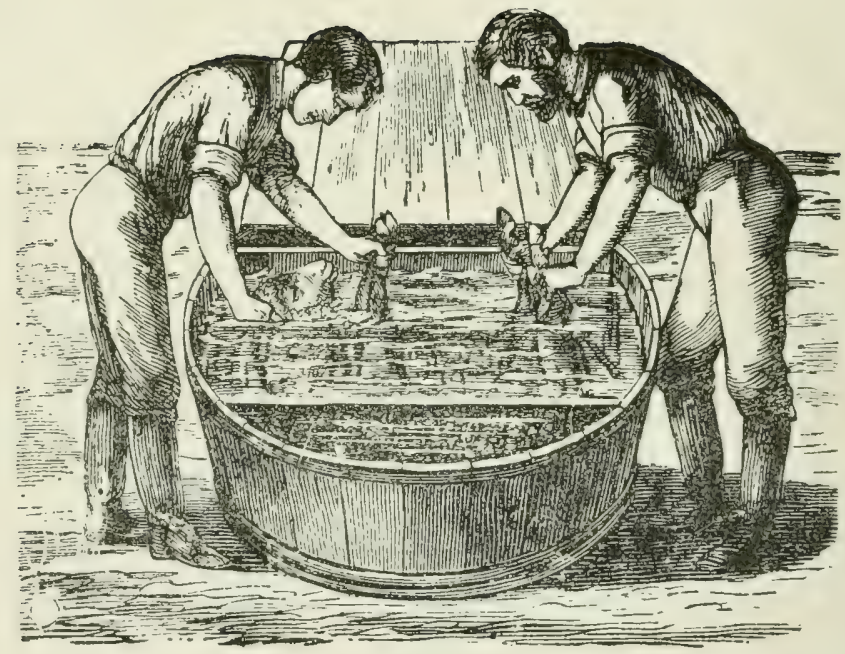




\section{DISEASES OF SHEEP,}

THEIR

CAUSES, SYMPTONS AND TREATMENT.

\section{CATARRH, OR COLD.}

Catarrh, or cold is quite a common complaint among sheep, during the fall, winter and spring. Indeed it is rarely absent in any flock where any great number is kept.

Causes:-It is most often catused by being confined in "lose, damp yards or by housing in stables or sheds, too closely together, without sufficient ventilation. The heat arising from the sheep canses a high temperature, thus over-heating the animals and when turned out into the bleak winds they catch cold very easily: in fact the liability to take cold is greater, than when allowed to roam in the open fields, exposed to the weather. It is well to have dry, open sheds whre sheep can lie down at night or to protect them from storms, but close housing is detrimental rather than beneficial. When allowed plenty of fresh air, coorl ventilation, dry yards and clean, dry bedding, catarrh will seldom be seen. A wet or damp cold or a close, moist atmosphere is very productive of this trouble.

Symptoms:-Nore or less ferer is present; the sheep snuffles considerably and mucous is discharged from the nostrils. There will be very little coughing but if the lungs become inrolred, the cough will become dry and 
deep-seated. The appetite is partially suspended, and the animal appears weak and stupid.

Treatment:-Remove the caluse as quickly as possible, to protect the balance of the flock, then remove the affected ones to warm, dry. well-ventilated quarters. If the bowels are constipated, give

Epsom Salts.......... three ounces.

Ginger............. two drachms.

Water..................

Give at one dose, to be followed with

Quinine............. two drachms.

Dover Powder......... two drachms.

Cayenne Pepper..... . two drachms. Mix.

Make twelve powders. Give three powders each day, or

Ginger.............. two ounces.

Gentian ............. two ounces.

Saltpeter............ two ounces. Mix.

Place in a quart of water and simmer over a slow fire for twenty minutes, then add half pint of good whisky and bottle. Give half wineglassful four times a day. Give good, nourishing food and occasional draughts of flaxseed tea. Good nursing is very important. Without this, medicines are useless. Keep the animal dry and comfortable, with plenty of fresh air.

\section{PNEUMONIA-LUNG FEVER, OR INFLAMMATION OF THE LUNGS.}

This disease is an inflammation of the lung substance and often follows catarrh. It is a very serious affection and is more frequent than is generally supposed, many sheep dying from this trouble and the owner imagining some other malady the cuuse of death, on account of its 
course heing so rapic and fatal. It is generally caused by exposure of solue sort, as washing in cold spring water on a raw. "old day, or exposure to cold rains in the fall and surang; by shearing too early or exposure to cold storms after shearing: becoming over-heated while driving or ly being too closely confined without. sufficient ventilation, etc.

Symptoms:-The first symptoms ohserved by the owner or keeper is loss of appetite and suspended rumination, closely followed by grinding of the teeth, with a high ferer and an inordinate desire for drinking water, or if in the winter, the eating of snow. The pulse is hard and quick, the breathing labored and rapid, accompanied by a hearing at the thanks and a painful "mol, with a flow of thick, yellow mucous from the nostrils.

Treatment:-While bleeding is strongly condemned ly some and as thorounhly upheld by others, I believe that both extremes are attended with baneful results. If block-letting is resorted to, it should be done early and ouly in young, strong, plethoric animals, when the liest of results are often obtained; but in aged and weakly anmals this manner of treatment should not be attempted. Whether the patient is bled or not, gire three unces of epsom salts in a quart of water. If this loes not operate freely in four or five hours, repeat the lose, as a grood, free physic is demanded and is not attended with any evil results. Immediately following the physic, administer-

Quinine Sulphate.....three drachms.

Saltpetre.............. one ounce. Mix.

Make nine powders. Give one powder every three or four hours. Or,

Liquor Ammonia Acetatis... one pint.

Fluid Ext. Aconite...... half drachm. Mix. 
Give two tablespoonfuls every three hours. As the sheep begin to improve, a pint of gruel or flaxseed tea may be given three times a day, to which may be added a drachm of powdered gentian or ginger. Keep the sheep in a comfortable, airy place, as good nursing is of as great importance as effective medicines.

\section{COSTIVENESS-STRETCHES-CONSTIPATION.}

Costiveness is most often seen where sheep are thanged from pasture to dry food. The regular exercise afforded while grazing, together with the succulent grasses eaten, will tend to keep the bowels in a moist condition, but when changed to dry food, they are usually kept in yards where they eat from racks, which requires no exercise. Then the dung becomes scanty, dry and hard and is passed at irregular intervals. The sheep grunts or moans from pain while passing manure, and the exposed part of the last bowel looks red and inflamed. If the constipation continues for any length of time, from neglect or otherwise, the sheep will be observed standing with forefeet extended and the hind feet set back, as if stretching itself; the head will be elevated and the back curved downward. The condition is now somewhat grave and a physic should be given immediately, as

Epsom Salts .......... three ounces.

Gentian, or Ginger....... one drachm. Mix.

Give at one dose in a pint of water as a drench. But. if, when changing sheep from pasture to dry food, they are allowed salt at will, costiveness will seldom be observed. Constipation in lambs is often noticed, especially in such as are not nursing the mother and are fed ipon cows' milk or the milk of other ewes. As soon as the bowels fail to act, the belly hecomes distended, the 
lamb lies down most of the time and appears as though in a stupor, the urine is suppressed or is very scanty and if the animal is not speedily relieved death will ensue. The best mode of treatment is to wive frequent injections of eastile soap and water, or inject a few ounces of melted lard into the last bowel. Also give a physic, as a spoonful of castor oil, or two or three spoonfuls of melted lard. After the bowels have responded, shoulil the lamb appear stupid and dull, wive a few sponnfuls of hot whisky three times a day.

\section{DIARRHOEA, OR SCOURS IN LAMBS.}

When sheep are first turned out to grass in the spring of the year, after having heen wintered on dry food, they are liable to have a looseness of the bowels or diarrhoea, which is seldom attended with any dan-

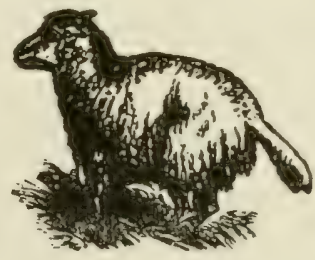

SCOURS IN IAABS.

grous results as regards the whole tock. Now and then a single individual will scour so badly, that the process of nutrition is seriously affected and dysentery is the result. In sucking lambs diarrhea is very fatal, if it does not receive the closest attention.

Causes:-It usually arises from exposure to cold, sudden changes of temperature, hot stabling in winter with the bad air evolred, the result of improper ventilation; a fat, full-blooded or plethoric coulition of the mother, nursing milk from caked bags, cold drinks, etr. 
Symptoms:-The lamb is not at all playful, but shums its playmates and appears languid and sad; it either lies down a considerable portion of the time or stands with its back arched. The dung, which is frequently passed, is thin and whitish in color, later on becoming mixed with mucous and finally bloody. It now ceases to nurse and eat and will bleat frequently. The belly becomes tucked up and if pressure is applied thereto, the animal evinces pain. It loses flesh very rapidly and if not relieved, will die in a few days.

Treatment:-First of all remove the cause if it can he clearly ascertained. Change the food immediately, have good ventilation, and as pure air as possible. Then take the white of an egg and beat it up thoroughly with a half cup of milk-warm water. Give three times a day. If this does not check the malady, take Prepared Chalk.......... one ounce.

Powdered Catechu...... four drachms.

Powdered Ginger....... two drachms.

Powd. Opium...........half drachm.

Peppermint Water........half pint. Mix.

Shake well before using. Give tablespoonful to a lamb, and two tablespoonfuls to a grown sheep. Great benefit is sometimes derived by giving two or three grains of quinine, four or five times a day. In cases of white scours arising from non-digestion of the ewe's milk, a tablespoonful of raw linseed oil should be given to clear the bowels of the irritant, and should the prostration be very great, the following will be of excellent service:

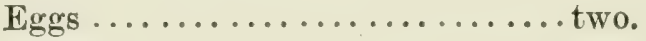

Whiskey .............. two ounces.

Essence of Ginger........ one drachm.

Beat up with a pint of flaxseed tea or milk and give three tablespoonfuls every three hours. 


\section{HOVEN, OR BLOATING.}

This disorder is a distension of the first stomach with ras, to an alarming extent, causing a bulging on the left side.

Causes:-The most common cause is feeding greedily upon green, succulent fool and more esperially, green clover that is wet with rain or lew. A rapid fermentation takes place and gas is quite liberally generated. Eating musty hay or blighted rorn, sometimes produces a disordered condition of the stomach and bloating; or it may be a sympton of some constitutional disturbance, whereby it causes a chemical change in the alkaline secretions of the stomach.

Symptoms:- Swelling of the belly on the left side, to a creater or less extent. Sometimes the skin is drawn so tightly over the swelling, that if pounderl with the end of the fingers, it will sound like a drum. These swellings appear shortly after the animal has been feeding. The breathing is seriously affected, being short, quick and accompanied with a grunt. The bowels are ronstipated and symptoms of pain are manifested by the anxious expression of the eyes and countenance.

Treatment:-The animal must receive immediate attention. If the swelling is great, there must be no time lost. Take

Ammonia Water (Hartshorn)......

$$
\ldots \ldots \ldots \ldots \ldots \text { one teaspoonful. }
$$

Water...................... Mix.

Give at one dose. Or,

Baking Soda......... one teaspoonful.

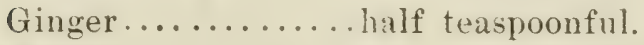

Water..................... Mix.

Give at one dose. If this does not give relief, do not wait too long but use a trocar and canula if you 
have one, if not, use a penknife. Either cut off or separate the wool on the left side over the most proninent part of the swelling, between the last rib and hip bone, and plunge the knife blade into the stomach. A zonse-quill or hollow elder may be inserted, to assist the escripe of gas. Often the was escapes sufficiently through the wound, withont placing a hollow tube into it. After the immediate symptoms are relieved, the following should be given :

Epsom Salts ........... two ounces.

Ginger................. one drachm.

Water..................... Mine pint.

Give at one dose, or give two or three ounces of raw linseed oil. If the sheep is somewhat prostrated, give one ounce of hot whiskey, to which may be adrled a drachm of ginger, after which feed on hran mash for a few days, and in a gradual manner, return to pasture. fometines mechanical means will aid materially in relieving of the gas. Manipulate the stomach with the hands, pressing it quite severely and the gas will escape by the mouth. Or by placing a stick as large as a man's wrist, cross-wise in the mouth and fastening it there, the gas will frequently belch up, quite heavily. Or a rubber hose may be passed down the throat into the stomach, and the gas allowed to escape in this manner.

\section{BRAXY-CHARBON-SPLENIC APOPLEXY.}

Causes:-This is a disease of the blood and is caused from over-feeding on very rich or unhealthy food, and exposure to changeable weather, consequently it is most frequently seen in the spring and fall of the year when the grass is either Juxuriant, succulent and sappy, or rovered with mildew and rust, as the season may be. It 
is also caused from high feeding with grain and hay and being confined in warm stables, without suficient exercise. Also from feeding on bottom lands, where good water is not obtainable or easy of access.

Symptoms:- II hen the termination is rapid and fatal, it might properly be called splenic Apoplexy, as after death the spleen will be found enlarged and gormed with blood and not infrequently ruptured, softened and

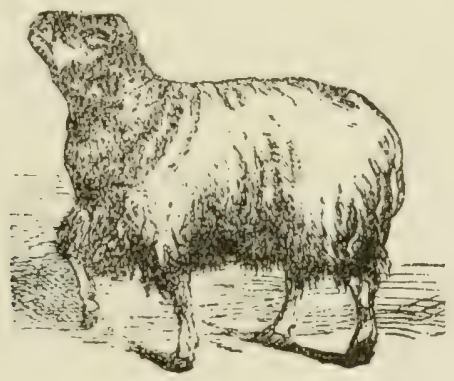

CHARHON.

ulcerated. When this state of atlairs is observed the attack will be sudden, the sherpl refusing food and shivering as if affected with a chill. Weakness is now manifested by stagrering or reeling movements, in its attempt to walk. The breathing is rery rapid, the eyes bloodshot and the mouth injected with blood of a dull, red color; the urine is passed freely and is tinged with hlood; a bloody froth emanates from the mouth and nostrils, the head and neck is bent backward during the conrulsive struggles, a gurgling or rattling is heard in the throat and death ends the scene. The slower furm is properly called Charbon and while it is called s'ower, it seldom lasts to exceed three days. The symptoms are pains in the bowels, the animal lying down and rising up frequently; later, it will stand with its lead down and ears dromping and its back humped 
The ares are bloodshot and the bolly somewhat swollen; red or purple spots appear on the skin, presenting an angry appearance. These pots swell and blisters form, filled with a watery or bloody fluid. A very offensive odor permeates the atmosphere around the sick animal, and a bloody fluid appears around the mouth, eyes, nose and anus. Convulsions now quickly claim the patient, or it passes into a stupor and dies.

Treatment:-Notwithstanding a large percentage perish, yet we are justified in trying to save what we possibly can. Immediately give a good physic as

Epsom Salts ............two ounces.

Water................. Mine pint. Mix. Or,

Castor, or Raw Linseed Oil........ ..................three ounces.

Ginger................ . one drachm. Mix.

Give at one dose. This should be followed immediately with drachm doses of chlorate of potash, every four hours; change pasture or mode of teeding and give light diet with good, fresh clean water. Avoid pasturing on low ground or where they have access to stagnant water.

\section{CONCRETIONS OF FOREIGN SUBSTANCES IN THE STOMACH.}

Foreign substances often find their way into the stomach, and in such a simple manner as to escape detection. There is no animal that grazes closer than the sheep, and in nipping the pasture so closely during dry seasons, they eat a certain amount of dirt and some times small stones are picked up and find their way into the -stomach, as well as small pieces of glass, "etc. Through the winter and spring months prior to shear- 
ing, when sheep are infested with ticks or any small, itching sores, they bite the parts and the wonl besomes entangled into the teeth, and later, is swallowed with the food. After it gains access to the stomach, it mily pis:s off with the excrement or it may form the nurlems of a ball which continues to increase daily and momthly, until it assumes immense proportions, sometimiss the size of a sugar bowl. These acrumulations are seldom detected until after death.

Symptoms:- When any of these substances rause derangement of the bowels, there will be violent puraring, the animal will grind its testh, the nose will be stretched out, and the aars hang pendulous. The appetite and rumination are suspended and the sheep will stand aloof from the balance of the flock, and possibly remain in one place, refraininy from moring for a lone period at a time. By pressing upon the stomaleh and bowels at the llanks, pain will be evinced and the animal will grunt or groan.

Treatment:-The only treatmont that will avail, consists in feeding soft food, as bran mash, oatmeal or flaxseed gruel, corm mush, atr., together with ounce doses daily of epsom or whuber salts administered with a pint of water. If the shere will not eat the soft food, it may be drenched quite liberally with the provender.

\section{CONGESTION OF THE LIVER.}

During the fall and winter season, when sheep are penned up without exercise and fed upon highly nutritious and stimulating food, preparing them either for exhibition or for sale, there is an excessive, amount of blood formed and as the liver is one of the purifying glands, it is liable to become gorwed with blood, or con- 
gested. It is caused by deranged digestion and if it partakes of a serious character, it will produce constipation, dullness and a yellowness of the mouth and eyes. When the symptoms are observed notime should be lost. Give immediately,

Epsom sialts..........two ounces.

Calomel.............three grains. Mix.

Give at one dose in a pint of warm water, to be followed every morning until the yellowish condition disappears, with two ounces of slauber salts in a pint of warm water. Allow plenty of water, slightly warmed, to drink. -

\section{INFLAMMATION OF THE LIVER.}

As congestion precedes inflammation, neglect of the former is liable to result in the latter. The same symptoms are also observed, but there is greater pain in the right side and if pressed upon heavily over the region of the liver, the animal will moan with pain. The nose and mouth are dry and hot, demonstrating a fevered condition. The breathing is slow and emits an offensive odor; the pulse is slow and the ears and limbs cold; the manure is dry, hard and black and covered with a yelluwish slime; the urine is scanty, high-colored and has an offensive smell.

Treatment:-The bowels should be unloaded as quickly as possible. Give

Epsom Salts........... two ounces.

Calomel.............. two grains. Mix.

Give at one dose in a pint of warm water. Frequent injections of warm water and castile soap are beneficial until the bowels act freely. Also give the following;

Glauber Salts........... one pound.

Powdered Saltpeter....... one ounce. Mix. 
Make eight powders. Give one powder morning and evening in a pint of warm water. Should the bowels become too loose, divide the powders arain. When convalescence takes place, with returning appetite. nothing but the most easily digested food should b. wiven, as boiled bran mash, flaxseed meal, turnips. rarrots, malt, etc., with crood, pure water and rooni should be allowed for plenty of exercise. Carefull! avoid too high, rich, stimulating food.

\section{INFLUENZA-EPIZOOTIC-DISTEMPER.}

This disease, though somewhat peculiar, is yet quite common in this country. It is not confined to any particular locality and the flocks over an extended area, may become affected at the same time. It is most preralent during continued cold, damp, changeable weather and the most fatal where the land is low and marshy or along river bottoms where heary fogs prevail in the morning and evening. It is always seen in the mildest form, where the herd is kept on high, dry elevations with good shelter for the niwht. It is neither contagjous nor infectious, but is produced through atmospheric influences and consists of an irritated and inflamed rondition of the air passages, causing a general disturbance of the system, with more or less prostration.

Symptoms:-The eyes look red, with a profusion of tears running down over the cheeks. There is a discharge of mucous from the nostrils, coughing and snee\%in to rear the nostrils, loss of appetite, suspended rumination, with emaciation and general weakness. The bowels are usually constipated and sometimes glight bloating is present. 
Treatment:- When first noticed, if on Iow grommi, change to high, dry pasture with groml shelter for the nights, and allow a liberal supply of chlorate of potassium in the drinking water, say on llachm three times. a day. If the bowels are constipated, wive

Épsom Salts ........... one ounce.

Ginger............. one drachm. Mix.

Give at one dose in half pint of water. When there is ronsiderable fever and much prostration, stimulants are indicated, as half ounce of whiskey or brandy and two ounces of hot water, to which should be added a teaspoonful of sugar. Give every four or five hours, or better still, give the following:

liquor ammonia acetatis, one ounce, every four hours. If the eyes are bally inflamed and mattering, bathe occasionally with warm water and salt. When the appetite is returning and recovery is indicated, give a soft, nutritious diet, as bran mash, flaxseed meal, boiled oats, etc., to which a little gentian may be adderl to tone up the system and assist digestion; or the following formula may be used:

Gentian............... one ounce.

Ginger................ one ounce.

Copperas............... one ounce.

Saltpetre............. one ounce. Mix.

Make sixteen powders. Give one powler norning and evening. Good nursing and shelter should always be provided for sick animals.

\section{DYSENTERY.}

This disease is caused in a similar manner to diarrhoa, and quite frequently results from neglected or a prolonged case of diarrhoea, as well as turning out on rich pasture after feeding on dry food. It may also 
be caused by drinking impure water, fwoding on poor, indigestible fodder. Excedingly hot and damp weather without shade or shelter, is a prolific cause.

Symptoms :-It diflers from diarrhoea in the severity of its symptoms. In diarrhoa there is little or no fever present, while in dysentery there is a high fever. The dung is passed in small, hard lumps and is covercd with blood and slime, and possesses a disagreable smeil. It is discharged often and is accompanied by heavy straining. The sheep humps up its back and moans or grunts during the act. The wool feels harsh and loosens its loold, and in the course of a few days is easily pulled out. There is more or less grinding of the teeth, a tucked-up appearance at the flank and the animal presents a discouraged and dejected appearance.

Treatment:- When a large number in a flock is affected, remove to a high and dry ground where there is different pasture, and pure water to drink, as there is more or less thirst. Such as do not recover quickly after the change, should be given a mild physic, as

Castor, or Raw Linseed Oil two ounces. Laudanum............. drache dix.

Give at one dose in half pint of thaxseed tea. They should now be separated from the balance of the fiock and kept in a cool, shady place, as the hot sun is very aggravating and fatal in this disease. The physic should be followed with frequent doses of flaxseed or slippery elm tea, to which should be added twice daily, a teaspoonful of laudanum. Gire one ounce of castor or raw linseed oil, every other day; it will do no harm and is often productive of much good. The pastures occupied by the flock while affected with dysentery, should not be used again, until after a couple of heavy rains, to destroy and wash away the poison from the manure, which would otherwise remain on the grasses and produce the disease. 


\section{BLACK-LEG-QUARTER-ILL-MURRAIN.}

This disease is not often observed in the Eastern or Middle States, from the fact that farmers depend upon wintering their flocks on stored provisions; but in the Western States, where large flocks are kept upon the prairies, many depend upon pasturing or lay in only a small supply of food for winter use; consequently, when a severe winter is passed through, the sheep being sparsely fed, are in a very thin condition. The spring () pens up with rains and hot sun, the grass grows luxuriantly and the half-starved sheep gorge themselves and take on flesh so rapidly that the blood becomes dis"rganized, so that while the owner or herder thinks the sheep are thriving finely, they commence to die very suddenly. It generally takes the young and thrifty sheep; the old ones are seldom attacked.

Symptoms:- When one or two die so suddenly, the herder generally looks over the flock, when he will observe some lame and limping. The sides or quarters will be found swollen, the wool comes off easily, and by pressing the hand down over the swollen spots, a crackling sound is heard under the skin, as thomgh filled with air or gas. The eyes look red and the mouth and tongue are inflamed and blistered. The urine is high colored, the bowels constipated, and if any dung is passed, it is covered with blood. In a short time the sheep can no longer maintain the standing position. It falls down upon its side, the tongue is protrucled, and hloating is ofserved. The limbs become outstretched and death claims the victim in a very few hours.

Treatment:-As prevention is the best cure, it is only necessary to remove the cause, and this is best done by arefully avoiding sudden changes from poor food to rich nutritious food or pastures. But when any of the 
afore mentioned symptoms are observed, the whole flock should be placed in a yard where there is no food to be obtained. Then take out to pasture for a couple of hours, morning and evening, until the stomach and entire system adapt themselves to the change, and the animal takes on flesh more moderately. Such as are sick, should have a physic immediately.

Glauber Salts ......... four ounces.

Sulphur...............half ounce.

Ginger............... one drachm. Mix.

Give at one dose in a pint of flaxseed tea or oatmeal gruel, or a little syrup and water. In the course of a few hours, a half teaspomful of turpentine in an oume of raw linseed oil or flaxseed tea may be given at intervals of five hours; or a teaspoonful of sweet spirits of nitre may be wiven every five hours, in a little water, as a drench. As before stated, this disease is very rapid and fatal; consequently treatment is not very satisfactory.

\section{SHEEP SMALL-POX.}

Small-pox is a contagious, eruptive fever, rery muh rosombling small-pox in the human family. It is not ilentical with the human-pox or cow-pox, but yet it is of the same type. It is not communicable to man or other animals, even by inoculation, and belongs exclusively to the sheep. Originally, it is produced by sheep inhaling impure air, or air contaminated with a virus, thus poisoning the blood, and the eruptions formex is nature's effort to rid the system of the imprisoned werms. It is most often contracted by coming in contuct with sheep affected with the disease, as it is equally as contagious and infectious with sheep, as small-pox is with the human family. 
Symptoms:- Sheep-pox assumes two forms, one malignant or dangerous, the other mild. But in either form there is a period of incubation, of from nine to eleren days, during which time no symptoms of the disease appear. At the termination of the period of incubation, fever is observed, the eyes look red and bloodshot, the breathing is quickened, the skin is dry and hot, there is loss of appetite, excessive thirst, and the animal will be noticed to lick at dirt. The eruption now makes its appearance in the form of small pimples on the skin, between the forelegs, which space is least covered with wool. These pimples soon run together, and in a few days a small blister appears, containing a fluid like water, which soon develops into matter and looks white. It is now at the stage when the matter should be collected and preserved for inoculation purposes. In a few days it dries up, the scabs scale off and leave a mark from which no wool will ever grow. The sheep now begins to improve and unless it receives some backset, a rapid recovery may be anticipated. When the malignant form is observed, there are no pustules found, but scarlet or purple spots are noticed. The skin rracks in various shapes, the wool falls off and a foul-smelling discharge comes from the nostrils; otherwise, the symptoms are the same as in the mild form. Death usually takes place within three weeks from the outset.

Treatment:-After the disease has once established itself, medicinal interference will avail but little, and instead of relying upon the use of medicines, greater attention should be given foward its prevention, which is done in two ways-first, by isolating the affected sheep from the healthy ones, as soon as noticed, and socondly by vaccination, which is performed by taking some of the white matter from the pustules of an 
affected sheep, and inserting a small portion of it beneath the skin of a healthy sheep, either at the ear or the end of the tail, care being taken that the matter is obtained from a sheep that is not affected with the scab or rot. Vaccination is the safest preventative, as all reports from France, Germany, Austria and Russia, where it has been thoroughly practiced, tends to prove. It has greatly lessened the mortality. It is needless to vaccinate sheep at the present time in this country, for as yet small-pox has not made its appearance, but owing to the rapid transit between Europe and this country, sheep might be imported and landed while the disease is in the incubative stage, and the malady would scatter very rapidly, then vaccination would he advisable, and when commenced, it should be continued until it has thoroughly taken on all. From the time smallpox is observed, there should be good nursing with plenty of fresh air, carefully avoiding draughts; there should be given a liberal amount of flaxseed or oatmeal gruel, in pure water, slightly warmed, into which may be placed a few drops of aromatic sulphuric acid. Give as a drink. If the sheep appear very weak, give the following:

Liquor Acetate of Ammonia........ .................... seven ounces.

Sweet Spirits Niter ......two ounces. Mix.

Give tablespoonful three times a day. When an animal dies of small-pox, it should be buried rery deeply and have plenty of lime thrown on the carcass, or else it should be burned up, as every portion of the dead animal will convey infection, if not completely destroyed. 


\section{APHTHA-FOOT AND MOUTH DISEASE.}

Aphtha has been observed in various parts of this country, but not to any great extent; only a few scattered cases have been seen. It has never affected whole districts, as it has in Europe. Seemingly, our climate is not suitable for its ravages, but rather tends to eradicate the disease than to encourage its develop-

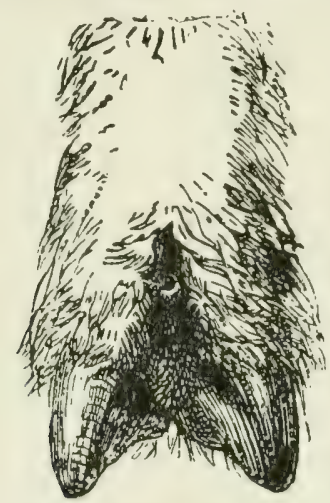

ULCERS BETWEEN THE TOES.

ment. Yet, some form of the disorder may develop in this climate and prove rery troublesome. In those climates which are favorable to its propagation, it is very contagious, and belongs to a class of eruptive fevers. It emanates from a ferment or poison in the blood.

Symptoms:-The ailment is usually ushered in with a chill, which is not always observed; a high fever follows accompanied with a cough. The appetite now becomes whimsical and the animal ceases to chew its cud. There is slight tenderness over the loins, and a grinding of the teeth with saliva drivelling from the mouth. Blisters containing a watery fluid, are now observed on the tongue, lips and mouth as also around and between the feet. These blisters eventually break 
and sometimes run together, leaving a raw sore, which is quite painful. The feet become so swollen and full of pain, that the animal lies down most of the time ano when attempting to walk, does so in a lame, unsteady manner. Like all other eruptive fevers, it runs its course, which is usually from ten to fifteen days, and if it terminates favorably, the animal is proof against the same disease, should it again be exposed thereto. But if unfavorable, the disease increases in force until the feet drop off, and the animal dies in great misery.

Treatment:- When not of a malignant type, the treatment is very simple and effective. Give one good physic but do not repeat it, as

Epsom Salts...........two ounces.

Jamaica Ginger......... one drachm.

Water...................... Mix.

Give at one dose. Wash the mouth twice daily, with the following lotion:

Powd. Alum............. one ounce.

Chlorate of Potassium...... one ounce.

Water................. one quart. Mix.

Wash the feet well, every other day with a mixture of bluestone and soft water, say an ounce of bluestone to two gallons of water. After bathing, use

Vaseline............ four ounces.

Oarbolic Acid..........two drachms. Mix.

Apply well, then wrap the feet in a cloth to protect them from dirt. Isolate all diseased animals from the healthy, and after the disorder has abated, fumigate the buildings thoroughly, by burning sulphur or scattering chlorinated lime. If the malady appears in the malignant form, the cheapest and most effective treatment is to kill and bury or burn all those affected, and remove such as have not taken the disease, to other quarters. 


\section{FOUL IN THE FOOT-SORE FEET-FOOT LAMENESS- GRAVEL.}

Causes:- Sheep that are kept in yards that have accumulations of manure, so arranged that it is very wet, causing a liquor to be formed by the manure and water, are liable to be afflicted with sore feet, as also such as are kept on low, boggy, marsh pasture. Diseases of this character are also observed during wet seasons, when pasturing where the grass is very tall and growthy; the cleft between the toes being irritated while walking through the grass. It is sometimes produced by a gravel or other substance becoming so firmly lodged between the toes, that the animal is unable to remove it, and it will produce a foul sore.

Symptoms:-It is seldom noticed until the sheep begin to show lameness, when upon examination, the cleft of the foot and the surrounding skin, will present a whitish appearance with some inflammation and swelling. These swellings soon develop into sores, greatly resembling ulcers, or ulcers may be present, and this condition should not be mistaken for foot-rot.

Treatment:-If attention is given to the trouble, it will soon disappear. If caused by filthy yards, remove to a dry, clean place, with plenty of dry straw to walk upon; or the flock may be turned upon hilly ground where the pasture is short. If they do not improve rapidly, take two pounds of copperas and dissolve it in ten gallons of soft water; put it in a tub and place the patient's feet in the solution; keep them there from two to three minutes, then turn on a dry place; it may be necessary to repeat this in three days, but one application will generally suffice. Or take,

Blue Vitriol............. one ounce,

Vaseline, or Tar.......... six ounces. Mix. 
Apply all around the sores. If gravel or some other substance causes the trouble, remove it, then trim off the diseased parts and apply the latter prescription.

\section{H00F-R0T--F00T-R0T.}

This disease is frequently observed in this country and the American shepherd who has charge of any great number of sheep, sooner or later becomes familiar with one of the greatest disturbing elements that befalls a flock.

Causes:-While there is no question about it being contagious, and in many instances contracted through

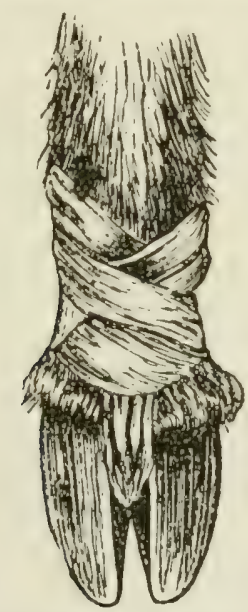

BANDAGE FOR FOOT-ROT.

the source of contagion, yet it will break out in a flock that has never been in contact with sheep affected with the disease, thus well demonstrating that it can arise spontaneously. These cases are usually found in sheep that pasture on wet, low ground; the feet become dis- 
eased and foul, the horn separates from the sensitive sole, and filth accumulating there, exerts its influence on the vascular tissues and softens the whole sole; if neglected at this period, germs are developed which destroy the whole structure of the foot very rapidly, and are highly contagious in character, as is observed in other sheep contracting the disease from the affected ones, although changed to higher and dryer pastures.

Symptoms:--The disease is usually observed in the forefeet, although the hind feet are not exempt and may become affected first. When attacking the forefeet first, the sheep will be very lame, possibly walking upon its knees while feeding, and when through feeding, it is almost constantly lying down. Upon examination, the feet will at first be found highly inflamed, looking red and moist, or rough and chafed. Then blisters will form around the heels and between the toes, which soon break and discharge. Ulcers are now formed, which suppurate and discharge a foul-smelling matter. The bottom of the foot soon separates from the sensitive parts, and a foul-smelling matter oozes out. Soon afterward the horny sole comes off, leaving the sensitive parts exposed, which look swollen, black, and spongy, forming a good nest for maggots, which are frequently found. The constitutional symptoms are fever, to a greater or less degree, and loss of appetite; the sheep failing to chew its cud and lying around in a stupid condition.

Treatment:-This should be prompt, from the fact that as the disease advances, the whole system becomes involved, and recovery is slow and tedious. The malady is dangerous and painful, but if immediate attention is given, with proper treatment and care it is always curable. As soon as lameness is observed, an examina- 
tion should be made, when all of the diseased parts must be removed with the knife, as a failure to do this, will prolong the disease and resist the action of all medicines that may be applied; but if the diseased parts are thoroughly removed, treatment is effective and certain. If there is an extra growth of horn at the toes, it should be cut down, and if matter is found, remove it and well expose the parts. If any portion of the horn looks whiter or softer than the balance of the foot, open it, as matter is likely concealed within. Should it bleed some, have no fear as there is no special danger. Clean the knife quite often, so that the matter from the affected parts will not come in contact with and adhere to the healthy tissues, carrying infection. After all diseased portions are removed, you are ready to apply medicines, and any caustic that will destroy germs, is beneficial. A cheap and easy method and one that is very effective, is to take a large tub or vat and put in it a strong solution of blue vitriol, say a pound to four gallons of water, and keep it as hot as can be borne by the hand. Have it about four inches deep, then put the feet of the sheep into the solution, holding them there about ten minutes, until thoroughly penetrating every crevice, then turn the animal on a dry, short pasture. One good application is generally all that is required. Or chloride of lime may be used. After paring the foot, cover it with the chloride and bandage. Inspect the foot in a couple of days, and if necessary apply again, repeating until a cure is completed. Or take,

Finely Powdered Blue Vitriol..... .................. one pound.

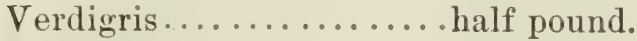

Linseed Oil.............. one pint.

Pine Tar.............. one quart. Mix.

Apply well all around the foot and allow it to dry, before turning out to pasture. Give internally, 
Epsom Salts........... two ounces. Baking Soda..........two drachms.

Water.................. half pint. Mix.

Give at one dose. Drachm doses of saltpetre given night and morning, will prove very beneficial, if there is much fever present. But it is seldom necessary to give medicines internally, the local applications being sufficient.

\section{SCAB-THE MANGE OR ITCH IN SHEEP.}

Causes:- Scab is a highly contagious disease, produced by an insect in or upon the skin. There are different forms of parasites that produce scab, concerning which it is here unnecessary to go into detail, as the treatmeut for all is the same. It has been asserted that.

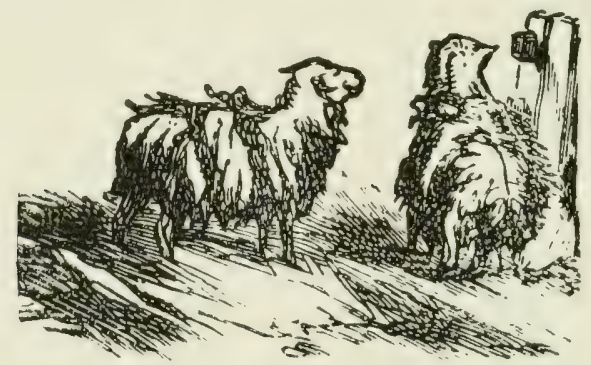

SCAB.

scab originates spontaneously, from neglect, exposure, etc. Such assertions are without foundation. It is now thoroughly understood that all animal life, owes its existence to a fecundated egg, and the scab insect is produced in the same manner. There is both a male and a female insect, the female being considerably the largest, and also exceeding the male in numbers, but living a shorter length of time. She dies very shortly after laying her eggs, which she deposits 
in the pores of the skin or around the scabs, and as they are very numerous and hatch in three days, there is a rapid increase. It has been truthfully asserted, that from one female, a million and a half of her progeny will be produced, in three months. This accounts for

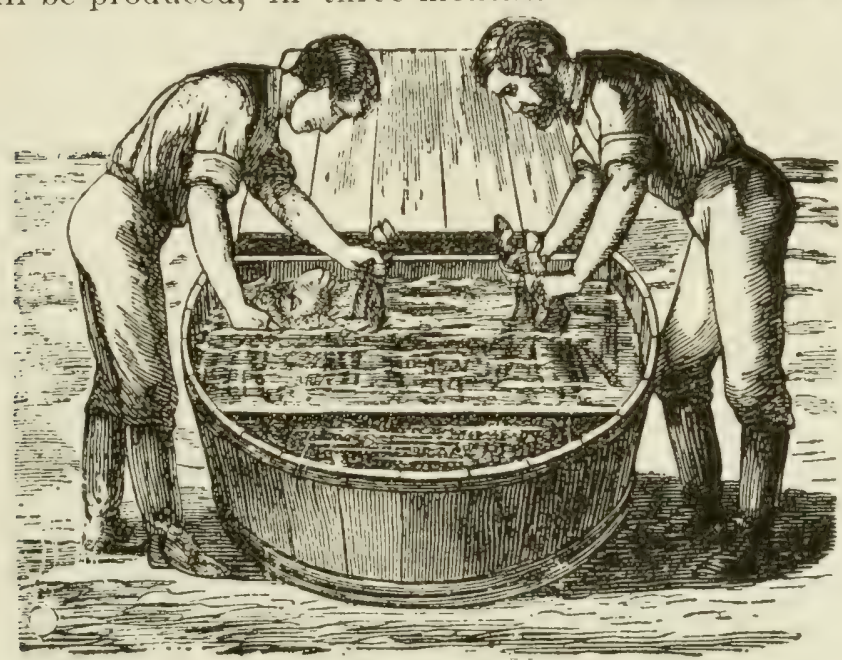

DIPPING SHEEP.

the rapidity with which the disease spreads, and also gives warning of the necessity for prompt and energetic treatment. That it sometimes appears in a flock under peculiar circumstances, none will deny, but flocks have been known to become affected by pasturing upon lands that had not been used for three years, after having grazed a flock of scably sheep, thus showing that it has great tenacity of life eren when not in its habitation, the sheep.

Symptoms:-The first symptom observed is restlessness accompanied by itching. The sheep will be noticed rubbing itself against a fence-post, stump, or stone, or any object to which it can get; the wool loosens and is 
rubbed off, leaving bare patches. They also frequently bite and nibble at their skin, thus pulling out the wool. The skin, upon examination, will appear somewhat thickened and whitish in color, and soon becomes covered with a yellowish liquid; later on, the scab is formed, which if left unattended, will so annoy the animal that it will rub the scabs off, causing them to bleed, and large sores or scabs are formed, from which the wool drops off, leaving the poor sheep an unsightly looking object. The sheep now rapidly falls off in flesh; the appetite is gone, the fleece is lost, and the animal dies from exhaustion and torment.

Treatment:-This can be acconplished in two waysfirst, by dipping the sheep into some solution that will kill parasites; second, rub ointment into the skin that will destroy the insect. But ointments are expensive and require more care, time and labor, as well as being a detriment to the sale of the wool for that season; for these reasons, it is seldom used. The first method, dipping, is both the cheapest and most effectual manner of disposing of the parasites. There are several formulas for making a solution; I will give the best, cheapest and safest for the shepherd or farmer to use. Take

Tobacco Leaves or Stems. . five pounds.

Flower of Sulphur..............

.......... one and one-half pounds.

Water..............twenty gallons.

Mix the tobacco and water and bring it to a boiling heat (but do not boil) until the strength is taken from the tobacco. Then into this liquid, put the sulphur and stir thoroughly. Allow the mixture to cool off, to about 118 or 120 degrees, then commence to dip the sheep. If there are any old, hard scabs, break them up and remove them, then dip the sheep and hold it 
under the water until it is well soaked, after which elevate it and let the water drip off, or place it on a rack where the liquid will drain back into the tub again. Cntinue in this manner until all have deen dipped that are affected. Stir the liquid frequently, to keep the sulphur floating, also add a little hot liquor occasionally, to keep up the temperature. The animals should be kept in a yard for a time, so that none of the liquid will drip upon the pasture. Repeat this process in about ten days, so as to destroy any new parasites that may have hatched out. Or take

Tobacco .................ten pounds.

Pearl Ash ............three pounds.

Sulphur............. three pounds.

Soft Soap.............three pounds.

Mix in ten gallons of water at boiling heat, (but do not boil) until the strength is extracted, then add water enough to make one hundred gallons. Use according to the foregoing instructions. It is usually estimated at one gallon to each sheep. Repeat in about ten days. Either of these recipes, if followed closely, will exterminate the scab insect, and result in a complete cure.

\section{MAGGOTS.}

Maggots are the product of the common flesh fly, the blow, or blue-bottle fly, and the meat tly, any of which deposit their eggs on a wound or injury; or the taglocks which accumulate around the tail of the sheep, will develop maggots so rapidly that within twenty-four hours they may be counted by the thousands. These maggots soon become live agents, which not only exist and subsist upon the sore or injured parts, but attack the skin, which becomes irritated and a serous fluid oozes out. In a little while the skin is pierced, or gives 
way, and these little pests keep on devouring the sheep until it is a living mass of corruption, and if not given prompt attention, it very soon succumbs. There is more trouble from this source during warm, wet weather, than during dry seasons. Prevention is always better than cure, so keep the tag-locks well trimmed, and the flies cannot then, deposit their eggs upon them, for they lay their eggs only upon a filthy spot. If the sheep gets bruised or cut badly enough to form a sore, attend to it and keep it clean, until thoroughly healed.

Sometimes at weaning time, sheep will suffer from caked bags, which break, causing ugly sores which the flies soon attack, resulting in the formation of maggots. Therefore, at weaning-time the ewes should receive extra attention, to prevent this trouble. Sheep that are affected with maggots, will stroll off by themselves, lie around considerably, and remain separate from the flock. When maggots are discovered, take turpentine, crude petroleum, or whale oil, any one of the three, and pour it freely upon them. This will kill the maggots, and prevent the flies from depositing more eggs. It will also assist in healing the wounds. These are the best remedies for this trouble.

\section{SHEEP TICK.}

The sheep tick has been observed by every farmer that owns or controls sheep. It is a dark red, toughskinned, plump-bodied insect, which developes from an egg, laid by the female tick. As the female deposits but one egg at a time, they do not multiply very rapidly, and if some attention is given to the sheep, it is easy to keep the flock comparatively free from them. When the sheep are shorn, in the spring of the year, the ticks emigrate to the lambs, for better shelter and 
foraging grounds, and if the sheep and lambs are dipped into a solution of tobacco and sulphur, it will rid them of these pests. Take

Tobacco (cheap leaves or stems)....

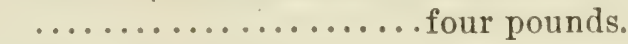

Sulphur............... one pound.

Water............twenty gallons. Mix.

Steep in hot water, a sufficient length of time to take the strength from the tobacco; then dip the sheep

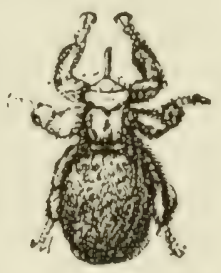

SHEEP TICK.

and lambs while the liquid is as hot as they can bear it. Hold the body in the liquid up to the head, for two or three minutes, then allow them to drain off'. Repeat the same operation in September and you will free your flock from this pest. The sheep will thrive better and on less food, and the wool will not be rubbed off, thus insuring a profit that will more than repay the cost of dipping, beside the comfort to the sheep.

\section{THE SHEEP LOUSE.}

Lice are seldom seen on sheep whose wool is very yolky. They are more often found on dry-fleeced sheep, that have very little yolk in the wool. The louse is a very small insect, with a red head and a pale yellow body, marked with dark bands, running cross-wise. It is generally seen along the sides of the neck, between 
the forelegs, also around the groin, on the inner side of the hind legs. - It is the cause of considerable annoyance to the sheep, and when the animals are observed rubbing their necks between bars or gates, or rubbing,

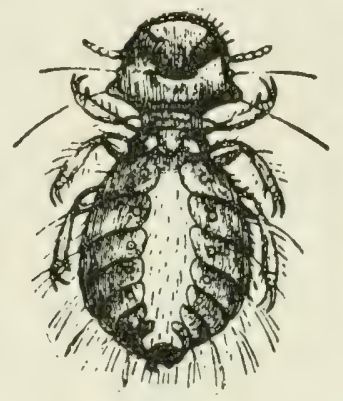

SHEEP LOUSE.

scratching and biting their legs on the inner surface, they should be examined, and if lice are found, use the following:

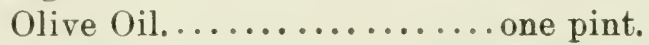

Sulphur.............. three ounces.

Creosote.............. one drachm. Mix.

Rub the parts infested with the lice; or take blue ointment and rub the parts sparingly. If the blue ointment is used, protect the sheep from storms for ten days. Sheep that are regularly dipped for scab or ticks are seldom troubled with lice.

\section{INTESTINAL WORMS-TAPE WORMS.}

The bowels of sheep are often the home of a great variety of worms, as round worms, pin worms, tape worms, thread worms, etc.

Causes:-Sheep that are running on pasture, may swallow some eggs that have been deposited there, by 
any animal that they may inhabit. The tape worm, for instance, may be dropped by man, dog, skunk, hog, rabhit, etc. But the most fresuent source from which they get them, is through the dog. Dogs are verv

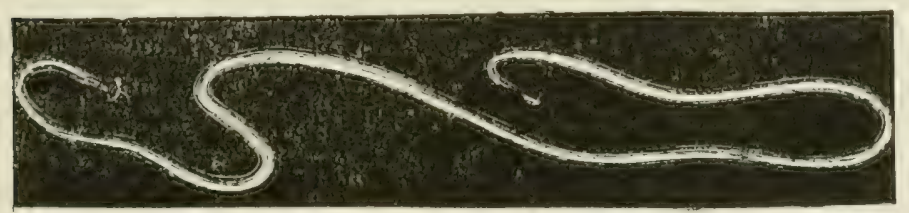

THREAD STRONGIF ENLARGED.

prone to worms, and when their excrement is left upon the pastures, these egrs from worms are liable to be piclied up by sheep, in whose intestines they soon

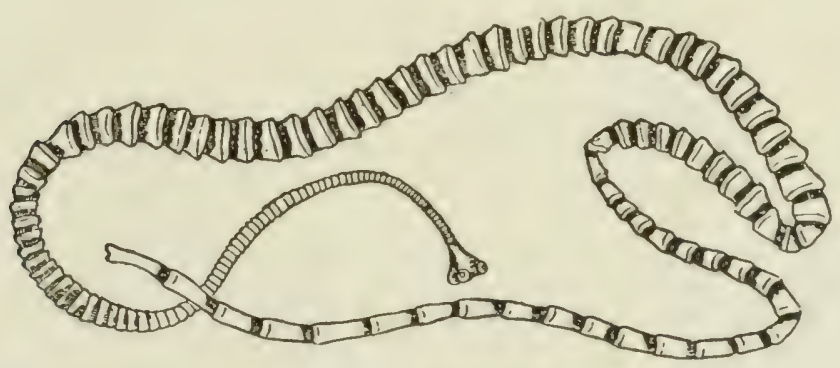

TAPE WORM.

develop, and sometimes cause great havoc among the flock.

Symptoms:-The stomach and howels are sometimes alive with worms, without any ohservable signs, until the animal is slaughtered, when myriads of them are found, and the sheep previously, to all appearances enjoyed excellent health. In general, the symptoms noticed are a ravenous appetite, alternating with a refusal of food: or there may be loss of appetite, with constipation alternating with diarrhœa, with a desire 
to eat dirt, sand, ashes, old mortar on walls, etc. There is also irritation around the hinder parts, as will be noticed by the animal rubbing its rump against fences, posts, or other objects. Sometimes worms or parts of worms are seen in the excrement that has recently passed. A short, dry cough is frequently a symptom of worms in the bowels. When there is a falling off in condition, from worms, it occurs very gradually, and when death claims the animal, it is greatly emaciated.

Treatment:-Old sheep are seldom troubled with worms. They generally find lodgment in young sheep and lambs. The medicinal doses should be varied somewhat, giving lambs one-half as much as required for a young sheep. For round, and thread worms, take

Raw Linseed Oil.........two ounces.

Spirits of Turpentine....... one ounce. Mix.

Give at one dose, to be repeated every four days until four doses are given. For the expulsion of tape worm, take

Powd. Areca Nut........ one drachm.

Oil Male Fern.......... fifteen drops. Mix.

Give at one dose in half pint of water, to be followed in twelve hours, with

Epsom Salts........... three ounces.

Ginger................. one drachm. Mix.

Give at one dose in a pint of water. Another remedy which is just as effective is to deprive the animal of food for three or four days, and during that time give a handful of hulled pumpkin seeds, ground, three times a day. At the end of the fourth day give a physic, as

Epsom Salts........... three ounces.

Ginger............... one drachm. Mix.

Give at one dose in a pint of water. When the worms are passed they should be destroyed by burning. 


\section{THE ROT-LIVER ROT-FLUKES IN THE LIVER.}

This disease is produced by a flat worm, of a whitish color, varying in size from a half inch to an inch in lencth and one-fourth to three-einhths of an inch in hreadth, which seels the intermal oriman for development, and more especially the liver. It is propagnted

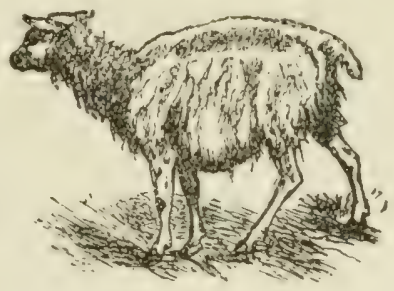

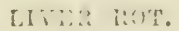

by means of eggs, which are deposited in the bowels and pass away with the manure, and throngh various sources it is conveyed into pools of stagnant water, where they pass one stage of their existence, possibly in the bodies of snails, water insects ant? shell-fish. As it is most prevalent duringrang suasons it is reatomable to presume that the meaduws berome inmmaterl, and as

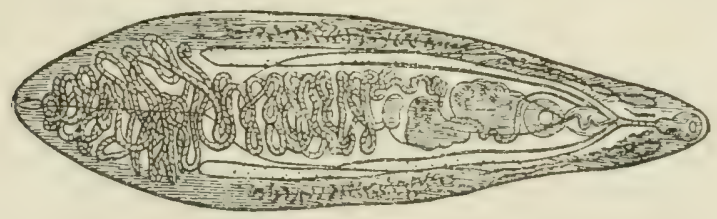

FLUKES.

the waters from these porls hecome scattered over considerable surface, these flukes are left upun the grasses, where they again enter the animal for final development. As they are taken into the stomach they pass on until they enter the gall inct, passing from that into the gall sack, then to the liver, where they complete 
their development. The disorder is rarely seen where sheep are grazed upon upland pastures, but is quite prevalant on lowland pasturage, especially during rainy seasons, when there is frequent inundation.

Symptoms:- When sheep are first affected with flukes they are inclined to take on flesh very rapidly for a short time; when this ceases, emaciation or failing in llesh, commences. An examination of the eye ball should now be made. In the healthy animal the membranes covering the eye-ball and eye-lids are a pink or reddish color, in fact, so much so that an amateur would suppose there was infiammation of the eyes; but when affected with flukes this membrane is of a pale or yellowish color. This is a symptom that never fails when the sheep is affected with flukes. As the disease progresses the appetite becomes irregular, but the demand for water increases. Dropsical swellings now appear; a large, watery tumor is noticed under the lower jaws, the back becomes prominently arched, the skin looks pale and bluodless and is tightly drawn to the frame. The wool is dry and harsh and separates very readily from the skin, leaving bare spots. A diarrhoea now sets in, caused by disoinanization of the liver. The flanks look hollow and there is a weakness or tenderness across the loins. The eyes are very yellow, or jaundiced. The whole system is now deranged. The breath imparts an offensive odor and the poor animal lies with its head outstretched, wearing a solitary and forlorn look, until death claims it as a victim. When the animal commences to run down the course of the disorder is very rapid and fatal.

Treatment:-The treatment should depend more upon prevention than upon a cure by medicinal agents, for when rot has reached the stage of thorough development and great emaciation, with a jaundiced appearance, 
rredicines will avail but little. As flukes cannot develop) at certain stages of their existence upon dry, uplanl pastures, it is well to graze the flock upon hilly pastures, fencing off all ponds of stagnant water, or draining them and watering the sheep from wells. should a flock become affected with flukes, but not to the extent of injuring them materialy, it is best to fatten and dispose of them. It is a well established fact that sheep aflected with flukes will live and thrive, but as to the amount their system can tolerate without serious injury cannot be definitely ascertained. That flukes are an established destructive factor 11 this country cannot now be denied as over one hundred of them were found in the liver of a single rabhit, in Minnesota, and they have also been found in deer and other animals, saying nothing of the number of sheep that have perished, the owners at a loss to know the cause. Common salt is known to be a preventative and destroyer of the flukes, at certain stages, and it should he given quite liberally; and as turpentine is very penetrating to the system, entering every crevice, and is is great destroyer of worms, it should enter into every mubination in the treatment of this disease. The sheep should not he allowed any fond for three or four hours either before or after wiving the medicine. The following formula has been used in England, with remarkable success :

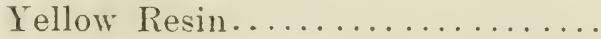

........ one and one-half drachms.

Spts. Turpentine.

........ one and one-half ounces.

Calomel ...........eighteen grains.

Tincture Iodine........ thirty drops. Mix.

Give at three doses, one every morning, for three consecutive mornings, in a little gruel. Or you may give 
Raw Linseed Oil.........two ounces.

Spts. Turpentine..........half ounce. Mix.

Give at one dose, and repeat every four days, until four doses are given. Or

Saltpetre.............two ounces.

Powd. Ginger............ one ounce.

Salt.................. one pound.

Boiling Water...........three quarts: Mix.

Dissolve thoroughly, then add spts. turpentine, ten ounces. Shake well before using. Give a wineglassful every fourth morning, until four doses are given. When sheep are known to have become affected with flukes from certain pastures, it is best to plow up those meadows and raise other crops for two or three years, to destroy the parasite, and where it is at all possible, it is best to graze sheep upon dry, upland lastures, with a provision of pure, fresh drinking water.

\section{LOCKJAW.}

It is seldom that lockjaw, or tetanus, is found among sheep, but as it is a nervous affection, it may be produced by fright from being chased with dogs, or by injuries or wounds from any source, when nearly or wholly healed upon the surface; or it may be caused in lambs, from cutting off the tail, or it may result from castration.

Symptoms:-The head is elevated and the eyewashers will protrude over the eye-ball to a greater or lesser extent. The animal will appear very nervous, and walk with a stiff, stilty gait. The jaws are closed but can be moved sidewise and sometimes opened a little; there is also some grinding of the teeth and more or less desire for drinking water, of which little can be 
swallowed, as the throat is affected. In some instances the head is drawn around to the side, while in others it is drawn upwards.

Treatment:- Remove to a dark, quiet place and first give a physic, as

Epsom Salts............two ounces.

Water....................... Mix.

Give at one dose, to be followed with

Bromide of Potassium .... two ounces.

Chloral Hydrate......... one ounce.

Water.................. que quart. Mix.

Give a wineglassful three times a day. If you cannot drench the sheep without too much excitement, take a syringe and squirt the preparation well back into the mouth. Use extra precautions not to excite the animal, and allow no one to go near it but yourself. If it can eat or suck any soft food, give all it desires, and also allow plenty of drinking water. If the disease results from a wound or from castration, grease the injured parts once daily with solid extract of belladonna. Quiet and warmth are very essential to effect a cure, as some will recover with good nursing, without the use of medicines.

\section{FRACTURES.}

Broken limbs sometimes occur where a flock of sheep is going over a fence; an animal ma become fastened between the bars and thus be crowded on hy the balance of the thock, or injuries of this character may result from being kicked or trampled upon by horses and cattle, and in various other ways, too numerous ' to mention. The bones will readily grow together, if fastened with splints and bandages, first getting the bones in proper position, the ends directly 
opposite each other. The splints should he wrapped in cloth or cotton batting, to prevent chafing and the bandages should not be so tight as to prevent free circulation of the blood; they should be examined frequently to see that they are in position and not too tightly drawn, and they should be worn for three or four weeks. It is not always necessary to confine the sheep, as they often do as well at pasture, but if confined, feed soft food.

\section{CONSTIPATION IN LAMBS.}

Constipation in lambs is generally brought about by feeding on cows' milk, or the milk of another ewe. When the disease occurs, the bowels cease to act, or that which does pass, is hard and lumpy, and there are long intervals between each passage, with considerahle pain, the back arched, and heavy straining, when the discharge does take place. The lamb lies down most of the time. The urine is scanty and the belly distended.

Treatment:-This consists of injections of warm milk or water into the rectum or last bowel; three or four ounces should be injected at a time, and this should be repeated every hour or two, until the bowels move freely. To do this properly, seize the lamb by the hind legs and elevate it so that it barely stands on its front. toes; hold it in this position while giving the injection, and also for a few ninutes afterwards. Give internally every six hours, until the bowels are relieved, a dessertspoonful of castor or raw linseed oil. If the lamb seems dull and inactive after the bowels have responded, rive small doses of sweetened hot brandy or whisky.

\section{DIARRHOEA, OR WHITE SCOURS IN LANBS.}

Diarrhœa in the suckling lamb, is often called "White Scours." 
Causes:-It may result from lying on cold, damp ground, exposure to storms, sudden changes of temperature, cold drinks, etc.; but the most frequent source, is

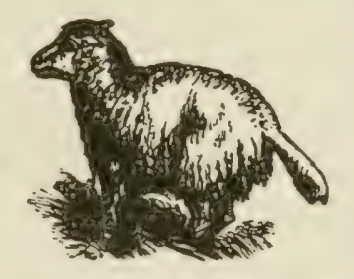

WHITE SCOURS IN IAMBS.

the altered condition of the mother's milk, when fed unon thrifty succulent urasses, or upon roots, as turnips, carrots and mangels. When not arising from these causes, there is a disordered condition of the lamb's stomach.

Symptoms:-There will be a watery dischare from the bowels, of a whitish color, which is the indigested milk passing straight through the intestines. Sometimes the discharge is of a pale, greenish color, very nuch resembling whey. This is produced by indigestion, the food becoming coagulated in the stomach, where it may accumulate in quite large quantities. The lamb now appears stupid and ceases to play with other lambs. It lies around considerably, and when standing, it is doubled up and the back is arched. It ceases to nurse or eat, but appears thirsty, and makes frequent efforts to pass dung. While lying around, it will bleat frecuently, and upon pressing on the belly, it evinces pain. The belly has a shrunken or tucked-up appearance; the animal loses flesh rapilly, and if not relieved, dies within a few days. Worms in the bowels will sometimes cause diarrhoea, but when this is the case, the dung will be mixed with slime or mucous. 
Treatment:- When causeri by the ewe's milk, change her diet immediately. Place her upon dryer food or poorer pasture and give the lamb, two or three times a day, the white of an egr heaten up with five times the amount of warm water, to which may be added a few drops of laudanum. Or,

Raw Linseed Oil.........two ounces.

Essence of Ginger........ one drachm. Mix.

Give a teaspoonful once or twice daily; or take

Prepared Chalk........... one ounce.

Powd. Catechu ........ four drachms.

Powd. Ginger.......... two drachms.

Powd. Opium...........half drachm.

Peppermint Water......... one pint. Mix.

Shake well before using and give two teaspoonfuls morning and evening. When resulting from worms, give

Raw Linseed Oil.......... one ounce.

Spts. Turpentine..........half ounce.

Laudanum..............ten drops. Mix.

Give at one dose. If at any time there is great weakness, give

The Whites of four Egg's.

Whisky .............. two ounces.

Tincture of Ginger ........ half ounce.

Linseed Tea .............. half pint. Mix.

Give a tablespoonful every three or four hours.

\section{LUNG WORMS-HOOSE IN LAMBS-PALE DISEASE- HUSK.}

Causes:-There is no question but that these worms gain access to the wind-pipe and lungs of lambs, but the manner in which they gain entrance to these organs of young animals, is not yet solved. The disorder usually 
makes its appearance during the fall months and is by far the most prevalent among lambs that graze upon lowland pastures which are subject to inundation from the rise of waters during the fall rains and which are covered with rich, succulent, rank grass.

Symptoms:-The presence of these worms in the wint-pipe causes much irritation and coughing. While they do not always inconvenience grown sheep, they are a frequent source of death among lambs. These worms often accumulate in such quantities in young lambs as to prevent proper aeration of the blood in the lungs, which causes a general disturbance of the whole system and produces a pale, bloodless condition. The appetite is partially or wholly lost, the lamb loses flesh rapidly and if not relieved, quickly succumbs. When these worms affert the bowels, as they sometimes do, dysentery will be observel, with bad-smelling passages, which are effected with much straining, and blood-clots will sometimes pass. The worms vary somewhat in size, being from one to three inches in length, threadlike and slender, and of a whitish color. When any number of lambs are affected, an examination of the passages of dung as well as the mucous from the nostrils, should be made, in order to detect the worms if possible; or if one of the animals should die, inspect the windpipe carefully and if worms are observed, commence treatment at once.

Treatment:-As preventative measures are always the best, the lambs should not be allowed to graze upon pastures that have received a toy-dressing of sheep manure, nor allowed to grize upon pastures which have recently been trodden upon by old sheep. Fresh pastures only should be used and in damp, rainy seasons such as are hilly or well drained should be chosen." An abundance of salt should be given, or better still, have 
rock salt in the field continuously. Medicinally, these worms must be attacked from two sources, viz., by the administration of remedies internally and by inhala. tions. Give internally,

Raw Linseed Oil........ seven ounces.

Spts. Turpentine.......... one ounce. Mix.

Give two tablespoonfuls every morning on an empt? stomach, until four doses have been given. Or,

Spts. Turpentine......... one ounce.

Whisky.............fifteen ounces. Mix.

Shake well and give a tahlespoonful every morning, for a week or ten days. Inhalations of sulphurous acid gas, should be given as follows: Place the lambs in a room where the ventilation is not too great, then take half pound of flour of sulphur and a cuart of pine tar, mix it with tow or waste and set it to burning. If the gas becomes too strong for you to endure, step out and remove the fire with you. Repeat this twice daily for a few days. The food should now be the best that can be procured, and if the appetite has completely failed, give linseed meal, oat meal, or corn meal gruel, as a drench, until the appearance of returning appetite and strength.

\section{SEDIMENT IN THE URINE-SAND IN THE URINE.}

Causes:-Formations and accumulations of a sandy deposit in the urine, are brought about by high feeding, or being grazed upon dry pastures without a sufficient supply of water. Resulting also from being fed upon. grain quite freely during the winter season, without a necessary amount of water, or from a changed conclition of the system, when recovering from lingering fevers, etc. 
Symptoms:- It seldom causes any inconvenience witl ewes, as their opening for the passage of urine, is large and free, but among rams and wethers, on account of the smallness of the urinary passage, it often causes considerable annoyance, by becoming deposited and adhering to the sides of the canal. Sandy or muddy deposits are observed in front of and around the sheath, clinging to the wool or hairs where the water has passed through, sometimes to the extent of forming small stones. Symptoms of retention of urine now appear. The sheep exhibits great uneasiness and pain, while trying to pass urine, and the passage will be a very small stream, or possibly only drop by drop.

Treatment:- When first noticed, allow plenty of fresh water to drink, to which may be added a teaspoonful of acetate of potash, once or twice daily, for a week, to increase the flow of urine and wash out the material. But if the urine passes only by drops, lay tlas sheep on its rump and draw out the penis, press the fingers along this organ and try to force out the accumulation. This should be repeated daily, until the passage of water is free. Give internally,

Raw Linseed Oil........ three ounces.

Laudanum.......... twenty drops. Mix.

Give at one dose. If this treatment fails in removal, the deposit may be cut out, the incision being made lengthwise along the penis; but unless the ram is valuable, it had best be slaughtered and used for mutton. In case of a wether being affected, use for mutton at once.

\section{CLAP, OR GONORRHOEA.}

This disease is brought about in sheep from want of cleanliness, with an excessive amount of ewes to attend to, or by serving a ewe that is affected with the whites. 
Symptoms:-The sheath and penis of the ram become swollen and sore, and intense pain is exhibited while passing the urine, which may pass by only a few drops at a time. Soon a whitish discharge comes from the penis, which, if left undisturbed, will cause ulcerous sores. There will be more or less stamping of the feet while passing water and more or less uneasiness is observed.

Treatment:-Remove the ram to a quiet place and do not allow him to serve any ewes, as the disease is very contagious and will be imparted to the ewes. Administer internally,

Balsam Copabia.......... two ounces.

Tr. Cubebs ............two ounces.

Tr. Kino..................... ounce.

Spts. Sweet Nitre .......... one ounce.

Laudanum .............. half ounce.

Syrup....... one and one-half ounces. Mix.

Give a teaspoonful three times a day. Withdraw the penis and bathe with the following lotion, after bathing with hot water:

Sugar of Lead ............ one ounce.

Sulphate of Zinc........... one ounce.

Water ...................... Mix.

Use once daily. In addition to the bathing, part of the solution may be injected into the penis once daily, until convalescence is established, when every third or fourth day will suffice. If any of the ewes become aflected syringe them once daily with the above solution, or use the following:

Permanganate of Potash....one ounce.

Water...................... Mix.

Inject either into rams or ewes, once daily. The food should be soft and easily digested, as bran mash, grass, etc: Stimulating diet should be avoided. Further, give ounce doses of raw linseed oil daily. 


\section{LAMBING IN THE EWE.}

Farmers residing in the Northern States, usually calculate to have their lambs yeaned in April and the forepart of May, as there is less danger from loss, than if yeaned earlier in the season.' But the percentage of loss during the lambing season, is crreater than there is any necessity for, through negligence or carelessness, and

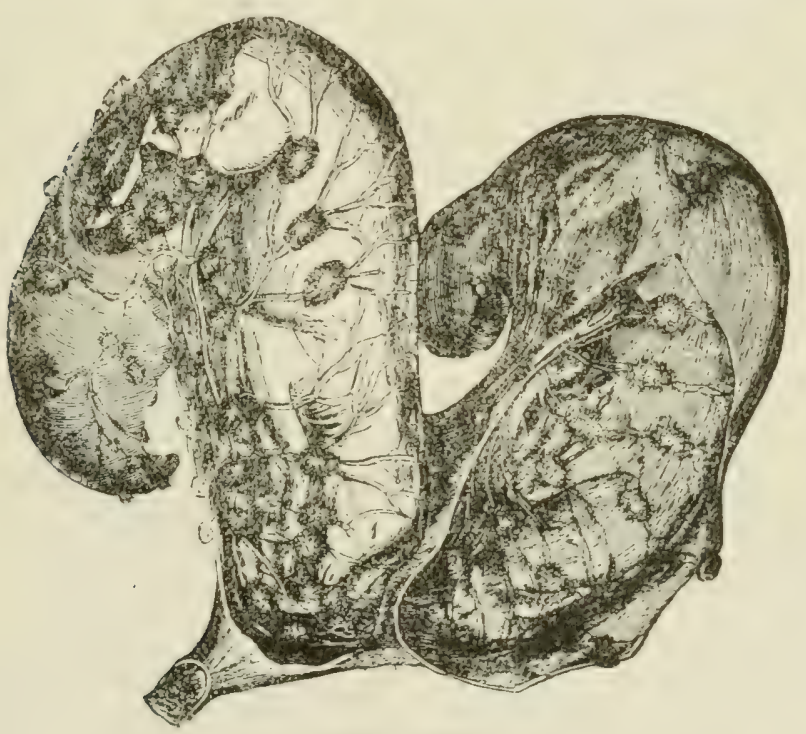

TWIN LAMIBS.

the want of places of protection for the ewe and lamb. Many lambs are lost each year by the ewe not being in a proper condition. There is much less danger from the ewe being in a good condition, even fat, than from being too poor. Dogs are also a source of great loss among lambs, and should not be allowed near a flock, at this season of the year. The flock should have a good, dry yard, so that they can move about in the sunlight, and a good shelter with plenty of rentila- 
tion, for the night. The owner or shepherd should be familiar and well acquainted with his sheep, thus insuring confidence and not fear, among them. Patience is required on the part of the shepherd. Do not be too meddlesome, but see to those that are in labor, and if the lamb is coming right, with forefeet and head first, leave her alone for a considerable time, then if you deem the lamb too large, render some assistance, but do not become rash and use too much force; gentle traction, with the parts well-oiled or greased, is much better. When the hind parts swell up and look red, and the bag swells and looks full, you may rely upon it, the ewe will drop her lambs within thirty-six hours. It is then the shepherd should be on the alert, to see that nothing goes wrong. Ewes as a rule, deliver their young without any serious difficulty, but occasionally a lamb is presented wrong, when it would be impossible for the ewe to deliver her young without assistance. Under these circumstances, the lamb should be pushed back i to the womb and straightened. If you can raise the hind feet first, do so, and deliver in that position. The various methods of delivery as laid down for mares and cows in another part of this work, will greatly aid the shepherd, and should be followed; the only exception being that in the attendance of a ewe, a small hand is required, and for this reason, a woman can do more effective work than a man. The hand should be well oiled before entering, and the movements should be slow and careful; hasty and rough help, is worse than none at all. Always raise the feet first. If the forefeet are raised, see that the head lies between them and is not doubled back. If the afterbirth does not come away voluntarily after the lamb is delivered, pull upon it gently, and it will usually be expelled in a short space of time. Should the ewe appear weak and exhausted, 
make and administer a gincer tea, of the same strength as you yourself would require.

\section{AFTER-PAINS IN EWES-PARTURIENT APOPLEXY.}

Parturient apoplexy occurs within four days after lambing and is most frequently seen in fat, thrifty

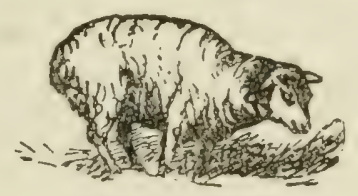

AFTER-PAINS IX LWES.

animals that are also heavy milcres. It is the result of a fevered condition at the time of lambing.

Symptoms:-The ewe is liwt noticed to be panting as if hot and feverish. Heary struming follows, with a

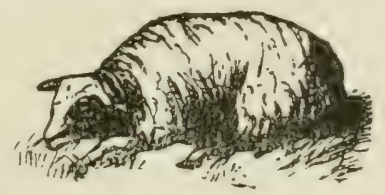

PARTURIENT APOPLEXY.

heaving at the flanks. The eyes look staring and glassy, the bowels are constipated, the urine is high colored and smells strong. The external hinder parts are swollen and red and later on become purple or black. After death the womb is noticed to contain pus ar matter; sufficient of this has been absorbed or taken into the system to poison the blood and cause death.

Treatment:-Prevention in these cases is always the best and safest. As it is most always found in very fat ewes, the safeguard lies in reducing their condition prior to lambing, but not to the extent of weakening 
them. Their food should be gradually reduced and a light bran mash should be given daily. After the disease has developed and pains are noticed, prepare the following :

Quinine Sulph......... drache dr.

Laudanum ............. one ounce,

Whisky.............three ounces.

Water.............. four ounces. Mix.

Give one ounce three or four times a day. If a badsmelling discharge comes from the hinder parts, they should be cleansed with

Permanganate of Potassiun.........

one drachm.

Water..................... Mix.

Syringe the vagina and womb with this mixture twice daily, until the discharge stops. Give one ounce of glauber salts daily to loosen the bowels, but do not physic. Apply warm cloths to the belly for a day, then in their stead apply a mustard plaster. The food should be light and easily digested, as bran mash, linseed meal or oat meal gruel.

\section{INVERSION OF THE WOMB.}

This is not a common affliction among sheep, but sometimes results from difficult labor, more especially in a weak ewe. The womb is turned inside out and hangs behind as a large, red sack, to which the afterbirth is sometimes attached. When the after-birth still clings to it, it should be gently removed and the womb washed in milk-warm water, to which may be added a little alum. Then elevate the hind parts and gradually work the womb back into its place, but first cut off the finger nails and oil or grease the hand thoroughly so as not to injure the delicate structure. After 
the organ has been replaced fusten a bandage around the hinder parts to prevent a recurrence, or as is sometimes practiced, put a deep stitch through the vulval or lips of the outer parts and allow it to stay so for twentyfour hours, when it may be removed, as the woml, will by this time have settled into its natural position and will there remain. Should the ewe exhibit signs of pain or attempt to strain, give thirty drops of laudanum every hour until the pain ceases. If she appears weak give ginger tea or half-ounce doses of hot brandy. While she is lying down see that she is well bedded and that her hind parts are elevated higher than her head, for a few days. For illustration, see inversion of the womb in cow.

\section{GARGET-INFLAMMATION OF THE UDDER.}

This consists of inflammation of the milk glands, and is caused from ewes lying on the cold, damp ground; resulting also in such as have lost their young and have not adopted an orphan or been supplied with a fosterlamb. It is also produced by a teat not being opened by a new-born lamb, or in ewes that give a greater flow of milk than the lamb will consume. The bag may also become gargeted at weaning time, when the owe is giving a strong flow of milk, and if neglected it becomes festered and is soon a tumbling, rolling mass of maggots.

Treatment:- When the disorder is first noticed grive a mild physic, as

Epsom Salts ............two ounces.

Ginger................. Me drachm. Mix.

Give at one dose in half pint of water, to be followed with drachm doses of saltpetre, morning and evening. Bathe the bay for thirty minutes with hot water, then apply camphor and lard twice daily, or take 
Copperas............. four ounces.

Water...............two quarts. Mix.

Apply frequently. Or,

Witchhazel........... five ounces.

Tr. Arnica.............. one ounce.

Tr. Belladonna........... one ounce.

Spts. Camphor........... one ounce. Mix.

Apply twice daily, after bathing with hot water. Injecting the teats with a solution of carbonate of soda, then milking out, will be productive of good. If the bag has festered, open it and allow the matter to escape, then wash the sore with

Carbolic Acid............. one ounce.

Soft Water ............. one quart. Mix. Or,

Permanganate of Potassium . one ounce.

Soft Water............. one quart. Mix.

Apply twice daily. If maggots have gained access to the sore, apply some spirits of turpentine to rout them, after which, keep clean, and use the carbolic acid solution. If the disease has resulted in the permanent loss of one or both teats, the animal should be discarded as a breeder, and either kept for her wool or sold to be slaughtered.

\section{ABORTION-SLIPPING OF LAMBS.}

Abortion is not of frequent occurrence among sheep, and when it does happen, it is generally traceable. It may result from being chased by dogs, or from injuries received while in the yard with other stock; from drinking an abundance of ice-cold water, also from eating large amounts of cold carrots, turnips and mangels. 'The stomach becoming distended with the cold food, lowers the temperature of the internal 
organs to such an extent that it causes the death of the young animal, and it is thus expelled from the womb. Feeding upon blasted or smutty rye, as well as slipping upon icy roads, will produce abortion.

Treatment:-In this "ase, also, prevention is the only safeguard against loss. Great care should be exercised when feeding roots; they should be moderately warm, or fed in limited quantities. See that the ewes are not chased, worried, or excited by dogs or any other sheepiestroying animal. Avoid feeding smutty rye, and driving over slippery roads. Allow them to drink regularly, to avoid engorgement with ice-cold water. When abortion does take place, see that the membranes all come away and if the sheep seems stupid and sluggish, vive a mild physic, as

Raw Linseed Oil.........two ounces.

Laudanum........... f irty drops. Mix.

Give at one dose, to be followed twice daily with irachm doses of saltpetre.

\section{PARTURIENT FEVER-PUERPURAL FEVER-MILK FEVER.}

This fever rarely attacks our common ewes, but is ceen among imported sheep, that are highly fed and in a fat condition, and are middle-aged, usually carrying twin lambs. It generally occurs a few days prior to lambing. The earliest symptoms, are loss of appetite, twitching of the hind legs, and of the ears. The ewe appears dull and stupid, with weakness, and staggering gait while walking. The eyes are drowsy, the head is carried low down; a dark, offensive, coffee-colored discharge comes from the vagina or hinder parts.i If the lamb is now dropped, it is dead, and possibly in a 
semi-putrid condition, but if the lamb is not passed, the patient grows steadily worse, the fever rises higher, the animal moans with pain and the discharge becoines more offensive. When the lamb does eventually come, it is almost rotten.

Treatment :-Remove the ewe from the balance of the herd to comfortable quarters, as soon as noticed. Then give a laxative to open the bowels, as

Epsom Salts........... two ounces.

Saltpetre............. one drachm.

Warm Water, or Linseed Gruel.....

$$
\text { .................... Mixe pint. }
$$

Give at one dose. Should she feel disposed to eat anything, give nothing but warm gruels or mashes. When the discharge is dark colored and offensive, the lamb is certainly dead, and should be removed as quickly as possible. Wash out the vagina with warm water, then place some solid extract of belladonna on the end of the finger, and smear it on the mouth of the womb, making an application in this manner every two hours. This will cause the mouth of the womb to dilate, when the putrid lamb will be expelled. The womb should then be washed out with,

Permanganate of Potash... one ounce.

Warm Water............ one quart. Mix. Or,

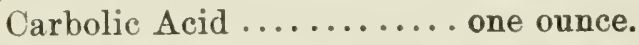

Warm Water............ one quart. Mix.

Use with a syringe. Also give internally,

Quinine Sulphate......three drachms.

Sulphate of Soda.........two ounces.

Saltpetre............... one ounce. Mix.

Make eighteen powders. Give one powder every three hours. Should the physic not operate in fifteen hours, repeat the dose. Mustard drafts along the beily 
are very beneficial. Should collapse seem imminent, give ounce doses of hot whisky. See that the position of the patient is as comfortable as possible; supply with a olean bed, at least twice a day.

\section{CASTRATION.}

This is a very necessary operation among lambs and should be performed as early as possible after lambing, a: there is less danger following than when the lambs are older. When the lamb is a week or ten days old the whole sack, seeds and all, may be cut off with one stroke of the knife or shears, without being attended with any danger, and only a few drops of blood will be observed to follow. But when the lamb has passed a few months the seeds develop very rapidly, the blood vessels and nerves are large, and the operation is attended with more danger. The sceds are to be drawn woll down, then one bold incision, cutting into the seerl and down to the point or bottom of the sack, should be made. The seed should then be withdrawn and pulled away forcibly, or the cord should be seraped with a knife until it separates; otherwise, the clamp should be used. If the latter method is chosen, fasten the clamp) very tightly, then cut of the seed half an inch below it and allow it to remain for three days, when it may be removed. The clamps may be hollowed out a little in the center and a small quantity of corrosive sublimate and lard placed in the groove. In castrating aged rams, scraping is not always safe, as the seeds are very large and the blood vessels correspondingly so and there is therefore danger of bleeding in this manner of operation. Neither is ligating or tying them with a string advisable, as it is sometimes impossible to tie tichtly enough to prevent bleeding. But when the limms are 
pressed on firmly bleeding seldom follows and for this reason this method is considered the safest and best mode of operation. Always see that the opening is clean down to the bottom so that all matter can escaie. To prevent too rapid healing, place a block of wool in the wound.

\section{DOCKING, OR CUTTING OFF THE TAIL.}

This operation is performed in order to keep the hind parts of the sheep in a clean condition, as those sheep which are allowed to carry a full tail hecome very dirty from accumulation of urine and manure, which adhere to the wool around these parts and form a nest for the blow-fly and maggot. The operation should be performed while the lamhs are very young-within two weeks after birth. The parts are not then very sensitive and consequently the operation is not very painf:il. The method is quite simple, yet mistakes are often made. When the lamb is caught the skin should be drawn up on the tail, toward the rump, so that after the tail is severed it will cover the bone, then with a sharp knife make a (juick cut, about two inches from the rump, and the opration is complete. A little pine tar placed on the stump of the tail will keep off flies and assist in healing. 


\section{DISEASES OF THE HOG,}

THEIR

CAUSES, SYMPTOMS AND TREATMENT.

\section{HOG CHOLERA.}

There are so many diseases of the swine which have never been any too thoroughly understood, that have been, for the want of a better term, called hog cholera. The loss to the firmers in this country alone, has been estimated at fifty millions in a single year, and all have

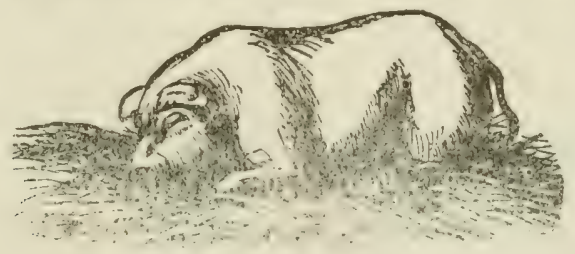

HOG CHOIERA.

been grouped under the one head-sholera. Let us for a few moments, briefly consider the manner in which swine have been treated, when deemed in health, and see what our own condition would be, under like circumstances. In the first place, the creature is considered-a hog-and that means, literally speaking, anything that is filthy and dirty. History tells us that the hog, in the natural or wild state, is one of the cleanliest of animals, so if the appallation, as applied to the hog, means filth and dirt, it must be an unnatural condition or innovation, brought about or inflicted upon 
the animal, through domestication. It is perfectly true, that hogs will wallow about in dirty water, in order to cool off the fevered or heated body, in summer, but if allowed to select for itself, it will not only drink of pure, clean water, but will lie in it also. Again, pen up a hog and give it good shelter and a bedding of clean straw to lie upon, and see if it will litter up its own bed; on the contrary, if a place is provided outside of its stall, it will go there to comply with the demands of nature, and deposit both manure and water. There are many other habits of the hog, that tend to cleanliness which might be mentioned, but space forbids, in a work of this kind. I believe that many of the diseases wh swine are heir to, have been brought about through domestication, and the manner in which they are cared for and fed, the so-called cholera included. The average hog is fed on sour swill, sour grain or corn, distillery slop, corn that has already been eaten and passed by cattle, that which has been trampled through dirt and dung, in fact anything that is of a dirty, sour nature, has been considered good enough to feed to the hog. Now the essence of sourness is rinegar, which is formed in the food after the alcoholic fermentation has run its course. The first effect from feeding this sour food, is constipation, followed by looseness or diarrhoa, superinduced by the paralyzing effects of the alcohol, vinegar, carbonic acid gas, etc., upon the bowels. After a time, the blood becomes affected through this vinegar yeast and various diseases are produced. Just so long as hogs are fed upon dirty, sour, indigestible food, trouble with the bowels must he expected, of a more or less severe nature, according to circumstances. When the hows are penned up, clean the pens daily, the same as you would your horse stables; feed them upon clean, sweet food, and disease will become less frequent. If 
the food is cooked thoroughly, the heat will destroy the alcohol and vinegar plants, and the danger of disease will be greatly lessened. To say that hogs fed upon weet, sound food could not contract diseases, would be mis-stating facts, but hogs that are fed on sound, sweet corn, well steamed, are not so liable to become diseased as those which are fed upon sour materials, and if those who are interested doubt these statements, they can easily prove or disprove them, by trials accurately conducted. The various formulas that have been tried with goor results, all have a tendency to tone up and correct the bowels; to prevent the formation of germs, and to destroy any that are already existing. I will now dwell somewhat upon the various forms of cholera, although the treatment tends to the same purpose-that of correcting the bowels, and lestroying and preventing the formation of germs.

\section{CHARBON, OR ANTHRAX.}

This disease, which effects both cattle and sheep, also finds a co-partner in the hog. It is brought about in a similar manner-hy taking very thin hogs and fattening them too rapidly, but more frequently in the hog, by focding upon sour material, or by being kept in filthy places. It is one of the various types of bloodpoisoning, caused by a bacilli or other germ finding ingress into and developing in the circulating fluids of the body. The malady is contagious, the contagion being given off by the blood, flesh and dung.

Symptoms:-The symptoms develon very rapidly. At first there will be loss of appetite and wreat prostration, with red and bluish spots appenring on various parts of the body. The ears and nose have a bluish cast, the breathing is quickened and painful and a 
pleading cry, or squealing, is often heard. Just as the low breaths its last, a passage from the bowels, coming involuntarily, is always observed. These symptoms, it will be noticed, last from one to forty-eight hours. ()thers in the apoplectic form, have been seen to step back from the feeding trough, utter a squeal, turn around, tumble over and die in less than a minute. As this disease has often been mistaken for contagions pneumo-enteritis, I will grive the distinguishing characteristics between the two, as laid down by Dr. Klein, of Iondon; then with the following table before him, the ordinary careful observer can scarcely make a mistake:

CHARBON.

Period of development or latency from a few hours to three days.

Easily transmissible to other species of animals.

Spleen always enlarged and often broken down.

Blood after death, dark anel linid.

Bacillus anthricus in the b!ock!.

Lungs and bowels frequently not implicated. Congh may be absent.

The discoloration local and of a true carbuncular appearance.
CONTAGIOUS PYEUMO-ENTERITIS.

Period of developinent from two to five days and more.

Rarely and with difficulty transmitted to other species.

Spleen rarely enlarged or otherwise changed.

Blood after death of ordinary appearance.

No bacillus anthricus in the bloot, but numberless bacilli in the serum of thorax and abdomen.

Lungs and bowels always both inflamed; cough always present.

The red or purple color diffused over the surface and of an crysipelatous appearance. 
Treatment:-As there is no specific remedy for this disease, the necessity for preventative measures hecomes apparent. The healthy should he separated from the sick at once and turned into a fresh pasture where there is an abundance of fresh water. The foor wiven should hes sweet and steamed or cooked, and limitod in quantity and the person attending them should not so near nor have anything to do with the afleceted ones.

As has been remarked before, it is rory contagious, being taken from the blood, flesh and manure. It is therefore necessary to bury all dead animals, very deeply: also throw line upun the arrates prior to cosering; or burn them ul', manure and all, then thomulny disinfect the place with carbolic acid or chloride of lime, and lo not allow any other liege near the lowality that season.

\section{CONTAGIOUS PNEUMO-ENTERITIS.}

This form of cholera consists of an inflammation of the lungs and bowels, causingered or purple spots on the skin and it is very contagious.

Causes:-There are many causes assigned to this discase, among which are ferding in close, filthy, foulsmelling pens and compelline the animals to drink water that is highly impremanted with dearing animal or vesetable matter: also foreling and keeping in low, damp, swampy, malarial places, feeding upon sour food, as swill from a sour barrel, distillery slop, sour or musty corn, or corn that has passed through cattle, or dirty food of any description. It may also result from feeding too highly on rich, concentrated, heating, nutritious food, morlucing a sullen fattening of the animal, whereby the blood hecomes surcharged with incompletely transformed material. Whether these are 
the real causes or not they are certainly conditions that should be remedied or avoided, as animals kept in such a state will have more or less bowel trouble, which renders them very susceptible to any contagious diseases, whether brought about by contact or through atmospheric causes. As the poison which produces this dis. ease is a vegetable germ, so small as not to be observed with the naked eye, it may be produced or developed in the filthy material, as administered to the hog, or carried for a considerable distance through the air and locating itself with those animals that are in a condi. tion to receive it. After it has once gained a foothold in a herd, its contagious character is soon developed and none need doubt the nature of the disease, as all hogs in the herd, regardless of age, size, sex or condition, fall a prey to the malady.

Symptoms:-The symptoms vary, according to the part of the animal affected, whether it is of a severe type or not. To this fact esperially I would refer the realler's attention so that the varying forms will not confuse him.

\section{The Erysipelatous Form.}

This is the most common of the different forms of the disease. The animal appears dull, with loss of appetite. If standing, the head hangs low down and it scarcely moves unless pushed by other hogs, and sometimes efforts at vomiting are observed. It generally lies down and when in that position can scarcely be moved. Cough is sometimes present, the urine is high colored and passed with great difficulty ; the bowels are somewhat constipated and what dung is passed is very hard and dark colored. In a few hours or possibly the n'xt day, the symptoms peculiar to this form develop themselves in the shape of dark red or purple blotches, 
gradually passing into a bluish black color. Whe' once seen they are not readily forgotten. 'These spots usually appear on the ears, throat, neck, breast and inside the forelegs. On white how the spots are readily observed. A discharge of dark purple fluid oozes from the notrils, the breathing becomes more difficult and labored, the hind parts become paralyzed and if the animal is forced to more it reels along with its head and hind legs drooping to the ground. At this stage a bad smelling diarhora sets in and the animal dies in from one to three days.

\section{Form of Malignant Sore Throat.}

This form occurs when the poison attacks the tissues beneath the lining membrane of the throat, instead of the tissues beneath the skin. The general symptoms at first, are the same as appear in the erysipelatous form. The dark red spots are seen mpon the throat, gradually changing to a dark purple. But the affection attacking the throat, causes great difficulty in breathing and swallowing and gives rise to another lot of symptoms peculiar to this form, which are not seen in the erysipelatous condition. From the very commencement, the animal makes frecuent attempts to vomit and there $i:$ difficulty in swallowing; there is such effort in breathing, that one would naturally suppose the animal was choking: in fact, it becomes so distressing, that the animal onnetimes sits upon its haunches and gasps for breath, while the swollen and livid tongue protrudes from the mouth. Sometimes the swelling in the throat is so great aud sudden, and the animal is choked to death in such a short space of time, that the other symptoms remain unohserved, or have hardly had time to develop themselves.

Treatment:- While the treatment of cholera has not been just as successful as we would desire it to be, yet 
many farmers allow their hogs to go mattended, and let them die. when a little time and the expenditure of a small amount of monoy, might save them hundreds of dollirs. When the symptoms are very pronounced, with a loss of appetite, there is but little hope for reovery, and owing to the eontagionsness of the trouble, they had better be knocked in the head, and burned or buried; but those that will eat, should be treated hy some of the remedies which have proved sucesseful in the hands of some of the greatest hog raisers in the anntry. First and above all things, separate the healthy from the sick, and place them upon fresh, wreen pilsture where there is an abundance of pure, fresh water. Feed them upon clean, sweet food, well steamed or conked, and the party attending the sick, should not feed nor wo near those in health. The sick animals should also he kept at a respectable distance. As the causes which favor development of this disease have alreally been mentioned, repetition is not necessary. The animals should be carefully removed, after which the attention should be turnad to the procuring of medicines for relief, and the destruction of the germs and their further development. There are two agents which possess great influence in this direction, and of which we have positive testimony in reard to their value, by their entering into all the varions combinations which have proved successful in the treatment of this disease. They are copperas and chlorate of potassium. The copperas is much the cheaper of the two, and just as effective as the chlorate. It has been more abundantly used, as it hastens chemical changes in the blood, and increases the firmness of the spleen. It has proven itself a raluable agent, and can almost be considered a specific. A remedy much praised and extensively used by western farmers, is the following: 
Copperas ............. one pound.

Soft Soap ............ me ralion. Mix.

Boil with four crallons of water. This should be well stirred with the forrl, and is considered one dose for twenty-five hogs. When the hogs are eating add a

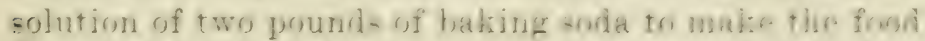
fram while it is being con-uned. Reyent the low every third day, until three droce have hesn anten. leve that wery hog fats a share of the renedy. If wo fail -

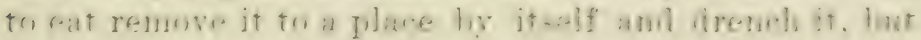
if too far cone, kill it and put it out of the way. Anrither netheul wit treatment much un-d in Kenturloy, and called an infallible remedy, i- componed of

Copperas............... pone pound.

Warm Water.........three gallons. Mix.

Stir until thoroughly dissolred. When the skin becrin-to look roulu, or a dark red color, commence inopdiately and afply every day, hy u-ing it waru--1nuz-

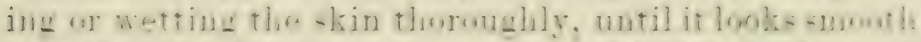
and the disculoration disargears. If this bathing an wetting of the skin be uesd in camnection with the admini-tration of the fromer renedy, better result- will undoubtedly be ohtained. Profk-cor Turner of Illinoi-. grive two recipes. either of whirh, he aty prositively preventi the disea-e. and, if criven freely in the fir-t stages of the malady, will also cure it. They are,

Flower of sulphur.......two pounds.

Copperas..............two jounds.

Madder ..............two pounds.

Black Antimony.........half pound.

Saltpetre ............. half pound.

Arsenic.............. two ounces.

Mix with twelve gallons of slop. This quantity is euflicient for one hundrell hor-, and whon criren sema- 
rately, the dose is one pint for each hog. The other recipe is

Common Salt......... four pounds.

Black Antimony......... one pound.

Copperas .............. one pound.

Sulphur............. one pound.

Saltpetre............ four ounces.

Wood Ashes (unleached).... one peck.

Mix thoroughly and place in a dry trough, where the hog's have free access to it. Moisten it a little to lieep from wasting. It has been reliably stated that where this has been used, prior to the breaking out of cholera in a herd, with a strict observance of cleanliness, it is a specific for the prevention of the disease. The other remedy which has been previously spoken of, is chlorate of potassium, but owing to the cost of the drug, it has not been so liberally used. However, I will give a recipe, which if made use of, and more especially when the throat is effected, will bring about remarkable and astonishing results.

Chlorate of Potassium.... one pound.

Water................... Mix gallons. Mix.

The dose is a half pint for each hog, given three or four times a day. The local treatment consists in bathing the swollen parts with hot water, which should have a liberal amount of copperas mixed with it. If sore spots break out, use

Sweet Oil............ four ounces.

Spirits Turpentine...... four ounces. Mix.

Apply twice daily to the sores. Common smart weed has been highly spoken of as a preventative of cholera. This plant has been used to a considerable extent by physicians for many years, for its curative properties in sore mouth, salivation, ulcers, etc., and I believe if it is used juliciously among hogs, it would not only act as a 
precentative of cholera, but would be beneficial in warding off many diseases known by that name. When the appetite is not what it should he, feed them enoun of the weed to make them cough and sneeze liberally, and the beneficial results will be obserred to follow. The way to use the weed, is to make a tea of it and mix it with the swill, in surh quantities as they will eat. If it is desired to keep the weed for use at any season of the year, it should be gathered when in bloom, tied up into small bundles, and hung in a dry, sheltered place. Charcoal, salt, and wood ashes are also efficient in warding off disease in hogs. They can be mixed and put in a special box or trough, where the hoge have free access, whenever they have a desire for it.

\section{MALIGNANT EPIZOOTIC CATARRH.}

Causes:-This disease, like all others that are produced by floating germs, is brought about by having the animals in a condition to receive it. It has often been observed that good sanitary conditions greatly assist in warding off all such diseases. Consequently, hregs that are kept clean, well housed and fed, and allowed an abundance of fresh water to drink and to bathe in, seldom contract this disease, and if they do, it is only in a very mild form. But where hogs are kept in filthy pens, and their skins are unhealthy and covered with a mass of foul dirt, the act of perspiration is checked and extra work is thrown upon the lungs. ' So also, when hogs are exposed to sudden changes of temperature, as cold rains, sleet or hail storms; the process of perspiration is checked, and by this means the other membranes of the body have a surplus of work to perform, when they become over-taxed and weak, thus furnishing the proper soil for these floating germs to take 
hold upon and develop themselves, which they do with extreme rapidity.

Symptoms:-The symptoms vary, according to the part of the animal which is the worst aflected. That form wherein the nostrils, windpipe, and lungs are the portions most affected, will develop a short, hoarse, hacking cough, with diflicult breathing and a panting motion of the tlanks. The head is held in a peculiarly stretched and somewhat drooping position. The animal moves along with a slow, stiff, or tottering gait, and if it squeals, a hoarseness of the throat is noticeable. Signs of fever are present all the time. Isually, the bowels aro constipated, but some have a diarrhoea and make frequent attempts at vomiting. When the bowels, spleen, liver and kidneys are the most affected, the cough is greatly lessened and may be absent; the breathing is not nearly so difficult, while the weakness and staggering gait of the hind parts, is greatly increased. The fever in the one form is equally as high as in the other. There is usually at first, constipation of the bowels, but as the disease advances toward a fatal issue, there is a change to a profuse and badly smelling diarrhoea. In some cases, and more especially when the kidneys are greatly aflected, the hog arches its back at the loins, to a very high degree. Sometimes the brain becomes affected, as is demonstrated by the animal staggering about in a wondering manner and running against objects as though wholly or partially blind. there may be ulcerating sores on different parts of the body, indicating an affection of the lymphatic system. The duration of the disease, is from five to fifteen days.

Treatment:-This implies good care as well as the judicious use of medicines. The sick animal should be separated from the balance of the herd and provirled with a clean, dry bed. The apartments should be well 
ventilated, avoiding drafts, guarding against undue heat and cold, wet and moisture. The sick always need pure air in abundance, with clear, fre: h water to drink, and casily digested, nutritious food to cat. If these directions are carefully followed, many will be cured which under reverse circumstances, die. The patient should be given, as soon as these symptoms make their appearance-

Powdered White Hellebore........ ..twenty grains.

Milk................. Malf pint. Mix.

This is a dose for a full-grown hog. For younger animals, give a little less hellehore. This will make the hog desperately sick for a timc, and it will sneak away and hide in a dark place. In the course of two or three hours, it will come out again and possibly receive a little food; it should then be riven three times a day for several days, or until a change for the better is observed, three grains of tartar emetic, if the trouble is located in the nose, windpipe and lunws, and three grains of calomel if the disease is mostly in the bowels. The medicine can easily be given in a boiled potato, a little milk or gruel. Externally apply to both sides, if in the lungs, and along the belly, if in the bowels, the following blister:

Powdered Cantharides.... one ounce.

Olive Oil............ four ounces. Mix.

Boil together very slowly, for half an hour. If the one application does not blister thoroughly, apply again the following day, with good friction. When convalescence has been estalblished, give from ten to thirty grains of copperas daily, to which may be added thirty grains of carbonate of potash, when the lungs have been greatly disturbed. In all cases where the bowels remain constipated, give 
Castor Oil.............. one ounce.

Spirits of Turpentine.... one drachm. Mix.

Give in half pint of milk or gruel. The reader should distinctly bear in mind that this disease attacks the delicate membranes and glands of the body, and it may Incalize itself in various places, while the symptoms just duccribed, are seldom all found in a single hog. Even the two forms just spoken of, may be so closely blended as to make it hard to distinguish which form predominates. However, the treatment is so similar, that no fatal mistake can occur.

\section{APOPLEXY.}

This disease occurs only in hogs that have been highly fed and are very fat. Hogs that are cared for in this manner, are very liable to congestion of the brain, which may pass rapidly on to effusion or apoplexy. During the congestive stage, which may last a few hours, the hog acts very stupid and moves around in a drowsy condition, but when it merges into effusion, and the apoplectic stroke occurs, the animal drops as suddenly as if knocked on the head with a sledge; the limbs straighten out and stiffen, the breathing is hard and stentorian, and there is a frothing and foaming at the mouth.

Treatment:-This should he very prompt. Dash cold water on the hog, and also hold a can six or eight feet. above it and let the cold water splash and strike the head for some time. Hasten the movements of the bowels. by wiving injections of warm water and castile snap, or if at hand, the following injection may be used:

Glycerine............. one drachm.

Water............... half pint. Mix.

Inject up the last bowel; or 
Epsom Salts.......... three ounces.

Spirits of Turpentine...two drachms.

Soapsuds ............. half pint. Mix.

Bleeding and blisterino do no special good, and should not be practiced. When the animal shows sigus of recovery, give

Epsom Salts.......... three ounces.

Water............... half pint. Mix.

Repeat every three or fuur hours, until a good physic is produced, then change the diet to a lighter quality, to reduce the flesh. If fattening for slaughter, after recovery takes place, butcher it immediately.

\section{EPILEPSY, OR STAGGERS.}

This disease is often confounded with apoplexy, to which it bears a striking resemblance, but as the treatment is about the same, it makes but little difference to the amateur practitioner.

Symptoms:-The disorder is caused by congestion of the brain, and during the stage of congestion, the animal is both stupid and restless. The eyes present an inflamed appearance, looking quite red. The pulse is hard and quick, and the bowels are usually constipated. These symptoms may gradually pass away, or they may increase in force, which latter phase will surely result, if the bloud pressure increases on the brain. A period of excitement is then brought on; the animal walks about or runs to and fro, sometimes moving around in a circle, frequently stepping very high, and running arainst various oljects as though it were blind; or, it may fall wer in an unconscious condition. The breathing is somewhat affected, being hard and laborious.

Treatment:-Dash cold water quite freely all over the body, splashing it upun the bead with considerable force. Give an injection immediately, composed of 
Glycerine............two drachms. Water...................... Malf pint. Inject this into the last bowel. Or,

Epsom Salts............ four ounces.

Spirits Turpentine......two drachms.

Water.................. pine pint. Mix.

Inject up the last bowel. Blisters applied to tho back of the neck, are frequently attended with beneficial results. The following will suffice:

Spts. Turpentine........ two ounces.

Hartshorn ............. one ounce.

Cantharides...........two drachms. Mix.

When applied, rub thoroughly. Hogs affected with epilepsy, should be fattened and slaughtered as early as possibly.

\section{CATARRH, OR SNUFFLES.}

This consists of an inflamed condition of the lining membranes of the nose and head, brought about by exposure. It requires but little attention further than good care by keeping in clean, dry, warm, well ventilated pens, carefully avoiding further exposure for the time. Open the bowels with a warm bran mash, to which may be added three or four ounces of epsom or glauber salts. There is, however, a chronic form of catarrh which affects hogs, called nasal gleet, in which there is a continuous discharge from one or both nostrils. It is not contagious and causes but little harm outside of the annoyance to the animal. If it has been running for a continued length of time, the nose may swell some and from the efforts to clean the nostrils by sneezing, streaks of blood will sometimes be observed. This is called by some, blue-nose," and is thought to be contagious and incurable, resembling glanders in the horse, which is a very erroneous and 
mistaken idea; but when the ailment arlvances to a condition where there is bleeding from the nostrils, surh animals so aflected do not thrive well and had hetter he destroyed.

\section{COUGHS AND COLDS.}

An ordinary cold is in some localities called a "rising of the lights," and is brought about by any of the ordinary measures, as surlden changes of temperiture, exposure, etc.

Symptoms:-The appetite is somewhat impairedsometimes wholly suspended. There is more or less coughing and often the cough hecones quick and severe. Fever is usually present, accompanied with panting or heaving at the flanks.

Treatment:-It is quite umecessary to bleed and purge, or administer calomel and tartar emetic, which is commonly atrised and frequently followed. The animal should be housed in comfortable quarters and cleanliness should be strictly observed. The throat and chest should be rubbed with mustard thinned with water to the consistancy of thick cream. Feed the animal upon light, warm, easily digested food, into which should be mixed thirty grains of copperas, three times a day. Also administer

Chlorate of Potassium...... one ounce.

Water................. one quart. Mix.

Give a wine glassful three times a day. Or,

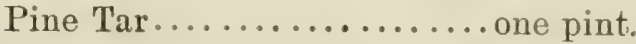

Water................ one gallon.

Mix thoroughly, and give a pint morning and evening. 


\section{QUINSY-STRANGLES-TONSILITIS.}

This is an inflammation of the glands in the upper part of the throat, known as the tonsils. It is quite a common complaint among hogs, and very often fatal, death being caused by the rapidity with which the swellings increase, thus completely closing the upper air passages, causing suffocation.

Symptoms:-The symptoms are well pronounced, distressing and rapid. Breathing and swallowing are performed with the greatest difficulty. Swellings appear under the neck and lower jaw, and as these swellings enlirge, the breathing is performed with increased labor. the tongue protrudes, and there is more or less slavering from the mouth.

Treatment:-As the disease progresses very rapidly, the treatment must be correspondingly so, otherwise a fital termination may be looked for. A number of incisions should be made into the skin with a sharppointed knife, immediately over the swellings, deep enough to draw the blood quite freely. These should be well bathed with hot water, or cloths wrung out of hot water should be applied. Internally administer,

Epsom Salts......... four ounces.

Water................. half pint. Mix.

Give at one dose, if the hog can swallow, to be followed with two-drachm doses of spirits of turpentine, every three hours, in a little milk or gruel, and if it cannot swallow this, take

Turpentine........... two ounces.

Sweet Oil............. two ounces. Mix.

Fasten a cloth on the end of a limber whip-stalk or green bough, dip it into the mixture, and force it well back, around the upper part of the throat. Repeat this two or three times a day. 


\section{CONSTIPATION.}

This affection is most frequently observed in young pigs that are too highly fed, and more especially upon dry grain food. Sows are sometimes constipated after the delivery of a lut of young pigs. In this case it is brought about hy the change of condition in the systen, the animal being more or less feverish. When hogs are constipated, they exhibit a great desire for drink, and the appetite is partially suspended.

Treatment:-This consists of a complete change of diet. Feed hot bran mashes, to which may be added some flaxseed meal. (iive some green food, as green clover; or turnips, carrots, apples, sweet beets, etc. Also allow a liberal amount of charcoal; it will greatly assist digestion, which is an auxiliary to the relief of constipation. If these fail to bring about the desired result, give two or three ounces of eysom or glauber salts, and repeat if necessary, until the bowels are relieved.

\section{DIARRHOEA, OR SCOURS.}

Hogs are very seldom troubled with this complaint, but young pigs are quite liable to attacks of diarrhoea, which often terminate fatally. It is usually brought on by a changed condition or bad quality of the mother"s milk.

Treatment:-The efforts should be directed toward eflecting a change in the condition of the milk. Change the sow's food immediately. If she has been kept upon dry, sour, or musty diet, grive her green food; or if from too much green food, give her clean, sweet, dry food, to which should be added 
Anise Seed............. one pound.

Gentian .............. half pound.

Baking Soda............ half pound.

Powdered Chalk......... one pound.

Sulphur............ half pound. Mix.

Give a tablespoonful in her slop, three times a day. Great care should be taken to keep the youngsters warm. It is very important that the pen be cleaned every day, and scattered over with slacked lime or dry earth, to absorb and destroy the smell arising from their passages, as it has a tendency to aggravate the complaint.

\section{AGUE CAKE-SPLEEN ENLARGEMENT-SPLENITIS.}

Hogs which are raised and kept in low, swampy, malarial districts, are subject to the same complaints as human beings and from continued attacks of ague are quite liable to have enlargement of the spleen or milt. While in this condition it is almost impossible to fatten them. Or again, the continuous fattened condition of hogs which are kept for exhibition purposes, in connection with high feeding and little or no exercise, eventually brings about enlargement of the splecn, whereupon the animals commence to lose flesh aud it will be found impossible to hold them in the condition the keeper or owner desires.

Symptoms:-There is a gradual and preceptible loss of flesh, apparently unaccountable. The hog eats fairly well, but fails to take on flesh and appears somewhat dull and languid. As the spleen enlarges, it is observed to lie always on the one side, or should it lie down on the opposite side it very quickly changes its position, and when walking it leans or doubles to one side as if the natural position caused internal pain. 
Treatment:-The object in treatment is the reduction of the size of the spleen. This can be accomplished by a change of climate, which is not a profitable proceeding in hog raising. The medicinal treatment calls for a brisk physic, as

Jalap.................ten grains.

Calomel..............ten grains. Mix.

Give in a boiled potato, on an empty stomach, to be followed with ten grain doses of quinine, three times a day for a week or ten days. Change the hogs to dry, upland pasture, also feed grains sparingly. Some veterinarians recommend very strongly in cases wherein the fattening process is interfered with, the use of

Wormwood Leaves........ one pound.

Liverwort Leaves......... one pound. Mix.

Boil slowly in two gallons of water for an hour, and give half pint to a pint daily, in soft food. Feed the hogs at regular intervals and be careful not to give too much at a time. If, after feeding, any food remains in the trough, remove it.

\section{DIPHTHERIA.}

This disease, like many others which affect hogs, has been classed under the head of cholera, presumably on account of the suddenness of its symptoms and its extreme fatality. It is more frequently caused by filth than any other source and once breaking out in a herd, owing to its contagiousuess, it spreads very rapidly and is very destructive.

Symptoms:-These appear rery suddenly. The animal ceases eating, the throat appears rery sore, accompanied with extreme weakness and stiffness across the back. The eyes appear dull and sunken, the hog moves around very slowly, with the head elevated and the mouth 
open. The tongue is dry and livid and the throat red and swollen, with grayish white spots scattered over the upper part of the throat. These increase very rapidly until the whole air passage is involved, threatening strangulation. There is more or less coughing and during a paroxysm of coughing, shreds of false membranes are expelled.

Treatment:-This must be prompt if attended with success. Separate the sick to a respectable distance from those unaffected, and see that the whole herd is put in a clean, dry place. Give those hogs that have been exposed,

Chlorate of Potash........ one pound.

Water...............two gallons. Mix.

This will make one dose for seventy-five hogs and should be given once daily for a week, in milk, slop or water. The sick hogs should have sulphur applied to their throats three times a day. This can be accomplished by tying a cloth or sheepskin with the woolly side out, upon a stick, filling it full of sulphur and forcing it back around the upper part of the mouth. Or use a spray for throwing powders. For those that still continue to eat a little, place drachm doses of chlorate of potash in their food, and give to such as do not eat,

Glauber Salts.............. six ounces.

Carbolic Acid..........two drachms.

Chlorate of Potash.......two ounces.

Water.................. one quart. Mix.

Give one ounce, three times a day. Feed upon warm, thin, sloppy food and keep in a dry, airy comfortable place.

\section{TRICHINOSIS-TRICHINA.}

As no remedy has yet been discovered which is capa. ble of destroying this parasite, it is only necessary to 
speak of it, to warn people against eating pork that has not been thoroughly cooked or fried. The hog does not mpear to suffer materially when affecter with this parasite, hence its prosence can only be determined hy microscopical examinations of the liesh. They are so

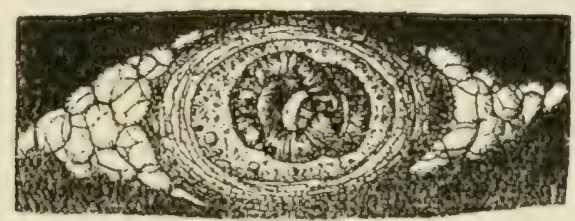

TRICHINA AND CYST GREATLY ENLARGED.

small as not to be observed with the naked eye, yet they increase with wonderful rapidity, dereloping alike in all animals, man included, upon gaining access to the stomach; here the worm becomes freed from the zyst that it has ocmpied, thromgh the process of diges.

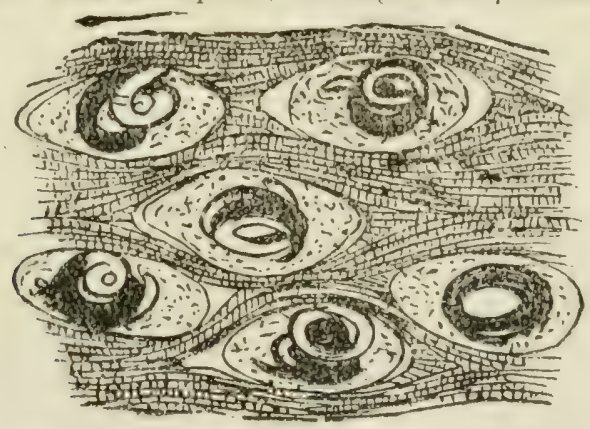

MUSCLE WITII ENCYSTED TRICHINA.

tion; the male and female pair and each female then lays a vast number of eggs, which develop into little worms that burrow around through the flesh until they become encysted. There they remain until they are eaten again and find their way back into the stomach, where they again reproduce. It has been estimated 
that from one million to five million have been discorered in a single pound of flesh. When man becomes affected with trichina, it produces symptoms very similar to typhoid fever.

\section{MEASLES.}

There hare been several causes assigned to this dis. ease, such as feeding upon certain articles of diet, as nuts, fruits, etc., as well as hereditary tendencies and contagion. It has lieen called measles, from the striking resemblance of the flesh of the hox. to the eruption of measlus on the human skin. The real cause

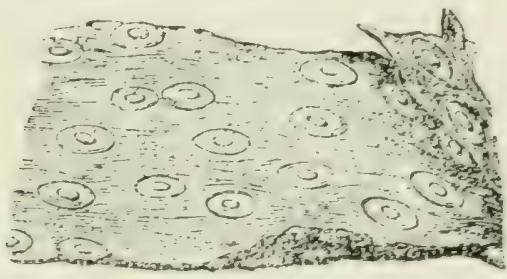

MEASLES IN PORK.

is tapeworm. which the hor prosibly eats while feeding on human excrement, or pasturing uyon wround which has heen fertilized with this kind of manure. After the eqgs are taken into the strmach, they develop into a worm and finl their way into the muscles, where they becone encysted. These cysts are about the size of a grain of wheat, and they are scatteres throughout the cutire srstem. Whon pork that has measles. is eaten without heine thoroughly corsked, so as to destroy thi; parasite, tapewrom will develuI in the human lowel, and grow to various lengths, from ten to sixty feet.

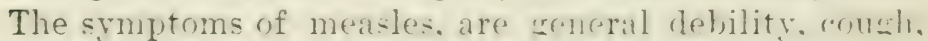
running from the eves; discharefo from the nose, and weakness of the hind legs. Small, watery pustules nf a 
reddish color, will he observed on various parts of the skin. The treatment consists in griving drachm doses of sulphur and saltpetre, every, day for several weeks, rogether with good, nutritious and easily digested food.

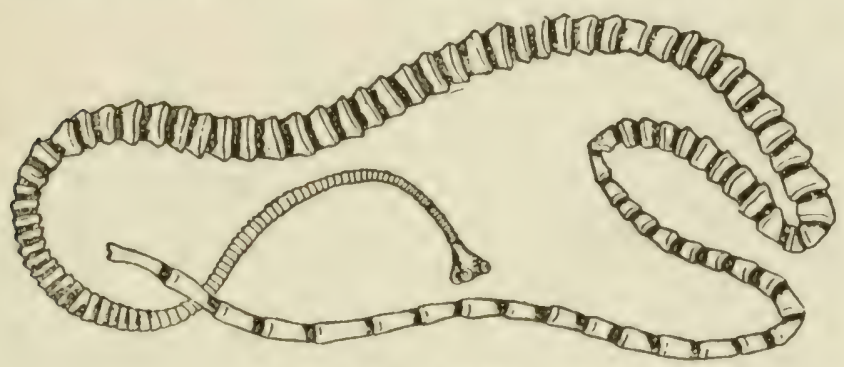

TAPE WORM.

But prevention is always hetter than medicinal treatment, therefore, keep hogs off from such pastures and away from human exerement, especially if any one of the houschold is known to have been aflected with tapeworm recently.

\section{PILES.}

This complaint is of more frequent occurrence than is usually supposed, but is rarely discovered until blood is seen passing away with the dung. It is caused by

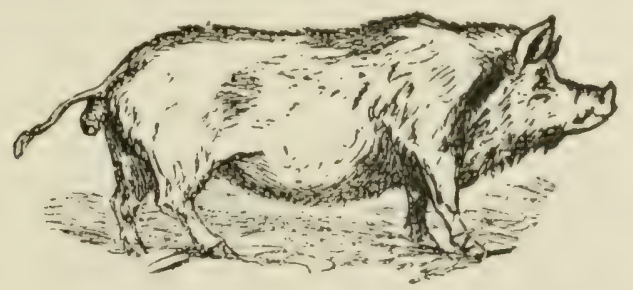

PILES.

indigestion, constipation, etc., and is generally considered incurable. But to reliere the animal as much as is possible, in order to fatten it for slaughter, an entiro 
change of food should be given, such as light, easily digested food, with some sour milk. Grease the parts frequently with benzoated vaseline, or

Vaseline............. two ounces.

Tannic Acid.......... four drachms. Mix.

Apply twice daily. Or inject the bowels with

Chlorate of Potassium....six drachms.

Laudanum............. two ounces.

Water............... four ounces. Mix.

Apply twice daily; or simply greasing the parts with vaseline or common lard, is beneficial. Such hogs should not be kept for any length of time.

\section{KIDNEY WORMS, OR LARD WORMS.}

The kidneys and the lard surrounding them, are liable to become affected with parasites, many of which are found in the fat in other parts of the body. These do not cause any serious harm, but sometimes a large worm called the Eustrongylus Gigas, makes its nest in the pelvis or center of the kidney from which the secreted urıne flows.

Symptoms:-The animal is inclined to lie down most of the time, and on attempting to rise, experiences difficulty in using its hind parts, which seem in a partially or wholly paralyzed condition. If it regains its feet, it moves about with an awkward, straddling gait.

Treatment :-That which has generally been practiced, is the application of turpentine across the loins, daily for several days; but as turpentine is good for the destruction of worms, it is better to give it internally, as a spoonful twice daily, mixed with oil or milk. Another remedy highly recommended, is

Copperas............ two drachms.

Sulphur........... two drachms. Mix. 
Give in the feed, erery night for ten days. However, the turpentine will reach them quicker and bo more effectual than anything else.

\section{LICE.}

There is no earthly reason why hows should be infested with this most disgusting parasite, when a little eare and attention will free them from the terrible annoyance resulting. As a usual thing, they are not found upon hogs that are healthy and thriving, hut may be

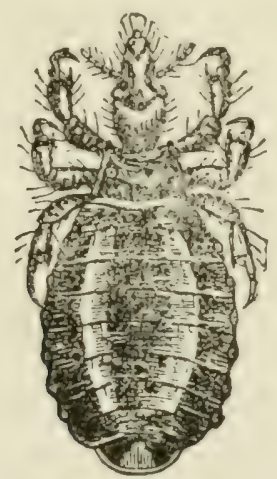

IIOG LOUSE.

found somewhat abundantly upon hoss that are half starved, or are badly out of condition. Their presence causes excessive irritation and itching, and prevents hogs from thriving that would otherwise do well on the same amount of food.

Treatment:-As lice belong to a low order of life, it is not only necessary to destroy them with medines, but if a permanent cure is to be expected, the whole pen will have to be renovated. The bedding must be taken out and burned, and the walls well white-washed. The floor should be covered with air-slacked lime, or wood 
ashes, and the animal kept clean and well fed. Also, to place the hogs in a better condition, give drachm doses of copperas and sulphur, once daily in their food. Externally use the following wash:

Stavesacre Seeds......... half pound.

White Hellebore......... two ounces.

Water.............. two gallons. Mix.

Break up the seeds and place over a fire, slowly simmering down to one gallon. Bathe the hows with this solution every third day, until three applications have been made, barely wetting the skin. As the nits hatch out every five days, you will effectually destroy them all, before there is time for a reproduction of more nits. Or use

Coal Oil .............. two quarts.

Lard Oil, or Whale Oil .... one quart. Mix.

Apply every three days, until three applications have been made. In some parts of the country, hogs are bathed in buttermilk, to exterminato lice. But the first recipe here given, will be found more effectual than anything else.

\section{THE MANGE-ITCH, OR SCAB.}

This disease is caused by the presence of a parasite called the Sarcoptes suis, and is transmissible to man. These small parasites burrow down into the skin, causing such annoyance that the animal keeps rubbing itself until scabs are formed. The insects have a great tenacity of life, and are communicated to other hogs by rubbing against posts where mangy hogs have rubbed and left some scabs.

Treatment:-This consists of external applications mainly, as there is no possibility of effecting a cure, until the insect is destroyed in its entirety. First, cover the whole body with soft soap, which has been 
made from lye of wood ashes, and let it remain on for two hours, then wash off and immediately apply,

Flower of Sulphur....... four ounces.

Spirits of Turpentine.... two ounces.

Oil of Tar............ two ounces.

Lard................ eight ounces. Mix.

Or the following wash may be used:

Flower of Sulphur.......two pounds.

Quicklime.............. one pound.

Water............. two gallons. Mix.

Boil together, continuously stirring it until it is well mixed. Mr. Richardson gives the following remedy which he says has never failed. He first washes the animal with soft soap and warm water, then dries it off and applies this ointment:

Train Oil ............... one pint.

Oil of Tar........... two drachms.

Oil Turpentine.........two drachms.

Petroleum .............ne drachm.

Mix with flower of sulphur, sufficient to form a thick paste, then thorourhly ruh the animal with this mixture, and allow it to remain on the skin for three days. (in the fourth day wash off with soft soap and water, and give in the food once daily for two weeks,

Flower of Sulphur..... four drachms.

Saltpetre........... one drachm. Mix.

Give at one dose. The pens should be washed with coal oil, and afterwards white-wash also those posto or rucks where the hogs have been in the habit of ruhbing.: The bedding should be burned, and a thorough renuvating system adopted. 


\section{DELIVERY AND CARE OF YOUNG-PIGGING.}

The uterus, or womb, of the sow differs but little in shape from that of other animals, that bear one or more off-spring at each time of delivery. But in all multiparous animals or animals that produce a number of young at each birth, conception does not take place in the body of the womb itself, but in various places along the channels which lead to the ovaries or female testicles. After conception has taken place these channels commence to enlarge, as also does the womb,

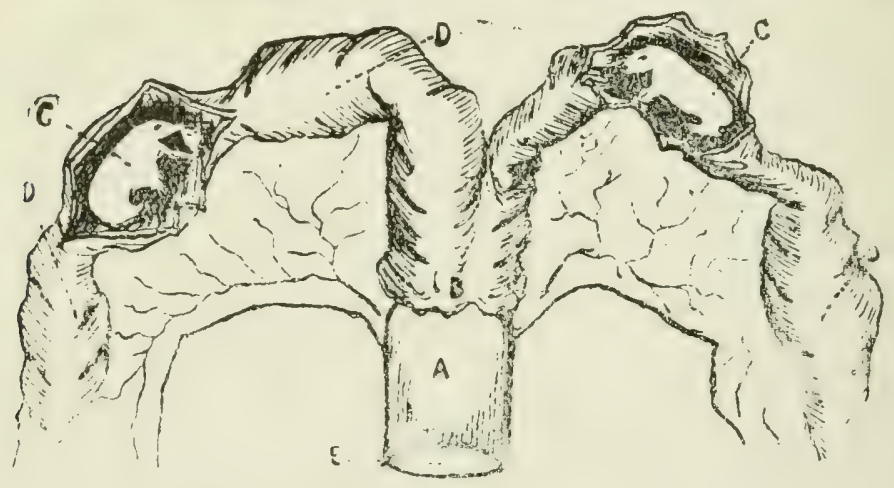

A.-Vagina, or Entrance.

B.-Body of Wornb.

C.-Fotus, or young pig exposed.

D. - Fœet us, or young pig covered.

to allow sufficient room for the perfect development of the young pigs, as well as to insure their safe delivery. They are covered with placental membranes or afterbirth, and are nourished through these and the umbilical cord, from the blood of the mother, the same as all other young animals, prior to birth. The period of pregnancy usually lasts about four months, but perfect. development and a safe delivery may take place at a much shorter period, as instances have been recorded where sows have delivered living and healthy young at 
one hundred and four days after service. When the time has about arrived for the delivery of her young, the sow should be placed in a separate pen or sty and a liberal amount of short straw or chaff allowed for bedding, as in a large bedding of long straw the youngsters may become entangled and not have the strength and activity to get out of the way of the mother, while changing her position; thus she might accidentally lie upon some of them and crush or kill them. It is a good plan to place a scantling or plank about five or six inches high, all around the pen, projecting outward from four to six inches, then should the sow lie too close to the wall and some of the young pigs should happen to be in that vicinity, instead of heing crushed they would slip into the space under the projection and thus escape injury. The sow should be kept in a place that is moderately warm, yet it should have good ventilation. When the day of delivery has arrived, if everything is normal she should not be disturbed, but given a good allowance of nourishing and easily digested food, as they generally suffer from weakness and prostration immediately after delivery. If this is allowed them and they are not irritated by the presence of people, they are less likely to devour their young. If, however, they cannot effect delivery alone, manual assistance becomes necessary and this should be rendered very cautiously and carefully. An examination should be made with the fingers, which hare previously been well oiled or greased with clean lard. If one of the young pigs lies crosswise it must he straightened, which can sometimes be accomplished by pressure along the belly and by assisting with the fingers in the ragina; or enlist some assistance from a person that has a small hand, as a boy or a woman. Hare the person oil or grease their hands well, then carefully pass it up the 
channel until the little creature is felt, then straighten it and also fasten a cord around its neck or hind feet, whichever is the most convenient, then apply gentle force or traction until it is delivered. Do not be rough or use too great force, but if need be, pass the hand at frequent intervals to be certain that it is straight. After it is delivered, no matter whether it be living or dead, allow sufficient time to elapse (say an hour or more) when she may proceed and deliver the remainder without assistance. If not, proceed in the manner as before described, until all are delivered. There are various instruments or forceps invented to assist in the performance of this act, but in the hands of an amateur they are somewhat unsafe as he might fasten onto the womb along with the young pig and tear it, thus endangering the life of the sow to a degree uncalled for. But if manipulated by a small hand and cord, there is much less danger. If there is much prostration in the sow following delivery, give half ounce doses of hot whisky every hour for a few hours, as well as the best nourishing, easily digested, warm food, to which may be added half ounce doses of glauber salts, three times a day. Keep her comfortable and warm and if constipation follows, give her a few injections of warm water and castile soap. If the womb is badly irritated, you may inject the following into the womb: Take one drachm of powdered opium and steep it thoroughly in a quart of hot water, then add one drachm of permanganate of potassium and inject four ounces every five or six hours. This will allay irritation and destroy any foul smell that may be present. At all times allow her what cool drinking water she may desire. 


\title{
DISEASES OF THE DOG,
}

THEIR

\author{
CAUSES, SYMPTOMS AND TREATHENT.
}

\section{DISTEMPER.}

Causes:-This is the most fiatil of the common diseases which affect young dogs. There is no doubt but that it is contagious, no matter as to the cause of its origin. It may be brought on by sudden changes of temperature, or exposure to cold and wet, and as it usually occurs about the time the animal is cutting its permanent teeth, the irritation produced from this source, may greatly assist in its development. Feeding upon raw meat, with close confinement, not being allowed the liberty of exercise at will, is also an auxiliary to the development of distemper. But as it usually attacks young dogs, and as they are seldom free from worms, I am greatly inclined to the opinion that they are one of the principal causes of this malady.

Symptoms:-The first symptoms observable, will be a slight dullness, the animal not appearing in its usual lively mood; the appetite begins to waver. Vomiting or attempts at vomiting, with light purging from the bowels, are among the earlier symptoms. The eyes now begin to look red and tears commence flowing down the cheeks. A light, short cough is now noticed, the pulse has quickened to possibly one hundred and twenty beats per minute, and as the disease advances, mattery 
accumulations are seen about the eyes and nose, increasing to a flow of mucous from one or both nostrils. The cough now becomes more severe, the hair begins to look rough and staring, and the nose is quite hot. If a change for the better does not take place at this period, the symptoms continue to increase in force; the eyes will become almost glued together with matter, the nostrils become closed up with a thick, mattery discharge, and the breath has an offensive smell. The dog now loses flesh very rapidly; blood streaks will be observed around the nose, and partial paralysis of the hind legs occurs, which gradually increases until it affects the whole body. The poor animal now lies prostrate and continues in this condition, until death relieves its sufferings. In those cases where favorable recoveries are looked for, the symptoms may appear very encouraging for three or four weeks, when a relapse occurs in a violent form, and it dies very suddenly; but when the acute symptoms subside and the dor appears to be doing well and gains somewhat in flesh, a speedy recovery may be looked for. If it fails to gain in flesh and the eyelids still present a red appearance, it is not free from dangerous consequences. After recovery has become complete, it is very seldom they take the disease again. There are cases known where distemper has occurred the second time, but this is the exception and not the rule.

Treatment:-Place the animal in comfortable quarters, where it will be clean and dry. Have the place moderately warm, carefully avoiding excessive heat and cold. Change the bedding frequently and allow plenty of pure, fresh air. As regards diet, refrain from giving meil altogether; give soft fond, as bread and milk, crackers and milk, boiled rice, or broth, and clean, fresh water to drink. If the lungs are affected, apply 
a mustard plaster to the chest, over which place a blanket. Keep it on for an hour, when it should be washed off and greased with a little sweet oil, but if considered necessary apply the mustard again; the dog should have a physic, for which the following will suffice :

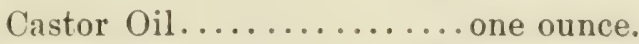

Santonine...........twelve grains. Mix.

Give half and repeat in four hours, if the first dose does not operate. This should be followed with

Quinine................ drachm.

Copperas............... one drachm.

Saltpetre.............. one drachm.

Gentian ............two drachms. Mix.

Make twenty powders or pills and give one three times a day. Should the bowels become costive, grive a tablespoonful of syrup of buckthorn every three or four hours until the bowels operate nicely. Sometimes, during the course of the disease, the brain becomes affected, as is demonstrated by delirium and fits. Procure the following as quickly as possible:

Bromide of Potassiun....two drachms.

Chloral Hydrate........ two scruples.

Water............... four ounces. Mix.

Give teaspoonful every two hours until the acute symptoms pass away, then give three times a day for a few days.

\section{DIARRHOEA AND DYSENTERY.}

This affection is generally brought on from overfeeding or the use of improper food. Severe work on a very hot day or sudden change from hot to cold, frequently affects the bowels, causing diarrhoea.

Symptoms:-The first symptom noticeable is the soft fluid condition of the passage of the bowels, instead of 
being solid, as in health. There is more or less sickness at the stomach, with an unusual desire for drinking water. The breath becomes somewhat offensive, as well as the passages from the bowels. The dog appears weak, the eyes presenting a sunken appearance, and if the animal is left alone it will go away to some cool place and lie down most of the time. If dysentery appears it will be observed by the heavy straining, while passing a thin, liquid substance, which is somewhat tinged with blood, the last bowel appearing considerably inflamed.

Treatment:-First give a mild physic, as a dessertspoonful of castor oil, to carry away any irritant that may be causing the diarrhea, after which allow the patient twenty-four hours rest without medicines, as it is always desirable to allow the bowels to resume their natural functions, which they will often do, without medical aid. If, after that time has elapsed and the disease continues, the following will suffice.

Laudanum ............two drachms.

Tr. Catechu.......... four drachms.

Tr. Ginger............ four drachms.

Tr. Cardamons .........two drachms.

Brandy............. four drachms. Mix.

Give teaspoonful erery two hours. If it nauseates the $\operatorname{dog}, \operatorname{mix}$ it with a little syrup, or take

Powd. Catechu.......... one drachm.

Powd. Opium ........... one scruple.

Prepared Chalk ........two drachms.

Powd. Ginger........... one drachm. Mix.

Make twelve powders. Give one powder in a little syrup every four hours. Should the bowels become too greatly constipated from the effects of the medicine, give a dessertspoonful of castor oil. 


\section{CONSTIPATION-COSTIVENESS.}

Causes:-Dogs are frequently troubled with constipation, without any good, assignable cause. Feeding rich food as meats, etc., without sufficient exercise will produce it. Occasionally doess swallow bones that have not been crushed thoroughly and they become lodged in the last bowel, thus preventing the passige. The dun: then dries out and becomes almost as hard as a stone.

Symptoms:-The dog will be noticed to make frequent efforts to stool, without anything passing. Wher: making the attempts at passage, they will often pant. At other times, the pain will be so severe as to cause it to yelp quite loudly. It will sometimes lie around considerably, often panting, and if taken out for a run, it will often stop, and strain violently to make its passage.

Treatment:-I, ections into the last bowel, will often remove the trouble. These may consist of castile soal. and warm water, or use some raw linseed oil, or

Glycerine............. half drachm.

Raw Linseed Oil.......... one ounce. Mix.

Or in the absence of the oil, use warm water and glycerine. Give internally, half an ounce of castor oil, or

Powdered Jalap........two drachms.

Tincture Gentian.......two drachms.

Simple Syrup.......... two ounces. Mix.

Give half, and repeat the dose if necessary, in six hours. Lay the dow upon its side and feel along the belly towards the hind parts, and you can frequently locate the hard lump. By using continuous pressure, it can sometimes be forced along the channel to the exit. Grease the finger and insert it up the last bowel. If you can feel the hardened matter, gradually break it to pieces. Or a spoon handle may be used for this purpose. 
If you feel a piece of bone, work around it carefully until it is loosened, then work it away. If you have tried for some time and have failed to remove all, give an injection of oil or grease, and allow the animal rest, for two or three hours, then try again. Continue in this manner, and you will eventually accomplish your object. After you have removed the obstruction, give a tablespoonful of castor oil, after which change its food, giving soft material for a few days, with plenty of exercise.

\section{COLIC-BELLY-ACHE.}

Causes:-It is usually brought about through something the animal has eaten, or exposure to cold and wet; from fatigue and fasting or being fed a heavy meal, after a hard day's run. Owing to the ease with which dogs can vomit, the overloaded stomach is emptied in this manner, thus avoiding many attacks of colic.

Symptoms:-The dog appears very uneasy and restless. It will draw its body all up in a heap and roll around. It will yelp or cry, without any assignable cause, also moan considerably and refuse to eat anything. The bowels are usually costive and bloating is sometimes observed.

Treatment:-If there is costiveness or bloating, injections will be beneficial, as

Glycerine........... half drachm.

Water, or Oil.......... two ounces. Mix.

Throw this up the last bowel, or in the absence of glycerine, use castile soap and hot water, frequently. Eathe the bowels with heary cloths wrung out of hot water, and give twenty drops of laudanum or a teaspoonful of paregoric in some warm milk or some ginger tea, to which may be added a little red pepper. Also give a physic, as 
Castor Oil............ half ounce.

Or,

Powdered Jalap.......... one drachm.

Simple Syrup............ one ounce. Mix.

Give at one dose and ropeat if necessary in five hours.

\section{INFLAMMATION OF THE BOWELS.}

This occurs from eating irritating substances in the food, also from exposure to cold, or sudden chilling when in a heated condition; from certain poisons, and from colic when not given proper attention.

Symptoms:-These very much resemble colic, but the pain is more continuous. There will be great uneasiness and whining, or brief disconnected cries. Pressure applied upon the belly, produces pain. The breathing is fast and the pulse is rapid, with a high fever.

Treatment:-Give twenty drops of laudanum every two or three hours, or half a grain of morphine will answer the same purpose. Apply mustard to the belly, and after it has acted, which will take an hour or more, apply hot cloths or a poultice made of hot bran, all along the belly. Aconite is grood in this disease, therefore give the following:

Fluid Extract Aconite Root........

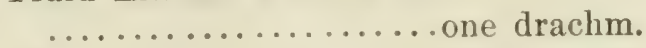

Water.............. eight ounces. Mix.

Give tablespoonful every hour. Allow ice-cold water to drink, in limited quantities. Do not be in too great haste to effect a movement of the bowels, but give slippery-elm tea or linseed jelly, and after recovery has taken place, you may give a tablespoonful of castor oil. If the bowels are still costive, give a few injections of warm water. 


\section{FITS-EPILEPSY.}

Fits are very often seen in the dog, on account of the high state of development of the nervous system, it being very tense and acute. Anything that will produce an irritation of the intestinal canal, is liable to produce fits, as indigestion, worms, etc., or it may be caused from some brain affection, as blood pressure, tumors, or injuries causing pressure upon the brain, etc.

Symptoms:-These, when once seen, are not readily forgotten. The dog will fall over and move its legs violently. There is champing of the jaws and frothing at the mouth. This may last for only a minute or possibly several minutes, when suddenly the animal rises, looks rather bewildered for a short time, then appears all right again, until another fit comes on. If the fits occur very frequently, the animal will likely die from exhaustion.

Treatment:- When fits are observed in young dogs, it is well to treat for intestinal worms. If tape-worm is suspected, give a wineglassful of pumpkin seed tea, on an empty stomach, every four hours until three doses have been given, then give half ounce of castor oil, or give a drachm of powdered areca nut, and follow it up with a physic. Or give two doses daily of finely powdered glass, for two or three days, then physic. If the fits are caused from some brain affection, use

Bromide of Potassium ...two drachms.

Chloral Hydrate......... one drachm.

Water.............. four ounces. Mix.

Give a teaspoonful every two hours until the patient is quieted, then give a teaspoonful twice daily for a week or ten days. Sometimes they will disappear and not return, but if there is structural change in the brain, they will likely terminate fatally. In all cases of fits the food should be of such a character as will be wasily digested and keep the bowels soft. 


\section{GOITRE.}

This consists of a gradual enlargement of the thyroid glands, situated on the sides of the neck a short distance behind the jaws. These glands in their natural condition are small, but when they begin to enlarace, which they frequently do, no inflammatory action is noticeable, but they gradually increase in size until they acquire the proportions of a large goose eqw. They do not always produce harm, but sometimes they press upon the windpipe to such an extent that they interfere with the preathing. The treatment consists of paintine the parts once daly with tineture of iodine, for a continued length of time. Also give internally

Iodide of Potassium .....two drachms.

Water.............. four ounces. Mix.

Give a teaspoonful twice laily, and the enlarement will usually disappear in the course of two months.

\section{COUGH-CATARRH-COLDS.}

Dogs that are subjected to exposures of wet and cold, more especially in the spring and fall of the year, are very liable to catch cold, when they will cough, sneeze and run at the nose, and sometimes considerable ferer will rise, which, if neglected, may derelop into more serious complications, as lung fever, etc.

Treatment:- This is very simple and if attended to early, no bad results follow. Give two grains of guinine three times a day, in a piece of fried or boiled meat, and rub the throat well with mustard. Or use

Raw Linseed Oil.........two ounces.

Hartshorn .............two ounces.

Turpentine...........two ounces. Mix.

Apply to the throat twice daily. If it appears a 
little too strong, which it may be for some dogs, add a little more oil. Should a bad cough be present, use

Syr. Squills ...........two drachms.

Syr. Tolu............ four drachms.

Syr. Ipecac........... two drachms.

Chloroform..............ten drops.

Honey, or Simple Syrup...two ounces. Mix.

Give a teaspoonful three times a day.

\section{LUNG FEVER-PNEUMONIA-INFLAMMATION OF THE LUNGS.}

This disease is generally brought on from exposure to cold and wet, changes of temperature, change of climate, sudden chilling when heated, neglected catarrh or colds, etc.

Symptoms:-The dog will appear somewhat stupid and not inclined to move about very much. The head will be held in an extended manner. There is an anxious look of countenance and a red or bloodshot appearance of the eyes. The breath is hot and the breathing painful and accompanied with a slight grunt at each expiration. The cough is short and somewhat distressing. The animal will frequently sit up for hours until through exhaustion, the forefeet slide forward and it falls down. By applying the ear to its sides the various sounds diflering from the healthy lungs, will be readily observed.

Treatment :-First place the dog in a good, comfortable place, where it is not too cold, and without draughts. Bandage the legs and body to keep it moderately warm. Allow it all the pure, cool, fresh water it will drink. Keep the bowels open and loose, feeding only such food as is soft and nourishing, as milk, broth, boiled rice, etc. Apply mustard drafts to the sides, rubbing well 
into the hair, and hanket immediately, to retain the fumes. Wash off in an homr and repeat again, as often as you deem it necessary. Internally, give

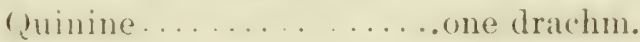

Dover Powder........... one drachm. Mix.

Make thirty powders. Give one powder every four to six hours. Also get six ounces of mindererus spirits. and give a teaspoonful every two hours. Change the bolding frepuently and keep the surromudings sweet and clean.

\section{LABIES-HYDROPHOBIA-MADNESS.}

As this disease is incurable nothing remains but to destroy the animal. For further particular: see treatios on this disease, amoner horses.

\section{MANGE.}

Causes:-This is gulte a common complaint amemo dogr amb is caused by a mite or parasite, of which there are diflerent varieties, that hurrows into the skin. some to a creater depth than whers. Persistent treatment is called for, and then sometimes the disorder batiles all treatment applied. Arain the parasites are more superficial, even worling around among the seabs, in which case they are more easily reached and destroyed. They are usually transferred from one dog to another by the animals ruming towethor, or by sleeping in a kemmel where a mangy dog las been kept, as they remain in a dirty kennel for an indefinte length of time.

Symptoms :-The dog appears out of condition and is not active and bright. The skin is itchy, the animal frequently and persistently scratching itself; or it sits down and rubs or scratches until sore spots are formed. The paracits neually makes its appearance in the form 
of red pimples or eruptions, around the inside of the hind legs, along the belly on the under side, around the ellows, about the eyes, and eventually spreading all over the whole body, when the hair either falls off or is scratched or rubbed off in patches, and scabs or scales are formed. The skin looks dry, harsh and scaly and emits a disagreeable smell, more especially on a damp day or if wetted a little.

Treatment:-As the disease is due to a parasite which affects the skin only, internal remedies are not required any further than to open the bowels nicely and keep them in that condition. For this purpose give half an ounce of castor oil, or

Powdered Jalap......... one drachm.

Syrup.................. Me ounce. Mix.

Give at one dose, then wash the dog thoroughly with soft soap and water, to remove all dirt and scabs. Dry off well and apply

Oil of Tar.............. one pint.

Flower of Sulphur........half pound.

Mix thoroughly and rub it well into the skin, every day until four or five applications have been made. Wash the dog with soft soap and warm water every other day, to allow the medicine to get well into the skin. Or use

Whale Oil ................. pint.

Sulphur............. eight ounces. Mix.

Apply in the same manner. Clean the kennel thoroughly and burn up all straw, old rags, etc., and wash the walls with

Corrosive Sublimate....... one ounce.

Water................ one gallon. Mix. 


\section{RED MANGE-ERYTHEMA-INFLAMMATION OF THE SKIN.}

This aflection usually breaks out on dogs that are pampered and highly fed, without sufficient exercise, and consists of a hreaking out or irritation of the skin, causing considerable itching. The scurf or material which oozes out, has a reddish cast, causing the skin and hair to assume that color. It is not contagious and yields readily to simple treatment, which consists in rolucing the dog somewhat in flesh. Give a physic, as castor oil, half an ounce; or epsom salts, a dessertspoonful; or

Powdered Jalap........two drachms.

Simple Syrup...........two ounces. Mix.

Give half and repeat the dose if necessary. Externally, wash the skin with tar soapl and water, then dry it thoroughly, and apply sparingly Friar's Balsam once daily for a few days, and a cure will result. Or use

Sugar of Lead .......... half ounce.

Sulphate of Zinc....... four drachms.

Water............ eight ounces. Mix.

Apply twice daily for a few days.

\section{CANKER OF THE EAR.}

This is cenerally caused from injuries to the ear, either by cufling it or by hitting it against some object. Or it may be caused by gretting something in the ear, or by being in the water too much.

' Symptoms:-The dog will be olserved to shake its head frequently and violently, making the ears llap and snap around its head. It will also dig and scratch in and around the ears with its paws, and sometimes it will carry its head to one sille while walking. A fou: smell emanates from the ear, and the surrounding parts look red, swollen and inflamed. 
Treatment:-If the disease has not been too long neglected, it yields readily to treatment. First, refrain from giving any meats to eat. Use only soft food, and that principally vegetables. Wash out the ear well, flaps and all, with either castile or tar soap, and warm water, dry it nicely then blow some iodoform into it, twice daily. Make a hood to go over the head, to confine the ears, so that the animal cannot flap or scratch them, and they will be well in a few days. Or you may pour the following into the ears, twice daily:

Lead Water...........three ounces.

Pure Soft Water....... three ounces. Mix.

Use just enough to fill the ears, then apply to the flaps of the ears,

Calomel.............. one drachm.

Powdered Camphor .....two drachms.

Oxide of Zinc Ointment.... one ounce. Mix.

Then fasten on the hood to prevent scratching.

\section{INLFAMMATION OF THE EYES.}

The eyes of dogs often become inflamed from injuries of various kinds, one of the most common being caused by the spanish needle, a species of burr. Hunting dogs frequently get these into their eyes, at the inner corner heneath the membrane that shoots out to cleanse the eye. They may also become inflamed from getting dust into them, or many other various ways.

Treatment:-Make strict search for anything that may possibly become lodged in these organs, and if found, remove it. The following lotion will assist greatly in the examination :

Cocaine...............ten grains.

Water...................... Malf ounce. Mix.

Apply a few drops every thirty seconds, until the eyo 
can be handled with ease. To reduce the inflammation, use

Sulphate of Zinc........ four grains.

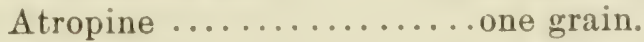

Water................ one ounce. Mix.

Apply a few drops three times a day; or,

Blue Vitriol........... two grains.

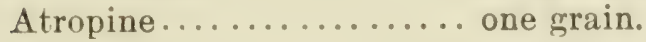

Water............... one ounce. Mix.

Apply a few drops twice a day.

\section{WARTS AND TUMORS ABOUT THE EYES.}

Small tumors sometimes appear at the inner corners of the eyes. They are generally fastened to the membrane which shoots out over the eye to cleanse it. They sometimes grow so large that they nearly or completely cover the eyeballs and they bleed quite easily and present an ugly appearance.

Treatment :-Apply to the eye

Cocaine...............ten grains.

Water................half ounce. Mix.

Put a few drops into the eye every half minute until it can be handled at will, then fasten a hook or pair of forceps into the tumor, or in the absence of these, run a needle and thread through it, then raise it up gently until you can see the whole of it. Take a pair of sharp scissors and clip it off, and if it is then left alone, the wound will quickly heal. If it does not, however, use

Lunar Caustic............ one grain.

Water.................. Mix.

Apply a few drops daily for a few days. 


\section{WOUNDS AND FRACTURES.}

The feet of dogs often become sore, especially hunting dogs that run through long, wet and coarse grasses; also thorns and stubbles, and injury likewise results from continued exposures to cold water. Not infrequently the toe nails are torn off. These troubles should be attended to as quickly as possible.

Treatment:-Remove any foreign substances immediately, then poultice the foot with flaxseed or slipueryelm until the inflammation has subsided, after which apply Friar's Balsam, twice daily, or

Carbolic Acid ...........two drachms.

Water ................. one pint. Mix.

Apply twice daily. Or,

Sugar of Lead............. one ounce.

White Vitriol............ half ounce.

Water ................. one pint. Mix.

Apply twice or thrice daily. If the skin is torn much it should be sewed up, and any of the foregoing solutions applied. When medicines are applied to dogs the parts should be bandaged loosely, to prevent the animal from licking at the wounds. But if the injury is not serious it is just as well to let the dog lick it and not apply medicines, as they keep it clean and it heals quite rapidly. If any bones are broken, set them straight and apply splints and bandage. Tise liwht, flexible splints and steep the bandage in thick starch water before applying. Allow the dog its liberty and they will heal in about a month.

\section{CHOREA.}

This consists of a derangement of the nerrous system, and, so far, has proven incurable, when existing to any great extent. It quite often follows debili- 
tating diseases, as distemper, etc. The symptoms olserverl are a twitrhing or quick jerking of a certain pirt or sot of murcles, more frequently aromol the face, nerk and forelimbs. Sometimes only one limb is allected and again the whole body. Occasionally it unfits a dog for any kind of work. After this discuse becomes chronic it is apparent!y painless. hut when dereloping, it is somewhat painful, as demonstrated by uneasiness and whining.

\section{WORMS IN THE INTESTINES.}

The intestines of the don are more or less troubled with worms. The two principal varieties that affect them, are the tape worm and the round worm. The latter are mostly observed, as about fifty perent. of

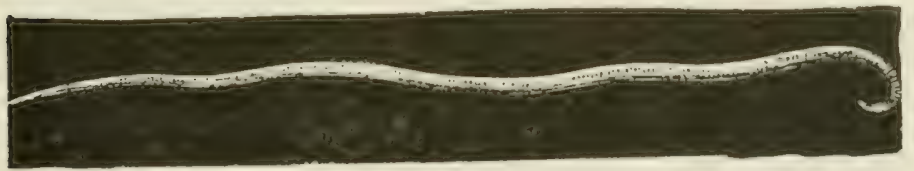

1:U:DEETEI) I:OUXI) WORM。

the dors cxamined atter death, arre found to be infested with them. These worms do not always remain in the bowels but often find their way into the stomach, where

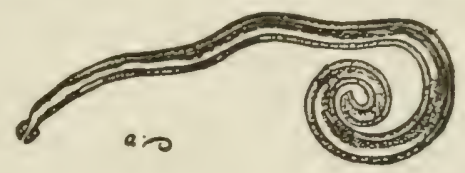

MALE BODKIT-TAILED ROUND WORM.

they produce irreat irritation. Sior do they stop when they find access to the stomach, but occasionally wend their way into the Iunge, throat, windpipe and nostrils, creating so much disturbance that they are frequently 
attended with fits or spasms. The tapeworm is the most injurious of all the intestinal worms. They find their way into the intestines of the dog, through eating the brains of sheep, also from eating rabbits, and through many other sources. The tapeworm is in appearance, flat, and made up of joints, each joint ranging from oneeiwth to a half inch in length. The entire length of the worm varies from a few inches to several feet. As fast as tiese joints mature, they pass away from the bowels,

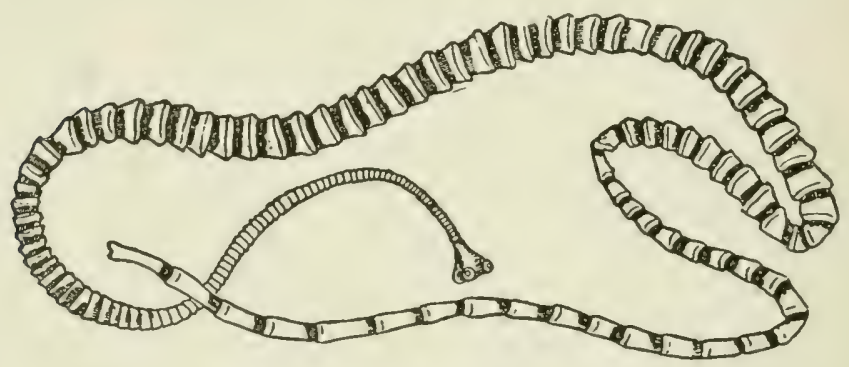

TAPEWORI.

when they deposit their ergs. These are in turn consumed by hogs, sheep, ralbits, or some other animal, in the intestinal orwans of which they hatch, setting free a small worm which burrows through the bowels and locates itself in some part of the flesh or brain, and there becomes encysted, until eaten by some other animal, or by some person, when it developes into another tapeworm. There are two leading species of tapeworm, which infest dogs, but as the treatment is the same, a description bere is quite unnecessary.

Symptoms:- Sometimes dogs will have an abmulance of worms within them, without any symptoms to indicate their presence, but when you observe a dry, short cough, a staring coat, a hot and bad smelling breath, a craving appetite with a fulure to take on flesh in proportion to the amount eaten, the bowels irregular, 
alternately with constipation and diarrhnea, with a desire to vomit, and sometimes vomiting worms followed by spasms, or frequent fits, the animal presenting a wor-legone appearance, rely upon it, it is troubled with worms. If the worms have gained access to the nose or throat, the eyes will look red and the countenance depressed, a frothy spume coming from the throat and mouth. The animal may sneeze, or bark and howl violently, or snap and bite at anything within reach.

Treatment:- When tapeworm is present, the hest mode to rid the patient of it, is not to allow anything to eat for twenty-four to forty-eight hours, then give a drachm of powlered areca nut with a little syrup. If it romits this, give another drachm and tie the mouth shut to prevent romiting. In the course of five or six hours, give a physic. as

Powdered Jalap........ two drachms.

Syrup...............two ounces. Mix.

Give half, and if the bowels do not move freely in four hours give the remainder. Or give

Castor Oil.......two tablespoonfuls.

If this fails to remore it entirely, repeat the treatment in ten days, or the following will answer equally as well: Take a guarter of a pound of hulled pumpkin serel and add a quart of water. Simmer down to a pint, and after fasting for a day or two, give a wineglassful every two hours, until four doses have been given. Immediately after giving the last dose, give a physic. Either of the above will suffice. For the round worm, take

Santonine ........... half drachm.

Copperas............ half drachm. Mix.

Make into seven powders. Give one powder every morning in a little milk or small piece of meat, on an empty stomach, until they are all given, then give a physic, as 
Castor Oil............... one ounce.

Repeat the treatment in two weeks, if necessary. When the worms are lodged in the throat or nose, inhalations of the fumes of burning sulphur, repeated every day for a few days, may dislodge them. Or for:ing some powdered sulphrr up into the parts, will greatly assist in removing them.

\section{WORMS IN THE KIDNEY.}

This worm, which is called the giant strongle, locates. itself in various parts of the body, but more especially in the kidneys. It measures from one to three feet in length, and from a quarter to a half inch in diameter.

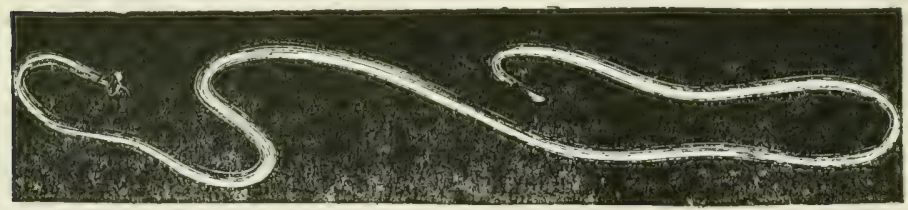

GIANT STHONGLE.

and is of a reddish pink color. Wherever it locates itself, it literally destroys that part. It destroys the kidneys completely, then drops into the abdominal cavity and causes the death of the animal. As yet all treatment has failed, although ten drop doses of turpentine given twice daily, if persisted in, might give the necessary relief desired.

\section{GONORRHOEA-CLAP.}

This disease is sometimes observed in the dog, but as a rule it causes very little inconvenience. It is oftell produced without any assignable cause, and since the ways in which it may originate are so various, I will 
not here designate any of them. The symptoms are observed by the discharge of matter coming from the penis or accumulating around the end of the sheath, the dog often licking at it to keep the parts clean.

Treatment:-Bathe and syringe the parts with wan water, once or twice daily, then wash with

White Vitriol............ one drachm.

Sugar of Lead........... one drachm.

Water................. Mine pint. Mix.

Apply twice daily, injecting a little at the sinme time. Use internally,

Balsam Copabia...........half ounce.

Tr. Cuherbs .............. one ounce.

Spts. Sweet Nitre.........half ounce.

Tincture Kino......... two drachms.

Sulphate Morphine ....... five grains.

Syrup...............two ounces. Mix.

Give a teaspoonful twice daily.

\section{PARTURITION-PUPPING.}

This generally takes plate between the sixty-seromit and sixty-fourth lay, and after it has commenced, from a quarter of an hour to an hour cenerally elapses between the birth of each pupIy. Many bitches are lost every year from this cause. There seems to, be a natural propensity for litches to co-habit with dogs much larger than themselves, and their lives are forfeited for so doing. Bitches should have considerahle exercise when pregnant, as delivery is much safer and easier than when they are penned up and exer'iser denied them. But when the time has arrived and the delivery is a little prolonged, do not medale too much ; give a little time. If, however, she cannot deliver : In I the puppy is either too large or is dead, oil the fingcis 
and go at the work of delivery gently. Use slight pressure along the belly until you can feel the head from bedind, then fasten a string over it or a pair of forceps and use gentle traction until it is delivered. After one is delivered, she may di "iver the balance herself, so allow her a little time and see, but if it is absolutely necessary, continue until you have gotten them all. The bitch should be kept in a warm, comfortable place and fed on soft, nutritious food. While giving milk and sucking her young, she should be well fed, allowing her some cooked meat every day. If, however, she should commence to fail rapidly in flesh and have a poor appetite, the puppies should be weaned at once and fed on boiled milk. When the time arrives to wean the puppies, care should be exercised to keep the teats from swelling and becoming hard and inflamed, or should the pups all die, the same trouble is to he expected. The bitch should be milked frequently and bathed with hot water, after which use

Camphor Gum.......... one ounce.

Lard...............three ounces. Mix.

Apply twice daily. Sometimes, but fortunately not often, the bitch has after-pains after pupping, and forces the womb out. This orwan should be washed in warm water, to which should be added a little alum, after which it should be returned with gentle pressure and the parts bandaged to prevent its coming out again. Give five drops of laudanum every half lowr until four or five doses are given, to prevent the pains. If, however, the womb has been out very iong and woten into such a condition that it cannot possibly be retuned, tio a strong silk cord around it, close up to the body, and in the course of a few hours cut the parts off about an inch back of the cord. Many hitehes may be saved by 
this operation. After the parts are cut off cover the wound with iodoform, and some iodoform and vaseline may be used as a dressing, for a few days afterwards.

\section{FLEAS.}

These are very lively insects. which infest dogs, and cause considerable annoyance and wory. The irrita-

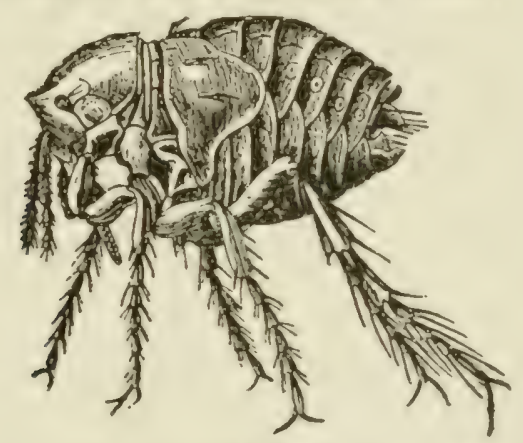

TII: DOG FLEA, OR OHIGGER.

tion produced by them causes the animal to scratch and dig at the parts attacked, considerably. They are very

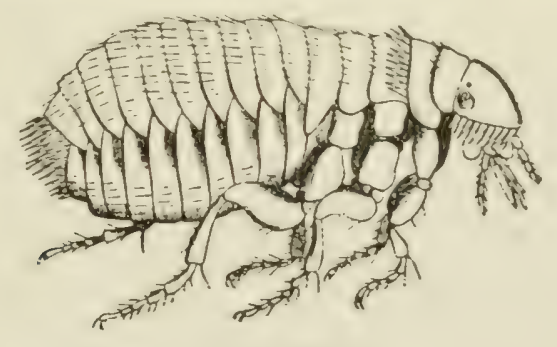

CAT FLES.

easily gotten rid of by rubbing Persian insect powder down into the roots of the hair, then wrapping the dog in a sheet for a few minutes. Or by taking tar soap 
and applying it to the wetted hair until a very heavy lather is produced. Allow it remain on for twenty minutes, then wash oft thoroughly. Coal oil or oil sassafras rubbed through the hair will also dispose of them. Cats are also troubled with fleas and they may be gotten rid of in the same manner.

\section{LICE.}

There are two kinds of lice which infest dogs; the blond-sucker and birl-louse. They are best destroyed by usine a strong solution of tobacen juice, or by sifting wood ashes into the hair. Whale oil will also kill them.

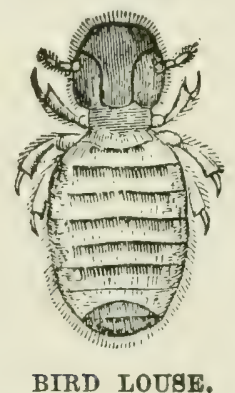

Or better still, take a quarter pound of stavesacre seeds, make a strong tea of them, and bathe the skin well every fourth day, for three or four applications. You will completely destroy the lice, and in that time the nits will all be hatched out. 


\section{USEFUL DRUGS AND MEDICINES}

PRESCRIBED IN THE

TREATMENT OF LIVE STOCK, WITH DOSES, FTC.

Acetate of Lead, Sugar of Lead-Checks hleeding, especially from the stomach and lungs. When comhined with opium it is used to rheck diarrhoea and dysentery. Externally, it heals burns, scalds, bruises and moist ulcers or sores, as well as itching skin comjlaints and cracked heels. When applied externally, mix half ounce in a pint of soft water.

Doses:-Horsesand cattle, one drachm: calres and sheep, fifteen grains: pigs, two grains: logs, one grain. To be given twice daily. I'rice per pound, 25 cts.

Aconite, Tincture of-Relieves pain and inflammation, lowers temperature and produces sweating.

Doses:-Horses and cattle, twenty to thirty drops; sheep and pigs, fire to ten drops; dogs, one to four drops. Price per pint, 60 cts.

Aloes, Barbadoes-Used as a phrsic, but should not be given to mares with foal. It is not much used for cattle.

Doses :- Horses, four to ten drachms; cattle, one to two ounces; sheep, half ounce to an ounce; hogs, two to five drachms; dogs, one drachm ; colts, about five grains to every week of its age. Tincture, per pint, 50 cts.; powder, per pound, 35 cts. 
Alum-Alum is procured in either lump or powdered form and is seldom given internally. It is used principally in sores and in ulcerated condition of the mouth. Also when mixed with wheat flour to arrest bleeding and the flow of joint oil from open joints. It is quite extensively used as a healing powder, when mixed with ioloform. It is a convenient gargle for sore throat. Occasionally it is given internally along with opium in diarrhea and dysentery.

Doses:-Horses and cattle, two to four drachms; sheep and pigs, one drachm; dogs ten to twenty grains. Price per pound, 8 cts.

Ammonia, Aqua or Liquid-It is used as a stimulant, relieves spasms of the bowels and bloating and also acts upon the kidneys, causing flow of urine. When griven internally it should be well diluted with water. It is principally used in making linaments.

Doses:-Horses, half ounce; cattle, one ounce; sheep and pigs, two drachms; dogs, ten drops. Price per pint, 10 cts.

Ammonia, Carbonate-This comes in the solid form and has the same action as the liquid ammonia, varyin: only in strength.

Doses:-Horses, two to four drachns; cattle, three co six drachms; sheep and pins, twenty mains; dows, five grains. It is given either in pills with linseed meal or dissolved in cold gruel. Price per pound, 17 ets.

Anise Seed-Soothes an irritable stomach, relieves indigestion and bloating, and acts as a mild stimulent.

Doses:-Horses, one ounce; cattle, one to two ounces; sheep and swine, two drachms; dogs, thirty grains. Can be repeated several times a day. Price per pound, 22 ets. 
Antimony, Tartrate, Tartar Emetic- ('auses a discharye from the nostrils, lowers the heart's action, and produces sweating. Externally it is used to cause small pustules or blisters, and acts as an irritant.

Dosis:-Horses and cattle, one to four crachms, given three or four times daily. Cansus vomiting, when given in loses of from four to ten grains, in hogs aud dogs. Price per pound, 55 cts.

Antimony, Butter of-Is used as a calistic for thrush and canker, and unhealthy granulations: for foul in the feet of cattle, aml foot-rot in sheep. It slon?h? be used with great caution, as it is very powerful, and cannot be diluted with water. Price per pint, 35 cts.

Animal Charcoal--Is prepare? from bonk. It is used to destroy foul smells, arrests putrefaction, purifies water when passed through it, and is an antidote in poisoning by arsenic, aconite and strychnine.

Dosks:- Horses, half ounce to an ounce: cattle, one ounce; sheep and pigs, two drachms: doers, twenty grains. It is usually given mixed with gruel. Price per pound, 10 cts.

Areca Nut-Is principally used to expel worms from dogs, proving distructive alike to tape and round worms.

Doses:-For dogs, from twenty grains iv two drachms. Prior to giving, the dog should fitst for two days. Price per pound, $40 \mathrm{cts}$.

Arnica, Tincture-It is principally used externally, in cases of strains, bruises and wounds, and especially in broken knees and sore shoulders. It is usually mixed in the proportion of one ounce of arnica to ten of water, to which is frequently added four drachms of sulphate of zinc. For painful wounds or bruises, it is often mixed with chloroform or laudanum, and diluted with water according to circumstances. Price per pint, 5úcts. 
Arsenic-Is used to alter the condition of the system, by toning up the nerves. It is useful in dyspepsia and indigestion, and in promoting recoveries from prolonged attacks of influenza or distemper. Externally, it is used to take off warts, and slough out fistula. In such cases, it is generally mixed one ounce of arsenic to four of lard or vaseline. It is much used in sheep dips.

Doses :- Horses and cattle, one to six grains; sheep, one to two grains; hogs, two to four grains; dogs, onefifteenth to one-tenth grain. It may be given once daily, and continued for a week or ten days. Price per pound, 10 cts.

Asafoetida-This is a mild stimulent, and relieves spasms and pain, as well as irritation of the stomach and bloating. It also causes the expulsion of worms.

Doses:-Horses, two to four drachms; cattle, one ounce; sheep and hogs, one drachm; dogs, fifteen grains. It may be given several times a day. Price per pound, 35 cts.

Atropine-This allays pain, soothes spasms of the bowels, and if given in large doses, causes paralysis. It is most generally used in eye washes. It is seldom used internally, being too powerful. Price per drachm, 50 cts.

Belladonna--Soothes spasms of the bowels, allays pain, and is frequently given in spasmodic colic, in half drachm doses of the extract, or ounce doses of the tincture. It is very useful in tetanus, or lockjaw. It also causes dilatation of the mouth of the womb, when applied. The doses of the extract are, for horses, one drachm; cattle, two drachms; sheep, ten grains; dogs, .wo grains. Solid extract per ounce, 40 cts.; tincture per pint, 90 cts.

Benzoin-U'sed as a mild stimulant, expectorant and arrests putrefaction. The compound tincture of ben- 
zoin, which is usually known as Fryer's Balsam, is quite extensively used as a stimulant and healing remedy for wounds, ulcers, and various skin complaints, in all classes of patients. Fryer's Balsam per pint, 70) cts. ; gum per pound, 60 cts.

Bluestone, Blue Vitriol-See copper Sulphate. Price per pound, 20 cents.

Buchu-Is a mild stimulant and tonic. It also has a soothing action upon the kidneys, causing an increased flow of urine. The doses of the tincture are, for horses and cattle, one ounce; sheep and hogs, four drachms; dogs, one drachm. Tincture, price per pint, j) (cts.

Calomel-When conjoined with aloes, it acts as a physic. It has a direct action upon the liver, causing an increased llow of bile, which it expels from the chodenum, or first bowel. Externally, when applied to old sores or indolent ulcers, it assists greatly in the healiner process. It also destroys the parisite of scial) or mange, kills lice and is useful in cracked hecls. It is one of the best remedies for thrush in the horse's frog.

Dosks:-Horses and cattle, one drachm; sheep and piors, ten grains, logse, two wrains. It is not used alone as a physic, but is combined with other drugs, as aloe's, epsom salts or oil. Price per pound, $\$ 1$.

Camphor-camphor is a nerve stimulant and assists in controlling spasms of the bowels. Externally, it is applied in the form of spirituous solution, to allay itchiner, and is soothing to bruises and sprains.

Doses:- Horses and cattle, one to two drachms; sheep and pigs, twenty grains; dogs, five grains. Gum, per pound, 70 cts. ; tincture, per pint, 60 cts.

Cantharides-Cantharides is a stimulant and acts upon the kilneys, causing an increased flow of urine. It also causes an increased desire for sexual intercourse; 
when given in small doses. Applied externally, it acts as a counter-irritant and causes blisters to form in from one to twelve hours.

Doses:- Horses and cattle, from four to twenty grains; sheep and swine, two to seren grains; does, half grain to two grains. To be given once or twice daily, usually in the form of a pill, or in flaxsecu cruel.

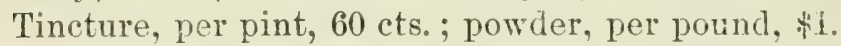

Capsicum, Cayenne Pepper, Red Peppar - This is useris n.s a stimulant and tonic, promoting digestion and impuring the appetite.

Doses:- Horses and cattle, three to four dracinns; sheep and hogs, one to two drachms; dogs, half draclim. Tincture, per pint, 50 cts.; powder, per pound, 30 ('ts.

Carbolic Asid-It arrests putrefaction and destroys parasites and germs, when applied locally. It is also a stimulant and caustic. It is employed mostly in liealing lotions, when it should be diluted with water, oil, or glycerine in the proportions of one to twenty or thirty.

Doses:-Horses and cattle, fifteen to thirty drous; sheep and pigs, six drops; dogs, one drop. It is leest given in a pill, mixed with linseed meal. Price per pint, 40 cts.

Cardamons-Has the same actions as anise seed, and is given in the same doses. See anise seed. Price per pound, $\$ 1$.

Castor 0il-This is principally used as a physic. As it causes no irritation or griping, it is frequently prescribed in diseases of the digestive organs and can be safely administered in advanced pregnancy. It is the best physic for foals and calves.

Doses:- Horses and cattle, one pint; sheep and pigs, two to four ounces; dogs, one to two ounces. Price per pint, 30 cts. 
Catechu-This is an astringent, and causes a hinding up of the bowels when administered, and is griven when there is bloody discharge from the bowels, in cases of diarrhœea and dysentery.

Doses:-(Powder.)-Horses, one to three drachms; cattle, one to five drachms; sheep and swine, one to two drachms; dogs, four to twenty grains. To be given three or four times a day, with gruel. Tincture, per pint, 60 cts. ; powder, per pound, 20 cts.

Chloral Hydrate-This lrug is quite extensively used in the treatment of live stock, to allay pain, quiet irritibility and cause sleep. It is used in cases of flatulent and spasmodic colic, brain troubles and nervous disorders, as tetanus, hysteria, etc.

I)oses:- For horses, two to eight drachms; for cattle, four drachms to one ounce; sheep and pigs, one drachm; dorgs, ten grains. Can be repeated ever two or three hours, and should be well diluted. Price per pound, $\$ 1.10$.

Chloroform-By whatever channel it enters the system, it slightly stimulates at first; excitement follows, then sleep. It allays pain, whether taken internally or applied locally, and is a valuable remedy when given for spasmodic colic.

Dosks:- Horses and cattle, one to two drachms; sheep and swine, twenty to forty drops; dogs, five to ten drops. It should be riven mixed with syrup, mucilage, whisked egg, or weik spirits, and may be repeated at intervals of from one to three hours. Price per pound, 75 cts.

Cocaine-When administered internally, small doses are stimulant and tonic. When applied externally, it diminishes sensibility to pain, and is most frequently used in making examinations of the eye. By mixing ten grains with an ounce of water and applying a few 
drops to the eye every thirty seconds, it deadens the pain so that an examination can be made without difficulty, when chaff and other foreign substances can be easily removed. Price per grain, 2 cts.

Cod-Liver 0il-This is prepared from the liver of the codfish and is a very nourishing tonic, changing the system in such a manner that it lays on flesh quite rapidly. It is very useful for weak and sickly colts and calves, and for animals convalescing from a lingering attack of distemper and influenza.

Doses:- Horses and cattle, two to three ounces; sheep and pigs, one ounce; dogs, one to four drachms. It may be given twice daily and continued for weeks, if required. Price per gallon, \$1.25.

Copaiba Balsam-This medicine is not much used in practice, save in diseases of the urinary organs, and in the treatment of gonorrhoea. Price per pound, 65 cts.

Copper Sulphate-Given in large doses, it is an irritant, but in medicinal doses, it is a valuable tonic and astringent. Externally it is used as a stimulant, astringent and caustic. It is used in eye washes, as a dressing for slugrish wounds, for the removal of warts, to cut off unhealthy granulations and to arrest bleeding.

Doses :-As a tonic and astringent, horses take one to two drachms; cattle, one to four drachms; sheep, twenty to thirty grains; pigs, five to ten grains; dogs, one grain. It should be given while eating or immediately thereafter. Price per pound, 20 cts.

Copperas-See Iron Sulphate. Price per pound, 4 cts.

Corrosive Sublimate-This is a preparation of mercury and is an irritant poison. It should not be given internally, except when prescribed by an expert. Externally it is used to arrest putrefaction, promote healthy granu- 
lations, to destroy indolent ulcers, for the destruction of parasites and as a caustic. It is used also, in many itching skin diseases. When used extermally, it should be diluted according to circumstances. Price per pound, $80 \mathrm{cts}$.

Cotton Seed 0il-This is obtained from the seeds of the cotton. It is a dark, lrownish red. fixed oil, and is principally used in maling liniment. Price per wallon, 70 cts.

Croton 0il-This is olitained from the croton sereds, and is a drastic physic, protureing wriping. It is seldom given alone but is usually mixed with other purwatives, when a quick action of the bowels is required. It is sometimes used externally, as a blister, but is more frequently mixed with other medicines, in the formation of liniments. It is used quite often to physic cattle.

Dosis :-Horses, fifteen to twenty drops; cattle, half drachm to two drachms: sheep and swine, five to ten drops; dogs, one to three drops. Price per pint. \$1.25.

Cubebs, Tincture-The chiof use of cubebs is for the relief of inllammation of the urinary passages, and like copaiba, it has long been userl for the cure of gonorrhea.

Doses:-Horses and cattle, one ounce; sheep and swine, half ounce; dogs, two drachms. To be given twice daily. Price per pint, $50 \mathrm{cts}$.

Digitalis-Digitalis grows wild in this country on gravelly, sandy soil, more especially hilly pastures. It has a direct action upon the heart and kidneys, wring force and strength to the former and causes an increased flow of urine from the latter.

Doses:-Of the powdered leares horses take ten to thirty grains; cattle, half drachm to a drachm; sheep and swine, eight to ten grains; dogs, one to four grains. 
Of the tincture, horses and cattle take two to four drachms; sheep and swine, one drachm; dogs, two to five drops. Tincture, price per pint, 50 cts.

Emetic Tartar-See Antimony Tartrate. Price per pound, 55 cts.

Epsom Salts, Magnesium Sulphate-This salt is used as a physic, to reduce fever and to alter the condition of the system. It is more extensively used for cattle and sheep, as its action upon the bowels gives better results than any other physic.

Doses :-As a physic for grown cattle, a pound to a pound and a half; calves, two to three months old, three to four ounces; sheep and pigs, four to six ounces; dogs, two to four drachms. For other purposes, as to lower fever and regulate the bowels, give one-fifth to one-eighth of the above doses. It should be well diluted with water. Price per pound, 7 cts.

Ergot of Rye-This is obtained from blasted rye, and is given to assist in difficult parturition, when the labor pains occur at long intervals. It is also of great benefit after parturition, to (ause contraction of the womb and arrest bleeding from this organ.

Doses:- Mare and cow, half an ounce to an ounce; sheep, swine and bitches, one drachm. Repeat at intervals of half an hour or an hour. Tincture, price per pint, 60 cts.

Ether Nitrous, Spirits of (Sweet Spirits of Nitre)-This drug is an excellent stimulant, produces sweating and also acts upon the kidneys, causing an increased flow of urine. It is very useful in the various forms of colic, acting as a stimulant and controlling the pain produced by spasms. It is also useful in loweringr fever.

Doses:- Horses and cattle, one to three ounces; sheep, two to four drachms: pigs, one to two drachms; dogs, one drachm. It should be well diluted with cold 
water, and should be given as quickly as possible : "ler it is diluted. Price per pint, $40 \mathrm{cts}$.

Ether, Sulphuric Ether-This is quite a valuable renedy and is used as a stimulant and to relieve spasms of the bowels. It is a useful remedy in flatulent colic, to relieve bloating. It is also used to produce sleep, the same as chloroform. It is also very useful where there is pain or cramping of the bowels.

Doses:- Horses and cattle, one to two ounces; sheep and pins, two to four drachms; dogs, one drachm. It should be well diluted with cold water hefore giving, but should not be diluted until ready for use. Price per pint, 75 cents.

Foenugreek-This has the same action and uses :16 anise secu, and is given in similar doses. See Anlsw Seed. Price per pound, $10 \mathrm{cts}$.

Gentian-This is a pure bitter, and is used to tone up the stomach and promote digestion. It improves the appetite and greneral tone. It is an exrellent stimulating tonic in intluenza and other epizontic discases. It is also beneficial for horses jaded and orer-workerl, or suffering from slight cold.

Doses:-Horses and cattle, half ounce to an ounce; sheep, one to two drachms; pim, one drachm; logs, ten to fifteen arains. To be wiven twice or thrice daily. Price per pound, 15 cts.

Gentian, Tincture of-Has the same ation at the powder, and is given for the same purposes. Doses about the same. Price per pint, 50 cts.

Ginger-Ginger is used as a stimulant, tones up the stomach and relieves bloating, and cramps of the bowels. It promotes digestion and increases the alppetite. When mixed with physic, it dimmishes the tendency to nauseate and gripe. 
Doses:-Horses and cattle, half ounce to one ounce; sheep, one to two drachms; pigs, half drachm, dogs, ten to twenty grains. Tincture, jer pint, 60 cts. ; powder, per pound, 30 ets.

Glauber Salts (Sulphate of Soda)-This is given as a physic; also to reduce fever; increases the secretion of bile in the liver, and has a slight action upon the kidneys.

Doses:- As a physic, cattle take a pound to a pound and a half; horses, one pound; sheep, two to four ounces; dogs, one ounce. It is usually combined with a little ginger, and should he well diluted with water. Price per pound, 5 cts.

Glycyrrhiza (Licorice Root)—This resembles sugar in its medicinal uses, and is occasionally used in irritation of the mucous membrane of the windpipe. Also to cover the disagreeable taste and odor of various drugs. Price per pound, 20 cts.

Goulard's Extract-This is used externally as an astringent. Also as a cooling or soothing application, to reduce inflammation of the testicles, when it is usually mixed with laudanum, in the proportions of one ounce of each to a pint of water. Price per pint, 20 cts.

Hellebore-Hellebore is used to expel worms from the bowels. When given as a physic it produces griping and in large doses produces vomiting. It is sometimes used externally and is a dangerous remedy when not used with great care. Price per pound, 30 ets.

Hemlock, Oil of-This is used in the formation of various liniments and is very fatal if given internally. Price per pint, 40 cts.

Hofiman's Anodyne-Consists of oil of wine, three parts; ether, sixty-four parts; rectified spirits, one hundred and twenty-eight parts. It is used to allay 
restlessness, sleeplessness and nervous disturbances, esperially in the absence of fever, and is a convenient renedy for flatulentey or bloating. It may be used in all cases in which sulphuric ether is given internally.

Doses:-Horses, one ounce; cattle, two ounces; sheep and pigs, two drachms; dogs, half a drachm. Price per pint, 65 cts.

Hypo-Sulphate of Soda-This remedy is used to destroy odors, arrest putrefaction and for the destruction of insects. It is beneficial in attarks of ferer, and relieves acidity of the stomach and bloating in bally fed young salves. It is also used to diminish the offensive secrerion and to abate the low fever occurring in distemper in dogs.

Doses:- Horses and cattle, two to four drachms; sheep and pigs, twenty to thirty grains; dogs, two to ive grains. It may be given several times daily. Pruce per pound, 7 cts.

Iodoform-This is used to prevent putrefartion and destroy foul smells. It is beneficial in healing wounds and sores, by being dusted upon the wound, but it is usually mixed with powdered alum for this purpose, in the proportion of one ounce of ioduform to four or six of the alum. Price per ounce, 40 cts.

Iodine-Indine is prepared from the semi-vitrified ashes of sea-weed. Medicinal doses arrest putrefaction, alter the condition of the system and act as a mild stimulant. It exerts its effects especially on mucous membranes, skin and lymphatic glands. Externally it is applied to arrest putrefaction and as a counterirritant or blister. It is used in chronic inflammation of the joints, wind-galls, bog spavin, strains of tendons and enlargement of the udder, and other glands.

Doses:-For horses and cattle, twenty grains to a 
drachm; sheep and pigs, ten to twenty grains; dogs, three to eight grains. To be repeated once or twice daily, and to be given a couple of hours after eating. When applied externally it is used in the form of a tincture. Powdered, per ounce, 35 cts.; tincture, per pint, 85 cts.

Iron Sulphate, Copperas-This is a good blood tonio and astringent, when given internally. Applied externally, it is an astringent and arrests bleeding and prevents putrefaction. It improves the appetite, diminishes exhausting discharges and abates glandular enlargements. It is especially beneficial when given to young horses, cattle or sheep which have been poorly kept, and whose blood is in a bad condition. It is also used to expel intestinal worms. Used externally, when dissolved in water, it is an excellent remedy for cracked heels and scratches.

Doses:-Horses and cattle, one to two drachms; sheep and hogs, ten to twenty grains; dogs, two to ten grains. Nay be given twice or thrice daily, and is usually combined with gentian or ginger. Price per pound, 4 ets.

Jalap-Jalap is principally used as a physic for dogs. It increases the flow of the bile from the liver, and assists in the expulsion of worms.

Doses:-Dogs, one to two drachms. It is best given combined with calomel, in the form of one drachm of jalip to three grains of calomel. Price, per pound, 60 its.

Kino-Kino is an astringent and causes a binding up of the bowels. It is used principally in the treatment of diarrhoea.

Doses:-Horses, half ounce; cattle, one ounco; sheep and pigs, two drachms; dogs, one drachm. Tincture, per pint, 60 ets. 
Lanolin - This is prepared by steeping and boiling sheep)'s wool in water. It is principally used as a basis for ointments and liniments, and mixes readily with fats and oils. Price per pound, $\$ 1.00$.

Laudanum-See Opium Tincture. Price per pint, $\$ 1.00$.

Liquor Ammonia Acetatis (Liq. Ammonia Acet.--Mindererus Spirits.) - This is prepared by taking one ounco of acetic acid and seven ounces of water. Mix towether and add carbonate of ammonia until it ceatses eflervescing. It should only be prepared shortly prior to use. It is a valuahle remedy to proluce sucating, increatse the flow of urine, reduce fovers and cause expectoration. It is neful in lungererer, influenza, strangles or distemper, scarletina, ereipelas, colds and it bronchitis. It is often combined with ofluel remedies, as epsom salts. chlorate and nitrate of potassium, gentian, ginger, quinine, etc. Where patients are weak and exhausted, it is usually combined with alcohol or sweet spirits of nitre.

Doses:-Horses and cattle, two to four ounces; sheep, one to two ounces: hogs, half an ounce to an ounce; $\log \mathrm{s}$, two to four drachms. Price per pint 20 cts.

Listerine-This is a proprietary medicine, and has about the same artions as carbolic acid. It is used in many healing lotions: also as a sargle for the mouth and throat. Price per bottle, 85 cts.

Liverwort-This herb arows wild in the shady woods, of North America, and is very selilom used in the treatment of disease. Price per pound, 40 cts.

Lime, Chloriae of-This preplatition of lime is sometimes known as bleaching powder, and is seldom griven internally. Externally, it is used as a stimulant, and 
to destroy the offensive smell from unhealthy wounds. It is more frequently used as a disinfectant, around stables where contagious or epizootic diseases prevail. Price per pound, 8 cts.

Lobelia-The chief use of lobelia, is in the treatment of asthma, by relieving the bronchial spasm. It is quite frequently administered to check or relieve heaves.

Doses of the Tincture-For Horses and cattle, half ounce to an ounce; sheep and hogs, one to three drachms; dogs, half drachm to a drachm. Tincture, price per pint, 50 cts.

Lunar Caustic-See Nitrate of Silver. Price per ounce, 70 cts.

Magnesia, Sulphate-See Epsom Salts. Price per pound, 5 cts.

Male Shield Fern, 0il of-This oil is principally used to expel worms. It is a very effectual remedy for tapeworm in dogs, sometimes dislodging them within three hours.

Doses:-Horses and cattle, two to four drachms; sheep and hogs, one drachm; dogs, half drachm to one drachm. It is sometimes given with either turpentine or linseed oil, and may be repeated within three days. Price per ounce, 30 cts.

Mercury, Red or Biniodide-This form of mercury is selnom given internally, but externally it is used to produce superficial inflammation or blisters, and is generally mixed with one part of the mercury to eight of vaseline or lard. It is valuable in the treatment of spavin and ringbone, sprains or enlarged tendons, and when applied around the throat, it sometimes relieves a chronic cough and roaring, where other remedies have failed. It should be used with care, or blemishes may 
in the result, as it na: diestroy the hair roots, if used ioo freely. Price jer ounce, 30 cts.

Morphia, Sulphate and Muriate (Morphine.)-This is the active principle of opium, from which it is olstained. It may he used in all cases where opium is recpuired. See Orium.

Doses:-Horses and cattle, tron to thirty grains; sheep and pigs, one to two grains; dogs, one-eighth to one-half a grain. Price per ounce, $\$ 2.50$.

Nitrate of Potassium--See L'otassium Nitrate. Price pri jound, 12 cts.

Nitrate of Silver (Lunar Caustic)-It is sometimes used as a tonic in chorea and epilepsy amoner dogs. It is schom used internally, but externally, it is used as a coutic to promote healthy wranulations in indolent vlecers. Sticks of it are sometimes foreed to the bottom of jipes, in fistulous withers and poll evil. It is an שx.ellent dressing for olstinately sore teats in cows, as woll ats for foot-rot in sheep. It is useful in removing Warts and other enlargements of the skin. A solution rontaining two to five arains in an ounce of water, ib bates pain and congestion of the eyes. It is, therefore, userl in several eye lotions. P'rice per ounce, 70 cts.

Nitric Acid-This is an irritant and corrosive and leaves a yellow or brown stam on the slin. It is used internally as a stimulant and tonic to the liver, and can be wiven to horses convalescing from intluenza, jamndice and other debilitating disorders. Externally, it is used to remove warts or other malignant growths, such as jometime appear in a bad case of grease-heel. When alpplied for these purposes, the surrounding parts should be protected by being well oiled.

Doses:-Of the diluted medicinal acid, horses and crittle take one to two drachms; sheep and pigs, ten to 
twenty drops; dogs, two to ten drops. It should always be well diluted with water. Price per pint, 9 ets.

Nitro-Muriatic Acid-This very much resembles nitric acid, and is given for the same dikeases and in the same doses as nitric acid. Price per pint, 15 cts.

Nux Vomica-Nux Vomica is the best nerve stimulant and tonic that is prescribed in veterinary practice. It is useful in dyspepsia and any stomach or nervous derangement, and may be used with great benefit when convalescing from any exhausting or nervous diseases.

Doses:- Horses and cattle, half a drachm to two drachms; sheep, ten to forty grains ; pigs, ten to twenty grains; dogs, half grain to two grains. Of the tincture, horses and cattle take half ounce to an ounce; sheep and pigs, one to two drachms; dogs, five to ten drops. When twitching of the muscles is produced, cease giring. Tincture, per pint, 50 cts.; powder, price per pound, 25 cts.

Olive Oil (Sweet 0il)_Olive oil given in small doses, resembles cod-liver oil, becoming assimilated and assist. in 2 in the production of heat and fat. In large doses it acts as a physic, for which it is seldom used. It is more frequently used in the formation of liniments, but is usually superseded by cheaper oil, as cottonseed oil, lard, linseed oil or vaseline. Price per gallon, 75 cts.

Opium, Powdered-No one remedy is more frequently prescribed thin opium in its various forms. It can be given as a stimulant. Small and frequent doses produce sweating; larger doses relieve pain and spasms of the bowels, depress the activity of the brain and produce sleep. It also relieves congestion and inflammation and controls pain. It is useful in inflammation of the lungs, bowels, kidneys, bladder, or any internal organ. "It is useful in diarhoea and dysentery and relieves 
cough, whether acute or chronic. It is very effectual in arresting premature labor pains. Externally it is used to relieve painful wounds, bruises, boils, or blistered surfaces, as well as inflammation of the eyes, skin or joints. For such purposes, ten drops each of laudanum and Goulard's Extract, may he mixed with an ounce of water and freely applied.

Doses:-(I'owhered or rum opium.) Horses, one to two drachms: (attle, fwo to four drachms: sheep), ten to forty grains: dogs, one to five grains. Price per ounce, 38 ets.

Opium, Tincture of (Laudanum)--Tincture of opium and laudanum are one and the same thing, and are preparel from the sum of powlered opium. They may be used for all complaints where oprum is indieated, such as colic, cramps, etc. See Opium.

Doses:- Horses and cattle, one to two ounces; sheep, two to fomr dratehms; hogs, one to tlio drachms; dogs, five to twenty drops. Price per pint, $\$ 1$.

Origanum, Dil of-This oil is sometimesusent to relieve the pain of carions teeth and nemralgia, hut is more frequently used in liniments. I'rice per pint, fo cts.

oxide of Zinc-See Zinc Oxide. I'rice per pound, 15 cts.

Podophyllin-This resin is taken from the mayapple or mandrake, and is used as a physic, hut is rery uncertain, unless (ombined with aloes, jalap or calomel; it then relieves confestion of the liver. It is sometimes used to lower the heart's action, in acute rheumatism and other intlammatory lisorders in robust patients.

a Doses:-To cause an increased flow of hile from the liver, horses and cattle take one to two drachms, mixed with aloes, calomel or cysom salts. Dows repuire one to two grains, with calomel one to two grains, which 
may be mixed with an ounce of oil. Price per ounce, 40 cts.

Potassium, Bicarbonate-Given before meals, it increases the secretion of the juices of the stomach, and is sometimes given for rheumatism. The action upon the kidneys is not so certain as the acetate or nitrate.

DosEs:- Horses and cattle, half ounce to an ounce; sheep and pigs, half a drachm to a drachm; dogs, ten to forty grains. May be repeated sereral times a day, well diluted with water. Price per pound, $20 \mathrm{cts}$.

Potassium, Sulphuret-Large doses are irritant. Small doses stimulate the secretions of the skin and alter the condition of the system. Externally, it is sometimes used as a substitute for sulphur, in skin diseases. It has been used in chronic cough and rheumatism.

Doses:-Horses and cattle, one to three drachms; dogs, two to ten grains. Price per pound, $30 \mathrm{cts}$.

Potassium, Iodide of-This closely resembles iodine, but is less powerful. It promotes absorption of deposits, reduces enlarged glands and lung consolidation. It is useful in rheumatism and skin diseases. Externally used, it is usually conjoined with soap liniment and laudanum, and is applied to swollen, rheumatie joints and to inflamed udders in cows and ewes.

Doses:- Horses and cattle, two to five drachms; sheep and pigs, twenty to forty grains; dogs, five to fifteen grains. May be given two or three times a day. Price per ounce, 25 ets.

Potassium, Bromide of-This form of potassium controls irritability or excitement, whether exhibited in restlessness or convulsions. It is often given mixed with alcohol, chloral or opium. It quiets irritability from tetanus or lockjaw, in horses, and wards off epileptic convulsions in dogs. 
Doses:-Horses and cattle, half ounce to an ounce; dogs, five to twenty grains. May be given every two or three hours. Price per pound, 40 cts.

Potassium, Nitrate of (Saltpetre)-This drug is quite extensively used in veterinary practice. Large doses are irritant and slimhty laxative. Medicinal doses arrest putrefaction, alter the condition of the system, reduce fever and act upon the kidneys by causing an inereased flow of urine. It is used quite largely in the treatment of febrile and infiammatory complaints, both in horses and cattle, and lessens the pain in fever and founder. Externally, when dissolred in water, it is an excellent application for hruises by dispersing the inereased flow of blood to the parts, thus exerting its cooling effects.

Doses:-IIorses and cattle, half ounce to an ounce; sheep, one to two drathms: pigs half a trichm; dogs, ten to thirty grains. May he wiren thee or four times daily. Price per pound, 12 cts.

Potassium, Chlorate of-This Irug is quite extensively used in reterinary practice. It arrests putrefiaction, alters the contition of the system, increases the llow from secreting wands and also the action of the kiclneys. It soothes and heals eruptions and ulcerations of the mouth and throat, while in catarrh, sore throat and distemper, it thins the surotions and promotes the discharge from the nostrils. It lowers the pulse and fevers, rleans the tongue, iniposes the appetite: crently stimulintes the howels and renders their evacuations more natural and less coated with mucous. It is useful in distemper, epizontic and the rarious forms of influenza, couchs and colds, as well as in the treatment of purpura.

Doses:-Horses and cattle, two to four drachms; sheep and pirs, twenty to forty grains; dogs, five to 
fifteen grains. May be given three or four times daily. Price per pound, 30 ets.

Potassium, Permanganate of-This remedy is seldom given internally, but is used externally as a mild stimulant, and to destroy the odors of foul and unhealthy sores. It is used frequently as an injection into the vagina and womb, to destroy offensive odors, and in the treatment of leucorrhoea or whites, mixed in the proportion of one drachm of the permanganate to a quart of water. Price per pound, $40 \mathrm{cts}$.

Potassium, Acetate of-Is used the same as the nitrate potassium, which it closely resembles in its actions, and is given in the same doses. Price per pound, $40 \mathrm{cts}$.

Potash, Caustic-Caustic potash is attained from ashes and is used to cauterize snake bites and to remove warts and fungus growths. It is seldom used internally. It is now used quite extensively in preventing the growth of horns on calves. This result is obtained by taking the stick potash and applying it over the parts where the horns grow, when the calf is two or three weeks old. First cut off the hair over the parts, then moisten them a little, then apply the potash sparingly, and no horns will grow afterwards. Price per pound, 60 ets.

Prussic Acid-This drug is such a deadly poison that it is unsafe in the hands of an amateur and should only be given where it has been prescribed by a professional man. Dilute, price per pint, $50 \mathrm{cts}$.

Quassia-Quassia is a bitter, which increases the appetite, tones up the system and expels worms. It is prescribed for dyspepsia, loss of appetite and in convalescence from debilitating diseases.

Doses:-It is usually given in the form of an infusion, which is made by soaking for an hour, one part of 
the chips to eighty parts of cold water. Of the infusion, horses and cattle take two to four ounces; sheep and pigs, four drachms; dogs, one drachm. Price per pound, 15 cts.

Quinine, Sulphate-Quinine is obtained from the bark of cinchona, of which there are different varieties, yiclding various amounts. It is used for the same diseases as cinchona bark, but being the extract is much stronger, it is given in smaller doses. It is given to tone up the system, improve the appetite and check fermentation within the bowels. It reduces fevers and hastens convalescence from debilitating diseases. Large doses sometimes check congestion of the lungs, thus cutting short an attack of lung fever. It is useful in influenza, protracted cases of strangles, and purpura, and other feverish attacks in horses; also in fevered conditions in cows and ewes after delivering their young, and in bad cases of distemper in dogs; or any disease which is caused from malaria.

Doses :- Horses and cattle, half drachm to a drachm; sheep and pigs, five to twenty grains; dogs, one to eight grains. May be given two or three times daily. Price per ounce, 40 cts.

Rectified Spirits-Rectified spirits very much resembles alcohol and is used for making all the spirits and a large number of the tinctures and extracts now in use. It is quite extensively used in its various forms. It stimulates the secretions of the stomach and improves the appetite in indigestion; it relieves bloating and controls the spasms of bowels, colic or intestinal colic; also checks persistent diarrhœa, equalizes irregular circulation in chills and strengthens the heart's action; it is used in epizootic catarrh, sore throat, congestion of the lungs, bronchitis and pneumonia, and in all patients during lingering or debilitating disorders, or in any 
case where stimulants are indicated. Used externally, it causes redness of the skin and acts as a mild irritant, entering, also, into the formation of various liniments.

Doses:-Of the rectified spirits, horses take about one ounce; cattle, one to three ounces; sheep, half an ounce; pigs, two drachms; dogs, one drachm. Whisky, gin and brandy, which are made from rectified spirits, are not nearly so strong and may be given in much larger doses, as often as occasion demands. Price per pint, 35 cts.

Resin-This is obtained as a residue, in the preparation of turpentine. It is a mild stimulant and acts upon the kidneys, causing an increased flow of urine. Externally, it is used in the formation of various plasters.

Doses:-Horses and cattle, one to two ounces; sheep and pigs, half an ounce; dogs, two drachms. Price per pound, 5 cts.

Rhubarb-Rhubarb increases the tone of the stomach, causes an increased flow of bile from the liver and acts as a mild physic. Small and repeated doses, improve the appetite. It is usually given combined with aloes, epsom salts or some other cathartic, as it scarcely produces any purging effect upon the bowels of norses or cattle, when given alone.

Doses:-To increase the tone of the stomach, horses take one ounce; cattle two ounces; sheep, one drachm; dogs, ten to twenty grains. Price per pound, $\$ 1.00$.

Salicylate of Soda-This is prepared from salicylic acid and soda. It is a whitish powder and is used considorably in the treatment of all kinds of rheumatism.

Doses :- Horses and cattle take two to four drachms; steep and dogs, ten to fifteen grains. Price per ounce, 18 cts.

Salicylic Acid-Salicylic acid is a soft, light, white pow der. It is odorless, but when inhaled, irritates the 
nostrils and has an action upon the liver which renders the bile more watery. It is used in dyspepsia where bloating occurs, and in diarrhou in young animals to arrest fermentation. It is used in all classes of patients for rhematism, whether acute or chronic. Externally, it is user conjoined with alcohol, in the treatment of skin discases, where itching is a predominant symptom.

Dosis:-Horses and cattle, one to two drachms; sheep, hogs and dogs, ten to fifteen grains. May be given every one to two hours, but should always be mixed with either baking soda or borax. Price per ounce, 15 ets.

Santonine-rintonine is userl almost exclusirely for the destruction of worms and is one of the hest remedies for their extermination. When wiven at night, it should be followed by a brisk physic in the morning, or it may be mixed with the physic and given at the same time, upon an empty stomach.

Doses:- Horses and cattle, one to two drachms; sheep and hoss, two to ten wranins: dogs, one to three grains. Price pcr ounce, 30 cts.

Soda, Bi-Carbonate of, and Carbonate (Baking Soda)These two preparations of sola counteract acidity and alter the condition of the system. When given a half hour before meals, they increase the secretion of the gastric juice. They are very serviceable in reliering. indigestion and bloating, hence are given to relieve flatulent colic.

Doses:-Horses and eattle, one to three drachms; sheep and pigs, twenty to forty rrains; dogs, five to fifteen grains. The bi-carbonate may be giren in double the amount above mentioned. Bi-Carbonate, per pound, 7 cts; Carbonate, per pound, 5 cts.

Sodium, Chloride-Common 'lable Salt. 
Sodium, Sulphate-See Glauber Salts. Price, per pound, 5 cts.

Spanish Brown-This is used in various mixtures and contains a light percentage of sesquioxide of iron. Price, per pound, 5icts.

Spirits Frumenti-Whiskey.

Squills, Syrup of-Then given in large doses, squills causes vomiting and purring; in small doses, it lowers the pulse rate and raises the blood pressure. It also causes expectoration, and increases the action of the kidneys. It is chiefly given in diseases of the air passages and lungs, in the form of syrup.

Doses:-Horses and cattle, two to four drachms; sheep, hog and dog, ten to twenty drops. Price, per pint, 45 cts.

Stavesacre Seeds-This seed is used for the destruction of lice, and is the best remedy known for this purpose. It is prepared by taking one pound of the seeds, adding two gallons of water. Simmer over a slow fire, for two hours, then add water sufficient to make two gallons. It is then ready for use. Price, per pound, 25 cts.

Strychnia, Strychnine-This is prepared from nux vomica and is given in all diseases where nux vomica is useful. See Nux Vomica.

Doses:-Horses, one to two grains; cattle, two to four grains; sheep, one-fifth to one grain; $h \mathrm{og}$ and $\mathrm{dog}$, one-fiftieth to one-twentieth of a grain. May be given twice daily. Price, per ounce, $\$ 1.50$.

Sulphuric Acid-This acid is a corrosive, irritant poison, but is used to arrest putrefaction, to tone up and cool off the system. It is also used as an astringent, and is given in chronic diarrhoa and dysentery. It is also useful in influenza tending to purpura. It is used 
externally in various liniments and as a hlisterıng ointment, when combined with other remedies.

Doses:-Of the medicinal acid, horses take one to two drachms; cattle, two to four drachms; sheep, half a drachm to a drachm; pigs, ten to twenty drops; dogs, two to five drops. May be given several times a day, freely diluted with water. Price, per pint, o cts.

Sulphur-Flower of sulphur is quite extensively used. It acts as a mild physic, when griven internally. Externally, it is used in various slin diseases, being very effectual in the cure of mange and scab, and is usually combined with tar oils.

Dosfs:-To loosen the bowels, horses take one to four ounces; cattle, three to six ounces; sheep and pigs, one ounce; dogs, two drachms. Ointments used externally usually consist of one part of sulphur to four of vaseline or lard. Price, per pound, 8 cts.

Sugar of Lead-see Acetate of Learl. Price per pound, 20 cts.

Sweet Oil-See Olive Oil. Price per gallon, 75 ets.

Tannin, Tannic Acid-This is used principally as an astringent in chronic diarrhea and dysentery. It is useful in preventing bleeding in the stomach, bowels or womb, also from the lungs. Externally it is used on ulcers of the mouth, sore teats, and piles. It is sometimes used as an injection, in gleet and whites.

Dosis:-Horses and cattle, one to two drachms; sheep, hog and dog, three to ten grains. Price, per pound, $\$ 1.50$.

Tansy, 0il of-This is used principally in the formation of liniments. It is a useful application for bruises, sprains, muscular rheumatism and chronic ulcers. Internally it is sometimes used to relieve flatulent colic. It is also employed to produce abortion. 
Doses:-Horses and cattle, half a drachm to a drachm; sheep, hog and dog, one to four drops. Price, per ounce, 25 cts.

Taraxacum--This remedy is taken from the dandelion, and is a good blood purifier, stimulates the liver, and is an excellent remedy for jaundice. Price, per pound, \$1.35.

Tartar Emetic-See Antimony Tartrate. Price, per pound, 55 cts.

Tincture 0pii Camph., Paregoric-This is a preparation of camphor and opium, benzoic acid, oil of anise, clarified honey and diluted alcohol. It is used to relive abdominal pains, or irritability of the stomach or bowels; also for allaying coumll.

Doses:-Horses and cattle, half ounce to an ounce; sheep, hog and dog, one to three drachms. Price, per pint, 60 cts.

Tolu, Syrup of-This is rincipally used for cougls colds, sore throat, or any chronic disease of the throat and lungs.

Doses:-Horses and cattle, half ounce to an ounce; sheep, hog and dog, two to four drachus. Price, per pint, 50 cts.

Verdigris, Copper Subacetate-This is an irritant poison and is seldom used internally. Externally it is used as a caustic stimu...nt, astringent and antiseptic. It is applied in the form of a powder, solution or ointment, and is made of one part of verdigris to eicht or ten of lard, vaseline or water. Price, per pound, $50 \mathrm{cts}$.

Vitriol, 0il of-See Sulphuric Acid. Price, per pint, 5 cts.

Vitriol, White-See Zine Sulphate. Price, per pound, 10 cts.

Whiskey-See Rectified Spirits. 
Witchhazel, Hamamelis-This is quite a raluable remedy, for sprains, bruises, wounds, inflammations, congestions, or injuries from any source, in soothing and controlling the inflammation. It also enters into the formation of many liniments. It is used also as a soothing application for piles. Price, per gallon, fo cts.

Wormwood, oil of-This oil is used in the form. tion of various liniments. Price, per ounce, $40 \mathrm{cts}$.

Zinc, Chloride-This remedy is starcely ever given internally. Externally, it is used as a stimulant, astringent, caustic, and for the destruction of paracites, and is a valuable remedy in the treatment of canker of the foot. Price, per pound, 50 cents.

Zinc, 0xide-Zine oxide is mostly used extemally, in the treatment of skin diseases. It is useful in relieving tenderness and itching, and assists in healing excoriattions of the skin, leing also frequently mixed with vaseline or crlycerine, in the form of an ointment. Price, per pound, $15 \mathrm{cts}$.

Zinc, Sulphate-This is seldom used internally, but externally it is used as a stimulant and astringent, to promote the healing of wounds and foul ulcers, and when combined with sugar of lead and water, makes the famous white lotion, a healing preparation. It is sometimes used in the treatment of diseases of the eyes. Price, per pound, $10 \mathrm{cts}$. 



\section{INDEX,}

\section{HORSE DEPARTMENT.}

PAGE.

Absoesses.................................................. 174

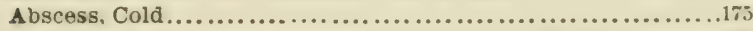

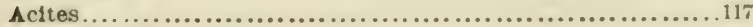

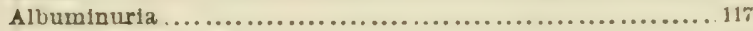

Amaurosis ................................................ 134

Amputation of Penis..................................... 187

Aneurism ...............................................199

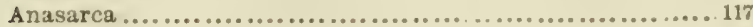

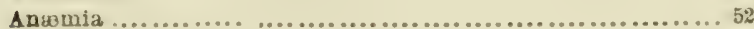

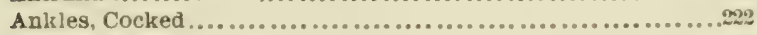

Apoplexy ....................................................

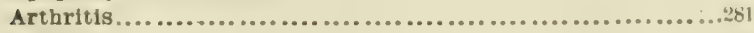

Asthma ............................................. 72

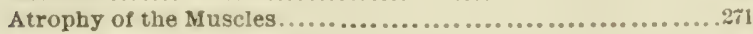

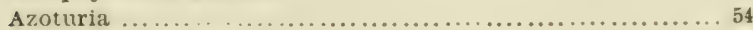

B Barbed Wire, wounds from............................

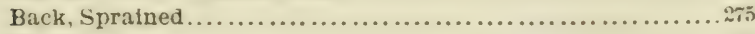

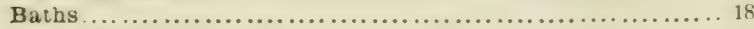

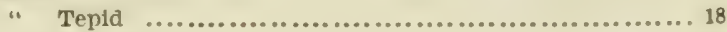

" Warm....................................... 19

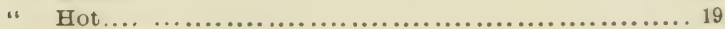

" Medicated................................... 19

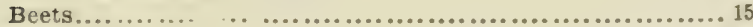

Bladder, Paralysts of .......................................

Inflammation of .................................

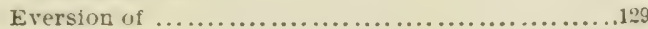

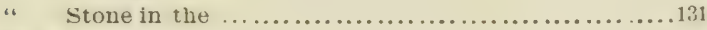

Bleeding ....... ....................................

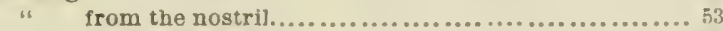

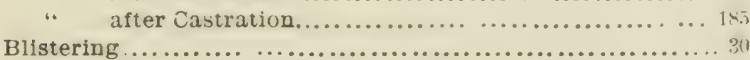

Blood Spavin ........................................

Bloody Urine.......................................... 119

Bog Spavin.........................................

Boluses.........................................

Bone Spavin .................................... $\because{ }^{4}$

Bots....................................................

Brain, Inflammation of.......................................

(607) 
Bran............................................. 14

Bright's Disease......................................117

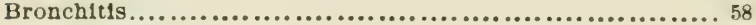

Bronchocele..............................................239

Broken or Bruised Knees. . ..............................267

Bruises....................................................172

of the Frog...................................... ...199

Burns or Scalds.........................................163

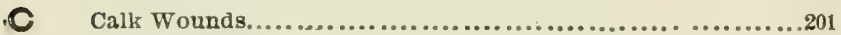

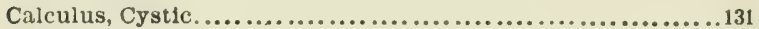

Canker... ...............................................193

Capped Hock.........................................227

Carpltis..............................................266

Carrots ................................................... 15

Caries of the Teeth........................................234

Castration............................................ 183

Bleeding After...............................185

Catarrh, Chronic.......................................246

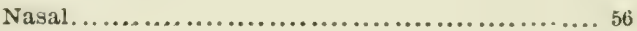

Chaff................................................ 13

Chafing by the Harness. ................................ 154

Choking............................................... 166

Chronic Roaring.................................. 57

Clap....................................................128

Cocked Ankles...........................................22

Coftin Jolnt Lameness... . . . . . . . . . . . . . . . . . . . . . . . . . . 210

Colis, Flatulent................................. 49

Spasmodic.................................. 45

Cold in the Head..................................... 56

" Abscess .......................................... 175

Congestion of the Lungs ........................... 70

" " " Liver................................ 99

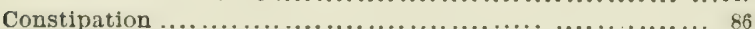

Contracted Heels .................................... 202

Contused and Incised Wounds .........................

Corn ............................................. 5

Corns ...................................................... 19

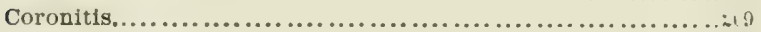

Cough, Chronlc........................................ c

Cramps.......................................... 45

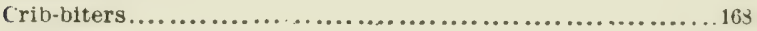

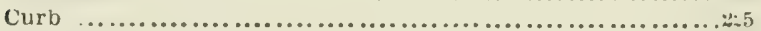

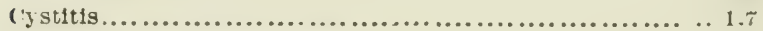

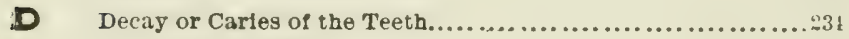

Dlaphragm, Spasm of.............................. 75

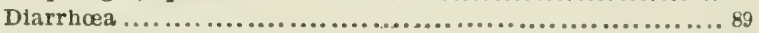

Diabetes, Insipldus....................................116

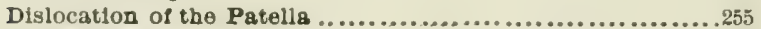

Disinfection........................................ 30

Vistemper or Strangles............................. 41 


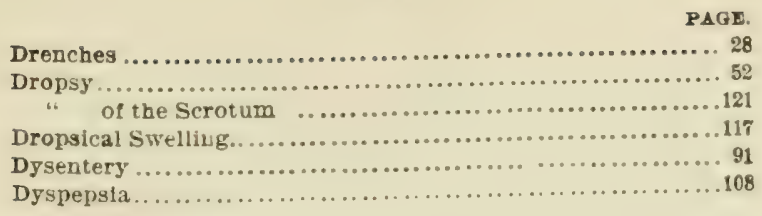

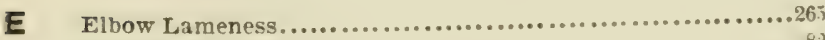

Enteritis

Enteritis-Muco ....................................... 84

Epllepsy............................................... 139

Eplstaxis.... ........................................... 53

Epizootic........................................... 43

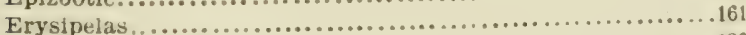

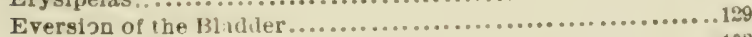

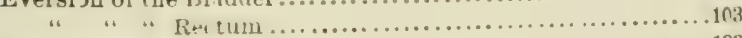

Eyeg, Infl immation of ..................................133

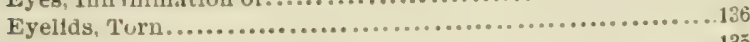

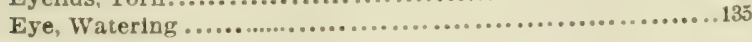

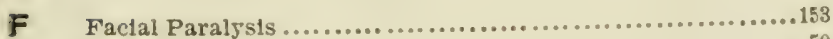

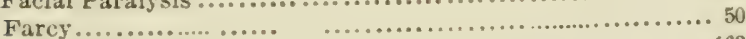

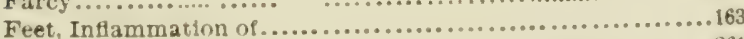

Fetlock Joint, Sprain of......... . . ...................281

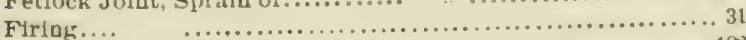

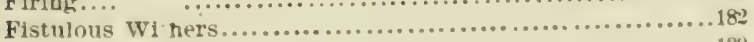

Fits, Falling

Flatulent Colic. ................................... 49

Flexor Tendons, Spraln of .............................. 26

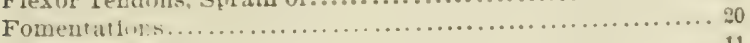

Foods aud Freding . ................................. 11

Foot, Grivel iu.

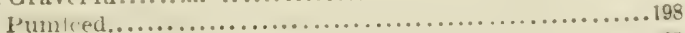

Foul She

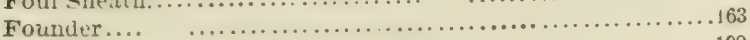

Frog, Bruises of .......................................... 199

G Galls, Saddle or Collar................................ 154

Gastritis.

Glanders .........................................

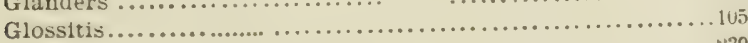

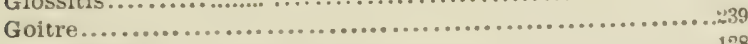

Gonorrbur

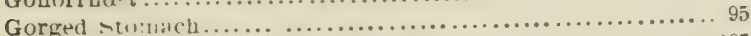

Gravel in Funt..................... . . ................ 197

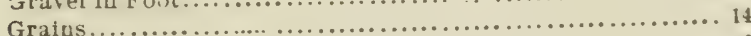

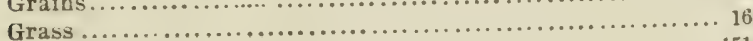

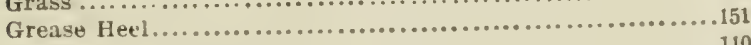

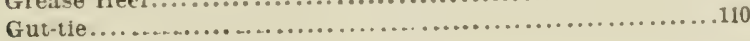


Homorrhagia Purpura........................................106

Homaturea .............................................119

Hæmorrhoids ...........................................240

Harness................................................ 17

Hay............................................ 12

Heaves............................................... 76

Heels, Contracted......................................202

Hemipleg1a.......................................... 97

Hepatitis ................................................. 101

Hernia.............................................. 188

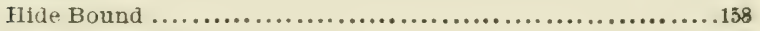

Hiud Limbs, Faralysis of. .................................. 102

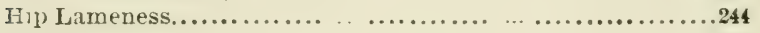

Hock. Capped........................................2.

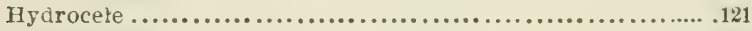

Hy ùrophobia........................................241

Hysteria............................................

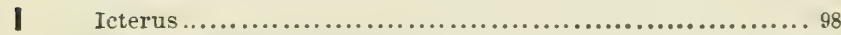

Incised and Contused Wounds...........................231

Indigest $t$ n. ...................................... 95

Inflammation of Bladder............................... 127

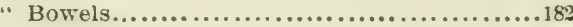

" Brain ....................................144

" Coronet....................................209

" Eyes.......................................133

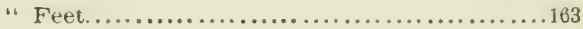

". Joints.............................

" Kidueys ............................... 12t

" Fuees.................................266

"Liver ...................................101

" Lining Membrane of the Abdominal Cavity..111

"Lungs ... .......................... 63

" Lymphaties. ......................... 77

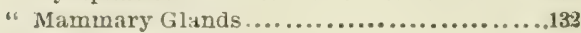

" Membrane and Spinal (n)

" Mouth ............................... $7 \theta$

"Stomach.............................. 92

" Testicles................................. 130

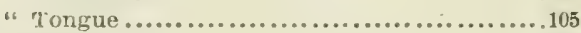

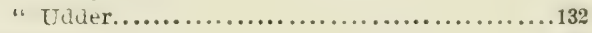

Influenza, Epizootic................................ 43

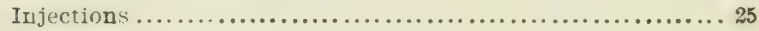

Intestinal Obstructions.................................... 87

Introductory........................................ 9

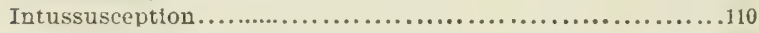

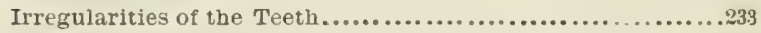

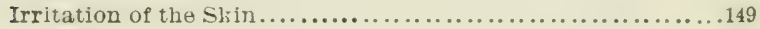

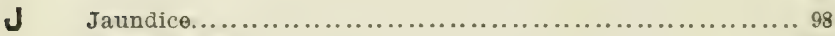

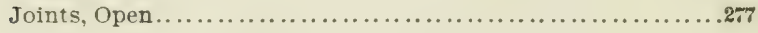


PAGE.

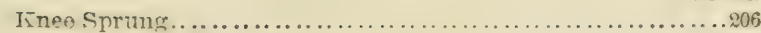

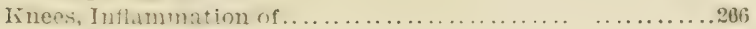

" Jroken or Bruised..............................267

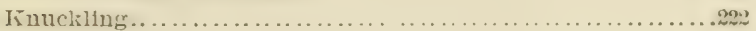

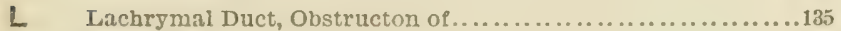

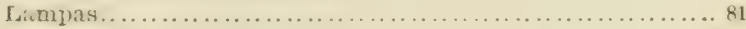

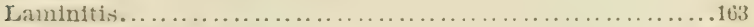

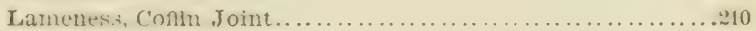

" Ellow

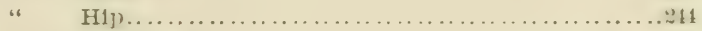

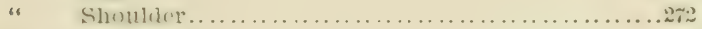

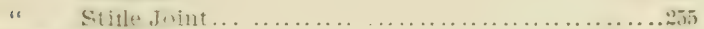

Laryngo Pharymeitis... . . . . . . . . . . . . . . .

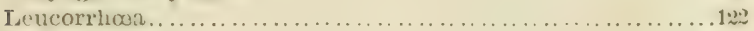

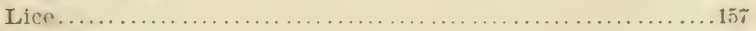

Linsved . . . . . . . . . . . . . . . . . . . . . . . . . . 15

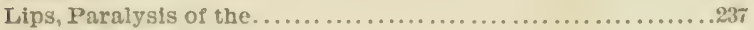

Liver, congerition of ................................

" Inflammation of ...................................

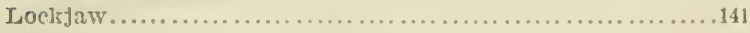

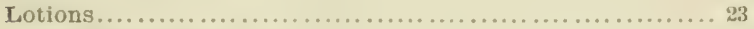

Lungs, Inflammation of .......................... 63

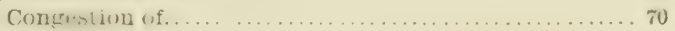

Lymphangitis........................................

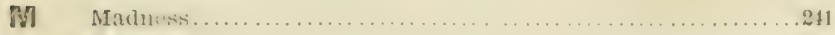

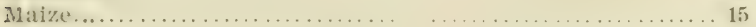

Mammary Glands, Infiammatiou of ......................

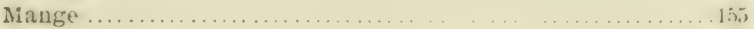

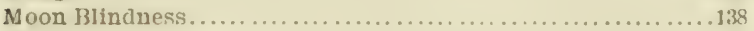

Mouth, Inflammation $(1, \ldots \ldots \ldots \ldots \ldots \ldots \ldots \ldots \ldots \ldots \ldots \ldots \ldots \ldots$

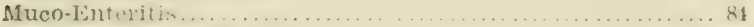

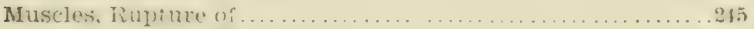

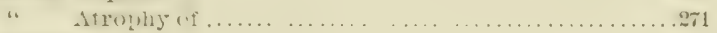

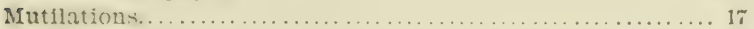

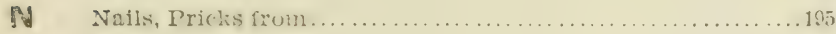

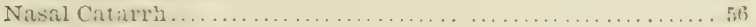

* Glect.....................................

" Polyj

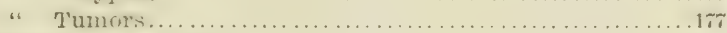

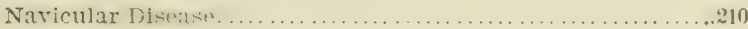

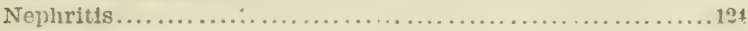

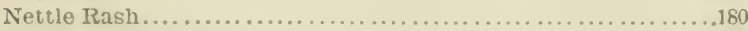

Nostrils, Blectine from tist . . . . . . . . . . . . . . . . 53

Wmt!? . .

Nostril, False, Tumol in . . . . . . . . . . . . . . . . . . .

O Obstructions, Intestinal............................ $8 \gamma^{r}$

Obstruction of the Lachrymal Duct........................ 135

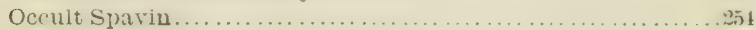

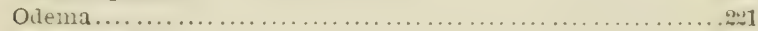

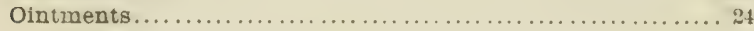


PAGE.

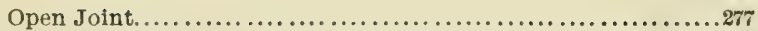

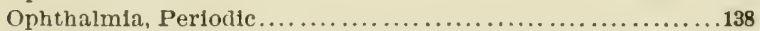

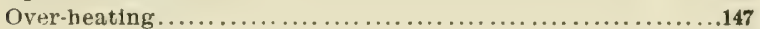

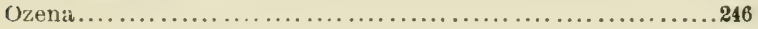

Partial Paralysts................................ 54

Paralysis...................................... 97

" of the Bladder............................... 120

" Facial .................................... 153

" of the Hind Limbs............................ 102

"6 "Lips.....................................237

" "Nerve of Sight ........................... 134

Parrot Mouth........................................235

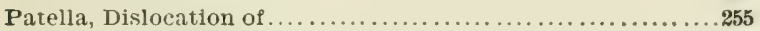

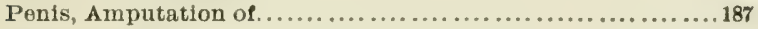

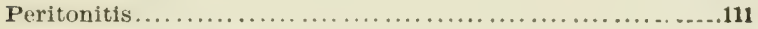

Periodic Ophthalmia ................................... 138

Pharyngeal Polypus.................................. 178

Phrenitis . . . . . . . . . . . . . . . . . . . . . . . . . . . . . 144

Phymosis and Paraphymosis............................ 186

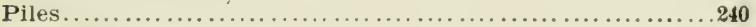

Plasters. . . . . . . . . . . . . . . . . . . . . . . . . . . . . . 23

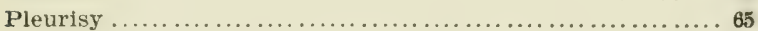

Pneumonia..................................... 63

Poll wvil ............................................ 169

Polypus, Nasal......................................177

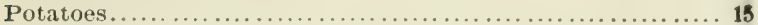

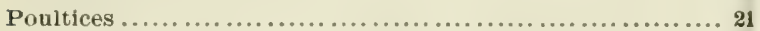

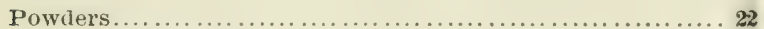

Pricks from Nails................................. 195

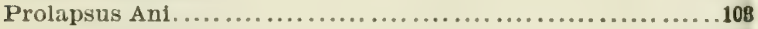

Prurigo-Pruritus ..................................... 149

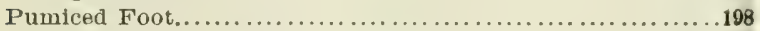

Purpura Hremorrhagia................................ 106

Q Quinsy...................................... 74

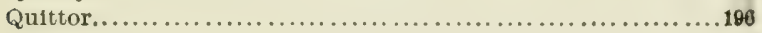

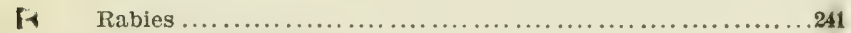

Rectum, Eversion of .......................................

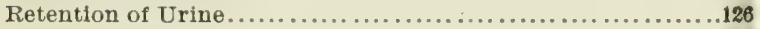

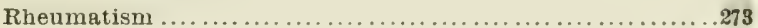

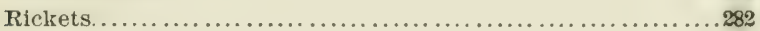

Ringworm ....................................

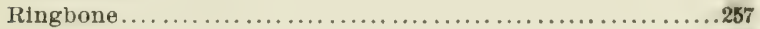

Roaring, Chronic ........................ 5 \%

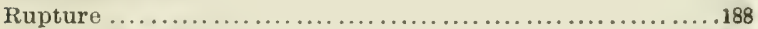

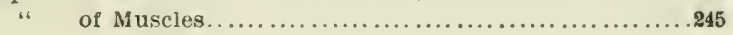

S Saddle or Collar Galls...................................154

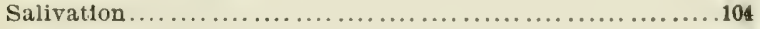

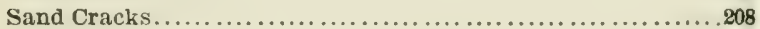

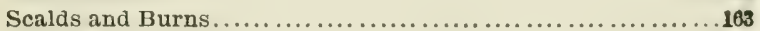

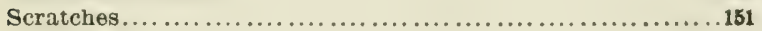




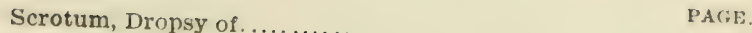

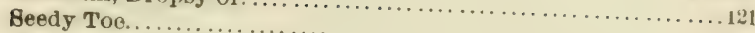

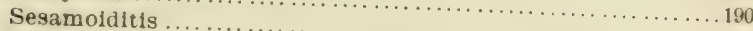

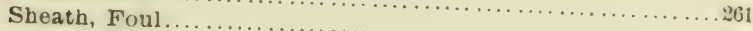

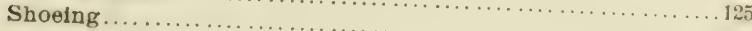

Shoulder Lameness . . . . . . . . . . . . . . . . . . . . . 31

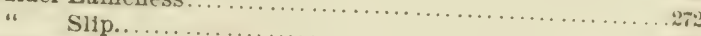

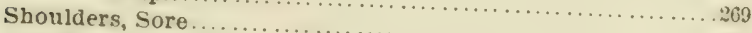

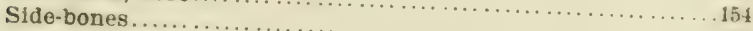

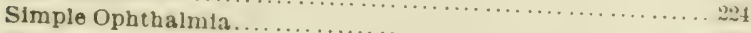

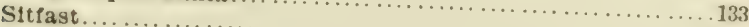

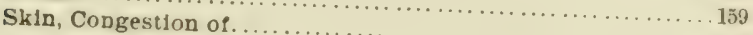

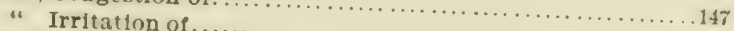

Slings.....

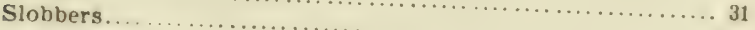

Sore Shins... . . . . . . . . . . . . . 104

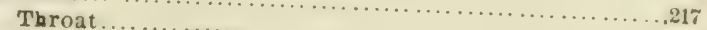

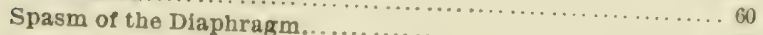

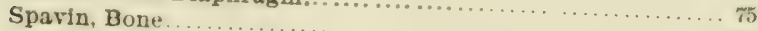

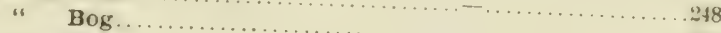

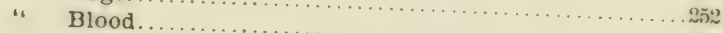

" Occult . . . . . . .

Spinal Meningitís . . . . . . . . . . . . . . . . . . . . . . . .

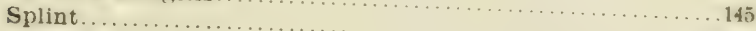

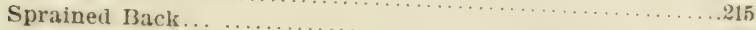

Sprain of the Fetlock Joint . . . . . . . . . . . . . . . . . . .

" " $"$ " Flexor Tendons............................ 261

Sprung In

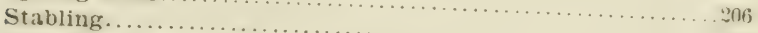

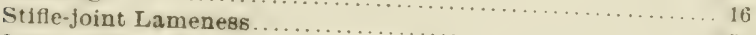

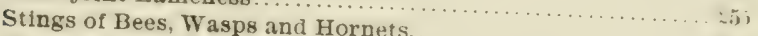

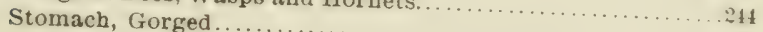

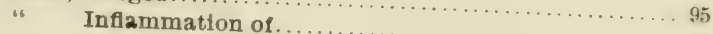

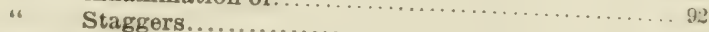

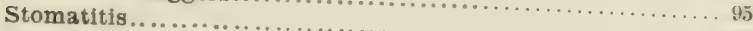

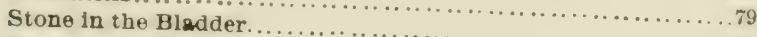

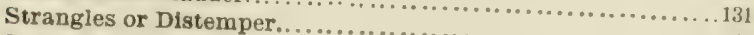

Straw. ...

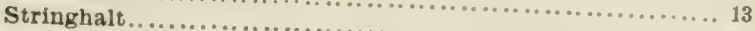

Stricture of the Urethra. . . . . . . . . . . . . . . . . . . . . . 279

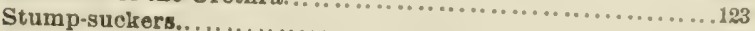

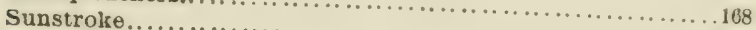

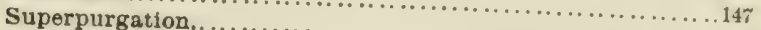

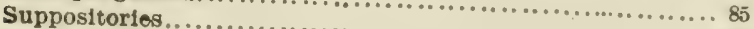

Surfeit. ............................................ 27

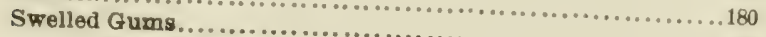

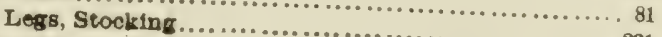

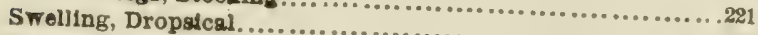

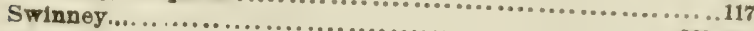

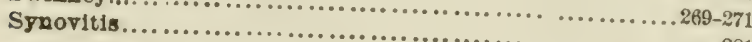


PAGE.

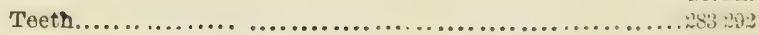

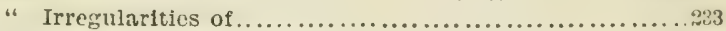

" Wolf or Supernumerary........................ 206

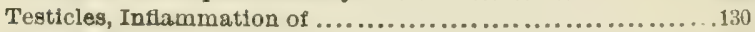

Totanus............................................... 141

Thorough Pin ........................................

Thrush.....................................

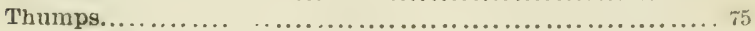

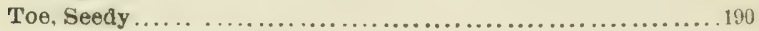

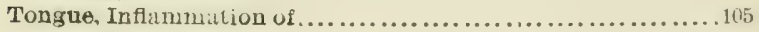

Injuries.................................. 80

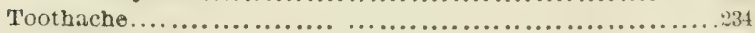

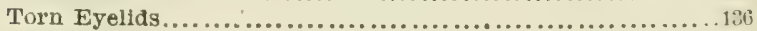

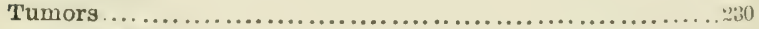

" Nasal ...................................

" In False Nostr1l................................ 17

" "the Throat...............................

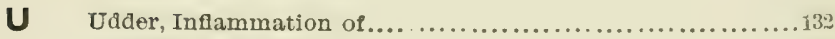

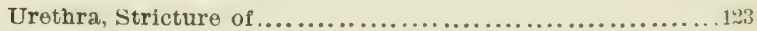

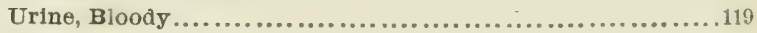

Urine, Retention of...................................

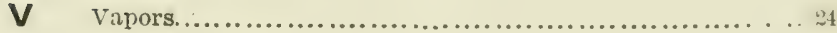

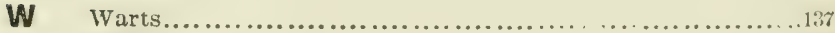

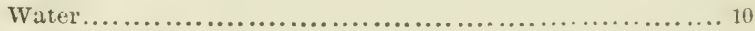

Watering Eye..........................................

Wheat and Rye...................................... 11

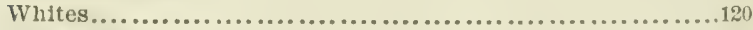

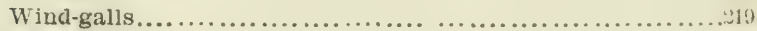

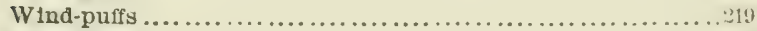

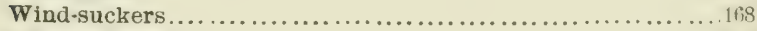

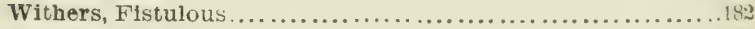

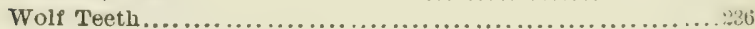

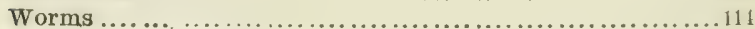

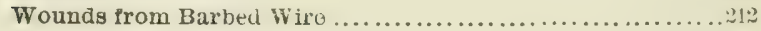

" Calk.....................................201

" Incised and Contused.................................

" at the Joints................................ 277

" About the Nostrils................................ 179

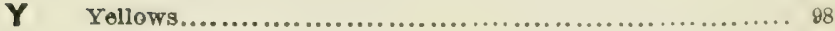

\section{CATTLE DEPARTMENT.}

A Affections of the Second Stomach......................... 995

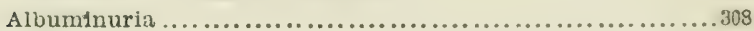
Apoplexy, Splonic .......................................... . 358

Aptha.....................................................205 


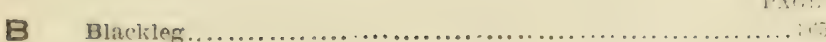

Bladder, Eversion of....................................

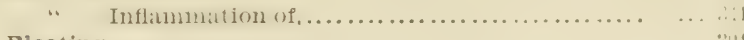

Bloating............................................

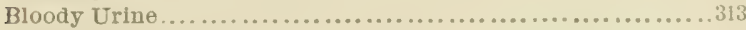

Bowels, Inflammation of ................................. 321

Brain, Inflammation of ..................................... 314

Bright's Disease...................................... 308

Buckeyes, Fits'from Eatiog......................................

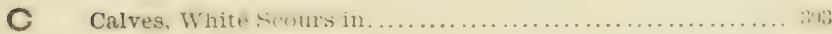

Cancerous Llews ..................................

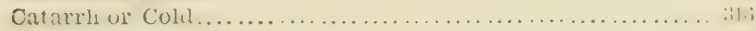

Cholking.............................................. 318

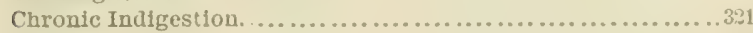

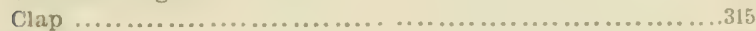

Colic................................................

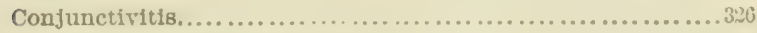

Contaglous Pleuro-Pneumonia ............................ 307

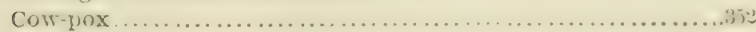

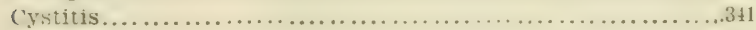

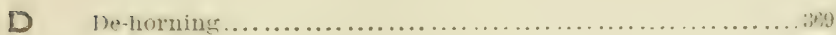

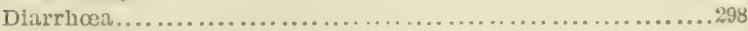

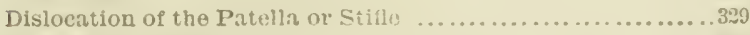

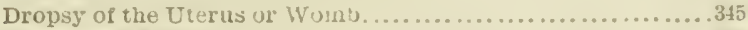

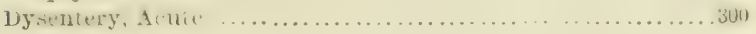

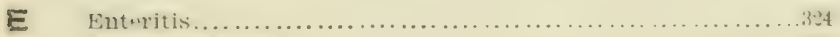

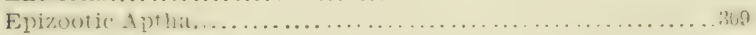

Eversion of Vagina ....................................... 344

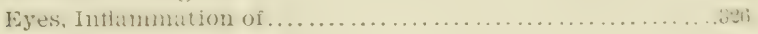

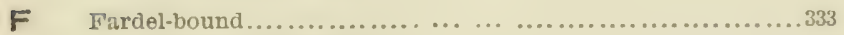

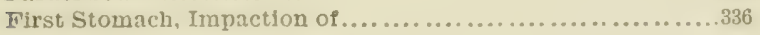

Fits from Eating Junclieyes............................ 331

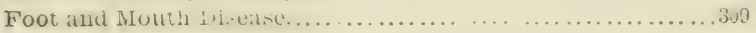

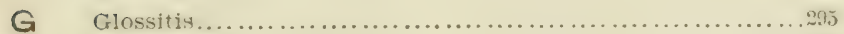

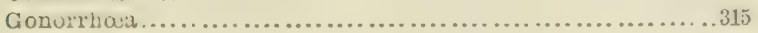

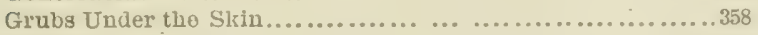

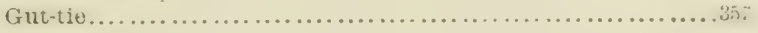

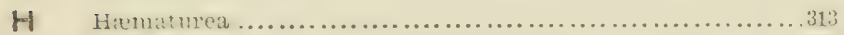

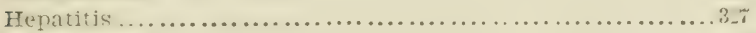

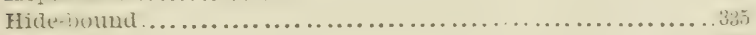

Hoven............................................

I Impaction of the Omasum................................... 333

.. $\quad$. .. Rumen................................

Indigestion, Chronic....................................881 
Inflammation of the Bladder..........................8.

" " " Bowels........................................

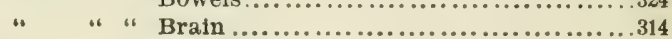

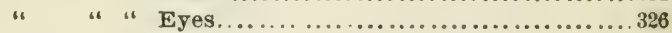

" " " Kidneys,.................................... " "

" " " Liver.............................827

" " " " " "

" " " "Tongue...........................295

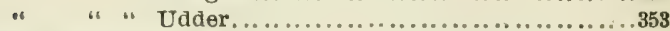

Intestines, Strangulation of........................ 357

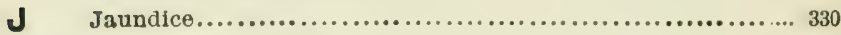

K Kidneys, Inflammation of...................................

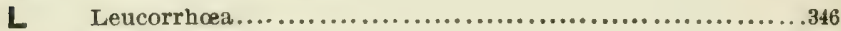

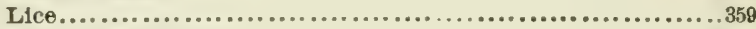

Liver, Inflammation of .....................................

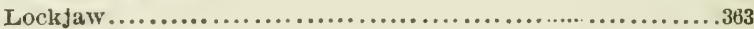

Lump-Jaw..............................................

Langs, Inflammation of ..................................... 339

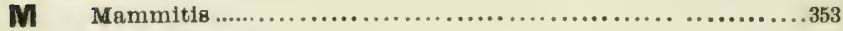

Mouth, Sore............................................295

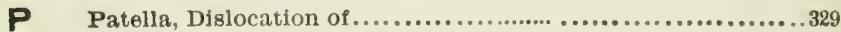

Phrenitis ..............................................

Pleurisy........................................... 347

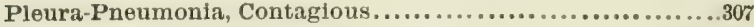

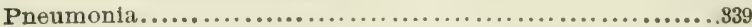

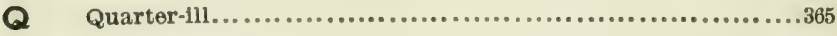

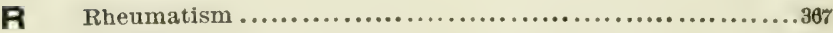

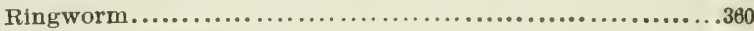

Rumen, Impaction of...................................

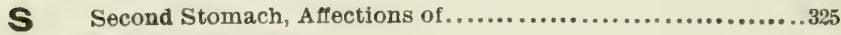

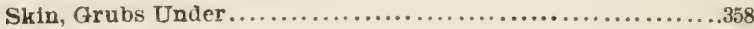

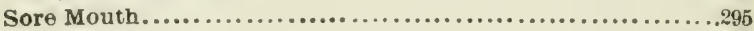

" Teats..............................................

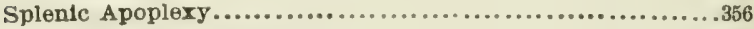

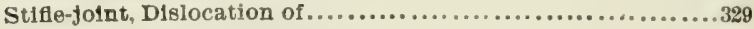

Strangulation of the Intestines................................

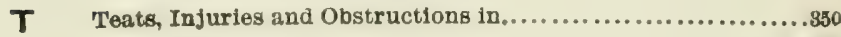

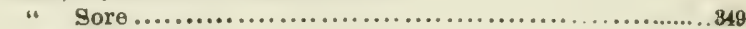

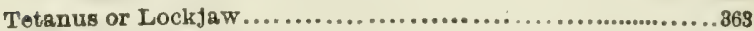

Tongue, Infiammation of ...............................295

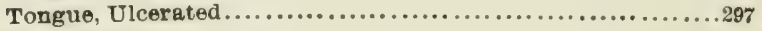

Tympanitis................................................

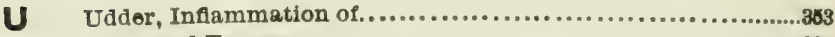

Ulcerated Tongue............................................

Ulcarts, Cancerouk....................................

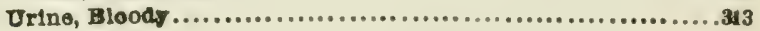


PAGE.

V Vagina, Eversion of ..................................314

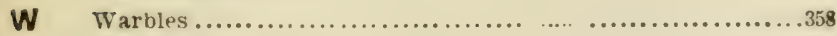

Warts.............................................................

White Scours in Calvets....................................303

Whites............................................. 316

Womb, Dropsy of.......................................345

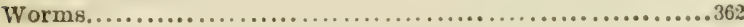

\section{OBSTETRICAL DEPARTMENT.}

A Abdominal and Breast Presentation.......................458

A bortion .................................................

Acites or Abdominal Dropsy...................................480

After-birth, Retention of..................................399

After-patns ..........................................

Anterlor Presentation..................................417

Apoplexy, Parturlent..................................... 388

B Barren Horses and Cattle .................................371

Bleeding from the Navel.................................408

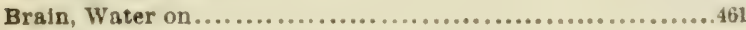

Breast and Abdominal Presentation .....................459

C Care and Feeding of Pregnant Anlmals...................... 383

Colts, Dlarrboea in......................................418

Constipation shortly After Birth..........................

Croup and Thigh Presentation ..........................445

D Deviation of Head Upward and Backward ..................433

Dlarrhoe in Colts...........................................413

Double-headed Monstrosity .........................458

Downward Deriation of the Head ........................427

Dropsy, Abdominal.................................480

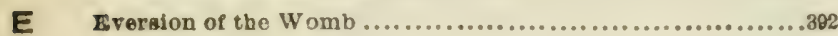

F Feeding and Care of Pregnant Animals.......................385

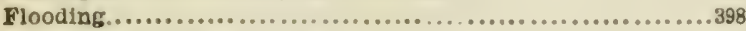

Footus, Twin, in Different Presentations ........................458

"Presentation and Position of......................116

Forelimbs Completely Retained............................4\&4

" Flexed at the Knees............................421

Forelimb Crossed Over the Neck........................418

H Fead, Downward Deviation of.........................487

" Deviation of, Upward and Backward.................483

" Lateral Deviation of, to the Right or Left................428

Hernia of the Uterus or TVomb.............................401

Hind-leg Deviation, Anterior Presentation....................434

Hind-legs, Deviation of, Into Genital Canal, Anterior Part of

the Body Presenting...................................436

Hock Presentation.....................................442

Hydrocephalus, Water on the Braln......................460 
PAGP.

Inflammation of the Peritoneum.......................... 406

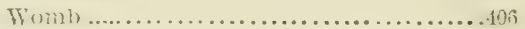

L Labor Pains, Premature......................................

Lateral Deviation of the Head to tho Right or Left...........429

Loin and shomb?r. Presentiation.........................44d

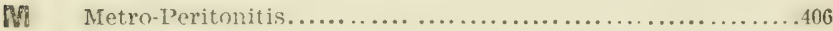

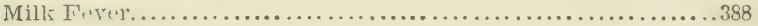

Monstrosity, Double-headed.............................458

Ny Navel, Flpeding from the......................... 408

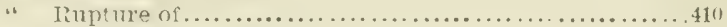

"Urine Passing from the........................409

Normal Parturition, Necessary Aid in......................

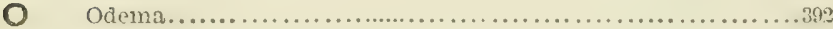

P Parturition, Its Symptoms, etc........................... 88?

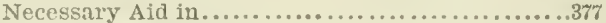

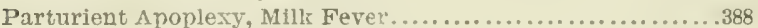

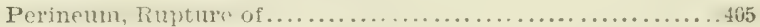

Persistence of the Irachus ...........................40 40

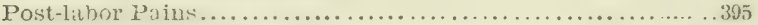

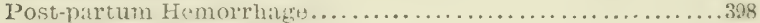

Posteriol Freseritition . . . . . . . . . . . . . . . . . . . . . .43s

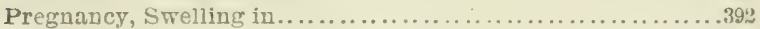

Pregnant Animals, Care and Feediny of ....................... 385

Premature Labor Pains...................................

Presentation and Postion of the Frotus...................416

Retention of the Meconium ............................. 411

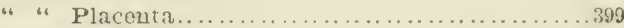

Rupture of the Navel...........................410

Perincum..............................40,

3 Shoulder and Loin Presentation.........................4. 40

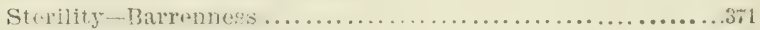

Swelling in Pregnancy............ .......................392

T Thigh and Croup Presentation.............................445

Transterse ['resentati.1. ............................ 18

Twin Fotus in Different Presentations.....................45t

U Umbilical Hemorrhase................................. 408

" Hernia ...................................410

Urine Passing from the Navel............................408

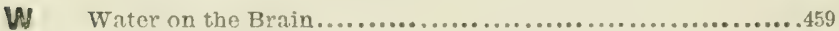

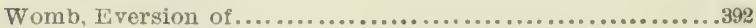

" Hernia op....................................401

" Iuflammation of...................................408 


\section{SHEEP DEPARTMENT.}

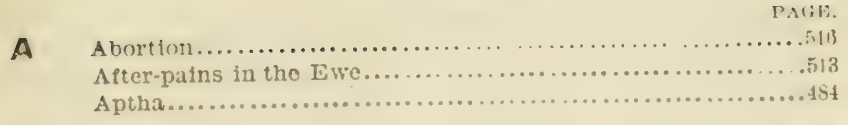

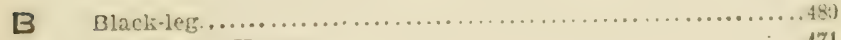

Bloutlug or Inoven.......... ...................... 471

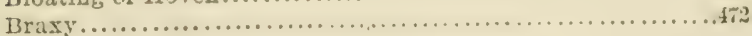

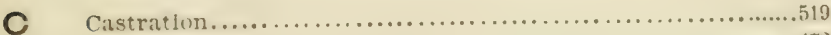

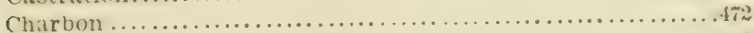

Clap or Gomorrhooil................................... 509

Cold or Citturh........................................... 465

Concretions in the Stomach ...............................4t

Coustipation ...........................................

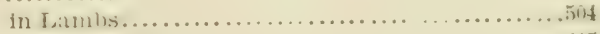

Congestion of the Liver................................475

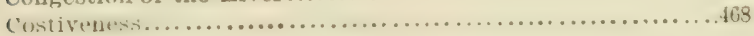

D Diarrhcon or Scours in Lambs...........................469

" "White Scours in Limbs.......................504

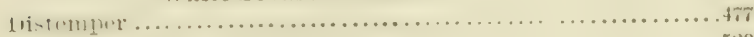

Docking or Cutting Off the Tail.........................5:0

Dysentery ...........................................

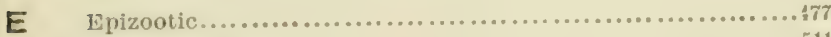

Ewe, Lambing in, .................................... . . . .

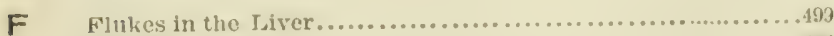

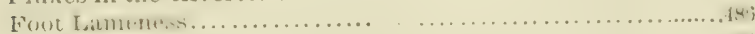

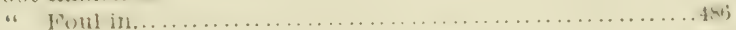

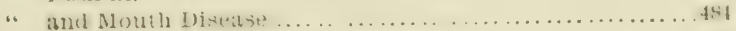

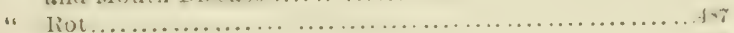

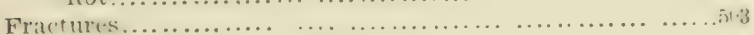

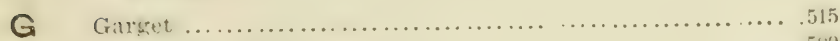

Gonorrhoea or Clapi...................................5uy

Gravel in Foot......................................... 486

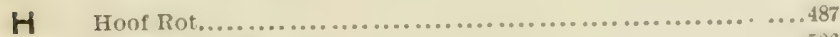

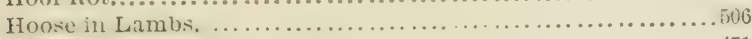

Hoven or Bloating..................................471

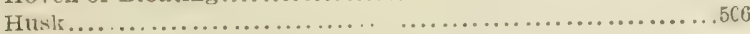

Inflammation of the Liver..............................

" $\quad$ ". $\quad$ Lungs........................... 460

" $\quad$ " Udder............................. 515

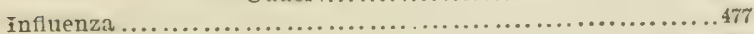

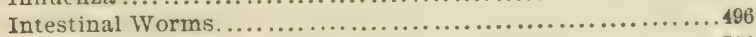

Inversion of the Womb................................

Itoh or Mango....................................4 400 
L Lambing in the Ewe......................................

Lambs, Constipation in..............................504

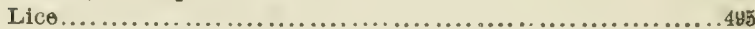

Liver, Congestion of ................................. 475

" Inflammation of ...............................476

Liver-rot. ......................................... 499

Lockjaw...............................................

Lung Fever........................................460

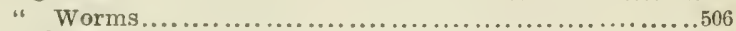

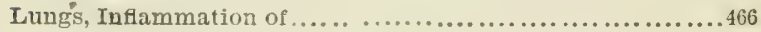

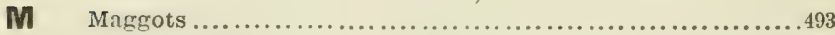

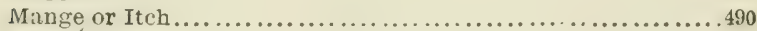

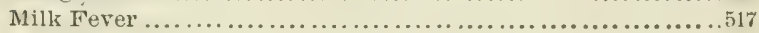

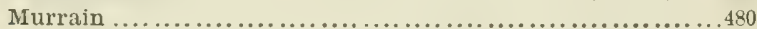

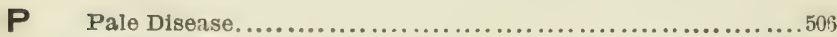

Pneumonia ..................................... 466

Parturtent Apoplexy ...................................... 513

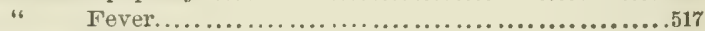

Puerperal Fever....................................... 517

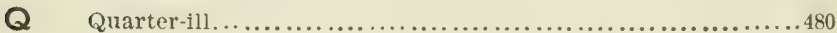

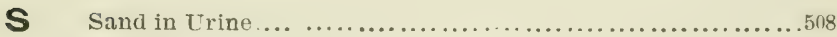

Scab ......................................... 490

Scours in Lambs, or Diarlhera ........................449

Sediment in the Urive................................. 508

Sherptick ... ....................................

Slippiug of Lambs ............................ 516

Small-pox ............................................ 481

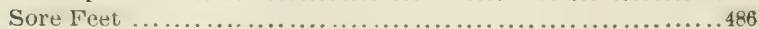

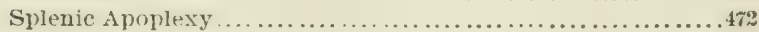

Stomach, Foreign Substances in.........................474

Stretches ........................................ 468

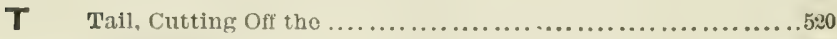

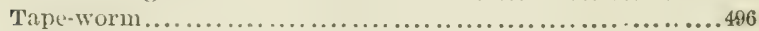

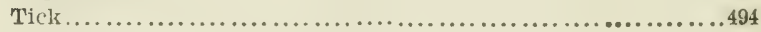

U Udder, Inflammation of .............................. 513

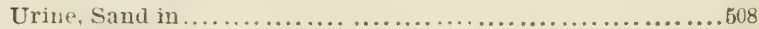

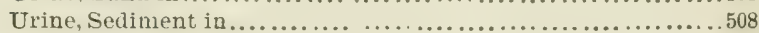

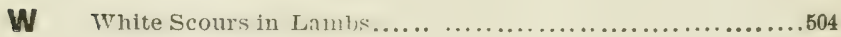

Womb, Inverslon of.................................514 


\section{HOG DEPARTMENT.}

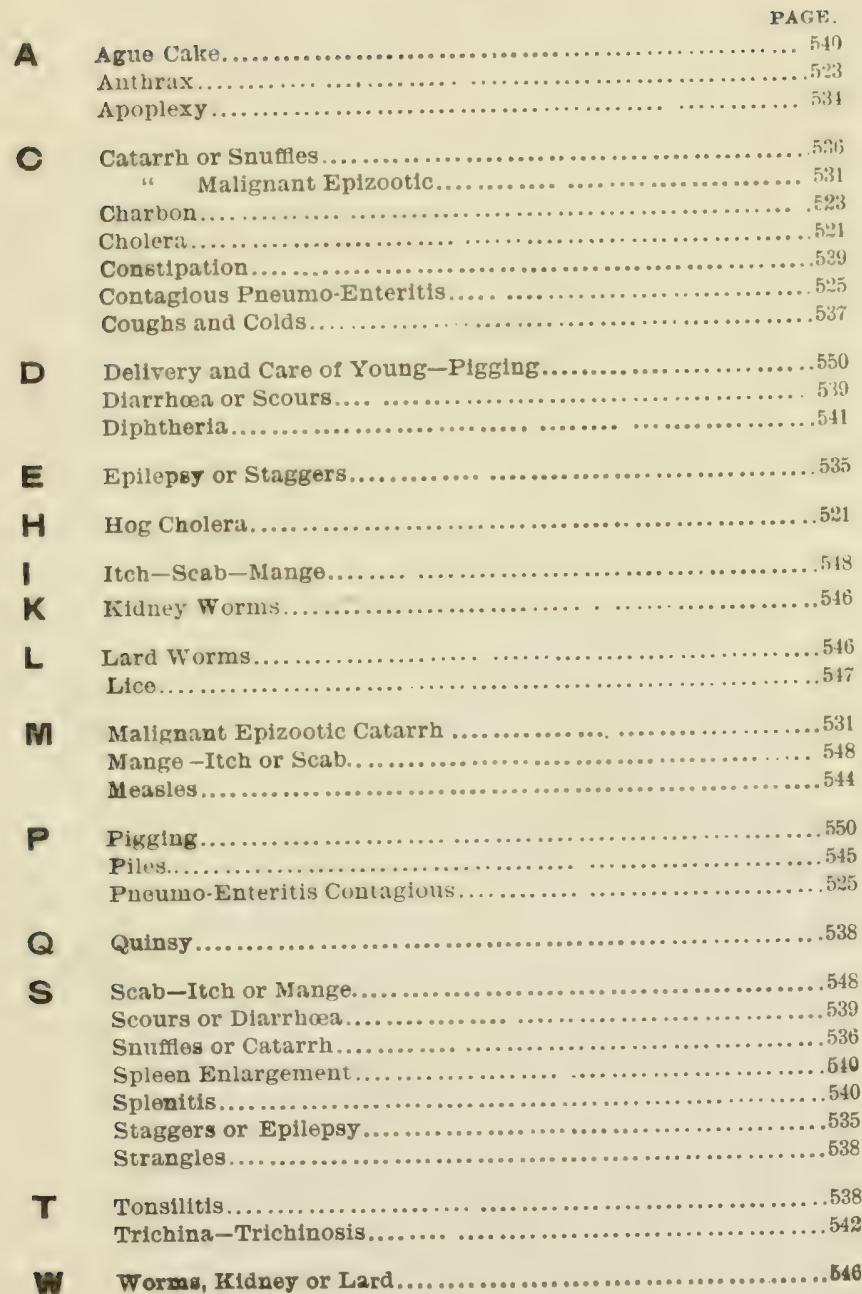




\section{DOG DEPARTMENT.}

PAGE.

B

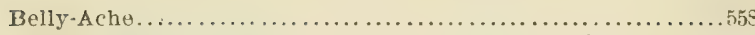

Bowels. Inflammation of ........................... 559

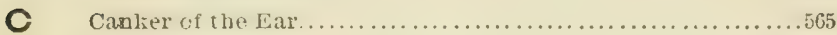

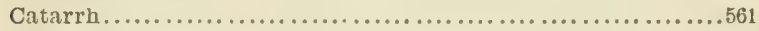

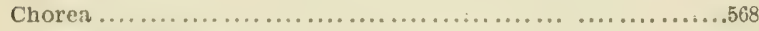

Clap or Gonorihue.................................

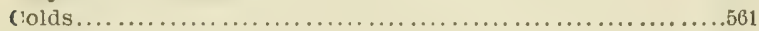

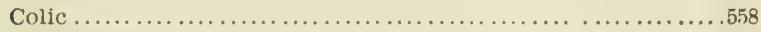

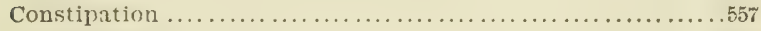

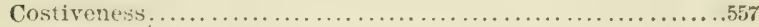

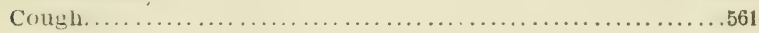

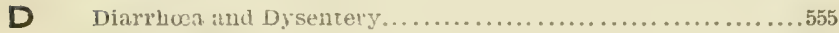

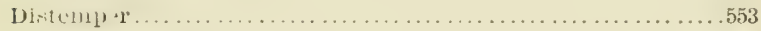

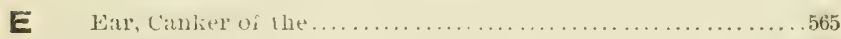

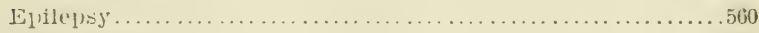

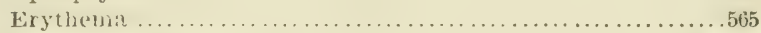

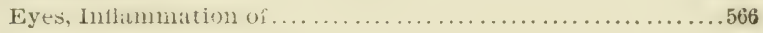

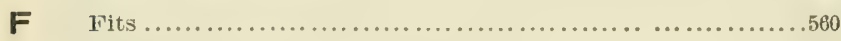

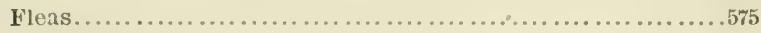

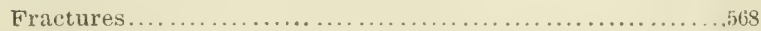

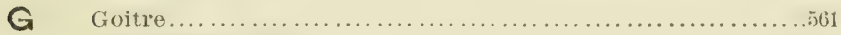

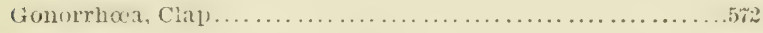

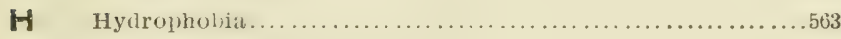

I Inflammation of the Bowels .......................... 559

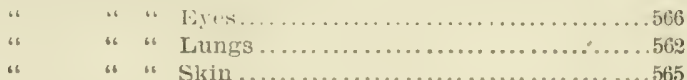

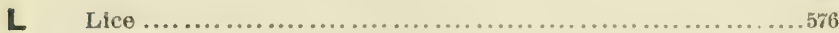

Lung Fever.............................

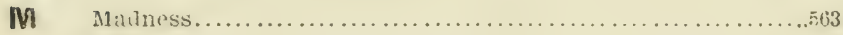

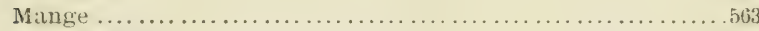

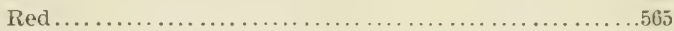

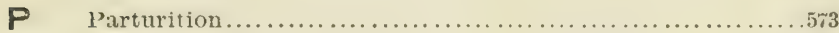

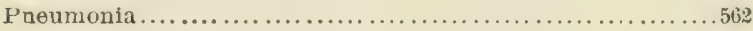

Pupping.....................................................

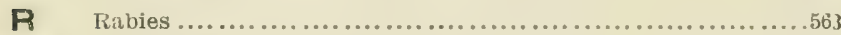

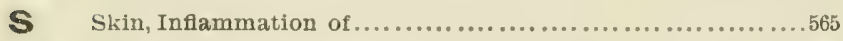

T Tumors About the Eyes............................... 567

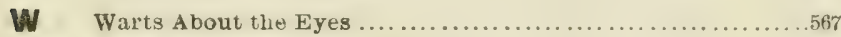

Worms in the Intestines..............................569

" " " Killn: y+ ........................ 572

Wounds and Fractures.................................568 






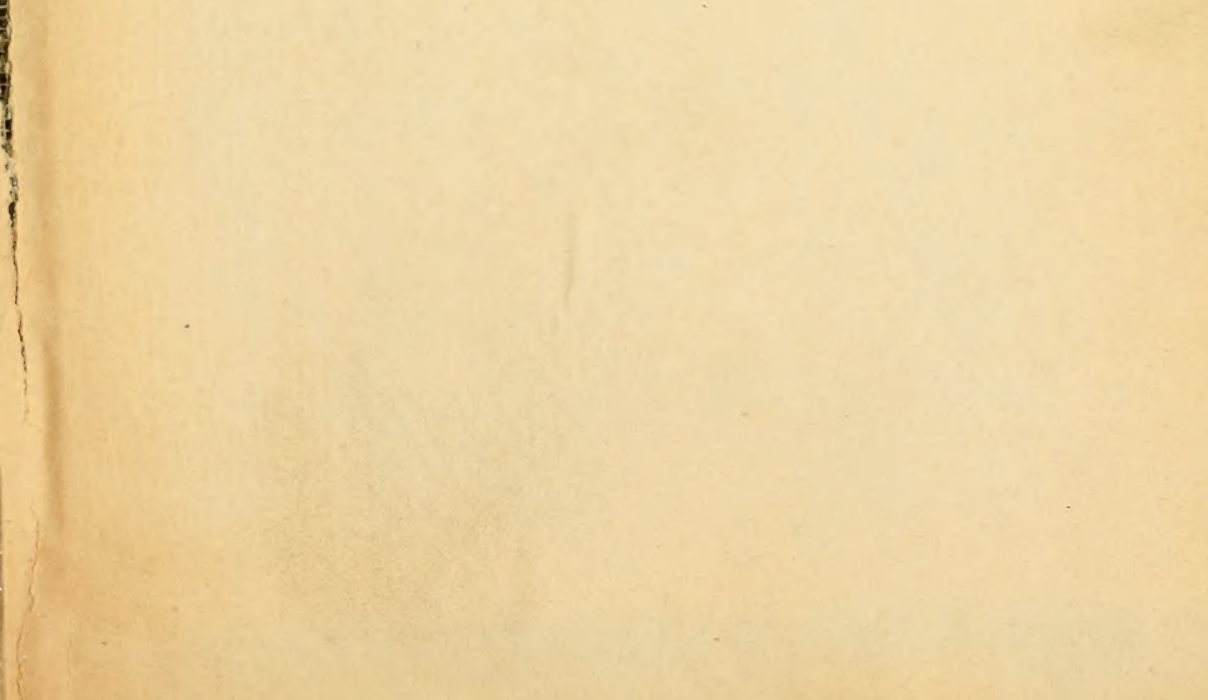




\section{LIBRARY OF CONGRESS

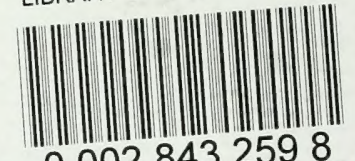 00028432598}

